Supporting Information

\title{
Alkene-chelated Ruthenium Alkylidenes - A Missing Link to New Catalysts
}

Saswata Gupta ${ }^{\ddagger}$, Venkata R. Sabbasani ${ }^{\ddagger}$, Siyuan Su, Donald J. Wink and Daesung Lee* Department of Chemistry, University of Illinois at Chicago, 845 W. Taylor St., Chicago, Illinois 60607 (USA)

*E-mail: dsunglee@uic.edu (D.L.)

\section{Table of Contents}

Materials and Methods s2

Experimental Details. s2

Characterization Data for Alkene-Chelates. s19

Catalytic activity: Structure-Activity studies. s30

i. Kinetic profile for different $\eta^{3}$-vinyl chelates s31

ii. Catalytic activity of oxygen chelates $\mathbf{1 3} \mathbf{p}^{\prime}$ and $\mathbf{1 3} \mathbf{q}^{\prime}$...... s37

iii. Kinetic profile for latent trans-1,5-chelates. s40

iv. High temperature NMR study of complex $\mathbf{1 0 I}$ s45

Benchmarking catalytic activity

i. RCM of dimethyl diallylmalonate s47

ii. RCM of dimethyl allymethallylmalonate. s60

iii. CM of 5-hexynyl acetate and methyl acrylate. s71

iv. ROMP of 1,5-cyclooctadiene. s75

NMR Spectra of Starting Materials. s91

NMR Spectra of Alkene Chelates. s131

X-Ray Crystallographic Data. s174

References. s187 


\section{Materials and Methods}

All Reactions were carried out in oven-dried glassware under inert atmosphere unless otherwise noted. All organometallic reactions were performed under argon using glovebox and standard Schlenk techniques. All reagents and compounds were purchased from Sigma-Aldrich, Acros, TCl America or Oakwood and used as received, unless otherwise noted. Grubbs $2^{\text {nd }}$ generation catalyst was purchased from Aldrich and CAPOT Chemicals (Sanghai, China) and used without any further purification. Toluene, dichloromethane, triethylamine, and acetonitrile were distilled over calcium hydride $\left(\mathrm{CaH}_{2}\right)$ under nitrogen. Tetrahydrofuran was dried over sodium benzophenone ketyl. Column chromatography was performed using silica gel $60 \AA$ (32-63 mesh) purchased from Silicycle Inc. Analytical thin layer chromatography (TLC) was performed on $0.25 \mathrm{~mm}$ E. Merck precoated silica gel 60 (particle size 0.040$0.063 \mathrm{~mm}$ ). Yields refer to chromatographically and spectroscopically pure isolated compounds unless otherwise stated. ${ }^{1} \mathrm{H}$ NMR and ${ }^{13} \mathrm{C}$ NMR spectra were recorded on a Bruker AV-500 spectrometer. ${ }^{1} \mathrm{H}$ NMR chemical shifts $(\delta)$ are reported in parts per million (ppm) downfield of TMS and are referenced relative to the residual solvent peak $\left(\mathrm{CDCl}_{3}(7.26 \mathrm{ppm})\right) .{ }^{13} \mathrm{C}$ chemical shifts $(\delta)$ are reported in parts per million downfield of TMS and are referenced to the carbon resonance of the solvent $\left(\mathrm{CDCl}_{3}(77.02 \mathrm{ppm})\right.$ ). Multiplicities are indicated by $s$ (singlet), d (doublet), $t$ (triplet), q (quartet), $p$ (quintet), $h$ (sextet), dd (doublet of doublet), dt (doublet of triplet), $\mathrm{m}$ (multiplet) and so on. ${ }^{1} \mathrm{H}$ NMR signals that fall within a ca. $0.3 \mathrm{ppm}$ range are generally reported as a multiplet, with a range of chemical shift values corresponding to the peak or center of the peak. Coupling constants, J, are reported in $\mathrm{Hz}$ (Hertz). Electrospray ionization (ESI) and Electron impact (EI) mass spectra were recorded on a Waters Micromass Q-Tof Ultima and Micromass 70-VSE, respectively in the University of Illinois at Urbana-Champaign.

\section{Experimental Details}

\section{General Procedure 1: Alkylation of nitriles ${ }^{1}$}

A round bottom flask was charged with $60 \mathrm{~mL}$ of anhydrous THF and dry diisopropylamine $(6.35 \mathrm{~mL}, 45$ $\mathrm{mmol}$ ) under argon and cooled to $-78^{\circ} \mathrm{C} . n$-BuLi $(16.8 \mathrm{~mL}, 42 \mathrm{mmol}$ ) solution ( $2.5 \mathrm{M}$ in hexanes) was added dropwise and stirred for $30 \mathrm{~min}$. Isobutyronitrile $(2.7 \mathrm{~mL}, 30 \mathrm{mmol})$ in $10 \mathrm{~mL}$ THF was added to the reaction to form a yellow solution. The mixture was stirred for $45 \mathrm{~min}$ at $-78{ }^{\circ} \mathrm{C}$ followed by addition of the alkyl halide $(20 \mathrm{mmol})$. The reaction was stirred at $-78{ }^{\circ} \mathrm{C}$ for $1 \mathrm{~h}$, warmed up to room temperature and stirred for $14 \mathrm{~h}$. The reaction was quenched with $1 \mathrm{M} \mathrm{HCl}(50 \mathrm{~mL})$ and extracted with EtOAc $(50 \mathrm{~mL} \times 2)$. The combined organic layer was washed with sat. $\mathrm{NaHCO}_{3}(50 \mathrm{~mL})$ and brine $(50 \mathrm{~mL})$, dried over dried over anhydrous $\mathrm{Na}_{2} \mathrm{SO}_{4}$, filtered, concentrated and purified by flash chromatography $\left(\mathrm{SiO}_{2}\right.$, hexanes-EtOAc, $10: 1$ ) to give the desired alkylated nitrile in $60-80 \%$ yield.

\section{General Procedure 2: Etherification reaction}

The alcohol ( $1.0 \mathrm{mmol}$ ) was dissolved in $5 \mathrm{~mL}$ anhydrous DMF and cooled to $0{ }^{\circ} \mathrm{C}$. $\mathrm{NaH}(60 \%$ suspension in mineral oil) (44 mg, $1.1 \mathrm{mmol}$ ) was added to the reaction mixture carefully and stirred for about $15 \mathrm{~min}$ followed by addition of the bromide or iodide $(1.1 \mathrm{mmol})$. The reaction was warmed to room temperature and stirred until completion (as monitored by TLC). The reaction was quenched with water $(2 \mathrm{~mL}$ ) diluted 
with EtOAc and the organic layer was washed with water $10 \mathrm{~mL}(\mathrm{x} 2)$ and brine $10 \mathrm{~mL}(\mathrm{x} 2)$, dried over anhydrous $\mathrm{Na}_{2} \mathrm{SO}_{4}$, filtered and concentrated. The crude product was purified by column chromatography $\left(\mathrm{SiO}_{2}\right.$, hexanes-EtOAc, 20:1) to obtain the corresponding ethers as colorless to yellow oils in $60-80 \%$ yield. For etherification of alcohols having multiynes or other sensitive functional groups the alcohol and the alkyl halide were first premixed in DMF followed by addition of $\mathrm{NaH}$ to obtain higher yields.

\section{General Procedure 3: Mitsunobu reaction²}

A THF solution of the alcohol ( $1 \mathrm{mmol})$ was cooled to $0{ }^{\circ} \mathrm{C}$ followed by addition of $\mathrm{PPh}_{3}(524.6 \mathrm{mg}, 2 \mathrm{mmol})$, the sulfonamide $(2 \mathrm{mmol})$ and finally dropwise addition of diisopropyl azodicarboxylate $(0.34 \mathrm{~mL}, 1.7$ $\mathrm{mmol})$. The reaction mixture was warmed to room temperature and further stirred until completion (monitored by $\mathrm{TLC}$ ). Then, the reaction mixture was concentrated and loaded on a silica gel column to get pure product in $80-95 \%$ yield.

\section{General Procedure 4: Boc deprotection}

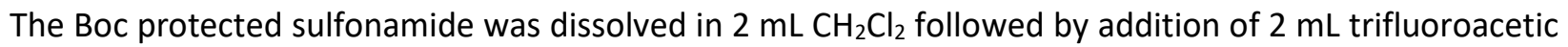
acid and stirred for $3 \mathrm{~h}$. The reaction was carefully quenched with satd. $\mathrm{NaHCO}_{3}$ soln. and extracted with DCM (10 $\mathrm{mL} \times 2)$. The combined organic layer was washed with water $(10 \mathrm{~mL})$ and brine $(10 \mathrm{~mL})$, dried over anhydrous $\mathrm{Na}_{2} \mathrm{SO}_{4}$, filtered, concentrated and purified by column chromatography $\left(\mathrm{SiO}_{2}\right.$, hexanesEtOAc, 5:1 $\rightarrow$ 3:1) to give the free sulfonamide as white solid in $75-90 \%$ yield.

\section{General Procedure 5: Copper catalyzed amidation of alkynyl bromide ${ }^{3}$}

To a solution of a tosylamide $(3 \mathrm{mmol})$ in toluene $(5 \mathrm{~mL})$ was added $\mathrm{CuSO}_{4} \cdot 5 \mathrm{H}_{2} \mathrm{O}(71.8 \mathrm{mg}, 0.45 \mathrm{mmol})$, phenanthroline $(162.2 \mathrm{mg}, 0.9 \mathrm{mmol}), \mathrm{K}_{2} \mathrm{CO}_{3}(912 \mathrm{mg}, 6.6 \mathrm{mmol})$ followed by the alkynyl bromide (5 $\mathrm{mmol}$ ) in toluene. The reaction mixture was heated at $65{ }^{\circ} \mathrm{C}$ for $16 \mathrm{~h}$. After completion, the reaction mixture was concentrated, filtered through a short pad of silica, and washed with EtOAc $(20 \mathrm{~mL})$. The crude mixture was then purified by column chromatography $\left(\mathrm{SiO}_{2}\right.$ hexanes-EtOAc, 10:1 $\left.\rightarrow 5: 1\right)$ to obtain the ynamide in $60-80 \%$ yield as a yellow oil.

\section{General Procedure 6: Sonogashira coupling ${ }^{4}$}

Aryl iodide (1 mmol) was dissolved in $5 \mathrm{~mL}$ anhydrous THF under argon atmosphere. $\mathrm{Pd}\left(\mathrm{PPh}_{3}\right)_{4}(23 \mathrm{mg}$, $0.02 \mathrm{mmol})$, the alkyne $(1.2 \mathrm{mmol})$ in $3 \mathrm{~mL} \mathrm{THF}, 2 \mathrm{~mL} \mathrm{Et}{ }_{3} \mathrm{~N}$ and Cul $(7.6 \mathrm{mg}, 0.04 \mathrm{mmol})$ were added sequentially and the mixture was stirred at room temperature for $12 \mathrm{~h}$. After completion, the reaction mixture was diluted with EtOAc $(20 \mathrm{~mL})$ followed by addition of satd. $\mathrm{NH}_{4} \mathrm{Cl}(10 \mathrm{~mL})$. The organic layer was extracted with $10 \mathrm{~mL} \mathrm{H}_{2} \mathrm{O}(\times 2)$ and $10 \mathrm{~mL}$ brine, dried over anhydrous $\mathrm{Na}_{2} \mathrm{SO}_{4}$, filtered and concentrated. The crude product was purified by column chromatography to obtain pure product in 65$75 \%$ yield. For bromides, the reactions were performed in a similar manner at $50{ }^{\circ} \mathrm{C}$. 


\section{Preparation of $6 a$}
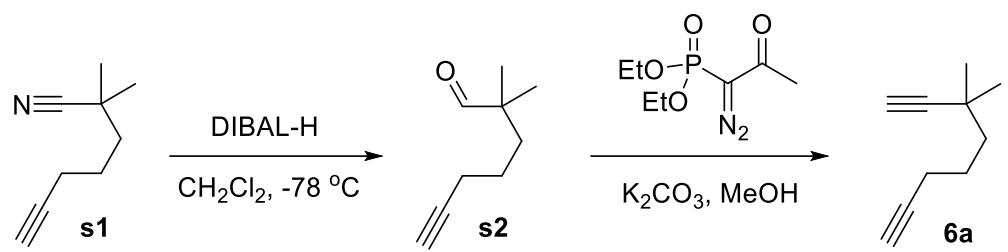

2,2-Dimethylhept-6-ynenitrile (prepared by following General Procedure 1), s1 (500 mg, 3.7 mmol) in dry

$\mathrm{CH}_{2} \mathrm{Cl}_{2}(15 \mathrm{~mL})$ was treated with DIBAL-H $(0.8 \mathrm{~mL}, 4.43 \mathrm{mmol})$ at $-78^{\circ} \mathrm{C}$. The reaction mixture was stirred at $-78{ }^{\circ} \mathrm{C}$ for $1 \mathrm{~h}$ and quenched with $\mathrm{MeOH}(1 \mathrm{~mL})$ followed by sat. potassium sodium tartrate solution (10 $\mathrm{mL}$ ) warmed to room temperature and stirred for $30 \mathrm{~min}$ until two clear layers were formed. The mixture was extracted with $\mathrm{CH}_{2} \mathrm{Cl}_{2}(10 \mathrm{~mL} \times 3)$, dried over anhydrous $\mathrm{MgSO}_{4}$, filtered, and concentrated under reduced pressure to provide the crude aldehyde s2. To a $\mathrm{MeOH}$ solution $(15 \mathrm{~mL})$ of aldehyde $\mathbf{s 2}(511 \mathrm{mg}$, $3.7 \mathrm{mmol}$ ) was added the Bestmann-Ohira reagent ${ }^{5}(1.2 \mathrm{~g}, 5.6 \mathrm{mmol})$ and $\mathrm{K}_{2} \mathrm{CO}_{3}(1.02 \mathrm{~g}, 7.4 \mathrm{mmol})$ at room temperature. The reaction mixture was stirred at the same temperature for $12 \mathrm{~h}$ and diluted with water $(10 \mathrm{~mL})$. The mixture was then extracted with pentane $(20 \mathrm{~mL} \times 3)$, dried over anhydrous $\mathrm{MgSO} 4$, filtered, and concentrated carefully under reduced pressure to provide the analytical pure 3,3dimethylocta-1,7-diyne, $6 \mathbf{6 a}\left(280 \mathrm{mg}, 56 \%\right.$ yield over 2 steps) as colorless oil. ${ }^{1} \mathbf{H} \mathbf{~ N M R}\left(500 \mathrm{MHz}, \mathrm{CDCl}_{3}\right) \delta$ $2.20(\mathrm{dt}, J=7.1,2.8 \mathrm{~Hz}, 2 \mathrm{H}), 2.06(\mathrm{~s}, 1 \mathrm{H}), 1.94(\mathrm{t}, J=2.6 \mathrm{~Hz}, 1 \mathrm{H}), 1.71-1.65(\mathrm{~m}, 2 \mathrm{H}), 1.50-1.46(\mathrm{~m}, 2 \mathrm{H}), 1.20$ (s, 6H); ${ }^{13} \mathrm{C} \mathrm{NMR}\left(125 \mathrm{MHz}, \mathrm{CDCl}_{3}\right) \delta 91.5,84.3,68.5,68.0,42.2,30.8,29.1,24.4,18.8$; HRMS (ESI) calcd for $\mathrm{C}_{10} \mathrm{H}_{13}[\mathrm{M}-\mathrm{H}]^{+} 133.1012$, found 133.1018.

\section{Preparation of $6 \mathrm{e}$ and $6 f$}

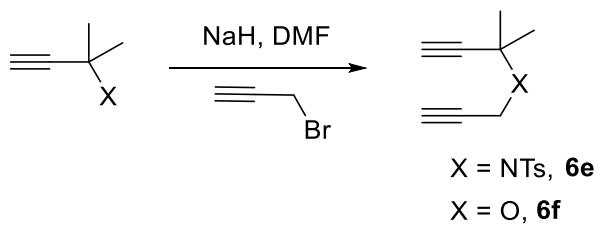

Compounds $\mathbf{6 e}$ and $\mathbf{6} \mathbf{f}$ were synthesized following the literature reports. ${ }^{6,7}$ Characterization data for $\mathbf{6 f}$ is available in the literature. ${ }^{7}$

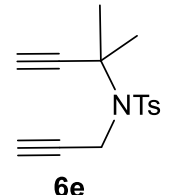

$6 e$

6e: colorless oil; ${ }^{1} \mathrm{H}$ NMR $\left(500 \mathrm{MHz}, \mathrm{CDCl}_{3}\right) \delta 7.81(\mathrm{~d}, J=8.3 \mathrm{~Hz}, 2 \mathrm{H}), 7.27(\mathrm{~d}, J=7.6 \mathrm{~Hz}, 2 \mathrm{H})$, $4.38(\mathrm{~d}, J=2.3 \mathrm{~Hz}, 2 \mathrm{H}), 2.41(\mathrm{~s}, 3 \mathrm{H}), 2.39(\mathrm{~s}, 1 \mathrm{H}), 2.31(\mathrm{t}, J=2.3 \mathrm{~Hz}, 1 \mathrm{H}), 1.72(\mathrm{~s}, 6 \mathrm{H}) ;{ }^{13} \mathrm{C}$ NMR $\left(125 \mathrm{MHz}_{2} \mathrm{CDCl}_{3}\right) \delta 143.2,139.6,129.4,127.3,85.8,81.2,72.4,72.3,56.7,36.9,30.1$; HRMS (ESI) calcd for $\mathrm{C}_{15} \mathrm{H}_{18} \mathrm{NO}_{2} \mathrm{~S}[\mathrm{M}+\mathrm{H}]^{+} 276.1058$, found 276.1055 .

\section{Preparation of $6 \mathrm{~g}$}

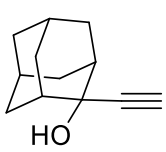

s3

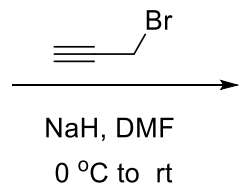

$0^{\circ} \mathrm{C}$ to rt

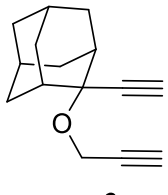

$6 \mathrm{~g}$ 
$\mathbf{6 g}$ was synthesized from s3 by etherification reaction (General Procedure 2 ) with propargyl bromide. Compound $\mathbf{s} \mathbf{3}$ was synthesized from 2 -adamantanone and ethynyl magnesium bromide by following typical literature procedures. ${ }^{8}$

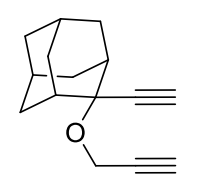

$6 \mathrm{~g}$

6g: yellow oil; ${ }^{1} \mathrm{H}$ NMR $\left(500 \mathrm{MHz}, \mathrm{CDCl}_{3}\right) \delta 4.29(\mathrm{~d}, J=2.5 \mathrm{~Hz}, 2 \mathrm{H}), 2.57(\mathrm{~s}, 1 \mathrm{H}), 2.40$ (t, $J=2.5$ $\mathrm{Hz}, 1 \mathrm{H}), 2.19-2.03(\mathrm{~m}, 6 \mathrm{H}), 1.83(\mathrm{p}, J=3.1 \mathrm{~Hz}, 1 \mathrm{H}), 1.80-1.75(\mathrm{~m}, 3 \mathrm{H}), 1.71-1.66(\mathrm{~m}, 2 \mathrm{H}), 1.52$ (ddt, $J=12.7,3.4,1.9 \mathrm{~Hz}, 2 \mathrm{H}) ;{ }^{13} \mathrm{C}$ NMR $\left(125 \mathrm{MHz}, \mathrm{CDCl}_{3}\right) \delta 84.45,80.96,78.73,75.43,73.30$, 51.16, 37.52, 35.92, 35.06, 31.53, 27.03, 26.56; HRMS (EI) calcd for $\mathrm{C}_{15} \mathrm{H}_{18} \mathrm{O}[\mathrm{M}]^{+} 214.1358$, found 214.1366 .

\section{Preparation of $6 \mathrm{~h}$}

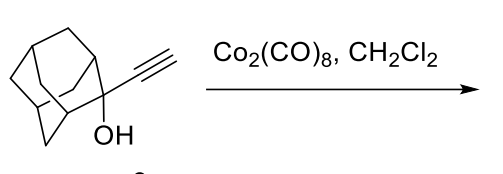

s3

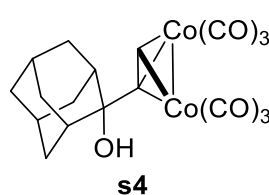

s4

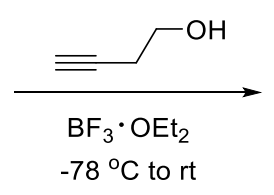

$-78{ }^{\circ} \mathrm{C}$ to rt

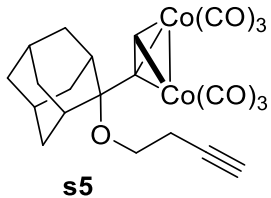

s5

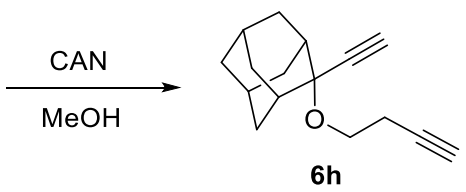

Substrate $\mathbf{6 h}$ cannot be synthesized by typical etherification reaction thus, it was synthesized by Nicholas reaction from s3. ${ }^{9}$ The tertiary alcohol $\mathbf{s} 3(440 \mathrm{mg}, 2.5 \mathrm{mmol})$ was dissolved in anhydrous $\mathrm{CH}_{2} \mathrm{Cl}_{2}(5 \mathrm{~mL})$ followed by addition of dicobaltoctacarbonyl $(855 \mathrm{mg}, 2.5 \mathrm{mmol}$ ) and stirred for $1 \mathrm{~h}$. The dark red mixture was concentrated and purified quickly by flash chromatography $\left(\mathrm{SiO}_{2}\right.$, hexanes-EtOAc, 20:1) to give the red complex s4. It was dissolved in anhydrous $\mathrm{CH}_{2} \mathrm{Cl}_{2}$ and cooled to $-78{ }^{\circ} \mathrm{C}$ followed by addition of $\mathrm{BF}_{3} \cdot \mathrm{OEt}_{2}$ $\left(31 \mu \mathrm{L}, 0.25 \mathrm{mmol}\right.$ ) and a solution of 3-butyn-1-ol $\left(0.39 \mathrm{~mL}, 5.2 \mathrm{mmol}\right.$ ) in $5 \mathrm{~mL} \mathrm{CH} \mathrm{Cl}_{2}$. The mixture was stirred at this temperature for $30 \mathrm{~min}$ followed by warming to room temperature and stirred until completion (monitored by TLC). The reaction was quenched with satd. $\mathrm{NaHCO}_{3}(5 \mathrm{~mL})$ and extracted with $\mathrm{CH}_{2} \mathrm{Cl}_{2}(10 \mathrm{~mL} \times 2)$. The combined organic layer was washed with brine $(2 \times 10 \mathrm{~mL})$, dried over anhydrous $\mathrm{Na}_{2} \mathrm{SO}_{4}$, filtered, concentrated, and purified by column chromatography ( $\mathrm{SiO}_{2}$, hexanes-EtOAc, 20:1) to give s5. Oxidation of $\mathbf{s 5}$ was carried out by stirring a MeOH solution $(0.25 \mathrm{M})$ of $\mathbf{s 5}$ with CAN (4.4 g, 8 $\mathrm{mmol})$. MeOH was removed under reduced pressure followed by extraction with EtOAc $(10 \mathrm{~mL} \times 2)$ and combined organic layer was washed with water $(10 \mathrm{~mL})$ and brine $(10 \mathrm{~mL})$. The combined organic layer was dried over anhydrous $\mathrm{Na}_{2} \mathrm{SO}_{4}$, filtered, concentrated, and purified by column chromatography $\left(\mathrm{SiO}_{2}\right.$, hexanes-EtOAc, 20:1 $\rightarrow 10: 1$ ) to give $6 \mathrm{~h}$ in $30 \%$ yield over 3 steps.

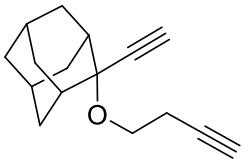

6h: yellow oil; ${ }^{1} \mathrm{H}$ NMR $\left(500 \mathrm{MHz}, \mathrm{CDCl}_{3}\right) \delta 3.68(\mathrm{t}, J=7.2 \mathrm{~Hz}, 2 \mathrm{H}), 2.69-2.36(\mathrm{~m}, 3 \mathrm{H})$, $2.11(\mathrm{~d}, J=12.6 \mathrm{~Hz}, 4 \mathrm{H}), 2.05(\mathrm{~d}, J=4.3 \mathrm{~Hz}, 2 \mathrm{H}), 2.00-1.94(\mathrm{~m}, 1 \mathrm{H}), 1.86-1.81(\mathrm{~m}, 1 \mathrm{H})$, $1.80-1.74(\mathrm{~m}, 3 \mathrm{H}), 1.72-1.65(\mathrm{~m}, 2 \mathrm{H}), 1.51(\mathrm{~d}, \mathrm{~J}=12.3 \mathrm{~Hz}, 2 \mathrm{H}) ;{ }^{13} \mathrm{C} \mathrm{NMR}\left(125 \mathrm{MHz}, \mathrm{CDCl}_{3}\right)$ $\delta$ 85.30, 81.77, 74.45, 69.00, 60.68, 37.58, 35.89, 34.96, 31.49, 27.12, 26.62, 20.29; HRMS (EI) calcd for $\mathrm{C}_{16} \mathrm{H}_{20} \mathrm{O}[\mathrm{M}]^{+} 228.1514$, found 228.1513 . 


\section{Preparation of $6 \mathbf{i}, \mathbf{6 j}$, and $6 \mathrm{I}$}

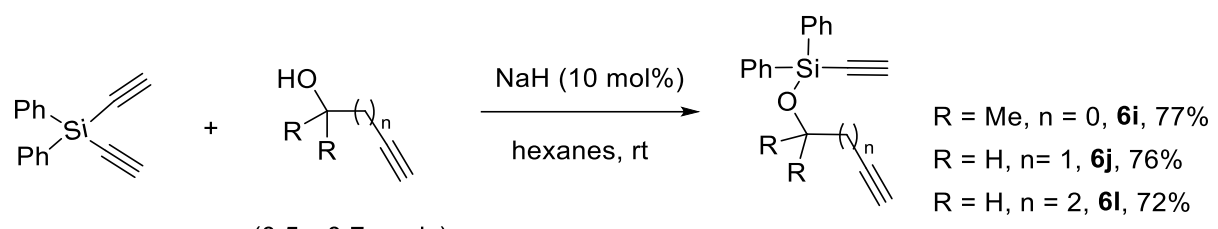

(0.5- 0.7 equiv)

To a stirrerd solution of each alcohol $(2.4 \mathrm{mmol})$ and diethynyldiphenylsilane $(3.6-4.8 \mathrm{mmol})$ in hexanes was added $\mathrm{NaH}$ ( $60 \%$ dispersion in mineral oil, $10 \mathrm{mg}, 0.24 \mathrm{mmol}$ ), and stirred at room temperature for 30 min. ${ }^{10}$ The reaction mixture was filtered through a pad of Celite, concentrated in vacuo, and the residue purified by gravity column chromatography $\left(\mathrm{SiO}_{2}\right.$, hexane: EtOAc $\left.=50: 1\right)$ to give compounds $\mathbf{6 i}, \mathbf{6} \mathbf{j}$ and $\mathbf{6 I}$ in $72-77 \%$ yield.
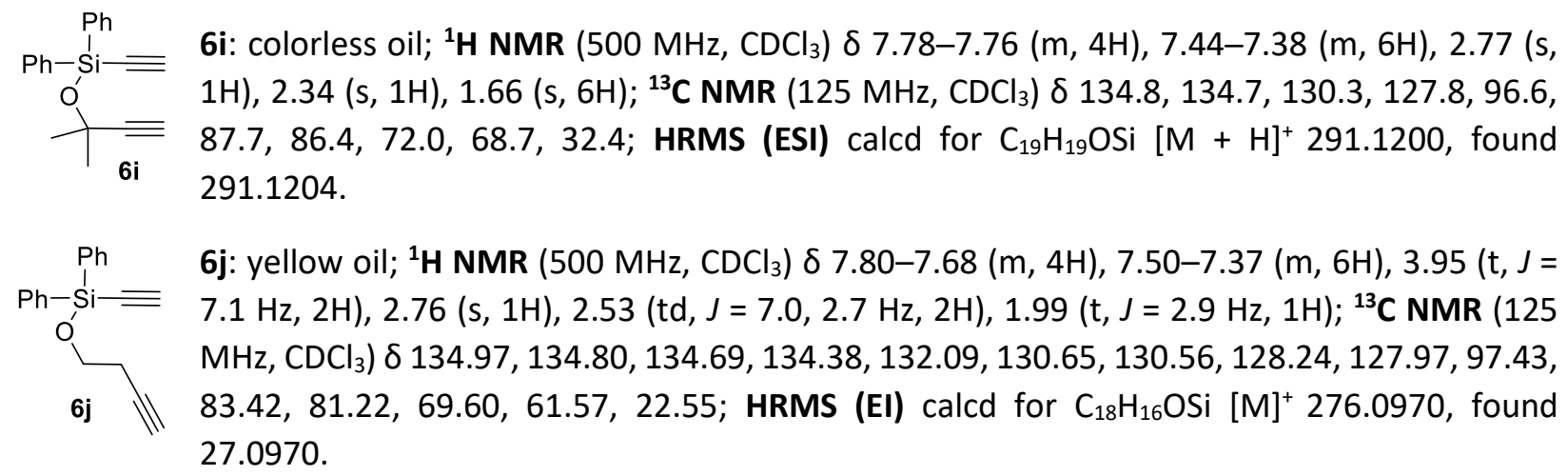

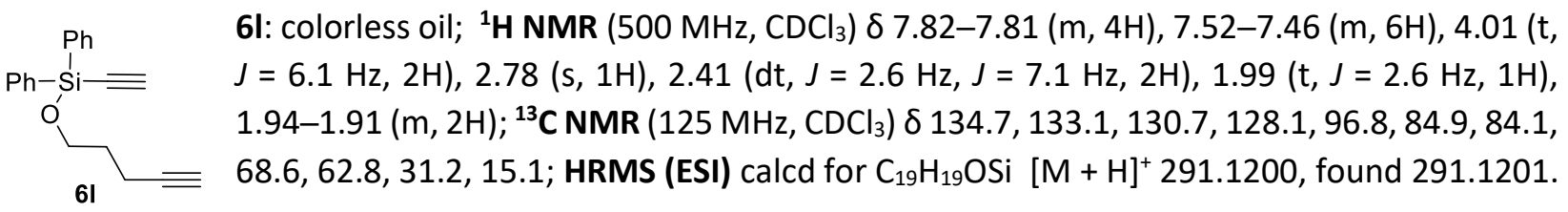

\section{Preparation of $6 \mathbf{k}$}

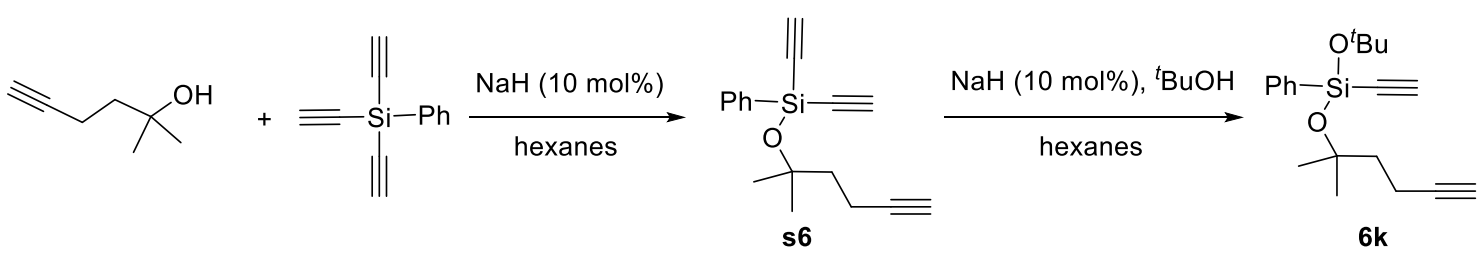

Compound $\mathbf{6 k}$ was synthesized from 2-methylhex-5-yn-2-ol and triethynyl(phenyl)silane in two steps (48\% yield in 2 steps) by following the procedure same described above for synthesis of $6 \mathbf{6}$. 


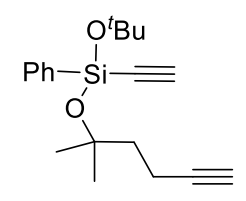

6k

6k: colorless oil; ${ }^{1} \mathbf{H}$ NMR $\left(500 \mathrm{MHz}, \mathrm{CDCl}_{3}\right) \delta$ 7.75-7.73 (m, 2H), 7.45-7.40 (m, 3H), 3.91 (q, J = 5.0 Hz, 2H), $2.55(\mathrm{~s}, 1 \mathrm{H}), 2.39-2.35(\mathrm{~m}, 2 \mathrm{H}), 1.94(\mathrm{t}, J=2.6 \mathrm{~Hz}, 1 \mathrm{H}), 1.90-1.86(\mathrm{~m}$, $2 \mathrm{H}), 1.41(\mathrm{~s}, 6 \mathrm{H}), 1.27(\mathrm{t}, J=5.0 \mathrm{~Hz}, 3 \mathrm{H}) ;{ }^{13} \mathrm{C} \mathrm{NMR}\left(125 \mathrm{MHz}, \mathrm{CDCl}_{3}\right) \delta 134.1,133.8,130.5$, 127.9, 93.8, 85.6, 85.1, 75.9, 67.9, 59.3, 43.2, 29.4, 29.3, 18.1, 13.7; HRMS (ESI) calcd for $\mathrm{C}_{17} \mathrm{H}_{22} \mathrm{O}_{2} \mathrm{SiNa}[\mathrm{M}+\mathrm{Na}]^{+} 309.1281$, found 309.1282 .

\section{Preparation of $6 \mathrm{~m}$}
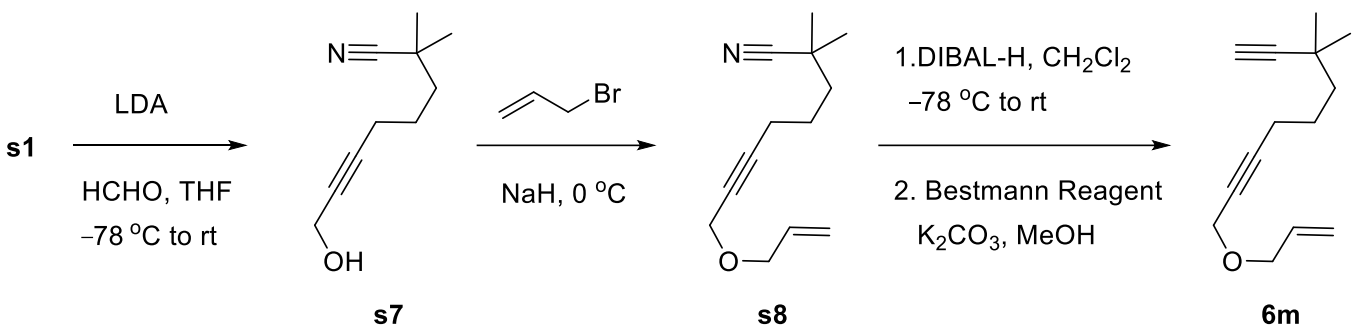

Compound $\mathbf{s} 1$ is treated with LDA followed by paraformaldehyde to form the alcohol $\mathbf{s} 7$ which underwent etherification with allyl bromide (General Procedure 2) to form $\mathbf{8 8}$. The cyano group in $\mathbf{8} 8$ was reduced with DIBAL-H followed by treatment with the Bestmann reagent following the procedure described above (preparation of 6 a) to form $6 \mathrm{~m}$ as yellow oil in $58 \%$ yield from $\mathbf{s} 8 .{ }^{1} \mathbf{H} \mathbf{~ N M R}\left(500 \mathrm{MHz}, \mathrm{CDCl}_{3}\right) \delta 5.93-5.85$ $(\mathrm{m}, 1 \mathrm{H}), 5.28(\mathrm{dd}, J=1.6 \mathrm{~Hz}, J=17.2 \mathrm{~Hz}, 1 \mathrm{H}), 5.18(\mathrm{dd}, J=1.5 \mathrm{~Hz}, J=10.4 \mathrm{~Hz}, 1 \mathrm{H}), 4.12(\mathrm{~m}, 2 \mathrm{H}), 4.04-4.02$ $(\mathrm{m}, 2 \mathrm{H}), 2.25-2.22(\mathrm{~m}, 2 \mathrm{H}), 2.06(\mathrm{~s}, 1 \mathrm{H}), 1.69-1.65(\mathrm{~m}, 2 \mathrm{H}), 1.49-1.46(\mathrm{~m}, 2 \mathrm{H}), 1.19(\mathrm{~s}, 6 \mathrm{H}) ;{ }^{13} \mathrm{C}$ NMR $(125$ $\left.\mathrm{MHz}, \mathrm{CDCl}_{3}\right) \delta 134.2,117.6,91.5,86.7,76.1,70.4,68.0,57.7,42.4,37.5,29.1,14.6,19.2$; HRMS (ESI) calcd for $\mathrm{C}_{14} \mathrm{H}_{21} \mathrm{O}[\mathrm{M}+\mathrm{H}]^{+}$205.1587, found 205.1588 .

\section{Preparation of $6 n$}

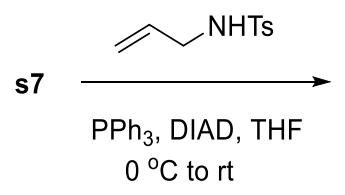

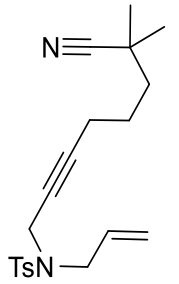

s9

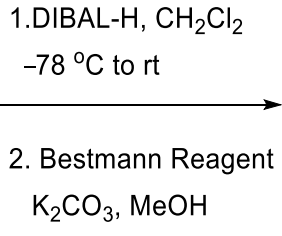

Compound S7 was subjected to a Mitsunobu reaction (General Procedure 3) with $N$-allyl-ptoluenesulfonamide to form $\mathbf{s} \mathbf{9}$ which was subsequently reduced with DIBAL-H followed by treatment the Bestmann reagent to give $\mathbf{6 n}$ as light yellow oil (46\% in 2 steps from $\mathbf{s 9}$ ). ${ }^{1} \mathbf{H} \mathbf{~ N M R}\left(500 \mathrm{MHz}, \mathrm{CDCl}_{3}\right) \delta 7.69$ $(\mathrm{d}, J=8.1 \mathrm{~Hz}, 2 \mathrm{H}), 7.25(\mathrm{~d}, J=8.0 \mathrm{~Hz}, 2 \mathrm{H}), 5.73-5.65(\mathrm{~m}, 1 \mathrm{H}), 5.23(\mathrm{~d}, J=17.2 \mathrm{~Hz}, 1 \mathrm{H}), 5.16(\mathrm{~d}, J=10.1 \mathrm{~Hz}$, $1 \mathrm{H}), 4.01(\mathrm{~s}, 2 \mathrm{H}), 3.75(\mathrm{~d}, J=6.3 \mathrm{~Hz}, 2 \mathrm{H}), 2.37(\mathrm{~s}, 3 \mathrm{H}), 2.03(\mathrm{~s}, 1 \mathrm{H}), 1.88(\mathrm{t}, J=6.9 \mathrm{~Hz}, 2 \mathrm{H}), 1.41-1.39(\mathrm{~m}$, 2H), 1.27-1.25 (m, 2H), 1.12 (s, 6H); ${ }^{13} \mathrm{C}$ NMR $\left(125 \mathrm{MHz}, \mathrm{CDCl}_{3}\right) \delta 143.2,136.2,132.2,129.3,127.8,119.5$, 91.3, 85.9, 72.6, 68.1, 48.9, 42.3, 36.3, 30.7, 29.1, 24.3, 21.5, 18.8; HRMS (ESI) calcd for $\mathrm{C}_{21} \mathrm{H}_{28} \mathrm{NO}_{2} \mathrm{~S}$ [M + $\mathrm{H}]^{+} 358.1835$, found 358.1837 . 


\section{Preparation of 60}

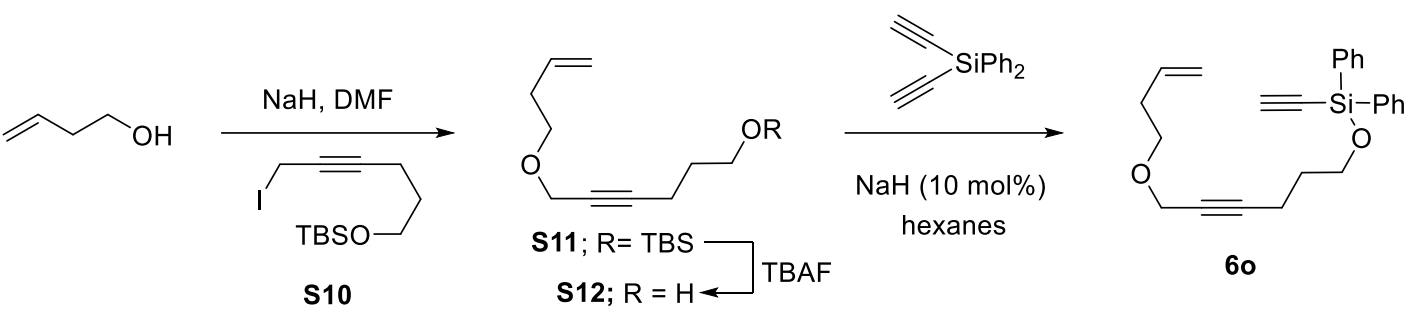

Synthesis of 60 commenced with but-3-enol through an etherification reaction with the propargylic iodide $\mathbf{s 1 0 ^ { 1 1 }}$ (General Procedure 2) forming the ether s11. The TBS group in s11 was removed using TBAF to form alcohol $\mathbf{s 1 2}$ by following standard literature procedures. Alcohol s12 was treated with diethynyldiphenyl silane and catalytic $\mathrm{NaH}$ to form compound 60 (colorless oil, $68 \%$ ) following the procedure described above for synthesis of $6 \mathbf{6 i}{ }^{1}{ }^{\mathbf{H}} \mathbf{N M R}\left(500 \mathrm{MHz}, \mathrm{CDCl}_{3}\right) \delta 7.73-7.72(\mathrm{~m}, 4 \mathrm{H}), 7.43-7.39(\mathrm{~m}, 6 \mathrm{H}), 5.85-5.80(\mathrm{~m}$, $1 \mathrm{H}), 5.13-5.09(\mathrm{~m}, 1 \mathrm{H}), 5.08-5.04(\mathrm{~m}, 1 \mathrm{H}), 4.10(\mathrm{~s}, 2 \mathrm{H}), 3.92(\mathrm{t}, J=5.9 \mathrm{~Hz}, 2 \mathrm{H}), 3.53(\mathrm{t}, J=6.6 \mathrm{~Hz}, 2 \mathrm{H})$, 2.75-2.72 (m, 2H), 2.37-2.33 (m, 3H), 1.86-1.83 (m, 2H); ${ }^{13} \mathrm{C} \mathrm{NMR}\left(125 \mathrm{MHz}, \mathrm{CDCl}_{3}\right) \delta 134.6,133.0,130.6$, 128.0, 97.4, 96.6, 86.2, 84.8, 76.3, 69.1, 62.8, 58.6, 34.0, 31.2, 15.4; HRMS (ESI) calcd for $\mathrm{C}_{24} \mathrm{H}_{26} \mathrm{O}_{2} \mathrm{SiNa}$ [M $+\mathrm{Na}]^{+}$397.1594, found 397.1596.

\section{Preparation of $6 p$}

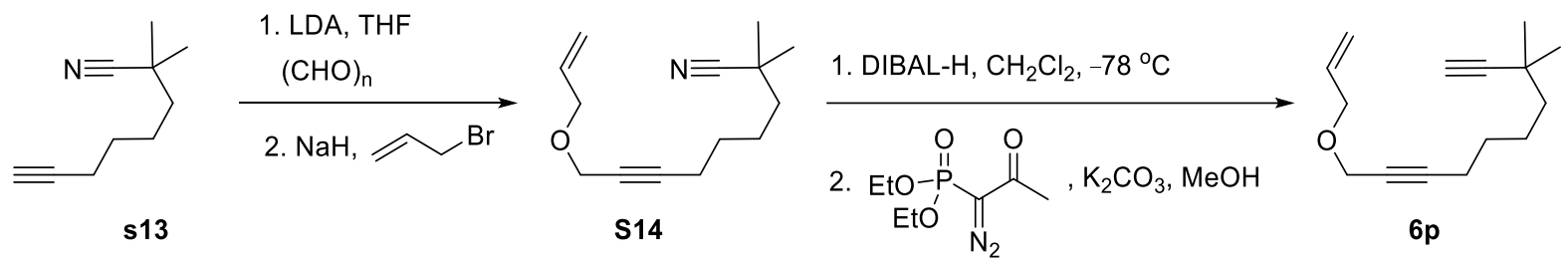

Compound S13 was synthesized by alkylation of isobutyronitrile with 6-iodo-hexyne (General Procedure 1). S13 was converted to $\mathbf{S 1 4}$ by deprotonation with LDA and trapping with paraformaldehyde followed by etherification with allyl bromide (General Procedure 2). Compound s14 was reduced with DIBAL-H followed by treatment with the Bestmann reagent to give $6 \mathrm{p}$ as colorless oil (46\% yield from $\mathbf{s} 14$ ). ${ }^{1} \mathbf{H}$ NMR $\left(500 \mathrm{MHz}, \mathrm{CDCl}_{3}\right) \delta 5.93-5.85(\mathrm{~m}, 1 \mathrm{H}), 5.30-5.17(\mathrm{~m}, 2 \mathrm{H}), 4.12(\mathrm{t}, J=2.0 \mathrm{~Hz}, 2 \mathrm{H}), 4.03(\mathrm{~d}, J=5.7 \mathrm{~Hz}, 2 \mathrm{H})$, 2.25-2.23 (m, 2H), $2.05(\mathrm{~s}, 1 \mathrm{H}), 1.56-1.52(\mathrm{~m}, 4 \mathrm{H}), 1.40-1.37(\mathrm{~m}, 2 \mathrm{H}), 1.19(\mathrm{~s}, 6 \mathrm{H}) ;{ }^{13} \mathrm{C}$ NMR (125 MHz, $\left.\mathrm{CDCl}_{3}\right) \delta 134.3,117.6,91.8,86.9,76.0,67.8,57.7,42.6,31.0,29.1,29.0,24.6,18.8$; HRMS (ESI) calcd for $\mathrm{C}_{15} \mathrm{H}_{22} \mathrm{ONa}[\mathrm{M}+\mathrm{Na}]^{+} 241.1563$, found 241.1578 . 


\section{Preparation of $s 17$}
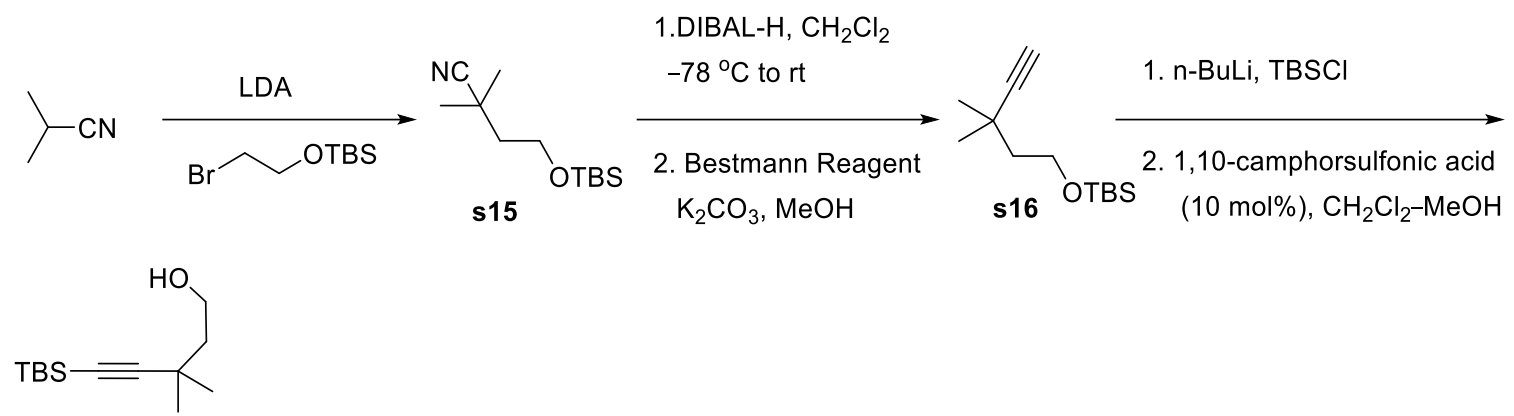

S17

Alkylation of isobutyronitrile with (2-bromoethoxy)(tert-butyl)dimethylsilane gave s15 (General Procedure 1). Nitrile s15 was reduced by DIBAL-H followed by treating with the Bestmann reagent to form s16 following the procedure described above (preparation of 6a). Compound s16 (905 mg, $4 \mathrm{mmol}$ ) was dissolved in anhydrous THF $(10 \mathrm{~mL})$, cooled to $-78{ }^{\circ} \mathrm{C}$ followed by dropwise addition of $3.6 \mathrm{~mL}$ of $n$-BuLi (2.5 $\mathrm{M}$ in hexanes). The reaction was stirred for $30 \mathrm{~min}$ at that temperature followed by addition of TBSCl (633 $\mathrm{mg}, 4.2 \mathrm{mmol}$ ). The reaction was slowly warmed to room temperature over an hour and stirred for another $30 \mathrm{~min}$. The reaction was quenched with satd. $\mathrm{NH}_{4} \mathrm{Cl}$ solution and extracted with EtOAc $(10 \mathrm{~mL} \mathrm{x}$ 2). The combined organic layer was washed with water $(10 \mathrm{~mL})$ and brine $(10 \mathrm{~mL})$, dried over anhydrous $\mathrm{Na}_{2} \mathrm{SO}_{4}$, filtered, concentrated and purified by column chromatography $\left(\mathrm{SiO}_{2}\right.$, hexanes-EtOAc, 20:1) to give TBS substituted alkyne. The TBS protection on the alcohol was selectively removed using catalytic 1,10camphorsulfonic acid in $\mathrm{MeOH}-\mathrm{CH}_{2} \mathrm{Cl}_{2}$ (1:1) following standard literature procedure to give s17 as colorless oil ( $58 \%$ yield in two steps from s16).

\section{Preparation of $6 q$}

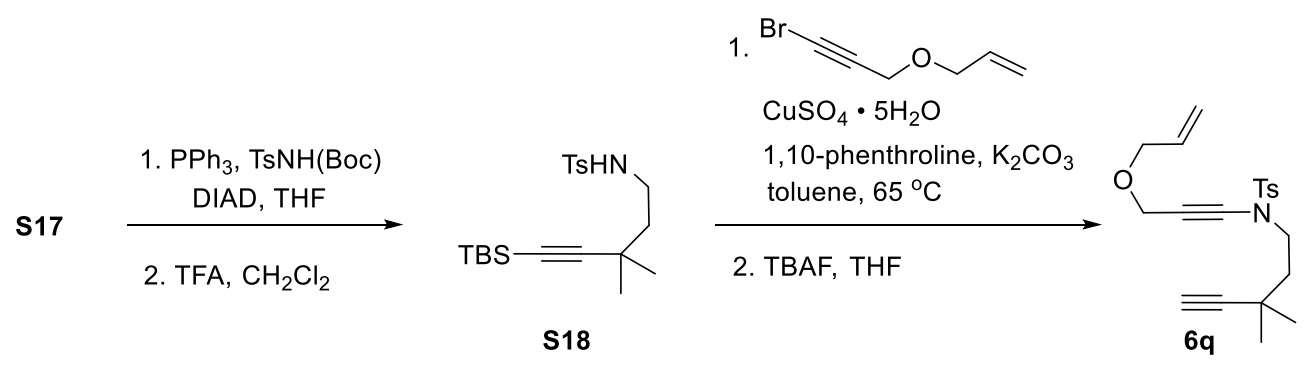

Compound s17 was subjected to a Mitsunobu reaction followed by Boc deprotection (General Procedures $\mathbf{3}$ and 4) to give sulfonamide s18. Compound s18 was subjected to copper catalyzed amidation of alkynyl bromide (General Procedure 5 ) followed by TBAF mediated removal of TBS group and gave $\mathbf{6 q}$ as colorless oil in $52 \%$ yield from s18. ${ }^{1} \mathbf{H}$ NMR $\left(500 \mathrm{MHz}, \mathrm{CDCl}_{3}\right) \delta 7.78(\mathrm{~d}, J=8.1 \mathrm{~Hz}, 2 \mathrm{H}), 7.33(\mathrm{~d}, J=8.0 \mathrm{~Hz}, 2 \mathrm{H})$, 5.91-5.83 (m, 1H), 5.29-5.18 (m, 2H), $4.27(\mathrm{~s}, 2 \mathrm{H}), 3.98(\mathrm{~d}, J=5.6 \mathrm{~Hz}, 2 \mathrm{H}), 3.53-3.50(\mathrm{~m}, 2 \mathrm{H}), 2.43(\mathrm{~s}, 3 \mathrm{H})$, $2.11(\mathrm{~s}, 1 \mathrm{H}), 1.75-1.71(\mathrm{~m}, 2 \mathrm{H}), 1.20(\mathrm{~s}, 6 \mathrm{H}) ;{ }^{13} \mathrm{C} \mathrm{NMR}\left(125 \mathrm{MHz}, \mathrm{CDCl}_{3}\right) \delta$ 144.7, 134.7, 134.1, 129.8, 127.6, $117.8,89.8,79.7,70.1,69.1,67.4,57.6,48.8,40.4,29.6,29.2,21.2$; HRMS (ESI) calcd for $\mathrm{C}_{20} \mathrm{H}_{26} \mathrm{NO}_{3} \mathrm{~S}[\mathrm{M}+$ $\mathrm{H}]^{+} 360.1628$, found 360.1628 . 


\section{Preparation of $6 r$}
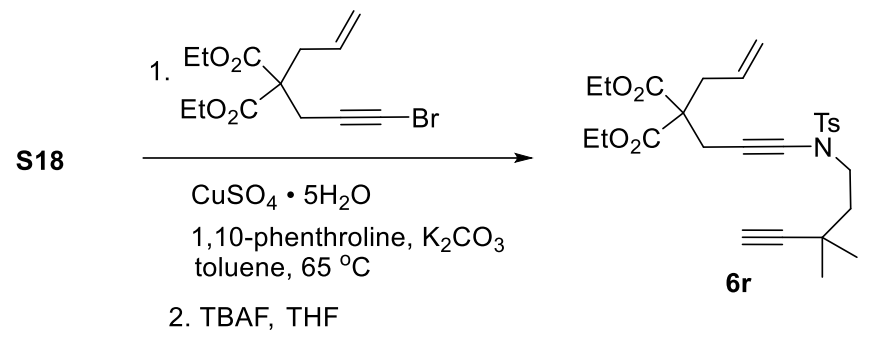

Compound s18 was subjected to copper catalyzed amidation of alkynyl bromide (General Procedures 5) followed by TBAF mediated deprotection to give $6 \mathrm{r}$ as yellow oil (42\% yield in 2 steps from $\mathbf{s} 18) .{ }^{1} \mathbf{H} \mathbf{~ N M R}$ $\left(500 \mathrm{MHz}, \mathrm{CDCl}_{3}\right) \delta 7.76(\mathrm{~d}, J=8.1 \mathrm{~Hz}, 2 \mathrm{H}), 7.33(\mathrm{~d}, J=8.1 \mathrm{~Hz}, 2 \mathrm{H}), 5.03-5.55(\mathrm{~m}, 1 \mathrm{H}), 5.14-5.03(\mathrm{~m}, 2 \mathrm{H})$, $4.16(\mathrm{q}, J=7.1 \mathrm{~Hz}, 4 \mathrm{H}), 3.45-3.41(\mathrm{~m}, 2 \mathrm{H}), 2.83(\mathrm{~s}, 2 \mathrm{H}), 2.71(\mathrm{~d}, J=7.4 \mathrm{~Hz}, 2 \mathrm{H}), 2.43(\mathrm{~s}, 3 \mathrm{H}), 2.09(\mathrm{~s}, 1 \mathrm{H})$, $1.89-1.85(\mathrm{~m}, 2 \mathrm{H}), 1.21(\mathrm{t}, J=7.1 \mathrm{~Hz}, 6 \mathrm{H}), 1.18(\mathrm{~s}, 6 \mathrm{H}) ;{ }^{13} \mathrm{C}$ NMR $\left(125 \mathrm{MHz}, \mathrm{CDCl}_{3}\right) \delta 169.8,144.5,134.8$, 131.9, 129.8, 127.6, 119.7, 89.8, 75.9, 69.1, 65.0, 61.6, 57.0, 48.7, 40.2, 36.5, 29.5, 29.1, 22.8, 21.6, 14.1; HRMS (ESI) calcd for $\mathrm{C}_{27} \mathrm{H}_{36} \mathrm{NO}_{6} \mathrm{~S}[\mathrm{M}+\mathrm{H}]^{+} 502.2258$, found 502.2259 .

\section{Preparation of $6 s$ and $6 t$}
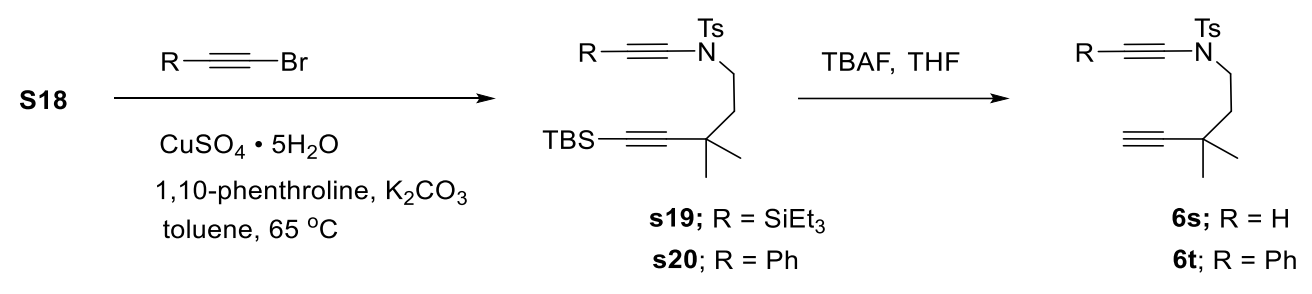

Compound s18 was subjected to coupling with triethylsilylacetylene bromide (General Procedure 5) to form s19 which was treated with 2 equiv. of TBAF to remove both silyl groups generating $6 \mathbf{s}$. In the same vein, $\mathbf{6 t}$ was synthesized by coupling $\mathbf{s 1 8}$ with phenylacetylene bromide (General Procedure 5) followed by desilylation with TBAF ( 1 equiv).

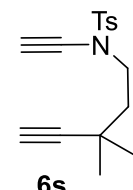

$6 s$

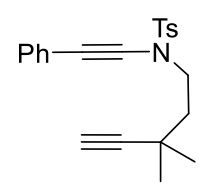

$6 t$

6s: white solid; ${ }^{1} \mathbf{H}$ NMR $\left(500 \mathrm{MHz}, \mathrm{CDCl}_{3}\right) \delta 7.82(\mathrm{~d}, J=8.2 \mathrm{~Hz}, 2 \mathrm{H}), 7.36(\mathrm{~d}, J=8.1 \mathrm{~Hz}, 2 \mathrm{H})$, 3.54-3.50 (m, 2H), $2.74(\mathrm{~s}, 1 \mathrm{H}), 2.46(\mathrm{~s}, 3 \mathrm{H}), 2.12(\mathrm{~s}, 1 \mathrm{H}), 1.77-1.74(\mathrm{~m}, 2 \mathrm{H}), 1.22(\mathrm{~s}, 6 \mathrm{H}) ;{ }^{13} \mathrm{C}$ NMR $\left(125 \mathrm{MHz}, \mathrm{CDCl}_{3}\right) \delta$ 144.8, 134.7, 129.9, 127.7, 89.7, 76.1, 69.1, 59.2, 48.6, 40.2, 29.6, 29.1, 21.7, 15.6; HRMS (ESI) calcd for $\mathrm{C}_{16} \mathrm{H}_{20} \mathrm{NO}_{2} \mathrm{~S}[\mathrm{M}+\mathrm{H}]^{+} 290.1209$, found 290.1217.

6t: yellow oil; ${ }^{1} \mathbf{H}$ NMR $\left(500 \mathrm{MHz}, \mathrm{CDCl}_{3}\right) \delta 7.85(\mathrm{~d}, J=7.7 \mathrm{~Hz}, 2 \mathrm{H}), 7.42-7.32(\mathrm{~m}, 4 \mathrm{H}), 7.31-7.26$ $(\mathrm{m}, 3 \mathrm{H}), 3.69-3.52(\mathrm{~m}, 2 \mathrm{H}), 2.46(\mathrm{~s}, 3 \mathrm{H}), 2.14(\mathrm{~s}, 1 \mathrm{H}), 1.88-1.77(\mathrm{~m}, 2 \mathrm{H}), 1.24(\mathrm{~s}, 6 \mathrm{H}) ;{ }^{13} \mathrm{C} \mathrm{NMR}$ $\left(125 \mathrm{MHz}, \mathrm{CDCl}_{3}\right) \delta 144.68,134.67,131.38,129.83,128.29,127.81,127.73,122.89,89.85$, 82.41, 70.78, 69.11, 49.03, 40.52, 29.66, 29.19, 21.69; HRMS (ESI) calcd for $\mathrm{C}_{22} \mathrm{H}_{24} \mathrm{NO}_{2} \mathrm{~S}[\mathrm{M}+$ $\mathrm{H}]^{+} 366.1528$, found 366.1527 . 


\section{Preparation of $6 u$}

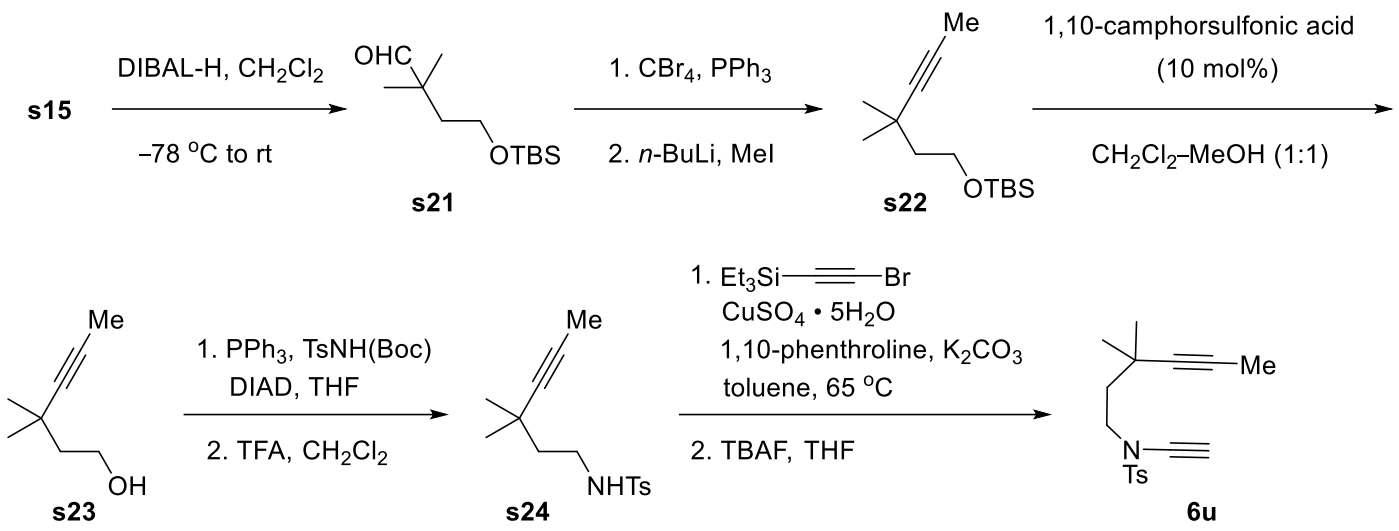

Synthesis of $6 \mathrm{u}$ commenced from DIBAL-H reduction of $\mathbf{s 1 5}$ to $\mathbf{s 2 1}$ following the procedure described above (preparation of 6a). s21 (1.15 g, $5 \mathrm{mmol}$ ) was dissolved in $20 \mathrm{~mL}$ anhydrous DCM and cooled to 0 ${ }^{\circ} \mathrm{C}$ followed by addition of $\mathrm{CBr}_{4}(3.31 \mathrm{~g}, 10 \mathrm{mmol})$ and $\mathrm{PPh}_{3}(5.24 \mathrm{~g}, 20 \mathrm{mmol})$. The dark orange solution was stirred at $0{ }^{\circ} \mathrm{C}$ for $1 \mathrm{~h}$ and hexanes was added. The orange-brown precipitate was filtered over silica, filtrate was concentrated and purified through column chromatography $\left(\mathrm{SiO}_{2}\right.$, hexanes-EtOAc, 20:1 $\rightarrow$ $10: 1)$ to obtain the vinylic dibromide as colorless oil. The dibromide was dissolved in dry THF, cooled to $-78{ }^{\circ} \mathrm{C}$ followed by dropwise addition of $4.4 \mathrm{~mL}$ of $n$-BuLi. The mixture was stirred for $1 \mathrm{~h}$ at $-78{ }^{\circ} \mathrm{C}$ followed by addition of Mel $(0.75 \mathrm{~mL}, 12 \mathrm{mmol}){ }^{12}$ The reaction was warmed to room temperature and stirred for $1 \mathrm{~h}$. The reaction was quenched with sat. $\mathrm{NH}_{4} \mathrm{Cl}$ soln. and extracted with EtOAc $(10 \mathrm{~mL} \times 2)$. The combined organic layer was washed was washed with water $(10 \mathrm{~mL})$ and brine $(10 \mathrm{~mL})$, dried over anhydrous $\mathrm{Na}_{2} \mathrm{SO}_{4}$, filtered, concentrated and purified by column chromatography $\left(\mathrm{SiO}_{2}\right.$, hexanes-EtOAc, 20:1) to give s22. The TBS group was deprotected using catalytic 1,10-camphorsulfonic acid in $\mathrm{MeOH}-\mathrm{CH}_{2} \mathrm{Cl}_{2}$ (1:1) to s23. Compound s23 was subjected to Mitsunobu reaction and Boc deprotection (General Procedure 3 and 4) to give s24 which underwent coupling with triethylsilylacetylene brmomide (General Procedure 5) followed by deprotection of the silyl group using TBAF to give $6 \mathrm{u}$ as colorless oil.

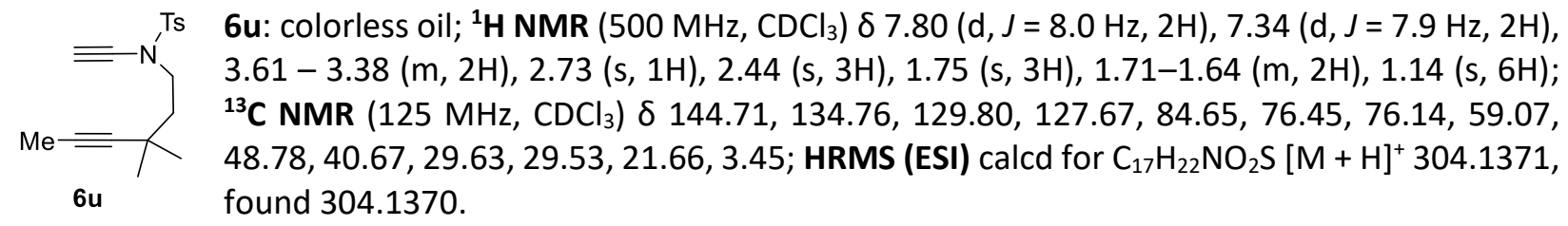


Preparation of 11a, 11b, 11e, 11f, and 11g

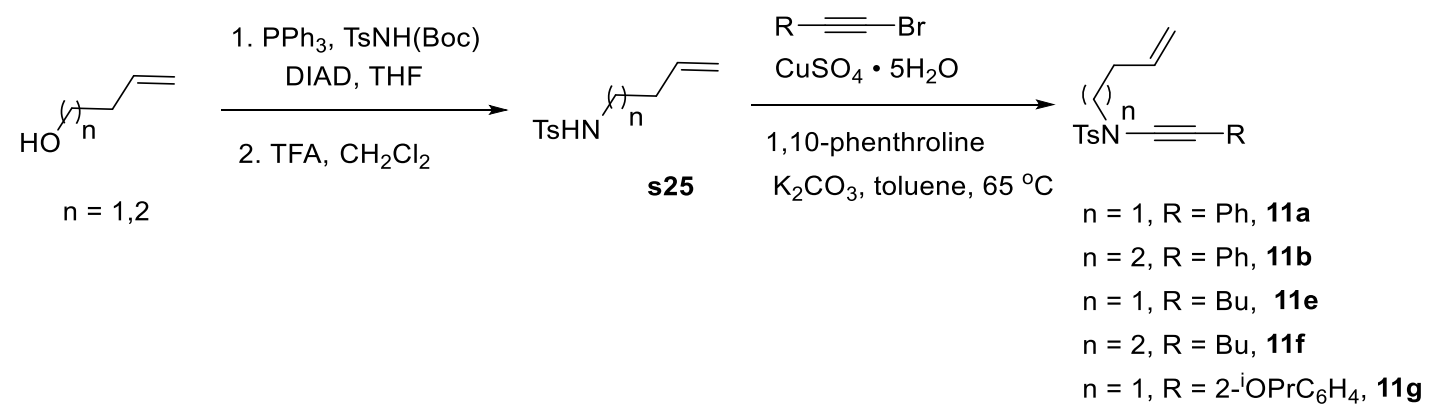

1,n-Enynes 11a, 11b, 11e, 11f and 11g were synthesized from 3-buten-1-ol or 4-penten-1-ol through a Mitsunobu reaction and Boc deprotection (General Procedure $\mathbf{3}$ and $\mathbf{4}$ ) to $\mathbf{s} 25$ followed by coupling with different alkynyl bromide (General Procedure 5).

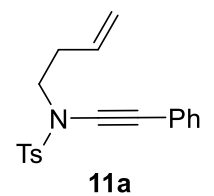

$1 \mathbf{a}$

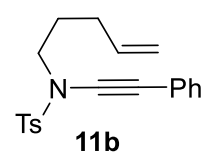

11a: colorless oil; ${ }^{1} \mathbf{H}$ NMR $\left(500 \mathrm{MHz}, \mathrm{CDCl}_{3}\right) \delta 7.86(\mathrm{~d}, J=8.0 \mathrm{~Hz}, 2 \mathrm{H}), 7.41-7.33(\mathrm{~m}, 4 \mathrm{H})$, 7.32-7.25 (m, 3H), $5.77(\mathrm{ddt}, J=17.1,10.5,6.8 \mathrm{~Hz}, 1 \mathrm{H}), 5.185 .01(\mathrm{~m}, 2 \mathrm{H}), 3.49(\mathrm{t}, J=7.4$ $\mathrm{Hz}, 2 \mathrm{H}), 2.48(\mathrm{q}, J=7.2 \mathrm{~Hz}, 2 \mathrm{H}), 2.45(\mathrm{~s}, 3 \mathrm{H}) ;{ }^{13} \mathrm{C}$ NMR $\left(126 \mathrm{MHz}, \mathrm{CDCl}_{3}\right) \delta$ 144.79, 134.60, $133.73,131.40,129.85,128.36,127.90,127.73,122.89,117.81,82.29,71.04,50.99,32.33$, 21.68; HRMS (ESI) calcd for $\mathrm{C}_{19} \mathrm{H}_{20} \mathrm{NO}_{2} \mathrm{~S}[\mathrm{M}+\mathrm{H}]^{+} 326.1215$, found 326.1209.

11b: colorless oil; ${ }^{1} \mathrm{H}$ NMR $\left(500 \mathrm{MHz}, \mathrm{CDCl}_{3}\right) \delta 7.84(\mathrm{~d}, J=8.4 \mathrm{~Hz}, 2 \mathrm{H}), 7.39-7.33(\mathrm{~m}, 4 \mathrm{H})$, 7.31-7.26 (m, 3H), $5.79(\mathrm{ddt}, J=17.0,10.4,6.8 \mathrm{~Hz}, 1 \mathrm{H}), 5.12-4.90(\mathrm{~m}, 2 \mathrm{H}), 3.41(\mathrm{t}, J=7.2$ $\mathrm{Hz}, 2 \mathrm{H}), 2.45(\mathrm{~s}, 3 \mathrm{H}), 2.14(\mathrm{q}, J=7.2 \mathrm{~Hz}, 2 \mathrm{H}), 1.82(\mathrm{p}, J=7.4 \mathrm{~Hz}, 2 \mathrm{H}) ;{ }^{13} \mathrm{C}$ NMR $(125 \mathrm{MHz}$, $\left.\mathrm{CDCl}_{3}\right) \delta 144.64,137.11,134.59,131.37,129.79,128.29,127.79,127.71,122.91,115.67$, 82.40, 70.70, 51.08, 30.32, 27.18, 21.68; HRMS (ESI) calcd for $\mathrm{C}_{20} \mathrm{H}_{22} \mathrm{NO}_{2} \mathrm{~S}[\mathrm{M}+\mathrm{H}]^{+} 340.1371$, found 340.1372 .

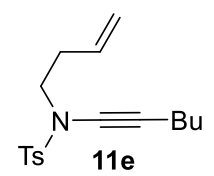

11e: colorless oil; ${ }^{1} \mathbf{H}$ NMR $\left(500 \mathrm{MHz}, \mathrm{CDCl}_{3}\right) \delta 7.77(\mathrm{~d}, J=8.0 \mathrm{~Hz}, 2 \mathrm{H}), 7.32(\mathrm{~d}, J=7.9 \mathrm{~Hz}$, $2 \mathrm{H}), 5.71(\mathrm{ddt}, J=17.0,10.2,6.8 \mathrm{~Hz}, 1 \mathrm{H}), 5.14-4.96(\mathrm{~m}, 2 \mathrm{H}), 3.31(\mathrm{t}, J=7.4 \mathrm{~Hz}, 2 \mathrm{H}), 2.43(\mathrm{~s}$, $3 \mathrm{H}), 2.36(\mathrm{q}, J=7.3 \mathrm{~Hz}, 2 \mathrm{H}), 2.25(\mathrm{t}, J=7.0 \mathrm{~Hz}, 2 \mathrm{H}), 1.46(\mathrm{p}, J=7.1 \mathrm{~Hz}, 2 \mathrm{H}), 1.35(\mathrm{dt}, J=16.9$, $7.4 \mathrm{~Hz}, 2 \mathrm{H}), 0.89$ (t, $J=7.3 \mathrm{~Hz}, 3 \mathrm{H}) ;{ }^{13} \mathrm{C}$ NMR $\left(125 \mathrm{MHz}, \mathrm{CDCl}_{3}\right) \delta 144.28,134.67,133.90$, $129.58,127.65,117.46,72.87,70.51,50.81,32.16,31.00,21.84,21.62,18.15,13.57$; HRMS (ESI) calcd for $\mathrm{C}_{17} \mathrm{H}_{24} \mathrm{NO}_{2} \mathrm{~S}[\mathrm{M}+\mathrm{H}]^{+} 306.1528$, found 306.1527.

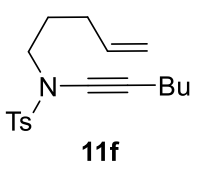

11f: colorless oil; ${ }^{1} \mathbf{H}$ NMR $\left(500 \mathrm{MHz}, \mathrm{CDCl}_{3}\right) \delta 7.76(\mathrm{~d}, J=7.9 \mathrm{~Hz}, 2 \mathrm{H}), 7.32(\mathrm{~d}, J=7.9 \mathrm{~Hz}$, $2 \mathrm{H}), 5.76(\mathrm{ddt}, J=16.9,10.3,6.7 \mathrm{~Hz}, 1 \mathrm{H}), 4.99(\mathrm{dd}, J=21.3,13.7 \mathrm{~Hz}, 2 \mathrm{H}), 3.25(\mathrm{t}, J=7.2 \mathrm{~Hz}$, $2 \mathrm{H}), 2.43(\mathrm{~s}, 3 \mathrm{H}), 2.25(\mathrm{t}, J=7.0 \mathrm{~Hz}, 2 \mathrm{H}), 2.08(\mathrm{q}, J=7.3 \mathrm{~Hz}, 2 \mathrm{H}), 1.71(\mathrm{p}, J=7.4 \mathrm{~Hz}, 2 \mathrm{H}), 1.45$ $(p, J=7.0 \mathrm{~Hz}, 2 \mathrm{H}), 1.36(\mathrm{p}, J=7.3 \mathrm{~Hz}, 2 \mathrm{H}), 0.88(\mathrm{t}, J=7.4 \mathrm{~Hz}, 3 \mathrm{H}) ;{ }^{13} \mathrm{C}$ NMR $\left(125 \mathrm{MHz}, \mathrm{CDCl}_{3}\right)$ $\delta 144.25,137.27,134.62,129.58,127.63,115.43,73.02,70.20,50.87,31.01,30.30,27.00,21.84,21.62$, 18.14, 13.58; HRMS (ESI) calcd for $\mathrm{C}_{18} \mathrm{H}_{26} \mathrm{NO}_{2} \mathrm{~S}[\mathrm{M}+\mathrm{H}]^{+} 320.1684$, found 320.1681 . 


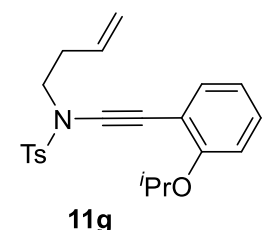

$11 \mathrm{~g}$

11g: yellow oil; ${ }^{1} \mathbf{H}$ NMR $\left(500 \mathrm{MHz}, \mathrm{CDCl}_{3}\right) \delta 7.90(\mathrm{~d}, \mathrm{~J}=8.3 \mathrm{~Hz}, 2 \mathrm{H}), 7.39-7.28(\mathrm{~m}, 3 \mathrm{H})$, 7.25-7.14 (m, 1H), 6.89-6.75 (m, 2H), $5.77(\mathrm{ddt}, J=17.1,10.2,6.8 \mathrm{~Hz}, 1 \mathrm{H}), 5.21-4.97$ $(\mathrm{m}, 2 \mathrm{H}), 4.57$ (hept, $J=6.1 \mathrm{~Hz}, 1 \mathrm{H}), 3.47(\mathrm{t}, J=7.4 \mathrm{~Hz}, 2 \mathrm{H}), 2.58-2.47(\mathrm{~m}, 2 \mathrm{H}), 2.43(\mathrm{~s}$, $3 \mathrm{H}), 1.34(\mathrm{~d}, J=6.1 \mathrm{~Hz}, 6 \mathrm{H}) ;{ }^{13} \mathrm{C} \mathrm{NMR}\left(125 \mathrm{MHz}, \mathrm{CDCl}_{3}\right) \delta 158.18,144.53,134.81,133.85$, $132.65,129.74,128.76,127.74,120.47,117.69,114.25,113.89,85.63,71.12,68.06$, 51.00, 32.07, 22.18, 21.65; HRMS (ESI) calcd for $\mathrm{C}_{22} \mathrm{H}_{26} \mathrm{NO}_{3} \mathrm{~S}[\mathrm{M}+\mathrm{H}]^{+} 384.1633$, found 384.1628.

\section{Preparation of 11c and 11d}

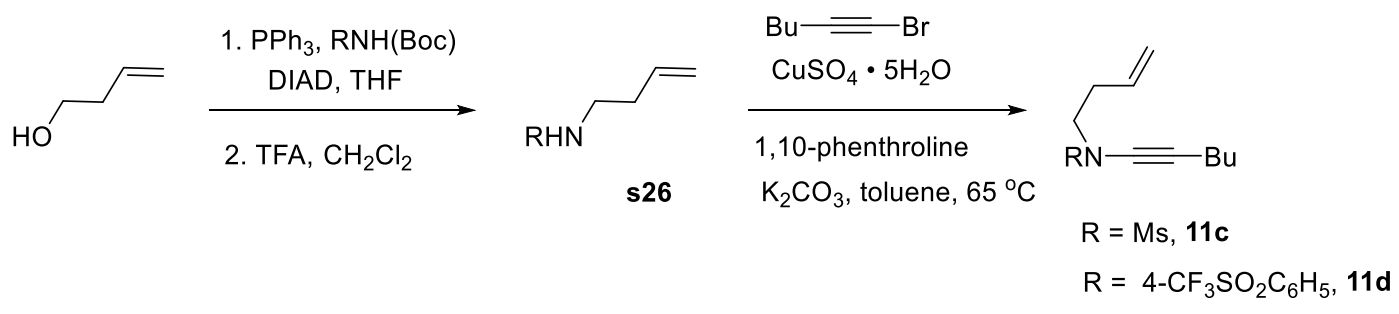

Compounds $\mathbf{1 1} \mathbf{c}$ and $\mathbf{1 1} \mathbf{d}$ were synthesized following the above scheme (similar to the preparation of 11a and 11b) wherein the Mitsunobu reaction was carried out with methanesulfonamide and 4trifluorobenzene sulfonamide, respectively.

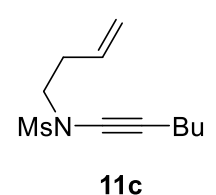

$11 \mathrm{c}$

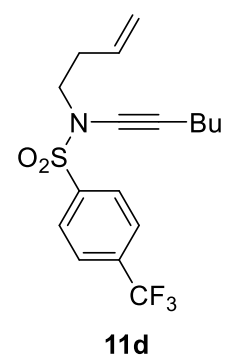

11c: colorless oil; ${ }^{1} \mathbf{H}$ NMR (500 MHz, $\left.\mathrm{CDCl}_{3}\right) \delta 5.86-5.61(\mathrm{~m}, 1 \mathrm{H}), 5.20-4.94(\mathrm{~m}, 2 \mathrm{H}), 3.42$ (td, $J=7.2,1.5 \mathrm{~Hz}, 2 \mathrm{H}), 2.99(\mathrm{~d}, J=1.6 \mathrm{~Hz}, 3 \mathrm{H}), 2.52-2.33(\mathrm{~m}, 2 \mathrm{H}), 2.29-2.16(\mathrm{~m}, 2 \mathrm{H}), 1.46$ $(\mathrm{p}, J=7.1 \mathrm{~Hz}, 2 \mathrm{H}), 1.38(\mathrm{p}, J=7.3 \mathrm{~Hz}, 2 \mathrm{H}), 0.87(\mathrm{td}, J=7.3,1.5 \mathrm{~Hz}, 3 \mathrm{H}) ;{ }^{13} \mathrm{C}$ NMR $(125 \mathrm{MHz}$, $\left.\mathrm{CDCl}_{3}\right) \delta 133.93,117.78,72.30,70.99,50.62,37.73,32.43,30.98,21.88,18.11,13.54$; HRMS (ESI) calcd for $\mathrm{C}_{11} \mathrm{H}_{20} \mathrm{NO}_{2} \mathrm{~S}[\mathrm{M}+\mathrm{H}]^{+} 230.1215$, found 230.1210 .

11d: yellow oil; ${ }^{1} \mathbf{H}$ NMR $\left(500 \mathrm{MHz}, \mathrm{CDCl}_{3}\right) \delta 8.02(\mathrm{~d}, J=8.2 \mathrm{~Hz}, 2 \mathrm{H}), 7.81(\mathrm{~d}, J=8.3 \mathrm{~Hz}, 2 \mathrm{H})$, $5.70(\mathrm{ddt}, J=17.1,10.2,6.8 \mathrm{~Hz}, 1 \mathrm{H}), 5.11-4.99(\mathrm{~m}, 2 \mathrm{H}), 3.37(\mathrm{t}, J=7.3 \mathrm{~Hz}, 2 \mathrm{H}), 2.38(\mathrm{q}, J=$ $7.1 \mathrm{~Hz}, 2 \mathrm{H}), 2.26(\mathrm{t}, J=7.0 \mathrm{~Hz}, 2 \mathrm{H}), 1.51-1.40(\mathrm{~m}, 2 \mathrm{H}), 1.41-1.23(\mathrm{~m}, 2 \mathrm{H}), 0.89(\mathrm{t}, J=7.3$ $\mathrm{Hz}, 3 \mathrm{H}) ;{ }^{13} \mathrm{C}$ NMR $\left(125 \mathrm{MHz}, \mathrm{CDCl}_{3}\right) \delta 141.03,135.34135 .10,134.83,134.57,133.53$, $128.12,126.15,126.12,117.80,72.08,71.21,51.08,32.15,30.89,21.84,18.06,13.50 ;{ }^{19} \mathrm{~F}$ NMR (376 MHz, CDCl ${ }_{3} \delta$-63.65; HRMS (ESI) calcd for $\mathrm{C}_{17} \mathrm{H}_{21} \mathrm{NO}_{2} \mathrm{SF}_{3}[\mathrm{M}+\mathrm{H}]^{+} 360.1245$, found 360.1238 .

\section{Preparation of $11 \mathrm{~h}$}

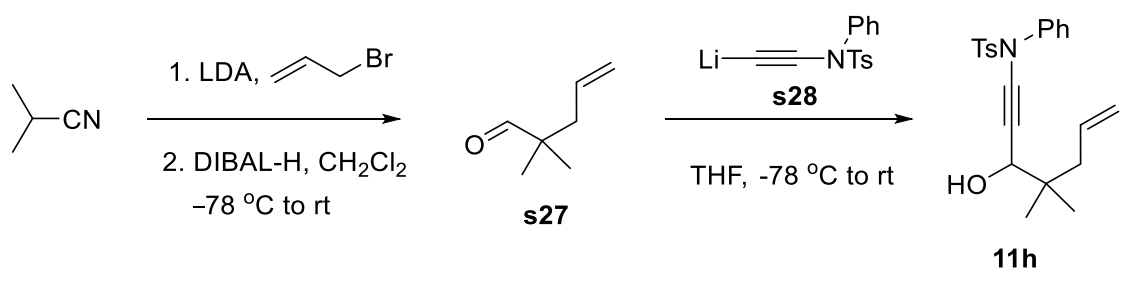


Synthesis of $11 \mathrm{~h}$ commenced with allylation of isobutyronitrile (General Procedure 1) followed by reduction of the nitrile using DIBAL-H to generate aldehyde $\mathbf{s 2 7}$ which was reacted with $\mathbf{2} 28$ in THF to form 11h. The synthesis of protonated form of $\mathbf{s} 28$ can be found in the literature. ${ }^{13}$

11h: yellow oil; ${ }^{1} \mathrm{H}$ NMR $\left(500 \mathrm{MHz}, \mathrm{CDCl}_{3}\right) \delta 7.55(\mathrm{~d}, J=8.0 \mathrm{~Hz}, 2 \mathrm{H}), 7.32-7.23(\mathrm{~m}, 7 \mathrm{H}), 5.87-5.79(\mathrm{~m}, 1 \mathrm{H})$, 5.06-5.03 (m, 2H), $4.21(\mathrm{~s}, 1 \mathrm{H}), 2.41(\mathrm{~s}, 3 \mathrm{H}), 2.19-2.06(\mathrm{~m}, 2 \mathrm{H}), 0.97(\mathrm{~s}, 3 \mathrm{H}), 9,95(\mathrm{~s}, 3 \mathrm{H}) ;{ }^{13} \mathrm{C}$ NMR $(125$ $\left.\mathrm{MHz}, \mathrm{CDCl}_{3}\right) \delta 145.1,138.8,134.9,133.0,129.6,129.1,128.3,128.2,126.2,117.7,79.8,70.4,70.2,42.9$, 39.2, 22.9, 22.8, 21.7; HRMS (ESI) calcd for $\mathrm{C}_{22} \mathrm{H}_{26} \mathrm{NO}_{3} \mathrm{~S}[\mathrm{M}+\mathrm{H}]^{+} 384.1628$, found 384.1626.

\section{Preparation of 11i-H}

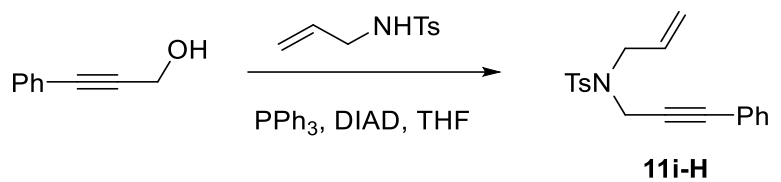

Compounds $\mathbf{1 1} \mathbf{i}-\mathbf{H}$ was obtained as a yellow oil in $74 \%$ yield through a Mitsunobu reaction of 3phenylprop-2-yn-ol with $\mathrm{N}$-tosyl-allylamine following the General Procedure $3 .{ }^{1} \mathbf{H} \mathbf{N M R}\left(500 \mathrm{MHz}, \mathrm{CDCl}_{3}\right)$ $\delta 7.78(\mathrm{~d}, J=7.8 \mathrm{~Hz}, 2 \mathrm{H}), 7.33-7.15(\mathrm{~m}, 5 \mathrm{H}), 7.06(\mathrm{~d}, J=7.4 \mathrm{~Hz}, 2 \mathrm{H}), 5.80$ (ddt, $J=16.7,9.9,6.5 \mathrm{~Hz}, 1 \mathrm{H}$ ), $5.33(\mathrm{~d}, J=17.1 \mathrm{~Hz}, 1 \mathrm{H}), 5.26(\mathrm{~d}, J=10.1 \mathrm{~Hz}, 1 \mathrm{H}), 4.31(\mathrm{~s}, 2 \mathrm{H}), 3.89(\mathrm{~d}, J=6.4 \mathrm{~Hz}, 2 \mathrm{H}), 2.33(\mathrm{~s}, 3 \mathrm{H}) ;{ }^{13} \mathrm{C} \mathrm{NMR}$ $\left(125 \mathrm{MHz}, \mathrm{CDCl}_{3}\right) \delta 143.55,135.99,132.10,131.51,129.56,128.42,128.15,127.84,122.23,119.96,85.74$, 81.67, 49.31, 36.75, 21.44; HRMS (ESI) calcd for $\mathrm{C}_{19} \mathrm{H}_{20} \mathrm{NO}_{2} \mathrm{~S}[\mathrm{M}+\mathrm{H}]^{+} 326.1215$, found 326.1209.

\section{Preparation of 11i}
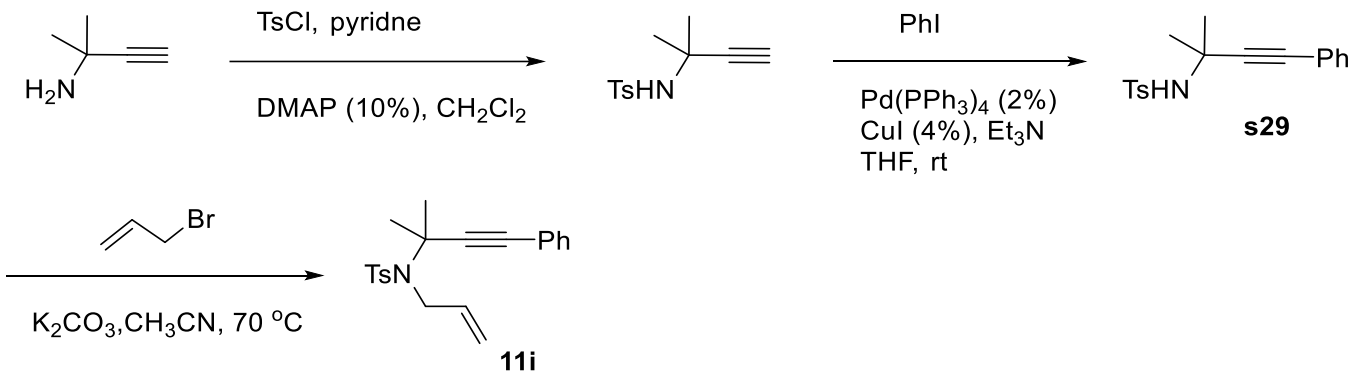

Synthesis of 11i commenced with tosylation of commercially available 2-methylbut-3-yn-2-amine by known procedures. A Sonogashira coupling of the tosylamide with iodobenzene generated $\mathbf{5} \mathbf{2 9}$ (General Procedure 6). Allylation of $\mathbf{s} 29$ under reported conditions ${ }^{6}$ generated $\mathbf{1 1 i}$ as yellow oil in $82 \%$ yield. ${ }^{1} \mathbf{H}$ NMR $(500 \text { MHz, CDCl })_{3} \delta 7.87-7.63(\mathrm{~m}, 2 \mathrm{H}), 7.31-7.24(\mathrm{~m}, 3 \mathrm{H}), 7.21$ (td, $\left.J=5.8,2.8 \mathrm{~Hz}, 4 \mathrm{H}\right), 6.07$ (ddd, $J=$ $16.1,10.6,5.4 \mathrm{~Hz}, 1 \mathrm{H}), 5.53-5.25(\mathrm{~m}, 1 \mathrm{H}), 5.23-5.06(\mathrm{~m}, 1 \mathrm{H}), 4.27-4.09(\mathrm{~m}, 2 \mathrm{H}), 2.35(\mathrm{~s}, 3 \mathrm{H}), 1.76(\mathrm{~s}, 6 \mathrm{H})$. ${ }^{13} \mathrm{C}$ NMR $\left(125 \mathrm{MHz}, \mathrm{CDCl}_{3}\right) \delta 142.89,139.48,136.93,131.52,129.39,128.33,128.19,127.39,122.52$, $116.96,91.55,83.66,57.12,50.82,30.93,21.44$; HRMS (ESI) calcd for $\mathrm{C}_{21} \mathrm{H}_{24} \mathrm{NO}_{2} \mathrm{~S}[\mathrm{M}+\mathrm{H}]^{+} 354.1528$, found 354.1524. 


\section{Preparation of $\mathbf{1 1 j}$}

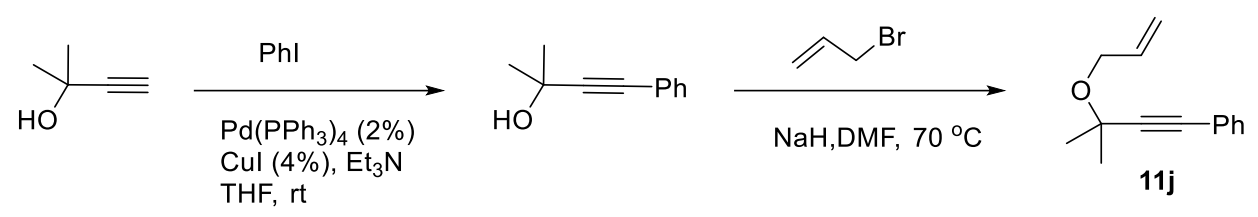

11j (light yellow oil) was synthesized from 2-methylbut-3-yn-2-ol in 65\% yield (2 steps) through a Sonogashira coupling (General procedure 6) with iodobenzene followed by etherification of the tertiary alcohol with allyl bromide (General procedure 2). ${ }^{1} \mathrm{H} \mathrm{NMR}\left(500 \mathrm{MHz}, \mathrm{CDCl}_{3}\right) \delta 7.43(\mathrm{dt}, J=5.2,2.9 \mathrm{~Hz}, 2 \mathrm{H})$, $7.31(\mathrm{dd}, J=3.9,2.3 \mathrm{~Hz}, 3 \mathrm{H}), 6.00(\mathrm{ddt}, J=16.0,10.9,5.6 \mathrm{~Hz}, 1 \mathrm{H}), 5.40-5.28(\mathrm{~m}, 1 \mathrm{H}), 5.21-5.12(\mathrm{~m}, 1 \mathrm{H})$, $4.34-3.98(\mathrm{~m}, 2 \mathrm{H}), 1.58(\mathrm{~s}, 6 \mathrm{H}) ;{ }^{13} \mathrm{C}$ NMR $\left(125 \mathrm{MHz}, \mathrm{CDCl}_{3}\right) \delta 135.57,131.70,128.26,128.22,122.89$, 116.40, 91.37, 84.21, 70.79, 65.63, 28.97; HRMS (ASAP) calcd for $\mathrm{C}_{14} \mathrm{H}_{17} \mathrm{O}[\mathrm{M}+\mathrm{H}]^{+} 202.1279$, found 202.1272.

\section{Preparation of 11k and 11l}
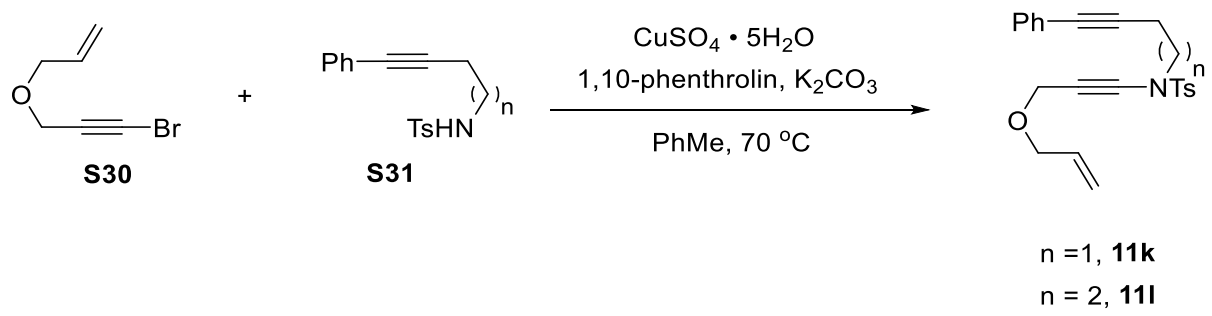

Compounds $\mathbf{1 1 k}$ and $\mathbf{1 1 l}$ are synthesized through a coupling between s30 and s31 (General Procedure 5). S31 was synthesized from but-3-yn-1-ol or pent-3-yn-ol through Sonogashira coupling with Phl followed by a Mitsunobu reaction and Boc deprotection (General Procedure 6 and 3).

$\mathrm{Ph}=11 \mathrm{k}$ : yellow oil; ${ }^{1} \mathrm{H}$ NMR $\left(500 \mathrm{MHz}, \mathrm{CDCl}_{3}\right) \delta 7.81(\mathrm{~d}, J=8.2 \mathrm{~Hz}, 2 \mathrm{H}), 7.36-7.26(\mathrm{~m}, 7 \mathrm{H})$, 5.89-5.84 (m, 1H), 5.30-5.19 (m, 2H), $4.28(\mathrm{~s}, 2 \mathrm{H}), 3.98(\mathrm{~d}, J=5.7 \mathrm{~Hz}, 2 \mathrm{H}), 3.61(\mathrm{t}, J=7.5$ $\mathrm{Hz}, 2 \mathrm{H}), 2.76(\mathrm{t}, J=7.5 \mathrm{~Hz}, 2 \mathrm{H}), 2.41(\mathrm{~s}, 3 \mathrm{H}) ;{ }^{13} \mathrm{C}$ NMR $\left(125 \mathrm{MHz}, \mathrm{CDCl}_{3}\right) \delta 144.9,134.7$, 131.7, 129.9, 128.2, 128.0, 127.7, 123.2, 117.8, 85.2, 82.7, 79.2, 70.2, 68.0, 57.6, 50.2, 21.7, 19.5; HRMS (ESI) calcd for $\mathrm{C}_{23} \mathrm{H}_{24} \mathrm{NO}_{3} \mathrm{~S}[\mathrm{M}+\mathrm{H}]^{+} 394.1477$, found 394.1474.

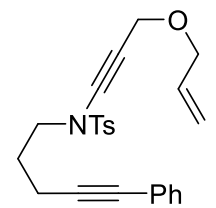

11I: yellow oil; ${ }^{1} \mathbf{H}$ NMR (500 MHz, $\left.\mathrm{CDCl}_{3}\right) \delta 7.81(\mathrm{~d}, J=8.3 \mathrm{~Hz}, 2 \mathrm{H}), 7.43-7.36(\mathrm{~m}, 2 \mathrm{H}), 7.32$ $(\mathrm{d}, J=8.1 \mathrm{~Hz}, 2 \mathrm{H}), 7.29-7.23(\mathrm{~m}, 3 \mathrm{H}), 5.88(\mathrm{ddt}, J=16.4,10.8,5.7 \mathrm{~Hz}, 1 \mathrm{H}), 5.28(\mathrm{dd}, J=17.3$, $1.6 \mathrm{~Hz}, 1 \mathrm{H}), 5.25-5.14(\mathrm{~m}, 1 \mathrm{H}), 4.29(\mathrm{~s}, 2 \mathrm{H}), 4.09-3.93(\mathrm{~m}, 2 \mathrm{H}), 3.52(\mathrm{t}, J=7.0 \mathrm{~Hz}, 2 \mathrm{H}), 2.46$ (t, $J=6.9 \mathrm{~Hz}, 2 \mathrm{H}), 2.43(\mathrm{~s}, 3 \mathrm{H}), 1.95(\mathrm{p}, J=7.0 \mathrm{~Hz}, 2 \mathrm{H}) .{ }^{13} \mathrm{C} \mathrm{NMR}\left(125 \mathrm{MHz}, \mathrm{CDCl}_{3}\right) \delta 144.75$, 111 134.57, 134.11, 131.60, 130.34, 129.83, 128.25, 127.79, 127.66, 123.62, 117.76, 88.20, 81.68, 79.45, 70.15, 67.55, 57.59, 50.38, 27.12, 21.66, 16.52; HRMS (ESI) calcd for $\mathrm{C}_{24} \mathrm{H}_{26} \mathrm{NO}_{3} \mathrm{~S}[\mathrm{M}+\mathrm{H}]^{+} 408.1633$, found 408.1628 . 


\section{Preparation of $11 \mathrm{~m}, 11 \mathrm{n}$, and 110}
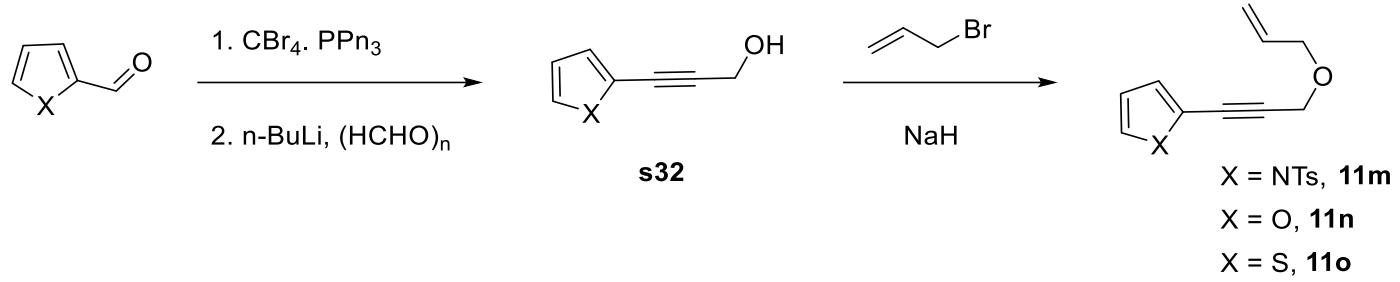

Synthesis of substrates $\mathbf{1 1} \mathbf{m}-\mathbf{1 1 0}$ commenced from commercially available 2-carboxaladehyde derivatives of pyrrole, furan and thiophene respectively through a Corey-Fuchs reaction followed by treatment of the vinylic dibromide with $n$-BuLi and trapping the alkynyl anion with paraformaldehyde (follow procedure for conversion of $\mathbf{s 2 1}$ to s22.). ${ }^{12}$ The propargylic alcohols $\mathbf{s 3 2}$ underwent etherification with allyl bromide (General Procedure 2) to give substrates 11m-110.

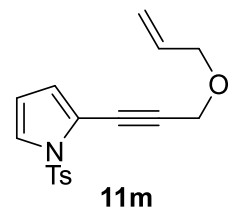

$11 \mathrm{~m}$ : brown solid; ${ }^{1} \mathrm{H}$ NMR $\left(500 \mathrm{MHz}, \mathrm{CDCl}_{3}\right) \delta 7.84(\mathrm{~d}, J=8.4 \mathrm{~Hz}, 2 \mathrm{H}), 7.34$ (dd, $J=3.4$, $1.7 \mathrm{~Hz}, 1 \mathrm{H}), 7.29(\mathrm{~d}, J=8.0 \mathrm{~Hz}, 2 \mathrm{H}), 6.51(\mathrm{dd}, J=3.5,1.7 \mathrm{~Hz}, 1 \mathrm{H}), 6.20(\mathrm{t}, J=3.4 \mathrm{~Hz}, 1 \mathrm{H})$, 5.94 (ddt, $J=17.2,10.3,5.7 \mathrm{~Hz}, 1 \mathrm{H}), 5.36(\mathrm{dt}, J=17.2,1.7 \mathrm{~Hz}, 1 \mathrm{H}), 5.25$ (dq, $J=10.4,1.4$ $\mathrm{Hz}, 1 \mathrm{H}), 4.40(\mathrm{~s}, 2 \mathrm{H}), 4.12(\mathrm{dt}, \mathrm{J}=5.8,1.5 \mathrm{~Hz}, 2 \mathrm{H}), 2.41(\mathrm{~s}, 3 \mathrm{H}) ;{ }^{13} \mathrm{C} \mathrm{NMR}\left(125 \mathrm{MHz}, \mathrm{CDCl}_{3}\right)$ $\delta$ 145.30, 135.37, 134.06, 129.82, 127.79, 123.53, 121.55, 117.89, 114.78, 111.50, 91.12, 76.58, 70.61, 57.93, 21.68; HRMS (ESI) calcd for $\mathrm{C}_{17} \mathrm{H}_{18} \mathrm{NO}_{3} \mathrm{~S}[\mathrm{M}+\mathrm{H}]^{+} 316.1007$, found 316.1001.

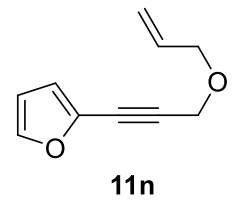

11n: colorless oil; ${ }^{1} \mathrm{H}$ NMR $\left(500 \mathrm{MHz}, \mathrm{CDCl}_{3}\right) \delta 7.38(\mathrm{~d}, J=2.0 \mathrm{~Hz}, 1 \mathrm{H}), 6.60(\mathrm{~d}, J=3.4 \mathrm{~Hz}$, $1 \mathrm{H}), 6.38(\mathrm{dd}, J=3.4,1.9 \mathrm{~Hz}, 1 \mathrm{H}), 5.93(\mathrm{ddt}, J=17.3,10.4,5.8 \mathrm{~Hz}, 1 \mathrm{H}), 5.34(\mathrm{dq}, J=17.3$, $1.6 \mathrm{~Hz}, 1 \mathrm{H}), 5.24(\mathrm{dq}, J=10.5,1.4 \mathrm{~Hz}, 1 \mathrm{H}), 4.39(\mathrm{~s}, 2 \mathrm{H}), 4.12(\mathrm{dt}, J=5.8,1.4 \mathrm{~Hz}, 2 \mathrm{H}) ;{ }^{13} \mathrm{C}$ NMR $\left(125 \mathrm{MHz}, \mathrm{CDCl}_{3}\right) \delta 143.68,136.54,133.90,118.03,115.70,110.85,89.67,76.60$, 70.73, 57.74; HRMS (EI) calcd for $\mathrm{C}_{10} \mathrm{H}_{10} \mathrm{O}_{2}[\mathrm{M}]^{+}$162.0681, found 162.0676 .

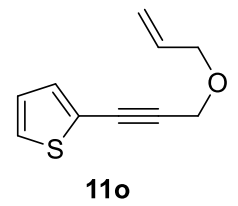

110: yellow oil; ${ }^{1} \mathbf{H}$ NMR (500 MHz, $\left.\mathrm{CDCl}_{3}\right) \delta$ 7.32-7.11 (m, 2H), 7.02-6.89 (m, 1H), 5.94 (ddt, $J=16.5,10.8,5.8 \mathrm{~Hz}, 1 \mathrm{H}), 5.44-5.29(\mathrm{~m}, 1 \mathrm{H}), 5.24(\mathrm{~d}, J=10.4 \mathrm{~Hz}, 1 \mathrm{H}), 4.39(\mathrm{~d}, J=$ $3.2 \mathrm{~Hz}, 2 \mathrm{H}), 4.20-3.98(\mathrm{~m}, 2 \mathrm{H}) ;{ }^{13} \mathrm{C}$ NMR $\left(125 \mathrm{MHz}, \mathrm{CDCl}_{3}\right) \delta 134.03,132.43,127.37$, 126.96, 122.60, 117.94, 89.18, 79.57, 70.77, 58.01; HRMS (EI) calcd for $\mathrm{C}_{10} \mathrm{H}_{10} \mathrm{OS}[\mathrm{M}]^{+}$ 178.0452 , found 178.0455 . 


\section{Preparation of 11p and 11q}

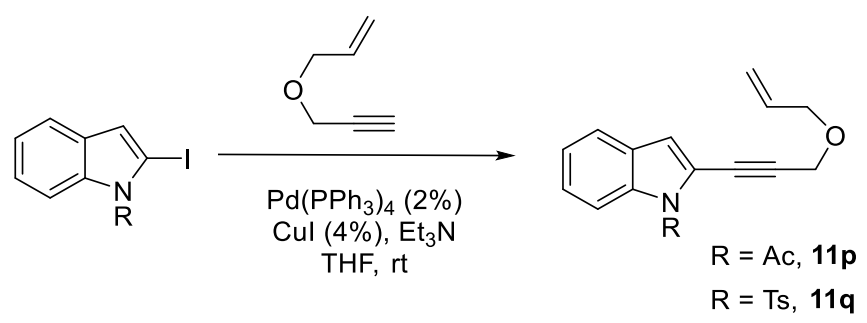

$\mathrm{N}$-Acetyl and $\mathrm{N}$-tosyl-2-iodoindole were subjected to a Sonogashira coupling with allyl propargyl ether (General Procedure 6) to form 11p and 11q, respectively.

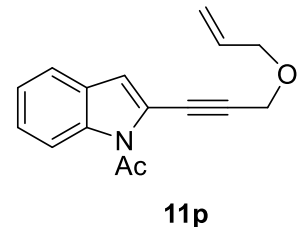

11p: yellow oil; ${ }^{1} \mathbf{H}$ NMR $\left(500 \mathrm{MHz}, \mathrm{CDCl}_{3}\right) \delta 8.45(\mathrm{~d}, J=8.4 \mathrm{~Hz}, 1 \mathrm{H}), 7.51(\mathrm{~d}, J=7.7$ $\mathrm{Hz}, 1 \mathrm{H}), 7.38(\mathrm{t}, J=7.8 \mathrm{~Hz}, 1 \mathrm{H}), 7.28(\mathrm{t}, J=7.6 \mathrm{~Hz}, 1 \mathrm{H}), 6.99(\mathrm{~s}, 1 \mathrm{H}), 5.95$ (ddt, $J=16.5$, 11.0, $5.7 \mathrm{~Hz}, 1 \mathrm{H}), 5.43-5.31(\mathrm{~m}, 1 \mathrm{H}), 5.27(\mathrm{~d}, J=10.3 \mathrm{~Hz}, 1 \mathrm{H}), 4.45(\mathrm{~s}, 2 \mathrm{H}), 4.14(\mathrm{~d}, J=$ $5.8 \mathrm{~Hz}, 2 \mathrm{H}), 2.88(\mathrm{~s}, 3 \mathrm{H}) ;{ }^{13} \mathrm{C}$ NMR $\left(125 \mathrm{MHz}, \mathrm{CDCl}_{3}\right) \delta 170.25,136.73,133.76,128.47$, $126.58,124.12,120.55,119.02,118.89,118.25,117.15,94.00,79.13,71.12,57.97$, 26.71; HRMS (ESI) calcd for $\mathrm{C}_{16} \mathrm{H}_{16} \mathrm{NO}_{2}[\mathrm{M}+\mathrm{H}]^{+} 254.1181$, found 254.1174 .

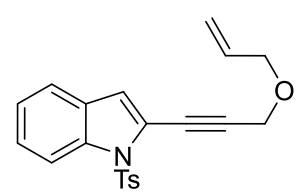

$11 q$

11q: yellow oil; ${ }^{1} \mathrm{H}$ NMR (500 MHz, $\left.\mathrm{CDCl}_{3}\right) \delta 8.23(\mathrm{~d}, J=8.5 \mathrm{~Hz}, 1 \mathrm{H}), 7.84(\mathrm{~d}, J=8.4 \mathrm{~Hz}$, $2 \mathrm{H}), 7.45(\mathrm{~d}, J=7.8 \mathrm{~Hz}, 1 \mathrm{H}), 7.41-7.34(\mathrm{~m}, 1 \mathrm{H}), 7.24(\mathrm{t}, J=7.6 \mathrm{~Hz}, 1 \mathrm{H}), 7.20(\mathrm{~d}, J=8.0$ $\mathrm{Hz}, 2 \mathrm{H}), 6.88(\mathrm{~s}, 1 \mathrm{H}), 5.99$ (ddt, $J=16.3,10.7,5.7 \mathrm{~Hz}, 1 \mathrm{H}), 5.46-5.36(\mathrm{~m}, 1 \mathrm{H}), 5.30-$ $5.25(\mathrm{~m}, 1 \mathrm{H}), 4.51(\mathrm{~s}, 2 \mathrm{H}), 4.23(\mathrm{~d}, J=5.7 \mathrm{~Hz}, 2 \mathrm{H}), 2.32(\mathrm{~s}, 3 \mathrm{H}) ;{ }^{13} \mathrm{C}$ NMR $(125 \mathrm{MHz}$, $\left.\mathrm{CDCl}_{3}\right) \delta 145.10,136.44,135.67,134.04,129.79,128.77,127.05,126.09,123.93$, 121.16, 120.21, 118.09, 117.69, 114.71, 93.27, 70.85, 58.00, 21.60; HRMS (ESI) calcd for $\mathrm{C}_{21} \mathrm{H}_{20} \mathrm{NO}_{3} \mathrm{~S}[\mathrm{M}+$ $\mathrm{H}]^{+}$366.1164, found 366.1159 .

Preparation of 11r, 11s, and 11t

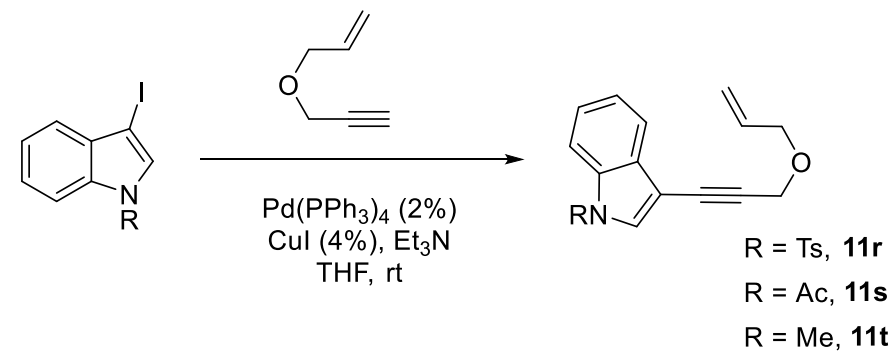

$\mathrm{N}$-Tosyl, $\mathrm{N}$-acetyl and $\mathrm{N}$-methyl-3-iodoindole were subjected to Sonogashira coupling conditions with allyl propargyl ether (General Procedure 6) to form 11r, 11s and 11t respectively.

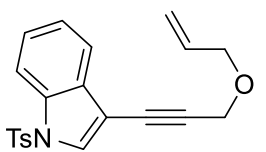

$11 \mathrm{r}$ 11r: orange solid; ${ }^{1} \mathrm{H}$ NMR $\left(500 \mathrm{MHz}, \mathrm{CDCl}_{3}\right) \delta 7.98(\mathrm{~d}, J=8.2 \mathrm{~Hz}, 1 \mathrm{H}), 7.81-7.70(\mathrm{~m}, 3 \mathrm{H})$, $7.63(\mathrm{~d}, J=7.8 \mathrm{~Hz}, 1 \mathrm{H}), 7.35(\mathrm{t}, J=7.7 \mathrm{~Hz}, 1 \mathrm{H}), 7.28(\mathrm{t}, J=7.5 \mathrm{~Hz}, 1 \mathrm{H}), 7.19(\mathrm{~d}, J=7.9 \mathrm{~Hz}$, $2 \mathrm{H}), 5.97$ (ddt, $J=16.3,10.7,5.6 \mathrm{~Hz}, 1 \mathrm{H}), 5.44-5.32(\mathrm{~m}, 1 \mathrm{H}), 5.25(\mathrm{~d}, J=9.9 \mathrm{~Hz}, 1 \mathrm{H}), 4.44$ $(\mathrm{s}, 2 \mathrm{H}), 4.17(\mathrm{~d}, J=5.7 \mathrm{~Hz}, 2 \mathrm{H}), 2.30(\mathrm{~s}, 3 \mathrm{H}) ;{ }^{13} \mathrm{C} \mathrm{NMR}\left(125 \mathrm{MHz}, \mathrm{CDCl}_{3}\right): \delta 145.40,134.86$, $134.18,134.05,130.79,130.04,129.35,126.94,125.52,123.81,120.53,117.98,113.62$, 
104.66, 89.52, 77.40, 70.77, 58.03, 21.57; HRMS (ESI) calcd for $\mathrm{C}_{21} \mathrm{H}_{20} \mathrm{NO}_{2}[\mathrm{M}+\mathrm{H}]^{+} 366.1164$, found 366.1156 .

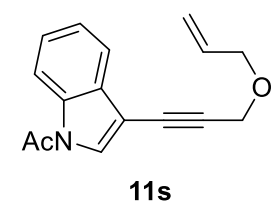

11s: yellow oil; ${ }^{1} \mathrm{H}$ NMR $\left(500 \mathrm{MHz}, \mathrm{CDCl}_{3}\right) \delta 8.40(\mathrm{~d}, J=8.2 \mathrm{~Hz}, 1 \mathrm{H}), 7.69-7.62(\mathrm{~m}, 1 \mathrm{H})$, $7.59(\mathrm{~s}, 1 \mathrm{H}), 7.42-7.35(\mathrm{~m}, 1 \mathrm{H}), 7.37-7.30(\mathrm{~m}, 1 \mathrm{H}), 6.05-5.88(\mathrm{~m}, 1 \mathrm{H}), 5.37(\mathrm{dq}, J=17.2$, $1.7 \mathrm{~Hz}, 1 \mathrm{H}), 5.26(\mathrm{dd}, J=10.3,1.7 \mathrm{~Hz}, 1 \mathrm{H}), 4.45(\mathrm{~s}, 2 \mathrm{H}), 4.18(\mathrm{dt}, J=5.8,1.5 \mathrm{~Hz}, 2 \mathrm{H})$, $2.60(\mathrm{~s}, 3 \mathrm{H}) ;{ }^{13} \mathrm{C}$ NMR $\left(125 \mathrm{MHz}, \mathrm{CDCl}_{3}\right) \delta 168.17,134.95,134.06,130.35,128.42$, $126.07,124.19,119.93,118.00,116.62,104.62,89.31,77.81,70.76,58.06,23.86$;

HRMS (ESI) calcd for $\mathrm{C}_{16} \mathrm{H}_{16} \mathrm{NO}_{2}[\mathrm{M}+\mathrm{H}]^{+} 254.1181$, found 254.1175 .

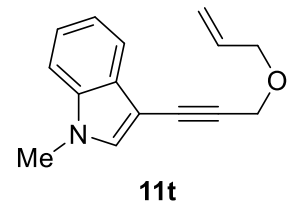

11t: yellow oil; ${ }^{1} \mathbf{H}$ NMR (500 MHz, $\left.\mathrm{CDCl}_{3}\right) \delta$ 7.68-7.53 (m, 1H), 7.31-7.27 (m, 2H), 7.14 (ddd, $J=8.0,4.7,3.2 \mathrm{~Hz}, 1 \mathrm{H}$ ), $6.80(\mathrm{~s}, 1 \mathrm{H}$ ), 5.99 (ddt, $J=17.3,10.4,5.8 \mathrm{~Hz}, 1 \mathrm{H}$ ), 5.39 (dq, $J=17.2,1.6 \mathrm{~Hz}, 1 \mathrm{H}), 5.29(\mathrm{dq}, J=10.4,1.4 \mathrm{~Hz}, 1 \mathrm{H}), 4.49(\mathrm{~s}, 2 \mathrm{H}), 4.19(\mathrm{dt}, J=5.8$, $1.4 \mathrm{~Hz}, 2 \mathrm{H}), 3.82(\mathrm{~s}, 3 \mathrm{H}) ;{ }^{13} \mathrm{C}$ NMR $\left(125 \mathrm{MHz}, \mathrm{CDCl}_{3}\right) \delta 137.19,134.00,127.07,123.15$, $121.38,121.04,120.12,118.12,109.47,107.87,91.29,78.17,70.86,58.02,30.65$; HRMS (ESI) calcd for $\mathrm{C}_{15} \mathrm{H}_{16} \mathrm{NO}[\mathrm{M}+\mathrm{H}]^{+} 226.1232$, found 226.1232 .
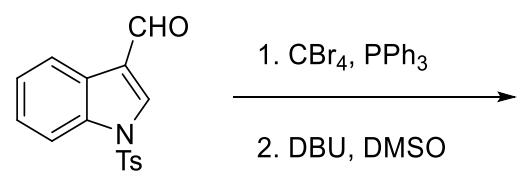

\section{Preparation of 11v}

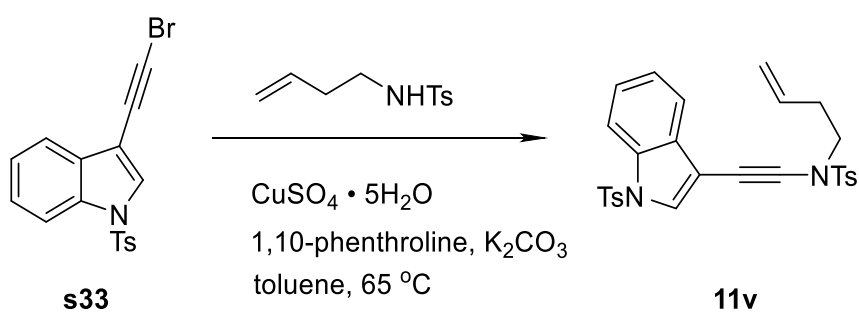

$\mathrm{N}$-tosylindole-3-carboxaldehyde was subjected to Corey-Fuchs reaction followed by elimination of the vinylic dibromide with DBU in DMSO following literature procedure. ${ }^{14}$ The alkynyl bromide $\mathbf{s 3}$ underwent coupling with the N-tosyl-but-3-enamine to form $11 \mathbf{v}$ (General Procedure 5). ${ }^{1} \mathbf{H}$ NMR $\left(500 \mathrm{MHz}, \mathrm{CDCl}_{3}\right) \delta$ $7.97(\mathrm{~d}, J=8.3 \mathrm{~Hz}, 1 \mathrm{H}), 7.85(\mathrm{~d}, J=7.9 \mathrm{~Hz}, 2 \mathrm{H}), 7.82-7.74(\mathrm{~m}, 2 \mathrm{H}), 7.68(\mathrm{~d}, J=2.7 \mathrm{~Hz}, 1 \mathrm{H}), 7.49(\mathrm{~d}, J=7.8$ $\mathrm{Hz}, 1 \mathrm{H}), 7.39-7.33(\mathrm{~m}, 3 \mathrm{H}), 7.27-7.22(\mathrm{~m}, 3 \mathrm{H}), 5.76(\mathrm{ddt}, J=17.3,10.7,6.8 \mathrm{~Hz}, 1 \mathrm{H}), 5.10$ (dd, $J=25.4,13.6$ $\mathrm{Hz}, 2 \mathrm{H}), 3.51(\mathrm{t}, J=7.2 \mathrm{~Hz}, 2 \mathrm{H}), 2.54-2.41(\mathrm{~m}, 5 \mathrm{H}), 2.35(\mathrm{~s}, 3 \mathrm{H}) ;{ }^{13} \mathrm{C} \mathrm{NMR}\left(125 \mathrm{MHz}, \mathrm{CDCl}_{3}\right) \delta 145.39,144.81$, 134.93, 134.66, 134.19, 133.60, 131.20, 130.06, 129.85, 128.97, 127.73, 126.98, 125.44, 123.69, 120.46, $117.91,113.58,104.56,85.88,61.93,51.05,32.31,21.70,21.62$; HRMS (ESI) calcd for $\mathrm{C}_{28} \mathrm{H}_{27} \mathrm{~N}_{2} \mathrm{O}_{4} \mathrm{~S}_{2}$ [M + $\mathrm{H}]^{+} 519.112$, found 519.1409 . 


\section{General procedure for synthesis of alkene-chelated ruthenium alkylidenes}

A Schlenk tube was charged with the 1,n-diyne/endiyne or 1,n-enyne $(0.07 \mathrm{mmol}), \mathrm{G}$-II catalyst (50 $\mathrm{mg}$, $0.06 \mathrm{mmol}$ ) and $5 \mathrm{~mL}$ dry $\mathrm{CH}_{2} \mathrm{Cl}_{2}(0.015 \mathrm{M})$ inside a glove box. The Schlenk tube was taken out, degassed, and refilled with argon by freeze, pump, and thaw process (three cycles). The reaction mixture was then heated at $45{ }^{\circ} \mathrm{C}$ for $3 \mathrm{~h}$. After completion, the reaction mixture was concentrated, and the residue was quickly purified by flash chromatography under $\mathrm{N}_{2}$. Details of physical appearance, yield, amount of complex prepared, solvent system used for purification and characterization data for each complex is provided underneath.

Stability and storage of the complexes: Most of the complexes are stored in normal glass vials in freezer $\left(-20^{\circ} \mathrm{C}\right)$ under neat conditions and remains good for few months. The complexes are not stored in solution which might lead to quicker degradation. Some complexes like $10 \mathrm{u}, \mathbf{1 3 f}, \mathbf{1 3 k}, \mathbf{1 3 l}$ and $13 \mathrm{t}$ are relatively less stable and are stored in glove box freezer.

\section{Characterization Data for Alkene-Chelates}

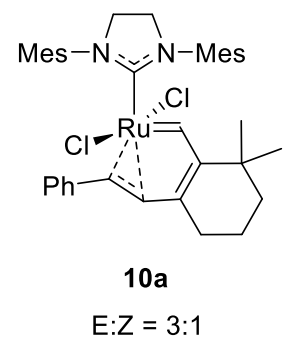

10a: $91 \%$ yield; E/Z-mixture in a 3:1 ratio; red solid; $57 \mathrm{mg}$ obtained; Purification: Flash Chromatography ( $\mathrm{SiO}_{2}$, hexanes-EtOAc, $\left.10: 1 \rightarrow 4: 1\right) ;{ }^{1} \mathbf{H}$ NMR $\left(500 \mathrm{MHz}, \mathrm{CDCl}_{3}\right)$ Eisomer: $\delta 16.56(\mathrm{~s}, 1 \mathrm{H}), 7.20(\mathrm{t}, J=7.1 \mathrm{~Hz}, 1 \mathrm{H}), 7.12-7.02(\mathrm{~m}, 4 \mathrm{H}), 7.01-6.96(\mathrm{~m}, 2 \mathrm{H})$, $6.55(\mathrm{~s}, 2 \mathrm{H}), 5.41(\mathrm{~d}, J=13.0 \mathrm{~Hz}, 1 \mathrm{H}), 4.48(\mathrm{~d}, J=13.0 \mathrm{~Hz}, 1 \mathrm{H}), 4.04-3.85(\mathrm{~m}, 4 \mathrm{H}), 2.54$ $-2.41(\mathrm{~m}, 6 \mathrm{H}), 2.32(\mathrm{~s}, 3 \mathrm{H}), 2.29-2.24(\mathrm{~m}, 6 \mathrm{H}), 2.23(\mathrm{~s}, 3 \mathrm{H}), 1.70-1.59(\mathrm{~m}, 3 \mathrm{H}), 1.35-$ $1.28(\mathrm{~m}, 3 \mathrm{H}), 0.71(\mathrm{~s}, 6 \mathrm{H})$; Z-isomer: $\delta 15.31(\mathrm{~s}, 1 \mathrm{H}), 7.20(\mathrm{t}, J=7.1 \mathrm{~Hz}, 1 \mathrm{H}), 7.12-7.02$ $(\mathrm{m}, 4 \mathrm{H}), 6.99(\mathrm{~s}, 1 \mathrm{H}), 6.83(\mathrm{~s}, 1 \mathrm{H}), 6.79(\mathrm{~s}, 1 \mathrm{H}), 6.41(\mathrm{~s}, 1 \mathrm{H}), 5.70(\mathrm{~d}, J=13.2 \mathrm{~Hz}, 1 \mathrm{H})$, $4.26(\mathrm{~d}, J=13.2 \mathrm{~Hz}, 1 \mathrm{H}), 4.18-4.05(\mathrm{~m}, 2 \mathrm{H}), 4.04-3.85(\mathrm{~m}, 2 \mathrm{H}), 2.82(\mathrm{~s}, 3 \mathrm{H}), 2.32(\mathrm{~s}, 3 \mathrm{H}), 2.26(\mathrm{~s}, 3 \mathrm{H}), 2.39$ $(\mathrm{s}, 3 \mathrm{H}), 2.11(\mathrm{~s}, 3 \mathrm{H}), 2.03(\mathrm{~s}, 3 \mathrm{H}), 1.54-1.49(\mathrm{~m}, 2 \mathrm{H}), 1.43-1.37(\mathrm{~m}, 2 \mathrm{H}), 1.35-1.30(\mathrm{~m}, 2 \mathrm{H}), 0.98(\mathrm{~s}, 3 \mathrm{H})$, 0.91 (s, 3H); ${ }^{13} \mathrm{C} \mathrm{NMR}\left(125 \mathrm{MHz}, \mathrm{CDCl}_{3}\right) \delta$ (all discernable signals for both isomers) 313.52, 312.68, 207.22, 207.13, 167.90, 167.03, 160.43, 158.24, 138.42, 138.19, 137.78, 137.69, 137.63, 137.51, 137.28, 137.13, 137.07, 135.26, 134.78, 134.06, 129.99, 129.94, 129.72, 129.49, 129.17, 128.97, 128.63, 128.55, 126.56, $126.38,126.21,123.31,122.42,107.65,106.77,52.24,51.85,51.72,51.57,38.35,38.13,37.90,34.50$, 33.55, 28.58, 28.24, 27.43, 27.18, 21.15, 21.02, 20.97, 19.93, 19.74, 19.55, 19.33, 19.24, 18.82, 18.58, 18.43; HRMS (ESI) calcd for $\mathrm{C}_{38} \mathrm{H}_{46} \mathrm{~N}_{2} \mathrm{Cl}_{2} \mathrm{Ru}[\mathrm{M}]^{+} 702.2076$ found, 702.2083 .

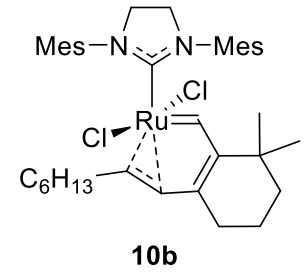

10b: $96 \%$ yield, only E-isomer; red solid; $50 \mathrm{mg}$ obtained; Purification: Flash Chromatography $\left(\mathrm{SiO}_{2}\right.$, hexanes-EtOAc, $\left.10: 1 \rightarrow 5: 1\right) ;{ }^{1} \mathbf{H}$ NMR $\left(500 \mathrm{MHz}, \mathrm{CDCl}_{3}\right) \delta$ $16.53(\mathrm{~s}, 1 \mathrm{H}), 6.96(\mathrm{~s}, 2 \mathrm{H}), 6.92(\mathrm{~s}, 2 \mathrm{H}), 4.79(\mathrm{~d}, \mathrm{~J}=12.9 \mathrm{~Hz}, 1 \mathrm{H}), 4.10(\mathrm{~s}, 4 \mathrm{H}), 3.73(\mathrm{dt}$, $J=13.5,6.9 \mathrm{~Hz}, 1 \mathrm{H}), 2.57(\mathrm{~s}, 6 \mathrm{H}), 2.55-2.37(\mathrm{~m}, 6 \mathrm{H}), 2.29(\mathrm{~s}, 3 \mathrm{H}), 2.27(\mathrm{~s}, 3 \mathrm{H}), 1.60-$ $1.54(\mathrm{~m}, 2 \mathrm{H}), 1.32-1.21(\mathrm{~m}, 5 \mathrm{H}), 1.21-1.07(\mathrm{~m}, 6 \mathrm{H}), 0.96-0.81(\mathrm{~m}, 6 \mathrm{H}), 0.64(\mathrm{~s}, 6 \mathrm{H})$; ${ }^{13} \mathrm{C}$ NMR $\left(125 \mathrm{MHz}, \mathrm{CDCl}_{3}\right) \delta 311.05,208.33,167.21,158.12,139.20,138.77,138.46$, $137.58,136.89,133.84,129.77,129.24,128.00,113.13,52.28,51.15,38.18,33.98,33.47,31.66,30.72$, $30.60,29.10,28.64,22.68,21.02,20.95,20.56,18.69,18.57,14.10 ;$ HRMS (ESI) calcd for $\mathrm{C}_{38} \mathrm{H}_{53} \mathrm{~N}_{2} \mathrm{Ru}$ [M $2 \mathrm{Cl}-\mathrm{H}]^{+} 639.3247$ found, 639.3285 . 


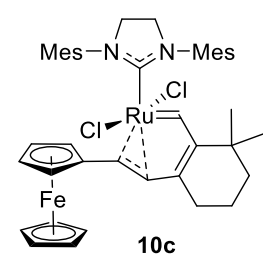

38.4, 34.9, 21.2, 21.0, 20.0, 18.8, 18.7; HRMS (ESI) calcd for $\mathrm{C}_{42} \mathrm{H}_{50} \mathrm{~N}_{2} \mathrm{Cl}_{2} \mathrm{FeRu}[\mathrm{M}]^{+} 810.1738$, found 810.1743 .

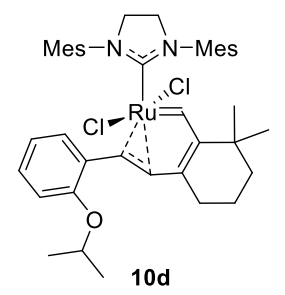

10d: $88 \%$ yield; $E / Z$-mixture in 14:1 ratio; red solid; 55 mg obtained; Purification: Flash Chromatography $\left(\mathrm{SiO}_{2}\right.$, hexanes-EtOAc, 10:1 $\left.\rightarrow 4: 1\right)$; ${ }^{1} \mathrm{H}$ NMR $\left(500 \mathrm{MHz}, \mathrm{CDCl}_{3}\right) \delta 16.65$ $(\mathrm{s}, 1 \mathrm{H}), 7.15(\mathrm{~d}, J=7.1 \mathrm{~Hz}, 1 \mathrm{H}), 7.08(\mathrm{t}, J=7.8 \mathrm{~Hz}, 1 \mathrm{H}), 6.98(\mathrm{~s}, 2 \mathrm{H}), 6.68(\mathrm{~d}, J=8.2 \mathrm{~Hz}$, $1 \mathrm{H}), 6.62(\mathrm{~s}, 2 \mathrm{H}), 6.54(\mathrm{t}, J=7.4 \mathrm{~Hz}, 1 \mathrm{H}), 5.22-5.21(\mathrm{~m}, 2 \mathrm{H}), 4.41-4.38(\mathrm{~m}, 1 \mathrm{H}), 4.02-3.95$ $(\mathrm{m}, 4 \mathrm{H}), 2.49(\mathrm{~s}, 6 \mathrm{H}), 2.32(\mathrm{~s}, 3 \mathrm{H}), 2.30(\mathrm{~s}, 6 \mathrm{H}), 2.26(\mathrm{~s}, 3 \mathrm{H}), 1.72-1.63(\mathrm{~m}, 4 \mathrm{H}), 1.36-1.33$ $(\mathrm{m}, 2 \mathrm{H}), 1.24(\mathrm{~s}, 3 \mathrm{H}), 1.23(\mathrm{~s}, 3 \mathrm{H}), 0.71(\mathrm{~s}, 6 \mathrm{H}) ;{ }^{13} \mathrm{C} \mathrm{NMR}\left(125 \mathrm{MHz}, \mathrm{CDCl}_{3}\right) \delta 312.2,212.3$, $207.5,166.9,160.6,154.8,138.3,137.9,137.5,137.3,134.6,130.0,129.7,127.5$, 126.7, 126.4, 125.1, 121.3, 113.5, 102.0, 70.2, 52.3, 51.9, 38.5, 34.7, 33.6, 28.7, 22.0, 21.2, 21.0, 19.9, 18.9, 18.7; HRMS (ESI) calcd for $\mathrm{C}_{41} \mathrm{H}_{52} \mathrm{Cl}_{2} \mathrm{~N}_{2} \mathrm{ORu}[\mathrm{M}]^{+} 760.2495$, found 760.2486 .

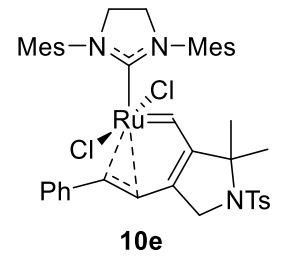

10e: $5 \%$ yield; greenish yellow solid; $3 \mathrm{mg}$ obtained; Purification: Flash Chromatography ( $\mathrm{SiO}_{2}$, hexanes-EtOAc, $\left.10: 1 \rightarrow 3: 1 \rightarrow 1: 1\right) ;{ }^{1} \mathrm{H}$ NMR $\left(500 \mathrm{MHz}, \mathrm{CDCl}_{3}\right)$ $\delta 16.16(\mathrm{~s}, 1 \mathrm{H}), 7.69(\mathrm{~d}, J=8.0 \mathrm{~Hz}, 2 \mathrm{H}), 7.26-7.20(\mathrm{~m}, 3 \mathrm{H}), 7.14(\mathrm{~d}, J=7.6 \mathrm{~Hz}, 2 \mathrm{H}), 7.08$ $(\mathrm{t}, J=7.6 \mathrm{~Hz}, 2 \mathrm{H}), 7.03-6.74(\mathrm{~m}, 2 \mathrm{H}), 6.63-6.40(\mathrm{~m}, 2 \mathrm{H}), 5.35(\mathrm{~d}, J=14.0 \mathrm{~Hz}, 1 \mathrm{H}), 5.06$ $(\mathrm{d}, J=14.0 \mathrm{~Hz}, 1 \mathrm{H}), 4.09-3.74(\mathrm{~m}, 4 \mathrm{H}), 3.13(\mathrm{~s}, 2 \mathrm{H}), 2.69-2.01(\mathrm{~m}, 21 \mathrm{H}), 1.29(\mathrm{~s}, 6 \mathrm{H})$;

${ }^{13} \mathrm{C}$ NMR $\left(125 \mathrm{MHz}, \mathrm{CDCl}_{3}\right) \delta 297.04,203.16,170.77,149.02,143.21,137.69,137.64$, 133.26, 129.66, 129.56, 129.06, 128.64, 127.55, 127.39, 127.27, 126.66, 124.32, 114.27, 69.35, 57.77, 51.83, 35.64, 35.16, 27.85, 27.00, 26.91, 26.63, 26.36, 26.17, 21.48, 21.05, 19.63, 18.68.; HRMS (ESI) calcd for $\mathrm{CHO}[\mathrm{M}+\mathrm{H}]^{+}$; HRMS (ESI) calcd for $\mathrm{C}_{43} \mathrm{H}_{48} \mathrm{~N}_{3} \mathrm{O}_{2} \mathrm{SCl}_{2} \mathrm{Ru}[\mathrm{M}-\mathrm{H}]^{+} 842.1882$, found 842.1920.

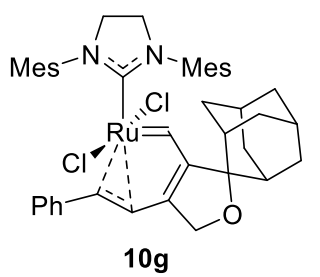

10g: 54\% yield; yellowish brown solid; $42 \mathrm{mg}$ obtained; Purification: Flash Chromatography $\left(\mathrm{SiO}_{2}\right.$, hexanes-EtOAc, $\left.20: 1 \rightarrow 10: 1\right) ;{ }^{1} \mathbf{H}$ NMR $\left(500 \mathrm{MHz}, \mathrm{CDCl}_{3}\right) \delta$ $17.13(\mathrm{~s}, 1 \mathrm{H}), 7.30-7.25(\mathrm{~m}, 1 \mathrm{H}), 7.16(\mathrm{~d}, J=7.7 \mathrm{~Hz}, 2 \mathrm{H}), 7.09(\mathrm{t}, J=7.7 \mathrm{~Hz}, 2 \mathrm{H}), 6.94$ $(\mathrm{s}, 2 \mathrm{H}), 6.51(\mathrm{~s}, 2 \mathrm{H}), 5.29-4.95(\mathrm{~m}, 2 \mathrm{H}), 3.97-3.84(\mathrm{~m}, 4 \mathrm{H}), 3.63(\mathrm{~s}, 2 \mathrm{H}), 2.48(\mathrm{~s}, 6 \mathrm{H})$, 2.33-2.09 (m, 12H), $2.14(\mathrm{~d}, J=12.7 \mathrm{~Hz}, 2 \mathrm{H}), 1.86-1.71(\mathrm{~m}, 4 \mathrm{H}), 1.69-1.63(\mathrm{~m}, 2 \mathrm{H})$, 1.53-1.36 (m, 4H), $0.97(\mathrm{~d}, J=13.3 \mathrm{~Hz}, 2 \mathrm{H}) ;{ }^{13} \mathrm{C}$ NMR $\left(125 \mathrm{MHz}, \mathrm{CDCl}_{3}\right) \delta 300.81$, 204.15, 172.30, 164.64, 138.28, 137.78, 137.08, 136.81, 134.43, 133.69, 130.21, 129.59, 128.65, 127.38, 126.79, 117.04, 112.03, 92.25, 75.15, 53.07, 51.55, 38.23, 37.67, 34.12, 33.32, 27.10, 27.01, 26.92, 26.69, 26.38, 26.18, 21.18, 19.77, 18.79; HRMS (ESI) calcd for $\mathrm{C}_{43} \mathrm{H}_{49} \mathrm{~N}_{2} \mathrm{ORu}[\mathrm{M}-2 \mathrm{Cl}$ $-\mathrm{H}]^{+} 711.2888$, found 711.2900 .

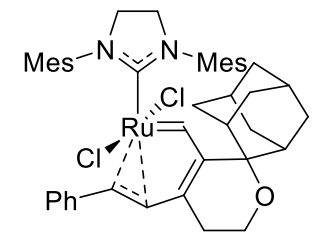

$10 \mathrm{~h}$

10h: 78\% yield; yellowish brown solid; $58 \mathrm{mg}$ obtained; Purification: Flash Chromatography $\left(\mathrm{SiO}_{2}\right.$, hexanes-EtOAc, $\left.10: 1 \rightarrow 4: 1\right) ;{ }^{1} \mathbf{H}$ NMR $\left(500 \mathrm{MHz}, \mathrm{CDCl}_{3}\right) \delta$ $16.89(\mathrm{~s}, 1 \mathrm{H}), 7.22(\mathrm{t}, J=7.3 \mathrm{~Hz}, 1 \mathrm{H}), 7.13-7.00(\mathrm{~m}, 5 \mathrm{H}), 6.84(\mathrm{~s}, 1 \mathrm{H}), 6.55(\mathrm{~s}, 1 \mathrm{H}), 6.41$ $(\mathrm{s}, 1 \mathrm{H}), 5.24(\mathrm{~d}, J=12.3 \mathrm{~Hz}, 1 \mathrm{H}), 4.71(\mathrm{~d}, J=12.3 \mathrm{~Hz}, 1 \mathrm{H}), 4.05-3.69(\mathrm{~m}, 6 \mathrm{H}), 2.79(\mathrm{~s}$, $3 \mathrm{H}), 2.42(\mathrm{~s}, 3 \mathrm{H}), 2.33-2.05(\mathrm{~m}, 14 \mathrm{H}), 1.80-1.68(\mathrm{~m}, 3 \mathrm{H}), 1.66(\mathrm{~s}, 2 \mathrm{H}), 1.64-1.53(\mathrm{~m}$, $2 \mathrm{H}), 1.52-1.31(\mathrm{~m}, 6 \mathrm{H}), 1.11(\mathrm{~d}, J=13.2 \mathrm{~Hz}, 1 \mathrm{H}) ;{ }^{13} \mathrm{C}$ NMR $\left(125 \mathrm{MHz}, \mathrm{CDCl}_{3}\right) \delta 300.81$, 204.15, 172.30, 164.64, 138.28, 137.78, 137.08, 136.81, 134.43, 133.69, 130.21, 
129.59, 128.65, 127.38, 126.79, 117.04, 112.03, 92.25, 75.15, 53.07, 51.55, 38.23, 37.67, 34.12, 33.32, 27.10, 27.01, 26.92, 26.69, 26.38, 26.18, 21.18, 19.77, 18.79; HRMS (ESI) calcd for $\mathrm{C}_{44} \mathrm{H}_{51} \mathrm{~N}_{2} \mathrm{ORu}[\mathrm{M}-2 \mathrm{Cl}$ $-\mathrm{H}]^{+} 725.3045$, found 725.3053 .

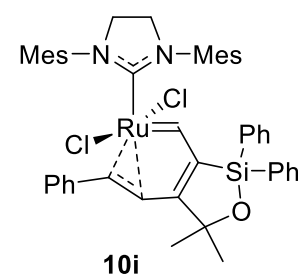

10i: $82 \%$ yield; red solid; $44 \mathrm{mg}$ obtaind; Purification: Flash Chromatography $\left(\mathrm{SiO}_{2}\right.$, hexanes-EtOAc, 10:1 $\rightarrow$ 4:1); ${ }^{1} \mathrm{H}$ NMR $\left(500 \mathrm{MHz}, \mathrm{CDCl}_{3}\right) \delta 16.02(\mathrm{~s}, 1 \mathrm{H}), 7.51-7.49$ $(\mathrm{m}, 2 \mathrm{H}), 7.42(\mathrm{~m}, 8 \mathrm{H}), 7.23(\mathrm{t}, J=7.0 \mathrm{~Hz}, 1 \mathrm{H}), 7.09-7.03(\mathrm{~m}, 4 \mathrm{H}), 6.94(\mathrm{~s}, 2 \mathrm{H}), 6.26$ (bs, $2 \mathrm{H}), 5.77(\mathrm{~d}, J=13.5 \mathrm{~Hz}, 1 \mathrm{H}), 4.79(\mathrm{~d}, J=13.5 \mathrm{~Hz}, 1 \mathrm{H}), 4.02-3.99(\mathrm{~m}, 4 \mathrm{H}), 2.32(\mathrm{~s}, 9 \mathrm{H})$, $2.21(\mathrm{~s}, 6 \mathrm{H}), 2.01(\mathrm{~s}, 3 \mathrm{H}), 1.53(\mathrm{~s}, 6 \mathrm{H}) ;{ }^{13} \mathrm{C}$ NMR $\left(125 \mathrm{MHz}, \mathrm{CDCl}_{3}\right) \delta 311.9,204.9,182.6$, $159.8,138.0,137.9,137.2,137.0,135.1,134.3,133.6,130.6,129.8,129.4,128.8$, 128.0, 126.9, 126.3, 123.7, 107.8, 86.2, 51.8, 51.6, 28.2, 21.3, 21.1, 19.7, 18.8; HRMS (ESI) calcd for $\mathrm{C}_{47} \mathrm{H}_{49} \mathrm{~N}_{2} \mathrm{OSiRu}[\mathrm{M}-2 \mathrm{Cl}-\mathrm{H}]^{+} 787.2652$, found 787.2637.

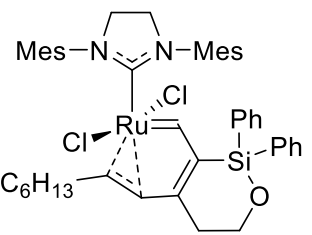

$10 \mathbf{j}$

10j: 93\% yield; red solid; $43 \mathrm{mg}$ obtained; Purification: Flash Chromatography $\left(\mathrm{SiO}_{2}\right.$, hexanes-EtOAc, 10:1 $\rightarrow$ 4:1); ${ }^{1} \mathrm{H}$ NMR $\left(500 \mathrm{MHz}, \mathrm{CDCl}_{3}\right) \delta 16.43(\mathrm{~s}, 1 \mathrm{H}), 7.47(\mathrm{t}, \mathrm{J}=$ $7.4 \mathrm{~Hz}, 2 \mathrm{H}), 7.37(\mathrm{t}, J=7.4 \mathrm{~Hz}, 4 \mathrm{H}), 7.31(\mathrm{~d}, J=7.1 \mathrm{~Hz}, 4 \mathrm{H}), 6.94(\mathrm{~s}, 2 \mathrm{H}), 6.20(\mathrm{~s}, 2 \mathrm{H})$, $5.07(\mathrm{~d}, J=13.0 \mathrm{~Hz}, 1 \mathrm{H}), 4.09-4.05(\mathrm{~m}, 4 \mathrm{H}), 3.97-3.86(\mathrm{~m}, 3 \mathrm{H}), 2.56(\mathrm{~s}, 6 \mathrm{H}), 2.34(\mathrm{~s}$, $3 \mathrm{H}), 2.30(\mathrm{~s}, 6 \mathrm{H}), 2.02(\mathrm{t}, J=4.9 \mathrm{~Hz}, 2 \mathrm{H}), 1.73(\mathrm{~s}, 3 \mathrm{H}), 1.60(\mathrm{~m}, 2 \mathrm{H}), 1.28-1.12(\mathrm{~m}$, $8 \mathrm{H}), 0.88(\mathrm{t}, J=7.2 \mathrm{~Hz}, 3 \mathrm{H}) ;{ }^{13} \mathrm{C}$ NMR $\left(125 \mathrm{MHz}, \mathrm{CDCl}_{3}\right) \delta 312.2,206.7,171.7,155.8$, $139.3,138.9,138.1,136.9,136.4,135.2,133.7,133.4,131.7,130.4,130.0,129.5$, 129.4, 127.0, 112.7, 60.6, 52.3, 51.1, 37.9, 31.7, 30.8, 30.7, 29.1, 27.8, 26.3, 22.7, 20.9, 20.6, 18.8, 14.2; HRMS (ESI) calcd for $\mathrm{C}_{46} \mathrm{H}_{55} \mathrm{~N}_{2} \mathrm{OSiRu}[\mathrm{M}-2 \mathrm{Cl}-\mathrm{H}]^{+} 781.3122$, found 781.3052.

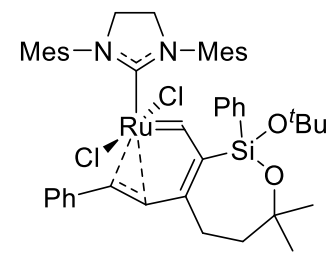

$10 \mathrm{k}$

10k: $83 \%$ yield; red solid; $44 \mathrm{mg}$ obtained; Purification: Flash Chromatography $\left(\mathrm{SiO}_{2}\right.$, hexanes-EtOAc, 10:1 $\rightarrow$ 4:1 $\left.\rightarrow 2: 1\right) ;{ }^{1} \mathrm{H}$ NMR $\left(500 \mathrm{MHz}, \mathrm{CDCl}_{3}\right) \delta 16.07(\mathrm{~s}, 1 \mathrm{H})$, 7.45-7.37 (m, 5H), 7.21-7.19 (m, 1H), 7.06-7.03 (m, 4H), $6.86(\mathrm{~s}, 2 \mathrm{H}), 6.35(\mathrm{~s}, 1 \mathrm{H})$, $5.67(\mathrm{~d}, J=12.6 \mathrm{~Hz}, 1 \mathrm{H}), 5.22(\mathrm{~s}, 1 \mathrm{H}), 4.45(\mathrm{~d}, J=12.6 \mathrm{~Hz}, 1 \mathrm{H}), 4.04-3.86(\mathrm{~m}, 4 \mathrm{H})$, $2.74(\mathrm{~s}, 3 \mathrm{H}), 2.44(\mathrm{~s}, 3 \mathrm{H}), 2.30(\mathrm{~s}, 3 \mathrm{H}), 1.98(\mathrm{~s}, 3 \mathrm{H}), 1.87(\mathrm{~s}, 3 \mathrm{H}), 1.83(\mathrm{~s}, 3 \mathrm{H}), 1.78(\mathrm{~m}$, $2 \mathrm{H}), 1.38(\mathrm{~m}, 5 \mathrm{H}), 1.21(\mathrm{~s}, 9 \mathrm{H}), 1.13(\mathrm{~s}, 3 \mathrm{H}) ;{ }^{13} \mathrm{C} \mathrm{NMR}\left(125 \mathrm{MHz}, \mathrm{CDCl}_{3}\right) \delta 317.5$, 206.4, 179.4, 158.4, 138.6, 138.3, 138.1, 137.6, 137.2, 136.0, 135.6, 134.6, 134.1, 130.2, 129.9, 129.7, 129.4, 129.1, 128.7, 127.4, 126.4, 126.2, 125.7, 104.9, 74.1, 73.5, 51.8, 40.2, 35.5, $31.8,31.7,31.5,31.3,31.2,28.2,27.7,26.6,21.2,21.1,20.1,19.3,19.2,18.4$; HRMS (ESI) calcd for $\mathrm{C}_{47} \mathrm{H}_{58} \mathrm{Cl}_{1} \mathrm{~N}_{2} \mathrm{O}_{2}$ RuSi $[\mathrm{M}-\mathrm{Cl}]^{+} 847.2994$, found 847.2999

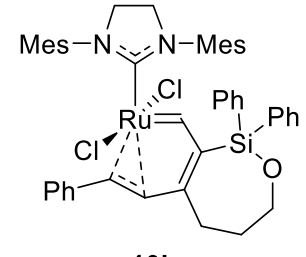

101

10l: $92 \%$ yield; red solid; $47 \mathrm{mg}$ obtained; Purification: Flash Chromatography $\left(\mathrm{SiO}_{2}\right.$, hexanes-EtOAc, 10:1 $\rightarrow$ 4:1); ${ }^{1} \mathbf{H}$ NMR $\left(500 \mathrm{MHz}, \mathrm{CDCl}_{3}\right) \delta 16.38(\mathrm{~s}, 1 \mathrm{H}), 7.50(\mathrm{t}, J=$ $7.2 \mathrm{~Hz}, 2 \mathrm{H}), 7.42(\mathrm{t}, J=7.3 \mathrm{~Hz}, 4 \mathrm{H}), 7.35(\mathrm{~d}, J=7.0 \mathrm{~Hz}, 4 \mathrm{H}), 7.24-7.23(\mathrm{~m}, 1 \mathrm{H}), 7.18$ $(\mathrm{d}, J=7.6 \mathrm{~Hz}, 2 \mathrm{H}), 7.09$ (t, J = $7.53 \mathrm{~Hz}, 2 \mathrm{H}), 6.55$ (bs, 2H), 6.05 (bs, 2H), 5.67 (d, $J=$ $12.7 \mathrm{~Hz}, 1 \mathrm{H}), 4.91(\mathrm{~d}, J=12.7 \mathrm{~Hz}, 1 \mathrm{H}), 3.92(\mathrm{bm}, 6 \mathrm{H}), 2.29-2.24(\mathrm{~m}, 17 \mathrm{H}), 1.94-1.77$ $(\mathrm{m}, 2 \mathrm{H}), 1.46(\mathrm{~s}, 3 \mathrm{H}) ;{ }^{13} \mathrm{C}$ NMR $\left(125 \mathrm{MHz}, \mathrm{CDCl}_{3}\right) \delta 315.1,206.2,181.2,157.5,137.8$, $137.8,136.3,135.3,135.1,134.6,133.9,133.9,130.2,129.8,129.5,128.7,128.3,128.1,128.0,126.8$, 126.6, 109.5, 65.3, 52.3, 51.7, 37.6, 28.9, 26.4, 26.2, 21.2, 20.5, 20.0, 19.9, 18.7; HRMS (ESI) calcd for $\mathrm{C}_{47} \mathrm{H}_{50} \mathrm{Cl}_{1} \mathrm{~N}_{2} \mathrm{OSiRu}[\mathrm{M}-\mathrm{Cl}]^{+} 823.2419$, found 823.2413. 


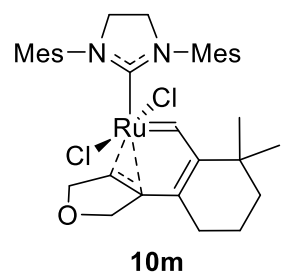

10m: 54\% yield; red solid; 28.5 mg obtained; Purification: Flash Chromatography $\left(\mathrm{SiO}_{2}\right.$, hexanes-EtOAc, 10:1 $\rightarrow$ 4:1 $\rightarrow$ 2:1); ${ }^{1} \mathrm{H}$ NMR $\left(500 \mathrm{MHz}, \mathrm{CDCl}_{3}\right) \delta 16.13(\mathrm{~s}, 1 \mathrm{H}), 7.07$ (s, $1 \mathrm{H}), 6.93(\mathrm{~s}, 1 \mathrm{H}), 6.90(\mathrm{~s}, 1 \mathrm{H}), 6.78(\mathrm{~s}, 1 \mathrm{H}), 5.48(\mathrm{~d}, J=10.0 \mathrm{~Hz}, 1 \mathrm{H}), 4.59(\mathrm{~d}, J=10.0 \mathrm{~Hz}$, $1 \mathrm{H}), 4.20-4.07(\mathrm{~m}, 5 \mathrm{H}), 3.71(\mathrm{~d}, J=11.9 \mathrm{~Hz}, 1 \mathrm{H}), 2.97(\mathrm{~s}, 3 \mathrm{H}), 2.75(\mathrm{~d}, J=11.9 \mathrm{~Hz}, 1 \mathrm{H})$, $2.65(\mathrm{~s}, 3 \mathrm{H}), 2.51(\mathrm{~m}, 3 \mathrm{H}), 2.27(\mathrm{~s}, 3 \mathrm{H}), 2.26(\mathrm{~s}, 3 \mathrm{H}), 2.22(\mathrm{~s}, 3 \mathrm{H}), 1.63-1.54(\mathrm{~m}, 3 \mathrm{H})$, 1.43-1.41 (m, 1H), 1.31-1.20 (m, 2H), $0.86(\mathrm{~s}, 3 \mathrm{H}), 0.36(\mathrm{~s}, 3 \mathrm{H}) ;{ }^{13} \mathrm{C}$ NMR (125 MHz, $\left.\mathrm{CDCl}_{3}\right) \delta 303.1,207.2,167.6,160.7,139.2,139.0,138.4,138.2,137.6,137.5,136.4,133.1,130.0,129.3$, 129.2, 127.5, 104.9, 73.5, 72.8, 52.1, 51.5, 38.2, 33.7, 33.1, 30.0, 28.2, 21.0, 20.9, 20.6, 20.4, 19.3, 18.7, 18.3; HRMS (ESI) calcd for $\mathrm{C}_{34} \mathrm{H}_{43} \mathrm{~N}_{2} \mathrm{ORu}[\mathrm{M}-2 \mathrm{Cl}-\mathrm{H}]^{+} 597.2413$ found, 597.2411.

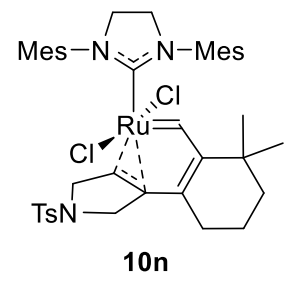

10n: 62\% yield; red solid; $38 \mathrm{mg}$ obtained; Purification: Flash Chromatography $\left(\mathrm{SiO}_{2}\right.$, hexanes-EtOAc, 10:1 $\rightarrow$ 4:1 $\rightarrow$ 1:1); ${ }^{1} \mathrm{H}$ NMR $\left(500 \mathrm{MHz}, \mathrm{CDCl}_{3}\right) \delta 16.10(\mathrm{~s}, 1 \mathrm{H}), 7.68(\mathrm{~d}$, $J=7.9 \mathrm{~Hz}, 2 \mathrm{H}), 7.32(\mathrm{~d}, J=7.9 \mathrm{~Hz}, 2 \mathrm{H}), 7.10(\mathrm{~s}, 1 \mathrm{H}), 7.06(\mathrm{~s}, 1 \mathrm{H}), 6.96(\mathrm{~s}, 1 \mathrm{H}), 6.77(\mathrm{~s}$, $1 \mathrm{H}), 5.00(\mathrm{~d}, J=10.9 \mathrm{~Hz}, 1 \mathrm{H}), 4.23-4.17(\mathrm{~m}, 1 \mathrm{H}), 4.17-4.14(\mathrm{~m}, 1 \mathrm{H}), 4.13-4.10(\mathrm{~m}, 1 \mathrm{H})$, 4.09-4.05 (m, 2H), 4.02-3.99 (m, 1H), $3.81(\mathrm{~d}, J=10.9 \mathrm{~Hz}, 1 \mathrm{H}), 3.05(\mathrm{~d}, J=12.9 \mathrm{~Hz}$, $1 \mathrm{H}), 2.95(\mathrm{~s}, 3 \mathrm{H}), 2.64(\mathrm{~s}, 3 \mathrm{H}), 2.58(\mathrm{~s}, 3 \mathrm{H}), 2.45-2.39(\mathrm{~m}, 5 \mathrm{H}), 2.32(\mathrm{~s}, 3 \mathrm{H}), 2.25(\mathrm{~s}, 3 \mathrm{H})$, $2.19(\mathrm{~s}, 3 \mathrm{H}), 1.49-1.42(\mathrm{~m}, 2 \mathrm{H}), 1.16(\mathrm{dd}, \mathrm{J}=23.5,10.8 \mathrm{~Hz}, 2 \mathrm{H}), 0.78(\mathrm{~s}, 3 \mathrm{H}), 0.34(\mathrm{~s}$, 3H); ${ }^{13} \mathrm{C}$ NMR $\left(125 \mathrm{MHz}, \mathrm{CDCl}_{3}\right) \delta 303.68,205.95,167.30,160.78,143.40,139.65,138.40,137.96,137.54$, $137.48,136.47,132.91,132.60,130.49,130.08,129.62$, 129.56, 129.39, 128.25, 124.36, 53.57, 53.17, 52.08, 51.65, 38.07, 33.62, 33.04, 29.83, 27.83, 21.55, 21.09, 20.89, 20.36, 20.18, 19.28, 18.63, 18.06; HRMS (ESI) calcd for $\mathrm{C}_{41} \mathrm{H}_{52} \mathrm{~N}_{3} \mathrm{O}_{2} \mathrm{SCl}_{2} \mathrm{Ru}[\mathrm{M}+\mathrm{H}]^{+} 822.2195$, found 822.2194.

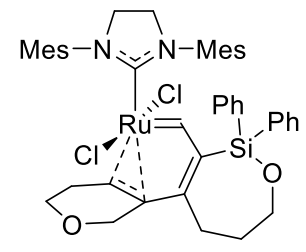

100

100: 84\% yield; red solid; $42 \mathrm{mg}$ obtained; Purification: Flash Chromatography $\left(\mathrm{SiO}_{2}\right.$, hexanes-EtOAc, 10:1 $\rightarrow$ 4:1 $\rightarrow$ 1:1); ${ }^{1} \mathbf{H}$ NMR $\left(500 \mathrm{MHz}, \mathrm{CDCl}_{3}\right) \delta 16.51(\mathrm{~s}, 1 \mathrm{H})$, 7.50-7.44 (m, 2H), 7.41-7.34 (m, 5H), 7.31-7.30 (m, 2H), $6.97(\mathrm{~s}, 1 \mathrm{H}), 6.87(\mathrm{~s}, 1 \mathrm{H})$, $6.30(\mathrm{~s}, 1 \mathrm{H}), 5.89(\mathrm{~s}, 1 \mathrm{H}), 5.45(\mathrm{~d}, J=14.4 \mathrm{~Hz}, 1 \mathrm{H}), 4.60(\mathrm{~d}, J=3.4 \mathrm{~Hz}, 1 \mathrm{H}), 4.52(\mathrm{~d}, J=$ $14.4 \mathrm{~Hz}, 1 \mathrm{H}), 4.16-3.98(\mathrm{~m}, 4 \mathrm{H}), 3.90-3.79(\mathrm{~m}, 3 \mathrm{H}), 3.29(\mathrm{td}, J=11.0,5.4 \mathrm{~Hz}, 1 \mathrm{H})$, 3.05-3.00 (m, 1H), $2.62(\mathrm{~s}, 3 \mathrm{H}), 2.59(\mathrm{~s}, 3 \mathrm{H}), 2.45(\mathrm{~s}, 3 \mathrm{H}), 2.27(\mathrm{~s}, 3 \mathrm{H}), 2.22(\mathrm{~s}, 3 \mathrm{H})$, 2.10-1.90 (m, 4H), 1.66-1.57 (m, 2H), $1.53(\mathrm{~m}, 3 \mathrm{H}) ;{ }^{13} \mathrm{C} \mathrm{NMR}\left(125 \mathrm{MHz}, \mathrm{CDCl}_{3}\right) \delta$ 309.8, 205.0, 177.6, 155.6, 139.7, 139.2, 138.9, 137.7, 136.6, 136.2, 135.6, 135.2, 135.0, 134.5, 133.4, 132.7, 130.2, 130.1, 129.9, 129.1, 127.0, 102.7, 63.7, 62.8, 52.4, 51.0, 34.2, 27.9, 24.7, 21.0, 20.7, 20.6, 20.5, 18.9, 18.7; HRMS (ESI) calcd for $\mathrm{C}_{44} \mathrm{H}_{49} \mathrm{~N}_{2} \mathrm{O}_{2}$ RuSi $[\mathrm{M}-2 \mathrm{Cl}-\mathrm{H}]^{+} 767.2601$, found 767.2575.

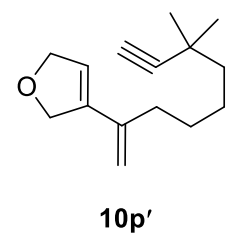

223.2.

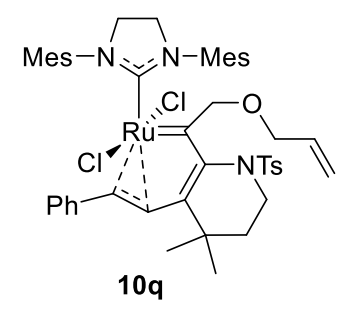

10p': $42 \%$ yield; colorless oil; $8 \mathrm{mg}$ obtained; Purification: Flash Chromatography $\left(\mathrm{SiO}_{2}\right.$, hexanes-EtOAc, 50:1 $\rightarrow$ 20:1); ${ }^{1} \mathrm{H}$ NMR $\left(500 \mathrm{MHz}, \mathrm{CDCl}_{3}\right) \delta 5.84(\mathrm{~s}, 1 \mathrm{H}), 5.00(\mathrm{~s}, 1 \mathrm{H})$, 4.77-4.70 (m, 5H), $2.30(\mathrm{t}, J=7.0,2 \mathrm{H}), 2.08(\mathrm{~s}, 1 \mathrm{H}), 1.52-1.41(\mathrm{~m}, 6 \mathrm{H}), 1.21(\mathrm{~s}, 6 \mathrm{H}) ;{ }^{13} \mathrm{C}$ NMR $\left(125 \mathrm{MHz}, \mathrm{CDCl}_{3}\right) \delta 140.7,121.5,118.6,112.9,92.0,76.6,75.3,67.7,43.0,34.1$, 31.0, 29.7, 29.2, 28.9, 25.2; LRMS (ESI) calcd for $\mathrm{C}_{15} \mathrm{H}_{20} \mathrm{OLi}[\mathrm{M}-2 \mathrm{H}+\mathrm{Li}]^{+} 223.2$, found,

10q: $88 \%$ yield; red solid; $61 \mathrm{mg}$ obtained; Purification: Flash Chromatography $\left(\mathrm{SiO}_{2}\right.$, hexanes-EtOAc, 10:1 $\rightarrow$ 4:1 $\rightarrow \mathrm{CH}_{2} \mathrm{Cl}_{2}$-EtOAc, $\left.1: 1\right) ;{ }^{1} \mathrm{H}$ NMR $\left(500 \mathrm{MHz}, \mathrm{CDCl}_{3}\right)$ $\delta 7.86(\mathrm{~d}, J=8.0 \mathrm{~Hz}, 2 \mathrm{H}), 7.29(\mathrm{~d}, J=8.0 \mathrm{~Hz}, 2 \mathrm{H}), 7.23(\mathrm{t}, J=7.3 \mathrm{~Hz}, 1 \mathrm{H}), 7.07(\mathrm{t}, J=$ $7.6 \mathrm{~Hz}, 2 \mathrm{H}), 7.00(\mathrm{bs}, 1 \mathrm{H}), 6.93(\mathrm{~m}, 4 \mathrm{H}), 6.33(\mathrm{~s}, 1 \mathrm{H}), 5.82(\mathrm{~m}, 1 \mathrm{H}), 5.71(\mathrm{~d}, J=13.4$ $\mathrm{Hz}, 1 \mathrm{H}), 5.21(\mathrm{~s}, 1 \mathrm{H}), 5.18(\mathrm{~s}, 1 \mathrm{H}), 5.08(\mathrm{~m}, 1 \mathrm{H}), 4.10-3.71(\mathrm{~m}, 8 \mathrm{H}), 2.97(\mathrm{dd}, J=20.3$, $7.9 \mathrm{~Hz}, 1 \mathrm{H}), 2.85(\mathrm{~s}, 3 \mathrm{H}), 2.58(\mathrm{~d}, J=15.0 \mathrm{~Hz}, 1 \mathrm{H}), 2.46(\mathrm{~s}, 3 \mathrm{H}), 2.39(\mathrm{~s}, 3 \mathrm{H}), 2.32(\mathrm{~s}$, $6 \mathrm{H}), 2.11(\mathrm{~s}, 3 \mathrm{H}), 1.83(\mathrm{~s}, 3 \mathrm{H}),(\mathrm{d}, J=13.8 \mathrm{~Hz}, 1 \mathrm{H}), 0.85(\mathrm{~s}, 6 \mathrm{H}), 0.81(\mathrm{~d}, J=3.7 \mathrm{~Hz}$, 
1H); ${ }^{13} \mathrm{C}$ NMR $\left(125 \mathrm{MHz}, \mathrm{CDCl}_{3}\right) \delta 318.5,205.7,162.6,155.4,143.3,139.1,138.0,137.7,137.2,136.7$, 136.1, 135.8, 133.9, 131.7, 131.0, 129.6, 129.4, 129.3, 128.8, 128.5, 127.1, 127.0, 115.2, 105.0, 98.7, 85.5, 72.2, 52.5, $52.344 .5,36.8,34.1,30.7,26.4,21.6,21.2,20.4,19.1,18.9,18.8$; HRMS (ESI) calcd for $\mathrm{C}_{48} \mathrm{H}_{57} \mathrm{~N}_{3} \mathrm{O}_{3} \mathrm{SClRu}[\mathrm{M}-\mathrm{Cl}]^{+} 892.2847$ found, 892.2845.

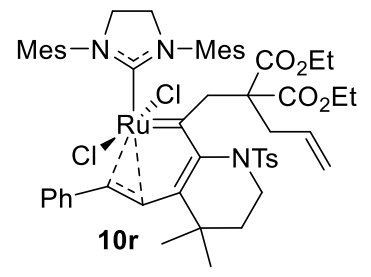

10r: $87 \%$ yield; red solid; $56 \mathrm{mg}$ obtained; Purification: Flash Chromatography $\left(\mathrm{SiO}_{2}\right.$, hexanes-EtOAc, 10:1 $\rightarrow$ 4:1 $\rightarrow \mathrm{CH}_{2} \mathrm{Cl}_{2}$-EtOAc, 1:1); ${ }^{1} \mathbf{H}$ NMR (500 MHz, $\left.\mathrm{CDCl}_{3}\right) \delta 7.92(\mathrm{~d}, J=7.1 \mathrm{~Hz}, 2 \mathrm{H}), 7.29(\mathrm{~d}, J=7.9 \mathrm{~Hz}, 2 \mathrm{H}), 7.19(\mathrm{t}, J=7.2 \mathrm{~Hz}, 1 \mathrm{H})$, 7.04-7.00 (m, 4H), $6.92(\mathrm{~d}, J=7.7 \mathrm{~Hz}, 2 \mathrm{H}), 6.84(\mathrm{~s}, 1 \mathrm{H}), 6.21(\mathrm{~s}, 1 \mathrm{H}), 5.90-5.81(\mathrm{~m}$, $1 \mathrm{H}), 5.66(\mathrm{~d}, J=13.2 \mathrm{~Hz}, 1 \mathrm{H}), 5.04-4.97(\mathrm{~m}, 2 \mathrm{H}), 4.42(\mathrm{~d}, J=15.9 \mathrm{~Hz}, 1 \mathrm{H})$, $4.27-4.16(\mathrm{~m}, 3 \mathrm{H}), 4.05-3.93(\mathrm{~m}, 5 \mathrm{H}), 3.74-3.73(\mathrm{~m}, 1 \mathrm{H}), 3.63(\mathrm{~d}, J=14.6 \mathrm{~Hz}, 1 \mathrm{H})$, 3.25-3.18 (m, 1H), $2.66(\mathrm{~s}, 3 \mathrm{H}), 2.69-2.67(\mathrm{~m}, 1 \mathrm{H}), 2.46(\mathrm{~s}, 3 \mathrm{H}), 2.40(\mathrm{~s}, 3 \mathrm{H}), 2.38(\mathrm{~s}, 3 \mathrm{H}), 2.30-2.27(\mathrm{~m}$, $4 \mathrm{H}), 2.20(\mathrm{~s}, 3 \mathrm{H}), 2.10-2.07(\mathrm{~m}, 1 \mathrm{H}), 1.98(\mathrm{~s}, 3 \mathrm{H}), 1.31-1.24(\mathrm{~m}, 6 \mathrm{H}), 0.91(\mathrm{~s}, 3 \mathrm{H}), 0.83(\mathrm{bm}, 2 \mathrm{H}), 0.71(\mathrm{~s}$, 3H); ${ }^{13} \mathrm{C}$ NMR $\left(125 \mathrm{MHz}, \mathrm{CDCl}_{3}\right) \delta 318.2,205.0,171.1,169.4,163.7,155.9,143.3,138.6,137.7,137.6$, 137.3, 137.0, 136.9, 136.2, 135.5, 133.9, 131.6, 130.7, 129.8, 129.4, 129.3, 128.5, 126.9, 126.6, 117.5, 104.0, 96.2, 61.7, 60.8, 60.7, 55.3, 53.0, 52.1, 45.1, 42.2, 37.2, 33.2, 30.4, 26.0, 21.5, 21.3, 20.5, 19.2, 18.8, 14.1, 13.9; HRMS (ESI) calcd for $\mathrm{C}_{55} \mathrm{H}_{67} \mathrm{~N}_{3} \mathrm{O}_{6} \mathrm{SCl}_{2} \mathrm{Ru}[\mathrm{M}]^{+} 1069.3166$ found, 1069.3180 .

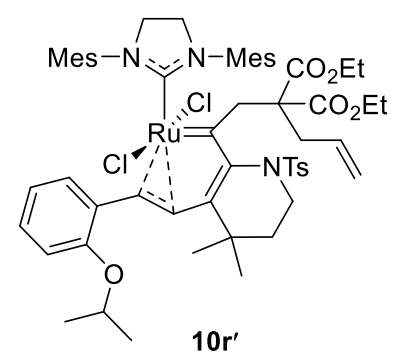

10r': 91\% yield; red solid; $61 \mathrm{mg}$ obtained; Purification: Flash Chromatography $\left(\mathrm{SiO}_{2}\right.$, hexanes-EtOAc, 10:1 $\rightarrow$ 4:1 $\left.\rightarrow \mathrm{CH}_{2} \mathrm{Cl}_{2}-\mathrm{EtOAc}, 1: 1\right) ;{ }^{1} \mathrm{H}$ NMR $(500 \mathrm{MHz}$, $\left.\mathrm{CDCl}_{3}\right) \delta 7.95(\mathrm{~d}, J=7.2 \mathrm{~Hz}, 2 \mathrm{H}), 7.29(\mathrm{~d}, J=8.1 \mathrm{~Hz}, 2 \mathrm{H}), 7.04-7.00(\mathrm{~m}, 1 \mathrm{H}), 6.96$ $(\mathrm{d}, J=2.8 \mathrm{~Hz}, 2 \mathrm{H}), 6.90(\mathrm{~s}, 1 \mathrm{H}), 6.67(\mathrm{~d}, J=8.2 \mathrm{~Hz}, 1 \mathrm{H}), 6.54(\mathrm{~d}, J=7.5 \mathrm{~Hz}, 1 \mathrm{H})$, $6.44(\mathrm{~s}, 1 \mathrm{H}), 6.41(\mathrm{t}, J=7.5 \mathrm{~Hz}, 1 \mathrm{H}), 5.84(\mathrm{dtd}, J=17.2,9.5,5.2 \mathrm{~Hz}, 1 \mathrm{H}), 5.75(\mathrm{~d}$, $J=13.3 \mathrm{~Hz}, 1 \mathrm{H}), 4.99(\mathrm{~d}, J=16.8 \mathrm{~Hz}, 1 \mathrm{H}), 4.92(\mathrm{dd}, J=1.9 \mathrm{~Hz}, 10.1 \mathrm{~Hz}, 1 \mathrm{H})$, 4.77-4.73 (m, 2H), 4.37-4.30 (m, 1H), 4.20-4.11 (m, 4H), 3.99-3.93 (m, 3H), 3.76-3.72 (m, 1H), 3.68-3.61 (m, 1H), 3.19-3.11 (m, 1H), $2.89(\mathrm{~s}, 3 \mathrm{H}), 2.53(\mathrm{~s}$, $3 \mathrm{H}), 2.39-2.34(\mathrm{~m}, 2 \mathrm{H}), 2.38(\mathrm{~s}, 3 \mathrm{H}), 2.36(\mathrm{~s}, 3 \mathrm{H}), 2.30(\mathrm{~s}, 3 \mathrm{H}), 2.28-2.23(\mathrm{~m}, 1 \mathrm{H}), 2.21(\mathrm{~m}, 3 \mathrm{H}), 2.13(\mathrm{~s}, 3 \mathrm{H})$, $1.93(\mathrm{~d}, J=16.2 \mathrm{~Hz}, 1 \mathrm{H}), 1.29(\mathrm{t}, J=7.1 \mathrm{~Hz}, 3 \mathrm{H}), 1.26-1.20(\mathrm{~m}, 6 \mathrm{H}), 1.12(\mathrm{~d}, J=6.1 \mathrm{~Hz}, 3 \mathrm{H}), 0.85-0.78(\mathrm{~m}$, 2H), $0.91(\mathrm{~s}, 3 \mathrm{H}), 0.68(\mathrm{~s}, 3 \mathrm{H}) ;{ }^{13} \mathrm{C}$ NMR $\left(125 \mathrm{MHz}, \mathrm{CDCl}_{3}\right) \delta$ 318.0, 205.1, 171.0, 170.2, 163.2, 156.2, 155.7, 143.3, 138.6, 138.1, 137.6, 137.2, 136.9, 136.7, 135.7, 135.0, 131.5, 130.8, 129.9, 129.5, 129.4, 128.1, 126.3, 121.1, 117.4, 113.7, 107.4, 94.1, 70.6, 61.2, 60.5, 56.7, 53.0, 52.5, 45.3, 40.5, 36.9, 33.0, 30.4, 26.0, 22.5, 22.0, 21.5, 21.3, 20.5, 19.7, 19.5, 18.9, 14.2, 13.8; HRMS (ESI) calcd for $\mathrm{C}_{58} \mathrm{H}_{73} \mathrm{Cl}_{2} \mathrm{~N}_{3} \mathrm{O}{ }_{7} \mathrm{RuS}[\mathrm{M}-\mathrm{H}]^{+}$ 1126.3506 , found 1126.3564 .

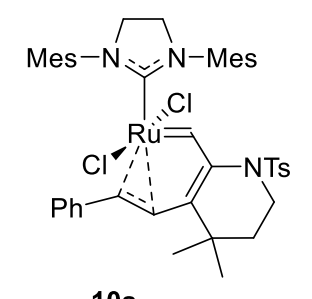

$10 \mathrm{~s}$

10s: $68 \%$ yield; red solid; $35 \mathrm{mg}$ obtained; Purification: Flash Chromatography $\left(\mathrm{SiO}_{2}\right.$, hexanes-EtOAc, 10:1 $\rightarrow$ 4:1 $\rightarrow \mathrm{CH}_{2} \mathrm{Cl}_{2}$-EtOAc, 1:1); ${ }^{1} \mathrm{H}$ NMR $\left(500 \mathrm{MHz}, \mathrm{CDCl}_{3}\right) \delta 16.34$ (s, $1 \mathrm{H}), 7.48(\mathrm{~d}, J=8.1 \mathrm{~Hz}, 2 \mathrm{H}), 7.27-7.25(\mathrm{~m}, 2 \mathrm{H}), 7.20-7.17(\mathrm{~m}, 4 \mathrm{H}), 7.11(\mathrm{t}, J=$ $7.6 \mathrm{~Hz}, 2 \mathrm{H}), 6.89(\mathrm{~s}, 1 \mathrm{H}), 6.55(\mathrm{~s}, 1 \mathrm{H}), 6.52(\mathrm{~s}, 1 \mathrm{H}), 5.23(\mathrm{~d}, J=13.2 \mathrm{~Hz}, 1 \mathrm{H}), 4.72(\mathrm{~d}, J$ $=13.2 \mathrm{~Hz}, 1 \mathrm{H}), 4.11-3.91(\mathrm{~m}, 4 \mathrm{H}), 3.74(\mathrm{td}, J=3.9 \mathrm{~Hz}, \mathrm{~J}=14.4 \mathrm{~Hz}, 1 \mathrm{H}), 3.26-3.20(\mathrm{~m}$, $1 \mathrm{H}), 2.77(\mathrm{~s}, 3 \mathrm{H}), 2.37(\mathrm{~s}, 3 \mathrm{H}), 2.33(\mathrm{~s}, 6 \mathrm{H}), 2.28(\mathrm{~s}, 3 \mathrm{H}), 2.25(\mathrm{~s}, 3 \mathrm{H}), 2.21(\mathrm{~s}, 3 \mathrm{H}), 1.10$ $(\mathrm{s}, 3 \mathrm{H}), 1.04-1.01(\mathrm{~m}, 1 \mathrm{H}), 0.78-0.72(\mathrm{~m}, 1 \mathrm{H}), 0.51(\mathrm{~s}, 3 \mathrm{H}) ;{ }^{13} \mathrm{C} \mathrm{NMR}\left(125 \mathrm{MHz}, \mathrm{CDCl}_{3}\right)$ $\delta$ 311.0, 205.6, 159.8, 152.2, 143.5, 138.7, 138.2, 137.7, 137.2, 137.1, 136.3, 136.1, 135.5, 134.7, 134.2, 131.0, 129.5, 129.4, 129.3, 128.6, 128.5, 127.3, 126.9, 115.2, 112.9, 52.4, 51.8, 44.1, 37.3, 33.8, 27.7, 27.2, 21.6, 21.2, 19.86, 19.81, 19.1, 18.7; HRMS (ESI) calcd for $\mathrm{C}_{44} \mathrm{H}_{51} \mathrm{~N}_{3} \mathrm{O}_{2} \mathrm{SClRu}[\mathrm{M}-\mathrm{Cl}]^{+} 822.2429$, found 822.2429. 


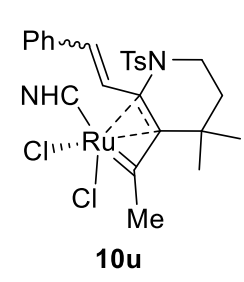

$E: Z=4: 1$

10u: $62 \%$ yield; mixture of $E / Z$-isomers in a $4: 1$ ratio; red solid; $40 \mathrm{mg}$ obtained; Purification: Flash Chromatography Chromatography $\left(\mathrm{SiO}_{2}\right.$, hexanes-EtOAc, 10:1 4:1 $\rightarrow \mathrm{CH}_{2} \mathrm{Cl}_{2}$-EtOAc, 1:1); ${ }^{1} \mathrm{H} \mathrm{NMR}\left(500 \mathrm{MHz}, \mathrm{CDCl}_{3}\right) \boldsymbol{E}$ isomer: $\delta 8.23(\mathrm{~d}, J=8.1 \mathrm{~Hz}, 2 \mathrm{H})$, $7.56(\mathrm{~d}, J=15.6 \mathrm{~Hz}, 1 \mathrm{H}), 7.34(\mathrm{~d}, J=7.2 \mathrm{~Hz}, 2 \mathrm{H}), 7.29-7.21(\mathrm{~m}, 5 \mathrm{H}), 7.06(\mathrm{~s}, 2 \mathrm{H}), 6.94(\mathrm{~s}$, $1 \mathrm{H}), 6.70(\mathrm{~s}, 1 \mathrm{H}), 5.36(\mathrm{~d}, J=15.6 \mathrm{~Hz}, 1 \mathrm{H}), 4.21-4.01(\mathrm{~m}, 4 \mathrm{H}), 3.66-3.53(\mathrm{~m}, 1 \mathrm{H}), 3.33-$ $3.23(\mathrm{~m}, 1 \mathrm{H}), 2.57(\mathrm{~s}, 3 \mathrm{H}), 2.44(\mathrm{~s}, 3 \mathrm{H}), 2.38(\mathrm{~s}, 3 \mathrm{H}), 2.36(\mathrm{~s}, 3 \mathrm{H}), 2.32(\mathrm{~s}, 6 \mathrm{H}), 2.19(\mathrm{~s}, 3 \mathrm{H})$, $1.77(\mathrm{~s}, 3 \mathrm{H}), 1.44(\mathrm{td}, J=13.2,4.3 \mathrm{~Hz}, 1 \mathrm{H}), 0.89(\mathrm{~s}, 3 \mathrm{H}), 0.80(\mathrm{~s}, 3 \mathrm{H}), 0.45-0.34(\mathrm{~m}, 1 \mathrm{H})$; $Z$ isomer: $\delta 8.16(\mathrm{~d}, J=8.0 \mathrm{~Hz}, 2 \mathrm{H}), 7.41(\mathrm{~d}, J=7.7 \mathrm{~Hz}, 2 \mathrm{H}), 7.29-7.20(\mathrm{~m}, 4 \mathrm{H}), 7.09(\mathrm{~s}, 1 \mathrm{H}), 7.06-7.04(\mathrm{~m}$, $1 \mathrm{H}), 7.04(\mathrm{~s}, 1 \mathrm{H}) .7 .02(\mathrm{~s}, 1 \mathrm{H}), 6.92(\mathrm{~s}, 1 \mathrm{H}), 6.05(\mathrm{~d}, J=13.1 \mathrm{~Hz}, 1 \mathrm{H}), 4.55(\mathrm{~d}, J=13.1 \mathrm{~Hz}, 1 \mathrm{H}), 4.39-4.22(\mathrm{~m}$, $4 \mathrm{H}), 3.39-3.32(\mathrm{~m}, 1 \mathrm{H}), 2.97-2.86(\mathrm{~m}, 1 \mathrm{H}), 2.67(\mathrm{~s}, 3 \mathrm{H}), 2.63(\mathrm{~s}, 3 \mathrm{H}), 2.57(\mathrm{~s}, 3 \mathrm{H}), 2.37-2.34(\mathrm{~m}, 9 \mathrm{H}), 2.26$ $(\mathrm{s}, 3 \mathrm{H}), 1.73(\mathrm{~s}, 3 \mathrm{H}), 1.67-1.54(\mathrm{~m}, 2 \mathrm{H}), 0.98(\mathrm{~s}, 3 \mathrm{H}), 0.84(\mathrm{~s}, 3 \mathrm{H}) ;{ }^{13} \mathrm{C} \mathrm{NMR}\left(125 \mathrm{MHz}, \mathrm{CDCl}_{3}\right)$ (all discernable peaks for both isomers) $\delta 258.18,257.30,217.17,214.30,146.29,146.11,140.37,140.12,140.04,139.75$, $139.41,138.99,138.31,137.85,137.80,137.28,137.23,136.80,136.41,136.36,133.37,131.44,131.20$, $130.62,130.47,130.38,130.12,130.11,129.94,129.87,129.73,129.65,129.59,128.86,128.56,128.25$, 128.10, 127.32, 127.12, 84.53, 84.13, 76.53, 76.10, 52.20, 52.13, 51.16, 50.97, 46.96, 46.74, 40.09, 39.54, 29.71, 29.45, 29.39, 29.21, 29.19, 28.66, 21.79, 21.38, 21.34, 21.10, 21.05, 20.13, 20.02, 19.38, 19.29, 18.77, 18.55, 18.48, 3.58, 3.54; HRMS (ESI) calcd for $\mathrm{C}_{45} \mathrm{H}_{52} \mathrm{~N}_{3} \mathrm{O}_{2} \mathrm{SRu}[\mathrm{M}-2 \mathrm{Cl}-\mathrm{H}]^{+} 800.2824$, found 800.2814 .

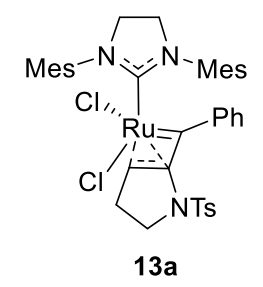

13a: $92 \%$ yield; green solid; $48 \mathrm{mg}$ obtained; Purification: Flash Chromatography $\left(\mathrm{SiO}_{2}\right.$, hexanes-EtOAc, 10:1 $\rightarrow$ 4:1 $\rightarrow \mathrm{CH}_{2} \mathrm{Cl}_{2}$-EtOAc, $\left.1: 1\right) ;{ }^{1} \mathrm{H}$ NMR $\left(500 \mathrm{MHz}, \mathrm{CDCl}_{3}\right) \delta 8.33-$ $7.56(\mathrm{~d}, J=8.0 \mathrm{~Hz}, 2 \mathrm{H}), 7.54(\mathrm{t}, J=7.5 \mathrm{~Hz}, 1 \mathrm{H}), 7.14(\mathrm{t}, J=7.7 \mathrm{~Hz}, 2 \mathrm{H}), 7.03(\mathrm{~s}, 1 \mathrm{H}), 7.00(\mathrm{~s}$, $1 \mathrm{H}), 6.95(\mathrm{~d}, J=8.1 \mathrm{~Hz}, 2 \mathrm{H}), 6.88(\mathrm{~d}, J=7.9 \mathrm{~Hz}, 2 \mathrm{H}), 6.28(\mathrm{~s}, 1 \mathrm{H}), 6.08(\mathrm{~s}, 1 \mathrm{H}), 4.25-4.16$ $(\mathrm{m}, 1 \mathrm{H}), 4.07-3.93(\mathrm{~m}, 2 \mathrm{H}), 3.87-3.58(\mathrm{~m}, 3 \mathrm{H}), 3.54(\mathrm{~d}, J=5.1 \mathrm{~Hz}, 1 \mathrm{H}), 2.62(\mathrm{~s}, 3 \mathrm{H}), 2.61$ $(\mathrm{s}, 3 \mathrm{H})$ 2.41-2.34 (m, 1H), $2.30(\mathrm{~s}, 3 \mathrm{H}), 2.29(\mathrm{~s}, 3 \mathrm{H}) 2.19(\mathrm{~s}, 3 \mathrm{H}), 1.94(\mathrm{~s}, 3 \mathrm{H}), 1.80-1.70$ $(\mathrm{m}, 1 \mathrm{H}), 1.69(\mathrm{~s}, 3 \mathrm{H}),{ }^{13} \mathrm{C}$ NMR $\left(125 \mathrm{MHz}, \mathrm{CDCl}_{3}\right) \delta$ 266.91, 209.92, 144.30, 143.58, 140.48, 139.82, 138.37, 136.96, 135.61, 133.43, 131.05, 130.07, 129.82, 129.44, 129.23, 128.96, 128.85, 128.62, 128.26, 127.92, $114.89,104.90,52.88,52.17,51.77,51.60,27.93,21.59,21.25,20.93,20.58,18.98,18.77,17.71$; HRMS (ESI) calcd for $\mathrm{C}_{39} \mathrm{H}_{42} \mathrm{~N}_{3} \mathrm{O}_{2} \mathrm{RuS}[\mathrm{M}-2 \mathrm{Cl}-\mathrm{H}]^{+} 718.2036$, found 718.2075.

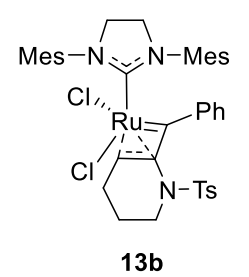

13b: $94 \%$ yield; green solid; $45 \mathrm{mg}$ obtained; Purification: Flash Chromatography $\left(\mathrm{SiO}_{2}\right.$, hexanes-EtOAc, 10:1 $\rightarrow$ 4:1 $\rightarrow \mathrm{CH}_{2} \mathrm{Cl}_{2}$-EtOAc, 1:1); ${ }^{1} \mathrm{H}$ NMR $\left(500 \mathrm{MHz}, \mathrm{CDCl}_{3}\right) \delta 7.64$ (d, $J=8.1 \mathrm{~Hz}, 2 \mathrm{H}), 7.18(\mathrm{~d}, J=8.1 \mathrm{~Hz}, 2 \mathrm{H}), 7.16-7.08(\mathrm{~m}, 2 \mathrm{H}), 7.07-7.01(\mathrm{~m}, 4 \mathrm{H}), 6.99(\mathrm{~s}$, $2 \mathrm{H}), 6.95(\mathrm{~s}, 1 \mathrm{H}), 6.08(\mathrm{t}, J=4.5 \mathrm{~Hz}, 1 \mathrm{H}), 4.30(\mathrm{q}, J=11.1 \mathrm{~Hz}, 1 \mathrm{H}), 4.23-4.12(\mathrm{~m}, 2 \mathrm{H})$, 4.11-4.03 (m, 1H), $3.96(\mathrm{q}, J=11.1 \mathrm{~Hz}, 1 \mathrm{H}), 3.85-3.74(\mathrm{~m}, 1 \mathrm{H}), 3.54-3.27(\mathrm{~m}, 1 \mathrm{H}), 3.01$ $-2.88(\mathrm{~m}, 1 \mathrm{H}), 2.57(\mathrm{~s}, 3 \mathrm{H}), 2.48(\mathrm{~s}, 3 \mathrm{H}), 2.41(\mathrm{~s}, 3 \mathrm{H}), 2.35(\mathrm{~s}, 3 \mathrm{H}), 2.32(\mathrm{~s}, 3 \mathrm{H}), 2.30(\mathrm{~s}$, $3 \mathrm{H}), 1.98(\mathrm{~s}, 3 \mathrm{H}), 1.28-1.24(\mathrm{~m}, 1 \mathrm{H}), 0.67$ (ddd, $J=24.7,13.2,5.4 \mathrm{~Hz}, 1 \mathrm{H}) ;{ }^{13} \mathrm{C} \mathrm{NMR}\left(125 \mathrm{MHz}, \mathrm{CDCl}_{3}\right) \delta$ 280.76, 215.28, 160.37, 154.46, 145.04, 140.66, 140.22, 139.15, 138.34, 137.77, 136.78, 136.58, 133.78, $130.44,130.36,130.06,129.60,129.52,128.49,128.39,126.26,125.60,122.18,52.08,51.29,45.78$, $23.42,21.67,21.33,21.14,20.60,19.36,19.09,18.75,17.17$; HRMS (ESI) calcd for $\mathrm{C}_{40} \mathrm{H}_{45} \mathrm{~N}_{3} \mathrm{O}_{2} \mathrm{SClRu}[\mathrm{M}-$ $\mathrm{Cl}]^{+}$768.1959, found 768.1977. 


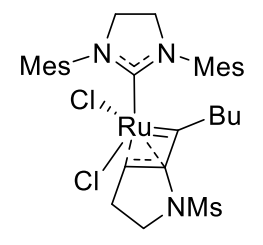

$13 c$

13c: $68 \%$ yield; green solid; $35 \mathrm{mg}$ obtained; Purification: Flash Chromatography $\left(\mathrm{SiO}_{2}\right.$, hexanes-EtOAc, 10:1 $\rightarrow$ 4:1 $\rightarrow \mathrm{CH}_{2} \mathrm{Cl}_{2}$-EtOAc, 1:1); ${ }^{1} \mathrm{H}$ NMR $\left(500 \mathrm{MHz}, \mathrm{CDCl}_{3}\right) \delta 7.05(\mathrm{~s}$, $1 \mathrm{H}), 7.03(\mathrm{~s}, 1 \mathrm{H}), 6.89(\mathrm{~s}, 1 \mathrm{H}), 6.83(\mathrm{~s}, 1 \mathrm{H}), 5.98(\mathrm{t}, J=3.6 \mathrm{~Hz}, 1 \mathrm{H}), 4.26-4.14(\mathrm{~m}, 2 \mathrm{H})$, $4.10-3.97(\mathrm{~m}, 2 \mathrm{H}), 3.80(\mathrm{td}, J=11.2,6.2 \mathrm{~Hz}, 1 \mathrm{H}), 3.63(\mathrm{td}, J=11.1,8.1 \mathrm{~Hz}, 1 \mathrm{H}), 2.76(\mathrm{~s}$, $3 \mathrm{H}), 2.51(\mathrm{~s}, 3 \mathrm{H}), 2.39(\mathrm{~s}, 3 \mathrm{H}), 2.34(\mathrm{~s}, 3 \mathrm{H}), 2.29(\mathrm{~s}, 6 \mathrm{H}), 2.17(\mathrm{~s}, 3 \mathrm{H}), 2.13-2.04(\mathrm{~m}, 2 \mathrm{H})$, 1.72-1.56 (m, 1H), 1.43-1.18 (m, 4H), 1.18-1.09 (m, $1 \mathrm{H}), 0.79(\mathrm{t}, J=7.3 \mathrm{~Hz}, 3 \mathrm{H}){ }^{13} \mathrm{C}$ NMR

$\left(125 \mathrm{MHz}, \mathrm{CDCl}_{3}\right) \delta 284.23,216.99,159.24,139.90,139.80,138.46,138.01,137.93$, 137.21, 136.35, 131.87, 130.29, 129.58, 129.49, 128.52, 103.64, 58.07, 51.58, 51.47, 48.64, 34.50, 29.44, $28.25,22.96,21.32,21.12,20.33,18.63,18.45,18.19,13.66$; HRMS (ESI) calcd for $\mathrm{C}_{31} \mathrm{H}_{42} \mathrm{~N}_{3} \mathrm{O}_{2} \mathrm{SRu}[\mathrm{M}-$ $2 \mathrm{Cl}-\mathrm{H}]^{+} 622.2041$, found 622.2049 .

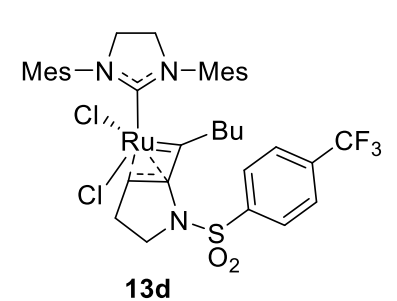

$13 d$

13d: $90 \%$ yield; green solid; $54 \mathrm{mg}$ obtained; Purification: Flash Chromatography $\left(\mathrm{SiO}_{2}\right.$, hexanes-EtOAc, 10:1 $\rightarrow$ 4:1 $\rightarrow \mathrm{CH}_{2} \mathrm{Cl}_{2}-$ EtOAc, 1:1); ${ }^{1} \mathbf{H}$ NMR $\left(500 \mathrm{MHz}, \mathrm{CDCl}_{3}\right) \delta 7.87(\mathrm{~d}, J=8.3 \mathrm{~Hz}, 2 \mathrm{H}), 7.65$ (d, $\left.J=8.4 \mathrm{~Hz}, 2 \mathrm{H}\right), 7.11-$ $7.06(\mathrm{~m}, 1 \mathrm{H}), 7.02-6.97(\mathrm{~m}, 1 \mathrm{H}), 6.91(\mathrm{~s}, 1 \mathrm{H}), 6.87(\mathrm{~s}, 1 \mathrm{H}), 6.10(\mathrm{t}, J=3.6 \mathrm{~Hz}, 1 \mathrm{H})$, $4.42-4.31(\mathrm{~m}, 1 \mathrm{H}), 4.30-4.21(\mathrm{~m}, 2 \mathrm{H}), 4.16(\mathrm{td}, J=10.5,5.2 \mathrm{~Hz}, 1 \mathrm{H}), 4.09-4.00$ $(\mathrm{m}, 1 \mathrm{H}), 3.75(\mathrm{td}, J=11.4,8.7 \mathrm{~Hz}, 1 \mathrm{H}), 3.43(\mathrm{td}, J=11.3,5.0 \mathrm{~Hz}, 1 \mathrm{H}), 2.49(\mathrm{~s}, 6 \mathrm{H})$, $2.44(\mathrm{~s}, 3 \mathrm{H}), 2.33-2.26(\mathrm{~m}, 3 \mathrm{H}), 2.31(\mathrm{~s}, 3 \mathrm{H}), 2.29(\mathrm{~s}, 3 \mathrm{H}), 2.19(\mathrm{~s}, 3 \mathrm{H}), 1.41-1.23$ $(\mathrm{m}, 2 \mathrm{H}), 1.14$ (ddt, $J=19.6,11.1,3.8 \mathrm{~Hz}, 2 \mathrm{H}), 0.86(\mathrm{t}, J=7.3 \mathrm{~Hz}, 3 \mathrm{H}) ;{ }^{13} \mathrm{C}$ NMR $\left(125 \mathrm{MHz}, \mathrm{CDCl}_{3}\right) \delta 284.31$, 216.56, 156.32, 140.10, 139.96, 138.85, 137.97, 137.77, 137.15, 136.96, 136.39, 131.30, 130.42, 129.70, 129.66, 129.20, 128.54, 128.50, 126.27, 126.23, 106.43, 57.61, 51.81, 51.27, 48.38, 29.29, 28.06, 23.07, $21.29,21.10,20.39,18.87,18.64,18.48,13.64 ;{ }^{19} \mathrm{~F} \mathrm{NMR} \mathrm{(376} \mathrm{MHz,} \mathrm{CDCl} 3$ ) $\delta-63.86$; HRMS (ESI) calcd for $\mathrm{C}_{37} \mathrm{H}_{43} \mathrm{~N}_{3} \mathrm{O}_{2} \mathrm{SRuF}_{3}[\mathrm{M}-2 \mathrm{Cl}-\mathrm{H}]^{+} 822.2429$, found 822.2429.

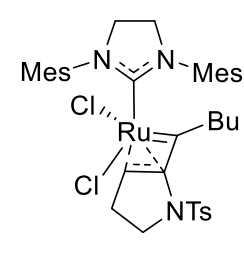

$13 e$

13e: $89 \%$ yield; green solid; $41 \mathrm{mg}$ obtained; Purification: Flash Chromatography $\left(\mathrm{SiO}_{2}\right.$, hexanes-EtOAc, 10:1 $\rightarrow$ 4:1 $\rightarrow \mathrm{CH}_{2} \mathrm{Cl}_{2}$-EtOAc, 1:1); ${ }^{1} \mathrm{H}$ NMR $\left(500 \mathrm{MHz}, \mathrm{CDCl}_{3}\right) \delta 7.57$ (d, J $=8.0 \mathrm{~Hz}, 2 \mathrm{H}), 7.15(\mathrm{~d}, J=8.1 \mathrm{~Hz}, 2 \mathrm{H}), 7.08(\mathrm{~s}, 1 \mathrm{H}), 7.00(\mathrm{~s}, 1 \mathrm{H}), 6.90(\mathrm{~s}, 1 \mathrm{H}), 6.86(\mathrm{~s}, 1 \mathrm{H})$, $6.04(\mathrm{t}, J=3.5 \mathrm{~Hz}, 1 \mathrm{H}), 4.39-4.31(\mathrm{~m}, 1 \mathrm{H}), 4.28-4.19(\mathrm{~m}, 1 \mathrm{H}), 4.14(\mathrm{td}, J=10.6,4.8 \mathrm{~Hz}$, $1 \mathrm{H}), 4.02(\mathrm{q}, J=11.2 \mathrm{~Hz}, 1 \mathrm{H}), 3.67(\mathrm{td}, J=11.3,8.7 \mathrm{~Hz}, 1 \mathrm{H}), 3.43(\mathrm{td}, J=11.3,4.9 \mathrm{~Hz}, 1 \mathrm{H})$, $2.49(\mathrm{~s}, 6 \mathrm{H}), 2.45(\mathrm{~s}, 3 \mathrm{H}), 2.33-2.25(\mathrm{~m}, 13 \mathrm{H}), 2.19(\mathrm{~s}, 3 \mathrm{H}), 1.33(\mathrm{tq}, J=13.6,6.8 \mathrm{~Hz}, 2 \mathrm{H})$, 1.21-1.13 (m, 1H), 1.12-1.03 (ddt, $J=15.5,10.4,4.8 \mathrm{~Hz}, 1 \mathrm{H}), 0.86(\mathrm{t}, J=7.4 \mathrm{~Hz}, 3 \mathrm{H}) ;{ }^{13} \mathrm{C}$ NMR $(126 \mathrm{MHz}$, $\left.\mathrm{CDCl}_{3}\right) \delta 285.42,217.14,156.56,144.94,139.94,139.89,138.79,137.95,137.80,137.15,136.47,131.50$, 130.64, 130.34, 129.69, 129.57, 129.39, 128.65, 128.55, 106.00, 57.48, 51.81, 51.21, 48.21, 29.34, 28.05, 23.11, 21.58, 21.30, 21.09, 20.40, 18.93, 18.69, 18.53, 13.68; HRMS (ESI) calcd for $\mathrm{C}_{37} \mathrm{H}_{46} \mathrm{~N}_{3} \mathrm{O}_{2} \mathrm{SRu}[\mathrm{M}-2 \mathrm{Cl}$ $-\mathrm{H}]^{+}$698.2354, found 698.2364 .

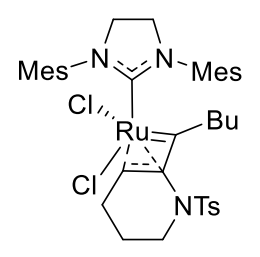

$13 f$

13f: $78 \%$ yield; green solid; 38 mg obtained; Purification: Flash Chromatography $\left(\mathrm{SiO}_{2}\right.$, hexanes-EtOAc, 10:1 $\rightarrow 4: 1 \rightarrow \mathrm{CH}_{2} \mathrm{Cl}_{2}$-EtOAc, 1:1); The ${ }^{1} \mathrm{H}$ NMR for this complex at 298K gave broad signals due to fluxionality in the complex but sharper NMR with distinct signals was obtained at $253 \mathrm{~K} ;{ }^{1} \mathrm{H}$ NMR $\left(400 \mathrm{MHz}, \mathrm{CDCl}_{3}, 253 \mathrm{~K}\right) \delta 7.45(\mathrm{~d}, J=8.1 \mathrm{~Hz}, 2 \mathrm{H})$, $7.15(\mathrm{~s}, 1 \mathrm{H}), 7.07-7.02(\mathrm{~m}, 3 \mathrm{H}), 6.91(\mathrm{~s}, 1 \mathrm{H}), 6.82(\mathrm{~s}, 1 \mathrm{H}), 5.49(\mathrm{t}, J=4.4 \mathrm{~Hz}, 1 \mathrm{H}), 4.54(\mathrm{~d}$, $J=13.3 \mathrm{~Hz}, 1 \mathrm{H}), 4.32-4.08(\mathrm{~m}, 1 \mathrm{H}), 4.07-3.78(\mathrm{~m}, 4 \mathrm{H}), 3.62(\mathrm{t}, J=12.6 \mathrm{~Hz}, 1 \mathrm{H}), 3.32(\mathrm{t}, J$ $=12.9 \mathrm{~Hz}, 1 \mathrm{H}), 2.90(\mathrm{~s}, 3 \mathrm{H}), 2.46(\mathrm{~s}, 6 \mathrm{H}), 2.34(\mathrm{~s}, 3 \mathrm{H}), 2.30(\mathrm{~s}, 3 \mathrm{H}), 2.27(\mathrm{~s}, 3 \mathrm{H}), 2.00-1.89$ $(\mathrm{m}, 2 \mathrm{H}), 1.94(\mathrm{~s}, 3 \mathrm{H}), 1.88-1.73(\mathrm{~m}, 2 \mathrm{H}), 1.51-1.23(\mathrm{~m}, 4 \mathrm{H}), 0.48(\mathrm{t}, J=6.7 \mathrm{~Hz}, 3 \mathrm{H}) ;{ }^{13} \mathrm{C} \mathrm{NMR}(125 \mathrm{MHz}$, $\left.\mathrm{CDCl}_{3}, 298 \mathrm{~K}\right) \delta 318.00,210.60,154.36,145.04,143.94,139.37,138.37,138.16,137.07,135.16,133.68$, $130.33,129.89,129.83,129.68,129.38,129.28,128.93,128.53,127.98,112.35,99.26,65.16,52.89$, 
$50.79,46.62,28.13,25.14,23.31,22.48,21.79,21.46,21.27,21.04,18.67,13.43$; HRMS (ESI) calcd for $\mathrm{C}_{38} \mathrm{H}_{48} \mathrm{~N}_{3} \mathrm{O}_{2} \mathrm{SRu}[\mathrm{M}-2 \mathrm{Cl}-\mathrm{H}]^{+} 712.2511$, found 712.2493. (The reason for the unusual downfield shift of the carbene signal comparable to that of trans-1,5-chelate might be due to combined effects of the presence of alkyl substituent (Bu group) and a 6-membered ring. These two factors usually make the carbene signal downfield shifted for $\eta^{3}$-vinyl alkylidene complexes, for example 13a vs. 13b and 13a vs. 13e).

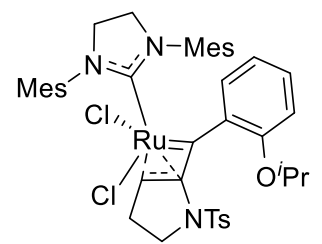

$13 \mathrm{~g}$

13g: 97\% yield; green solid; $58 \mathrm{mg}$ obtained; Purification: Flash Chromatography $\left(\mathrm{SiO}_{2}\right.$, hexanes-EtOAc, 10:1 $\rightarrow$ 4:1 $\rightarrow \mathrm{CH}_{2} \mathrm{Cl}_{2}$-EtOAc, $\left.1: 1\right) ;{ }^{1} \mathrm{H}$ NMR $\left(500 \mathrm{MHz}, \mathrm{CDCl}_{3}\right)$ $\delta 9.11(\mathrm{dd}, J=7.8,1.8 \mathrm{~Hz}, 1 \mathrm{H}), 7.57-7.43(\mathrm{~m}, 1 \mathrm{H}), 7.08(\mathrm{~d}, J=8.1 \mathrm{~Hz}, 2 \mathrm{H}), 7.01-6.84$ $(\mathrm{m}, 4 \mathrm{H}), 6.81(\mathrm{t}, J=7.5 \mathrm{~Hz}, 1 \mathrm{H}), 6.60(\mathrm{~d}, J=8.4 \mathrm{~Hz}, 1 \mathrm{H}), 6.30(\mathrm{~s}, 1 \mathrm{H}), 6.28(\mathrm{~s}, 1 \mathrm{H}), 4.57$ $(p, J=6.1 \mathrm{~Hz}, 1 \mathrm{H}), 4.24(\mathrm{q}, J=10.6 \mathrm{~Hz}, 1 \mathrm{H}), 4.03-3.91(\mathrm{~m}, 1 \mathrm{H}), 3.84-3.78(\mathrm{~m}, 2 \mathrm{H})$, $3.42(\mathrm{dt}, J=11.9,8.5 \mathrm{~Hz}, 1 \mathrm{H}), 3.27(\mathrm{~d}, J=5.3 \mathrm{~Hz}, 1 \mathrm{H}), 2.67(\mathrm{~s}, 3 \mathrm{H}), 2.53(\mathrm{~s}, 3 \mathrm{H}), 2.52$ $(\mathrm{s}, 3 \mathrm{H}), 2.26(\mathrm{~s}, 3 \mathrm{H}), 2.23-2.16(\mathrm{~m}, 1 \mathrm{H}) 2.20(\mathrm{~s}, 4 \mathrm{H}), 1.94(\mathrm{~s}, 3 \mathrm{H}), 1.70(\mathrm{~s}, 3 \mathrm{H}), 1.67-$ $1.60(\mathrm{~m}, 1 \mathrm{H}), 1.59(\mathrm{~d}, J=6.0 \mathrm{~Hz}, 3 \mathrm{H}), 1.55(\mathrm{~d}, J=6.0 \mathrm{~Hz}, 3 \mathrm{H}) ;{ }^{13} \mathrm{C} \mathrm{NMR}\left(125 \mathrm{MHz}, \mathrm{CDCl}_{3}\right) \delta 261.11,209.89$, 143.44, 140.36, 139.91, 137.65, 137.32, 136.96, 135.71, 135.43, 134.35, 133.95, 133.20, 132.42, 131.43, 131.03, 130.02, 129.11, 129.00, 128.94, 121.01, 111.64, 108.77, 71.52, 52.73, 51.49, 51.16, 46.35, 27.12, 22.91, 22.26, 21.66, 21.28, 20.99, 20.63, 19.25, 18.69, 17.95; HRMS (ESI) calcd for $\mathrm{C}_{42} \mathrm{H}_{49} \mathrm{~N}_{3} \mathrm{O}_{3} \mathrm{SClRu}[\mathrm{M}-$ Cl] $]^{+}$812.2227, found 812.2250.

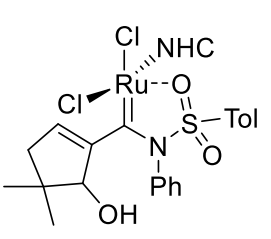

$13 h^{\prime}$

13h': 86\% yield; green solid; $44 \mathrm{mg}$ obtained; Purification: Flash Chromatography $\left(\mathrm{SiO}_{2}\right.$, hexanes-EtOAc, 10:1 $\rightarrow$ 4:1 $\rightarrow \mathrm{CH}_{2} \mathrm{Cl}_{2}$-EtOAc, 1:1); ${ }^{1} \mathrm{H}$ NMR $\left(500 \mathrm{MHz}, \mathrm{CDCl}_{3}\right) \delta 7.76$ $(\mathrm{d}, J=8.2 \mathrm{~Hz}, 2 \mathrm{H}), 7.22-7.14(\mathrm{~m}, 3 \mathrm{H}), 7.10-7.02(\mathrm{~m}, 4 \mathrm{H}), 6.86-6.82(\mathrm{~m}, 2 \mathrm{H}), 6.86-6.57$ $(\mathrm{m}, 2 \mathrm{H}), 5.53(\mathrm{~d}, J=2.0 \mathrm{~Hz}, 1 \mathrm{H}), 4.31-4.04(\mathrm{~m}, 4 \mathrm{H}), 3.90(\mathrm{~s}, 1 \mathrm{H}), 3.62(\mathrm{~d}, J=4.2 \mathrm{~Hz}, 1 \mathrm{H})$, $2.59(\mathrm{~s}, 3 \mathrm{H}), 2.56(\mathrm{~s}, 3 \mathrm{H}), 2.42(\mathrm{~s}, 3 \mathrm{H}), 2.36(\mathrm{~s}, 3 \mathrm{H}), 2.34(\mathrm{~s}, 3 \mathrm{H}), 2.29(\mathrm{~s}, 3 \mathrm{H}), 2.24(\mathrm{~s}, 3 \mathrm{H})$, $1.79(\mathrm{~d}, J=16.6 \mathrm{~Hz}, 1 \mathrm{H}), 1.38(\mathrm{~d}, J=16.4 \mathrm{~Hz}, 1 \mathrm{H}), 0.96(\mathrm{~s}, 3 \mathrm{H}), 0.46(\mathrm{~s}, 3 \mathrm{H}) ;{ }^{13} \mathrm{C} \mathrm{NMR}$ $\left(125 \mathrm{MHz}, \mathrm{CDCl}_{3}\right) \delta 262.6,214.7,153.9,146.2,140.5,139.0,138.4,138.3,137.2,137.0$, 136.3, 132.9, 130.6, 130.5, 130.0, 129.9, 129.7, 129.3, 129.1, 128.5, 127.8, 122.1, 88.1, 52.7, 51.1, 47.2, 41.7, 27.7, 23.5, 21.8, 21.4, 21.1, 20.5, 19.2, 19.0, 18.7; HRMS (ESI) calcd for $\mathrm{C}_{43} \mathrm{H}_{50} \mathrm{~N}_{3} \mathrm{O}_{4} \mathrm{RuS}[\mathrm{M}-2 \mathrm{Cl}+\mathrm{H}$ $+\mathrm{CO}^{+} 806.2560$ found, 806.2483 .

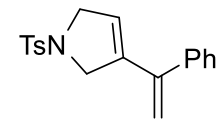

$13 \mathrm{i}^{\prime}-\mathrm{H}$

13i'-H: 52\% yield; pale-yellow oil; Purification: Flash Chromatography $\left(\mathrm{SiO}_{2}\right.$, hexanesEtOAc, 10:1 $\rightarrow 7: 1) ;{ }^{1} \mathrm{H}$ NMR $\left(500 \mathrm{MHz}, \mathrm{CDCl}_{3}\right) \delta 7.76(\mathrm{~d}, J=8.0 \mathrm{~Hz}, 2 \mathrm{H}), 7.35(\mathrm{~d}, J=8.0 \mathrm{~Hz}$, $2 \mathrm{H}), 7.32-7.28(\mathrm{~m}, 3 \mathrm{H}), 7.23-7.18(\mathrm{~m}, 2 \mathrm{H}), 5.46(\mathrm{~s}, 1 \mathrm{H}), 5.17(\mathrm{~s}, 1 \mathrm{H}), 5.07(\mathrm{~s}, 1 \mathrm{H}), 4.35(\mathrm{~s}$, $2 \mathrm{H}), 4.20(\mathrm{~d}, J=3.2 \mathrm{~Hz}, 2 \mathrm{H}), 2.44(\mathrm{~s}, 3 \mathrm{H}) ;{ }^{13} \mathrm{C}$ NMR $\left(125 \mathrm{MHz}, \mathrm{CDCl}_{3}\right) \delta 143.57,143.01$, $140.06,138.35,134.21,129.86,128.50,128.22$, 127.83, 127.53, 124.03, 115.95, 55.58, 54.71, 21.56; HRMS (ESI) calcd for $\mathrm{C}_{19} \mathrm{H}_{20} \mathrm{NO}_{2} \mathrm{~S}[\mathrm{M}+\mathrm{H}]^{+} 326.1215$, found 326.1227 .

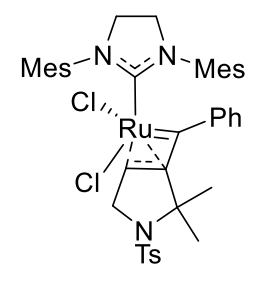

13i

13i: 66\% yield; green solid; 35 mg obtained; Purification: Flash Chromatography $\left(\mathrm{SiO}_{2}\right.$, hexanes-EtOAc, 10:1 $\rightarrow$ 4:1 $\rightarrow \mathrm{CH}_{2} \mathrm{Cl}_{2}$-EtOAc, 1:1); ${ }^{1} \mathrm{H}$ NMR $\left(500 \mathrm{MHz}, \mathrm{CDCl}_{3}\right) \delta 9.13-$ $8.90(\mathrm{~m}, 1 \mathrm{H}), 7.61(\mathrm{~d}, J=7.9 \mathrm{~Hz}, 2 \mathrm{H}), 7.55(\mathrm{t}, J=7.6 \mathrm{~Hz}, 1 \mathrm{H}), 7.24-7.12(\mathrm{~m}, 3 \mathrm{H}), 7.09(\mathrm{~d}, J$ $=7.9 \mathrm{~Hz}, 2 \mathrm{H}), 6.98(\mathrm{~s}, 1 \mathrm{H}), 6.95(\mathrm{~s}, 1 \mathrm{H}), 6.20(\mathrm{~s}, 1 \mathrm{H}), 6.18(\mathrm{~s}, 1 \mathrm{H}), 4.39-4.30(\mathrm{~m}, 1 \mathrm{H}), 4.25$ (q, $J=10.7 \mathrm{~Hz}, 1 \mathrm{H}), 4.15-4.04(\mathrm{~m}, 1 \mathrm{H}), 3.90(\mathrm{dd}, J=13.3,3.8 \mathrm{~Hz}, 1 \mathrm{H}), 3.86-3.73(\mathrm{~m}, 2 \mathrm{H})$, $2.67(\mathrm{~d}, J=13.3 \mathrm{~Hz}, 1 \mathrm{H}), 2.62(\mathrm{~s}, 3 \mathrm{H}), 2.57(\mathrm{~s}, 3 \mathrm{H}), 2.37(\mathrm{~s}, 3 \mathrm{H}), 2.31(\mathrm{~s}, 3 \mathrm{H}), 2.22(\mathrm{~s}, 3 \mathrm{H})$, $2.01(\mathrm{~s}, 3 \mathrm{H}), 1.85(\mathrm{~s}, 3 \mathrm{H}), 1.56(\mathrm{~s}, 3 \mathrm{H}), 1.00(\mathrm{~s}, 3 \mathrm{H}) ;{ }^{13} \mathrm{C} \mathrm{NMR}\left(125 \mathrm{MHz}, \mathrm{CDCl}_{3}\right) \delta 272.81$, $208.75,142.72$, 142.10, 140.08, 139.33, 138.32, 137.58, 137.27, 134.77, 134.44, 133.76, 132.59, 130.99, 
130.27, 130.09, 129.05, 128.89, 128.76, 127.76, 123.72, 94.22, 66.04, 58.64, 52.82, 52.48, 51.86, 32.19, $22.32,21.53,20.95,20.84,20.59,19.10,19.02,17.39$; HRMS (ESI) calcd for $\mathrm{C}_{41} \mathrm{H}_{48} \mathrm{~N}_{3} \mathrm{O}_{2} \mathrm{SRu}[\mathrm{M}-2 \mathrm{Cl}+\mathrm{H}]^{+}$ 748.2511 , found 748.2504 .

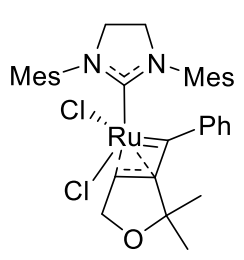

13j

13j: 62\% yield; green solid; $35 \mathrm{mg}$ obtained; Purification: Flash Chromatography $\left(\mathrm{SiO}_{2}\right.$, hexanes-EtOAc, 10:1 $\rightarrow$ 4:1 $\rightarrow \mathrm{CH}_{2} \mathrm{Cl}_{2}$-EtOAc, 1:1); ${ }^{1} \mathbf{H}$ NMR $\left(500 \mathrm{MHz}, \mathrm{CDCl}_{3}\right) \delta 9.64-$ $8.71(\mathrm{~m}, 1 \mathrm{H}), 7.57(\mathrm{t}, J=7.4 \mathrm{~Hz}, 1 \mathrm{H}), 7.40-7.11(\mathrm{~m}, 3 \mathrm{H}), 7.08(\mathrm{~s}, 1 \mathrm{H}), 7.04(\mathrm{~s}, 1 \mathrm{H}), 6.32(\mathrm{~s}$, $1 \mathrm{H}), 6.08(\mathrm{~s}, 1 \mathrm{H}), 4.51(\mathrm{~s}, 1 \mathrm{H}), 4.32-4.10(\mathrm{~m}, 3 \mathrm{H}), 3.88(\mathrm{q}, J=10.9 \mathrm{~Hz}, 1 \mathrm{H}), 3.76(\mathrm{q}, J=$ $11.2 \mathrm{~Hz}, 1 \mathrm{H}), 3.69(\mathrm{~d}, J=12.9 \mathrm{~Hz}, 1 \mathrm{H}), 2.76(\mathrm{~s}, 3 \mathrm{H}), 2.58(\mathrm{~s}, 3 \mathrm{H}), 2.31(\mathrm{~s}, 6 \mathrm{H}), 1.86(\mathrm{~s}, 3 \mathrm{H})$, $1.76(\mathrm{~s}, 3 \mathrm{H}), 1.62(\mathrm{~s}, 3 \mathrm{H}), 0.77(\mathrm{~s}, 3 \mathrm{H}) ;{ }^{13} \mathrm{C} \mathrm{NMR}\left(125 \mathrm{MHz}, \mathrm{CDCl}_{3}\right) \delta 271.77,211.35,141.59$, $140.16,139.33,138.43,137.64,135.17,134.89,134.20,132.64,131.00,130.43,130.08$, 129.36, 128.77, 91.14, 81.19, 70.99, 66.09, 52.30, 52.23, 29.72, 22.90, 21.20, 20.82, 20.51, 19.24, 19.01, 17.75; HRMS (ESI) calcd for $\mathrm{C}_{34} \mathrm{H}_{40} \mathrm{~N}_{2} \mathrm{OCIRu}[\mathrm{M}-\mathrm{Cl}]^{+} 629.1873$, found 629.1879.

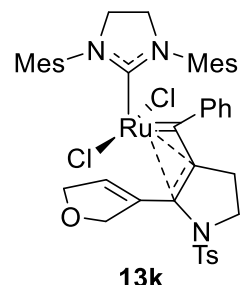

13k: 71\% yield; yellow-brown solid; $34 \mathrm{mg}$ obtained; Purification: Flash Chromatography $\left(\mathrm{SiO}_{2}\right.$, hexanes-EtOAc, 10:1 $\left.\rightarrow 4: 1 \rightarrow \mathrm{CH}_{2} \mathrm{Cl}_{2}-\mathrm{EtOAc}, 1: 1\right)$; ${ }^{1} \mathbf{H} \mathbf{N M R}$ $\left(500 \mathrm{MHz}, \mathrm{CDCl}_{3}\right) \delta 7.65(\mathrm{~d}, J=7.9 \mathrm{~Hz}, 2 \mathrm{H}), 7.28(\mathrm{~d}, J=8.2 \mathrm{~Hz}, 2 \mathrm{H}), 7.14-7.06(\mathrm{~m}, 2 \mathrm{H})$, $6.95(\mathrm{dd}, J=16.6,9.0 \mathrm{~Hz}, 3 \mathrm{H}), 6.86(\mathrm{~s}, 1 \mathrm{H}), 6.45(\mathrm{~s}, 1 \mathrm{H}), 6.29(\mathrm{~s}, 1 \mathrm{H}), 6.27(\mathrm{~s}, 1 \mathrm{H}), 4.92$ $(\mathrm{d}, J=12.0 \mathrm{~Hz}, 1 \mathrm{H}), 4.67(\mathrm{~s}, 1 \mathrm{H}), 4.49(\mathrm{~d}, J=12.2 \mathrm{~Hz}, 1 \mathrm{H}), 4.22(\mathrm{~d}, J=18.3 \mathrm{~Hz}, 1 \mathrm{H}), 4.04-$ $3.87(\mathrm{~m}, 4 \mathrm{H}), 3.79(\mathrm{dt}, J=16.5,9.0 \mathrm{~Hz}, 2 \mathrm{H}), 3.38(\mathrm{q}, J=11.5 \mathrm{~Hz}, 1 \mathrm{H}), 2.77(\mathrm{~s}, 3 \mathrm{H}), 2.62$ (s, 3H), 2.49-2.40 (m, 4H), $2.34(\mathrm{~s}, 3 \mathrm{H}), 2.30(\mathrm{~s}, 3 \mathrm{H}), 2.24(\mathrm{~s}, 3 \mathrm{H}), 1.81(\mathrm{~s}, 3 \mathrm{H}), 1.50(\mathrm{~d}, J=18.3 \mathrm{~Hz}, 1 \mathrm{H}) ;{ }^{13} \mathrm{C}$ NMR $\left(125 \mathrm{MHz}, \mathrm{CDCl}_{3}\right) \delta 282.17,203.93,169.24,144.91,139.18,139.02,138.86,138.26,138.13,137.39$, 136.65, 134.59, 134.22, 133.17, 130.94, 130.21, 129.91, 129.38, 128.80, 128.17, 127.64, 125.57, 112.24, $96.11,87.55,69.76,53.10,52.84,51.91,26.84,21.74,21.19,21.12,20.51,19.25,19.08,18.75 ;$ HRMS (ESI) calcd for $\mathrm{C}_{43} \mathrm{H}_{46} \mathrm{~N}_{3} \mathrm{O}_{3} \mathrm{SRu}[\mathrm{M}-2 \mathrm{Cl}-\mathrm{H}]^{+} 786.2298$ found, 786.2277 .

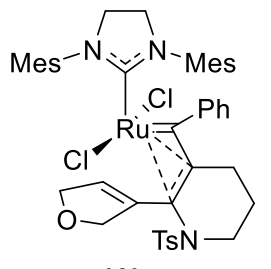

13I: 78\% yield; yellow-brown solid; $44 \mathrm{mg}$ obtained; Purification: Flash Chromatography $\left(\mathrm{SiO}_{2}\right.$, hexanes-EtOAc, 10:1 $\rightarrow 4: 1 \rightarrow \mathrm{CH}_{2} \mathrm{Cl}_{2}$-EtOAc, 1:1); ${ }^{1} \mathbf{H}$ NMR $\left(500 \mathrm{MHz}, \mathrm{CDCl}_{3}\right) \delta 7.72(\mathrm{~d}, J=8.1 \mathrm{~Hz}, 2 \mathrm{H}), 7.47(\mathrm{~d}, J=7.3 \mathrm{~Hz}, 2 \mathrm{H}), 7.37-7.33(\mathrm{~m}, 3 \mathrm{H})$, 7.29-7.27 (m, 2H), $7.11(\mathrm{~s}, 1 \mathrm{H}), 7.06(\mathrm{~s}, 1 \mathrm{H}), 6.98(\mathrm{~s}, 1 \mathrm{H}), 6.83(\mathrm{~s}, 1 \mathrm{H}), 4.64-4.57(\mathrm{~m}$, $2 \mathrm{H}), 4.38-4.26(\mathrm{~m}, 1 \mathrm{H}), 4.20-3.98(\mathrm{~m}, 4 \mathrm{H}), 3.96(\mathrm{~s}, 1 \mathrm{H}), 3.65(\mathrm{td}, J=13.7,4.3 \mathrm{~Hz}, 1 \mathrm{H})$, $3.09(\mathrm{dt}, J=14.9,7.6 \mathrm{~Hz}, 1 \mathrm{H}), 2.82(\mathrm{~d}, J=12.3 \mathrm{~Hz}, 1 \mathrm{H}), 2.70(\mathrm{~s}, 3 \mathrm{H}), 2.64(\mathrm{~s}, 3 \mathrm{H}), 2.63-$ $2.60(\mathrm{~m}, 1 \mathrm{H}), 2.57(\mathrm{~s}, 3 \mathrm{H}), 2.53-2.48(\mathrm{~m}, 1 \mathrm{H}), 2.41(\mathrm{~s}, 3 \mathrm{H}), 2.35-2.28(\mathrm{~m}, 5 \mathrm{H}), 2.22(\mathrm{~s}, 3 \mathrm{H}), 1.46(\mathrm{~s}, 3 \mathrm{H}) ;{ }^{13} \mathrm{C}$ NMR $\left(125 \mathrm{MHz}, \mathrm{CDCl}_{3}\right) \delta$ 231.82, 207.22, 146.67, 139.87, 138.86, 137.82, 137.62, 134.92, 134.89, 134.27, 133.99, 131.65, 131.48, 131.02, 130.63, 129.96, 129.77, 128.47, 128.40, 127.67, 124.12, 116.89, 89.55, 80.97, 75.90, 73.61, 67.03, 66.06, 52.18, 52.13, 52.09, 26.66, 21.79, 21.08, 20.91, 20.72, 19.73, 18.82, 17.32, 17.18; HRMS (ESI) calcd for $\mathrm{C}_{44} \mathrm{H}_{48} \mathrm{~N}_{3} \mathrm{O}_{3} \mathrm{SRu}[\mathrm{M}-2 \mathrm{Cl}-\mathrm{H}]^{+} 800.2460$, found 800.2452. 


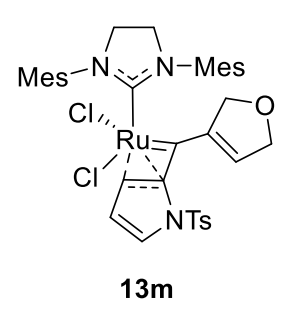

13m: This compound was obtained as a 3:1 mixture with sulfonamide chelate byproduct; 95\% yield; green solid; $62 \mathrm{mg}$ obtained; Purification: Flash Chromatography $\left(\mathrm{SiO}_{2}\right.$, hexanes-EtOAc, 10:1 $\rightarrow$ 4:1 $\rightarrow \mathrm{CH}_{2} \mathrm{Cl}_{2}$-EtOAc, $\left.1: 1\right) ;{ }^{1} \mathbf{H}$ NMR $\left(500 \mathrm{MHz}, \mathrm{CDCl}_{3}\right) \delta$ (the spectral description for only the alkene chelate is given) 8.62 (dd, $J=4.0,1.7 \mathrm{~Hz}, 1 \mathrm{H}), 7.38(\mathrm{dd}, J=3.2,1.7 \mathrm{~Hz}, 1 \mathrm{H}), 7.25-7.18(\mathrm{~m}, 4 \mathrm{H}), 7.11(\mathrm{~s}, 1 \mathrm{H})$, $7.05(\mathrm{~d}, J=5.4 \mathrm{~Hz}, 2 \mathrm{H}), 6.57(\mathrm{~s}, 1 \mathrm{H}), 6.51(\mathrm{~s}, 1 \mathrm{H}), 6.30(\mathrm{t}, \mathrm{J}=3.5 \mathrm{~Hz}, 1 \mathrm{H}), 4.24-4.09(\mathrm{~m}$, $3 \mathrm{H}), 4.00-3.87(\mathrm{~m}, 5 \mathrm{H}), 2.69(\mathrm{~s}, 3 \mathrm{H}), 2.61(\mathrm{~s}, 3 \mathrm{H}), 2.56(\mathrm{~s}, 3 \mathrm{H}), 2.36(\mathrm{~s}, 3 \mathrm{H}), 2.30(\mathrm{~s}, 3 \mathrm{H})$, $2.06(\mathrm{~s}, 3 \mathrm{H}), 1.70(\mathrm{~s}, 3 \mathrm{H}) ;{ }^{13} \mathrm{C} \mathrm{NMR}\left(125 \mathrm{MHz}, \mathrm{CDCl}_{3}\right) \delta$ (all discernable signals for product and byproduct) 249.76, 209.16, 146.39, 145.41, 140.97, 140.52, 140.29, 139.13, 138.20, 137.46, 137.34, 136.86, 135.65, $135.12,134.98,134.26,132.66,131.21,130.57,130.40,130.07,129.96,129.71,129.47,129.39,129.28$, 127.46, 125.06, 124.78, 124.41, 118.87, 117.44, 112.05, 109.06, 88.15, 76.88, 76.64, 74.20, 66.46, 63.51, 52.50, 51.91, 51.30, 51.24, 26.49, 26.37, 26.16, 26.03, 21.57, 21.16, 20.90, 20.65, 19.26, 18.99, 18.96, 18.87, 18.83, 17.56; HRMS (ESI) calcd for $\mathrm{C}_{37} \mathrm{H}_{42} \mathrm{~N}_{3} \mathrm{O}_{3} \mathrm{SRu}[\mathrm{M}-2 \mathrm{Cl}+\mathrm{H}]^{+} 710.1990$ found, 710.1965.

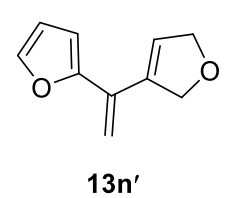

13n': $58 \%$ yield; colorless oil; $10 \mathrm{mg}$ obtained; Purification: Flash Chromatography $\left(\mathrm{SiO}_{2}\right.$, hexanes-EtOAc, 40:1 $\rightarrow$ 20:1); ${ }^{1} \mathrm{H}$ NMR $\left(500 \mathrm{MHz}, \mathrm{CDCl}_{3}\right) \delta 7.42(\mathrm{~d}, J=1.9 \mathrm{~Hz}, 1 \mathrm{H}), 6.46(\mathrm{~d}$, $J=3.2 \mathrm{~Hz}, 1 \mathrm{H}), 6.42(\mathrm{dd}, J=3.5,1.8 \mathrm{~Hz}, 1 \mathrm{H}), 6.22-6.17(\mathrm{~m}, 1 \mathrm{H}), 5.60(\mathrm{~s}, 1 \mathrm{H}), 5.02(\mathrm{~s}, 1 \mathrm{H})$, 4.89-4.84 (m, 2H), 4.84-4.78 (m, 2H); $\left.{ }^{13} \mathrm{C} \mathrm{NMR} \mathrm{(125} \mathrm{MHz,} \mathrm{CDCl}_{3}\right) \delta 143.17,142.02,136.25$, $131.06,124.84,113.18,111.08,107.89,76.88,75.75$; LRMS (EI) calcd for $\mathrm{C}_{10} \mathrm{H}_{10} \mathrm{O}_{2}[\mathrm{M}]^{+}$ 162.0, found 162.0 .

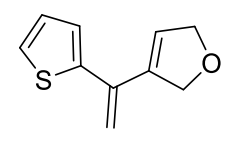

$130^{\prime}$

13o': 76\% yield; yellow oil; $16 \mathrm{mg}$ obtained; Purification: Flash Chromatography $\left(\mathrm{SiO}_{2}\right.$, hexanes-EtOAc, 40:1 $\rightarrow$ 20:1); ${ }^{1} \mathrm{H}$ NMR $\left(500 \mathrm{MHz}, \mathrm{CDCl}_{3}\right) \delta 7.25(\mathrm{~d}, J=5.5 \mathrm{~Hz}, 1 \mathrm{H}), 7.12$ (d, J = 3.5 Hz, 1H), $7.03(\mathrm{~d}, J=4.3 \mathrm{~Hz}, 1 \mathrm{H}), 6.09(\mathrm{~s}, 1 \mathrm{H}), 5.40(\mathrm{~s}, 1 \mathrm{H}), 5.00(\mathrm{~s}, 1 \mathrm{H}), 4.87$ (d, J $=5.0 \mathrm{~Hz}, 2 \mathrm{H}), 4.81(\mathrm{~d}, J=4.6 \mathrm{~Hz}, 2 \mathrm{H}) ;{ }^{13} \mathrm{C}$ NMR $\left(125 \mathrm{MHz}, \mathrm{CDCl}_{3}\right) \delta 143.17,142.02,136.25$, 131.06, 124.84, 113.18, 111.08, 107.89, 76.88, 75.75; LRMS (EI) calcd for $\mathrm{C}_{10} \mathrm{H}_{10} \mathrm{OS}[\mathrm{M}]^{+}$178.0, found 178.0 .

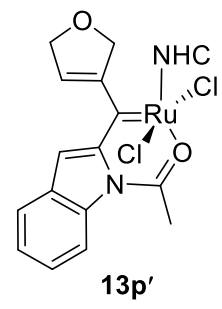

13p': 52\% yield; green solid; $36 \mathrm{mg}$ obtained; Purification: Flash Chromatography $\left(\mathrm{SiO}_{2}\right.$, hexanes-EtOAc, 10:1 $\rightarrow$ 4:1 $\rightarrow \mathrm{CH}_{2} \mathrm{Cl}_{2}$-EtOAc, $\left.1: 1\right) ;{ }^{1} \mathrm{H}$ NMR $\left(500 \mathrm{MHz}, \mathrm{CDCl}_{3}\right) \delta 7.79$ (d, $J=$ $7.7 \mathrm{~Hz}, 1 \mathrm{H}), 7.71(\mathrm{~d}, J=7.5 \mathrm{~Hz}, 1 \mathrm{H}), 7.67(\mathrm{~d}, J=8.4 \mathrm{~Hz}, 1 \mathrm{H}), 7.42(\mathrm{t}, J=7.5 \mathrm{~Hz}, 1 \mathrm{H}), 7.20(\mathrm{~s}$, $1 \mathrm{H}), 7.11(\mathrm{~s}, 1 \mathrm{H}), 6.89(\mathrm{~s}, 1 \mathrm{H}), 6.84(\mathrm{~s}, 1 \mathrm{H}), 5.52-5.32(\mathrm{~m}, 3 \mathrm{H}), 4.76(\mathrm{~s}, 1 \mathrm{H}), 4.66(\mathrm{~d}, J=13.5$ $\mathrm{Hz}, 2 \mathrm{H}), 4.31-4.07(\mathrm{~m}, 2 \mathrm{H}), 3.87(\mathrm{~d}, J=11.0 \mathrm{~Hz}, 2 \mathrm{H}), 3.02(\mathrm{~s}, 3 \mathrm{H}), 2.65(\mathrm{~s}, 3 \mathrm{H}), 2.49(\mathrm{~s}, 3 \mathrm{H})$, $2.38(\mathrm{~s}, 3 \mathrm{H}), 2.14(\mathrm{~s}, 3 \mathrm{H}), 2.04(\mathrm{~s}, 3 \mathrm{H}), 1.70(\mathrm{~s}, 3 \mathrm{H}) ;{ }^{13} \mathrm{C} \mathrm{NMR}\left(125 \mathrm{MHz}, \mathrm{CDCl}_{3}\right) \delta 216.06$, $177.22,159.29,144.37,140.39,138.00,136.75,135.54,133.92,132.15,130.80,129.80$, 129.69, 128.22, 126.67, 126.05, 123.86, 115.64, 113.09, 101.04, 76.93, 51.59, 50.74, 26.86, 26.38, 21.37, 20.96, 20.40, 18.85, 18.62, 17.04; HRMS (ESI) calcd for $\mathrm{C}_{36} \mathrm{H}_{39} \mathrm{~N}_{3} \mathrm{O}_{2} \mathrm{ClRu}[\mathrm{M}-\mathrm{Cl}]^{+} 682.1774$ found, 682.1782 . 


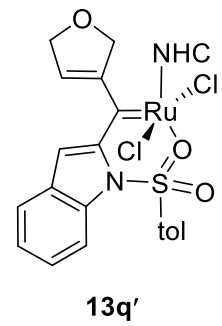

13q': 76\% yield; green solid; $41 \mathrm{mg}$ obtained; Purification: Flash Chromatography $\left(\mathrm{SiO}_{2}\right.$, hexanes-EtOAc, 10:1 $\rightarrow$ 4:1 $\rightarrow \mathrm{CH}_{2} \mathrm{Cl}_{2}$-EtOAc, 1:1); ${ }^{1} \mathbf{H}$ NMR $\left(500 \mathrm{MHz}, \mathrm{CDCl}_{3}\right) \delta 7.97$ (d, $J$ $=8.4 \mathrm{~Hz}, 1 \mathrm{H}), 7.70(\mathrm{t}, J=7.9 \mathrm{~Hz}, 1 \mathrm{H}), 7.61(\mathrm{~d}, J=7.7 \mathrm{~Hz}, 1 \mathrm{H}), 7.51(\mathrm{~d}, J=8.0 \mathrm{~Hz}, 2 \mathrm{H}), 7.33-$ $7.25(\mathrm{~m}, 1 \mathrm{H}), 7.21(\mathrm{~s}, 1 \mathrm{H}), 7.13(\mathrm{~s}, 1 \mathrm{H}), 7.06(\mathrm{~s}, 1 \mathrm{H}), 6.96(\mathrm{~d}, J=8.1 \mathrm{~Hz}, 2 \mathrm{H}), 6.75(\mathrm{~s}, 1 \mathrm{H})$, 5.62-5.47 (m, 2H), $5.36(\mathrm{~s}, 1 \mathrm{H}), 4.84-4.67(\mathrm{~m}, 2 \mathrm{H}), 4.59(\mathrm{t}, J=8.3 \mathrm{~Hz}, 1 \mathrm{H}), 4.38-4.24(\mathrm{~m}$, $2 \mathrm{H}), 4.15(\mathrm{td}, J=11.3,5.8 \mathrm{~Hz}, 1 \mathrm{H}), 3.98(\mathrm{q}, J=11.2 \mathrm{~Hz}, 1 \mathrm{H}), 2.57(\mathrm{~s}, 3 \mathrm{H}), 2.46(\mathrm{~s}, 3 \mathrm{H}), 2.35$ $(\mathrm{s}, 3 \mathrm{H}), 2.24(\mathrm{~s}, 3 \mathrm{H}), 2.15(\mathrm{~s}, 3 \mathrm{H}), 2.09(\mathrm{~s}, 3 \mathrm{H}), 1.97(\mathrm{~s}, 3 \mathrm{H}) ;{ }^{13} \mathrm{C}$ NMR $\left(125 \mathrm{MHz}, \mathrm{CDCl}_{3}\right) \delta$ 259.98, 213.79, 160.53, 155.56, 146.18, 140.69, 140.51, 138.05, 137.59, 137.40, 137.17, 135.73, 132.78, $131.13,130.56,130.15,129.84,129.63,129.08,128.50,127.54,126.78,126.52,125.21,122.66,116.50$, 113.01, 105.24, 76.91, 76.70, 51.25, 51.21, 21.54, 21.37, 20.93, 20.44, 18.89, 18.54; HRMS (ESI) calcd for $\mathrm{C}_{41} \mathrm{H}_{43} \mathrm{~N}_{3} \mathrm{O}_{3} \mathrm{SClRu}[\mathrm{M}-\mathrm{Cl}]^{+} 794.1757$ found, 794.1763.

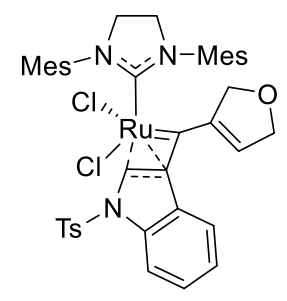

$13 r$

13r: The reaction was performed at $35^{\circ} \mathrm{C}$ for $1 \mathrm{~h}$. This compound was isolated in $40 \%$ yield alongwith $42 \%$ (NMR yield) metathesis product; green solid; $38 \mathrm{mg}$ obtained; Purification: Flash Chromatography $\left(\mathrm{SiO}_{2}\right.$, hexanes-EtOAc, 10:1 $\rightarrow 4: 1 \rightarrow \mathrm{CH}_{2} \mathrm{Cl}_{2}-$ EtOAc, 1:1); The ${ }^{1} \mathrm{H}$ NMR for this complex at $298 \mathrm{~K}$ gave broad signals due to fluxionality in the complex but sharper NMR with distinct signals was obtained at $253 \mathrm{~K} ;{ }^{1} \mathbf{H} \mathbf{N M R}$ (400 MHz, $\left.\mathrm{CDCl}_{3}, 253 \mathrm{~K}\right) \delta 9.43(\mathrm{~s}, 1 \mathrm{H}), 8.05-7.76(\mathrm{~m}, 3 \mathrm{H}), 7.44-7.36(\mathrm{~m}, 3 \mathrm{H}), 7.32(\mathrm{~d}, J$ $=8.1 \mathrm{~Hz}, 2 \mathrm{H}), 7.13(\mathrm{~s}, 1 \mathrm{H}), 7.12(\mathrm{~s}, 1 \mathrm{H}), 6.49(\mathrm{~s}, 1 \mathrm{H}), 5.76(\mathrm{~s}, 1 \mathrm{H}), 4.61-4.51(\mathrm{~m}, 2 \mathrm{H})$, $4.46(\mathrm{~s}, 1 \mathrm{H}), 4.27(\mathrm{td}, J=30.9,28.1,9.8 \mathrm{~Hz}, 3 \mathrm{H}), 3.88(\mathrm{~d}, J=12.3 \mathrm{~Hz}, 3 \mathrm{H}), 2.70(\mathrm{~s}, 3 \mathrm{H}), 2.66(\mathrm{~s}, 3 \mathrm{H}), 2.57(\mathrm{~s}$, $3 \mathrm{H}), 2.37$ (s, 3H), 2.33 (s, 3H), 1.65 (s, 3H), 1.50 (s, 3H); ${ }^{13} \mathrm{C} \mathrm{NMR}\left(125 \mathrm{MHz}, \mathrm{CDCl}_{3}\right) \delta 255.26,210.56,146.23$, 140.64, 139.60, 138.64, 137.50, 137.05, 135.60, 134.26, 134.18, 131.19, 130.61, 130.47, 130.17, 129.43, 128.88, 127.62, 125.30, 124.37, 119.83, 113.59, 74.44, 68.79, 66.78, 52.36, 51.84, 21.71, 21.23, 20.50, 20.27, 19.10, 18.94, 17.65; HRMS (ESI) calcd for $\mathrm{C}_{41} \mathrm{H}_{44} \mathrm{~N}_{3} \mathrm{O}_{3} \mathrm{SRu}[\mathrm{M}-2 \mathrm{Cl}+\mathrm{H}]^{+} 760.2147$ found, 760.2112.

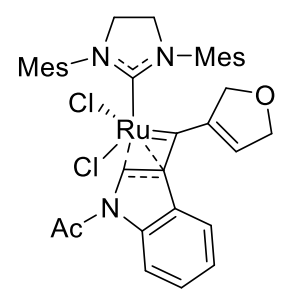

$13 \mathrm{~s}$

13s: The reaction was performed at $35{ }^{\circ} \mathrm{C}$ for $1 \mathrm{~h}$. This compound was isolated in $55 \%$ yield alongwith $25 \%$ (NMR yield) metathesis product; green solid; $40 \mathrm{mg}$ obtained; Purification: Flash Chromatography $\left(\mathrm{SiO}_{2}\right.$, hexanes-EtOAc, 10:1 $\rightarrow 4: 1 \rightarrow \mathrm{CH}_{2} \mathrm{Cl}_{2}-$ EtOAc, 1:1); ${ }^{1} \mathrm{H}$ NMR (500 MHz, $\left.\mathrm{CDCl}_{3}\right) \delta 9.39(\mathrm{~s}, 1 \mathrm{H}), 8.43(\mathrm{~d}, J=7.7 \mathrm{~Hz}, 1 \mathrm{H}), 7.58-7.44$ $(\mathrm{m}, 1 \mathrm{H}), 7.44-7.33(\mathrm{~m}, 2 \mathrm{H}), 7.12(\mathrm{~d}, J=8.4 \mathrm{~Hz}, 2 \mathrm{H}), 6.52(\mathrm{~s}, 1 \mathrm{H}), 5.65(\mathrm{~s}, 1 \mathrm{H}), 4.64(\mathrm{~d}, J$ $=12.4 \mathrm{~Hz}, 1 \mathrm{H}), 4.49(\mathrm{~s}, 1 \mathrm{H}), 4.47-4.39(\mathrm{~m}, 2 \mathrm{H}), 4.35(\mathrm{q}, J=11.1 \mathrm{~Hz}, 1 \mathrm{H}), 4.18(\mathrm{q}, J=10.5$ $\mathrm{Hz}, 1 \mathrm{H}), 3.89(\mathrm{p}, J=9.5,8.1 \mathrm{~Hz}, 2 \mathrm{H}), 3.64(\mathrm{~d}, J=11.7 \mathrm{~Hz}, 1 \mathrm{H}), 2.73(\mathrm{~s}, 3 \mathrm{H}), 2.70(\mathrm{~s}, 3 \mathrm{H})$, $2.67(\mathrm{~s}, 3 \mathrm{H}), 2.58(\mathrm{~s}, 3 \mathrm{H}), 2.34(\mathrm{~s}, 3 \mathrm{H}), 1.53(\mathrm{~d}, \mathrm{~J}=6.1 \mathrm{~Hz}, 6 \mathrm{H}) ;{ }^{13} \mathrm{C} \mathrm{NMR}\left(125 \mathrm{MHz}, \mathrm{CDCl}_{3}\right)$ $\delta 256.60,209.38,169.47,140.75,138.94,138.60,137.27,137.07,135.25,135.10,134.40,131.63,131.44$, $129.99,129.55,129.03,128.85,126.08,125.05,123.02,118.91,117.02,74.43,68.69,66.33,52.38,51.76$, $23.75,21.22,20.41,20.31,19.32,19.04,17.31$; HRMS (ESI) calcd for $\mathrm{C}_{36} \mathrm{H}_{38} \mathrm{~N}_{3} \mathrm{O}_{2} \mathrm{Ru}[\mathrm{M}-2 \mathrm{Cl}-\mathrm{H}]^{+} 646.2008$ found, 646.1986 .

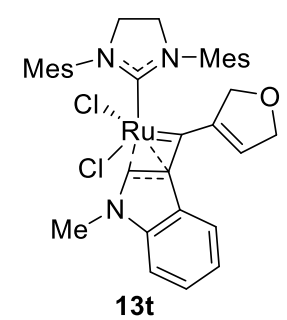

$13 t$ 13t: $88 \%$ yield; red solid; $55 \mathrm{mg}$ obtained; Purification: Flash Chromatography $\left(\mathrm{SiO}_{2}\right.$, hexanes-EtOAc, 10:1 $\rightarrow$ 4:1 $\rightarrow \mathrm{CH}_{2} \mathrm{Cl}_{2}$-EtOAc, 1:1); ${ }^{1} \mathrm{H}$ NMR $\left(500 \mathrm{MHz}, \mathrm{CDCl}_{3}\right) \delta 8.88$ (s, 1H), $7.45(\mathrm{~d}, J=7.5 \mathrm{~Hz}, 1 \mathrm{H}), 7.35-7.26(\mathrm{~m}, 3 \mathrm{H}), 7.11(\mathrm{~s}, 1 \mathrm{H}), 7.07(\mathrm{~s}, 1 \mathrm{H}), 6.42(\mathrm{~s}$, $1 \mathrm{H}), 5.73(\mathrm{~s}, 1 \mathrm{H}), 4.65-4.54(\mathrm{~m}, 1 \mathrm{H}), 4.47(\mathrm{~d}, J=11.4 \mathrm{~Hz}, 1 \mathrm{H}), 4.39(\mathrm{~d}, J=11.9 \mathrm{~Hz}, 1 \mathrm{H})$, 4.36-4.22 (m, 2H), 4.20-4.06 (m, 1H), 3.91-3.82 (m, 1H), $3.78(\mathrm{~d}, J=10.8 \mathrm{~Hz}, 2 \mathrm{H}), 3.73$ $(\mathrm{s}, 3 \mathrm{H}), 2.71(\mathrm{~s}, 3 \mathrm{H}), 2.67(\mathrm{~s}, 3 \mathrm{H}), 2.56(\mathrm{~s}, 3 \mathrm{H}), 2.33(\mathrm{~s}, 3 \mathrm{H}), 1.62(\mathrm{~s}, 3 \mathrm{H}), 1.47(\mathrm{~s}, 3 \mathrm{H}) ;{ }^{13} \mathrm{C}$ NMR $\left(125 \mathrm{MHz}, \mathrm{CDCl}_{3}\right) \delta 250.51,211.56,144.52,140.16,139.10,137.85,137.41$, 
136.34, 135.41, 135.33, 134.83, 132.34, 131.17, 129.76, 129.49, 128.76, 128.35, 127.97, 123.44, 123.14, 121.07, 119.59, 110.04, 74.62, 67.07, 66.89, 52.36, 51.80, 34.05, 21.22, 20.46, 20.34, 19.30, 19.10, 17.56; HRMS (ESI) calcd for $\mathrm{C}_{35} \mathrm{H}_{40} \mathrm{~N}_{3} \mathrm{ORu}[\mathrm{M}-2 \mathrm{Cl}+\mathrm{H}]^{+} 620.2215$ found, 620.2184 .

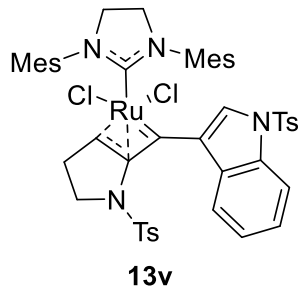

13v: $64 \%$ yield; green solid; $40 \mathrm{mg}$ obtained; Purification: Flash Chromatography $\left(\mathrm{SiO}_{2}\right.$, hexanes-EtOAc, 10:1 $\rightarrow$ 4:1 $\rightarrow \mathrm{CH}_{2} \mathrm{Cl}_{2}$-EtOAc, 1:1); ${ }^{1} \mathrm{H}$ NMR $\left(500 \mathrm{MHz}, \mathrm{CDCl}_{3}\right) \delta 9.65(\mathrm{~s}$, $1 \mathrm{H}), 7.96-7.70(\mathrm{~m}, 3 \mathrm{H}), 7.55(\mathrm{~s}, 1 \mathrm{H}), 7.34-7.25(\mathrm{~m}, 3 \mathrm{H}), 7.20-7.13(\mathrm{~m}, 2 \mathrm{H}), 7.06-6.98$ $(\mathrm{m}, 2 \mathrm{H}), 6.97-6.84(\mathrm{~m}, 3 \mathrm{H}), 6.50(\mathrm{~s}, 1 \mathrm{H}), 6.30(\mathrm{~s}, 1 \mathrm{H}), 5.60(\mathrm{~s}, 1 \mathrm{H}), 4.26-4.17(\mathrm{~m}, 1 \mathrm{H})$, 3.82-3.67 (m, 4H), 3.48-3.37 (m, $1 \mathrm{H}), 2.67(\mathrm{~s}, 3 \mathrm{H}), 2.59-2.51(\mathrm{~m}, 6 \mathrm{H}), 2.28-2.20(\mathrm{~m}$, 9H), 1.96-1.86 (m, 2H), $\left.1.72(\mathrm{~s}, 3 \mathrm{H}), 1.55(\mathrm{~s}, 3 \mathrm{H}) ;{ }^{13} \mathrm{C} \mathrm{NMR} \mathrm{(125} \mathrm{MHz,} \mathrm{CDCl}\right) \delta 250.97$, $207.46,145.42,143.59,140.76,139.95,138.24,136.58,135.95,135.19,134.69,134.31$, $133.82,133.22$, 131.18, 130.12, 129.71, 129.22, 128.83, 128.57, 128.05, 127.69, 126.95, 124.91, 123.34, $122.22,113.15,111.83,105.80,53.18,51.36,51.15,49.27,27.51,21.68,21.53,21.29,20.81,20.48,19.01$, 18.72, 17.64; HRMS (ESI) calcd for $\mathrm{C}_{48} \mathrm{H}_{51} \mathrm{~N}_{4} \mathrm{O}_{4} \mathrm{~S}_{2} \mathrm{Ru}[\mathrm{M}-2 \mathrm{Cl}+\mathrm{H}]^{+} 913.2395$ found, 913.2360.

\section{Catalytic activity: Structure-activity relationship studies ${ }^{15,16}$}

Different types of alkene-chelates were tested for their RCM activity on dimethyl diallylmalonate. Based on their reactivity, the structure-activity relationships of these catalysts were established and various factors affecting the catalytic activity were determined. The general trend of catalytic activities for the trans-1,5-chelates and $\eta^{3}$-vinyl chelates along with the factors affecting catalytic activity is enlisted below. The kinetic data (raw data and plot) for catalytic activities of $\eta^{3}$-vinyl chelates and trans-1,5-chelates are also provided in the subsections underneath.

\section{Reactivity trends for trans-1,5-chelates}

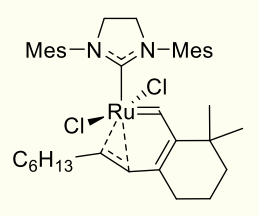

$10 \mathrm{~b}$

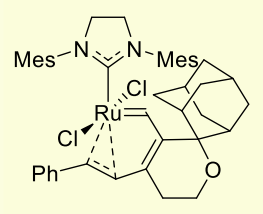

$10 \mathrm{~h}$

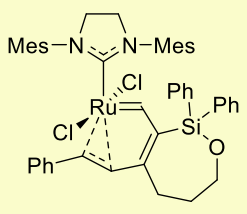

101

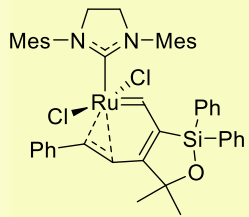

$10 \mathrm{i}$

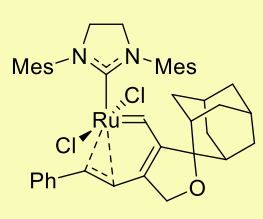

$10 \mathrm{~g}$

Increasing catalytic activity

Factors affecting catalytic-activity of trans-1,5-chelates:

- 1,5-chelates associated with 5-membered ring like $10 \mathrm{i}$ and $10 \mathrm{~g}$ are highly reactive and are metathesis active at room temperature while complexes associated with 6 or 7-membered rings like $10 \mathrm{~b}, 10 \mathrm{~h}$ and $10 \mathrm{l}$ are inactive at room temperature and show catalytic activity at higher temperature $\left(60{ }^{\circ} \mathrm{C}\right.$ or higher).

- Catalytic activity increases drastically with variation in the tethering group. For instance, in the $\mathrm{RCM}$ of dimethyl diallylmalonate catalyzed by these latent chelates at $80^{\circ} \mathrm{C}$ in $\mathrm{C}_{6} \mathrm{D}_{6}$, complex $10 \mathrm{~b}$ 
containing a gem-dimethyl group on the fused 6-membered ring is much less active (12\% conversion) than the corresponding adamantyl-containing complex $10 \mathrm{~h}$ ( $57 \%$ conversion), yet 7membered ring-fused complex $\mathbf{1 0 l}$ containing a diphenylsilyl group is much more reactive $\mathbf{9 9 . 1 \%}$ conversion).

\section{Reactivity trends for $\eta^{3}$-vinyl chelates}

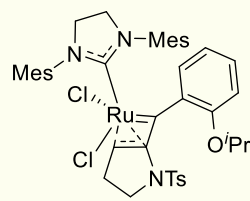

$13 \mathrm{~g}$

Inactive at $45^{\circ} \mathrm{C}$

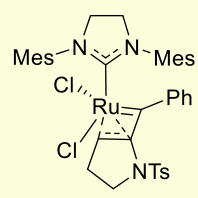

$13 a$

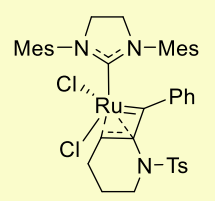

$13 b$

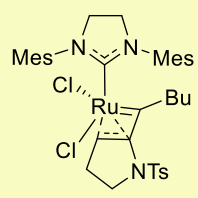

$13 \mathrm{e}$

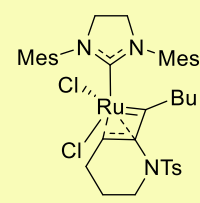

$13 f$

Increasing catalytic activity

Factors affecting catalytic-activity of $\eta^{3}$-vinyl chelates:

- The $\eta^{3}$-vinyl chelates are generally highly active catalysts reactive at room temperature. The catalytic activity depends on the substituent on the carbenic carbon. The lesser is the steric hindrance by the substituent the more is the catalytic-activity due to easier [2+2] cycloaddition with the substrate alkene. Thus, butyl substituted catalysts like $\mathbf{1 3 e}$ and $\mathbf{1 3 f}$ are more reactive than phenyl substituted congeners $\mathbf{1 3 a}$ and $\mathbf{1 3 b}$.

- Size of the fused ring also affects the catalytic activity. Six-membered ring fused complexes like $\mathbf{1 3 b}$ and $\mathbf{1 3 f}$ are more reactive than their 5-membered ring congeners like $13 \mathrm{a}$ and $\mathbf{1 3 e}$. The reason for higher reactivity of six-membered fused complexes lies to the fact that the six-membered ring of the bicyclo[4.1.0]heptane moiety is highly distorted to assume a boat conformation (revealed from X-ray structure) thereby having a lower dissociation barrier.

- Interestingly complex $\mathbf{1 3 g}$ is catalytically inactive. The reason could be the presence of an alternate chelation site which is the 2-isopropxy group. The free Ru-alkylidene might form a continuous equilibrium between the alkene-chelate and the oxygen-chelate and renders the complex inactive.

\section{Catalytic activities of $\eta^{3}$-vinyl chelates for RCM reaction of dimethyldiallyl malonate at $45^{\circ} \mathrm{C}$}

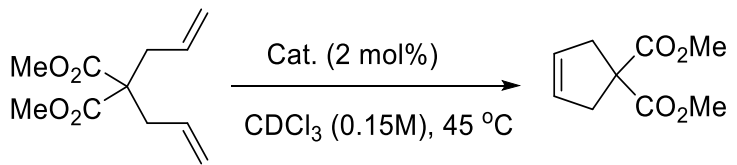

An NMR tube with a screw-cap septum top was charged inside a glovebox with catalyst stock solution (0.1 $\mathrm{M}, 24 \mu \mathrm{L}, 2.4 \mu \mathrm{mol}, 2 \mathrm{~mol} \%)$ in $0.3 \mathrm{~mL}$ of $\mathrm{CDCl}_{3}$. The NMR tube was degassed by freeze, pump, thaw method (x 3) and refilled with argon. The sample was equilibrated at $45^{\circ} \mathrm{C}$ in the NMR probe before dimethyl diallyl malonate $\left(25 \mathrm{mg}, 0.12 \mathrm{mmol}\right.$ ) solution in $0.5 \mathrm{~mL}$ of $\mathrm{CDCl}_{3}$ was added via a syringe. Data points were collected over an appropriate time interval using the Bruker pseudo 2D kinetics method. The 
conversion of the product was determined by comparing the ratio of the integrals of the methylene protons in the starting material, $\delta 2.67-2.57(\mathrm{~m})$, with those of the product, $\delta 3.02(\mathrm{~s})$. Three independent experiments were performed for each complexes and mean conversion was determined. The conversion versus time graph was plotted for different complexes to have a comparison of their reactivities.

\section{Catalyst 13a}

\begin{tabular}{|c|c|c|c|c|c|}
\hline \multirow{2}{*}{$\begin{array}{l}\text { Time } \\
\text { (min) }\end{array}$} & \multicolumn{2}{|c|}{ Conversion (\%) } & \multirow[b]{2}{*}{ Trial 3} & \multirow[b]{2}{*}{ Avg. } & \multirow[b]{2}{*}{ Std. Dev. } \\
\hline & Trial 1 & Trial 2 & & & \\
\hline 0.35 & 1.1 & 0.8 & 1.6 & 1.2 & 0.33 \\
\hline 1.03 & 2.0 & 2.4 & 2.6 & 2.3 & 0.27 \\
\hline 1.72 & 3.3 & 3.5 & 3.7 & 3.5 & 0.17 \\
\hline 2.40 & 5.1 & 6.4 & 6.6 & 6.0 & 0.67 \\
\hline 3.08 & 7.5 & 7.8 & 8.1 & 7.8 & 0.25 \\
\hline 3.77 & 10.5 & 11.1 & 9.4 & 10.3 & 0.71 \\
\hline 4.45 & 14.3 & 13.2 & 14.7 & 14.1 & 0.63 \\
\hline 5.13 & 18.6 & 17.5 & 18.9 & 18.3 & 0.61 \\
\hline 5.82 & 23.4 & 25.4 & 22.3 & 23.7 & 1.28 \\
\hline 6.50 & 28.7 & 29.6 & 28.3 & 28.9 & 0.55 \\
\hline 7.18 & 34.1 & 34.5 & 35.3 & 34.6 & 0.50 \\
\hline 7.87 & 39.7 & 40.5 & 41.3 & 40.5 & 0.67 \\
\hline 8.55 & 45.2 & 45.6 & 44.1 & 45.0 & 0.64 \\
\hline 9.23 & 50.6 & 51.3 & 49.2 & 50.4 & 0.87 \\
\hline 9.92 & 55.8 & 57.6 & 54.4 & 55.9 & 1.31 \\
\hline 10.60 & 60.6 & 62.4 & 58.2 & 60.4 & 1.72 \\
\hline 11.28 & 65.1 & 67.2 & 65.3 & 65.9 & 0.94 \\
\hline 11.97 & 69.3 & 70.5 & 69 & 69.6 & 0.65 \\
\hline 12.65 & 73.1 & 72.4 & 71.1 & 72.2 & 0.82 \\
\hline 13.33 & 76.5 & 76.8 & 74.3 & 75.9 & 1.11 \\
\hline 14.02 & 79.6 & 78.9 & 80.3 & 79.6 & 0.57 \\
\hline 14.70 & 82.3 & 82.5 & 81.7 & 82.2 & 0.34 \\
\hline 15.38 & 84.7 & 85.8 & 81.2 & 83.9 & 1.97 \\
\hline 16.07 & 86.8 & 83.1 & 85.4 & 85.1 & 1.54 \\
\hline 16.75 & 88.5 & 89.2 & 87.4 & 88.4 & 0.74 \\
\hline 17.43 & 90.2 & 90.8 & 89.1 & 90.0 & 0.70 \\
\hline 18.12 & 91.3 & 93.4 & 90.6 & 91.8 & 1.19 \\
\hline
\end{tabular}




\section{Catalyst 13b}

\begin{tabular}{|r|r|r|r|r|r|}
\hline $\begin{array}{l}\text { Time } \\
\text { (min) }\end{array}$ & \multicolumn{2}{|l|}{ Conversion (\%) } & & & \\
\hline & \multicolumn{1}{|l|}{ Trial 1 } & \multicolumn{1}{l|}{ Trial 2 } & \multicolumn{1}{l|}{ Trial 3 } & \multicolumn{1}{l|}{ Avg. } & Std. Dev. \\
\hline 0.35 & 2.3 & 4.4 & 3.7 & 3.5 & 0.89 \\
\hline 1.03 & 13.2 & 10.2 & 16.4 & 13.3 & 2.53 \\
\hline 1.72 & 42.9 & 38.6 & 54.1 & 45.2 & 6.53 \\
\hline 2.40 & 74.2 & 68.4 & 75.3 & 72.6 & 3.02 \\
\hline 3.08 & 90.2 & 88.3 & 87.6 & 88.7 & 1.11 \\
\hline 3.77 & 96.0 & 94.2 & 93.1 & 94.4 & 1.18 \\
\hline 4.45 & 98.0 & 96.2 & 95.4 & 96.5 & 1.07 \\
\hline 5.13 & 98.8 & 97.3 & 97.0 & 97.7 & 0.79 \\
\hline 5.82 & 99.1 & 98.3 & 99.1 & 98.8 & 0.37 \\
\hline 6.50 & 99.1 & 99.2 & 98.7 & 99.0 & 0.22 \\
\hline 7.18 & 99.2 & 99.1 & 98.3 & 98.9 & 0.40 \\
\hline 7.87 & 99.2 & 98.3 & 99.1 & 98.9 & 0.40 \\
\hline 8.55 & 99.2 & 99.2 & 99.1 & 99.2 & 0.05 \\
\hline & & & & & \\
\hline
\end{tabular}

\section{Catalyst 13c}

\begin{tabular}{|c|c|c|c|c|c|}
\hline \multirow{2}{*}{$\begin{array}{l}\text { Time } \\
\text { (min) }\end{array}$} & \multicolumn{2}{|c|}{ Conversion (\%) } & \multirow[b]{2}{*}{ Trial 3} & \multirow[b]{2}{*}{ Avg. } & \multirow[b]{2}{*}{ Std. Dev. } \\
\hline & Trial 1 & Trial 2 & & & \\
\hline 0.35 & 1.2 & 1.5 & 1.7 & 1.5 & 0.20 \\
\hline 1.03 & 1.5 & 2.2 & 2.6 & 2.1 & 0.46 \\
\hline 1.72 & 21.8 & 19.1 & 25.6 & 22.2 & 2.67 \\
\hline 2.40 & 74.0 & 64.2 & 61.6 & 66.6 & 5.33 \\
\hline 3.08 & 93.0 & 89.1 & 91.4 & 91.2 & 1.61 \\
\hline 3.77 & 97.1 & 95.2 & 96.4 & 96.2 & 0.78 \\
\hline 4.45 & 97.8 & 94.3 & 97.0 & 96.4 & 1.48 \\
\hline 5.13 & 98.1 & 97.1 & 98.2 & 97.8 & 0.49 \\
\hline 5.82 & 98.1 & 97.6 & 98.4 & 98.0 & 0.33 \\
\hline 6.50 & 97.9 & 98.2 & 97.1 & 97.7 & 0.46 \\
\hline 7.18 & 97.8 & 98.4 & 97.7 & 98.0 & 0.32 \\
\hline 7.87 & 97.7 & 98.5 & 97.7 & 98.0 & 0.37 \\
\hline 8.55 & 97.8 & 97.8 & 97.6 & 97.7 & 0.09 \\
\hline 9.23 & 97.8 & 98.3 & 98.5 & 98.2 & 0.31 \\
\hline 9.92 & 97.8 & 97.1 & 97.3 & 97.4 & 0.30 \\
\hline 10.60 & 97.8 & 98.2 & 97.1 & 97.7 & 0.46 \\
\hline 11.28 & 97.9 & 97.4 & 98.2 & 97.8 & 0.33 \\
\hline
\end{tabular}




\begin{tabular}{|r|r|r|r|r|r|}
\hline \multicolumn{1}{l}{$\begin{array}{l}\text { Time } \\
\text { (min) }\end{array}$} & \multicolumn{2}{|l|}{ Conversion (\%) } & & & \\
\hline & Trial 1 & \multicolumn{1}{|l|}{ Trial 2 } & \multicolumn{1}{l|}{ Trial 3 } & Avg. & Std. Dev. \\
\hline 0.35 & 5.7 & 4.8 & 8.3 & 6.3 & 1.49 \\
\hline 1.03 & 34.5 & 27.6 & 36.1 & 32.7 & 3.69 \\
\hline 1.72 & 61.1 & 51.6 & 54.3 & 55.7 & 3.99 \\
\hline 2.40 & 77.5 & 68.3 & 71.6 & 72.5 & 3.81 \\
\hline 3.08 & 87.0 & 85.4 & 83.2 & 85.2 & 1.54 \\
\hline 3.77 & 91.5 & 89.4 & 90.3 & 90.4 & 0.84 \\
\hline 4.45 & 93.9 & 91.8 & 93.4 & 93.0 & 0.91 \\
\hline 5.13 & 95.6 & 93.4 & 94.5 & 94.5 & 0.88 \\
\hline 5.82 & 96.3 & 95.5 & 96.0 & 95.9 & 0.32 \\
\hline 6.50 & 96.9 & 96.1 & 96.2 & 96.4 & 0.35 \\
\hline 7.18 & 97.4 & 97.1 & 97.4 & 97.3 & 0.14 \\
\hline 7.87 & 97.4 & 97.4 & 98.0 & 97.6 & 0.28 \\
\hline 8.55 & 97.7 & 97.2 & 98.9 & 97.9 & 0.72 \\
\hline 9.23 & 96.5 & 96.8 & 96.6 & 96.6 & 0.12 \\
\hline 9.92 & 98.3 & 97.8 & 98.1 & 98.1 & 0.21 \\
\hline 10.60 & 98.1 & 96.8 & 97.3 & 97.4 & 0.55 \\
\hline
\end{tabular}

\section{Catalyst 13e}

\begin{tabular}{|r|r|r|r|r|r|}
\hline $\begin{array}{l}\text { Time } \\
\text { (min) }\end{array}$ & \multicolumn{2}{|l|}{ Conversion (\%) } & & & \\
\hline & Trial 1 & \multicolumn{1}{|l|}{ Trial 2 } & Trial 3 & \multicolumn{1}{|l|}{ Avg. } & Std. Dev. \\
\hline 0.35 & 2.3 & 3.2 & 4.6 & 3.4 & 0.95 \\
\hline 1.03 & 31.0 & 28 & 32.5 & 30.5 & 1.87 \\
\hline 1.72 & 63.2 & 61.2 & 65.6 & 63.3 & 1.80 \\
\hline 2.40 & 83.0 & 78.2 & 81.6 & 80.9 & 2.03 \\
\hline 3.08 & 92.9 & 90.8 & 91.6 & 91.8 & 0.87 \\
\hline 3.77 & 96.1 & 95.6 & 95.3 & 95.7 & 0.35 \\
\hline 4.45 & 98.2 & 97.2 & 98 & 97.8 & 0.43 \\
\hline 5.13 & 98.1 & 98.1 & 97.6 & 97.9 & 0.24 \\
\hline 5.82 & 99.5 & 98.3 & 99.1 & 99.0 & 0.51 \\
\hline 6.50 & 98.7 & 99.2 & 98.7 & 98.9 & 0.24 \\
\hline 7.18 & 98.9 & 99.4 & 99.1 & 99.1 & 0.21 \\
\hline 7.87 & 99.7 & 98.6 & 99.3 & 99.2 & 0.45 \\
\hline
\end{tabular}




\begin{tabular}{|r|r|r|r|r|r|}
\hline \multicolumn{1}{l|}{$\begin{array}{l}\text { Time } \\
\text { (min) }\end{array}$} & \multicolumn{2}{|l|}{ Conversion (\%) } & & & \\
\hline & \multicolumn{1}{|c|}{ Trial 1 } & \multicolumn{1}{l|}{ Trial 2 } & \multicolumn{1}{|c|}{ Trial 3 } & \multicolumn{1}{l|}{ Avg. } & Std. Dev. \\
\hline 0.35 & 57.8 & 48.2 & 51.3 & 52.4 & 4.01 \\
\hline 1.03 & 92.8 & 81.6 & 87.3 & 87.2 & 4.57 \\
\hline 1.72 & 98.0 & 92.6 & 94.7 & 95.1 & 2.22 \\
\hline 2.40 & 98.8 & 97.6 & 98.1 & 98.2 & 0.49 \\
\hline 3.08 & 98.9 & 97.4 & 98.4 & 98.2 & 0.63 \\
\hline 3.77 & 99.2 & 99.2 & 98.4 & 98.9 & 0.38 \\
\hline 4.45 & 99.2 & 99.1 & 99.4 & 99.2 & 0.12 \\
\hline 5.13 & 99.2 & 98.8 & 99.1 & 99.0 & 0.17 \\
\hline 5.82 & 99.3 & 98.7 & 98.6 & 98.9 & 0.33 \\
\hline
\end{tabular}

\section{Catalyst 13g}

\begin{tabular}{|c|c|c|c|c|c|}
\hline \multirow{2}{*}{$\begin{array}{l}\text { Time } \\
\text { (min) }\end{array}$} & \multicolumn{2}{|c|}{ Conversion (\%) } & \multirow[b]{2}{*}{ Trial 3} & \multirow[b]{2}{*}{ Avg. } & \multirow[b]{2}{*}{ Std. Dev. } \\
\hline & Trial 1 & Trial 2 & & & \\
\hline 0.35 & 0.4 & 0.3 & 0.2 & 0.3 & 0.09 \\
\hline 1.03 & 0.4 & 0.6 & 0.4 & 0.5 & 0.09 \\
\hline 1.72 & 0.4 & 0.4 & 0.5 & 0.4 & 0.05 \\
\hline 2.40 & 0.4 & 0.5 & 0.5 & 0.5 & 0.03 \\
\hline 3.08 & 0.4 & 0.5 & 0.5 & 0.5 & 0.03 \\
\hline 3.77 & 0.4 & 0.5 & 0.5 & 0.5 & 0.05 \\
\hline 4.45 & 0.5 & 0.5 & 0.4 & 0.5 & 0.04 \\
\hline 5.13 & 0.4 & 0.4 & 0.4 & 0.4 & 0.02 \\
\hline 5.82 & 0.5 & 0.3 & 0.3 & 0.4 & 0.08 \\
\hline 6.50 & 0.4 & 0.3 & 0.3 & 0.3 & 0.06 \\
\hline 7.18 & 0.4 & 0.3 & 0.3 & 0.3 & 0.06 \\
\hline 7.87 & 0.4 & 0.2 & 0.3 & 0.3 & 0.10 \\
\hline 8.55 & 0.4 & 0.4 & 0.2 & 0.3 & 0.10 \\
\hline 9.23 & 0.5 & 0.5 & 0.4 & 0.5 & 0.04 \\
\hline 9.92 & 0.5 & 0.4 & 0.2 & 0.4 & 0.12 \\
\hline 10.60 & 0.5 & 0.4 & 0.6 & 0.5 & 0.08 \\
\hline 11.28 & 0.5 & 0.4 & 0.2 & 0.4 & 0.11 \\
\hline 11.97 & 0.5 & 0.6 & 0.2 & 0.4 & 0.17 \\
\hline 12.65 & 0.5 & 0.2 & 0.2 & 0.3 & 0.13 \\
\hline 13.33 & 0.5 & 0.3 & 0.4 & 0.4 & 0.08 \\
\hline 14.02 & 0.5 & 0.5 & 0.4 & 0.5 & 0.05 \\
\hline
\end{tabular}




\begin{tabular}{|r|r|r|r|r|r|}
\hline 14.70 & 0.5 & 0.5 & 0.4 & 0.5 & 0.05 \\
\hline 15.38 & 0.5 & 0.5 & 0.4 & 0.5 & 0.04 \\
\hline 16.07 & 0.5 & 0.5 & 0.5 & 0.5 & 0.01 \\
\hline 16.75 & 0.5 & 0.3 & 0.3 & 0.4 & 0.09 \\
\hline 17.43 & 0.5 & 0.4 & 0.2 & 0.4 & 0.12 \\
\hline 18.12 & 0.5 & 0.3 & 0.5 & 0.4 & 0.09 \\
\hline
\end{tabular}
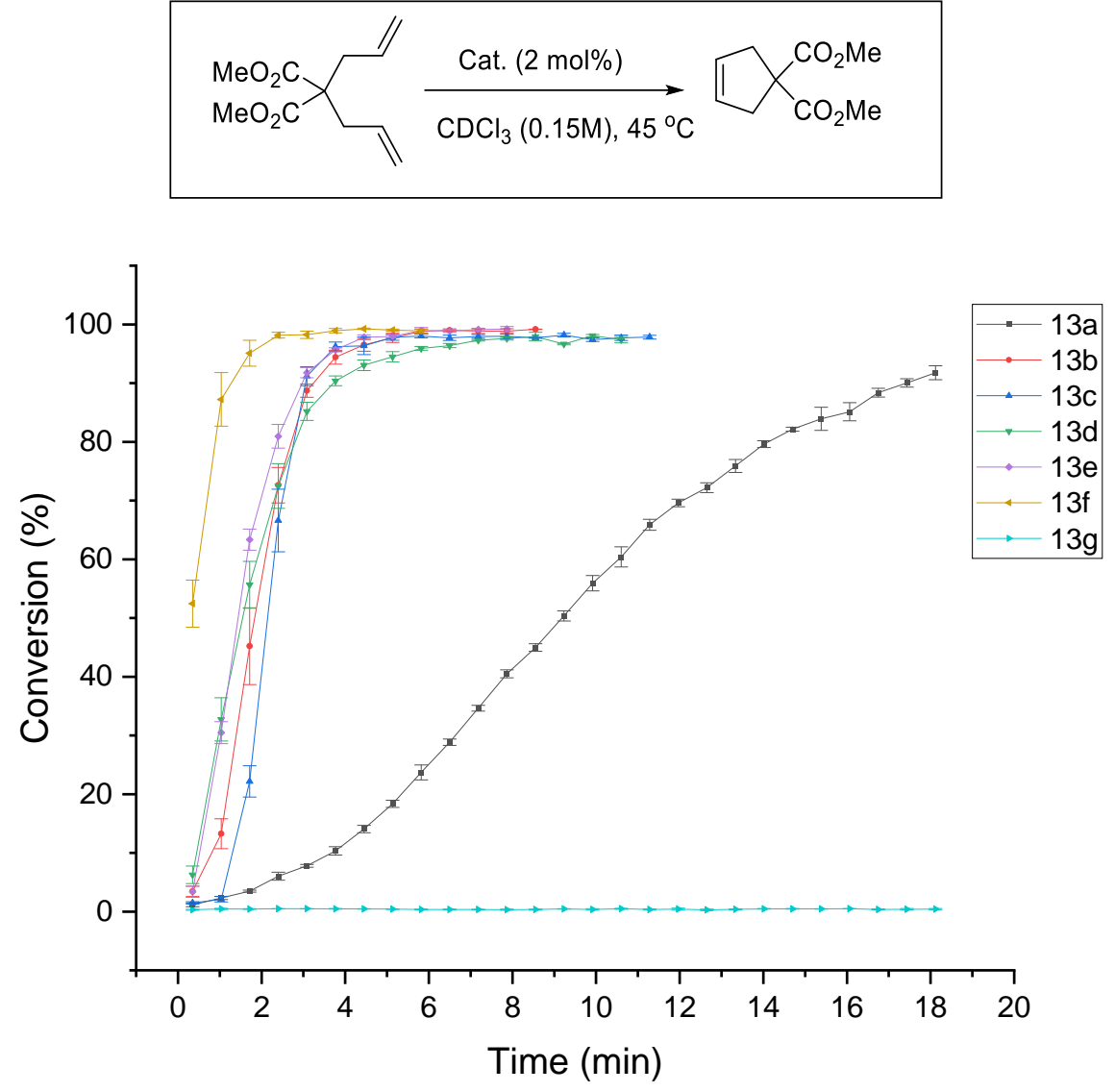

Fig. S3. Kinetic profile for RCM reactions to disubstituted olefins using $\eta^{3}$-vinyl chelates as catalysts at 45 ${ }^{\circ} \mathrm{C}$. Each data point represents the average of three independent experiments. Error bar represents standard error of the triplicate experiments. 
Comparison of RCM activity of oxygen chelates 13p' and 13q' with alkene chelates

Catalyst 13p'

\begin{tabular}{|r|r|r|r|r|r|}
\hline \multicolumn{1}{|l|}{$\begin{array}{l}\text { Time } \\
\text { (min) }\end{array}$} & \multicolumn{2}{|l|}{ Conversion (\%) } & & & \\
\hline & Trial 1 & Trial 2 & Trial 3 & Avg. & Std. Dev. \\
\hline 0.35 & 2.6 & 0.5 & 1.3 & 1.5 & 0.85 \\
\hline 1.03 & 1.7 & 1.2 & 1.8 & 1.6 & 0.26 \\
\hline 1.72 & 1.2 & 0.8 & 1.5 & 1.2 & 0.29 \\
\hline 2.40 & 1.2 & 1.4 & 1.1 & 1.2 & 0.13 \\
\hline 3.08 & 1.2 & 0.9 & 1.0 & 1.0 & 0.13 \\
\hline 3.77 & 1.2 & 1.4 & 1.0 & 1.2 & 0.16 \\
\hline 4.45 & 1.2 & 1.4 & 1.3 & 1.3 & 0.07 \\
\hline 5.13 & 1.2 & 1.4 & 1.5 & 1.4 & 0.11 \\
\hline 5.82 & 1.3 & 1.6 & 1.5 & 1.5 & 0.14 \\
\hline 6.50 & 1.2 & 1.6 & 1.4 & 1.4 & 0.15 \\
\hline 7.18 & 1.3 & 1.5 & 1.4 & 1.4 & 0.09 \\
\hline 7.87 & 1.3 & 1.5 & 1.4 & 1.4 & 0.09 \\
\hline 8.55 & 1.3 & 1.5 & 1.4 & 1.4 & 0.10 \\
\hline 9.23 & 1.3 & 1.4 & 1.4 & 1.4 & 0.05 \\
\hline 9.92 & 1.3 & 1.4 & 1.4 & 1.4 & 0.05 \\
\hline 10.60 & 1.3 & 1.1 & 1.4 & 1.3 & 0.12 \\
\hline 11.28 & 1.3 & 1.2 & 1.3 & 1.3 & 0.05 \\
\hline 11.97 & 1.3 & 1.0 & 1.3 & 1.2 & 0.14 \\
\hline 12.65 & 1.3 & 1.2 & 1.3 & 1.3 & 0.04 \\
\hline 13.33 & 1.3 & 1.3 & 1.6 & 1.4 & 0.14 \\
\hline 14.02 & 1.3 & 1.5 & 1.3 & 1.4 & 0.09 \\
\hline 14.70 & 1.3 & 1.5 & 1.6 & 1.5 & 0.11 \\
\hline 15.38 & 1.3 & 1.5 & 1.6 & 1.5 & 0.11 \\
\hline 16.07 & 1.3 & 1.4 & 1.6 & 1.4 & 0.12 \\
\hline 16.75 & 1.4 & 1.4 & 1.5 & 1.4 & 0.05 \\
\hline 17.43 & 1.4 & 1.4 & 1.5 & 1.4 & 0.05 \\
\hline 18.12 & 1.4 & 1.4 & 1.5 & 1.4 & 0.06 \\
\hline & & & & & \\
\hline & & 1.3 & & &
\end{tabular}

Catalyst 13q'

\begin{tabular}{|r|r|r|r|r|r|}
\hline $\begin{array}{l}\text { Time } \\
\text { (min) }\end{array}$ & \multicolumn{2}{|l|}{ Conversion (\%) } & & & \\
\hline & \multicolumn{1}{|l|}{ Trial 1 } & \multicolumn{1}{|c|}{ Trial 2 } & \multicolumn{1}{|l|}{ Trial 3 } & \multicolumn{1}{l|}{ Avg. } & Std. Dev. \\
\hline 0.35 & 0.1 & 0.3 & 0.5 & 0.3 & 0.16 \\
\hline 1.03 & 0.3 & 0.4 & 0.2 & 0.3 & 0.08 \\
\hline 1.72 & 1.0 & 2.1 & 3.4 & 2.2 & 0.98 \\
\hline 2.40 & 2.8 & 3.6 & 4.5 & 3.6 & 0.68 \\
\hline
\end{tabular}




\begin{tabular}{|c|c|c|c|c|c|}
\hline \multirow{2}{*}{$\begin{array}{l}\text { Time } \\
\text { (min) }\end{array}$} & \multicolumn{2}{|c|}{ Conversion (\%) } & \multirow[b]{2}{*}{ Trial 3} & \multirow[b]{2}{*}{ Avg. } & \multirow[b]{2}{*}{ Std. Dev. } \\
\hline & Trial 1 & Trial 2 & & & \\
\hline 3.08 & 7.0 & 5.1 & 6.7 & 6.3 & 0.83 \\
\hline 3.77 & 14.5 & 12.2 & 15.8 & 14.2 & 1.49 \\
\hline 4.45 & 25.3 & 22.1 & 23.6 & 23.7 & 1.31 \\
\hline 5.13 & 37.6 & 35.3 & 34.5 & 35.8 & 1.33 \\
\hline 5.82 & 50.3 & 48.4 & 47.5 & 48.7 & 1.17 \\
\hline 6.50 & 61.8 & 62.4 & 58.3 & 60.8 & 1.81 \\
\hline 7.18 & 71.4 & 68.2 & 69.5 & 69.7 & 1.32 \\
\hline 7.87 & 79.5 & 77.3 & 78.2 & 78.3 & 0.92 \\
\hline 8.55 & 85.7 & 81.2 & 83.8 & 83.6 & 1.86 \\
\hline 9.23 & 90.1 & 88.7 & 89.4 & 89.4 & 0.55 \\
\hline 9.92 & 93.2 & 91.2 & 92.4 & 92.3 & 0.83 \\
\hline 10.60 & 95.1 & 93.4 & 95.2 & 94.6 & 0.83 \\
\hline 11.28 & 96.3 & 95.2 & 96.7 & 96.1 & 0.63 \\
\hline 11.97 & 97.2 & 97.4 & 97.6 & 97.4 & 0.16 \\
\hline 12.65 & 97.6 & 98.1 & 98.4 & 98.0 & 0.31 \\
\hline 13.33 & 98.3 & 98.4 & 98.2 & 98.3 & 0.08 \\
\hline 14.02 & 98.6 & 98.7 & 98.6 & 98.6 & 0.06 \\
\hline 14.70 & 98.6 & 99.2 & 99.0 & 98.9 & 0.23 \\
\hline 15.38 & 98.5 & 98.7 & 99.2 & 98.8 & 0.30 \\
\hline 16.07 & 98.6 & 98.8 & 99.0 & 98.8 & 0.16 \\
\hline 16.75 & 99.0 & 99.2 & 98.9 & 99.0 & 0.12 \\
\hline 17.43 & 98.8 & 99.1 & 99.3 & 99.1 & 0.19 \\
\hline 18.12 & 98.9 & 98.8 & 99.2 & 99.0 & 0.17 \\
\hline
\end{tabular}



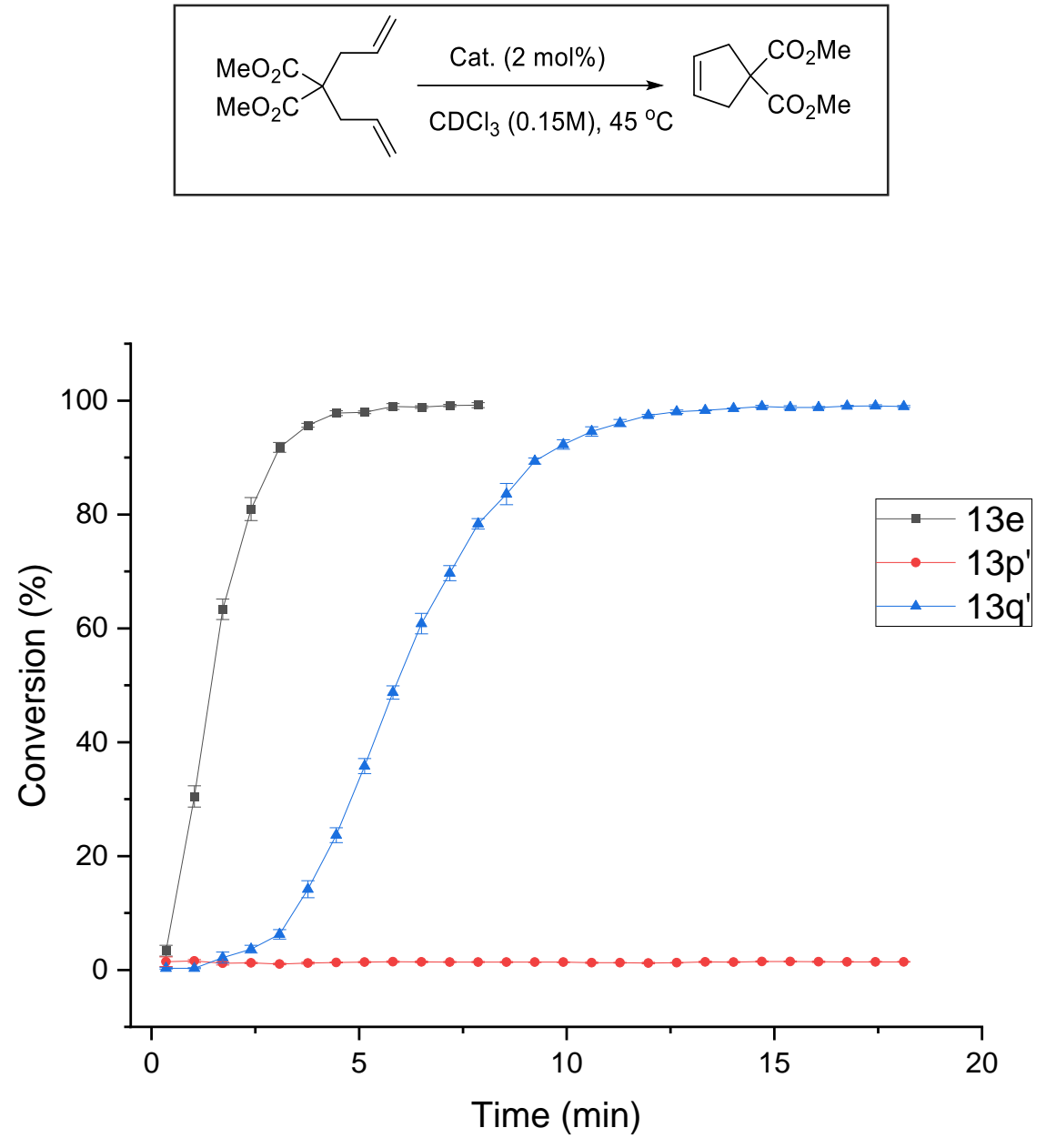

Fig. S4. Kinetic profile for RCM reactions to disubstituted olefins using oxygen-chelated complexes and its comparison with a $\eta^{3}$-vinyl chelate $\mathbf{1 3 e}$. The error bar represents the standard error for the triplicate experiments.

Notes: The acetamide chelated complex $13 p^{\prime}$ did not show any catalytic activity at $45^{\circ} \mathrm{C}$ compared to the sulfonamide chelated complex $\mathbf{1 3} \mathbf{q}^{\prime}$ which showed decent catalytic activity. The reason for this might be

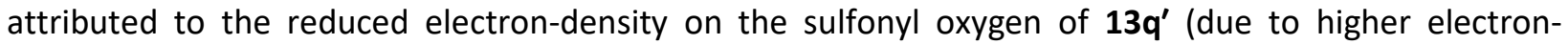
withdrawing nature of S) which makes the oxygen chelation weaker thereby increasing the catalytic activity. On the other hand, the acetamide oxygen in $\mathbf{1 3} \mathbf{p}^{\prime}$ has much higher electron-density as it is connected to a much less electronegative element "carbon", thus oxygen chelation is much stronger, and no catalytic activity is observed at $45^{\circ} \mathrm{C}$. The catalytic activity of $13 p^{\prime}$ was also tested at higher temperature $\left(60^{\circ} \mathrm{C}\right)$ but lead to less than $5 \%$ conversion after $20 \mathrm{~h}$. 


\section{Catalytic activities of trans-1,5-chelate for RCM reaction of dimethyl diallylmalonate at $80{ }^{\circ} \mathrm{C}$}

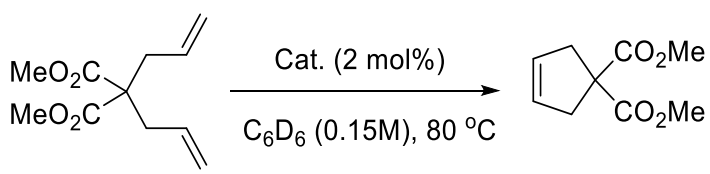

An NMR tube with a screw-cap septum top was charged inside a glovebox with catalyst stock solution (0.1 $\mathrm{M}, 24 \mu \mathrm{L}, 2.4 \mu \mathrm{mol}, 2 \mathrm{~mol} \%)$ in $0.3 \mathrm{~mL}$ of $\mathrm{C}_{6} \mathrm{D}_{6}$ and a solution of dimethyl diallylmalonate $(25 \mathrm{mg}, 0.12$ $\mathrm{mmol}$ ) in $0.5 \mathrm{~mL}$ of $\mathrm{C}_{6} \mathrm{D}_{6}$. The NMR tube was degassed by freeze, pump, thaw method ( $\times 3$ ) and refilled with argon. An initial NMR was taken to get the initial conversion which is zero at room temperature (as these complexes are latent at room temperature). The NMR tube was heated in an oil bath at $80^{\circ} \mathrm{C}$. The NMR tube was removed at regular intervals and cooled immediately in an ice-bath and ${ }^{1} \mathrm{H}$ NMR was taken to determine the conversion. The conversion of the product was determined by comparing the ratio of the integrals of the methylene protons in the starting material, $\delta 2.63$ (d), with those of the product, $\delta$ 3.02 (s). Three independent experiments were performed for each complexes and mean conversion was determined. The conversion versus time graph was plotted for different complexes to have a comparison of their reactivities.

\section{Catalyst 10b}

\begin{tabular}{|r|r|r|r|r|r|}
\hline Time (hr) & \multicolumn{2}{|l|}{ Conversion (\%) } & & \\
\hline & Trial 1 & Trial 2 & Trial 3 & Average & Std dev \\
\hline 0 & 0 & 0 & 0 & 0 & 0 \\
\hline 0.16 & 0.9 & 1.7 & 1.5 & 1.4 & 0.34 \\
\hline 0.5 & 1.9 & 1.3 & 2.8 & 2.0 & 0.62 \\
\hline 1 & 3.2 & 3.6 & 4.1 & 3.6 & 0.37 \\
\hline 2 & 4.2 & 4.7 & 5.8 & 4.9 & 0.67 \\
\hline 3 & 5 & 5.5 & 4.8 & 5.1 & 0.29 \\
\hline 5.5 & 6.6 & 6.8 & 7.2 & 6.9 & 0.25 \\
\hline 10.5 & 9.3 & 9.5 & 10.1 & 9.6 & 0.34 \\
\hline 16.4 & 12.1 & 12.8 & 13.7 & 12.9 & 0.65 \\
\hline
\end{tabular}




\section{Catalyst 10h}

\begin{tabular}{|c|c|c|c|c|c|}
\hline Time (hr) & Conversion (\%) & & & & \\
\hline & Trial 1 & Trial 2 & Trial 3 & Average & Std dev \\
\hline 0 & 0 & 0 & 0 & 0 & 0 \\
\hline 0.16 & 3.8 & 3.4 & 4.7 & 4.0 & 0.54 \\
\hline 0.5 & 7.9 & 8.2 & 7.4 & 7.8 & 0.33 \\
\hline 1 & 14.9 & 14.5 & 15.8 & 15.1 & 0.54 \\
\hline 2 & 23.5 & 20.2 & 25.5 & 23.1 & 2.19 \\
\hline 3 & 33.3 & 32.1 & 36.5 & 34.0 & 1.86 \\
\hline 5.5 & 42.2 & 41.5 & 45.5 & 43.1 & 1.74 \\
\hline 10.5 & 50.2 & 50.5 & 54.3 & 51.7 & 1.87 \\
\hline 16.4 & 56.8 & 53.2 & 57.2 & 55.7 & 1.80 \\
\hline
\end{tabular}

\section{Catalyst 10I}

\begin{tabular}{|r|r|r|r|r|r|r|}
\hline Time (hr) & \multicolumn{2}{|l|}{ Conversion (\%) } & & \\
\hline & Trial 1 & Trial 2 & Trial 3 & Average & Std dev \\
\hline 0 & 0 & 0 & 0 & 0 & 0 \\
\hline 0.08 & 3.1 & 4.5 & 3.9 & 3.8 & 0.57 \\
\hline 0.25 & 6.2 & 7.5 & 7.7 & 7.1 & 0.66 \\
\hline 0.42 & 10.8 & 12.5 & 11.4 & 11.6 & 0.70 \\
\hline 0.92 & 29.9 & 32.2 & 26.5 & 29.5 & 2.34 \\
\hline 1.5 & 52.6 & 55.3 & 53.2 & 53.7 & 1.16 \\
\hline 2 & 74.6 & 77.2 & 73.1 & 75.0 & 1.69 \\
\hline 2.6 & 92.5 & 94.6 & 90.1 & 92.4 & 1.84 \\
\hline 3.6 & 99.1 & 98.6 & 98.1 & 98.6 & 0.41 \\
\hline
\end{tabular}




\section{Catalyst 10n}

\begin{tabular}{|r|r|r|r|r|r|}
\hline Time (hr) & \multicolumn{2}{|l|}{ Conversion (\%) } & \\
\hline & Trial 1 & Trial 2 & Trial 3 & Average & Std dev \\
\hline 0 & 0 & 0 & 0 & 0 & 0 \\
\hline 0.16 & 7.8 & 8.2 & 6.5 & 7.5 & 0.73 \\
\hline 0.33 & 9.5 & 9.3 & 10.7 & 9.8 & 0.62 \\
\hline 0.5 & 11.1 & 12.5 & 12.7 & 12.1 & 0.71 \\
\hline 1 & 21.9 & 24.5 & 19.3 & 21.9 & 2.12 \\
\hline 1.5 & 32.2 & 35.6 & 34.3 & 34.0 & 1.40 \\
\hline 2 & 41.8 & 44.2 & 43.6 & 43.2 & 1.02 \\
\hline 2.6 & 50 & 50.5 & 51.8 & 50.8 & 0.76 \\
\hline 3.6 & 59.1 & 60.4 & 59.3 & 59.6 & 0.57 \\
\hline 7.5 & 67 & 67.8 & 69 & 67.9 & 0.82 \\
\hline 11 & 76 & 78.5 & 77.1 & 77.2 & 1.02 \\
\hline 16 & 80.6 & 83.2 & 81 & 81.6 & 1.14 \\
\hline & & & & & \\
\hline
\end{tabular}



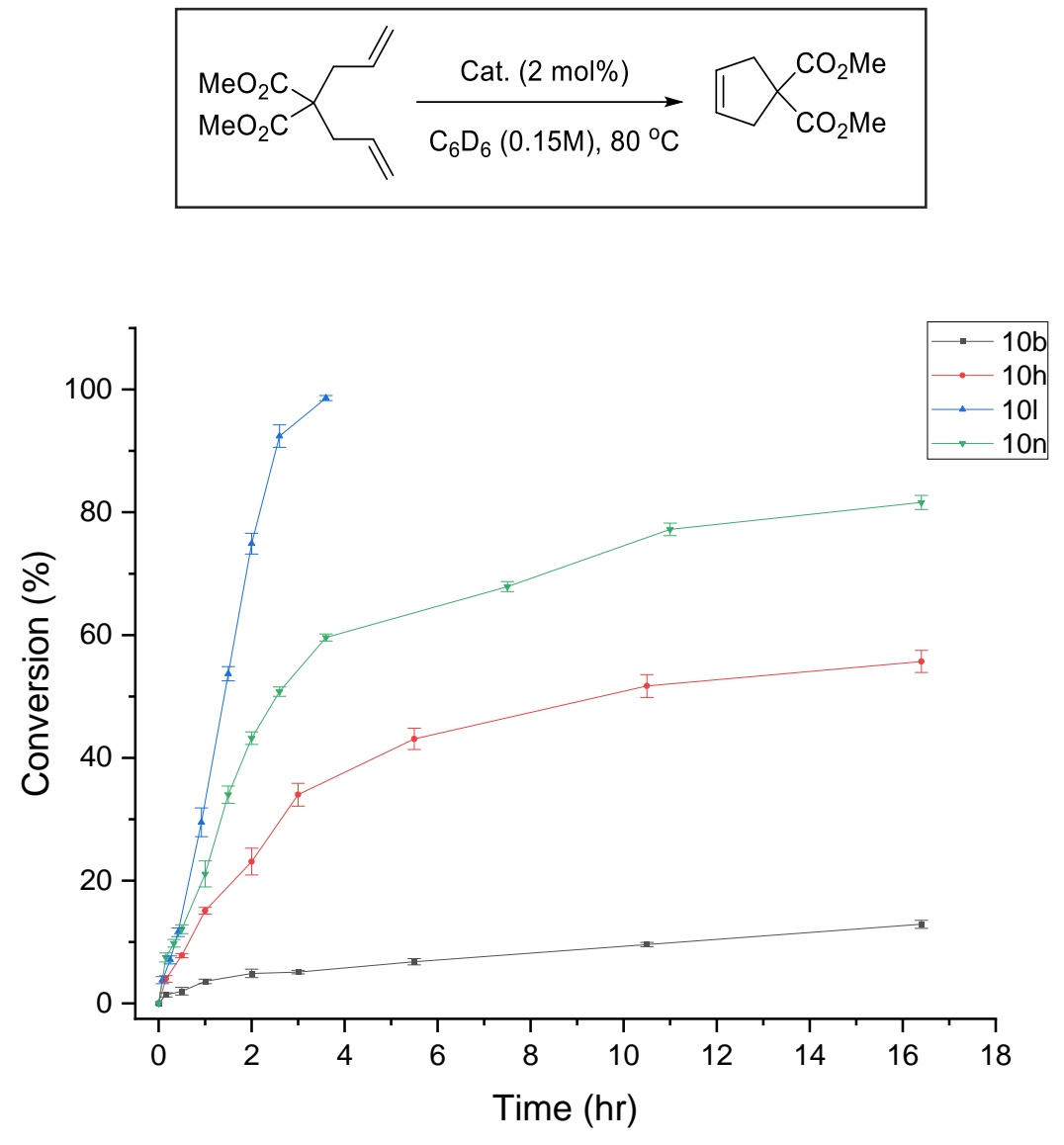

Fig. S5. Kinetic profile for RCM reactions to disubstituted olefins using the latent trans-1,5-chelates as catalysts at $80^{\circ} \mathrm{C}$. Each data point represents the average of three independent experiments. Error bar represents standard error of the triplicate experiments. 
Catalytic Activity for latent 1,5-chelates in $\mathrm{CDCl}_{3}$ at $60^{\circ} \mathrm{C}$
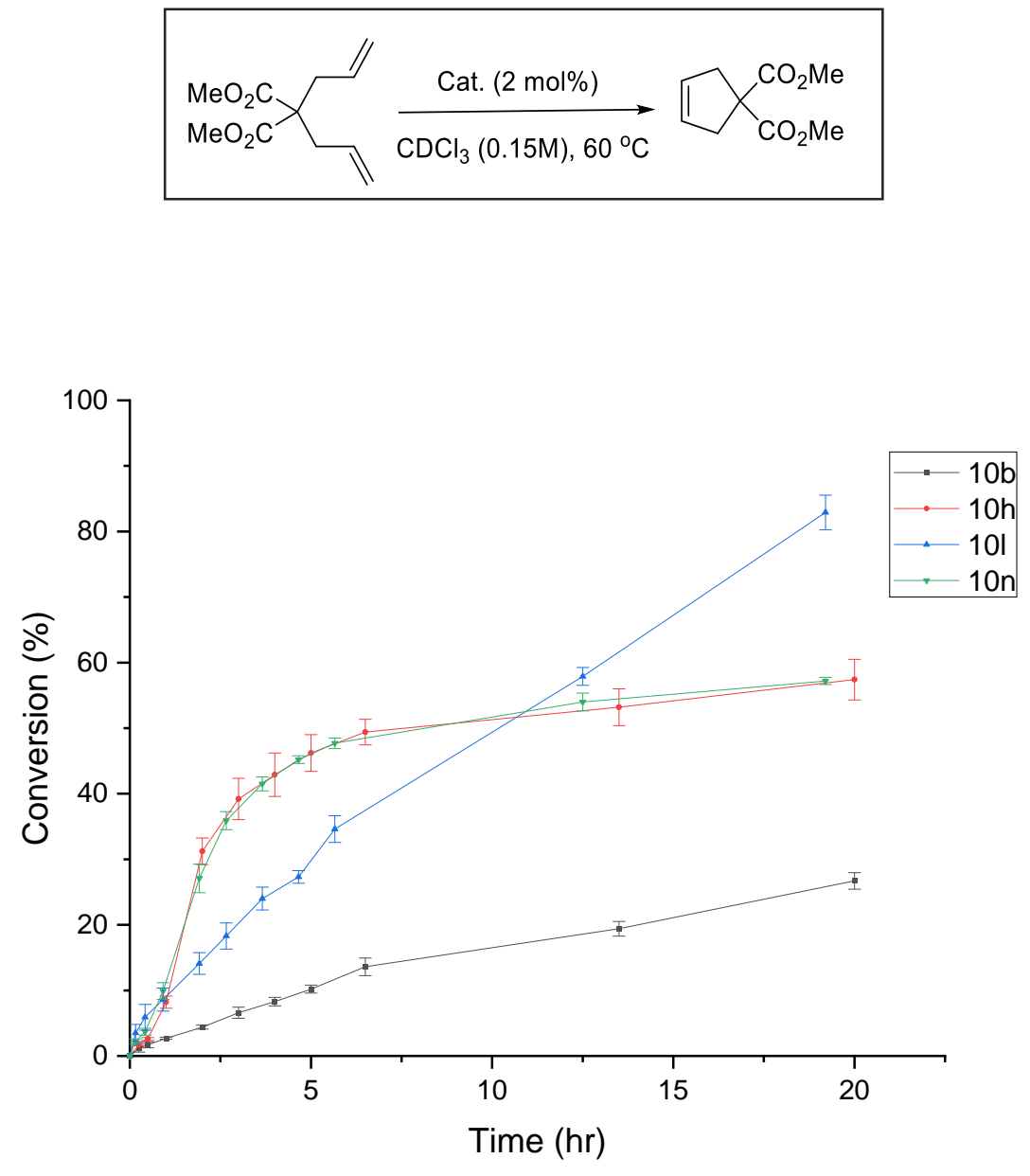

Fig. S6 Kinetic Profile for RCM activity of latent 1,5-chelates in $\mathrm{CDCl}_{3}$ at $60{ }^{\circ} \mathrm{C}$. Each data point represents the average of three independent experiments. Error bar represents standard error of the triplicate experiments.

The catalytic activity of the latent 1,5-chelates were also monitored in $\mathrm{CDCl}_{3}$ to have consistency with other experiments. Since the monitoring was done at $60^{\circ} \mathrm{C}$ compared to the one done in $\mathrm{C}_{6} \mathrm{D}_{6}$ at $80{ }^{\circ} \mathrm{C}$, the net conversion of the complexes is much lower after a period of $20 \mathrm{~h}$. However, the relative differences of reactivities of these four complexes did not change much. Although some striking differences can be noticed, for instance in $\mathrm{CDCl}_{3}$ the complex $10 \mathrm{l}$ initiates at a slow rate compared to its initiation in $\mathrm{C}_{6} \mathrm{D}_{6}$ although the final conversion is $85 \%$, the highest among the four complexes. The reactivity of $10 \mathrm{~h}$ and $10 \mathrm{n}$ is similar in $\mathrm{CDCl}_{3}$ although in $\mathrm{C}_{6} \mathrm{D}_{6}$ the reactivity of $10 \mathrm{n}$ is higher than 10 l. 


\section{NMR spectras of $10 \mathrm{l}$ at $353 \mathrm{~K}\left(80^{\circ} \mathrm{C}\right)$ at different time interval for $3 \mathrm{~h}$}
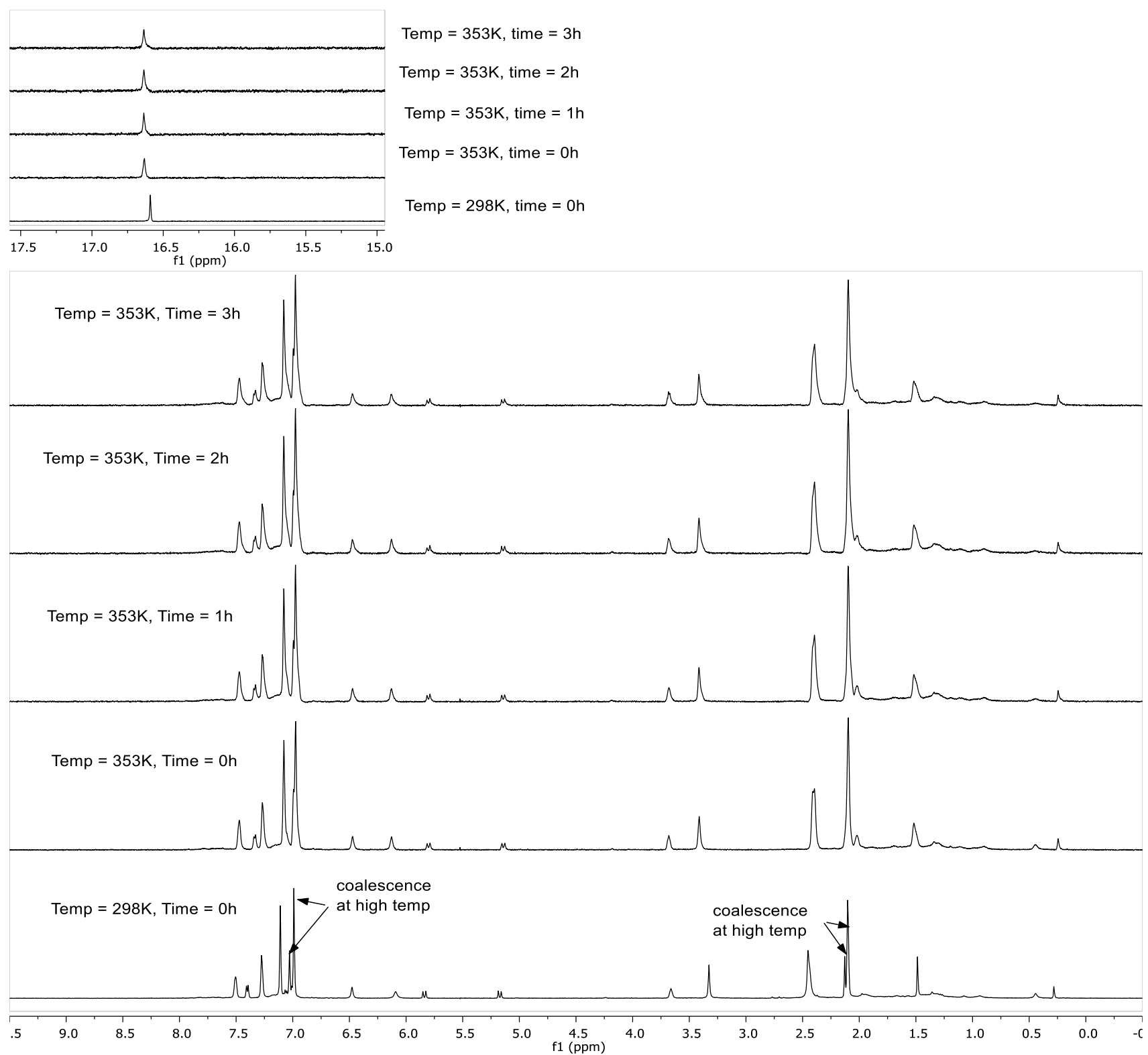


\section{NMR spectras of $10 \mathrm{l}$ at different temperatures from $298 \mathrm{~K}$ to $373 \mathrm{~K}$}
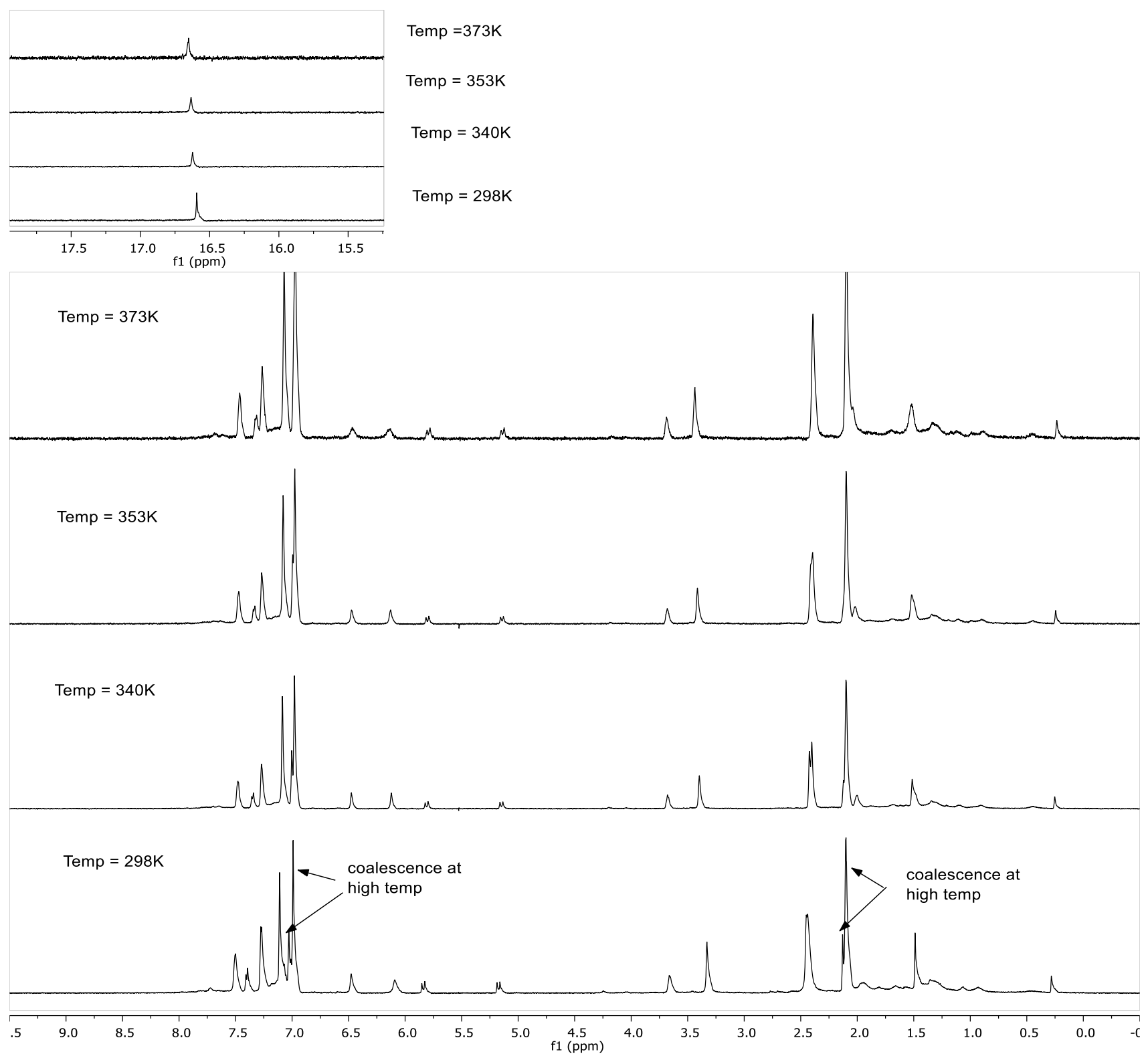


\section{Benchmarking Catalytic-Activity}

After establishing a structure-activity relationship for different types of alkene-chelates we selected a subset of relatively more active complexes to benchmark their catalytic activity for various metathesis process with respect to standard metathesis catalysts. The kinetics of catalytic activity of the alkenechelates were studied for different reactions using NMR techniques (Bruker pseudo 2D kinetics method). The experimental details, raw data of conversion with time, plots showing reaction profile and calculations of kinetic parameters like $k_{o b s,} t_{1 / 2}$, and $k_{o b s}$ (w.r.t G-II) for various metathesis process are given in the subsections underneath.

\section{Catalytic activities of selected alkene-chelates for RCM reaction of dimethyl diallylmalonate at $30^{\circ} \mathrm{C}$}

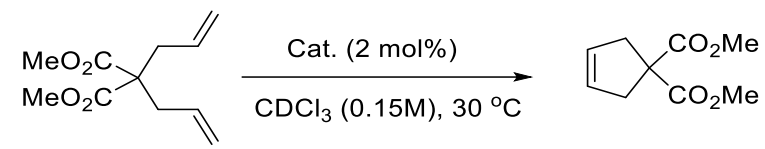

An NMR tube with a screw-cap septum top was charged inside a glovebox with catalyst stock solution (0.1 $\mathrm{M}, 24 \mu \mathrm{L}, 2.4 \mu \mathrm{mol}, 2 \mathrm{~mol} \%)$ in $0.3 \mathrm{~mL}$ of $\mathrm{CDCl}_{3}$. The NMR tube was degassed by freeze, pump, thaw method (cycle of 3 ) and refilled with argon. The sample was equilibrated at $30^{\circ} \mathrm{C}$ in the NMR probe before dimethyl diallylmalonate $\left(25 \mathrm{mg}, 0.12 \mathrm{mmol}\right.$ ) solution in $0.5 \mathrm{~mL}$ of $\mathrm{CDCl}_{3}$ was added via a syringe. Data points were collected over an appropriate time interval using the Bruker pseudo 2D kinetics method. The conversion of the product was determined by comparing the ratio of the integrals of the methylene protons in the starting material, $\delta 2.67-2.57(\mathrm{~m})$, with those of the product, $\delta 3.02(\mathrm{~s})$. Three independent experiments were performed for each complex and the mean conversion was determined. The conversion versus time graph was plotted for different complexes to have a comparison of their reactivities. Kinetic parameter $k_{\text {obs }}$ was determined from the slope of $\ln ([S M])$ vs time plot, where, $\ln ([S M])=\ln ((100-a v g$. conv.) $/ 100 *$ conc. $(0.15 \mathrm{M}))$.

\section{Catalyst 10g}

\begin{tabular}{|r|r|r|r|r|r|r|}
\hline $\begin{array}{l}\text { Time } \\
\text { (min) }\end{array}$ & \multicolumn{2}{|l|}{ Conversion (\%) } & & & & \\
\hline & Trial 1 & Trial 2 & \multicolumn{1}{|c|}{ Trial 3 } & Avg. & Std. Dev. & LN([SM]) \\
\hline 0.35 & 5.3 & 5.8 & 4.1 & 5.1 & 0.71 & -1.95 \\
\hline 1.03 & 31.8 & 25.5 & 27.6 & 28.3 & 2.64 & -2.23 \\
\hline 1.72 & 59.8 & 52.1 & 53.4 & 55.1 & 3.36 & -2.70 \\
\hline 2.40 & 74.6 & 75.6 & 71.3 & 73.8 & 1.84 & -3.24 \\
\hline 3.08 & 82.1 & 81.4 & 83.6 & 82.4 & 0.92 & -3.63 \\
\hline 3.77 & 86.7 & 85.4 & 87.8 & 86.6 & 0.98 & -3.91 \\
\hline 4.45 & 89.5 & 88.2 & 89.7 & 89.1 & 0.67 & -4.12 \\
\hline 5.13 & 91.4 & 89.8 & 91.6 & 90.9 & 0.80 & -4.30 \\
\hline 5.82 & 92.8 & 91.8 & 92.5 & 92.4 & 0.42 & -4.47 \\
\hline 6.50 & 94.0 & 93.6 & 94.7 & 94.1 & 0.46 & -4.73 \\
\hline
\end{tabular}




\begin{tabular}{|r|r|r|r|r|r|r|}
\hline $\begin{array}{l}\text { Time } \\
\text { (min) }\end{array}$ & \multicolumn{2}{|l|}{ Conversion (\%) } & & & & \\
\hline & Trial 1 & Trial 2 & \multicolumn{1}{l|}{ Trial 3 } & \multicolumn{1}{l|}{ Avg. } & Std. Dev. & LN([SM]) \\
\hline 7.18 & 94.7 & 94.5 & 95.6 & 94.9 & 0.47 & -4.88 \\
\hline 7.87 & 95.2 & 96.3 & 96.2 & 95.9 & 0.49 & -5.09 \\
\hline 8.55 & 95.6 & 96.8 & 96.4 & 96.3 & 0.48 & -5.19 \\
\hline 9.23 & 96.2 & 96.5 & 95.9 & 96.2 & 0.25 & -5.17 \\
\hline 9.92 & 96.4 & 96.6 & 95.7 & 96.2 & 0.38 & -5.17 \\
\hline 10.60 & 96.8 & 97.1 & 96.9 & 96.9 & 0.14 & -5.38 \\
\hline 11.28 & 96.9 & 97.3 & 97.1 & 97.1 & 0.15 & -5.44 \\
\hline 11.97 & 96.9 & 97.4 & 96.8 & 97.0 & 0.26 & -5.42 \\
\hline 12.65 & 97.2 & 97.2 & 97.3 & 97.2 & 0.04 & -5.49 \\
\hline 13.33 & 97.3 & 97.1 & 98.4 & 97.6 & 0.58 & -5.62 \\
\hline 14.02 & 97.5 & 98.2 & 98.6 & 98.1 & 0.48 & -5.85 \\
\hline 14.70 & 97.4 & 97.7 & 98.6 & 97.9 & 0.51 & -5.76 \\
\hline 15.38 & 97.7 & 98.5 & 98.7 & 98.3 & 0.43 & -5.97 \\
\hline
\end{tabular}

\section{Catalyst 10i}

\begin{tabular}{|r|r|r|r|r|r|r|}
\hline \multicolumn{1}{l|}{$\begin{array}{l}\text { Time } \\
\text { (min) }\end{array}$} & \multicolumn{2}{|l|}{ Conversion (\%) } & & & \\
\hline & Trial 1 & Trial 2 & Trial 3 & Avg. & Std. Dev. & LN([SM]) \\
\hline 0.35 & 1.0 & 1.5 & 1.3 & 1.0 & 0.18 & -1.91 \\
\hline 1.03 & 3.6 & 3.8 & 3.0 & 3.6 & 0.35 & -1.93 \\
\hline 1.72 & 7.9 & 8.1 & 8.3 & 7.9 & 0.15 & -1.98 \\
\hline 2.40 & 14.0 & 12.5 & 14.3 & 14.0 & 0.79 & -2.05 \\
\hline 3.08 & 21.3 & 19.2 & 21.5 & 21.3 & 1.03 & -2.14 \\
\hline 3.77 & 31.1 & 30.8 & 32.1 & 31.1 & 0.55 & -2.27 \\
\hline 4.45 & 40.3 & 39.8 & 40.7 & 40.3 & 0.37 & -2.41 \\
\hline 5.13 & 48.6 & 49.7 & 48.4 & 48.6 & 0.57 & -2.56 \\
\hline 5.82 & 57.4 & 55.3 & 58.6 & 57.4 & 1.36 & -2.75 \\
\hline 6.50 & 65.2 & 64.3 & 66.7 & 65.2 & 0.99 & -2.95 \\
\hline 7.18 & 71.9 & 72.5 & 71.3 & 71.9 & 0.49 & -3.17 \\
\hline 7.87 & 77.5 & 76.4 & 78.3 & 77.5 & 0.78 & -3.39 \\
\hline 8.55 & 81.8 & 81.6 & 82.1 & 81.8 & 0.21 & -3.60 \\
\hline 9.23 & 85.8 & 85.6 & 85.9 & 85.8 & 0.12 & -3.85 \\
\hline 9.92 & 88.6 & 88.2 & 89.1 & 88.6 & 0.37 & -4.07 \\
\hline 10.60 & 90.8 & 91.2 & 90.6 & 90.8 & 0.25 & -4.28 \\
\hline 11.28 & 92.7 & 92.5 & 93.1 & 92.7 & 0.25 & -4.51 \\
\hline 11.97 & 93.7 & 93.6 & 94.1 & 93.7 & 0.21 & -4.66 \\
\hline 12.65 & 95.9 & 96.1 & 95.7 & 95.9 & 0.16 & -5.09 \\
\hline 13.33 & 94.4 & 95.7 & 95.5 & 94.4 & 0.57 & -4.78 \\
\hline & & & & & & \\
\hline
\end{tabular}




\begin{tabular}{|r|r|r|r|r|r|r|}
\hline $\begin{array}{l}\text { Time } \\
\text { (min) }\end{array}$ & \multicolumn{2}{|l|}{ Conversion (\%) } & & & & \\
\hline & Trial 1 & \multicolumn{1}{l|}{ Trial 2 } & Trial 3 & \multicolumn{1}{|l|}{ Avg. } & Std. Dev. & LN([SM]) \\
\hline 14.02 & 95.9 & 96.6 & 96.3 & 95.9 & 0.29 & -5.09 \\
\hline 14.70 & 96.7 & 96.8 & 97.2 & 96.7 & 0.21 & -5.32 \\
\hline 15.38 & 97.3 & 97.5 & 97.7 & 97.3 & 0.16 & -5.51 \\
\hline 16.07 & 96.9 & 97.2 & 97.6 & 96.9 & 0.27 & -5.38 \\
\hline 16.75 & 97.8 & 98.2 & 98.4 & 97.8 & 0.24 & -5.73 \\
\hline 17.43 & 98.2 & 97.8 & 98.4 & 98.2 & 0.25 & -5.92 \\
\hline 18.12 & 98.0 & 98.2 & 98.5 & 98.0 & 0.21 & -5.80 \\
\hline 18.80 & 98.0 & 98.6 & 98.4 & 98.0 & 0.25 & -5.81 \\
\hline 19.48 & 96.6 & 98.3 & 98.5 & 96.6 & 0.86 & -5.27 \\
\hline
\end{tabular}

\section{Catalyst 13c}

\begin{tabular}{|c|c|c|c|c|c|c|}
\hline \multirow{2}{*}{$\begin{array}{l}\text { Time } \\
\text { (min) }\end{array}$} & \multicolumn{2}{|c|}{ Conversion (\%) } & \multirow[b]{2}{*}{ Trial 3} & \multirow[b]{2}{*}{ Avg. } & \multirow[b]{2}{*}{ Std. Dev. } & \multirow[b]{2}{*}{$\mathrm{LN}([\mathrm{SM}])$} \\
\hline & Trial 1 & Trial 2 & & & & \\
\hline 0.35 & 1.9 & 1.2 & 2.8 & 2.0 & 0.65 & -1.92 \\
\hline 1.03 & 7.4 & 6.4 & 7.9 & 7.2 & 0.62 & -1.97 \\
\hline 1.72 & 16.0 & 14.1 & 17.3 & 15.8 & 1.32 & -2.07 \\
\hline 2.40 & 26.4 & 22.4 & 27.1 & 25.3 & 2.07 & -2.19 \\
\hline 3.08 & 36.8 & 37.5 & 35.4 & 36.6 & 0.87 & -2.35 \\
\hline 3.77 & 46.6 & 42.1 & 48.4 & 45.7 & 2.65 & -2.51 \\
\hline 4.45 & 55.1 & 51.4 & 55.2 & 53.9 & 1.77 & -2.67 \\
\hline 5.13 & 62.8 & 61.6 & 63.8 & 62.7 & 0.90 & -2.88 \\
\hline 5.82 & 69.3 & 67.5 & 69.5 & 68.8 & 0.89 & -3.06 \\
\hline 6.50 & 74.5 & 73.1 & 75.1 & 74.2 & 0.84 & -3.25 \\
\hline 7.18 & 78.9 & 77.2 & 81.2 & 79.1 & 1.64 & -3.46 \\
\hline 7.87 & 82.5 & 83.1 & 82.2 & 82.6 & 0.37 & -3.65 \\
\hline 8.55 & 85.5 & 84.6 & 86.1 & 85.4 & 0.62 & -3.82 \\
\hline 9.23 & 87.9 & 86.3 & 88.1 & 87.4 & 0.81 & -3.97 \\
\hline 9.92 & 89.8 & 89.5 & 90.4 & 89.9 & 0.37 & -4.19 \\
\hline 10.60 & 91.3 & 90.7 & 91.8 & 91.3 & 0.45 & -4.34 \\
\hline 11.28 & 92.6 & 91.7 & 93.5 & 92.6 & 0.73 & -4.50 \\
\hline 11.97 & 93.5 & 93.1 & 93.8 & 93.5 & 0.29 & -4.63 \\
\hline 12.65 & 94.4 & 93.8 & 94.8 & 94.3 & 0.41 & -4.76 \\
\hline 13.33 & 94.9 & 94.1 & 94 & 94.3 & 0.43 & -4.77 \\
\hline 14.02 & 95.4 & 95.3 & 95.4 & 95.4 & 0.04 & -4.97 \\
\hline 14.70 & 95.8 & 96.2 & 96.8 & 96.3 & 0.43 & -5.18 \\
\hline
\end{tabular}




\begin{tabular}{|r|r|r|r|r|r|r|}
\hline $\begin{array}{l}\text { Time } \\
\text { (min) }\end{array}$ & \multicolumn{2}{|l|}{ Conversion (\%) } & & & & \\
\hline & \multicolumn{1}{|l|}{ Trial 1 } & \multicolumn{1}{l|}{ Trial 2 } & Trial 3 & \multicolumn{1}{|l|}{ Avg. } & Std. Dev. & LN([SM]) \\
\hline 15.38 & 96.2 & 95.7 & 96.8 & 96.2 & 0.45 & -5.18 \\
\hline 16.07 & 96.4 & 96.5 & 97.3 & 96.7 & 0.41 & -5.32 \\
\hline 16.75 & 96.7 & 97.3 & 97.6 & 97.2 & 0.36 & -5.48 \\
\hline
\end{tabular}

\section{Catalyst 13d}

\begin{tabular}{|c|c|c|c|c|c|c|}
\hline \multirow{2}{*}{$\begin{array}{l}\text { Time } \\
\text { (min) }\end{array}$} & \multicolumn{2}{|c|}{ Conversion (\%) } & \multirow[b]{2}{*}{ Trial 3} & \multirow[b]{2}{*}{ Avg. } & \multirow[b]{2}{*}{ Std. Dev. } & \multirow[b]{2}{*}{$\mathrm{L}([\mathrm{SM}])$} \\
\hline & Trial 1 & Trial 2 & & & & \\
\hline 0.35 & 6.1 & 4.8 & 7.3 & 6.1 & 1.02 & -1.96 \\
\hline 1.03 & 24.1 & 20.3 & 22.1 & 22.2 & 1.56 & -2.15 \\
\hline 1.72 & 37.5 & 32.0 & 35.4 & 35.0 & 2.27 & -2.33 \\
\hline 2.40 & 48.5 & 45.4 & 47.8 & 47.2 & 1.34 & -2.54 \\
\hline 3.08 & 57.4 & 54.2 & 56.3 & 56.0 & 1.34 & -2.72 \\
\hline 3.77 & 64.9 & 61.3 & 65.7 & 64.0 & 1.91 & -2.92 \\
\hline 4.45 & 71.0 & 69.3 & 73.4 & 71.2 & 1.68 & -3.14 \\
\hline 5.13 & 76.1 & 72.8 & 78.7 & 75.9 & 2.41 & -3.32 \\
\hline 5.82 & 80.2 & 79.5 & 80.7 & 80.1 & 0.49 & -3.51 \\
\hline 6.50 & 83.5 & 80.5 & 84.6 & 82.9 & 1.74 & -3.66 \\
\hline 7.18 & 86.2 & 84.8 & 87.8 & 86.3 & 1.23 & -3.88 \\
\hline 7.87 & 88.5 & 88.2 & 89.1 & 88.6 & 0.38 & -4.07 \\
\hline 8.55 & 90.3 & 89.8 & 90.5 & 90.2 & 0.29 & -4.22 \\
\hline 9.23 & 91.7 & 91.2 & 92.3 & 91.7 & 0.45 & -4.39 \\
\hline 9.92 & 92.9 & 92.5 & 92.7 & 92.7 & 0.18 & -4.52 \\
\hline 10.60 & 93.8 & 92.4 & 94.2 & 93.5 & 0.78 & -4.63 \\
\hline 11.28 & 94.7 & 93.8 & 95.2 & 94.6 & 0.58 & -4.81 \\
\hline 11.97 & 95.4 & 95.7 & 95.9 & 95.7 & 0.22 & -5.03 \\
\hline 12.65 & 95.8 & 95.9 & 96.2 & 96.0 & 0.15 & -5.11 \\
\hline 13.33 & 96.4 & 96.2 & 96.8 & 96.5 & 0.25 & -5.24 \\
\hline 14.02 & 96.7 & 96.4 & 97.0 & 96.7 & 0.25 & -5.31 \\
\hline 14.70 & 97.2 & 97.5 & 97.9 & 97.5 & 0.28 & -5.60 \\
\hline 15.38 & 97.3 & 97.7 & 98.0 & 97.7 & 0.30 & -5.65 \\
\hline 16.07 & 97.4 & 97.2 & 97.9 & 97.5 & 0.29 & -5.59 \\
\hline 16.75 & 97.6 & 98.3 & 98.5 & 98.1 & 0.39 & -5.88 \\
\hline 17.43 & 97.8 & 98.4 & 98.6 & 98.3 & 0.34 & -5.95 \\
\hline
\end{tabular}




\begin{tabular}{|c|c|c|c|c|c|c|}
\hline \multirow{2}{*}{$\begin{array}{l}\text { Time } \\
\text { (min) }\end{array}$} & \multicolumn{2}{|c|}{ Conversion (\%) } & \multirow[b]{2}{*}{ Trial 3} & \multirow[b]{2}{*}{ Avg. } & \multirow[b]{2}{*}{ Std. Dev. } & \multirow[b]{2}{*}{$\mathrm{LN}([\mathrm{SM}])$} \\
\hline & Trial 1 & Trial 2 & & & & \\
\hline 0.35 & 3.5 & 2.1 & 4.7 & 3.4 & 1.06 & -1.93 \\
\hline 1.03 & 17.2 & 14.8 & 19.6 & 17.2 & 1.96 & -2.09 \\
\hline 1.72 & 30.0 & 26.5 & 27.8 & 28.1 & 1.46 & -2.23 \\
\hline 2.40 & 40.8 & 38 & 37.3 & 38.7 & 1.51 & -2.39 \\
\hline 3.08 & 49.8 & 45.6 & 51.1 & 48.8 & 2.35 & -2.57 \\
\hline 3.77 & 57.6 & 55.4 & 58.3 & 57.1 & 1.24 & -2.74 \\
\hline 4.45 & 64.2 & 63.1 & 66.7 & 64.7 & 1.51 & -2.94 \\
\hline 5.13 & 69.8 & 65.4 & 70.3 & 68.5 & 2.20 & -3.05 \\
\hline 5.82 & 74.5 & 72.1 & 76.7 & 74.4 & 1.88 & -3.26 \\
\hline 6.50 & 78.6 & 77.5 & 79.5 & 78.5 & 0.82 & -3.44 \\
\hline 7.18 & 82.1 & 80.5 & 81.6 & 81.4 & 0.66 & -3.58 \\
\hline 7.87 & 84.9 & 83.1 & 85.4 & 84.5 & 0.98 & -3.76 \\
\hline 8.55 & 87.3 & 85.6 & 87.2 & 86.7 & 0.78 & -3.91 \\
\hline 9.23 & 89.4 & 88.6 & 89.7 & 89.2 & 0.46 & -4.13 \\
\hline 9.92 & 90.9 & 91.5 & 90.4 & 90.9 & 0.45 & -4.30 \\
\hline 10.60 & 92.5 & 91.9 & 92.8 & 92.4 & 0.38 & -4.48 \\
\hline 11.28 & 93.6 & 93.1 & 94.1 & 93.6 & 0.41 & -4.65 \\
\hline 11.97 & 94.6 & 94.1 & 94.8 & 94.5 & 0.29 & -4.79 \\
\hline 12.65 & 95.6 & 94.9 & 95.7 & 95.4 & 0.35 & -4.97 \\
\hline 13.33 & 96.1 & 96.3 & 96.7 & 96.4 & 0.26 & -5.21 \\
\hline 14.02 & 96.7 & 95.8 & 96.9 & 96.5 & 0.47 & -5.24 \\
\hline 14.70 & 96.8 & 97.3 & 97.5 & 97.2 & 0.28 & -5.48 \\
\hline 15.38 & 97.4 & 97.1 & 98.2 & 97.6 & 0.47 & -5.61 \\
\hline 16.07 & 97.7 & 98.3 & 97.1 & 97.7 & 0.49 & -5.66 \\
\hline 16.75 & 98.0 & 97.1 & 99.3 & 98.1 & 0.90 & -5.88 \\
\hline 17.43 & 98.1 & 99.4 & 98.6 & 98.7 & 0.52 & -6.25 \\
\hline 18.12 & 98.3 & 98.1 & 99.3 & 98.6 & 0.53 & -6.13 \\
\hline
\end{tabular}




\begin{tabular}{|r|r|r|r|r|r|r|}
\hline $\begin{array}{l}\text { Time } \\
(\mathrm{min})\end{array}$ & \multicolumn{2}{|l|}{ Conversion (\%) } & & & & \\
\hline & \multicolumn{1}{|l|}{ Trial 1 } & \multicolumn{1}{l|}{ Trial 2 } & \multicolumn{1}{l|}{ Trial 3 } & \multicolumn{1}{l|}{ Avg. } & Std. Dev. & LN([SM]) \\
\hline 0.35 & 39.6 & 34.6 & 35.3 & 36.5 & 2.20 & -2.35 \\
\hline 1.03 & 68.1 & 62.4 & 65.3 & 65.3 & 2.34 & -2.95 \\
\hline 1.72 & 81.1 & 78.8 & 80.3 & 80.1 & 0.97 & -3.51 \\
\hline 2.40 & 87.8 & 85.4 & 88.3 & 87.2 & 1.26 & -3.95 \\
\hline 3.08 & 91.8 & $90 . .8$ & 91.6 & 91.7 & 0.10 & -4.39 \\
\hline 3.77 & 94.0 & 93.0 & 93.7 & 93.6 & 0.43 & -4.64 \\
\hline 4.45 & 95.0 & 95.4 & 95.6 & 95.3 & 0.26 & -4.96 \\
\hline 5.13 & 96.8 & 97.4 & 97.2 & 97.1 & 0.23 & -5.45 \\
\hline 5.82 & 97.1 & 97.6 & 97.3 & 97.3 & 0.21 & -5.52 \\
\hline 6.50 & 97.8 & 97.4 & 98.2 & 97.8 & 0.33 & -5.71 \\
\hline 7.18 & 98.6 & 98.2 & 98.8 & 98.4 & 0.22 & -6.04 \\
\hline 7.87 & 97.8 & 98.1 & 98.5 & 98.1 & 0.31 & -5.87 \\
\hline 8.55 & 97.7 & 98.1 & 98.4 & 98.1 & 0.28 & -5.84 \\
\hline
\end{tabular}

Catalyst G-I

\begin{tabular}{|r|r|r|r|r|r|r|}
\hline $\begin{array}{l}\text { Time } \\
\text { (min) }\end{array}$ & \multicolumn{2}{|l|}{ Conversion (\%) } & & & & \\
\hline & \multicolumn{1}{|l|}{ Trial 1 } & \multicolumn{1}{l|}{ Trial 2 } & \multicolumn{1}{l|}{ Trial 3 } & \multicolumn{1}{l|}{ Avg. } & Std. Dev. & LN([SM]) \\
\hline 0.35 & 56.7 & 49.5 & 51.3 & 52.5 & 3.07 & -2.64 \\
\hline 1.03 & 76.6 & 72.5 & 71.8 & 73.6 & 2.13 & -3.23 \\
\hline 1.72 & 86.0 & 84.5 & 83.6 & 84.7 & 0.97 & -3.77 \\
\hline 2.40 & 90.7 & 88.6 & 89.1 & 89.5 & 0.90 & -4.15 \\
\hline 3.08 & 93.3 & 91.8 & 93.2 & 92.8 & 0.68 & -4.52 \\
\hline 3.77 & 94.8 & 93.5 & 94.6 & 94.3 & 0.56 & -4.76 \\
\hline 4.45 & 95.8 & 94.8 & 95.5 & 95.4 & 0.42 & -4.97 \\
\hline 5.13 & 96.5 & 96.2 & 97.1 & 96.6 & 0.38 & -5.27 \\
\hline 5.82 & 96.8 & 97.5 & 96.3 & 96.9 & 0.49 & -5.36 \\
\hline 6.50 & 97.2 & 97.4 & 97.0 & 97.2 & 0.16 & -5.48 \\
\hline 7.18 & 97.4 & 97.7 & 97.9 & 97.7 & 0.19 & -5.66 \\
\hline 7.87 & 97.7 & 97.1 & 97.4 & 97.4 & 0.23 & -5.54 \\
\hline 8.55 & 97.8 & 98.2 & 97.5 & 97.8 & 0.29 & -5.72 \\
\hline 9.23 & 97.9 & 98.3 & 98.5 & 98.2 & 0.25 & -5.93 \\
\hline 9.92 & 97.9 & 97.4 & 98.2 & 97.8 & 0.33 & -5.74 \\
\hline 10.60 & 98.1 & 98.6 & 98.3 & 98.3 & 0.21 & -5.99 \\
\hline & & & & & & \\
\hline
\end{tabular}




\begin{tabular}{|r|r|r|r|r|r|r|}
\hline $\begin{array}{l}\text { Time } \\
\text { (min) }\end{array}$ & \multicolumn{2}{|l|}{ Conversion (\%) } & & & & \\
\hline & Trial 1 & Trial 2 & Trial 3 & \multicolumn{1}{|l|}{ Avg. } & Std. Dev. & LN([SM]) \\
\hline 11.28 & 98.1 & 97.8 & 98.4 & 98.1 & 0.25 & -5.85 \\
\hline 11.97 & 98.1 & 97.6 & 98.3 & 98.0 & 0.29 & -5.81 \\
\hline
\end{tabular}

Catalyst G-II

\begin{tabular}{|c|c|c|c|c|c|c|}
\hline \multirow{2}{*}{$\begin{array}{l}\text { Time } \\
(\min )\end{array}$} & \multicolumn{2}{|c|}{ Conversion (\%) } & \multirow[b]{2}{*}{ Trial 3} & \multirow[b]{2}{*}{ Avg. } & \multirow[b]{2}{*}{ Std. Dev. } & \multirow[b]{2}{*}{$\mathrm{LN}([\mathrm{SM}])$} \\
\hline & Trial 1 & Trial 2 & & & & \\
\hline 0.35 & 1.8 & 1.3 & 2.7 & 1.9 & 0.58 & -1.92 \\
\hline 1.03 & 4.1 & 3.2 & 3.6 & 3.6 & 0.36 & -1.93 \\
\hline 1.72 & 7.2 & 5.8 & 6.7 & 6.6 & 0.59 & -1.97 \\
\hline 2.40 & 10.6 & 9.3 & 11.2 & 10.4 & 0.79 & -2.01 \\
\hline 3.08 & 14.0 & 14.5 & 15.3 & 14.6 & 0.54 & -2.05 \\
\hline 3.77 & 17.3 & 15.9 & 17.8 & 17.0 & 0.81 & -2.08 \\
\hline 4.45 & 20.6 & 19.3 & 20.8 & 20.2 & 0.67 & -2.12 \\
\hline 5.13 & 23.9 & 22.6 & 23.6 & 23.4 & 0.55 & -2.16 \\
\hline 5.82 & 27.0 & 25.8 & 26.3 & 26.4 & 0.51 & -2.20 \\
\hline 6.50 & 30.2 & 29.7 & 30.4 & 30.1 & 0.29 & -2.25 \\
\hline 7.18 & 33.1 & 32.3 & 34.3 & 33.2 & 0.82 & -2.30 \\
\hline 7.87 & 36.1 & 35.2 & 35.8 & 35.7 & 0.36 & -2.34 \\
\hline 8.55 & 38.8 & 36.8 & 37.3 & 37.6 & 0.87 & -2.37 \\
\hline 9.23 & 41.5 & 40.2 & 41.7 & 41.1 & 0.67 & -2.43 \\
\hline 9.92 & 44.1 & 42.6 & 45.6 & 44.1 & 1.22 & -2.48 \\
\hline 10.60 & 46.5 & 46.1 & 47.3 & 46.6 & 0.50 & -2.53 \\
\hline 11.28 & 48.8 & 49.5 & 48.1 & 48.8 & 0.57 & -2.57 \\
\hline 11.97 & 51.0 & 50.3 & 52.6 & 51.3 & 0.96 & -2.62 \\
\hline 12.65 & 53.1 & 55.7 & 54.2 & 54.3 & 1.08 & -2.68 \\
\hline 13.33 & 55.0 & 55.8 & 54.7 & 55.2 & 0.46 & -2.70 \\
\hline 14.02 & 56.9 & 57.5 & 56.1 & 56.8 & 0.57 & -2.74 \\
\hline 14.70 & 58.6 & 59.7 & 58.2 & 58.8 & 0.64 & -2.78 \\
\hline 15.38 & 60.2 & 60.6 & 61.6 & 60.8 & 0.59 & -2.83 \\
\hline 16.07 & 61.7 & 61.8 & 62.4 & 62.0 & 0.30 & -2.86 \\
\hline 16.75 & 63.2 & 63.7 & 63.5 & 63.5 & 0.22 & -2.90 \\
\hline 17.43 & 64.5 & 63.9 & 64.7 & 64.4 & 0.34 & -2.93 \\
\hline 18.12 & 65.8 & 66.3 & 66.5 & 66.2 & 0.32 & -2.98 \\
\hline 18.80 & 67.0 & 67.7 & 67.2 & 67.3 & 0.30 & -3.01 \\
\hline 19.48 & 68.1 & 68.5 & 67.8 & 68.1 & 0.29 & -3.04 \\
\hline 20.17 & 69.1 & 68.9 & 69.5 & 69.2 & 0.25 & -3.07 \\
\hline
\end{tabular}




\begin{tabular}{|c|c|c|c|c|c|c|}
\hline \multirow{2}{*}{$\begin{array}{l}\text { Time } \\
\text { (min) }\end{array}$} & \multicolumn{2}{|c|}{ Conversion (\%) } & \multirow[b]{2}{*}{ Trial 3} & \multirow[b]{2}{*}{ Avg. } & \multirow[b]{2}{*}{ Std. Dev. } & \multirow[b]{2}{*}{$\mathrm{LN}([\mathrm{SM}])$} \\
\hline & Trial 1 & Trial 2 & & & & \\
\hline 20.85 & 70.1 & 70.3 & 70.4 & 70.3 & 0.13 & -3.11 \\
\hline 21.53 & 71.0 & 71.6 & 71.6 & 71.4 & 0.27 & -3.15 \\
\hline 22.22 & 71.9 & 72.3 & 72.4 & 72.2 & 0.22 & -3.18 \\
\hline 22.90 & 72.7 & 73.0 & 73.6 & 73.1 & 0.36 & -3.21 \\
\hline 23.58 & 73.5 & 73.3 & 73.9 & 73.6 & 0.25 & -3.23 \\
\hline 24.27 & 74.2 & 74.3 & 74.3 & 74.3 & 0.03 & -3.26 \\
\hline 24.95 & 74.9 & 74.5 & 75.3 & 74.9 & 0.33 & -3.28 \\
\hline 25.63 & 75.6 & 76.3 & 75.4 & 75.8 & 0.38 & -3.32 \\
\hline 26.32 & 76.3 & 76.7 & 75.8 & 76.3 & 0.37 & -3.33 \\
\hline 27.00 & 76.9 & 77.2 & 76.5 & 76.9 & 0.29 & -3.36 \\
\hline 27.68 & 77.4 & 77.7 & 78.2 & 77.8 & 0.33 & -3.40 \\
\hline 28.37 & 78.1 & 78.5 & 77.9 & 78.2 & 0.25 & -3.42 \\
\hline 29.05 & 78.6 & 78.2 & 78.4 & 78.4 & 0.16 & -3.43 \\
\hline 29.73 & 79.1 & 79.5 & 79.3 & 79.3 & 0.17 & -3.47 \\
\hline 30.42 & 79.5 & 80.2 & 79.5 & 79.7 & 0.33 & -3.49 \\
\hline 31.10 & 80.0 & 80.2 & 81.3 & 80.5 & 0.56 & -3.53 \\
\hline 31.78 & 80.5 & 81.2 & 81.6 & 81.1 & 0.44 & -3.56 \\
\hline 32.47 & 81.0 & 81.8 & 81.5 & 81.4 & 0.34 & -3.58 \\
\hline 33.15 & 81.4 & 81.7 & 81.6 & 81.6 & 0.15 & -3.59 \\
\hline 33.83 & 81.8 & 82.3 & 82.5 & 82.2 & 0.29 & -3.62 \\
\hline 34.52 & 82.3 & 82.1 & 81.6 & 82.0 & 0.29 & -3.61 \\
\hline 35.20 & 82.7 & 82.9 & 82.5 & 82.7 & 0.16 & -3.65 \\
\hline
\end{tabular}

Catalyst G-III

\begin{tabular}{|r|r|r|r|r|r|}
\hline $\begin{array}{l}\text { Time } \\
\text { (min) }\end{array}$ & \multicolumn{2}{|l|}{ Conversion (\%) } & & & \\
\hline & \multicolumn{1}{|l|}{ Trial 1 } & \multicolumn{1}{l|}{ Trial 2 } & \multicolumn{1}{l|}{ Trial 3 } & \multicolumn{1}{l|}{ Avg. } & Std. Dev. \\
\hline 0.35 & 15.4 & 13.6 & 16.8 & 15.3 & 1.3 \\
\hline 1.03 & 23.5 & 21.2 & 24.5 & 23.1 & 1.4 \\
\hline 1.72 & 29.8 & 29.3 & 31.2 & 30.1 & 0.8 \\
\hline 2.40 & 33.6 & 35.7 & 33.1 & 34.1 & 1.1 \\
\hline 3.08 & 36.2 & 37.1 & 36.5 & 36.6 & 0.4 \\
\hline 3.77 & 38.1 & 38.3 & 38.5 & 38.3 & 0.2 \\
\hline 4.45 & 39.3 & 39.1 & 39.6 & 39.3 & 0.2 \\
\hline 5.13 & 39.6 & 39.7 & 39.2 & 39.5 & 0.2 \\
\hline 5.82 & 41.1 & 41.5 & 40.9 & 41.2 & 0.3 \\
\hline 6.50 & 41.2 & 41.7 & 41.3 & 41.4 & 0.2 \\
\hline
\end{tabular}




\begin{tabular}{|r|r|r|r|r|r|}
\hline $\begin{array}{l}\text { Time } \\
\text { (min) }\end{array}$ & \multicolumn{2}{|l|}{ Conversion (\%) } & & \\
\hline & Trial 1 & Trial 2 & Trial 3 & \multicolumn{1}{l|}{ Avg. } & Std. Dev. \\
\hline 7.18 & 40.8 & 40.6 & 41.5 & 41.0 & 0.4 \\
\hline 7.87 & 41.9 & 42.3 & 41.1 & 41.8 & 0.5 \\
\hline 8.55 & 41.9 & 42.1 & 41.3 & 41.8 & 0.3 \\
\hline 9.23 & 42.0 & 42.2 & 39.7 & 41.3 & 1.1 \\
\hline 9.92 & 42.4 & 42.1 & 42.8 & 42.4 & 0.3 \\
\hline 10.60 & 42.0 & 42.6 & 39.4 & 41.3 & 1.4 \\
\hline 11.28 & 42.3 & 42.5 & 42.9 & 42.6 & 0.2 \\
\hline 11.97 & 42.6 & 42.8 & 42.7 & 42.7 & 0.1 \\
\hline 12.65 & 41.5 & 41.7 & 41.9 & 41.7 & 0.2 \\
\hline 13.33 & 41.6 & 42.2 & 41.8 & 41.9 & 0.3 \\
\hline 14.02 & 44.1 & 43.2 & 43.0 & 43.4 & 0.5 \\
\hline 14.70 & 43.2 & 43.5 & 42.7 & 43.1 & 0.3 \\
\hline 15.38 & 42.6 & 42.7 & 42.9 & 42.7 & 0.1 \\
\hline 16.07 & 42.9 & 43.4 & 42.7 & 43.0 & 0.3 \\
\hline 16.75 & 42.8 & 42.9 & 43.2 & 43.0 & 0.2 \\
\hline 17.43 & 42.6 & 42.2 & 43.1 & 42.6 & 0.4 \\
\hline 18.12 & 42.5 & 43.1 & 43.4 & 43.0 & 0.4 \\
\hline 18.80 & 43.0 & 43.7 & 43.6 & 43.4 & 0.3 \\
\hline 19.48 & 42.3 & 42.8 & 43.1 & 42.7 & 0.3 \\
\hline 20.17 & 42.6 & 42.8 & 43.4 & 42.9 & 0.3 \\
\hline 20.85 & 41.4 & 42.6 & 42.9 & 42.3 & 0.6 \\
\hline 21.53 & 43.4 & 43.6 & 43.2 & 43.4 & 0.2 \\
\hline 22.22 & 41.0 & 43.5 & 42.3 & 42.3 & 1.0 \\
\hline 22.90 & 42.8 & 43.8 & 42.8 & 43.1 & 0.5 \\
\hline & & & & & \\
\hline
\end{tabular}



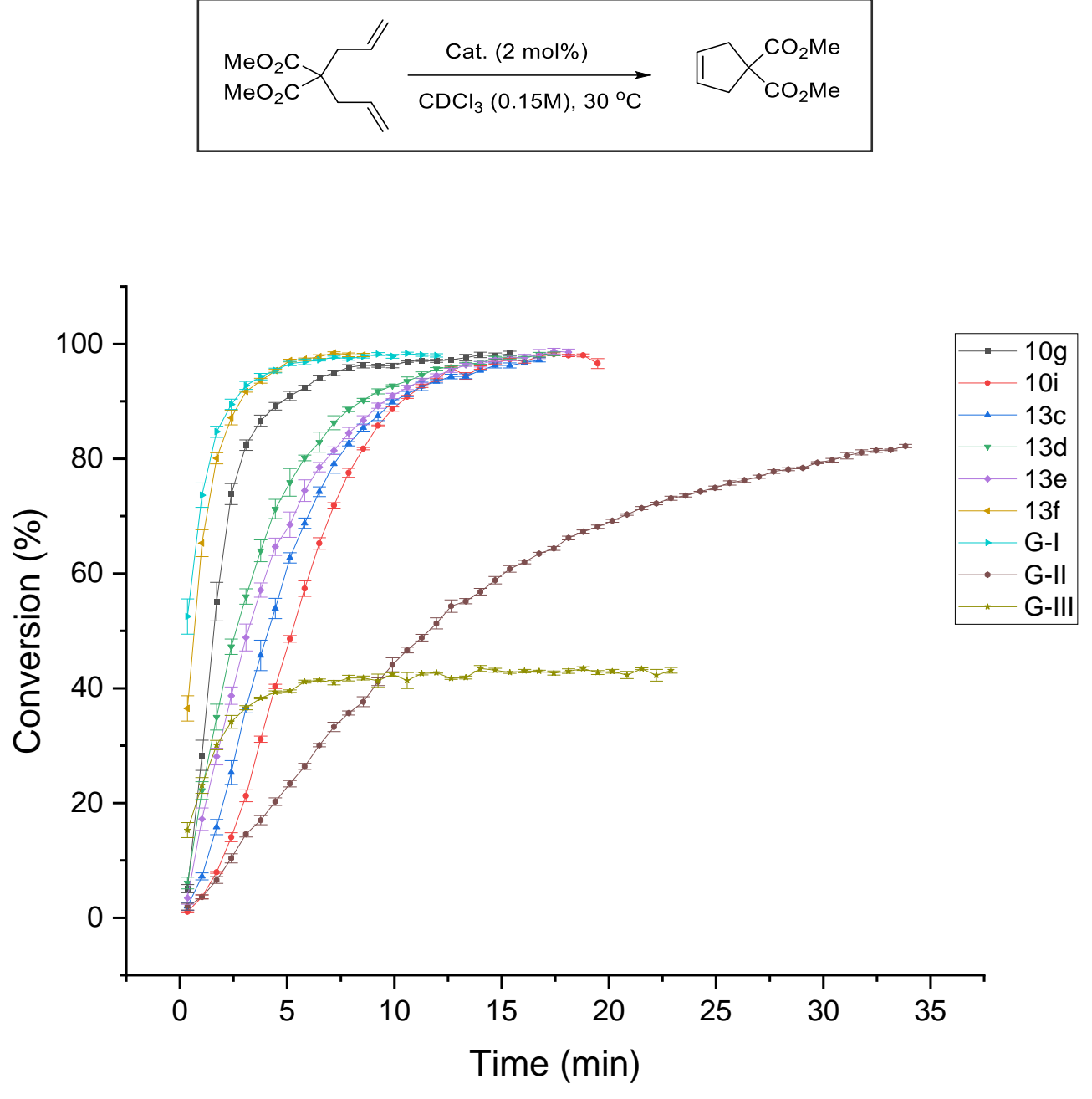

Fig. S7. Kinetic profiles for $\mathrm{RCM}$ reactions to disubstituted olefins in $\mathrm{CDCl}_{3}$ using selected alkene-chelates as catalysts at $30^{\circ} \mathrm{C}$. Each data point represents the average of three independent experiments. Error bar represents standard error of the triplicate experiments. 


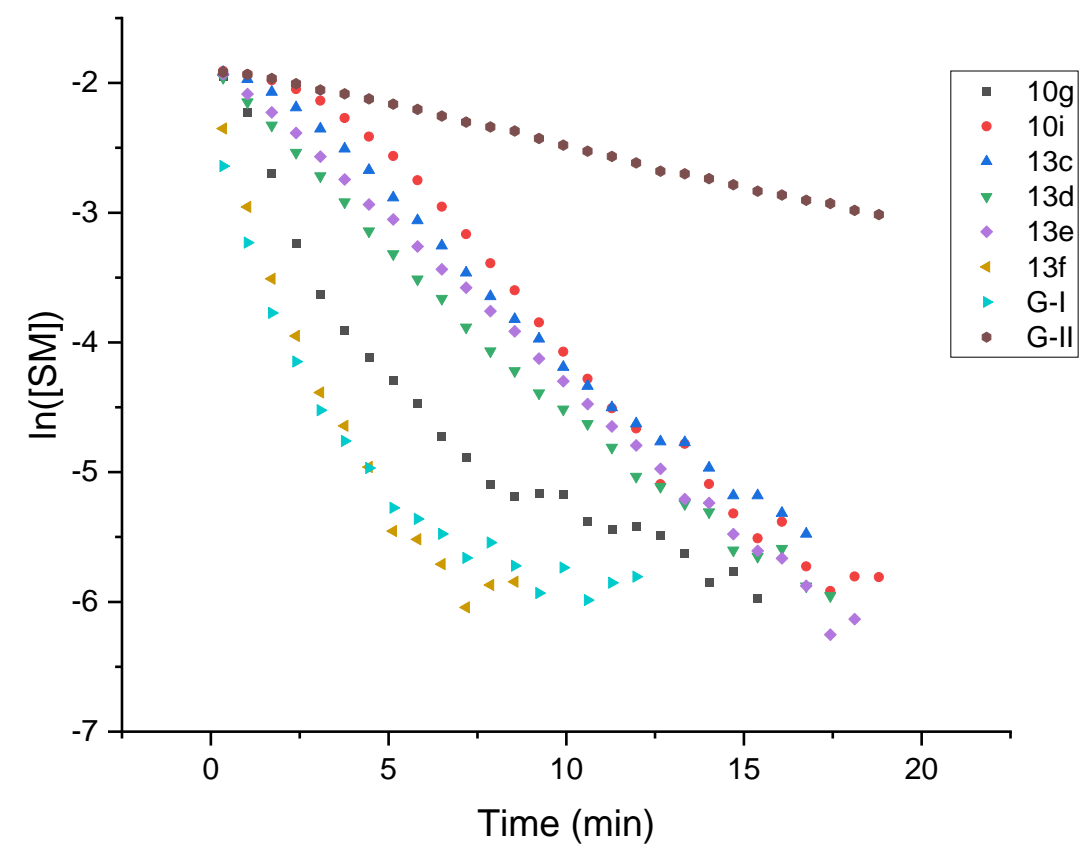

Fig. S8. Log plot of selected alkene-chelates for RCM reaction to disubstituted olefins in $\mathrm{CDCl}_{3}$. $\mathrm{K}_{\mathrm{obs}}$ was determined from the slope of these curves and $t_{1 / 2}$ values were accordingly determined as $\ln 2 / k_{\text {obs }}$

Kinetic parameters (in $\mathrm{CDCl}_{3}$ ) of selected catalysts for RCM to disubstituted alkenes ( $k_{\mathrm{obs}}, \mathrm{t}_{1 / 2}$ and $\mathrm{k}_{\text {rel }}$ )

\begin{tabular}{ccccc}
\hline Cat. & $\mathrm{k}_{\mathrm{obs}}\left(10^{-4} \mathrm{~s}^{-1}\right)$ & $\mathrm{t}_{1} / 2(\mathrm{~s})$ & $\mathrm{k}_{\mathrm{rel}}\left(\mathrm{CDCl}_{3}\right)$ & Linearity of plot \\
\hline $\mathbf{1 0 g}$ & 86.8 & 79.9 & 9.64 & Upto $90 \%$ conv. \\
$\mathbf{1 0 i}$ & 49.5 & 140.0 & 5.50 & Between $14 \%$ to $90 \%$ conv. \\
$\mathbf{1 3 c}$ & 43.2 & 160.5 & 4.80 & \\
$\mathbf{1 3 d}$ & 42.8 & 162.0 & 4.76 & \\
$\mathbf{1 3 e}$ & 41.8 & 165.8 & 4.64 & 11.48 \\
$\mathbf{1 3 f}$ & 103.3 & 67.1 & 9.83 \\
$\mathbf{G - I}$ & 88.5 & 57.2 & 1 \\
GII & 9 & 770.2 & \\
\hline
\end{tabular}




\section{Catalytic activities of selected alkene-chelates for RCM reaction of dimethyl diallylmalonate at $30{ }^{\circ} \mathrm{C}$ in $\mathrm{CD}_{2} \mathrm{Cl}_{2}$ solvent}

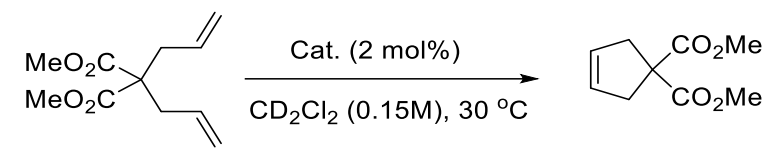

An NMR tube with a screw-cap septum top was charged inside a glovebox with catalyst stock solution (0.1 $\mathrm{M}, 16 \mu \mathrm{L}, 1.6 \mu \mathrm{mol}, 2 \mathrm{~mol} \%)$ in $0.2 \mathrm{~mL}$ of $\mathrm{CD}_{2} \mathrm{Cl}_{2}$. The NMR tube was degassed by freeze, pump, thaw method (cycle of 3 ) and refilled with argon. The sample was equilibrated at $30^{\circ} \mathrm{C}$ in the NMR probe before dimethyl diallylmalonate $(16.5 \mathrm{mg}, 0.078 \mathrm{mmol})$ solution in $0.3 \mathrm{~mL}$ of $\mathrm{CD}_{2} \mathrm{Cl}_{2}$ was added via a syringe. Data points were collected over an appropriate time interval using the Bruker pseudo 2D kinetics method. The conversion of the product was determined by comparing the ratio of the integrals of the methylene protons in the starting material, $\delta 2.65-2.61(\mathrm{~m})$, with those of the product, $\delta 3.01(\mathrm{~s})$. Three independent experiments were performed for each complex and the mean conversion was determined. The conversion versus time graph was plotted for different complexes to have a comparison of their reactivities. Kinetic parameter $k_{\text {obs }}$ was determined from the slope of $\ln ([S M])$ vs time plot, where, $\ln ([S M])=\ln ((100$-avg. conv.) $/ 100 *$ conc. $(0.15 \mathrm{M}))$.
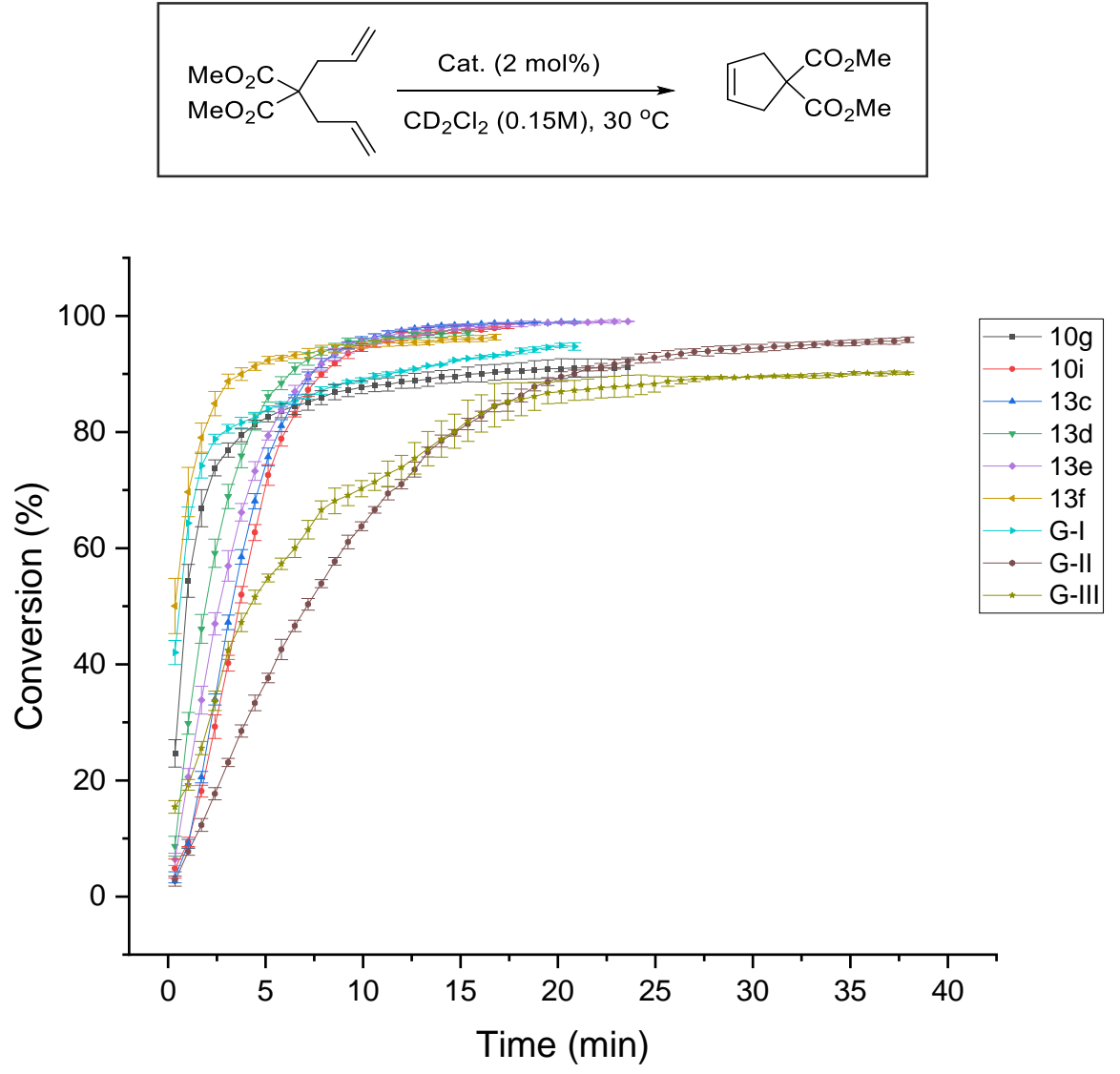

Fig. S9. Kinetic profiles for RCM reactions to disubstituted olefins using selected alkene-chelates as 
catalysts at $30{ }^{\circ} \mathrm{C}$ in $\mathrm{CD}_{2} \mathrm{Cl}_{2}$. Each data point represents the average of three independent experiments. Error bar represents standard error of the triplicate experiments.

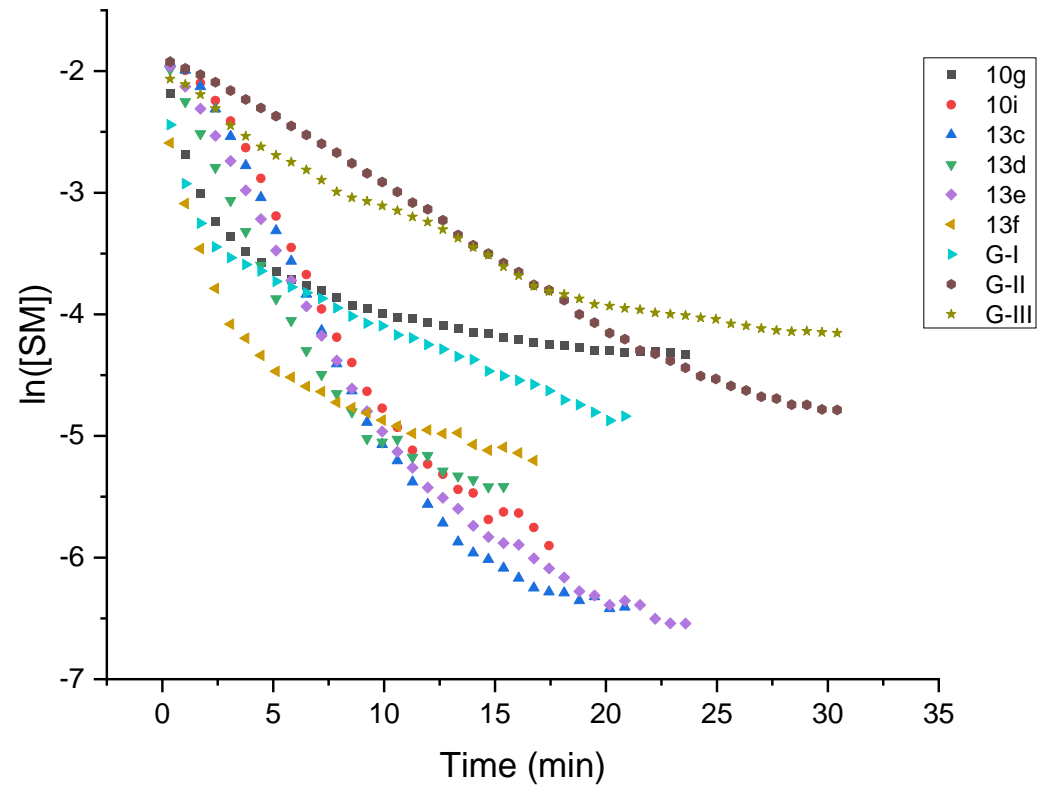

Fig. S10. Log plot of selected alkene-chelates for $\mathrm{RCM}$ reaction in $\mathrm{CD}_{2} \mathrm{Cl}_{2}$ to disubstituted olefins. $\mathrm{K}_{\mathrm{obs}}$ was determined from the slope of these curves and $t_{1 / 2}$ values were accordingly determined as $\ln 2 / \mathrm{k}_{\text {obs }}$

Kinetic parameters (in $\mathrm{CD}_{2} \mathrm{Cl}_{2}$ ) of selected catalysts for $\mathrm{RCM}$ to disubstituted alkenes $\left(\mathrm{k}_{\mathrm{obs}}, \mathrm{t}_{1 / 2}\right.$ and $\left.\mathrm{k}_{\text {rel }}\right)$

\begin{tabular}{ccccl}
\hline Cat. & $\mathrm{k}_{\text {obs }}\left(10^{-4} \mathrm{~s}^{-1}\right)$ & $\mathrm{t}_{1} / 2(\mathrm{~s})$ & $\mathrm{k}_{\text {rel }}\left(\mathrm{CD}_{2} \mathrm{Cl}_{2}\right)$ & Linearity of plot \\
\hline $\mathbf{1 0 g}$ & 53.5 & 129.6 & 3.57 & Upto $80 \%$ conv. \\
$\mathbf{1 0 i}$ & 54.6 & 127.2 & 3.63 & \\
$\mathbf{1 3 c}$ & 56.1 & 123.6 & 3.74 & \\
$\mathbf{1 3 d}$ & 57.5 & 120.5 & 3.83 & \\
$\mathbf{1 3 e}$ & 52.2 & 132.8 & 3.48 & \\
$\mathbf{1 3 f}$ & 89.7 & 77.3 & 5.98 & Upto $90 \%$ conv. \\
$\mathbf{G - I}$ & 66 & 105.0 & 4.40 & Upto $80 \%$ conv. \\
$\mathbf{G - I I}$ & 15 & 462.1 & 1 & \\
$\mathbf{G - I I I}$ & 16.3 & 425.2 & 1.09 & Upto $85 \%$ conv. \\
\hline
\end{tabular}


The kinetics of RCM of dimethyl diallylmalonate was also monitored in $\mathrm{CD}_{2} \mathrm{Cl}_{2}$ to have a consistency with previously reported experiments (ref. 15). It was found that in $\mathrm{CD}_{2} \mathrm{Cl}_{2}$ the reactivity of standard metathesis catalysts like G-II and G-III increases significantly giving higher conversion (95\% vs. $82 \%$ for G-II and $90 \%$ vs. $45 \%$ for G-III) and higher overall rate. For G-II there is 1.6 times rate enhancement. For alkene-chelates which showed excellent reactivity in $\mathrm{CDCl}_{3}$ also showed decent reactivity in $\mathrm{CD}_{2} \mathrm{Cl}_{2}$. In $\mathrm{CD}_{2} \mathrm{Cl}_{2}$ the rate increased for complexes 10i, 13c, 13d and 13e while complexes like $10 \mathrm{~g}, 13 \mathrm{f}$ and G-I showed a decrease in rate compared to that in $\mathrm{CDCl}_{3}$.

\section{Catalytic activities of selected alkene chelates for RCM reaction of dimethyl allylmethallylmalonate at $30^{\circ} \mathrm{C}$}

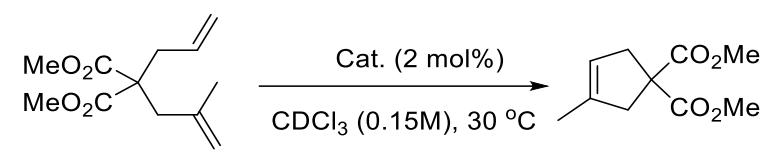

An NMR tube with a screw-cap septum top was charged inside a glovebox with catalyst stock solution (0.1 $\mathrm{M}, 22 \mu \mathrm{L}, 2.2 \mu \mathrm{mol}, 2 \mathrm{~mol} \%)$ in $0.3 \mathrm{~mL}$ of $\mathrm{CDCl}_{3}$. The NMR tube was degassed by freeze, pump, thaw method (cycle of 3 ) and refilled with argon. The sample was equilibrated at $30^{\circ} \mathrm{C}$ in the NMR probe before dimethyl allylmethallylmalonate $\left(25 \mathrm{mg}, 0.11 \mathrm{mmol}\right.$ ) solution in $0.5 \mathrm{~mL}$ of $\mathrm{CDCl}_{3}$ was added via a syringe. Data points were collected over an appropriate time interval using the Bruker pseudo 2D kinetics method. The conversion of the product was determined by comparing the ratio of the integrals of the methylene protons in the starting material, $\delta 2.70(\mathrm{~s}), 2.67(\mathrm{dt})$, with those of the product, $\delta 2.97(\mathrm{~s}), 2.91(\mathrm{~m})$. Three independent experiments were performed for each complexes and mean conversion was determined. The conversion versus time graph was plotted for different complexes to have a comparison of their reactivities. Kinetic parameter $k_{\text {obs }}$ was determined from the slope of $\ln ([\mathrm{SM}])$ vs time plot, where, $\ln ([\mathrm{SM}])$ $=\ln ((100$-avg. conv. $) / 100 *$ conc. (0.15 M)).

\section{Catalyst 10g}

\begin{tabular}{|r|r|r|r|r|r|r|}
\hline $\begin{array}{l}\text { Time } \\
\text { (min) }\end{array}$ & \multicolumn{2}{|l|}{ Conversion (\%) } & & & & \\
\hline & \multicolumn{1}{|l|}{ Trial 1 } & \multicolumn{1}{l|}{ Trial 2 } & \multicolumn{1}{l|}{ Trial 3 } & \multicolumn{1}{l|}{ Avg. } & Std. Dev. & In([SM]) \\
\hline 0.35 & 1.6 & 2.4 & 3.1 & 2.4 & 0.62 & -1.92 \\
\hline 1.03 & 12.1 & 11.4 & 13.7 & 12.4 & 0.97 & -2.03 \\
\hline 1.72 & 32.8 & 29.6 & 30.2 & 30.9 & 1.37 & -2.27 \\
\hline 2.40 & 49.2 & 43.1 & 45.4 & 45.9 & 2.50 & -2.51 \\
\hline 3.08 & 59.7 & 52.6 & 57.3 & 56.5 & 2.96 & -2.73 \\
\hline 3.77 & 66.5 & 62.1 & 68.3 & 65.6 & 2.60 & -2.96 \\
\hline 4.45 & 71.1 & 70.2 & 69.9 & 70.4 & 0.50 & -3.11 \\
\hline 5.13 & 74.6 & 73.8 & 74.8 & 74.4 & 0.43 & -3.26 \\
\hline 5.82 & 77.1 & 76.1 & 77.3 & 76.8 & 0.53 & -3.36 \\
\hline 6.50 & 79.3 & 78.7 & 78.1 & 78.7 & 0.48 & -3.44 \\
\hline 7.18 & 81.0 & 79.2 & 82.5 & 80.9 & 1.35 & -3.55 \\
\hline 7.87 & 82.4 & 83.6 & 83.8 & 83.3 & 0.64 & -3.68 \\
\hline
\end{tabular}




\begin{tabular}{|c|c|c|c|c|c|c|}
\hline \multirow{2}{*}{$\begin{array}{l}\text { Time } \\
\text { (min) }\end{array}$} & \multicolumn{2}{|c|}{ Conversion (\%) } & \multirow[b]{2}{*}{ Trial 3} & \multirow[b]{2}{*}{ Avg. } & \multirow[b]{2}{*}{ Std. Dev. } & \multirow[b]{2}{*}{$\ln ([\mathrm{SM}])$} \\
\hline & Trial 1 & Trial 2 & & & & \\
\hline 8.55 & 83.9 & 84.7 & 83.3 & 84.0 & 0.57 & -3.73 \\
\hline 9.23 & 84.9 & 84.4 & 85.3 & 84.9 & 0.37 & -3.78 \\
\hline 9.92 & 85.7 & 85.9 & 86.4 & 86.0 & 0.28 & -3.86 \\
\hline 10.60 & 86.3 & 85.7 & 86.6 & 86.2 & 0.37 & -3.88 \\
\hline 11.28 & 87.0 & 87.4 & 86.8 & 87.1 & 0.25 & -3.94 \\
\hline 11.97 & 87.7 & 87.9 & 87.6 & 87.7 & 0.12 & -4.00 \\
\hline 12.65 & 88.3 & 88.7 & 89.1 & 88.7 & 0.31 & -4.08 \\
\hline 13.33 & 88.8 & 89.3 & 89.4 & 89.2 & 0.29 & -4.12 \\
\hline 14.02 & 89.1 & 89.3 & 89.6 & 89.3 & 0.21 & -4.13 \\
\hline 14.70 & 89.6 & 91.4 & 91.2 & 90.7 & 0.81 & -4.28 \\
\hline 15.38 & 90.0 & 90.4 & 90.8 & 90.4 & 0.31 & -4.24 \\
\hline 16.07 & 90.4 & 91.3 & 91.6 & 91.1 & 0.53 & -4.31 \\
\hline 16.75 & 90.7 & 91.5 & 91.8 & 91.3 & 0.45 & -4.34 \\
\hline 17.43 & 90.9 & 91.4 & 91.7 & 91.3 & 0.32 & -4.34 \\
\hline 18.12 & 91.2 & 92.1 & 91.4 & 91.6 & 0.40 & -4.37 \\
\hline 18.80 & 91.4 & 91.7 & 92.1 & 91.7 & 0.30 & -4.39 \\
\hline 19.48 & 91.5 & 91.7 & 92.2 & 91.8 & 0.28 & -4.40 \\
\hline 20.17 & 91.8 & 92.1 & 92.6 & 92.2 & 0.33 & -4.44 \\
\hline 20.85 & 92.1 & 93.2 & 92.8 & 92.7 & 0.48 & -4.51 \\
\hline 21.53 & 92.5 & 93.1 & 93.4 & 93.0 & 0.36 & -4.56 \\
\hline 22.22 & 92.8 & 93.4 & 93.6 & 93.3 & 0.33 & -4.60 \\
\hline 22.90 & 92.8 & 93.5 & 92.6 & 93.0 & 0.39 & -4.55 \\
\hline 23.58 & 93.0 & 93.8 & 93.2 & 93.3 & 0.35 & -4.60 \\
\hline 24.27 & 93.2 & 92.8 & 93.7 & 93.2 & 0.37 & -4.59 \\
\hline 24.95 & 93.5 & 94.1 & 94.4 & 94.0 & 0.39 & -4.71 \\
\hline 25.63 & 93.6 & 93.8 & 94.2 & 93.9 & 0.26 & -4.69 \\
\hline
\end{tabular}

\section{Catalyst 10i}

\begin{tabular}{|r|r|r|r|r|r|r|}
\hline $\begin{array}{l}\text { Time } \\
\text { (min) }\end{array}$ & \multicolumn{2}{l|}{ Conversion (\%) } & \multicolumn{2}{l|}{} & \\
\hline & \multicolumn{1}{|l|}{ Trial 1 } & \multicolumn{1}{l|}{ Trial 2 } & \multicolumn{1}{l|}{ Trial 3 } & \multicolumn{1}{l|}{ Avg. } & Std. Dev. & $\ln$ ([SM]) \\
\hline 0.35 & 0.3 & 0.4 & 0.2 & 0.3 & 0.08 & -1.90 \\
\hline 1.03 & 0.4 & 0.6 & 0.5 & 0.5 & 0.10 & -1.90 \\
\hline 1.72 & 0.6 & 0.7 & 0.8 & 0.7 & 0.10 & -1.90 \\
\hline 2.40 & 1.0 & 1.2 & 0.9 & 1.0 & 0.13 & -1.91 \\
\hline 3.08 & 1.6 & 1.8 & 1.4 & 1.6 & 0.16 & -1.91 \\
\hline 3.77 & 2.6 & 2.8 & 2.9 & 2.8 & 0.10 & -1.93 \\
\hline
\end{tabular}




\begin{tabular}{|c|c|c|c|c|c|c|}
\hline \multirow{2}{*}{$\begin{array}{l}\text { Time } \\
\text { (min) }\end{array}$} & \multicolumn{2}{|c|}{ Conversion (\%) } & \multirow[b]{2}{*}{ Trial 3} & \multirow[b]{2}{*}{ Avg. } & \multirow[b]{2}{*}{ Std. Dev. } & \multirow[b]{2}{*}{$\ln ([\mathrm{SM}])$} \\
\hline & Trial 1 & Trial 2 & & & & \\
\hline 4.45 & 4.1 & 3.6 & 4.2 & 4.0 & 0.26 & -1.94 \\
\hline 5.13 & 6.0 & 5.5 & 6.3 & 5.9 & 0.33 & -1.96 \\
\hline 5.82 & 8.6 & 7.6 & 9.4 & 8.5 & 0.74 & -1.99 \\
\hline 6.50 & 11.7 & 12.2 & 12.8 & 12.2 & 0.45 & -2.03 \\
\hline 7.18 & 15.4 & 14.5 & 16.7 & 15.5 & 0.90 & -2.07 \\
\hline 7.87 & 19.6 & 20.5 & 20.7 & 20.3 & 0.47 & -2.12 \\
\hline 8.55 & 24.2 & 23.2 & 24.8 & 24.1 & 0.66 & -2.17 \\
\hline 9.23 & 29.1 & 27.8 & 30.4 & 29.1 & 1.06 & -2.24 \\
\hline 9.92 & 34.2 & 35.1 & 36.4 & 35.2 & 0.91 & -2.33 \\
\hline 10.60 & 39.3 & 40.2 & 41.9 & 40.5 & 1.08 & -2.42 \\
\hline 11.28 & 44.4 & 42.8 & 43.1 & 43.4 & 0.67 & -2.47 \\
\hline 11.97 & 49.3 & 47.6 & 50.2 & 49.0 & 1.08 & -2.57 \\
\hline 12.65 & 53.9 & 52.4 & 54.1 & 53.5 & 0.77 & -2.66 \\
\hline 13.33 & 58.3 & 58.6 & 59.7 & 58.9 & 0.60 & -2.79 \\
\hline 14.02 & 62.3 & 62.6 & 63.2 & 62.7 & 0.36 & -2.88 \\
\hline 14.70 & 66.0 & 65.2 & 66.4 & 65.9 & 0.50 & -2.97 \\
\hline 15.38 & 69.3 & 68.9 & 70.1 & 69.4 & 0.50 & -3.08 \\
\hline 16.07 & 72.3 & 72.7 & 73.1 & 72.7 & 0.33 & -3.20 \\
\hline 16.75 & 74.8 & 75.7 & 76.2 & 75.6 & 0.56 & -3.31 \\
\hline 17.43 & 77.1 & 78.2 & 77.2 & 77.5 & 0.51 & -3.39 \\
\hline 18.12 & 79.0 & 79.6 & 79.4 & 79.3 & 0.25 & -3.47 \\
\hline 18.80 & 80.7 & 80.8 & 80.4 & 80.6 & 0.17 & -3.54 \\
\hline 19.48 & 82.1 & 82.6 & 81.8 & 82.2 & 0.33 & -3.62 \\
\hline 20.17 & 83.2 & 83.8 & 84.2 & 83.7 & 0.40 & -3.71 \\
\hline 20.85 & 84.2 & 84.6 & 84.9 & 84.6 & 0.28 & -3.77 \\
\hline 21.53 & 85.1 & 85.3 & 85.7 & 85.4 & 0.27 & -3.82 \\
\hline 22.22 & 85.8 & 86.2 & 86.8 & 86.3 & 0.42 & -3.88 \\
\hline 22.90 & 86.4 & 86.7 & 87.1 & 86.7 & 0.29 & -3.92 \\
\hline 23.58 & 86.8 & 87.2 & 87.3 & 87.1 & 0.21 & -3.95 \\
\hline 24.27 & 87.2 & 87.4 & 87 & 87.2 & 0.16 & -3.95 \\
\hline 24.95 & 87.5 & 87.3 & 88.1 & 87.6 & 0.34 & -3.99 \\
\hline 25.63 & 87.8 & 88.4 & 88.6 & 88.3 & 0.36 & -4.04 \\
\hline 26.32 & 88.0 & 88.5 & 88.8 & 88.4 & 0.32 & -4.05 \\
\hline 27.00 & 88.2 & 88.6 & 88.9 & 88.6 & 0.27 & -4.07 \\
\hline 27.68 & 88.5 & 89.1 & 89 & 88.9 & 0.28 & -4.09 \\
\hline 28.37 & 88.6 & 89 & 89.2 & 88.9 & 0.26 & -4.10 \\
\hline 29.05 & 88.8 & 89.4 & 89.2 & 89.1 & 0.26 & -4.12 \\
\hline 29.73 & 88.9 & 89.5 & 89.6 & 89.3 & 0.32 & -4.13 \\
\hline 30.42 & 89.0 & 89.2 & 89.8 & 89.3 & 0.33 & -4.14 \\
\hline
\end{tabular}




\begin{tabular}{|r|r|r|r|r|r|r|}
\hline $\begin{array}{l}\text { Time } \\
\text { (min) }\end{array}$ & \multicolumn{2}{|l|}{ Conversion (\%) } & & & & \\
\hline & \multicolumn{1}{|l|}{ Trial 1 } & \multicolumn{1}{l|}{ Trial 2 } & \multicolumn{1}{|c|}{ Trial 3 } & \multicolumn{1}{l|}{ Avg. } & Std. Dev. & In([SM]) \\
\hline 31.10 & 89.3 & 89.8 & 90.1 & 89.7 & 0.34 & -4.17 \\
\hline 31.78 & 89.3 & 89.6 & 90.2 & 89.7 & 0.37 & -4.17 \\
\hline 32.47 & 89.5 & 90.1 & 90.3 & 90.0 & 0.35 & -4.20 \\
\hline
\end{tabular}

\section{Catalyst 13c}

\begin{tabular}{|c|c|c|c|c|c|c|}
\hline \multirow{2}{*}{$\begin{array}{l}\text { Time } \\
\text { (min) }\end{array}$} & \multicolumn{2}{|c|}{ Conversion (\%) } & \multirow[b]{2}{*}{ Trial 3} & \multirow[b]{2}{*}{ Avg. } & \multirow[b]{2}{*}{ Std. Dev. } & \multirow[b]{2}{*}{$\ln ([\mathrm{SM}])$} \\
\hline & Trial 1 & Trial 2 & & & & \\
\hline 0.35 & 1.2 & 0.9 & 0.8 & 1.0 & 0.18 & -1.91 \\
\hline 1.03 & 1.1 & 1.3 & 1.2 & 1.2 & 0.07 & -1.91 \\
\hline 1.72 & 1.2 & 1.4 & 1.3 & 1.3 & 0.07 & -1.91 \\
\hline 2.40 & 1.4 & 1.3 & 1.5 & 1.4 & 0.08 & -1.91 \\
\hline 3.08 & 1.4 & 1.7 & 1.6 & 1.6 & 0.12 & -1.91 \\
\hline 3.77 & 2.9 & 2.5 & 3.5 & 3.0 & 0.41 & -1.93 \\
\hline 4.45 & 7.8 & 6.1 & 8.7 & 7.5 & 1.08 & -1.98 \\
\hline 5.13 & 17.9 & 15.3 & 19.2 & 17.5 & 1.63 & -2.09 \\
\hline 5.82 & 31.1 & 34.2 & 31.3 & 32.2 & 1.43 & -2.29 \\
\hline 6.50 & 44.6 & 42.6 & 43.4 & 43.5 & 0.81 & -2.47 \\
\hline 7.18 & 55.6 & 54.6 & 53.1 & 54.4 & 1.04 & -2.68 \\
\hline 7.87 & 64.9 & 62.1 & 63.4 & 63.5 & 1.13 & -2.90 \\
\hline 8.55 & 72.1 & 70.4 & 69.8 & 70.8 & 0.98 & -3.13 \\
\hline 9.23 & 77.7 & 78.2 & 79.1 & 78.3 & 0.59 & -3.43 \\
\hline 9.92 & 82.0 & 81.2 & 82.7 & 82.0 & 0.61 & -3.61 \\
\hline 10.60 & 85.5 & 84.8 & 85.7 & 85.3 & 0.38 & -3.82 \\
\hline 11.28 & 88.0 & 87.2 & 87.6 & 87.6 & 0.32 & -3.98 \\
\hline 11.97 & 89.6 & 90.1 & 89.8 & 89.8 & 0.19 & -4.18 \\
\hline 12.65 & 91.2 & 90.7 & 91.3 & 91.1 & 0.27 & -4.31 \\
\hline 13.33 & 92.2 & 91.7 & 92.6 & 92.2 & 0.37 & -4.44 \\
\hline 14.02 & 93.3 & 93.1 & 93.8 & 93.4 & 0.29 & -4.62 \\
\hline 14.70 & 94.1 & 94.7 & 94.4 & 94.4 & 0.26 & -4.78 \\
\hline 15.38 & 94.6 & 95.2 & 94.8 & 94.9 & 0.26 & -4.86 \\
\hline 16.07 & 95.0 & 94.8 & 95.3 & 95.0 & 0.20 & -4.90 \\
\hline 16.75 & 95.4 & 95.7 & 95.1 & 95.4 & 0.25 & -4.97 \\
\hline 17.43 & 95.8 & 96.3 & 94.5 & 95.5 & 0.76 & -5.01 \\
\hline 18.12 & 96.1 & 96.6 & 96.4 & 96.4 & 0.21 & -5.21 \\
\hline 18.80 & 96.5 & 96.1 & 96.7 & 96.4 & 0.25 & -5.23 \\
\hline 19.48 & 96.7 & 97.3 & 96.9 & 97.0 & 0.26 & -5.39 \\
\hline
\end{tabular}




\begin{tabular}{|r|r|r|r|r|r|r|}
\hline $\begin{array}{l}\text { Time } \\
\text { (min) }\end{array}$ & \multicolumn{2}{|l|}{ Conversion (\%) } & & & & \\
\hline & \multicolumn{1}{|c|}{ Trial 1 } & \multicolumn{1}{|l|}{ Trial 2 } & \multicolumn{1}{l|}{ Trial 3 } & \multicolumn{1}{|l|}{ Avg. } & Std. Dev. & In([SM]) \\
\hline 20.17 & 96.7 & 97.1 & 96.8 & 96.9 & 0.19 & -5.36 \\
\hline 20.85 & 96.7 & 97.1 & 97.1 & 97.0 & 0.18 & -5.39 \\
\hline 21.53 & 97.0 & 97.4 & 97.2 & 97.2 & 0.15 & -5.48 \\
\hline
\end{tabular}

\section{Catalyst $13 e$}

\begin{tabular}{|c|c|c|c|c|c|c|}
\hline \multirow{2}{*}{$\begin{array}{l}\text { Time } \\
\text { (min) }\end{array}$} & \multicolumn{2}{|c|}{ Conversion (\%) } & \multirow[b]{2}{*}{ Trial 3} & \multirow[b]{2}{*}{ Avg. } & \multirow[b]{2}{*}{ Std. Dev. } & \multirow[b]{2}{*}{$\ln ([\mathrm{SM}]$} \\
\hline & Trial 1 & Trial 2 & & & & \\
\hline 0.35 & 0.6 & 0.7 & 0.4 & 0.6 & 0.12 & -1.90 \\
\hline 1.03 & 0.8 & 1.2 & 0.9 & 1.0 & 0.18 & -1.91 \\
\hline 1.72 & 0.9 & 1.1 & 1.3 & 1.1 & 0.16 & -1.91 \\
\hline 2.40 & 1.8 & 2.4 & 2.5 & 2.2 & 0.32 & -1.92 \\
\hline 3.08 & 12.5 & 10.2 & 15.4 & 12.7 & 2.13 & -2.03 \\
\hline 3.77 & 27.0 & 28.2 & 25.6 & 26.9 & 1.06 & -2.21 \\
\hline 4.45 & 38.5 & 39.6 & 36.3 & 38.1 & 1.37 & -2.38 \\
\hline 5.13 & 48.0 & 45.3 & 49.1 & 47.5 & 1.60 & -2.54 \\
\hline 5.82 & 56.0 & 58.1 & 56.4 & 56.8 & 0.92 & -2.74 \\
\hline 6.50 & 62.6 & 63.4 & 61.4 & 62.5 & 0.82 & -2.88 \\
\hline 7.18 & 68.1 & 66.2 & 69.4 & 67.9 & 1.31 & -3.03 \\
\hline 7.87 & 72.7 & 72.1 & 73.2 & 72.7 & 0.45 & -3.19 \\
\hline 8.55 & 76.3 & 76.9 & 77.2 & 76.8 & 0.37 & -3.36 \\
\hline 9.23 & 79.4 & 81.4 & 78.8 & 79.9 & 1.11 & -3.50 \\
\hline 9.92 & 82.0 & 83.6 & 81.4 & 82.3 & 0.93 & -3.63 \\
\hline 10.60 & 84.0 & 84.8 & 82.8 & 83.9 & 0.82 & -3.72 \\
\hline 11.28 & 85.7 & 85.1 & 86.2 & 85.7 & 0.45 & -3.84 \\
\hline 11.97 & 87.0 & 86.8 & 87.7 & 87.2 & 0.38 & -3.95 \\
\hline 12.65 & 88.3 & 87.6 & 89.2 & 88.4 & 0.65 & -4.05 \\
\hline 13.33 & 89.4 & 89.1 & 90.4 & 89.6 & 0.56 & -4.16 \\
\hline 14.02 & 90.1 & 90.7 & 91.3 & 90.7 & 0.48 & -4.27 \\
\hline 14.70 & 90.9 & 91.5 & 91.7 & 91.4 & 0.32 & -4.35 \\
\hline 15.38 & 91.6 & 92.5 & 93.1 & 92.4 & 0.61 & -4.47 \\
\hline 16.07 & 92.3 & 92.6 & 93.2 & 92.7 & 0.39 & -4.51 \\
\hline 16.75 & 92.8 & 93.4 & 93.6 & 93.3 & 0.33 & -4.60 \\
\hline 17.43 & 93.3 & 93.8 & 94.1 & 93.7 & 0.34 & -4.67 \\
\hline 18.12 & 93.7 & 94.2 & 94.6 & 94.2 & 0.37 & -4.74 \\
\hline 18.80 & 94.0 & 94.3 & 94.7 & 94.3 & 0.29 & -4.77 \\
\hline
\end{tabular}




\begin{tabular}{|r|r|r|r|r|r|r|}
\hline $\begin{array}{l}\text { Time } \\
\text { (min) }\end{array}$ & \multicolumn{2}{l|}{ Conversion (\%) } & & & \\
\hline & \multicolumn{1}{|l}{ Trial 1 } & \multicolumn{1}{l|}{ Trial 2 } & \multicolumn{1}{l|}{ Trial 3 } & \multicolumn{1}{l|}{ Avg. } & Std. Dev. & In([SM]) \\
\hline 19.48 & 94.6 & 95.2 & 94.8 & 94.9 & 0.25 & -4.87 \\
\hline 20.17 & 94.7 & 94.9 & 95.1 & 94.9 & 0.16 & -4.87 \\
\hline 20.85 & 95.1 & 95.5 & 95.7 & 95.4 & 0.26 & -4.98 \\
\hline 21.53 & 95.4 & 95.6 & 96.2 & 95.7 & 0.35 & -5.05 \\
\hline 22.22 & 95.7 & 96 & 96.1 & 95.9 & 0.19 & -5.10 \\
\hline 22.90 & 95.9 & 96.5 & 96.3 & 96.2 & 0.25 & -5.18 \\
\hline 23.58 & 96.2 & 97.4 & 96.8 & 96.8 & 0.48 & -5.34 \\
\hline 24.27 & 96.4 & 97.2 & 96.9 & 96.8 & 0.33 & -5.35 \\
\hline 24.95 & 96.6 & 97.3 & 96.7 & 96.9 & 0.30 & -5.36 \\
\hline 25.63 & 96.8 & 97.2 & 98.1 & 97.4 & 0.54 & -5.53 \\
\hline 26.32 & 96.8 & 97.1 & 97.8 & 97.2 & 0.42 & -5.48 \\
\hline 27.00 & 97.2 & 97.5 & 97 & 97.2 & 0.21 & -5.48 \\
\hline 27.68 & 97.2 & 97.6 & 97.7 & 97.5 & 0.23 & -5.58 \\
\hline 28.37 & 97.4 & 97.5 & 98.1 & 97.7 & 0.32 & -5.65 \\
\hline 29.05 & 97.6 & 97.8 & 98.2 & 97.9 & 0.26 & -5.74 \\
\hline
\end{tabular}

\section{Catalyst 13f}

\begin{tabular}{|r|r|r|r|r|r|r|}
\hline \multicolumn{1}{l}{$\begin{array}{l}\text { Time } \\
\text { (min) }\end{array}$} & \multicolumn{2}{|l|}{ Conversion (\%) } & & & \\
\hline & Trial 1 & \multicolumn{1}{l|}{ Trial 2 } & \multicolumn{1}{l|}{ Trial 3 } & \multicolumn{1}{l|}{ Avg. } & Std. Dev. & In([SM]) \\
\hline 0.35 & 22.1 & 16.4 & 17.3 & 18.6 & 2.50 & -2.10 \\
\hline 1.03 & 44.4 & 36.2 & 38.6 & 39.7 & 3.43 & -2.40 \\
\hline 1.72 & 55.3 & 48.3 & 50.2 & 51.3 & 2.94 & -2.62 \\
\hline 2.40 & 62.3 & 59.4 & 60.2 & 60.6 & 1.23 & -2.83 \\
\hline 3.08 & 67.2 & 65.4 & 69.3 & 67.3 & 1.59 & -3.01 \\
\hline 3.77 & 70.6 & 70.2 & 71.6 & 70.8 & 0.59 & -3.13 \\
\hline 4.45 & 72.7 & 72.5 & 73.1 & 72.8 & 0.25 & -3.20 \\
\hline 5.13 & 74.9 & 73.5 & 73.8 & 74.1 & 0.59 & -3.25 \\
\hline 5.82 & 76.4 & 75.4 & 76.6 & 76.1 & 0.53 & -3.33 \\
\hline 6.50 & 76.8 & 75.9 & 76.6 & 76.4 & 0.39 & -3.34 \\
\hline 7.18 & 79.0 & 79.2 & 79.6 & 79.3 & 0.25 & -3.47 \\
\hline 7.87 & 79.7 & 80 & 79.6 & 79.8 & 0.17 & -3.50 \\
\hline 8.55 & 80.8 & 80.4 & 81 & 80.7 & 0.25 & -3.54 \\
\hline 9.23 & 80.7 & 80.6 & 81.2 & 80.8 & 0.27 & -3.55 \\
\hline 9.92 & 82.6 & 81.8 & 82.4 & 82.3 & 0.33 & -3.63 \\
\hline 10.60 & 82.4 & 82.1 & 82.6 & 82.4 & 0.21 & -3.63 \\
\hline
\end{tabular}




\begin{tabular}{|r|r|r|r|r|r|r|}
\hline $\begin{array}{l}\text { Time } \\
\text { (min) }\end{array}$ & \multicolumn{2}{|l|}{ Conversion (\%) } & & & & \\
\hline & Trial 1 & Trial 2 & Trial 3 & \multicolumn{1}{l|}{ Avg. } & Std. Dev. & In([SM]) \\
\hline 11.28 & 82.9 & 83.1 & 83.3 & 83.1 & 0.15 & -3.68 \\
\hline 11.97 & 83.6 & 83.8 & 84 & 83.8 & 0.16 & -3.72 \\
\hline 12.65 & 83.5 & 83.7 & 83.3 & 83.5 & 0.16 & -3.70 \\
\hline 13.33 & 83.8 & 84.2 & 83.7 & 83.9 & 0.21 & -3.72 \\
\hline 14.02 & 84.2 & 84.6 & 84.7 & 84.5 & 0.24 & -3.76 \\
\hline 14.70 & 84.3 & 84.4 & 84.2 & 84.3 & 0.08 & -3.75 \\
\hline 15.38 & 84.7 & 84.8 & 84.6 & 84.7 & 0.08 & -3.77 \\
\hline 16.07 & 84.5 & 84.9 & 84.6 & 84.7 & 0.16 & -3.77 \\
\hline 16.75 & 86.0 & 86.2 & 85.7 & 86.0 & 0.20 & -3.86 \\
\hline 17.43 & 85.3 & 85.1 & 85.8 & 85.4 & 0.29 & -3.82 \\
\hline 18.12 & 85.8 & 86 & 86.2 & 86.0 & 0.15 & -3.86 \\
\hline 18.80 & 86.1 & 86.5 & 86.2 & 86.3 & 0.18 & -3.88 \\
\hline 19.48 & 85.9 & 86.2 & 86.1 & 86.1 & 0.12 & -3.87 \\
\hline 20.17 & 86.3 & 86.6 & 86.8 & 86.6 & 0.20 & -3.90 \\
\hline 20.85 & 86.1 & 86.5 & 86.9 & 86.5 & 0.32 & -3.90 \\
\hline 21.53 & 86.9 & 87.1 & 86.3 & 86.8 & 0.34 & -3.92 \\
\hline 22.22 & 86.4 & 86.6 & 87.1 & 86.7 & 0.30 & -3.91 \\
\hline 22.90 & 86.8 & 87.3 & 86.8 & 87.0 & 0.24 & -3.93 \\
\hline 23.58 & 86.6 & 86.4 & 86.8 & 86.6 & 0.16 & -3.91 \\
\hline 24.27 & 86.5 & 87 & 86.9 & 86.8 & 0.21 & -3.92 \\
\hline 24.95 & 86.8 & 87.2 & 86.7 & 86.9 & 0.22 & -3.93 \\
\hline 25.63 & 86.6 & 86.8 & 87.1 & 86.8 & 0.19 & -3.93 \\
\hline 26.32 & 86.6 & 86.8 & 86.6 & 86.7 & 0.10 & -3.91 \\
\hline 27.00 & 87.0 & 87.4 & 87.1 & 87.2 & 0.18 & -3.95 \\
\hline & & & & & & \\
\hline & & & & & & \\
\hline
\end{tabular}

\section{Catalyst G-I}

\begin{tabular}{|r|r|r|r|r|r|}
\hline $\begin{array}{l}\text { Time } \\
\text { (min) }\end{array}$ & \multicolumn{2}{|l|}{ Conversion (\%) } & & & \\
\hline & \multicolumn{1}{|c|}{ Trial 1 } & \multicolumn{1}{|c|}{ Trial 2 } & \multicolumn{1}{l|}{ Trial 3 } & \multicolumn{1}{l|}{ Avg. } & Std. Dev. \\
\hline 0.35 & 7.5 & 6.1 & 8.3 & 7.3 & 0.91 \\
\hline 1.03 & 18.1 & 18.4 & 19.6 & 18.7 & 0.65 \\
\hline 1.72 & 26.4 & 24.2 & 27.3 & 26.0 & 1.30 \\
\hline 2.40 & 32.1 & 34.4 & 33.1 & 33.2 & 0.93 \\
\hline 3.08 & 36.0 & 38 & 38.6 & 37.5 & 1.10 \\
\hline 3.77 & 38.7 & 38.8 & 39.2 & 38.9 & 0.21 \\
\hline 4.45 & 40.6 & 39.8 & 40.5 & 40.3 & 0.35 \\
\hline
\end{tabular}




\begin{tabular}{|c|c|c|c|c|c|}
\hline \multirow{2}{*}{$\begin{array}{l}\text { Time } \\
\text { (min) }\end{array}$} & \multicolumn{2}{|c|}{ Conversion (\%) } & \multirow[b]{2}{*}{ Trial 3} & \multirow[b]{2}{*}{ Avg. } & \multirow[b]{2}{*}{ Std. Dev. } \\
\hline & Trial 1 & Trial 2 & & & \\
\hline 5.13 & 41.9 & 41.7 & 42.1 & 41.9 & 0.16 \\
\hline 5.82 & 42.8 & 43.6 & 43.3 & 43.2 & 0.33 \\
\hline 6.50 & 43.6 & 43.8 & 44.2 & 43.9 & 0.25 \\
\hline 7.18 & 44.3 & 45.1 & 45.4 & 44.9 & 0.45 \\
\hline 7.87 & 44.7 & 44.9 & 44.6 & 44.7 & 0.12 \\
\hline 8.55 & 45.0 & 45.4 & 45.2 & 45.2 & 0.16 \\
\hline 9.23 & 45.3 & 45.7 & 45.9 & 45.6 & 0.26 \\
\hline 9.92 & 45.5 & 46.3 & 45.8 & 45.9 & 0.33 \\
\hline 10.60 & 45.7 & 46.2 & 45.9 & 45.9 & 0.21 \\
\hline 11.28 & 45.8 & 46.3 & 45.7 & 45.9 & 0.26 \\
\hline 11.97 & 45.9 & 46.1 & 47.1 & 46.4 & 0.52 \\
\hline 12.65 & 46.0 & 46.3 & 45.8 & 46.0 & 0.21 \\
\hline 13.33 & 46.2 & 46.5 & 46.8 & 46.5 & 0.25 \\
\hline 14.02 & 46.1 & 46.3 & 46.7 & 46.4 & 0.24 \\
\hline 14.70 & 46.1 & 46.2 & 46.4 & 46.2 & 0.11 \\
\hline 15.38 & 46.1 & 46.2 & 46.4 & 46.2 & 0.12 \\
\hline 16.07 & 46.1 & 46.1 & 46.4 & 46.2 & 0.13 \\
\hline 16.75 & 46.1 & 46.1 & 46.2 & 46.1 & 0.06 \\
\hline 17.43 & 46.2 & 45.8 & 46 & 46.0 & 0.16 \\
\hline 18.12 & 46.1 & 46.1 & 46.2 & 46.1 & 0.05 \\
\hline 18.80 & 46.0 & 46.1 & 46 & 46.0 & 0.05 \\
\hline 19.48 & 46.0 & 46.1 & 46 & 46.0 & 0.05 \\
\hline 20.17 & 46.0 & 45.8 & 46.4 & 46.1 & 0.25 \\
\hline 20.85 & 45.8 & 45.9 & 46.4 & 46.0 & 0.26 \\
\hline 21.53 & 45.6 & 45.9 & 46.4 & 46.0 & 0.33 \\
\hline 22.22 & 45.5 & 45.7 & 45.8 & 45.7 & 0.11 \\
\hline 22.90 & 45.5 & 45.9 & 46.2 & 45.9 & 0.28 \\
\hline 23.58 & 45.5 & 46.3 & 45.9 & 45.9 & 0.33 \\
\hline
\end{tabular}

Catalyst G-II

\begin{tabular}{|r|r|r|r|r|r|r|}
\hline $\begin{array}{l}\text { Time } \\
\text { (min) }\end{array}$ & \multicolumn{2}{|l|}{ Conversion (\%) } & & & \\
\hline & Trial 1 & Trial 2 & \multicolumn{1}{|l|}{ Trial 3 } & Avg. & Std. Dev. & In([SM]) \\
\hline 0.35 & 0.6 & 0.8 & 0.8 & 0.7 & 0.10 & -1.90 \\
\hline 1.03 & 0.7 & 0.9 & 1.1 & 0.9 & 0.15 & -1.91 \\
\hline 1.72 & 1.4 & 1.3 & 1.7 & 1.5 & 0.17 & -1.91 \\
\hline
\end{tabular}




\begin{tabular}{|c|c|c|c|c|c|c|}
\hline \multirow{2}{*}{$\begin{array}{l}\text { Time } \\
\text { (min) }\end{array}$} & \multicolumn{2}{|c|}{ Conversion (\%) } & \multirow[b]{2}{*}{ Trial 3} & \multirow[b]{2}{*}{ Avg. } & \multirow[b]{2}{*}{ Std. Dev. } & \multirow[b]{2}{*}{$\ln ([S M])$} \\
\hline & Trial 1 & Trial 2 & & & & \\
\hline 2.40 & 2.7 & 2.5 & 3.3 & 2.8 & 0.34 & -1.93 \\
\hline 3.08 & 4.4 & 4.2 & 4.8 & 4.5 & 0.25 & -1.94 \\
\hline 3.77 & 6.0 & 6.1 & 6.4 & 6.2 & 0.16 & -1.96 \\
\hline 4.45 & 8.1 & 8.3 & 8.7 & 8.4 & 0.27 & -1.98 \\
\hline 5.13 & 10.2 & 9.7 & 10.6 & 10.2 & 0.37 & -2.00 \\
\hline 5.82 & 12.5 & 12.8 & 13.1 & 12.8 & 0.23 & -2.03 \\
\hline 6.50 & 14.8 & 15.1 & 14.6 & 14.8 & 0.21 & -2.06 \\
\hline 7.18 & 16.9 & 17.2 & 17.5 & 17.2 & 0.24 & -2.09 \\
\hline 7.87 & 19.1 & 20.3 & 20.6 & 20.0 & 0.64 & -2.12 \\
\hline 8.55 & 21.6 & 21.8 & 22.4 & 21.9 & 0.33 & -2.14 \\
\hline 9.23 & 23.9 & 24.2 & 23.1 & 23.7 & 0.46 & -2.17 \\
\hline 9.92 & 26.1 & 28.2 & 27.5 & 27.3 & 0.87 & -2.22 \\
\hline 10.60 & 28.3 & 29.1 & 28.1 & 28.5 & 0.43 & -2.23 \\
\hline 11.28 & 30.5 & 31.4 & 32.4 & 31.4 & 0.78 & -2.27 \\
\hline 11.97 & 32.6 & 32.8 & 31.1 & 32.2 & 0.77 & -2.29 \\
\hline 12.65 & 34.7 & 33.8 & 34.9 & 34.5 & 0.47 & -2.32 \\
\hline 13.33 & 36.7 & 36.3 & 37.4 & 36.8 & 0.45 & -2.36 \\
\hline 14.02 & 38.7 & 39.3 & 39.6 & 39.2 & 0.36 & -2.39 \\
\hline 14.70 & 40.6 & 39.8 & 40.8 & 40.4 & 0.43 & -2.41 \\
\hline 15.38 & 42.5 & 41.6 & 43.4 & 42.5 & 0.73 & -2.45 \\
\hline 16.07 & 44.4 & 45.8 & 46.9 & 45.7 & 1.04 & -2.51 \\
\hline 16.75 & 46.1 & 48.2 & 47.2 & 47.2 & 0.88 & -2.53 \\
\hline 17.43 & 47.8 & 48.3 & 46.4 & 47.5 & 0.80 & -2.54 \\
\hline 18.12 & 49.5 & 50.1 & 50.5 & 50.0 & 0.41 & -2.59 \\
\hline 18.80 & 51.0 & 51.8 & 52.1 & 51.6 & 0.44 & -2.62 \\
\hline 19.48 & 52.6 & 53.4 & 53.1 & 53.0 & 0.33 & -2.65 \\
\hline 20.17 & 54.1 & 54.4 & 54.6 & 54.4 & 0.22 & -2.68 \\
\hline 20.85 & 55.5 & 55.2 & 55.9 & 55.5 & 0.29 & -2.71 \\
\hline 21.53 & 56.8 & 57 & 57.1 & 57.0 & 0.13 & -2.74 \\
\hline 22.22 & 58.1 & 58.4 & 58.3 & 58.3 & 0.13 & -2.77 \\
\hline 22.90 & 59.3 & 59.5 & 59.1 & 59.3 & 0.16 & -2.80 \\
\hline 23.58 & 60.5 & 60.7 & 60.1 & 60.4 & 0.25 & -2.82 \\
\hline 24.27 & 61.5 & 61.8 & 61.2 & 61.5 & 0.25 & -2.85 \\
\hline 24.95 & 62.6 & 62.9 & 63.1 & 62.9 & 0.23 & -2.89 \\
\hline 25.63 & 63.6 & 64.7 & 63.9 & 64.1 & 0.48 & -2.92 \\
\hline 26.32 & 64.5 & 64.7 & 64.5 & 64.6 & 0.11 & -2.93 \\
\hline 27.00 & 65.2 & 65.9 & 66.2 & 65.8 & 0.41 & -2.97 \\
\hline 27.68 & 66.2 & 66.7 & 67.2 & 66.7 & 0.40 & -3.00 \\
\hline 28.37 & 67.0 & 67.4 & 67.6 & 67.3 & 0.24 & -3.02 \\
\hline 29.05 & 67.7 & 67.9 & 67.8 & 67.8 & 0.08 & -3.03 \\
\hline
\end{tabular}




\begin{tabular}{|r|r|r|r|r|r|r|}
\hline $\begin{array}{l}\text { Time } \\
\text { (min) }\end{array}$ & \multicolumn{2}{|l|}{ Conversion (\%) } & & & & \\
\hline & \multicolumn{1}{|c|}{ Trial 1 } & \multicolumn{1}{|c|}{ Trial 2 } & \multicolumn{1}{|c|}{ Trial 3 } & \multicolumn{1}{|c|}{ Avg. } & Std. Dev. & In([SM]) \\
\hline 29.73 & 68.4 & 68.7 & 69.1 & 68.7 & 0.28 & -3.06 \\
\hline 30.42 & 69.1 & 69.5 & 69.8 & 69.5 & 0.29 & -3.08 \\
\hline 31.10 & 69.9 & 70.3 & 70.4 & 70.2 & 0.23 & -3.11 \\
\hline 31.78 & 70.6 & 71.5 & 71.2 & 71.1 & 0.38 & -3.14 \\
\hline 32.47 & 71.2 & 71.7 & 71.4 & 71.4 & 0.21 & -3.15 \\
\hline
\end{tabular}
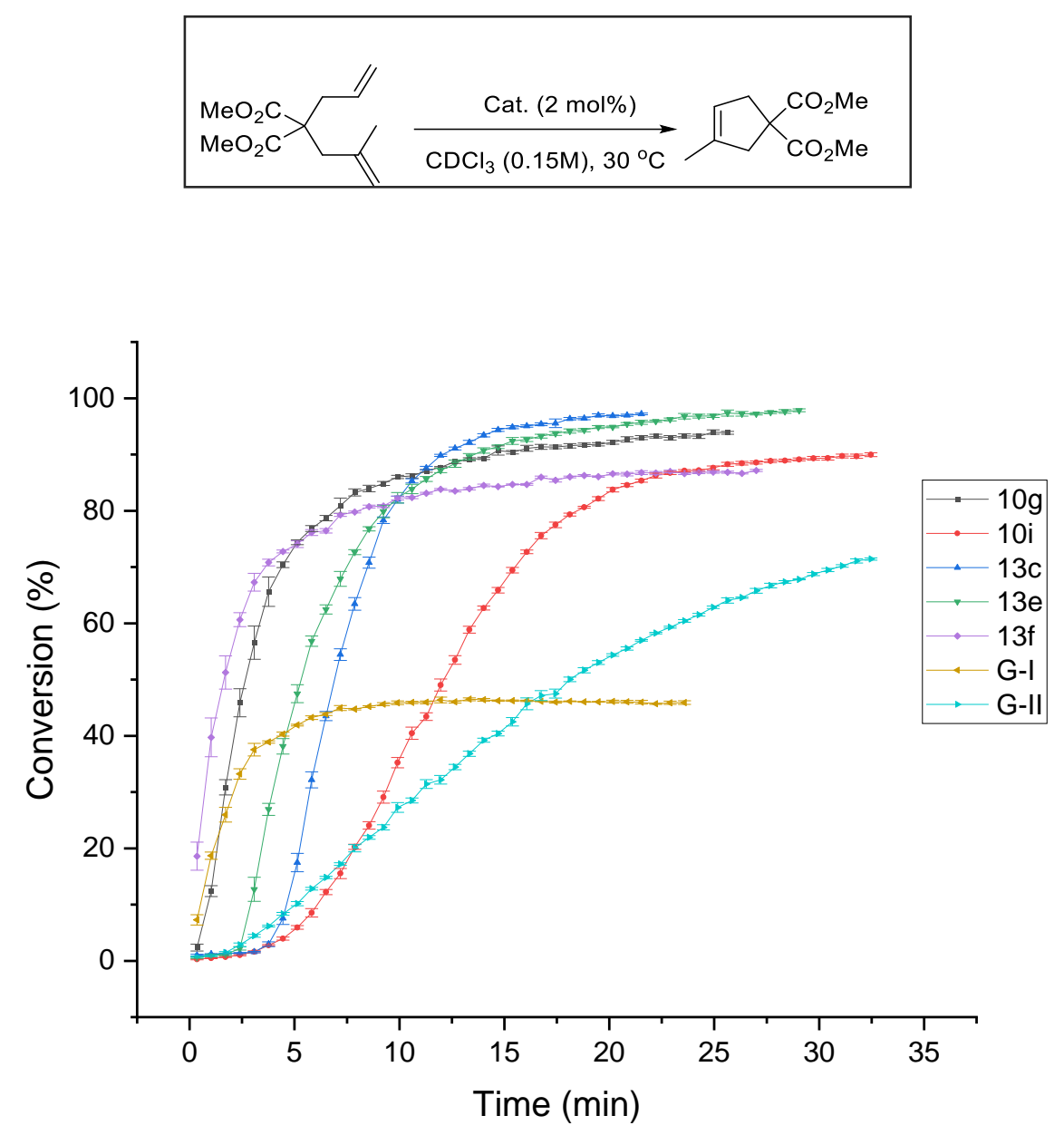

Fig. S11. Kinetic profiles for RCM reactions to trisubstituted olefins using selected alkene-chelates as catalysts at $30^{\circ} \mathrm{C}$. Each data point represents the average of three independent experiments. Error bar represents standard error of the triplicate experiments. 


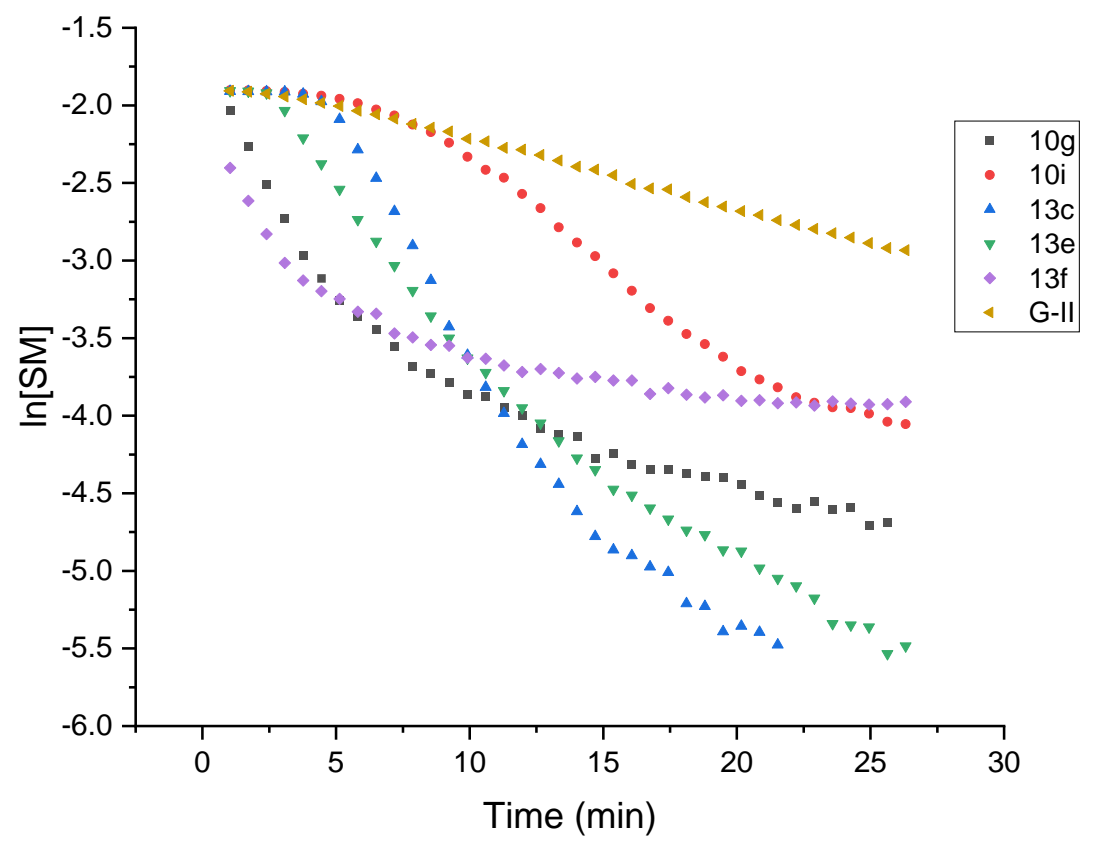

Fig. S12. Log plot of selected alkene-chelates for RCM reaction to trisubstituted olefins. Kobs was determined from the slope of these curve and $t_{1 / 2}$ values were accordingly determined. It is worth noting that for RCM to trisubstituted olefins the fastest catalysts like $13 \mathbf{f}$ and $\mathbf{1 0 g}$ the log plots are curved which indicates quicker catalytic decomposition which is also well reflected in their low conversion. To determine $k_{o b s}$ for these catalysts only the portions of the curve showing linearity is considered.

Kinetic parameters of selected catalysts for RCM to trisubstituted alkenes ( $k_{\text {obs }}, t_{1 / 2}$ and $k_{\text {rel }}$ )

\begin{tabular}{ccccc}
\hline Cat. & $\mathrm{k}_{\text {obs }}\left(10^{-4} \mathrm{~s}^{-1}\right)$ & $\mathrm{t}_{1} / 2(\mathrm{~s})$ & $\mathrm{k}_{\text {rel }}\left(\mathrm{CDCl}_{3}\right)$ & Linearity of plot \\
\hline $10 \mathrm{~g}$ & 44.3 & 156.5 & 6.15 & Upto $80 \%$ conv. \\
$\mathbf{1 0 i}$ & 22.5 & 308.1 & 3.13 & Between $12-85 \%$ conv. \\
$13 \mathrm{c}$ & 39.1 & 177.3 & 5.43 & \\
$13 \mathbf{1 3}$ & 29.8 & 232.6 & 4.14 & Above $12 \%$ conv. \\
$13 \mathrm{f}$ & 54.8 & 126.5 & 7.61 & \\
GII & 7.2 & 962.7 & 1 & \\
\hline
\end{tabular}




\section{Catalytic activities of selected alkene chelates for CM reaction of 5-hexenyl acetate and methyl acrylate}

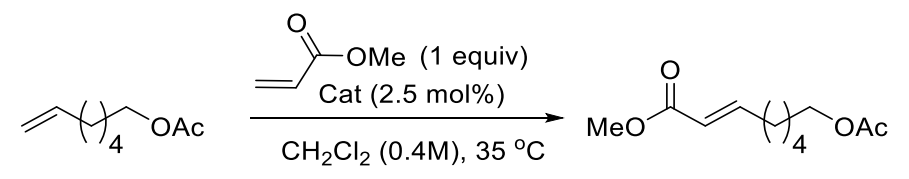

5-Hexenyl acetate $(30 \mathrm{mg}, 0.21 \mathrm{mmol})$ and methyl acrylate $(18.1 \mathrm{mg}, 0.21 \mathrm{mmol})$ and internal standard

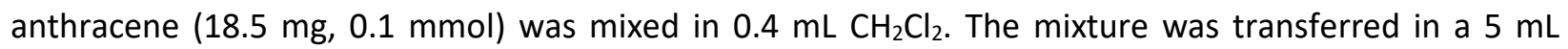
septum-topped vial filled with argon and $15 \mu \mathrm{mol}$ aliquot was removed and diluted with $0.5 \mathrm{~mL}$ of $\mathrm{CH}_{2} \mathrm{Cl}_{2}$ to take the initial $\mathrm{nmr}$. The catalyst stock solution $\left(5.25 \mu \mathrm{mol}, 104 \mu \mathrm{L}, 0.05 \mathrm{M}\right.$ in $\left.\mathrm{CH}_{2} \mathrm{Cl}_{2}\right)$ was added and the reaction mixture was heated at $35{ }^{\circ} \mathrm{C}$. An aliquot $(15 \mu \mathrm{L})$ was removed from the vial at desired time interval and diluted with $0.5 \mathrm{~mL}$ of $\mathrm{CDCl}_{3}$ in an NMR tube which was kept at $-78{ }^{\circ} \mathrm{C}$ until NMR was taken. Attempts to monitor this reaction in an NMR tube resulted to incomplete conversions due to ethylene build-up. All conversions were determined relative to anthracene which was the internal standard. The internal standard singlet at $8.43 \mathrm{ppm}$ was given an integration of 1.00 in the spectrum at each time point. The multiplet at 5.05-4.91 ppm $\left(2 \mathrm{H} ; \mathrm{C}=\mathrm{CH}_{2}\right.$ of 5-hexenyl acetate $)$ and the doublet of doublets at $6.13 \mathrm{ppm}$ $(1 \mathrm{H} ; J=17.3,1.7 \mathrm{~Hz}$; cis- $\mathrm{C}=\mathrm{CHH}$ of methyl acrylate) were used as peaks to monitor the disappearance of the starting materials. The conversion of the product was determined from the integration of the doublet of triplet at $6.95 \mathrm{ppm}(1 \mathrm{H} ; J=15.3,6.9 \mathrm{~Hz} ; \mathrm{C}=\mathrm{CHR})$. The experiment was performed twice and the mean $\%$ conversion was determined. (Attempt to perform the reaction in $\mathrm{CDCl}_{3}$ lead to much lower conversion with a maximum of $69 \%$ for $\mathrm{G}-\mathrm{II})$.

Catalyst $10 \mathrm{~g}$ (Isolated Yield of CM product: $54 \%$ )

\begin{tabular}{|c|c|c|c|c|}
\hline $\begin{array}{l}\text { Time } \\
\text { (min) }\end{array}$ & $\begin{array}{l}\% \text { hexenyl } \\
\text { acetate } \\
\text { cosumed }^{1}\end{array}$ & $\begin{array}{l}\% \text { acrylate } \\
\text { consumed }^{1}\end{array}$ & $\begin{array}{l}\% \text { product } \\
\text { formed }^{1}\end{array}$ & Std. Dev. ${ }^{2}$ \\
\hline 0 & 0.0 & 0.0 & 0.0 & 0.00 \\
\hline 2 & 33.2 & 25.7 & 27.8 & 1.65 \\
\hline 4 & 47.5 & 37.1 & 40.4 & 2.10 \\
\hline 6 & 53.0 & 42.6 & 44.8 & 2.40 \\
\hline 8 & 58.5 & 45.5 & 50.6 & 1.95 \\
\hline 10 & 64.0 & 47.3 & 52.6 & 1.20 \\
\hline 15 & 67.2 & 51.0 & 55.3 & 0.45 \\
\hline 20 & 69.0 & 54.2 & 57.3 & 0.55 \\
\hline 30 & 70.0 & 56.3 & 59.9 & 0.55 \\
\hline 40 & 73.4 & 58.1 & 61.7 & 0.10 \\
\hline 60 & 76.1 & 60.1 & 62.8 & 0.60 \\
\hline 80 & 77.7 & 62.6 & 62.6 & 0.05 \\
\hline
\end{tabular}

${ }^{1}$ Represents the mean conversion of two experiments. ${ }^{2}$ Standard deviation shown here is only calculated with respect to the mean value of $\%$ product formation. 
Catalyst 10i (Isolated Yield of CM product: 61\%)

\begin{tabular}{|r|r|r|r|r|}
\hline $\begin{array}{l}\text { Time } \\
\text { (min) }\end{array}$ & $\begin{array}{l}\text { \% hexenyl } \\
\text { acetate } \\
\text { consumed }\end{array}$ & $\begin{array}{l}\text { \% acrylate } \\
\text { consumed }^{1}\end{array}$ & $\begin{array}{l}\text { \% product } \\
\text { formed }^{1}\end{array}$ & Std. Dev. $^{2}$ \\
\hline 0 & 0.0 & 0.0 & 0.0 & 0.00 \\
\hline 2 & 60.3 & 51.5 & 45.8 & 2.65 \\
\hline 4 & 66.9 & 54.1 & 54.8 & 1.40 \\
\hline 6 & 69.5 & 58.4 & 57.1 & 1.50 \\
\hline 8 & 71.1 & 62.1 & 59.6 & 0.80 \\
\hline 10 & 74.3 & 63.1 & 61.0 & 0.90 \\
\hline 15 & 76.1 & 64.3 & 62.1 & 0.50 \\
\hline 20 & 78.0 & 65.5 & 64.4 & 0.25 \\
\hline 30 & 78.6 & 67.3 & 66.4 & 0.45 \\
\hline 40 & 79.3 & 68.4 & 66.8 & 0.55 \\
\hline 60 & 79.7 & 69.5 & 67.7 & 0.70 \\
\hline 80 & 81.7 & 71.4 & 69.4 & 0.25 \\
\hline & & & & \\
\hline
\end{tabular}

${ }^{1}$ Represents the mean conversion of two experiments. ${ }^{2}$ Standard deviation shown here is only calculated with respect to the mean value of $\%$ product formation.

Catalyst 13c (Isolated Yield of CM product: 76\%)

\begin{tabular}{|r|r|r|r|r|}
\hline $\begin{array}{l}\text { Time } \\
\text { (min) }\end{array}$ & $\begin{array}{l}\text { \% hexenyl } \\
\text { acetate } \\
\text { consumed }\end{array}$ & $\begin{array}{l}\text { \% acrylate } \\
\text { consumed }\end{array}$ & $\begin{array}{l}\text { \% product } \\
\text { formed }^{1}\end{array}$ & Std. Dev. $^{2}$ \\
\hline 0 & 0.0 & 0.0 & 0.0 & 0.00 \\
\hline 2 & 56.9 & 46.5 & 43.6 & 1.95 \\
\hline 4 & 70.5 & 65.1 & 61.6 & 1.10 \\
\hline 6 & 74.0 & 72.1 & 69.7 & 1.15 \\
\hline 8 & 79.8 & 75.1 & 72.1 & 1.35 \\
\hline 10 & 83.0 & 76.9 & 74.2 & 0.40 \\
\hline 15 & 87.6 & 79.5 & 77.2 & 0.55 \\
\hline 20 & 90.1 & 81.0 & 79.5 & 0.40 \\
\hline 30 & 90.9 & 82.5 & 80.7 & 0.60 \\
\hline 40 & 92.7 & 84.0 & 81.6 & 0.35 \\
\hline 60 & 97.1 & 84.1 & 82.0 & 0.20 \\
\hline 80 & 98.3 & 86.8 & 82.1 & 0.60 \\
\hline & & & & \\
\hline
\end{tabular}

${ }^{1}$ Represents the mean conversion of two experiments. ${ }^{2}$ Standard deviation shown here is only calculated with respect to the mean value of $\%$ product formation. 
Catalyst 13e (Isolated Yield of CM product: 63\%)

\begin{tabular}{|r|r|r|r|r|}
\hline $\begin{array}{l}\text { Time } \\
\text { (min) }\end{array}$ & $\begin{array}{l}\text { \% hexenyl } \\
\text { acetate } \\
\text { consumed }\end{array}$ & $\begin{array}{l}\text { \% acrylate } \\
\text { consumed }\end{array}$ & $\begin{array}{l}\text { \% product } \\
\text { formed }^{1}\end{array}$ & Std. Dev. $^{2}$ \\
\hline 0 & 0.0 & 0.0 & 0.0 & 0.00 \\
\hline 2 & 46.1 & 35.5 & 32.7 & 1.95 \\
\hline 4 & 53.9 & 46.3 & 45.3 & 1.10 \\
\hline 6 & 63.7 & 57.8 & 57.6 & 1.15 \\
\hline 8 & 69.4 & 63.6 & 60.5 & 1.35 \\
\hline 10 & 72.1 & 66.2 & 63.2 & 0.40 \\
\hline 15 & 75.3 & 69.7 & 65.3 & 0.55 \\
\hline 20 & 77.4 & 72.2 & 67.9 & 0.40 \\
\hline 30 & 79.3 & 74.2 & 67.7 & 0.60 \\
\hline 40 & 81.8 & 75.3 & 68.0 & 0.35 \\
\hline 60 & 83.3 & 76.3 & 69.4 & 0.20 \\
\hline 80 & 86.6 & 77.1 & 69.7 & 0.60 \\
\hline & & & & \\
\hline
\end{tabular}

${ }^{1}$ Represents the mean conversion of two experiments. ${ }^{2}$ Standard deviation shown here is only calculated with respect to the mean value of $\%$ product formation.

Catalyst G-II (Isolated Yield of CM product: 86\%)

\begin{tabular}{|r|r|r|r|r|}
\hline $\begin{array}{l}\text { Time } \\
\text { (min) }\end{array}$ & $\begin{array}{l}\text { \% hexenyl } \\
\text { acetate } \\
\text { consumed }\end{array}$ & $\begin{array}{l}\text { \% acrylate } \\
\text { consumption }\end{array}$ & $\begin{array}{l}\text { \% product } \\
\text { formed }^{1}\end{array}$ & Std. Dev. \\
\hline 0 & 0.0 & 0.0 & 0.0 & 0.00 \\
\hline 2 & 23.6 & 28.1 & 24.8 & 0.95 \\
\hline 4 & 35.9 & 39.2 & 33.5 & 1.40 \\
\hline 6 & 53.5 & 58.3 & 51.9 & 1.30 \\
\hline 8 & 61.3 & 67.0 & 62.5 & 2.15 \\
\hline 10 & 74.0 & 76.0 & 72.6 & 2.15 \\
\hline 15 & 80.5 & 81.1 & 76.5 & 1.80 \\
\hline 20 & 85.8 & 85.9 & 81.4 & 1.05 \\
\hline 30 & 89.2 & 89.7 & 86.1 & 0.90 \\
\hline 40 & 92.4 & 92.4 & 88.4 & 1.10 \\
\hline 60 & 95.4 & 93.5 & 90.7 & 0.30 \\
\hline 80 & 97.2 & 95.8 & 94.5 & 0.35 \\
\hline & & & & \\
\hline
\end{tabular}

${ }^{1}$ Represents the mean conversion of two experiments. ${ }^{2}$ Standard deviation shown here is only calculated with respect to the mean value of $\%$ product formation. 

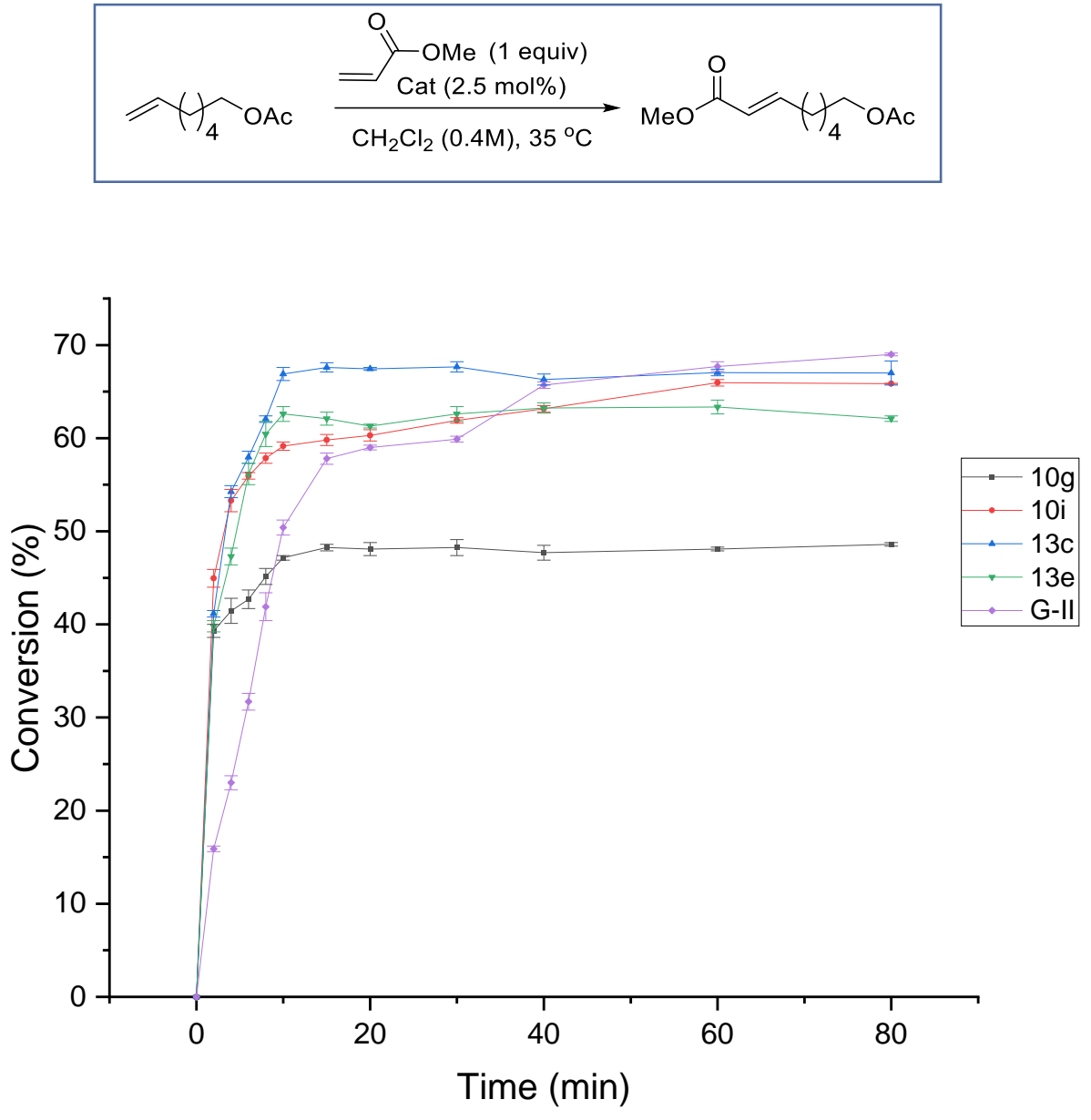

Fig. S13. Kinetic Profile for cross-metathesis of 5-hexenyl acetate with methyl acrylate using active alkenechelated ruthenium alkylidenes as catalysts. Each data point represents the average of two independent experiments. Error bars represents standard error of the duplicate experiment. The alkene-chelates initiates with a much higher rate compared to G-II but slows down leading to lower conversions compared to G-II. The low conversion for alkene-chelates can be due to either rapid catalyst decomposition or higher formation of homo metatathesis product from 5-hexenyl acetate which could be indicated by the higher consumption of 5-hexenyl acetate. 


\section{Catalytic activities of selected alkene chelates for ROMP of 1,5-cyclooctadiene}

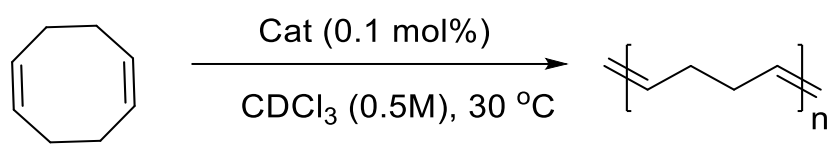

An NMR tube with a screw-cap septum top was charged inside a glovebox with catalyst stock solution $(0.01 \mathrm{M}, 27 \mu \mathrm{L}, 0.1 \mathrm{~mol} \%)$ in $0.3 \mathrm{~mL}$ of $\mathrm{CDCl}_{3}$. The NMR tube was degassed by freeze, pump, thaw method (cycle of 3) and refilled with argon. The sample was equilibrated at $30{ }^{\circ} \mathrm{C}$ in the NMR probe before $1,5-$ cyclooctadiene ( $30 \mathrm{mg}, 0.27 \mathrm{mmol}$ ) solution in $0.5 \mathrm{~mL}$ of $\mathrm{CDCl}_{3}$ was added via a syringe. Data points were collected over an appropriate time interval using the Bruker pseudo 2D kinetics method. The conversion of the polymer was determined by comparing the ratio of the integrals of the methylene protons in the starting material, $\delta 5.61-5.51(\mathrm{~m})$, with those of the polymer, $\delta 5.45-5.34(\mathrm{br} \mathrm{m})$. Three independent experiments were performed for each complexes and mean conversion was determined. The conversion versus time graph was plotted for different complexes to have a comparison of their reactivities. The kinetic parameter $k_{o b s}$ was determined from the slope of $\ln ([S M])$ vs time plot, where, $\ln ([S M])=\ln ((100$ avg. conv.) / $100 *$ conc. $(0.5 \mathrm{M})$ ).

\section{Catalyst $10 \mathrm{~g}$}

\begin{tabular}{|c|c|c|c|c|c|c|}
\hline \multirow{2}{*}{$\begin{array}{l}\text { Time } \\
\text { (min) }\end{array}$} & \multicolumn{2}{|c|}{ Conversion (\%) } & \multirow[b]{2}{*}{ Trial 3} & \multirow[b]{2}{*}{ Avg. } & \multirow[b]{2}{*}{ Std. Dev. } & \multirow[b]{2}{*}{$\ln ([S M])$} \\
\hline & Trial 1 & Trial 2 & & & & \\
\hline 0.35 & 1.1 & 0.9 & 1.7 & 1.2 & 0.34 & -0.71 \\
\hline 1.03 & 1.5 & 1.8 & 1.6 & 1.6 & 0.14 & -0.71 \\
\hline 1.72 & 2.1 & 2.3 & 2 & 2.1 & 0.12 & -0.71 \\
\hline 2.40 & 2.9 & 3.1 & 3.4 & 3.1 & 0.19 & -0.73 \\
\hline 3.08 & 3.6 & 3.8 & 4 & 3.8 & 0.15 & -0.73 \\
\hline 3.77 & 5.6 & 5.8 & 5.9 & 5.8 & 0.13 & -0.75 \\
\hline 4.45 & 8.1 & 8.4 & 9.1 & 8.5 & 0.43 & -0.78 \\
\hline 5.13 & 10.6 & 11.2 & 10.1 & 10.6 & 0.45 & -0.81 \\
\hline 5.82 & 13.7 & 13.1 & 13.9 & 13.6 & 0.35 & -0.84 \\
\hline 6.50 & 16.6 & 16.9 & 17.3 & 16.9 & 0.29 & -0.88 \\
\hline 7.18 & 19.9 & 20.5 & 19.2 & 19.9 & 0.53 & -0.91 \\
\hline 7.87 & 22.8 & 21.2 & 23.2 & 22.4 & 0.86 & -0.95 \\
\hline 8.55 & 25.8 & 25.1 & 29.2 & 26.7 & 1.80 & -1.00 \\
\hline 9.23 & 28.7 & 29.2 & 28 & 28.6 & 0.49 & -1.03 \\
\hline 9.92 & 31.5 & 29.6 & 32.4 & 31.2 & 1.17 & -1.07 \\
\hline 10.60 & 34.3 & 33.8 & 34.9 & 34.3 & 0.45 & -1.11 \\
\hline 11.28 & 36.9 & 37.4 & 36.5 & 36.9 & 0.37 & -1.15 \\
\hline 11.97 & 39.4 & 39.2 & 40.1 & 39.6 & 0.39 & -1.20 \\
\hline 12.65 & 41.6 & 41.9 & 42.1 & 41.9 & 0.19 & -1.24 \\
\hline 13.33 & 44.1 & 46.2 & 43.8 & 44.7 & 1.08 & -1.29 \\
\hline
\end{tabular}




\begin{tabular}{|c|c|c|c|c|c|c|}
\hline \multirow{2}{*}{$\begin{array}{l}\text { Time } \\
\text { (min) }\end{array}$} & \multicolumn{2}{|c|}{ Conversion (\%) } & \multirow[b]{2}{*}{ Trial 3} & \multirow[b]{2}{*}{ Avg. } & \multirow[b]{2}{*}{ Std. Dev. } & \multirow[b]{2}{*}{$\ln ([S M])$} \\
\hline & Trial 1 & Trial 2 & & & & \\
\hline 14.02 & 46.4 & 46.1 & 47.4 & 46.6 & 0.56 & -1.32 \\
\hline 14.70 & 48.5 & 48.2 & 48.9 & 48.5 & 0.29 & -1.36 \\
\hline 15.38 & 50.6 & 50.2 & 51.8 & 50.9 & 0.68 & -1.40 \\
\hline 16.07 & 52.3 & 52.4 & 52.9 & 52.5 & 0.25 & -1.44 \\
\hline 16.75 & 54.4 & 54 & 54.8 & 54.4 & 0.33 & -1.48 \\
\hline 17.43 & 56.2 & 56.7 & 57.1 & 56.7 & 0.39 & -1.53 \\
\hline 18.12 & 57.8 & 56.1 & 58.2 & 57.4 & 0.90 & -1.55 \\
\hline 18.80 & 59.7 & 60.5 & 59.1 & 59.8 & 0.57 & -1.60 \\
\hline 19.48 & 61.2 & 61.5 & 60.2 & 61.0 & 0.55 & -1.63 \\
\hline 20.17 & 62.8 & 63.2 & 62.2 & 62.7 & 0.41 & -1.68 \\
\hline 20.85 & 64.3 & 64.1 & 64.9 & 64.4 & 0.34 & -1.73 \\
\hline 21.53 & 65.7 & 65.1 & 66.3 & 65.7 & 0.49 & -1.76 \\
\hline 22.22 & 67.1 & 66.5 & 67.4 & 67.0 & 0.37 & -1.80 \\
\hline 22.90 & 68.2 & 67.6 & 68.5 & 68.1 & 0.37 & -1.84 \\
\hline 23.58 & 69.7 & 69.1 & 69.8 & 69.5 & 0.30 & -1.88 \\
\hline 24.27 & 70.6 & 70.1 & 71.2 & 70.6 & 0.45 & -1.92 \\
\hline 24.95 & 71.9 & 70.8 & 72.3 & 71.7 & 0.63 & -1.95 \\
\hline 25.63 & 73.0 & 73.6 & 73.8 & 73.5 & 0.36 & -2.02 \\
\hline 26.32 & 74.2 & 73.7 & 74.5 & 74.1 & 0.33 & -2.05 \\
\hline 27.00 & 75.3 & 75.1 & 75.8 & 75.4 & 0.29 & -2.10 \\
\hline 27.68 & 76.3 & 75.1 & 76.8 & 76.1 & 0.72 & -2.12 \\
\hline 28.37 & 77.0 & 76.4 & 77.4 & 76.9 & 0.41 & -2.16 \\
\hline 29.05 & 78.2 & 77.6 & 78.3 & 78.0 & 0.30 & -2.21 \\
\hline 29.73 & 79.1 & 79.5 & 78.9 & 79.2 & 0.25 & -2.26 \\
\hline 30.42 & 79.9 & 80.1 & 81.4 & 80.5 & 0.67 & -2.33 \\
\hline 31.10 & 80.6 & 80.3 & 80.9 & 80.6 & 0.24 & -2.33 \\
\hline 31.78 & 81.6 & 81.8 & 82.4 & 81.9 & 0.34 & -2.40 \\
\hline 32.47 & 82.2 & 82 & 82.9 & 82.4 & 0.38 & -2.43 \\
\hline 33.15 & 82.8 & 83.1 & 83.6 & 83.2 & 0.32 & -2.48 \\
\hline 33.83 & 83.6 & 84.2 & 83.9 & 83.9 & 0.23 & -2.52 \\
\hline 34.52 & 84.2 & 81.6 & 84.4 & 83.4 & 1.28 & -2.49 \\
\hline 35.20 & 85.1 & 85.4 & 85.7 & 85.4 & 0.26 & -2.62 \\
\hline 35.88 & 85.4 & 85.6 & 86 & 85.7 & 0.25 & -2.64 \\
\hline 36.57 & 86.2 & 86.4 & 86.8 & 86.5 & 0.25 & -2.69 \\
\hline 37.25 & 86.8 & 87.2 & 87.5 & 87.2 & 0.29 & -2.75 \\
\hline 37.93 & 87.2 & 87.5 & 87.7 & 87.5 & 0.22 & -2.77 \\
\hline 38.62 & 87.7 & 88.1 & 88.4 & 88.1 & 0.28 & -2.82 \\
\hline 39.30 & 88.5 & 88.9 & 89.2 & 88.9 & 0.31 & -2.89 \\
\hline 39.98 & 88.7 & 89.1 & 88.6 & 88.8 & 0.21 & -2.88 \\
\hline 40.67 & 89.3 & 89.5 & 89.7 & 89.5 & 0.17 & -2.95 \\
\hline
\end{tabular}




\begin{tabular}{|r|r|r|r|r|r|r|}
\hline $\begin{array}{l}\text { Time } \\
\text { (min) }\end{array}$ & \multicolumn{2}{|l|}{ Conversion (\%) } & & & & \\
\hline & \multicolumn{1}{|l|}{ Trial 1 } & \multicolumn{1}{l|}{ Trial 2 } & \multicolumn{1}{l|}{ Trial 3 } & \multicolumn{1}{l|}{ Avg. } & \multicolumn{1}{l|}{ Std. Dev. } & In([SM]) \\
\hline 41.35 & 89.6 & 89.4 & 89.8 & 89.6 & 0.16 & -2.96 \\
\hline 42.03 & 90.5 & 90.7 & 91.2 & 90.8 & 0.31 & -3.08 \\
\hline 42.72 & 90.5 & 90.8 & 91.4 & 90.9 & 0.36 & -3.09 \\
\hline 43.40 & 91.2 & 90.8 & 91.6 & 91.2 & 0.33 & -3.12 \\
\hline 44.08 & 91.7 & 91.5 & 92.1 & 91.8 & 0.25 & -3.19 \\
\hline 44.77 & 92.1 & 92.7 & 92.8 & 92.5 & 0.30 & -3.29 \\
\hline 45.45 & 91.9 & 92.5 & 93.1 & 92.5 & 0.47 & -3.29 \\
\hline 46.13 & 92.6 & 92.8 & 93.4 & 92.9 & 0.34 & -3.34 \\
\hline 46.82 & 92.7 & 92.6 & 93.1 & 92.8 & 0.22 & -3.32 \\
\hline 47.50 & 93.4 & 93.1 & 93.5 & 93.3 & 0.17 & -3.40 \\
\hline 48.18 & 93.8 & 93.6 & 93.3 & 93.6 & 0.19 & -3.43 \\
\hline
\end{tabular}

\section{Catalyst 10i}

\begin{tabular}{|c|c|c|c|c|c|c|}
\hline \multirow{2}{*}{$\begin{array}{l}\text { Time } \\
\text { (min) }\end{array}$} & \multicolumn{2}{|c|}{ Conversion (\%) } & \multirow[b]{2}{*}{ Trial 3} & \multirow[b]{2}{*}{ Avg. } & \multirow[b]{2}{*}{ Std. Dev. } & \multirow[b]{2}{*}{$\ln ([S M])$} \\
\hline & Trial 1 & Trial 2 & & & & \\
\hline 0.35 & 1.5 & 1.8 & 2.1 & 1.8 & 0.24 & -0.71 \\
\hline 1.03 & 2.4 & 2.8 & 3.2 & 2.8 & 0.32 & -0.72 \\
\hline 1.72 & 4.4 & 4.8 & 5.1 & 4.8 & 0.28 & -0.74 \\
\hline 2.40 & 7.3 & 7.4 & 7.8 & 7.5 & 0.22 & -0.77 \\
\hline 3.08 & 11.5 & 10.6 & 11.7 & 11.3 & 0.49 & -0.81 \\
\hline 3.77 & 16.5 & 16.1 & 17.4 & 16.7 & 0.55 & -0.88 \\
\hline 4.45 & 21.9 & 19.7 & 22.1 & 21.2 & 1.09 & -0.93 \\
\hline 5.13 & 27.5 & 27.8 & 26.2 & 27.2 & 0.69 & -1.01 \\
\hline 5.82 & 32.9 & 30.4 & 33.8 & 32.4 & 1.44 & -1.08 \\
\hline 6.50 & 38.1 & 36.1 & 40.2 & 38.1 & 1.67 & -1.17 \\
\hline 7.18 & 42.6 & 41.4 & 43.1 & 42.4 & 0.71 & -1.24 \\
\hline 7.87 & 46.6 & 44.2 & 47.3 & 46.0 & 1.33 & -1.31 \\
\hline 8.55 & 50.1 & 48.2 & 50.4 & 49.6 & 0.98 & -1.38 \\
\hline 9.23 & 53.1 & 52.1 & 54.4 & 53.2 & 0.94 & -1.45 \\
\hline 9.92 & 55.8 & 55.3 & 56.2 & 55.8 & 0.37 & -1.51 \\
\hline 10.60 & 58.2 & 59.1 & 59.4 & 58.9 & 0.51 & -1.58 \\
\hline 11.28 & 60.5 & 60.7 & 61.4 & 60.9 & 0.40 & -1.63 \\
\hline 11.97 & 62.5 & 62.8 & 63.1 & 62.8 & 0.26 & -1.68 \\
\hline 12.65 & 64.3 & 61.2 & 64.4 & 63.3 & 1.48 & -1.70 \\
\hline 13.33 & 66.0 & 68.1 & 67.8 & 67.3 & 0.94 & -1.81 \\
\hline 14.02 & 67.6 & 68 & 67.9 & 67.8 & 0.18 & -1.83 \\
\hline
\end{tabular}




\begin{tabular}{|c|c|c|c|c|c|c|}
\hline \multirow{2}{*}{$\begin{array}{l}\text { Time } \\
\text { (min) }\end{array}$} & \multicolumn{2}{|c|}{ Conversion (\%) } & \multirow[b]{2}{*}{ Trial 3} & \multirow[b]{2}{*}{ Avg. } & \multirow[b]{2}{*}{ Std. Dev. } & \multirow[b]{2}{*}{$\ln ([S M])$} \\
\hline & Trial 1 & Trial 2 & & & & \\
\hline 14.70 & 69.1 & 70.3 & 69.5 & 69.6 & 0.49 & -1.89 \\
\hline 15.38 & 70.7 & 70.2 & 71.6 & 70.8 & 0.58 & -1.93 \\
\hline 16.07 & 72.2 & 72.8 & 73.1 & 72.7 & 0.38 & -1.99 \\
\hline 16.75 & 73.7 & 73.5 & 74.4 & 73.9 & 0.39 & -2.03 \\
\hline 17.43 & 75.2 & 75.8 & 76.1 & 75.7 & 0.39 & -2.11 \\
\hline 18.12 & 76.6 & 77 & 77.3 & 77.0 & 0.30 & -2.16 \\
\hline 18.80 & 77.9 & 78.2 & 78.4 & 78.2 & 0.21 & -2.21 \\
\hline 19.48 & 79.1 & 80.1 & 81.6 & 80.3 & 1.01 & -2.32 \\
\hline 20.17 & 80.3 & 80.5 & 81.8 & 80.9 & 0.66 & -2.35 \\
\hline 20.85 & 81.5 & 82.6 & 82.1 & 82.1 & 0.46 & -2.41 \\
\hline 21.53 & 82.6 & 82.9 & 83.4 & 83.0 & 0.33 & -2.46 \\
\hline 22.22 & 83.6 & 84.5 & 83.8 & 84.0 & 0.38 & -2.52 \\
\hline 22.90 & 84.6 & 84.8 & 85.2 & 84.9 & 0.26 & -2.58 \\
\hline 23.58 & 85.4 & 85.7 & 86.1 & 85.7 & 0.28 & -2.64 \\
\hline 24.27 & 86.3 & 86.8 & 88.1 & 87.1 & 0.75 & -2.74 \\
\hline 24.95 & 87.2 & 87.8 & 88.3 & 87.8 & 0.45 & -2.79 \\
\hline 25.63 & 87.9 & 88.1 & 88.6 & 88.2 & 0.30 & -2.83 \\
\hline 26.32 & 88.6 & 89 & 89.3 & 89.0 & 0.28 & -2.90 \\
\hline 27.00 & 89.3 & 89.5 & 89.6 & 89.5 & 0.14 & -2.94 \\
\hline 27.68 & 90.0 & 90.2 & 89.8 & 90.0 & 0.16 & -3.00 \\
\hline 28.37 & 90.6 & 90.4 & 91.6 & 90.9 & 0.53 & -3.08 \\
\hline 29.05 & 91.1 & 92.6 & 91.5 & 91.7 & 0.64 & -3.18 \\
\hline 29.73 & 91.7 & 92.3 & 92.5 & 92.2 & 0.34 & -3.24 \\
\hline 30.42 & 92.1 & 92.4 & 92.8 & 92.4 & 0.29 & -3.27 \\
\hline 31.10 & 92.5 & 92.7 & 93.2 & 92.8 & 0.28 & -3.33 \\
\hline 31.78 & 92.9 & 93.4 & 92.6 & 93.0 & 0.33 & -3.35 \\
\hline 32.47 & 93.5 & 93.1 & 93.9 & 93.5 & 0.33 & -3.42 \\
\hline 33.15 & 93.8 & 94.5 & 94.2 & 94.2 & 0.29 & -3.53 \\
\hline 33.83 & 94.1 & 93.8 & 94.3 & 94.1 & 0.21 & -3.52 \\
\hline 34.52 & 94.5 & 94.7 & 95.3 & 94.8 & 0.34 & -3.66 \\
\hline 35.20 & 94.8 & 95.2 & 95.5 & 95.2 & 0.29 & -3.72 \\
\hline 35.88 & 95.1 & 95.4 & 95.7 & 95.4 & 0.24 & -3.77 \\
\hline 36.57 & 95.6 & 95.9 & 96.1 & 95.9 & 0.22 & -3.88 \\
\hline 37.25 & 95.9 & 96.2 & 96.5 & 96.2 & 0.25 & -3.96 \\
\hline 37.93 & 95.9 & 96.2 & 96.4 & 96.2 & 0.22 & -3.95 \\
\hline 38.62 & 96.3 & 96.5 & 96.7 & 96.5 & 0.17 & -4.04 \\
\hline 39.30 & 96.3 & 96.4 & 96.9 & 96.5 & 0.26 & -4.06 \\
\hline 39.98 & 96.7 & 96.6 & 97.1 & 96.8 & 0.21 & -4.14 \\
\hline 40.67 & 96.8 & 97 & 96.7 & 96.8 & 0.12 & -4.15 \\
\hline 41.35 & 96.9 & 97.2 & 96.8 & 97.0 & 0.17 & -4.19 \\
\hline
\end{tabular}




\begin{tabular}{|r|r|r|r|r|r|r|}
\hline $\begin{array}{l}\text { Time } \\
(\mathrm{min})\end{array}$ & \multicolumn{2}{|l|}{ Conversion (\%) } & & & & \\
\hline & Trial 1 & \multicolumn{1}{|l|}{ Trial 2 } & \multicolumn{1}{|c|}{ Trial 3 } & \multicolumn{1}{|c|}{ Avg. } & Std. Dev. & In([SM]) \\
\hline 42.03 & 97.1 & 97.3 & 97.4 & 97.3 & 0.12 & -4.29 \\
\hline 42.72 & 97.4 & 97.4 & 97.2 & 97.3 & 0.09 & -4.31 \\
\hline 43.40 & 97.5 & 97.8 & 97.7 & 97.7 & 0.13 & -4.45 \\
\hline 44.08 & 97.6 & 97.9 & 97.8 & 97.8 & 0.14 & -4.49 \\
\hline 44.77 & 97.6 & 97.9 & 97.6 & 97.7 & 0.14 & -4.47 \\
\hline 45.45 & 97.8 & 98.2 & 98 & 98.0 & 0.18 & -4.60 \\
\hline 46.13 & 97.9 & 98.4 & 98.3 & 98.2 & 0.23 & -4.71 \\
\hline 46.82 & 98.0 & 98.5 & 98.6 & 98.4 & 0.25 & -4.81 \\
\hline 47.50 & 98.0 & 98.6 & 98.7 & 98.4 & 0.29 & -4.86 \\
\hline 48.18 & 98.1 & 98.3 & 98.5 & 98.3 & 0.15 & -4.77 \\
\hline
\end{tabular}

\section{Catalyst 13c}

\begin{tabular}{|r|r|r|r|r|r|r|}
\hline $\begin{array}{l}\text { Time } \\
\text { (min) }\end{array}$ & \multicolumn{2}{|l|}{ Conversion (\%) } & & & & \\
\hline & Trial 1 & Trial 2 & Trial 3 & Avg. & Std. Dev. & In([SM]) \\
\hline 0.35 & 1.2 & 1.5 & 1.7 & 1.5 & 0.22 & -0.71 \\
\hline 1.03 & 1.4 & 1.6 & 1.8 & 1.6 & 0.17 & -0.71 \\
\hline 1.72 & 1.9 & 2.2 & 2.9 & 2.3 & 0.42 & -0.72 \\
\hline 2.40 & 2.7 & 3.1 & 3.4 & 3.1 & 0.27 & -0.72 \\
\hline 3.08 & 4.7 & 4.2 & 5.6 & 4.8 & 0.58 & -0.74 \\
\hline 3.77 & 8.4 & 7.2 & 9.1 & 8.2 & 0.79 & -0.78 \\
\hline 4.45 & 14.0 & 12.6 & 13.2 & 13.3 & 0.59 & -0.84 \\
\hline 5.13 & 20.3 & 18.1 & 19.6 & 19.3 & 0.93 & -0.91 \\
\hline 5.82 & 26.2 & 24.3 & 27.5 & 26.0 & 1.31 & -0.99 \\
\hline 6.50 & 31.8 & 32.9 & 30.6 & 31.8 & 0.94 & -1.08 \\
\hline 7.18 & 36.8 & 35.1 & 38.2 & 36.7 & 1.27 & -1.15 \\
\hline 7.87 & 41.3 & 40.8 & 42.6 & 41.6 & 0.76 & -1.23 \\
\hline 8.55 & 45.2 & 43.2 & 46.4 & 44.9 & 1.32 & -1.29 \\
\hline 9.23 & 48.8 & 46.4 & 49.9 & 48.4 & 1.46 & -1.35 \\
\hline 9.92 & 51.9 & 53.7 & 52.5 & 52.7 & 0.75 & -1.44 \\
\hline 10.60 & 54.7 & 54.5 & 54.2 & 54.5 & 0.22 & -1.48 \\
\hline 11.28 & 57.2 & 55.2 & 58.1 & 56.8 & 1.21 & -1.53 \\
\hline 11.97 & 59.4 & 60.1 & 58.2 & 59.2 & 0.78 & -1.59 \\
\hline 12.65 & 61.5 & 63.1 & 62.5 & 62.4 & 0.64 & -1.67 \\
\hline 13.33 & 63.3 & 64.2 & 63.8 & 63.8 & 0.38 & -1.71 \\
\hline 14.02 & 65.0 & 64.2 & 64.8 & 64.7 & 0.33 & -1.73 \\
\hline 14.70 & 66.5 & 67.2 & 66.1 & 66.6 & 0.45 & -1.79 \\
\hline & & & & & & \\
\hline
\end{tabular}




\begin{tabular}{|c|c|c|c|c|c|c|}
\hline \multirow{2}{*}{$\begin{array}{l}\text { Time } \\
\text { (min) }\end{array}$} & \multicolumn{2}{|c|}{ Conversion (\%) } & \multirow[b]{2}{*}{ Trial 3} & \multirow[b]{2}{*}{ Avg. } & \multirow[b]{2}{*}{ Std. Dev. } & \multirow[b]{2}{*}{$\ln ([S M])$} \\
\hline & Trial 1 & Trial 2 & & & & \\
\hline 15.38 & 67.9 & 67 & 67.3 & 67.4 & 0.37 & -1.81 \\
\hline 16.07 & 69.3 & 69.5 & 68.7 & 69.2 & 0.34 & -1.87 \\
\hline 16.75 & 70.4 & 70.1 & 70.9 & 70.5 & 0.33 & -1.91 \\
\hline 17.43 & 71.4 & 71.6 & 70.8 & 71.3 & 0.34 & -1.94 \\
\hline 18.12 & 72.5 & 71.8 & 72.3 & 72.2 & 0.29 & -1.97 \\
\hline 18.80 & 73.4 & 73.1 & 74.5 & 73.7 & 0.60 & -2.03 \\
\hline 19.48 & 74.4 & 75.2 & 75 & 74.9 & 0.35 & -2.07 \\
\hline 20.17 & 75.2 & 76.2 & 76.4 & 75.9 & 0.52 & -2.12 \\
\hline 20.85 & 75.8 & 76.4 & 76.1 & 76.1 & 0.24 & -2.12 \\
\hline 21.53 & 76.8 & 78.2 & 77.1 & 77.4 & 0.59 & -2.18 \\
\hline 22.22 & 77.5 & 77.1 & 78 & 77.5 & 0.37 & -2.19 \\
\hline 22.90 & 78.2 & 78 & 79.3 & 78.5 & 0.57 & -2.23 \\
\hline 23.58 & 78.9 & 79.3 & 79.4 & 79.2 & 0.21 & -2.26 \\
\hline 24.27 & 79.6 & 80.1 & 80.3 & 80.0 & 0.31 & -2.30 \\
\hline 24.95 & 80.1 & 80.4 & 81 & 80.5 & 0.36 & -2.33 \\
\hline 25.63 & 80.7 & 82.4 & 82.8 & 82.0 & 0.89 & -2.41 \\
\hline 26.32 & 81.3 & 81.5 & 81.2 & 81.3 & 0.12 & -2.37 \\
\hline 27.00 & 81.8 & 82.2 & 82.7 & 82.2 & 0.37 & -2.42 \\
\hline 27.68 & 82.3 & 82.6 & 82.9 & 82.6 & 0.24 & -2.44 \\
\hline 28.37 & 82.8 & 83.2 & 84.8 & 83.6 & 0.86 & -2.50 \\
\hline 29.05 & 83.5 & 83.8 & 84.2 & 83.8 & 0.29 & -2.52 \\
\hline 29.73 & 83.9 & 81.2 & 84.3 & 83.1 & 1.38 & -2.47 \\
\hline 30.42 & 84.1 & 82.8 & 83.6 & 83.5 & 0.54 & -2.50 \\
\hline 31.10 & 84.5 & 83.8 & 84.9 & 84.4 & 0.45 & -2.55 \\
\hline 31.78 & 85.2 & 85.3 & 85.7 & 85.4 & 0.22 & -2.62 \\
\hline 32.47 & 85.3 & 86.8 & 87.2 & 86.4 & 0.80 & -2.69 \\
\hline 33.15 & 85.7 & 86.2 & 86.5 & 86.1 & 0.33 & -2.67 \\
\hline 33.83 & 86.2 & 87.2 & 88.1 & 87.2 & 0.79 & -2.75 \\
\hline 34.52 & 86.4 & 88.2 & 87.5 & 87.4 & 0.73 & -2.76 \\
\hline 35.20 & 86.8 & 87.2 & 85.1 & 86.4 & 0.91 & -2.69 \\
\hline 35.88 & 87.1 & 88.2 & 87.4 & 87.6 & 0.47 & -2.78 \\
\hline 36.57 & 87.5 & 88.2 & 87.2 & 87.6 & 0.42 & -2.78 \\
\hline 37.25 & 88.0 & 88.5 & 88.8 & 88.4 & 0.34 & -2.85 \\
\hline 37.93 & 87.9 & 88.2 & 87.9 & 88.0 & 0.14 & -2.81 \\
\hline 38.62 & 88.5 & 88.9 & 89.1 & 88.8 & 0.26 & -2.88 \\
\hline 39.30 & 88.8 & 89.2 & 89.4 & 89.1 & 0.24 & -2.91 \\
\hline 39.98 & 89.0 & 89.2 & 89.5 & 89.2 & 0.21 & -2.92 \\
\hline 40.67 & 89.3 & 89.5 & 89.8 & 89.5 & 0.21 & -2.95 \\
\hline 41.35 & 89.5 & 90.2 & 90.6 & 90.1 & 0.45 & -3.01 \\
\hline 42.03 & 89.7 & 90.2 & 90.7 & 90.2 & 0.41 & -3.02 \\
\hline
\end{tabular}




\begin{tabular}{|r|r|r|r|r|r|r|}
\hline 42.72 & 90.2 & 90.4 & 90.8 & 90.5 & 0.26 & -3.04 \\
\hline 43.40 & 90.2 & 90.5 & 90.8 & 90.5 & 0.25 & -3.05 \\
\hline 44.08 & 90.4 & 90.8 & 91.3 & 90.8 & 0.36 & -3.08 \\
\hline 44.77 & 90.6 & 90.8 & 91.1 & 90.8 & 0.19 & -3.08 \\
\hline 45.45 & 91.0 & 91.5 & 92 & 91.5 & 0.41 & -3.16 \\
\hline 46.13 & 91.1 & 91.6 & 91.8 & 91.5 & 0.29 & -3.16 \\
\hline 46.82 & 91.2 & 91.7 & 92.3 & 91.7 & 0.45 & -3.19 \\
\hline 47.50 & 91.4 & 91.6 & 91.1 & 91.4 & 0.20 & -3.14 \\
\hline 48.18 & 91.5 & 91.8 & 92.7 & 92.0 & 0.50 & -3.22 \\
\hline 48.87 & 91.9 & 92.2 & 92.7 & 92.3 & 0.33 & -3.25 \\
\hline 49.55 & 92.1 & 92.5 & 92.8 & 92.5 & 0.29 & -3.28 \\
\hline
\end{tabular}

\section{Catalyst 13e}

\begin{tabular}{|c|c|c|c|c|c|c|}
\hline \multirow{2}{*}{$\begin{array}{l}\text { Time } \\
\text { (min) }\end{array}$} & \multicolumn{2}{|c|}{ Conversion (\%) } & \multirow[b]{2}{*}{ Trial 3} & \multirow[b]{2}{*}{ Avg. } & \multirow[b]{2}{*}{ Std. Dev. } & \multirow[b]{2}{*}{$\ln ([S M])$} \\
\hline & Trial 1 & Trial 2 & & & & \\
\hline 0.35 & 1.3 & 1.8 & 1.5 & 1.5 & 0.22 & -0.71 \\
\hline 1.03 & 1.4 & 1.7 & 2.2 & 1.8 & 0.32 & -0.71 \\
\hline 1.72 & 2.1 & 2.5 & 3.1 & 2.6 & 0.41 & -0.72 \\
\hline 2.40 & 2.9 & 3.2 & 3.7 & 3.3 & 0.34 & -0.73 \\
\hline 3.08 & 5.8 & 6.4 & 6.7 & 6.3 & 0.37 & -0.76 \\
\hline 3.77 & 8.4 & 9.2 & 8.8 & 8.8 & 0.31 & -0.79 \\
\hline 4.45 & 13.4 & 11.7 & 15.1 & 13.4 & 1.39 & -0.84 \\
\hline 5.13 & 18.9 & 17.2 & 21.1 & 19.1 & 1.60 & -0.90 \\
\hline 5.82 & 25.0 & 24.6 & 26.7 & 25.4 & 0.91 & -0.99 \\
\hline 6.50 & 33.4 & 31.4 & 34.5 & 33.1 & 1.28 & -1.09 \\
\hline 7.18 & 42.2 & 40.2 & 45.6 & 42.7 & 2.23 & -1.25 \\
\hline 7.87 & 50.8 & 49.1 & 53.4 & 51.1 & 1.77 & -1.41 \\
\hline 8.55 & 58.9 & 59.6 & 63.2 & 60.6 & 1.87 & -1.62 \\
\hline 9.23 & 65.2 & 66.1 & 67.2 & 66.2 & 0.81 & -1.78 \\
\hline 9.92 & 70.4 & 69.4 & 70.7 & 70.2 & 0.55 & -1.90 \\
\hline 10.60 & 74.0 & 73.6 & 74.5 & 74.0 & 0.37 & -2.04 \\
\hline 11.28 & 77.7 & 77.1 & 78.4 & 77.7 & 0.53 & -2.20 \\
\hline 11.97 & 80.6 & 79.8 & 80.7 & 80.4 & 0.40 & -2.32 \\
\hline 12.65 & 82.4 & 83.8 & 82.6 & 82.9 & 0.61 & -2.46 \\
\hline 13.33 & 85.0 & 86.3 & 85.4 & 85.6 & 0.54 & -2.63 \\
\hline 14.02 & 86.3 & 86.8 & 87.2 & 86.8 & 0.39 & -2.71 \\
\hline 14.70 & 87.4 & 88.1 & 87.6 & 87.7 & 0.30 & -2.79 \\
\hline 15.38 & 88.1 & 89.4 & 88.7 & 88.7 & 0.54 & -2.88 \\
\hline 16.07 & 89.5 & 88.5 & 89.8 & 89.3 & 0.56 & -2.93 \\
\hline
\end{tabular}




\begin{tabular}{|c|c|c|c|c|c|c|}
\hline \multirow{2}{*}{$\begin{array}{l}\text { Time } \\
\text { (min) }\end{array}$} & \multicolumn{2}{|c|}{ Conversion (\%) } & \multirow[b]{2}{*}{ Trial 3} & \multirow[b]{2}{*}{ Avg. } & \multirow[b]{2}{*}{ Std. Dev. } & \multirow[b]{2}{*}{$\ln ([S M])$} \\
\hline & Trial 1 & Trial 2 & & & & \\
\hline 16.75 & 90.1 & 90.5 & 90.8 & 90.5 & 0.28 & -3.04 \\
\hline 17.43 & 91.1 & 91.8 & 92.6 & 91.8 & 0.62 & -3.20 \\
\hline 18.12 & 92.8 & 93.1 & 93.6 & 93.2 & 0.31 & -3.38 \\
\hline 18.80 & 92.9 & 93.5 & 94.2 & 93.5 & 0.54 & -3.43 \\
\hline 19.48 & 94.0 & 94.7 & 94.9 & 94.5 & 0.40 & -3.60 \\
\hline 20.17 & 93.6 & 94.5 & 94.8 & 94.3 & 0.51 & -3.56 \\
\hline 20.85 & 95.2 & 96.1 & 95.4 & 95.6 & 0.38 & -3.81 \\
\hline 21.53 & 94.9 & 95.8 & 95.7 & 95.5 & 0.40 & -3.79 \\
\hline 22.22 & 94.9 & 95.4 & 95.7 & 95.3 & 0.35 & -3.76 \\
\hline 22.90 & 95.8 & 96.2 & 96.7 & 96.2 & 0.38 & -3.97 \\
\hline 23.58 & 95.4 & 95.7 & 96.2 & 95.8 & 0.32 & -3.86 \\
\hline 24.27 & 95.8 & 96.4 & 96.2 & 96.1 & 0.23 & -3.95 \\
\hline 24.95 & 96.4 & 96.7 & 96.9 & 96.7 & 0.21 & -4.09 \\
\hline 25.63 & 96.3 & 96.8 & 97 & 96.7 & 0.30 & -4.10 \\
\hline 26.32 & 96.6 & 96.8 & 97.2 & 96.9 & 0.26 & -4.15 \\
\hline 27.00 & 96.8 & 97 & 97.2 & 97.0 & 0.17 & -4.20 \\
\hline 27.68 & 96.9 & 96.8 & 97.4 & 97.0 & 0.26 & -4.21 \\
\hline 28.37 & 97.1 & 97.1 & 97.5 & 97.2 & 0.19 & -4.28 \\
\hline 29.05 & 97.3 & 97.6 & 97.9 & 97.6 & 0.25 & -4.42 \\
\hline 29.73 & 97.6 & 97.8 & 98 & 97.8 & 0.17 & -4.51 \\
\hline 30.42 & 97.8 & 98.4 & 98.2 & 98.1 & 0.25 & -4.68 \\
\hline 31.10 & 97.5 & 98.1 & 98.4 & 98.0 & 0.36 & -4.61 \\
\hline 31.78 & 97.6 & 98.4 & 98.5 & 98.2 & 0.39 & -4.70 \\
\hline 32.47 & 97.8 & 98.1 & 98.4 & 98.1 & 0.24 & -4.66 \\
\hline 33.15 & 97.6 & 98.3 & 98.6 & 98.2 & 0.42 & -4.69 \\
\hline 33.83 & 97.9 & 98.3 & 98.7 & 98.3 & 0.31 & -4.77 \\
\hline 34.52 & 98.0 & 98.5 & 98.4 & 98.3 & 0.22 & -4.77 \\
\hline 35.20 & 98.1 & 98.2 & 98.3 & 98.2 & 0.06 & -4.72 \\
\hline
\end{tabular}

\section{Catalyst $\mathbf{1 3 f}$}

\begin{tabular}{|r|r|r|r|r|r|r|}
\hline $\begin{array}{l}\text { Time } \\
\text { (min) }\end{array}$ & \multicolumn{2}{|l|}{ Conversion (\%) } & & & & \\
\hline & \multicolumn{1}{|l|}{ Trial 1 } & \multicolumn{1}{|c|}{ Trial 2 } & \multicolumn{1}{|c|}{ Trial 3 } & Avg. & Std. Dev. & In([SM]) \\
\hline 0.35 & 1.0 & 2.1 & 1.6 & 1.6 & 0.47 & -0.71 \\
\hline 1.03 & 4.1 & 3.6 & 4.3 & 4.0 & 0.29 & -0.73 \\
\hline 1.72 & 15.0 & 12.2 & 17.4 & 14.9 & 2.12 & -0.85 \\
\hline 2.40 & 32.8 & 30.1 & 34.6 & 32.5 & 1.85 & -1.09 \\
\hline
\end{tabular}




\begin{tabular}{|c|c|c|c|c|c|c|}
\hline \multirow{2}{*}{$\begin{array}{l}\text { Time } \\
\text { (min) }\end{array}$} & \multicolumn{2}{|c|}{ Conversion (\%) } & \multirow[b]{2}{*}{ Trial 3} & \multirow[b]{2}{*}{ Avg. } & \multirow[b]{2}{*}{ Std. Dev. } & \multirow[b]{2}{*}{$\ln ([S M])$} \\
\hline & Trial 1 & Trial 2 & & & & \\
\hline 3.08 & 47.3 & 44.2 & 49.6 & 47.0 & 2.21 & -1.33 \\
\hline 3.77 & 57.2 & 55.8 & 59.3 & 57.4 & 1.44 & -1.55 \\
\hline 4.45 & 64.9 & 63.2 & 65.8 & 64.6 & 1.08 & -1.73 \\
\hline 5.13 & 70.7 & 69.2 & 71.4 & 70.4 & 0.92 & -1.91 \\
\hline 5.82 & 75.1 & 73.8 & 76.2 & 75.0 & 0.98 & -2.08 \\
\hline 6.50 & 78.5 & 78.1 & 79.4 & 78.7 & 0.55 & -2.24 \\
\hline 7.18 & 81.4 & 80.6 & 82.7 & 81.6 & 0.86 & -2.38 \\
\hline 7.87 & 84.1 & 83.5 & 82.8 & 83.5 & 0.52 & -2.49 \\
\hline 8.55 & 85.8 & 84.1 & 86.2 & 85.4 & 0.91 & -2.61 \\
\hline 9.23 & 87.4 & 87.6 & 87.9 & 87.6 & 0.20 & -2.78 \\
\hline 9.92 & 88.9 & 89.4 & 90.1 & 89.5 & 0.48 & -2.95 \\
\hline 10.60 & 90.4 & 90.8 & 91.2 & 90.8 & 0.31 & -3.08 \\
\hline 11.28 & 90.9 & 91.4 & 91.7 & 91.3 & 0.32 & -3.14 \\
\hline 11.97 & 92.3 & 92.1 & 92.6 & 92.3 & 0.20 & -3.26 \\
\hline 12.65 & 92.9 & 93.4 & 93.6 & 93.3 & 0.31 & -3.39 \\
\hline 13.33 & 93.7 & 93.9 & 94 & 93.9 & 0.11 & -3.49 \\
\hline 14.02 & 94.5 & 94.2 & 94.7 & 94.5 & 0.20 & -3.59 \\
\hline 14.70 & 95.1 & 95.3 & 95.6 & 95.3 & 0.22 & -3.76 \\
\hline 15.38 & 95.4 & 95.2 & 94.8 & 95.1 & 0.25 & -3.72 \\
\hline 16.07 & 95.8 & 96.2 & 96.4 & 96.1 & 0.26 & -3.94 \\
\hline 16.75 & 96.1 & 96.6 & 96.2 & 96.3 & 0.20 & -3.99 \\
\hline 17.43 & 96.8 & 96.6 & 97 & 96.8 & 0.16 & -4.14 \\
\hline 18.12 & 96.9 & 97.2 & 97.4 & 97.2 & 0.19 & -4.26 \\
\hline 18.80 & 97.2 & 97.1 & 97.5 & 97.3 & 0.18 & -4.29 \\
\hline 19.48 & 97.4 & 97.3 & 97.5 & 97.4 & 0.08 & -4.34 \\
\hline 20.17 & 97.7 & 98.1 & 97.9 & 97.9 & 0.17 & -4.55 \\
\hline 20.85 & 98.0 & 98.3 & 98.5 & 98.3 & 0.22 & -4.74 \\
\hline 21.53 & 98.1 & 98.4 & 98.2 & 98.2 & 0.12 & -4.73 \\
\hline 22.22 & 98.0 & 98.6 & 98.8 & 98.5 & 0.33 & -4.88 \\
\hline 22.90 & 98.2 & 98.5 & 98.7 & 98.5 & 0.19 & -4.88 \\
\hline 23.58 & 98.4 & 98.6 & 98.9 & 98.6 & 0.22 & -4.98 \\
\hline 24.27 & 98.4 & 98.7 & 98.9 & 98.7 & 0.20 & -5.02 \\
\hline 24.95 & 98.6 & 98.5 & 98.8 & 98.6 & 0.12 & -4.99 \\
\hline 25.63 & 98.7 & 98.6 & 98.8 & 98.7 & 0.08 & -5.04 \\
\hline 26.32 & 98.7 & 98.5 & 98.7 & 98.6 & 0.10 & -4.99 \\
\hline 27.00 & 98.8 & 98.4 & 98.6 & 98.6 & 0.15 & -4.96 \\
\hline 27.68 & 98.7 & 98.9 & 99 & 98.9 & 0.11 & -5.18 \\
\hline 28.37 & 98.9 & 98.7 & 99.2 & 98.9 & 0.21 & -5.23 \\
\hline 29.05 & 98.6 & 98.7 & 98.9 & 98.7 & 0.12 & -5.07 \\
\hline 29.73 & 99.4 & 99.2 & 99.5 & 99.4 & 0.12 & -5.74 \\
\hline
\end{tabular}




\section{Catalyst G-I}

\begin{tabular}{|c|c|c|c|c|c|}
\hline \multirow{2}{*}{$\begin{array}{l}\text { Time } \\
\text { (min) }\end{array}$} & \multicolumn{2}{|c|}{ Conversion (\%) } & \multirow[b]{2}{*}{ Trial 3} & \multirow[b]{2}{*}{ Avg. } & \multirow[b]{2}{*}{ Std. Dev. } \\
\hline & Trial 1 & Trial 2 & & & \\
\hline 0.35 & 1.0 & 0.7 & 0.8 & 0.8 & 0.14 \\
\hline 1.03 & 1.0 & 0.8 & 1.1 & 1.0 & 0.13 \\
\hline 1.72 & 0.9 & 0.8 & 1.1 & 0.9 & 0.12 \\
\hline 2.40 & 1.4 & 1.2 & 1.3 & 1.3 & 0.06 \\
\hline 3.08 & 1.7 & 1.3 & 1.3 & 1.4 & 0.18 \\
\hline 3.77 & 1.7 & 1.4 & 1.6 & 1.6 & 0.14 \\
\hline 4.45 & 2.2 & 1.7 & 1.9 & 1.9 & 0.19 \\
\hline 5.13 & 2.2 & 2 & 2.1 & 2.1 & 0.10 \\
\hline 5.82 & 1.9 & 1.8 & 2 & 1.9 & 0.08 \\
\hline 6.50 & 2.5 & 2.3 & 2.7 & 2.5 & 0.16 \\
\hline 7.18 & 3.1 & 3.3 & 3 & 3.1 & 0.13 \\
\hline 7.87 & 2.9 & 3 & 3.2 & 3.0 & 0.13 \\
\hline 8.55 & 2.6 & 2.8 & 2.7 & 2.7 & 0.08 \\
\hline 9.23 & 3.4 & 3.2 & 3.5 & 3.4 & 0.13 \\
\hline 9.92 & 3.5 & 3.4 & 3.6 & 3.5 & 0.08 \\
\hline 10.60 & 3.6 & 3.7 & 3.6 & 3.6 & 0.04 \\
\hline 11.28 & 3.4 & 3.2 & 3.5 & 3.4 & 0.13 \\
\hline 11.97 & 3.7 & 3.6 & 3.8 & 3.7 & 0.08 \\
\hline 12.65 & 3.9 & 4 & 4.1 & 4.0 & 0.10 \\
\hline 13.33 & 4.2 & 4.4 & 4.3 & 4.3 & 0.07 \\
\hline 14.02 & 4.0 & 4.2 & 4.4 & 4.2 & 0.15 \\
\hline 14.70 & 4.4 & 4.5 & 4.3 & 4.4 & 0.08 \\
\hline 15.38 & 4.1 & 4.2 & 4.3 & 4.2 & 0.07 \\
\hline 16.07 & 4.6 & 4.7 & 4.4 & 4.6 & 0.12 \\
\hline 16.75 & 4.1 & 4.3 & 4.5 & 4.3 & 0.15 \\
\hline 17.43 & 5.4 & 4.8 & 4.7 & 5.0 & 0.29 \\
\hline 18.12 & 4.4 & 5 & 5.3 & 4.9 & 0.36 \\
\hline 18.80 & 4.1 & 4.6 & 5.2 & 4.6 & 0.47 \\
\hline 19.48 & 5.1 & 5.4 & 5.5 & 5.3 & 0.19 \\
\hline 20.17 & 5.3 & 5.5 & 5.8 & 5.5 & 0.20 \\
\hline 20.85 & 4.3 & 4.7 & 5.2 & 4.7 & 0.38 \\
\hline 21.53 & 3.9 & 3.7 & 4.6 & 4.1 & 0.38 \\
\hline 22.22 & 5.8 & 5.6 & 4.8 & 5.4 & 0.44 \\
\hline 22.90 & 4.9 & 5.2 & 4.7 & 4.9 & 0.20 \\
\hline 23.58 & 5.7 & 5.5 & 6 & 5.7 & 0.21 \\
\hline 24.27 & 6.1 & 5.5 & 5.7 & 5.8 & 0.23 \\
\hline 24.95 & 6.6 & 6.8 & 6.4 & 6.6 & 0.16 \\
\hline
\end{tabular}




\begin{tabular}{|r|r|r|r|r|r|}
\hline Time \\
(min)
\end{tabular}




\begin{tabular}{|c|c|c|c|c|c|c|}
\hline \multirow{2}{*}{$\begin{array}{l}\text { Time } \\
\text { (min) }\end{array}$} & \multicolumn{2}{|c|}{ Conversion (\%) } & \multirow[b]{2}{*}{ Trial 3} & \multirow[b]{2}{*}{ Avg. } & \multirow[b]{2}{*}{ Std. Dev. } & \multirow[b]{2}{*}{$\ln ([S M])$} \\
\hline & Trial 1 & Trial 2 & & & & \\
\hline 0.35 & 1.4 & 1.6 & 1.7 & 1.6 & 0.14 & -0.71 \\
\hline 1.03 & 1.7 & 2 & 2.2 & 2.0 & 0.22 & -0.71 \\
\hline 1.72 & 2.6 & 3.1 & 3.3 & 3.0 & 0.28 & -0.72 \\
\hline 2.40 & 4.3 & 4.5 & 5.1 & 4.6 & 0.36 & -0.74 \\
\hline 3.08 & 2.6 & 4.5 & 5.6 & 4.2 & 1.25 & -0.74 \\
\hline 3.77 & 4.7 & 4.3 & 5.7 & 4.9 & 0.59 & -0.74 \\
\hline 4.45 & 5.6 & 6.2 & 6.4 & 6.1 & 0.33 & -0.76 \\
\hline 5.13 & 6.6 & 7.2 & 7.4 & 7.1 & 0.34 & -0.77 \\
\hline 5.82 & 8.3 & 8.8 & 9.1 & 8.7 & 0.32 & -0.78 \\
\hline 6.50 & 11.1 & 12.2 & 11.6 & 11.6 & 0.45 & -0.82 \\
\hline 7.18 & 13.3 & 13.8 & 14.6 & 13.9 & 0.54 & -0.84 \\
\hline 7.87 & 15.0 & 16.1 & 16.4 & 15.8 & 0.60 & -0.87 \\
\hline 8.55 & 17.5 & 18.2 & 18.4 & 18.0 & 0.37 & -0.89 \\
\hline 9.23 & 20.0 & 21.2 & 20.6 & 20.6 & 0.47 & -0.92 \\
\hline 9.92 & 23.0 & 24.2 & 22.6 & 23.3 & 0.68 & -0.96 \\
\hline 10.60 & 26.6 & 25.1 & 27.2 & 26.3 & 0.88 & -1.00 \\
\hline 11.28 & 30.2 & 30.5 & 31.2 & 30.6 & 0.43 & -1.06 \\
\hline 11.97 & 33.4 & 34.4 & 35.1 & 34.3 & 0.70 & -1.11 \\
\hline 12.65 & 38.5 & 36.8 & 38.9 & 38.1 & 0.91 & -1.17 \\
\hline 13.33 & 42.8 & 43.1 & 44 & 43.3 & 0.51 & -1.26 \\
\hline 14.02 & 46.9 & 47.3 & 46.5 & 46.9 & 0.33 & -1.33 \\
\hline 14.70 & 51.2 & 51.7 & 52.1 & 51.7 & 0.37 & -1.42 \\
\hline 15.38 & 54.5 & 55.3 & 56.1 & 55.3 & 0.67 & -1.50 \\
\hline 16.07 & 57.7 & 58.2 & 59.1 & 58.3 & 0.58 & -1.57 \\
\hline 16.75 & 59.9 & 59.6 & 60.4 & 60.0 & 0.33 & -1.61 \\
\hline 17.43 & 63.0 & 62.1 & 63.7 & 62.9 & 0.65 & -1.69 \\
\hline 18.12 & 65.0 & 64.2 & 66.5 & 65.2 & 0.95 & -1.75 \\
\hline 18.80 & 67.1 & 66.7 & 67.6 & 67.1 & 0.37 & -1.81 \\
\hline 19.48 & 69.0 & 68.4 & 69.3 & 68.9 & 0.38 & -1.86 \\
\hline 20.17 & 70.9 & 71.6 & 72.3 & 71.6 & 0.57 & -1.95 \\
\hline 20.85 & 72.8 & 73.4 & 73.8 & 73.3 & 0.41 & -2.01 \\
\hline 21.53 & 74.8 & 75.2 & 75.7 & 75.2 & 0.38 & -2.09 \\
\hline 22.22 & 76.4 & 77.2 & 76.6 & 76.7 & 0.33 & -2.15 \\
\hline 22.90 & 78.2 & 79.4 & 79.8 & 79.1 & 0.69 & -2.26 \\
\hline 23.58 & 80.0 & 80.5 & 82.2 & 80.9 & 0.94 & -2.35 \\
\hline 24.27 & 81.5 & 82.6 & 83.4 & 82.5 & 0.77 & -2.44 \\
\hline
\end{tabular}




\begin{tabular}{|c|c|c|c|c|c|c|}
\hline \multirow{2}{*}{$\begin{array}{l}\text { Time } \\
\text { (min) }\end{array}$} & \multicolumn{2}{|c|}{ Conversion (\%) } & \multirow[b]{2}{*}{ Trial 3} & \multirow[b]{2}{*}{ Avg. } & \multirow[b]{2}{*}{ Std. Dev. } & \multirow[b]{2}{*}{$\ln ([S M])$} \\
\hline & Trial 1 & Trial 2 & & & & \\
\hline 24.95 & 83.1 & 84.2 & 84.6 & 84.0 & 0.61 & -2.52 \\
\hline 25.63 & 84.4 & 85.2 & 84.9 & 84.8 & 0.31 & -2.58 \\
\hline 26.32 & 85.6 & 85.9 & 86.1 & 85.9 & 0.19 & -2.65 \\
\hline 27.00 & 86.9 & 87.6 & 87.8 & 87.4 & 0.39 & -2.77 \\
\hline 27.68 & 88.0 & 88.7 & 88.9 & 88.5 & 0.39 & -2.86 \\
\hline 28.37 & 88.9 & 89.6 & 89.8 & 89.4 & 0.38 & -2.94 \\
\hline 29.05 & 89.9 & 90.5 & 90.3 & 90.2 & 0.27 & -3.02 \\
\hline 29.73 & 90.7 & 91.2 & 91.4 & 91.1 & 0.28 & -3.11 \\
\hline 30.42 & 91.5 & 92.3 & 91.7 & 91.8 & 0.34 & -3.20 \\
\hline 31.10 & 92.3 & 93.1 & 92.9 & 92.8 & 0.35 & -3.32 \\
\hline 31.78 & 92.8 & 93.3 & 93.5 & 93.2 & 0.30 & -3.38 \\
\hline 32.47 & 93.3 & 94.2 & 93.8 & 93.8 & 0.36 & -3.47 \\
\hline 33.15 & 93.9 & 94.6 & 94.2 & 94.2 & 0.28 & -3.55 \\
\hline 33.83 & 94.3 & 95.1 & 94.7 & 94.7 & 0.32 & -3.63 \\
\hline 34.52 & 94.7 & 95.2 & 95.4 & 95.1 & 0.28 & -3.71 \\
\hline 35.20 & 95.0 & 95.7 & 95.6 & 95.4 & 0.31 & -3.78 \\
\hline 35.88 & 95.4 & 96.2 & 95.8 & 95.8 & 0.31 & -3.87 \\
\hline 36.57 & 95.8 & 96.3 & 96.5 & 96.2 & 0.30 & -3.96 \\
\hline 37.25 & 96.1 & 96.7 & 97.1 & 96.6 & 0.42 & -4.08 \\
\hline 37.93 & 96.4 & 97.2 & 96.8 & 96.8 & 0.34 & -4.13 \\
\hline 38.62 & 96.7 & 97.3 & 97.6 & 97.2 & 0.39 & -4.26 \\
\hline 39.30 & 96.9 & 97.4 & 97.9 & 97.4 & 0.39 & -4.35 \\
\hline 39.98 & 97.1 & 97.5 & 98.2 & 97.6 & 0.47 & -4.42 \\
\hline 40.67 & 97.2 & 97.6 & 98.4 & 97.7 & 0.50 & -4.48 \\
\hline 41.35 & 97.5 & 98.2 & 98.5 & 98.1 & 0.43 & -4.63 \\
\hline 42.03 & 97.6 & 97.9 & 98.2 & 97.9 & 0.24 & -4.56 \\
\hline 42.72 & 97.7 & 98.2 & 98.5 & 98.1 & 0.34 & -4.67 \\
\hline 43.40 & 97.8 & 98.5 & 98.8 & 98.4 & 0.40 & -4.82 \\
\hline 44.08 & 98.0 & 98.4 & 98.9 & 98.4 & 0.37 & -4.85 \\
\hline 44.77 & 98.2 & 98.7 & 99.1 & 98.7 & 0.35 & -5.02 \\
\hline 45.45 & 98.3 & 98.6 & 98.9 & 98.6 & 0.25 & -4.96 \\
\hline 46.13 & 98.6 & 98.8 & 99 & 98.8 & 0.16 & -5.12 \\
\hline 46.82 & 98.4 & 98.6 & 99.1 & 98.7 & 0.31 & -5.03 \\
\hline 47.50 & 98.8 & 98.9 & 99.3 & 99.0 & 0.23 & -5.29 \\
\hline 48.18 & 98.6 & 98.8 & 99.2 & 98.9 & 0.26 & -5.16 \\
\hline 48.87 & 98.4 & 98.7 & 99.1 & 98.7 & 0.28 & -5.07 \\
\hline 49.55 & 98.8 & 99 & 99.2 & 99.0 & 0.15 & -5.31 \\
\hline
\end{tabular}




\section{Catalyst G-III}

\begin{tabular}{|c|c|c|c|c|c|}
\hline \multirow{2}{*}{$\begin{array}{l}\text { Time } \\
\text { (min) }\end{array}$} & \multicolumn{2}{|c|}{ Conversion (\%) } & \multirow[b]{2}{*}{ Trial 3} & \multirow[b]{2}{*}{ Avg. } & \multirow[b]{2}{*}{ Std. Dev. } \\
\hline & Trial 1 & Trial 2 & & & \\
\hline 0.35 & 85.7 & 83.4 & 85.2 & 84.8 & 1.00 \\
\hline 1.03 & 87.9 & 85.2 & 86.4 & 86.5 & 1.12 \\
\hline 1.72 & 87.4 & 86.4 & 87.1 & 87.0 & 0.40 \\
\hline 2.40 & 89.0 & 87.2 & 88.9 & 88.4 & 0.82 \\
\hline 3.08 & 90.2 & 90.7 & 91.2 & 90.7 & 0.39 \\
\hline 3.77 & 92.1 & 92.4 & 92.7 & 92.4 & 0.25 \\
\hline 4.45 & 93.7 & 94.2 & 94.8 & 94.2 & 0.47 \\
\hline 5.13 & 94.8 & 95.3 & 95.9 & 95.3 & 0.45 \\
\hline 5.82 & 95.6 & 96.2 & 96.8 & 96.2 & 0.50 \\
\hline 6.50 & 96.2 & 97.4 & 98.1 & 97.2 & 0.79 \\
\hline 7.18 & 96.7 & 97.4 & 97.2 & 97.1 & 0.31 \\
\hline 7.87 & 96.8 & 97.2 & 97.2 & 97.1 & 0.19 \\
\hline 8.55 & 97.4 & 97.6 & 97.8 & 97.6 & 0.14 \\
\hline 9.23 & 97.4 & 97.6 & 97.2 & 97.4 & 0.16 \\
\hline 9.92 & 97.5 & 97.7 & 98 & 97.7 & 0.19 \\
\hline 10.60 & 97.6 & 98 & 98.2 & 97.9 & 0.24 \\
\hline 11.28 & 97.9 & 98 & 98.4 & 98.1 & 0.20 \\
\hline 11.97 & 97.9 & 98.4 & 98.2 & 98.2 & 0.19 \\
\hline 12.65 & 98.1 & 98.3 & 98.5 & 98.3 & 0.15 \\
\hline 13.33 & 98.1 & 98.3 & 98.4 & 98.3 & 0.11 \\
\hline 14.02 & 98.1 & 98.4 & 98.6 & 98.4 & 0.21 \\
\hline
\end{tabular}



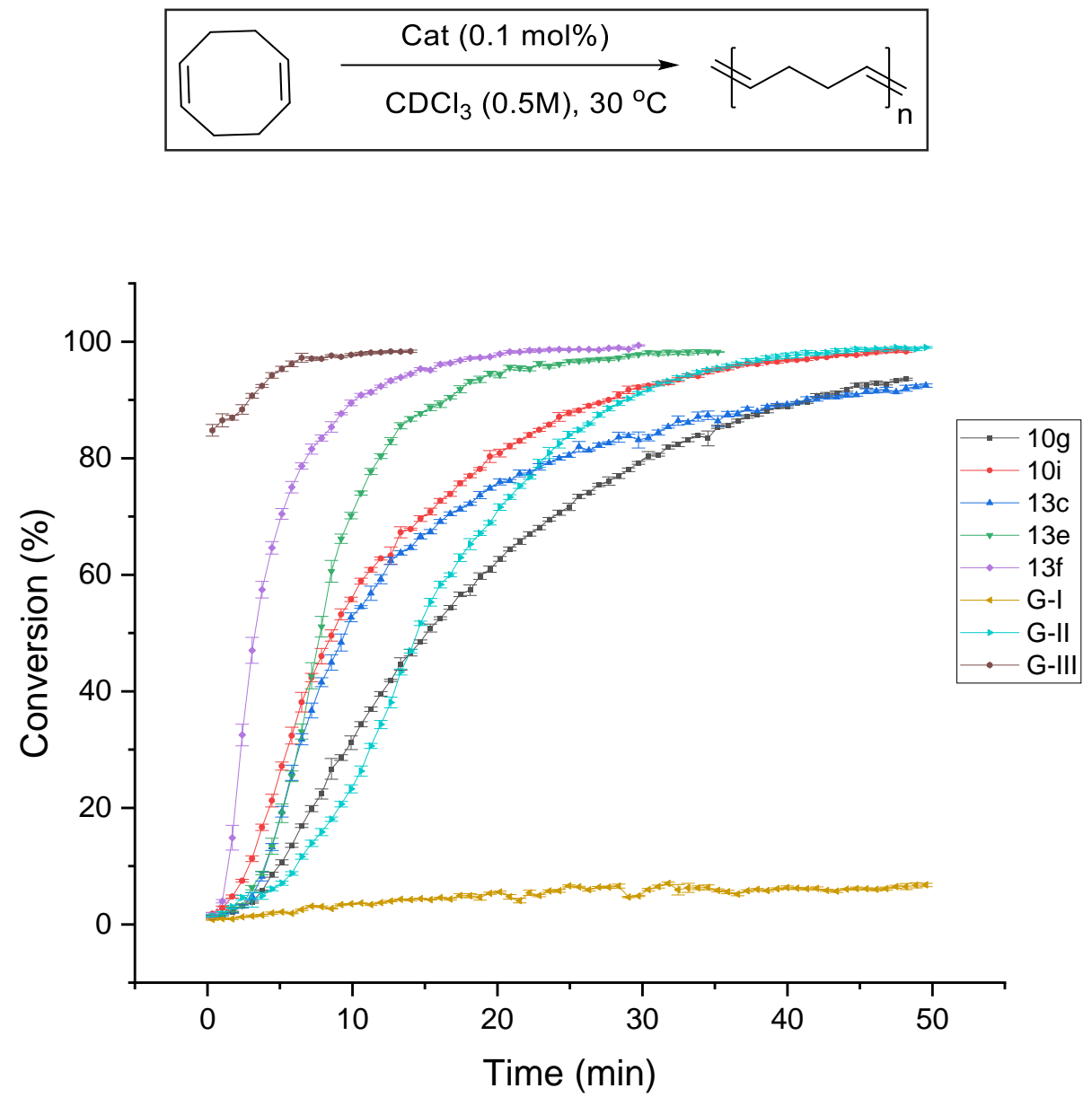

Fig. S14. Kinetic profiles for ROMP reaction of 1,5-cyclooctadiene using selected alkene-chelates as catalysts at $30^{\circ} \mathrm{C}$. Each data point represents the average of three independent experiments. Error bar represents standard error of the triplicate experiments. 


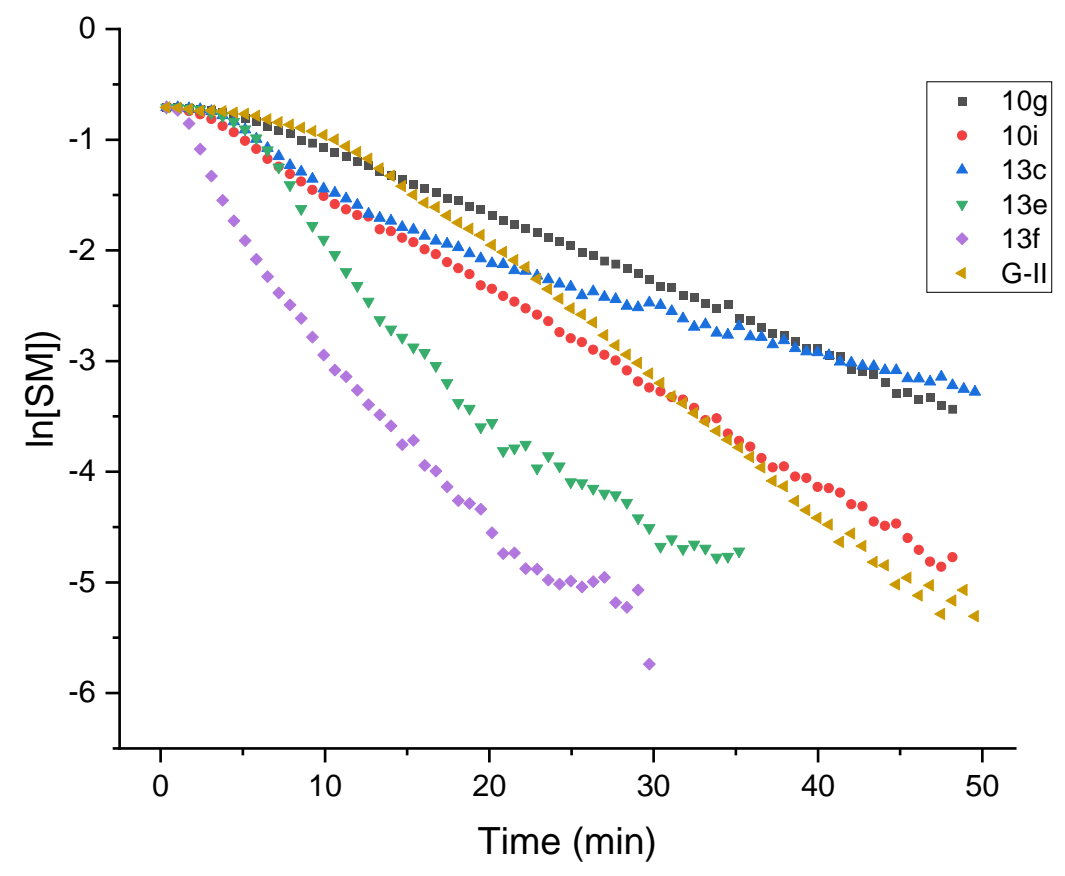

Fig. S15. Log plot of selected alkene-chelates for ROMP reaction of 1,5-cyclooctadiene. Kobs was determined from the slope of these curves and $t_{1 / 2}$ values were accordingly determined.

Kinetic parameters of selected catalysts for ROMP of 1,5-cyclooctadiene ( $k_{\text {obs }}, t_{1 / 2}$ and $\left.k_{\text {rel }}\right)$

\begin{tabular}{ccccc}
\hline Cat. & $\mathrm{k}_{\text {obs }}\left(10^{-4} \mathrm{~s}^{-1}\right)$ & $\mathrm{t}_{1} / 2(\mathrm{~s})$ & $\mathrm{k}_{\text {rel }}$ & Linearity of plot \\
\hline $\mathbf{1 0 g}$ & 10.2 & 679.6 & 0.52 & \\
$\mathbf{1 0 i}$ & 14.8 & 468.3 & 0.75 & \\
$\mathbf{1 3 C}$ & 8.2 & 845.3 & 0.42 & Above $13 \%$ conv. \\
$\mathbf{1 3 e}$ & 26.5 & 261.6 & 1.35 & Above $33 \%$ conv. \\
$\mathbf{1 3 f}$ & 32.7 & 212.0 & 1.66 & \\
GII & 19.7 & 351.9 & 1 & Above $26 \%$ conv. \\
\hline
\end{tabular}

Note: With G-III, $86.5 \%$ conversion was achieved within first 35s hence, log plot cannot be obtained and $\mathrm{k}_{\mathrm{obs}}$ was not determined. 


\section{${ }^{1} \mathrm{H},{ }^{13} \mathrm{C}$ NMR Spectra of Starting Materials}

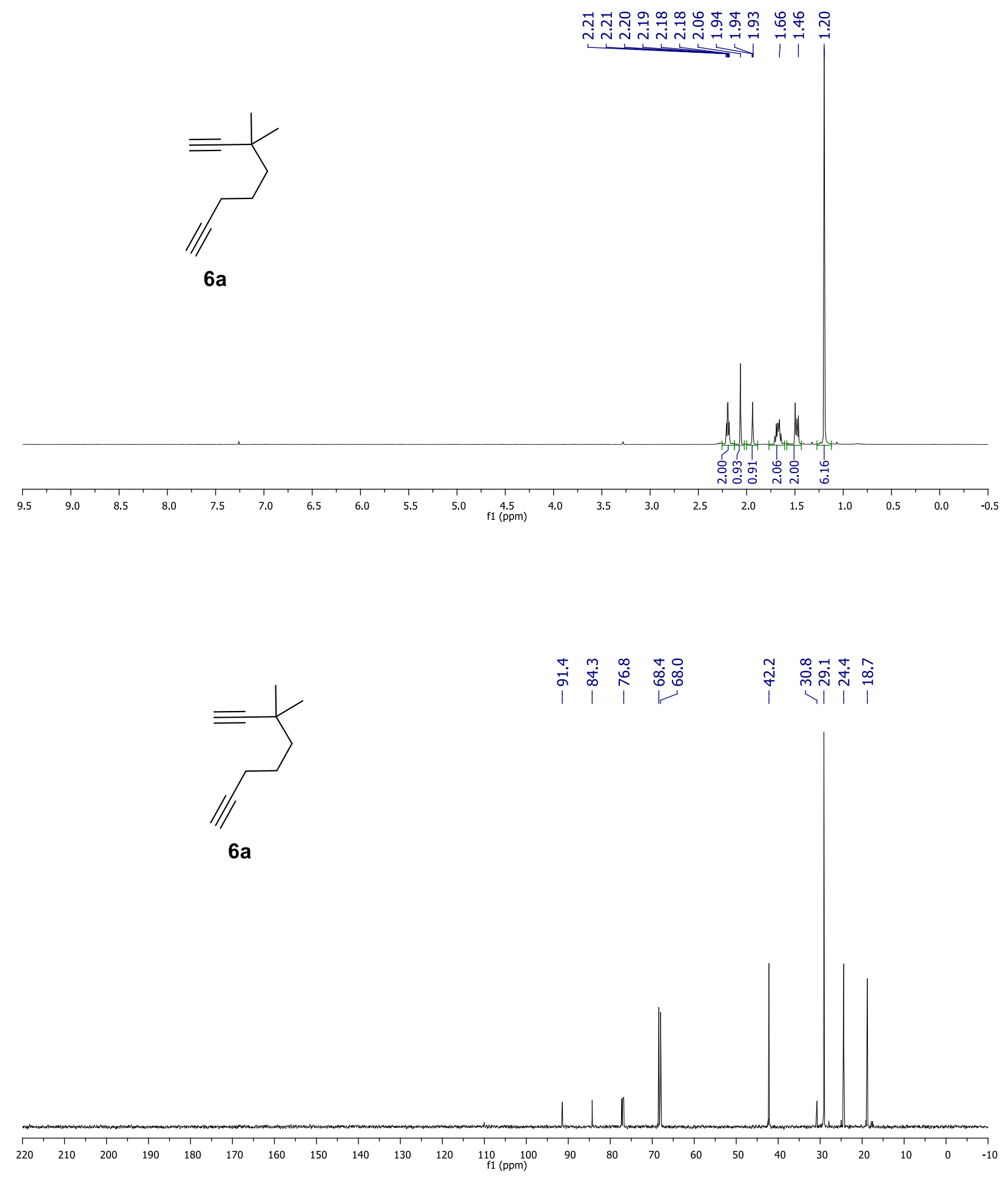



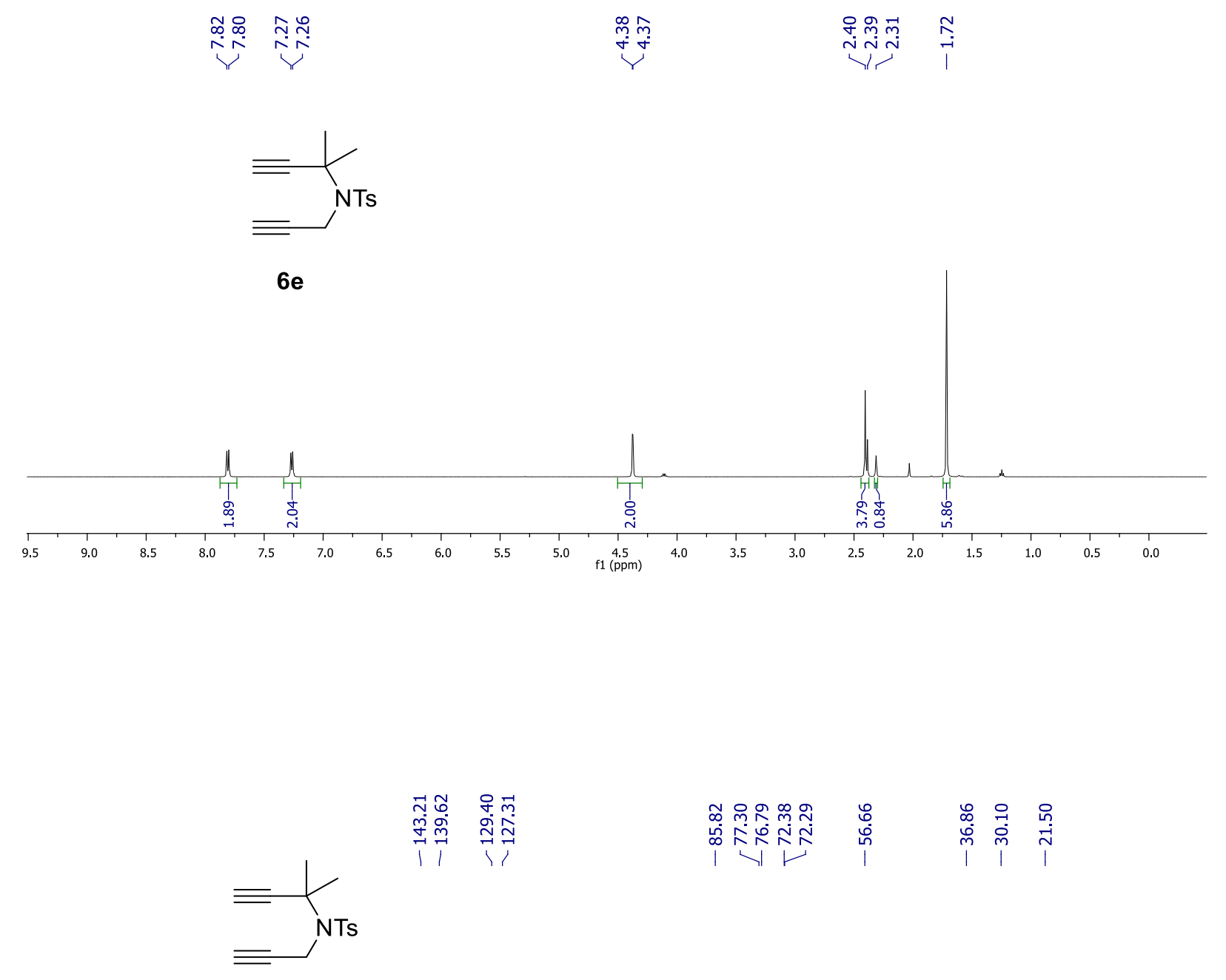

$6 e$

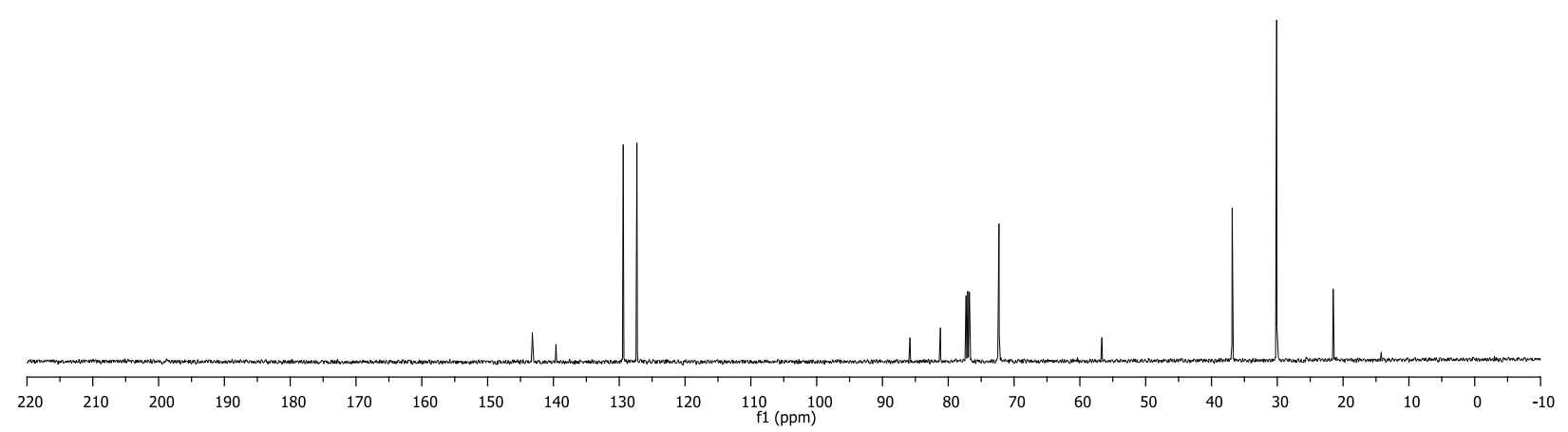




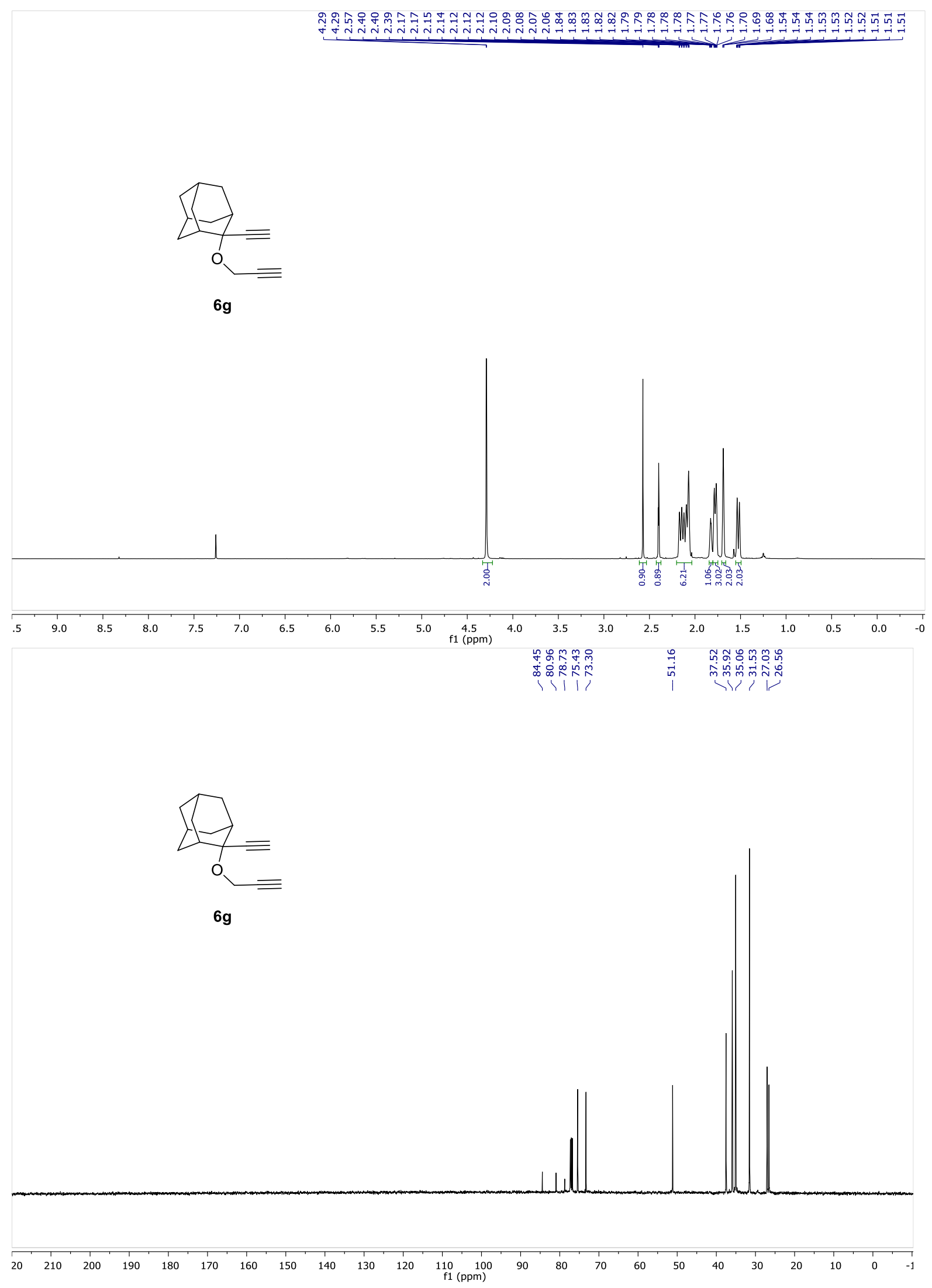



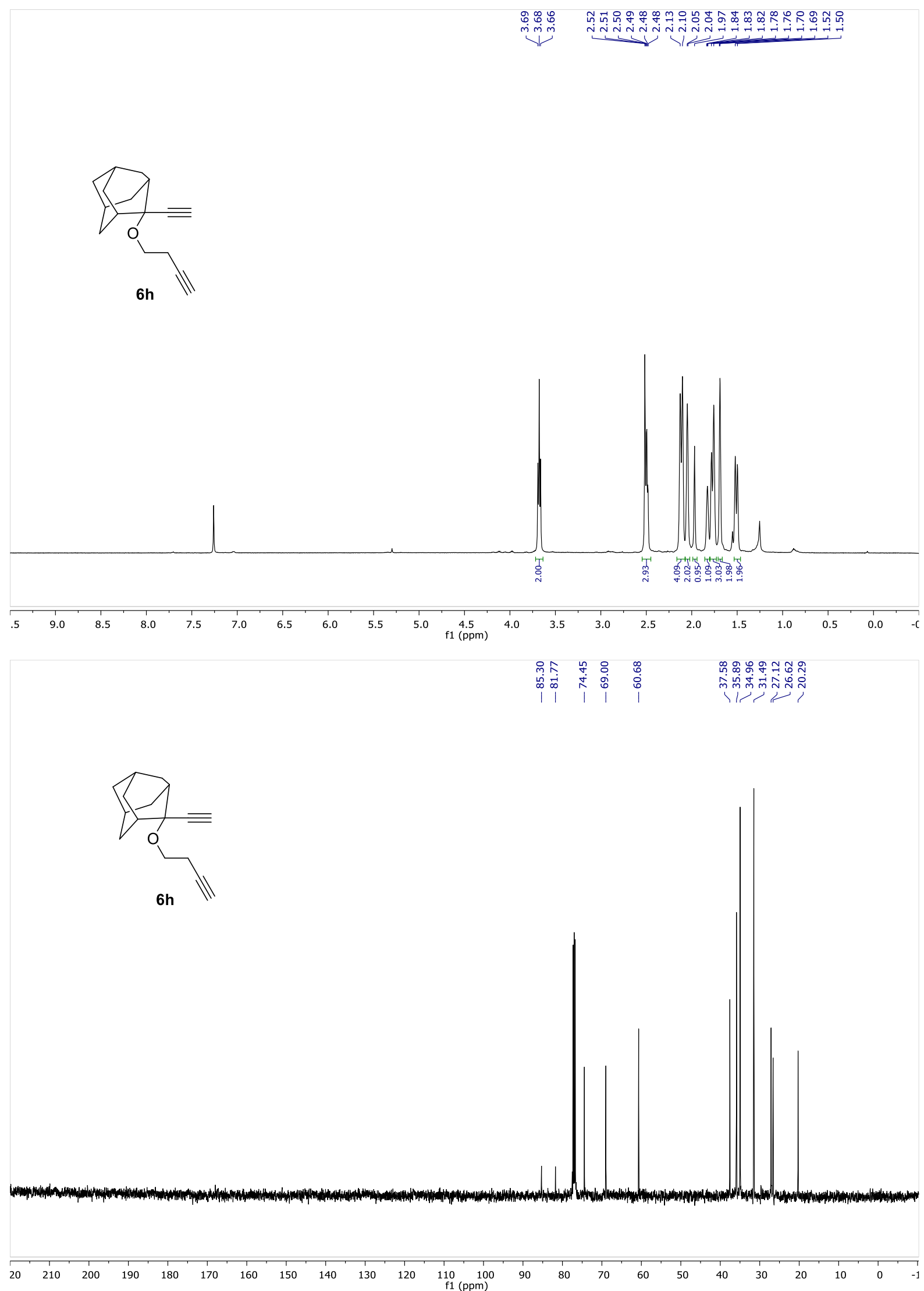


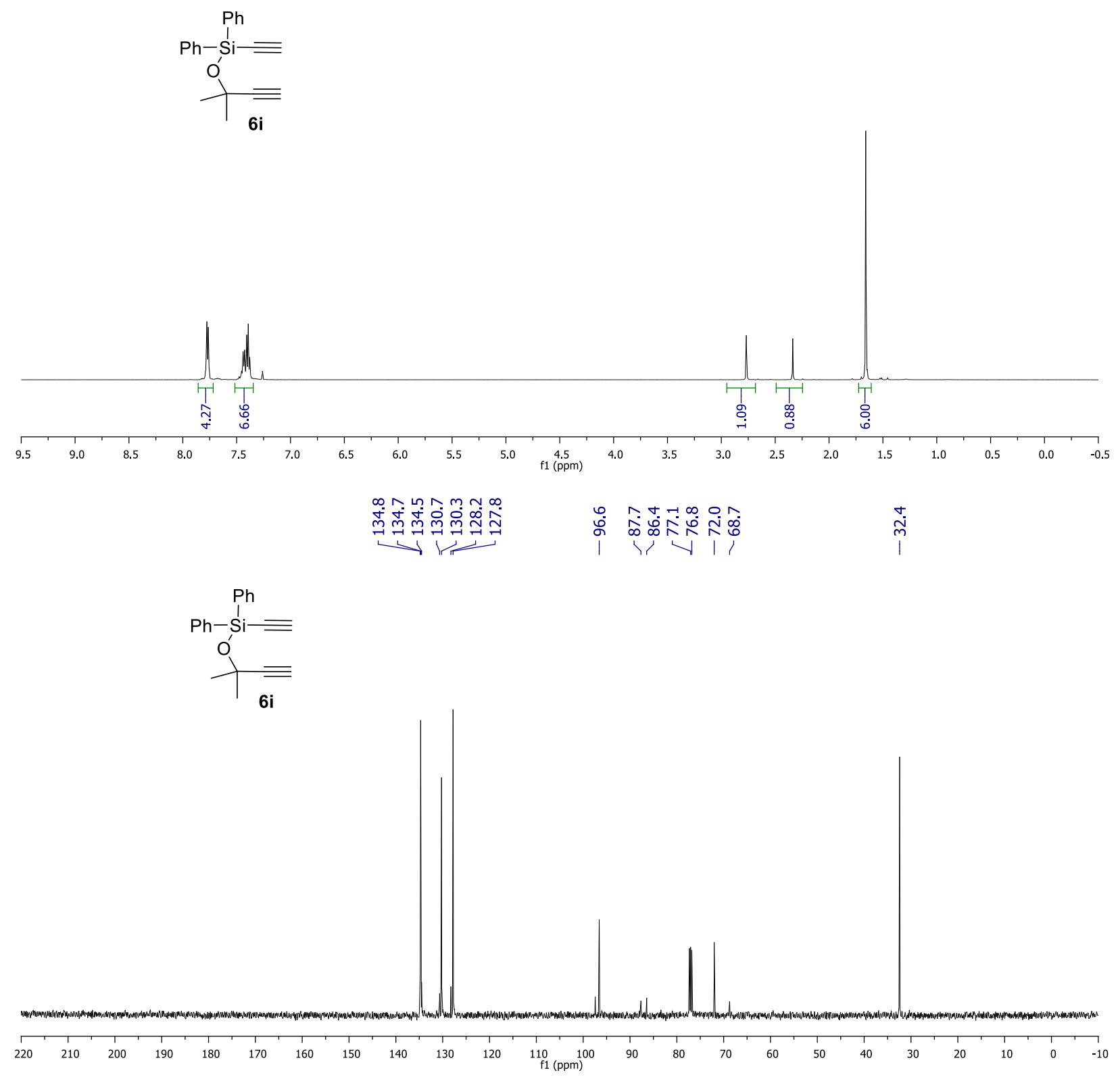



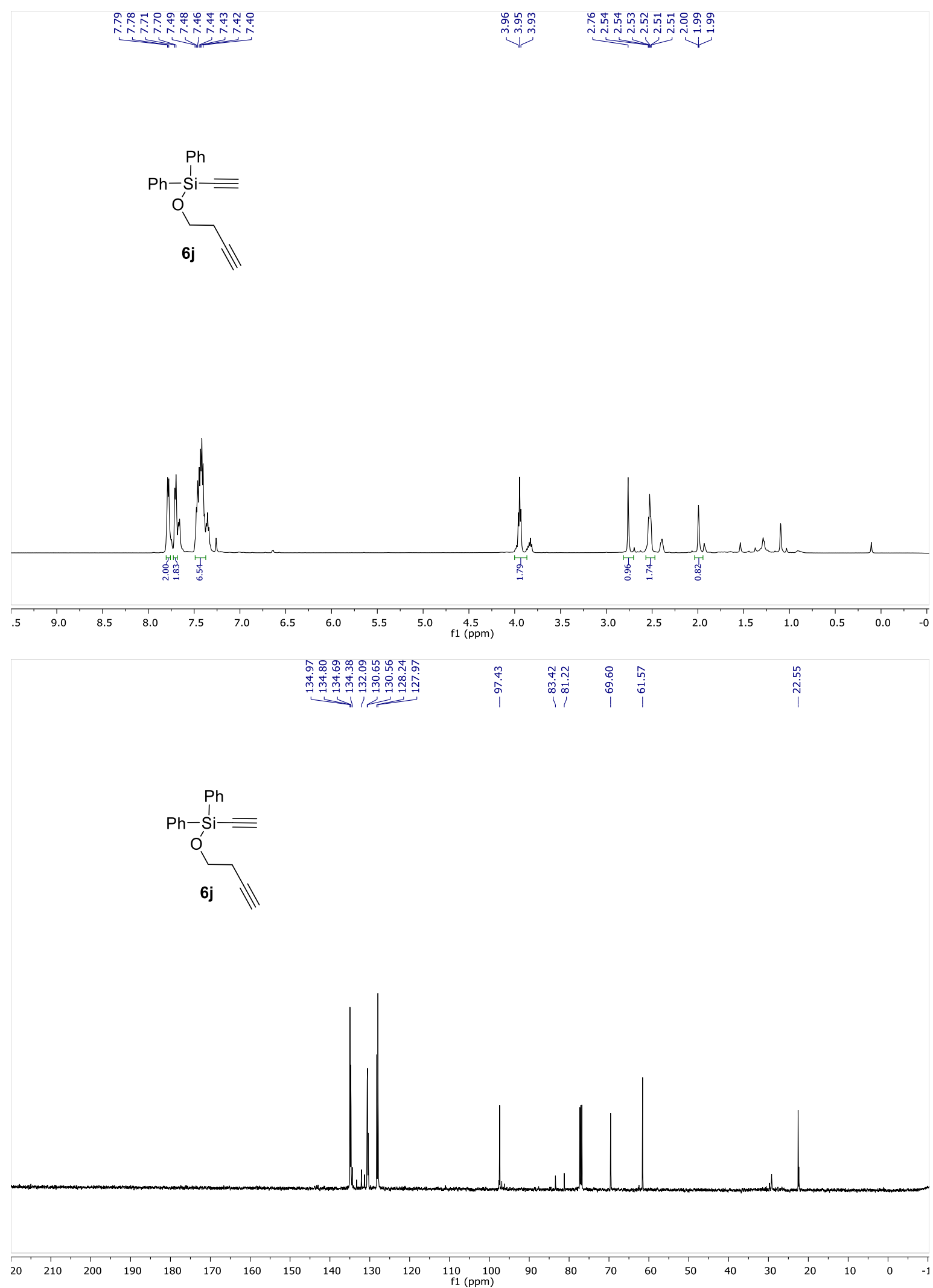


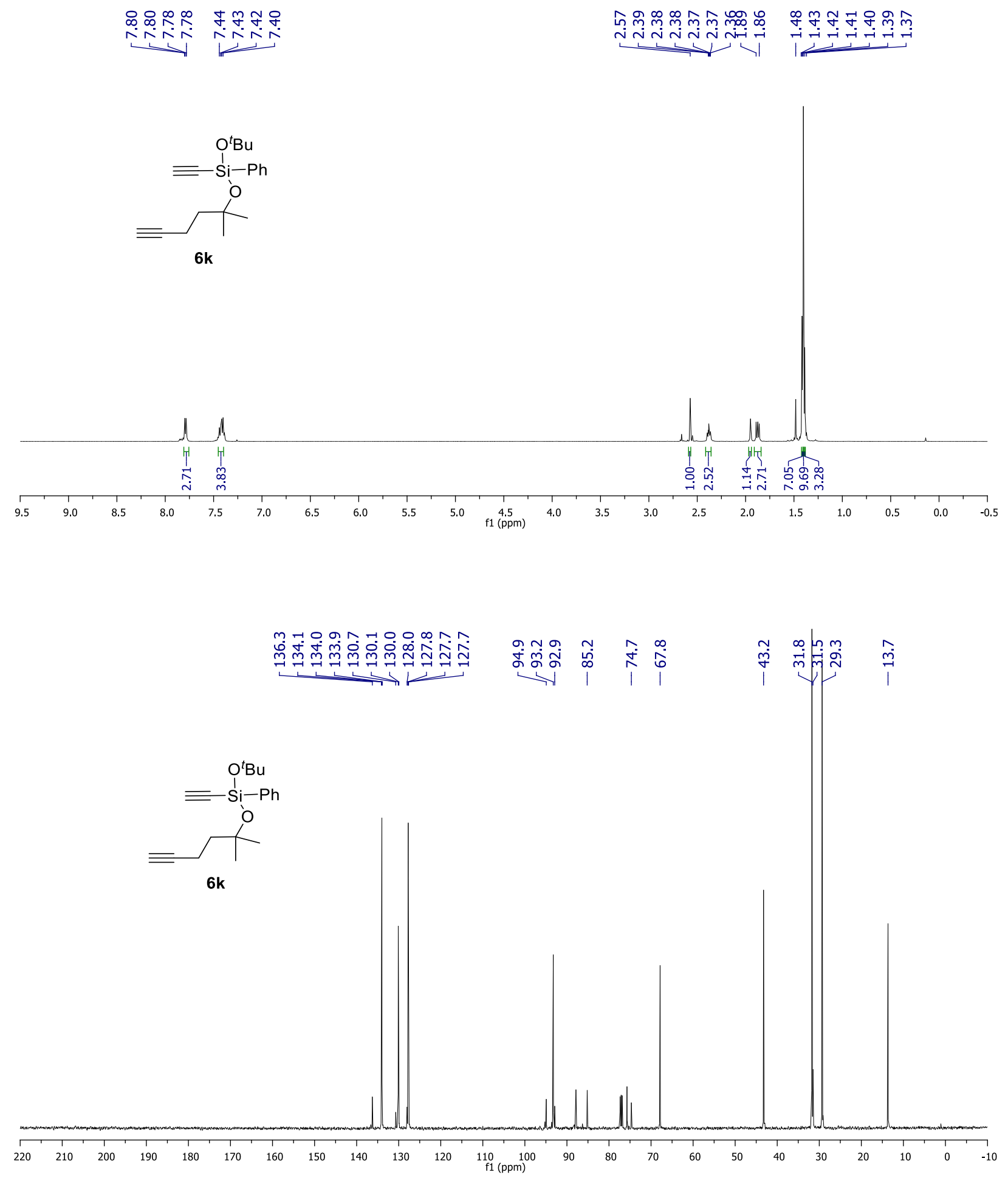




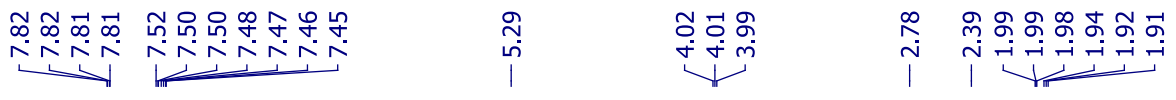
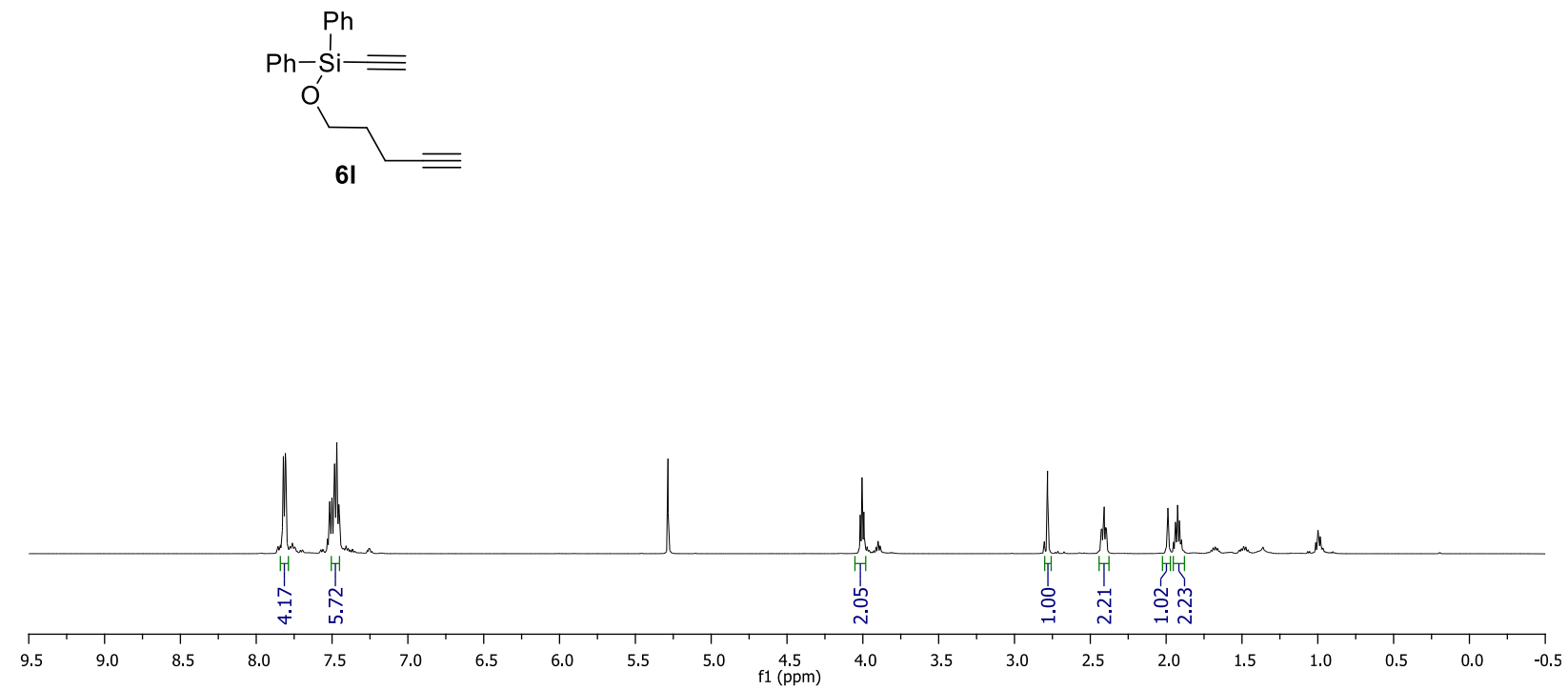

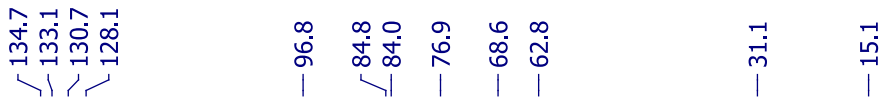
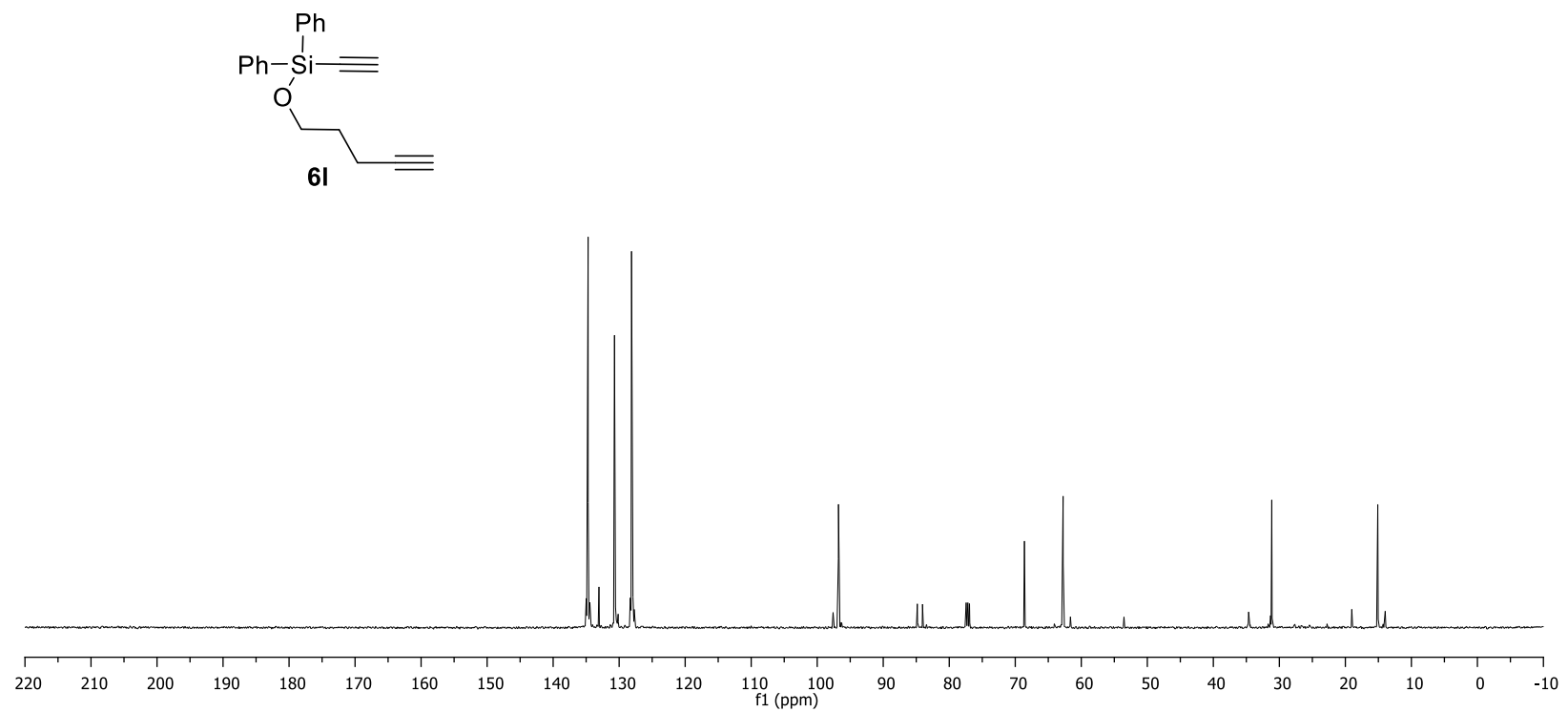


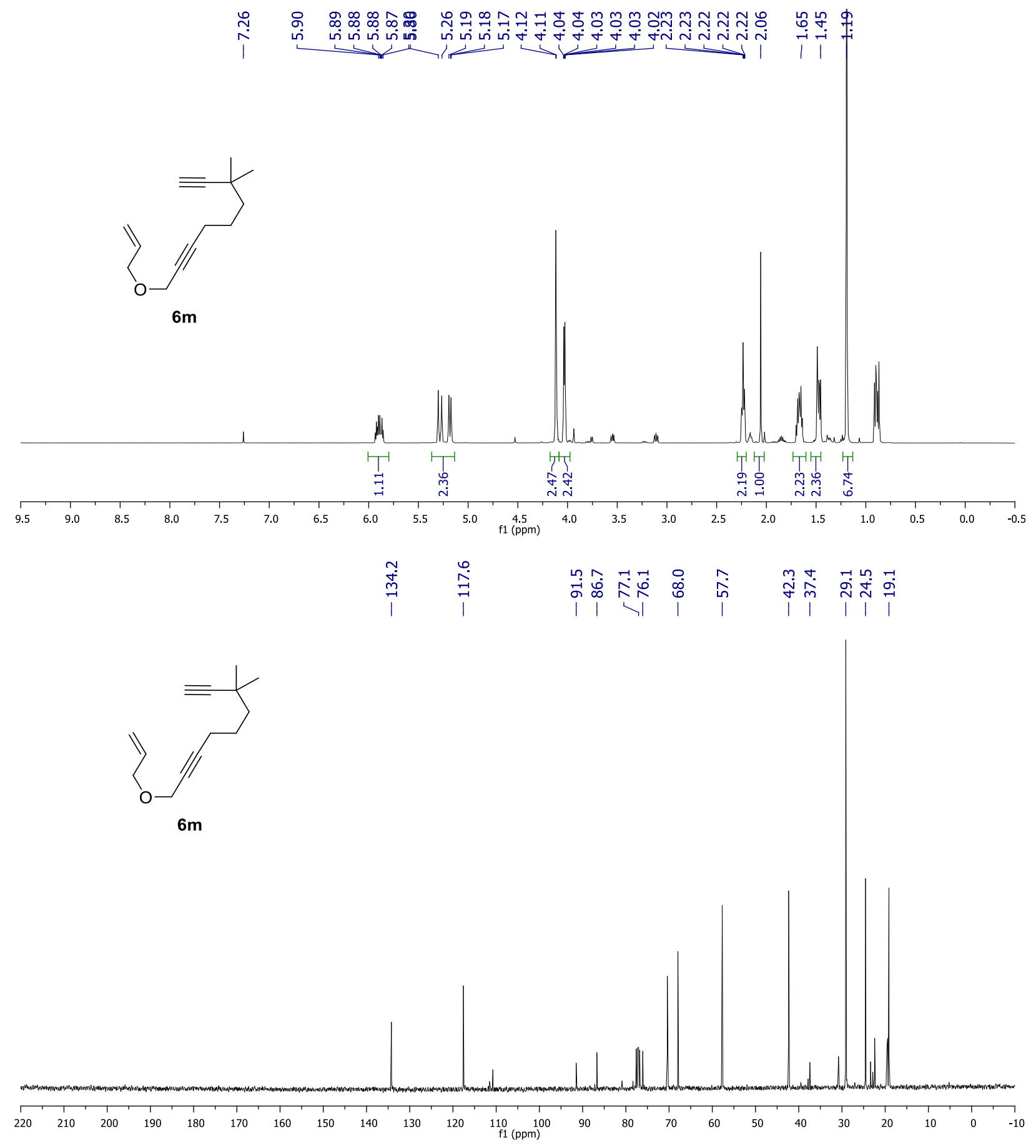




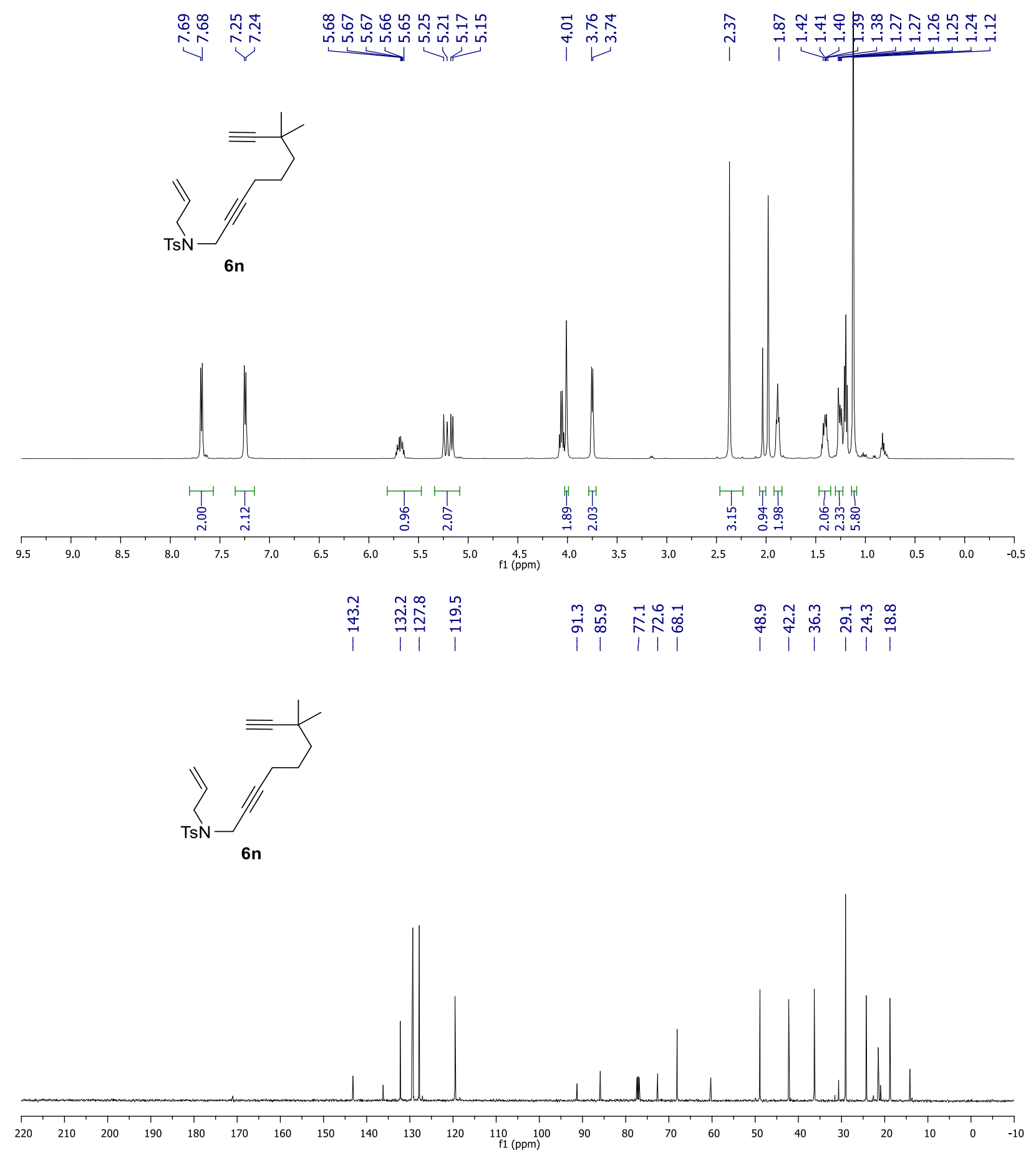




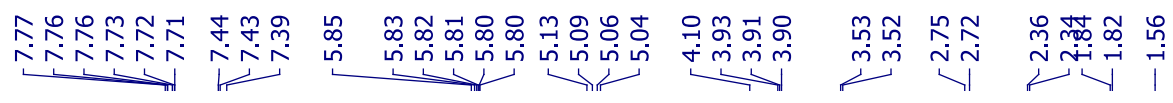

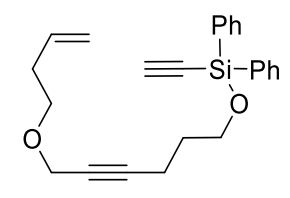

60

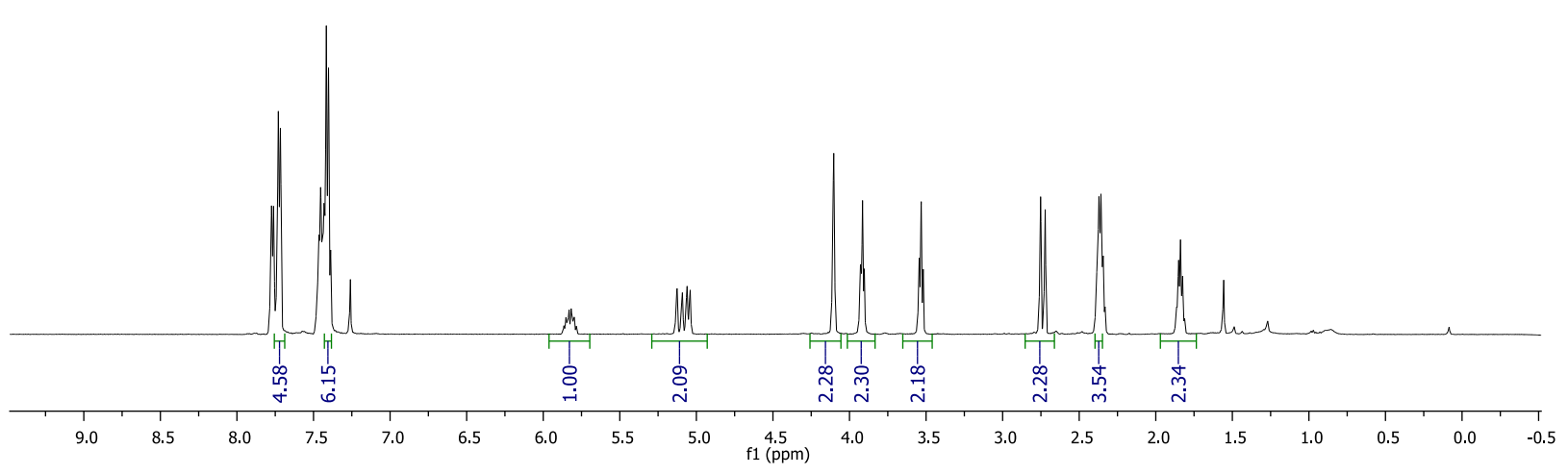

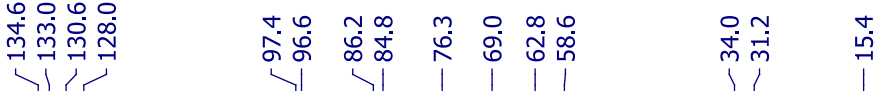

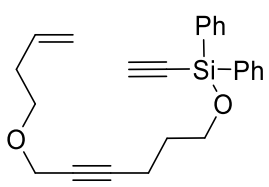

60

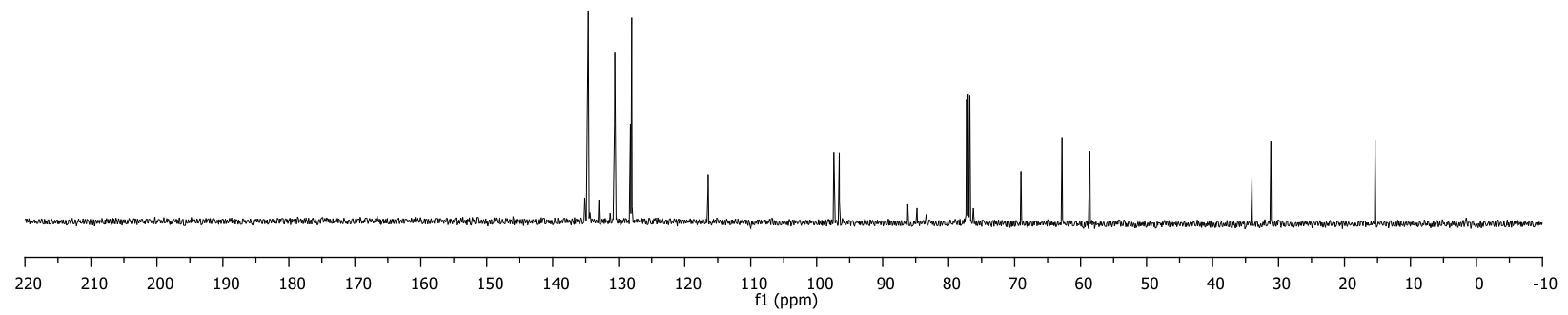




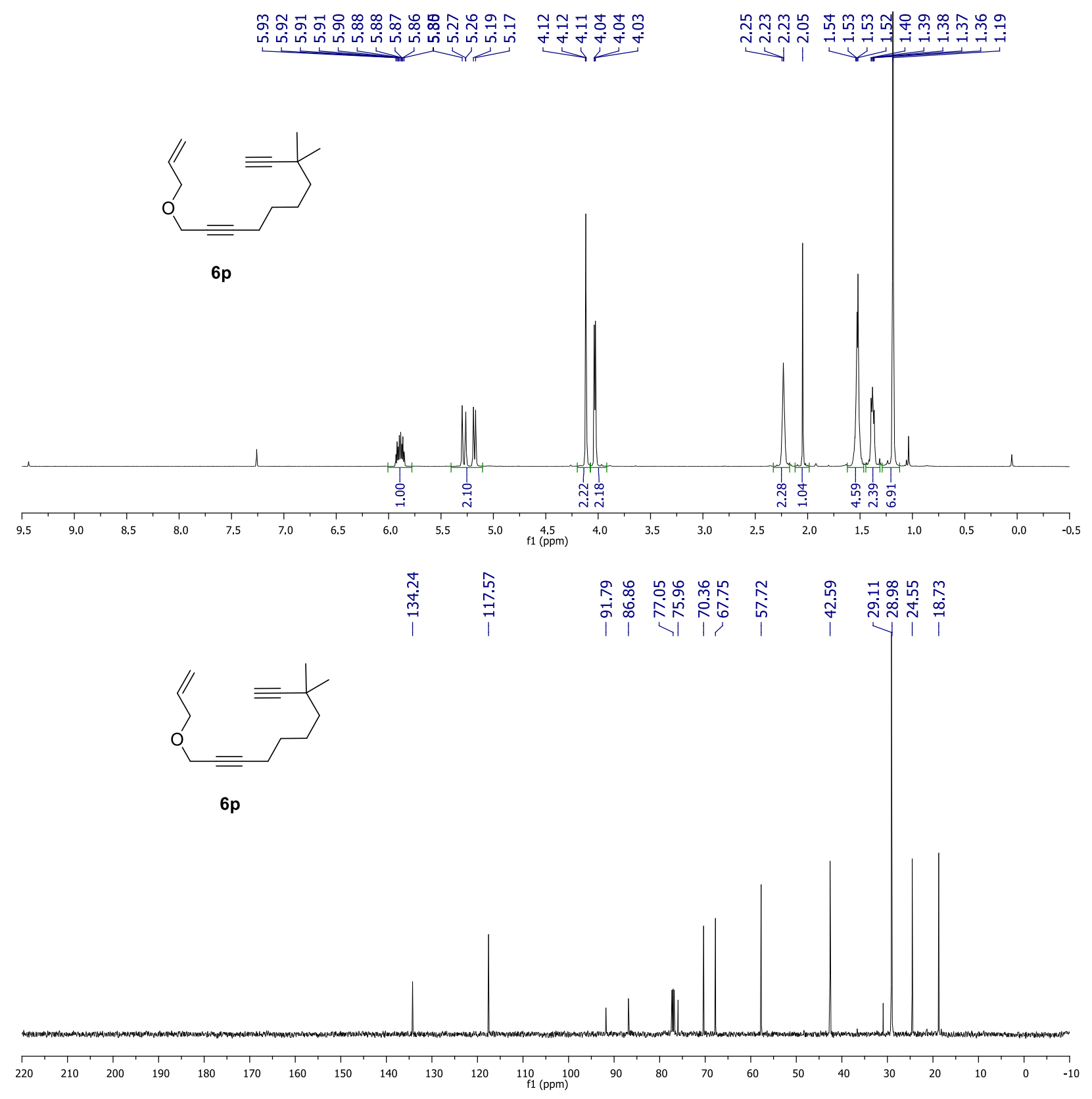




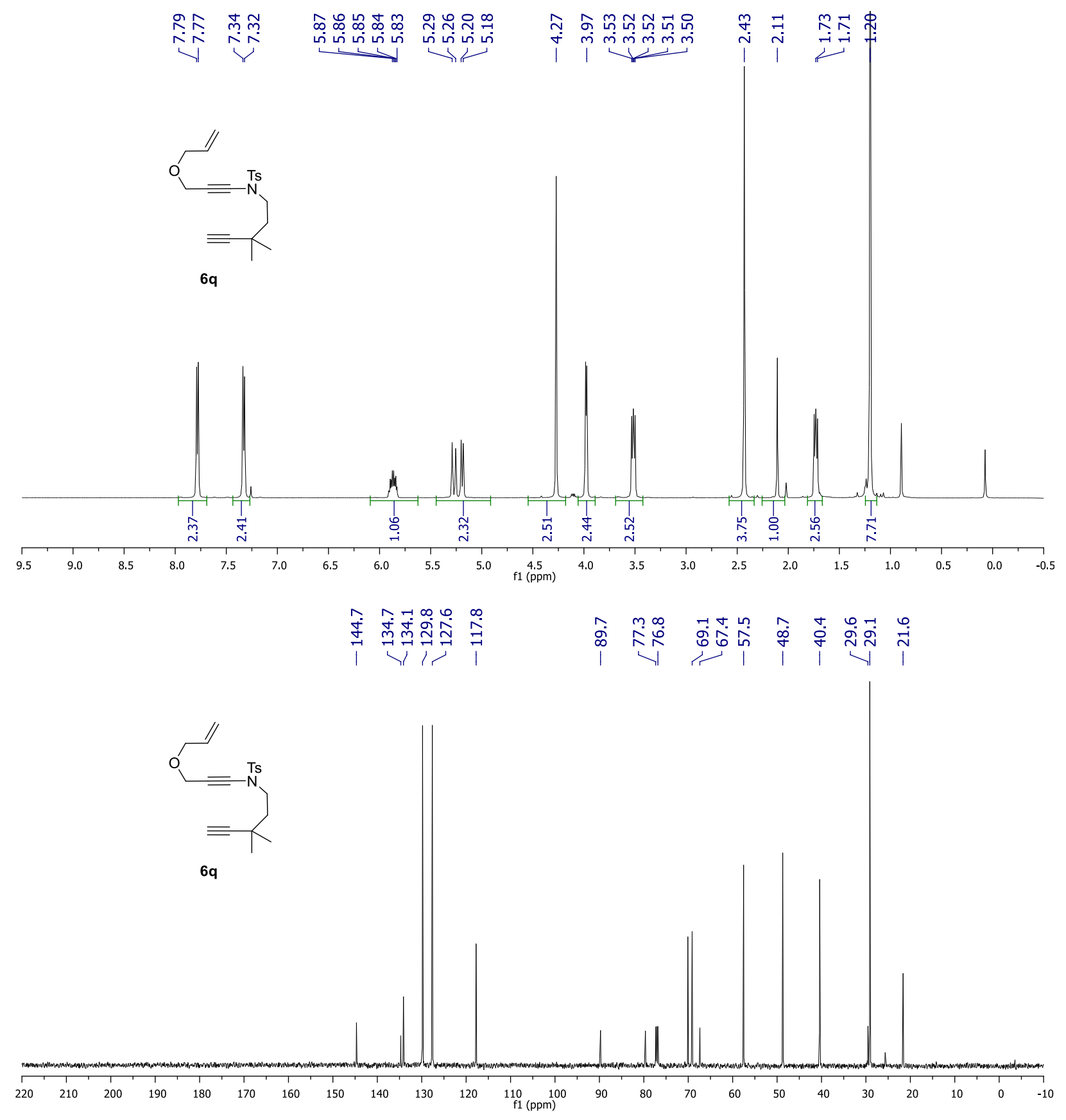



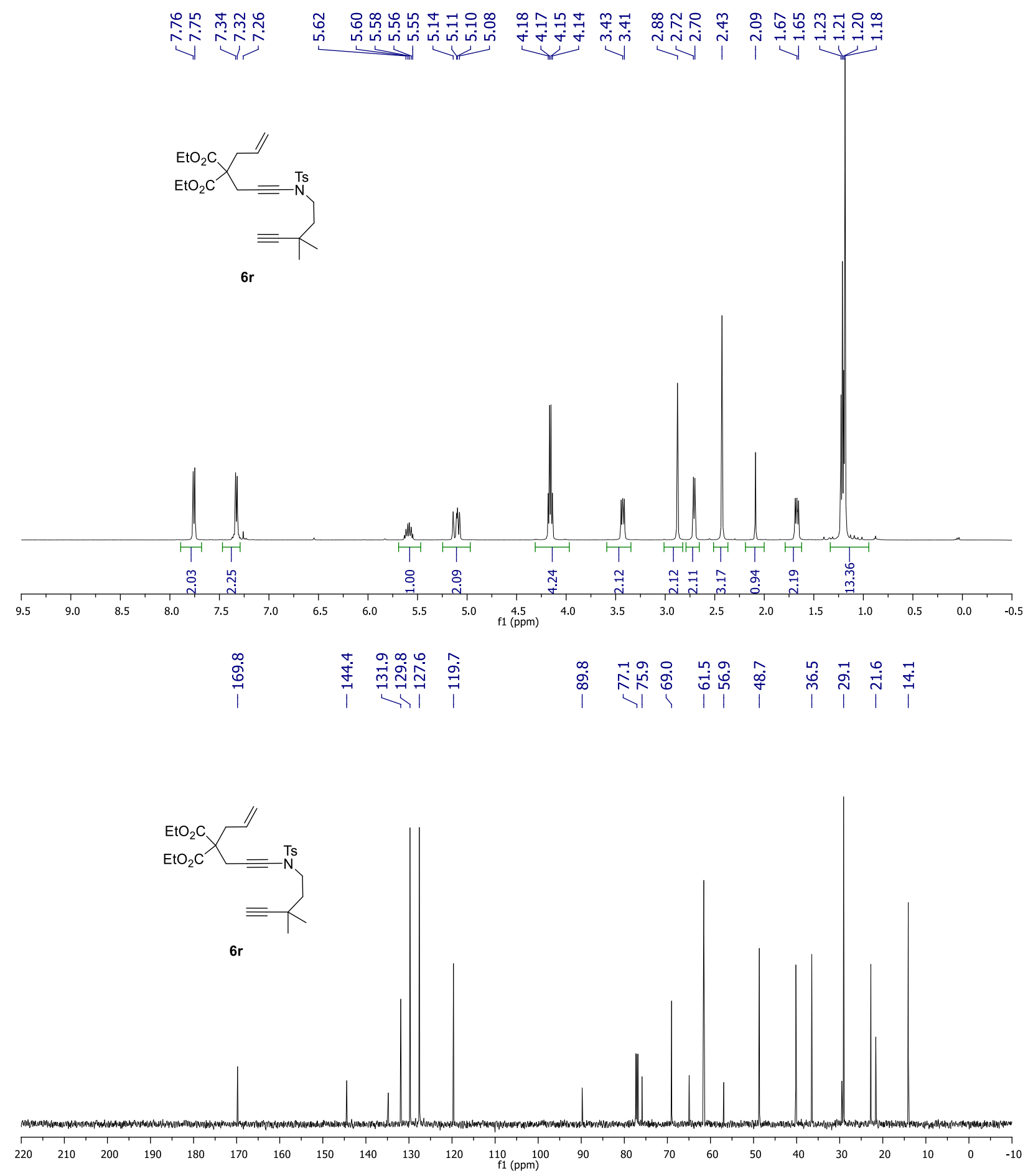


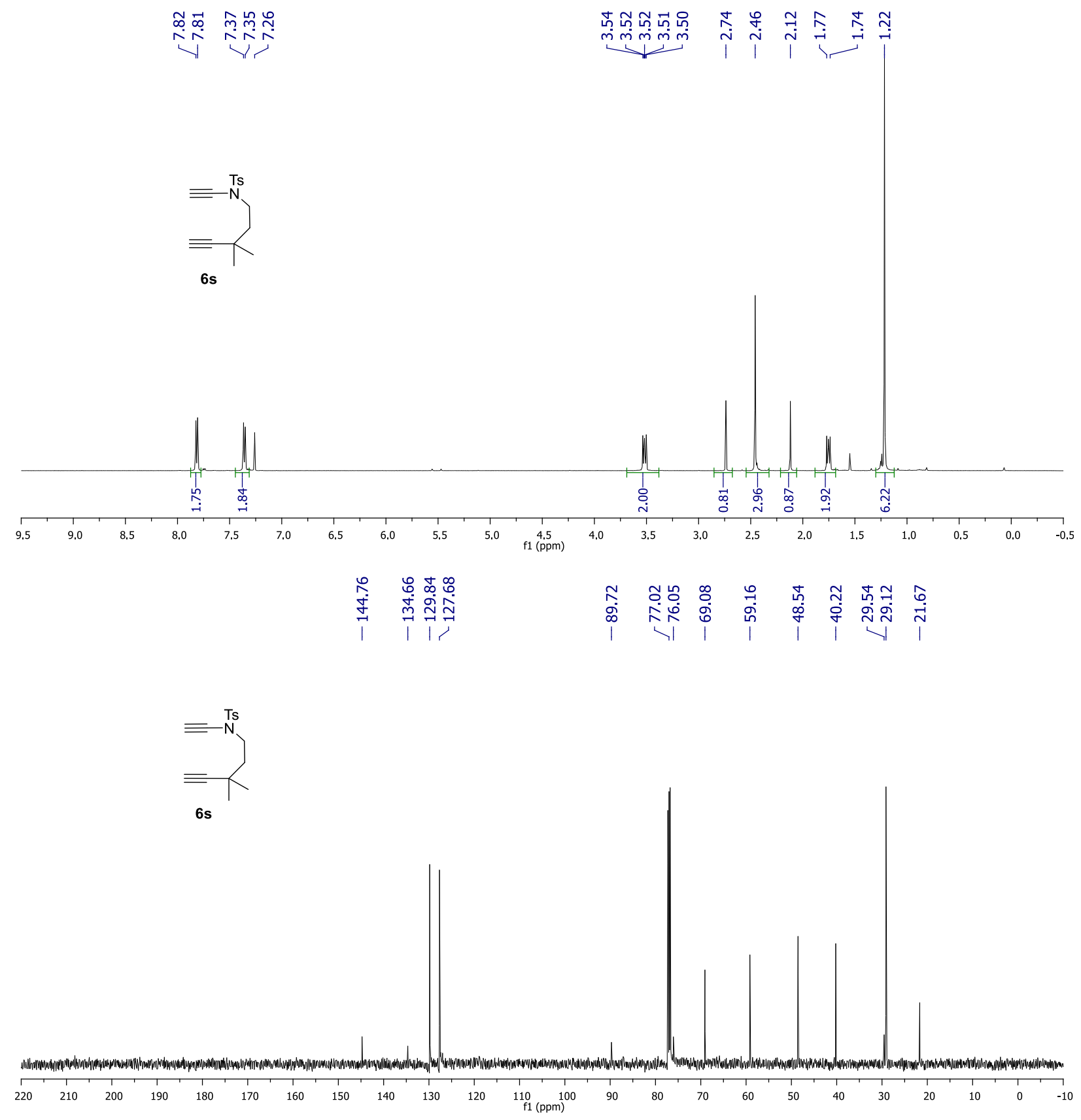




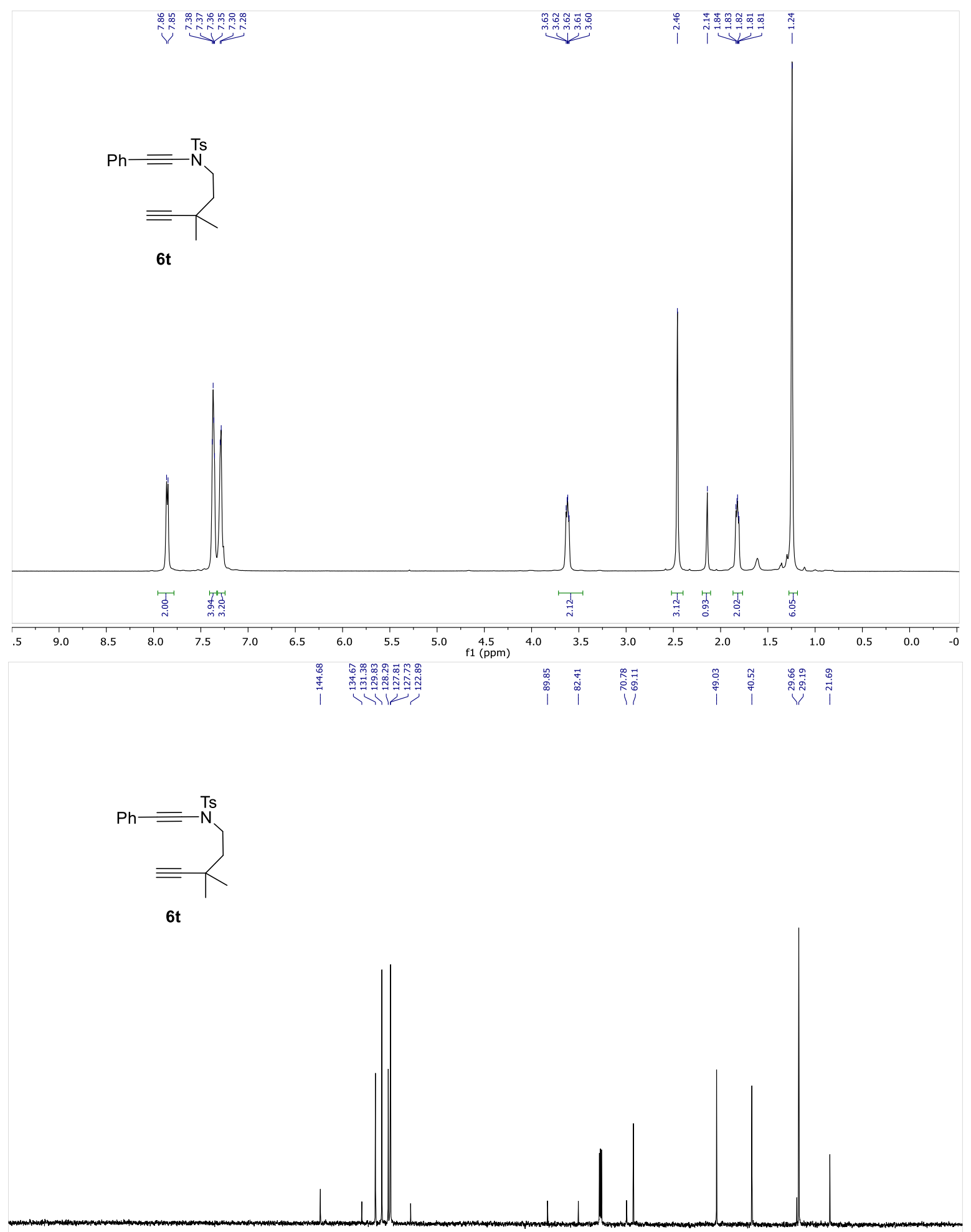

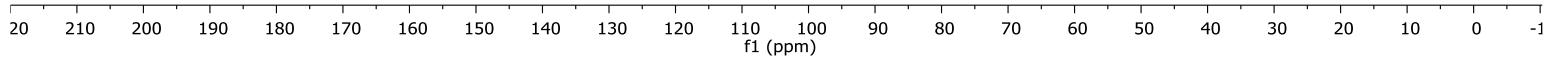



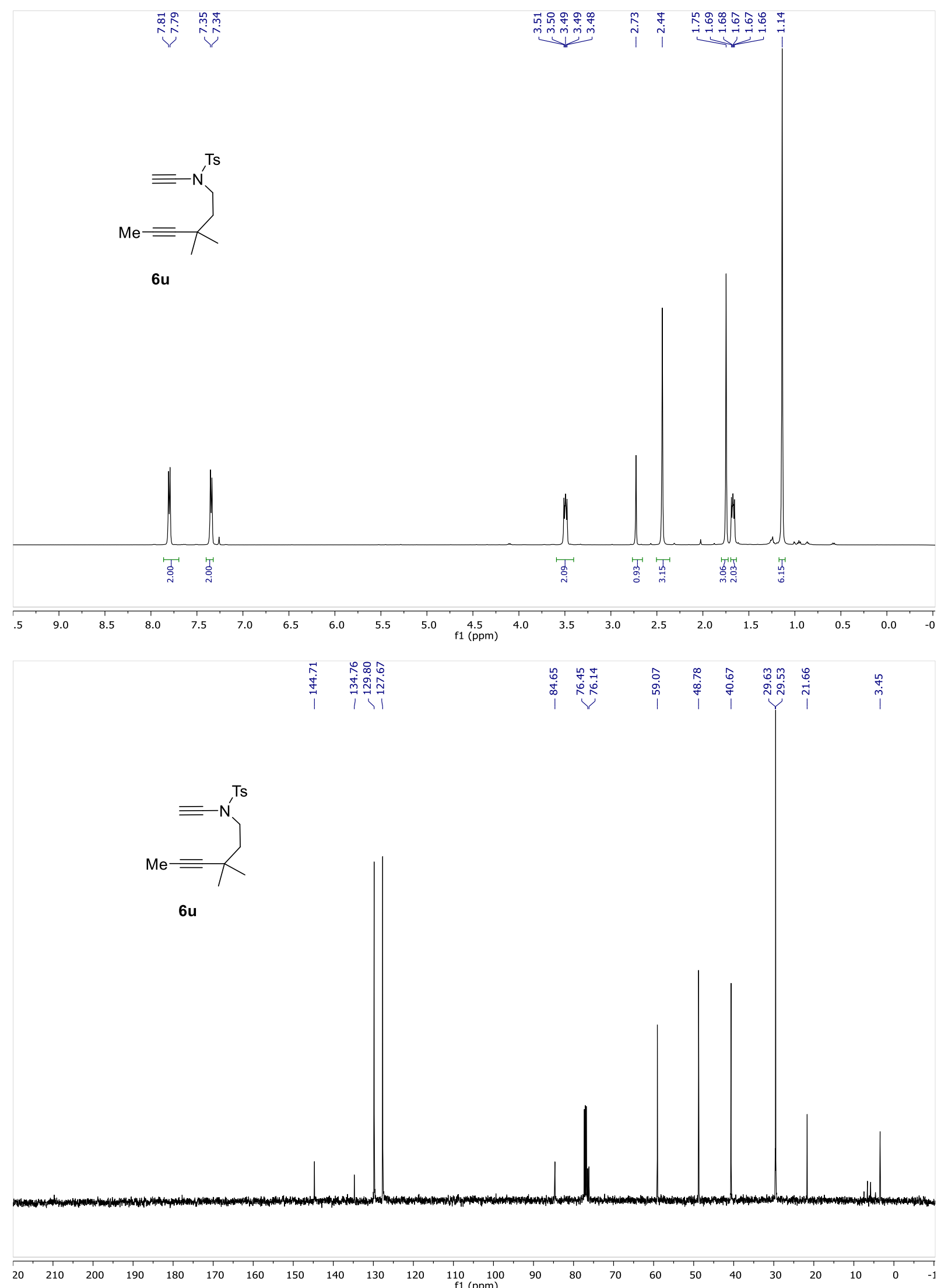

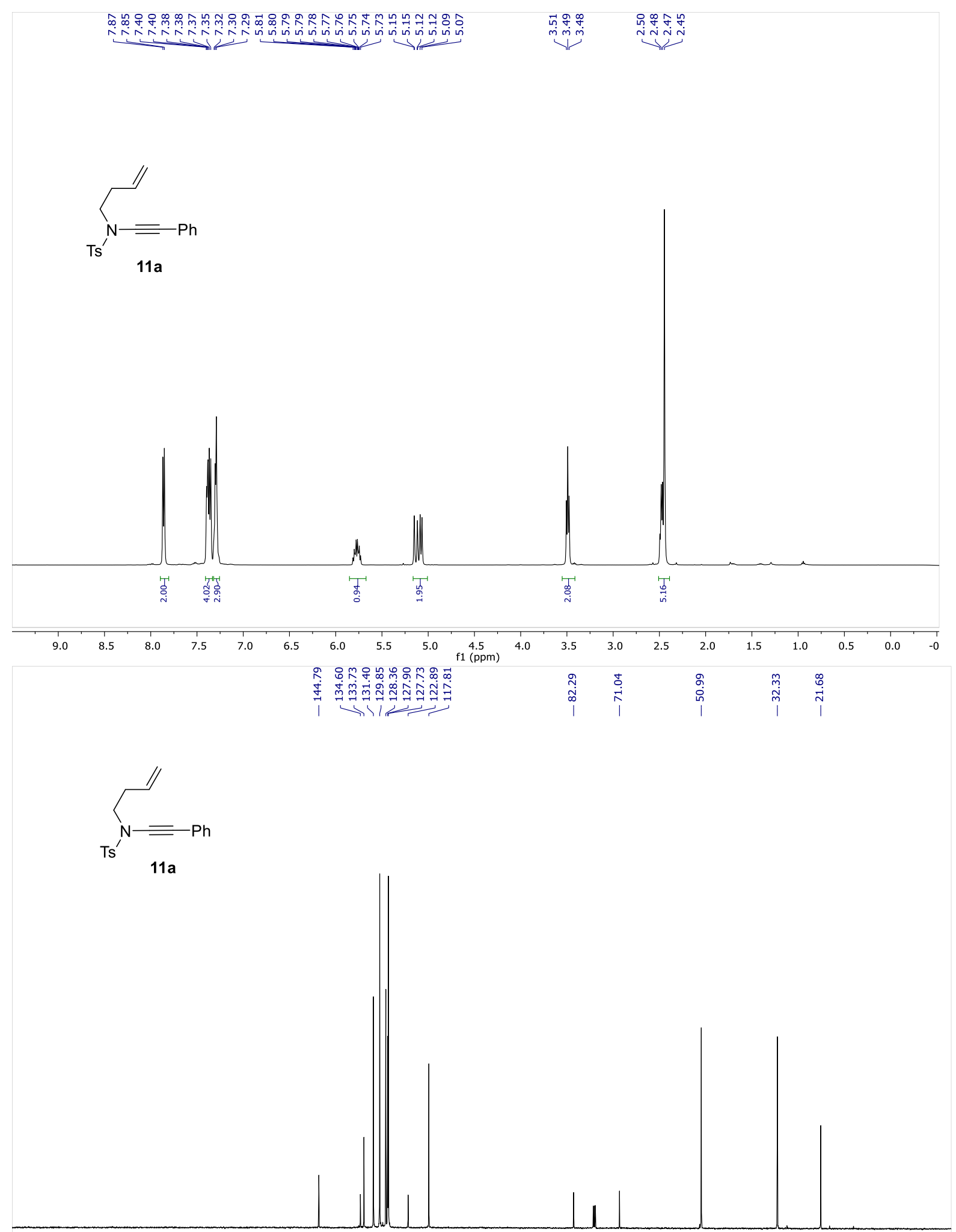

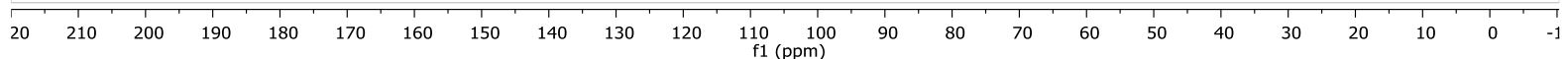




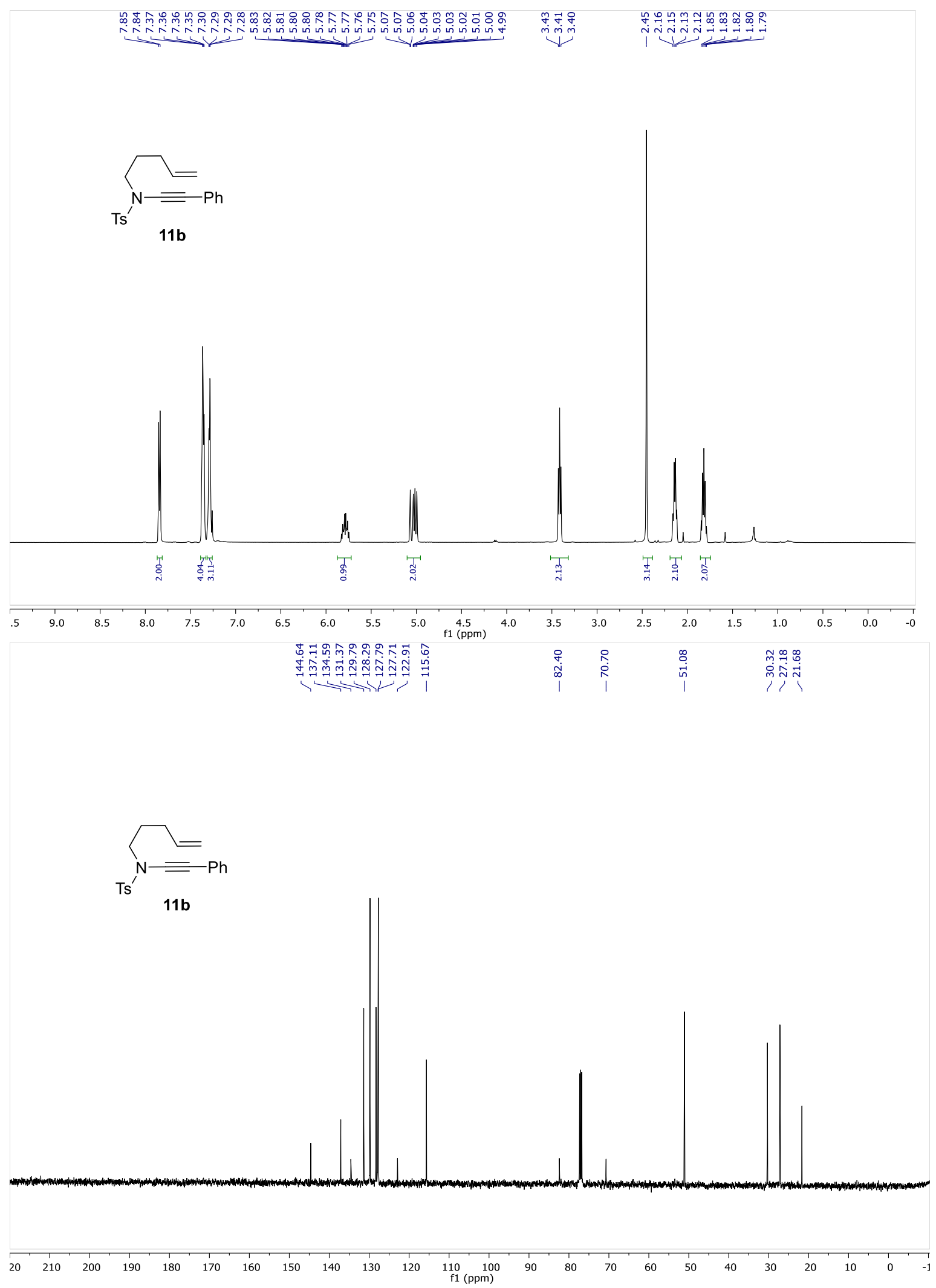




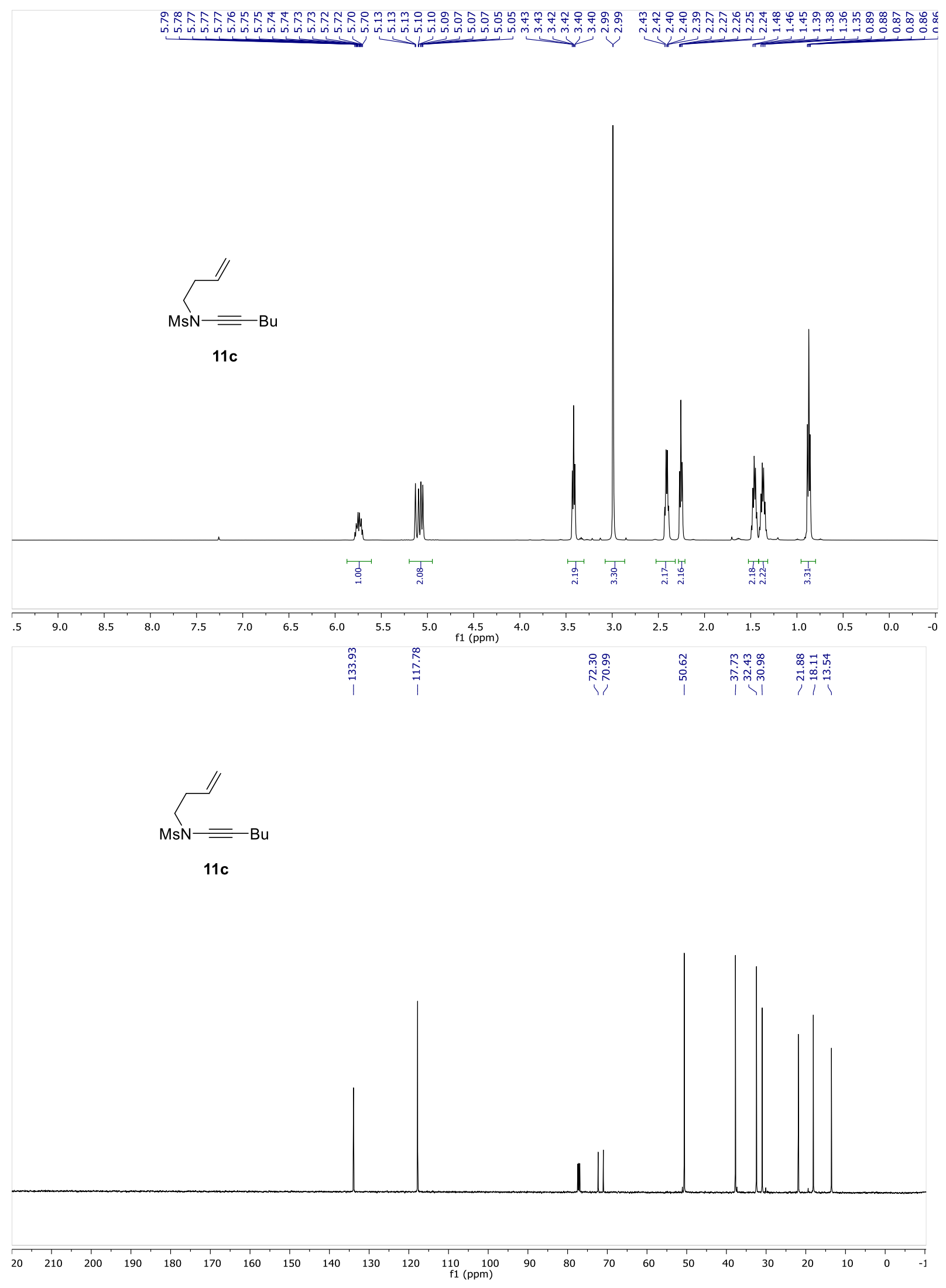




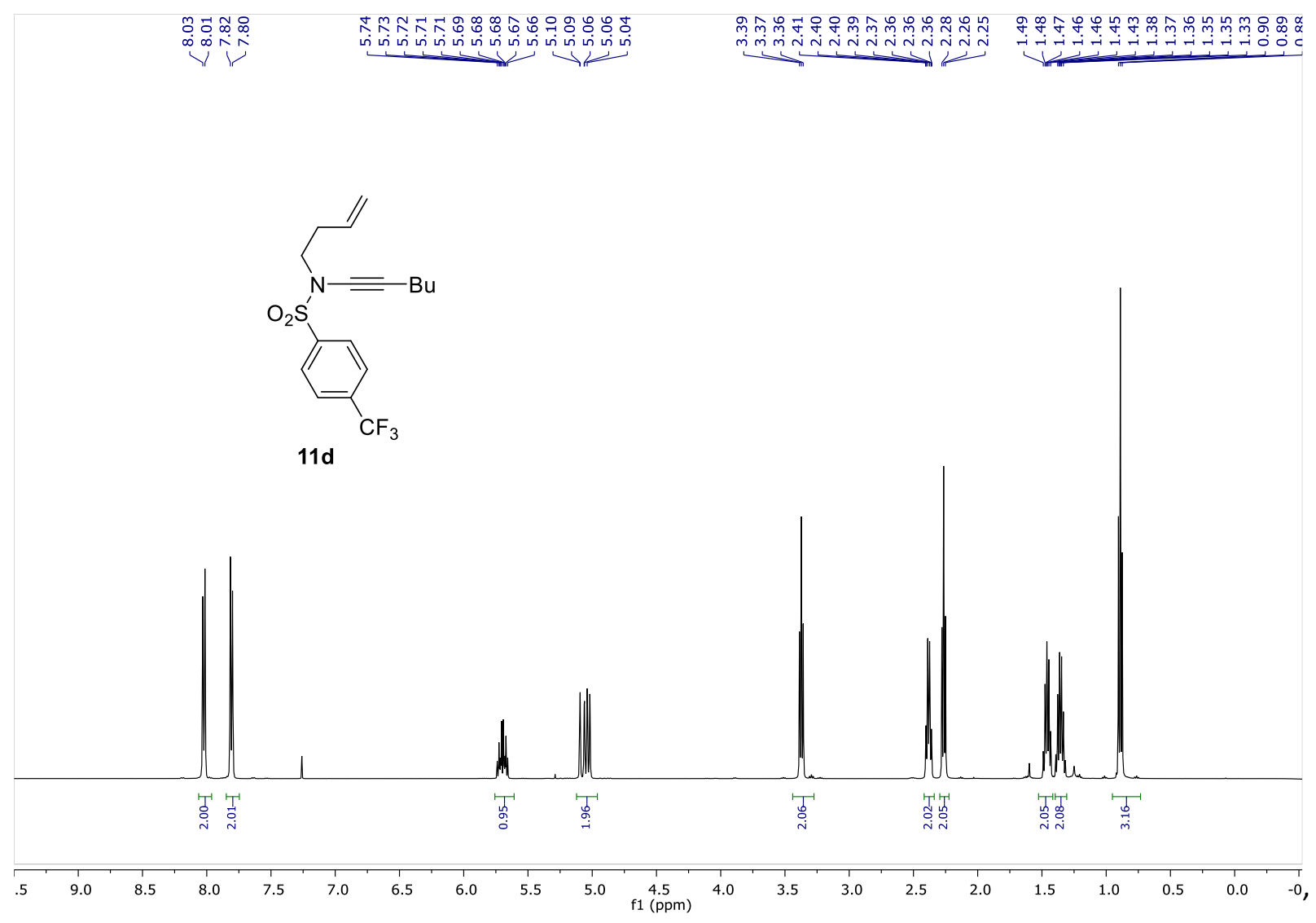

${ }^{19} \mathrm{~F}$ NMR $\left(\mathrm{CDCl}_{3}, 376 \mathrm{MHz}\right)$

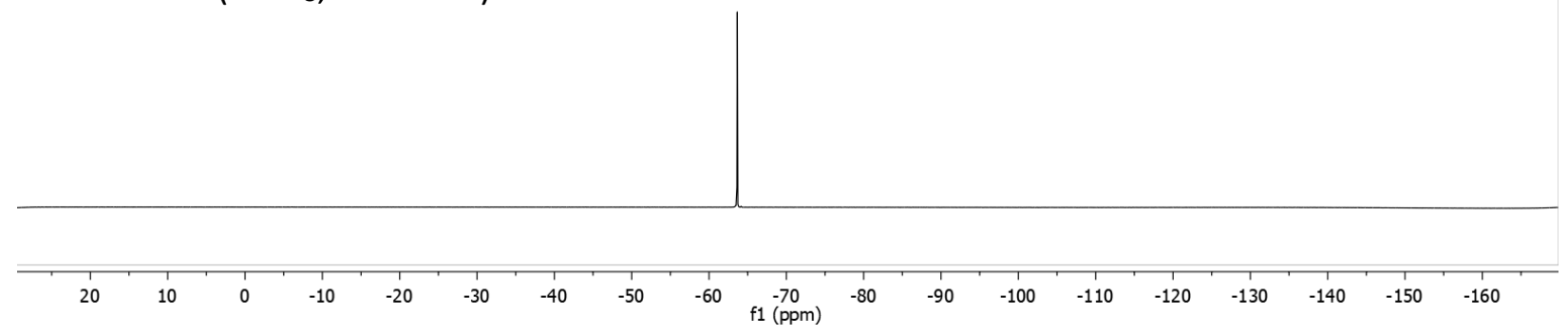




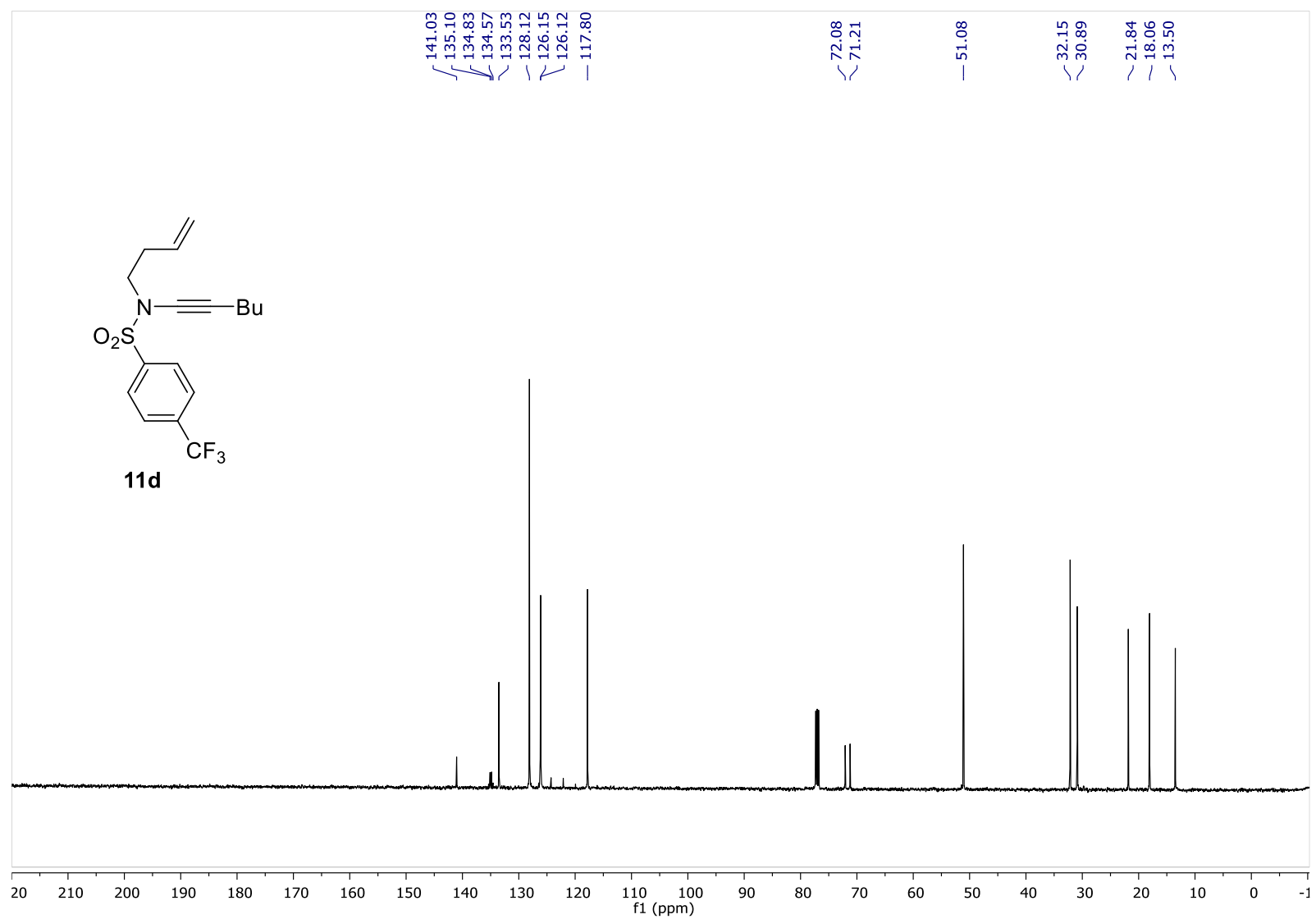



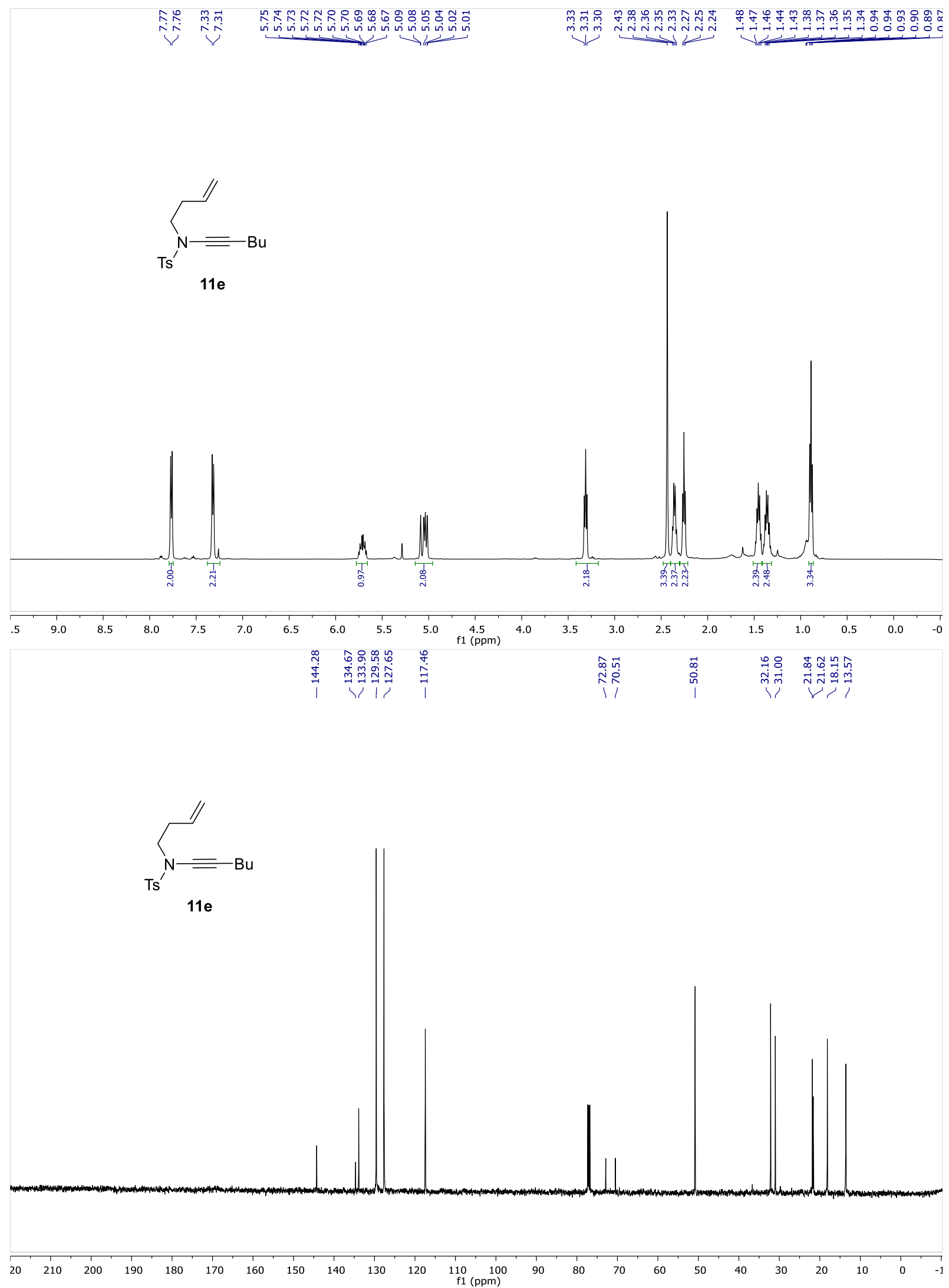


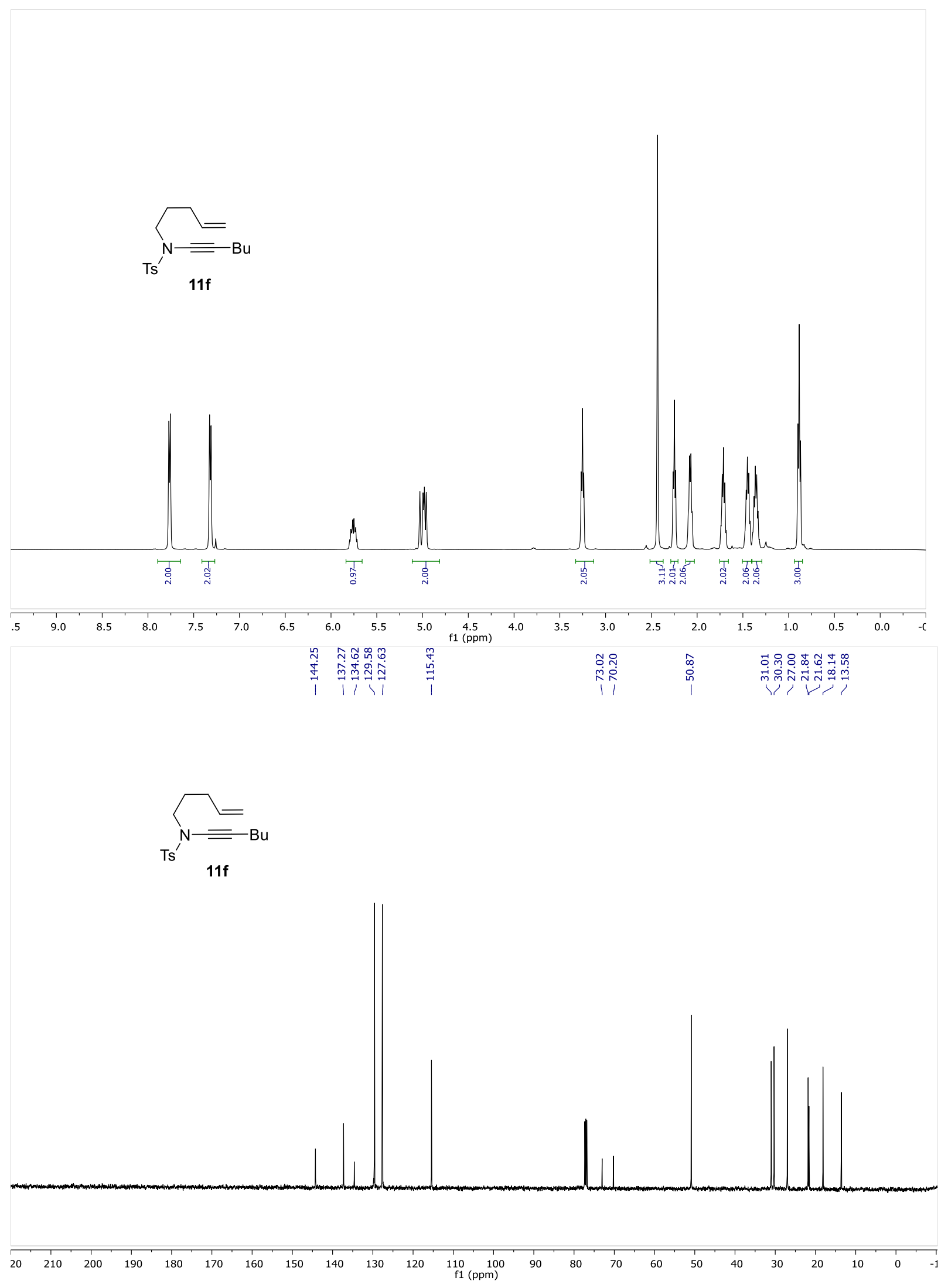



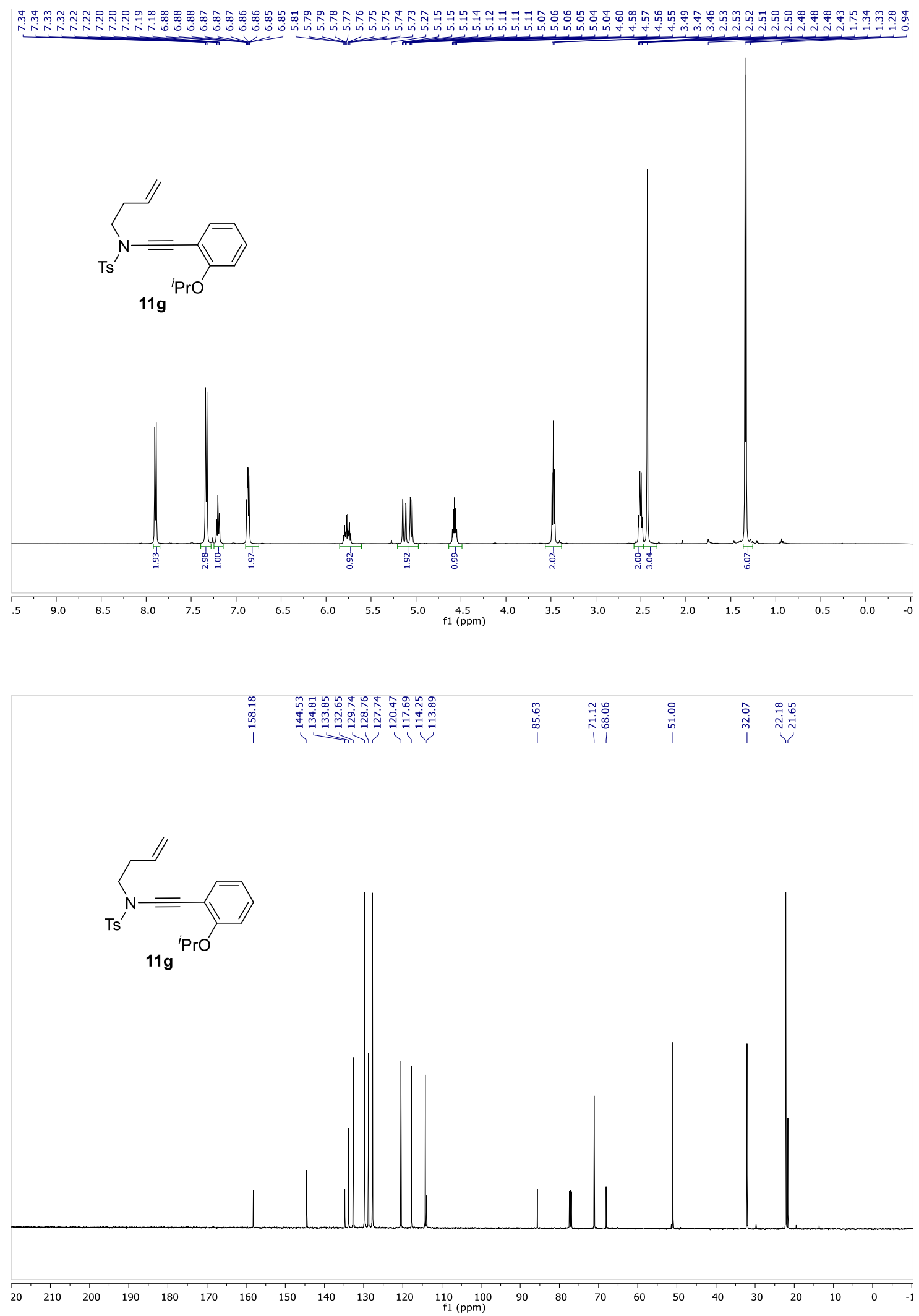

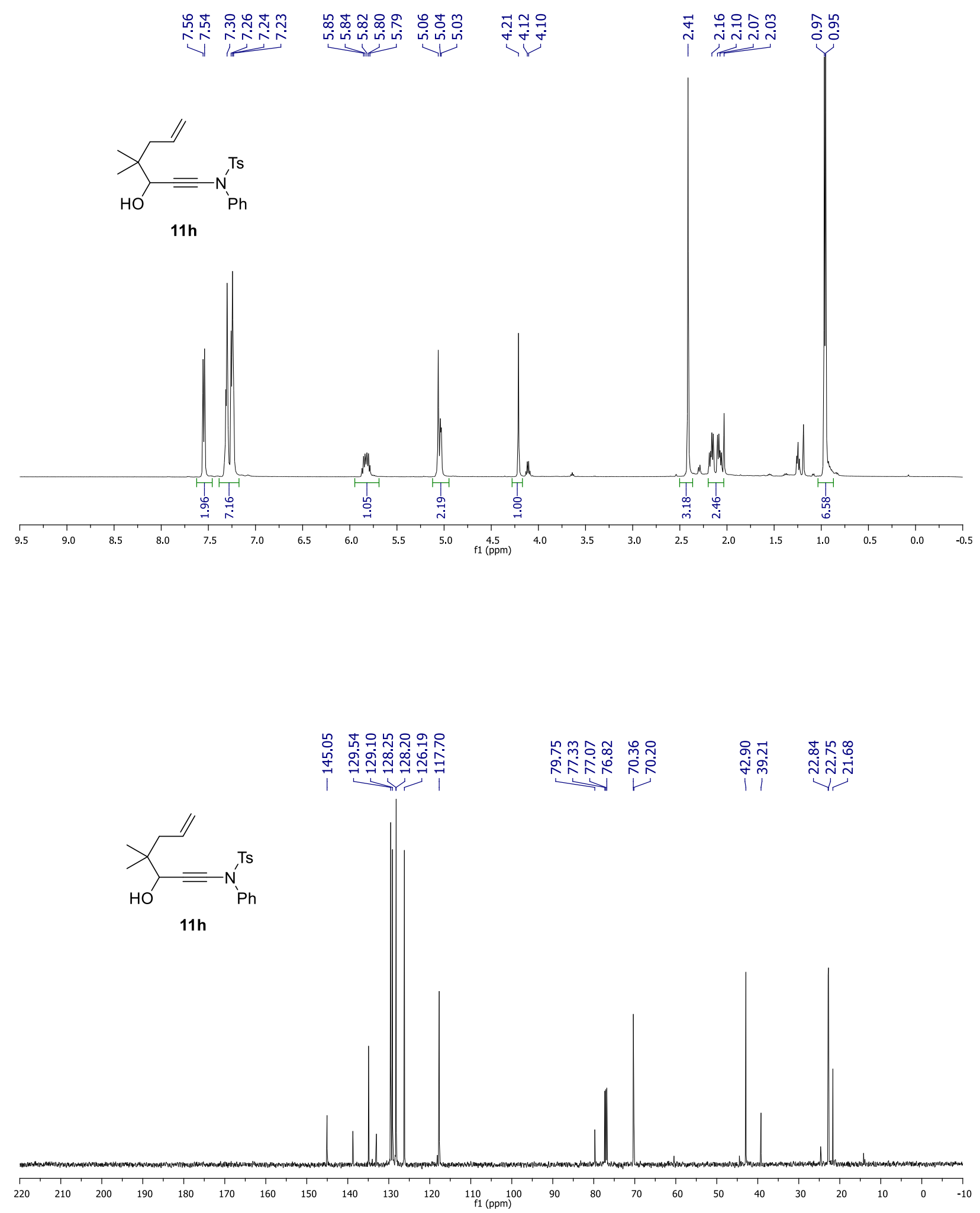

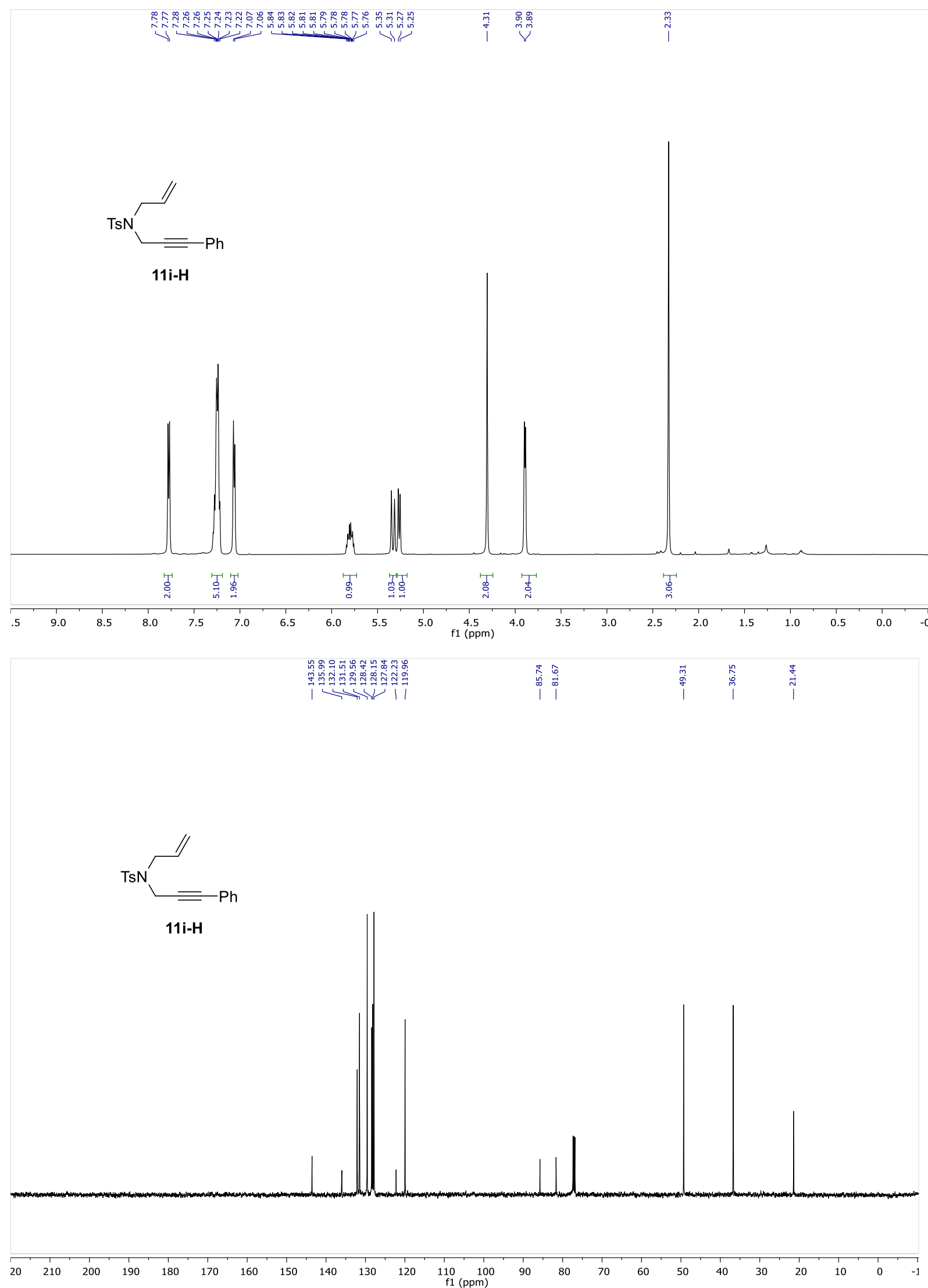


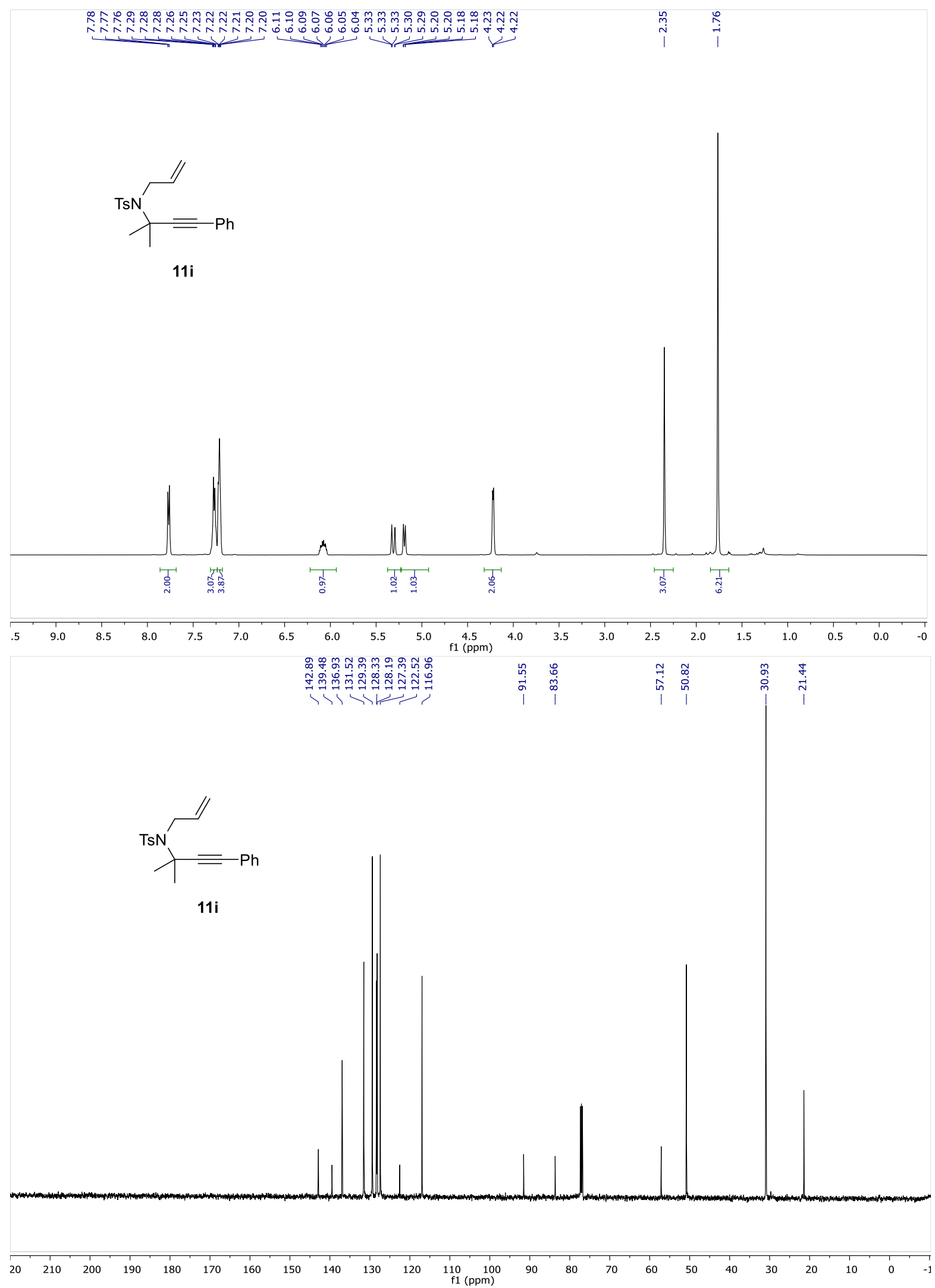




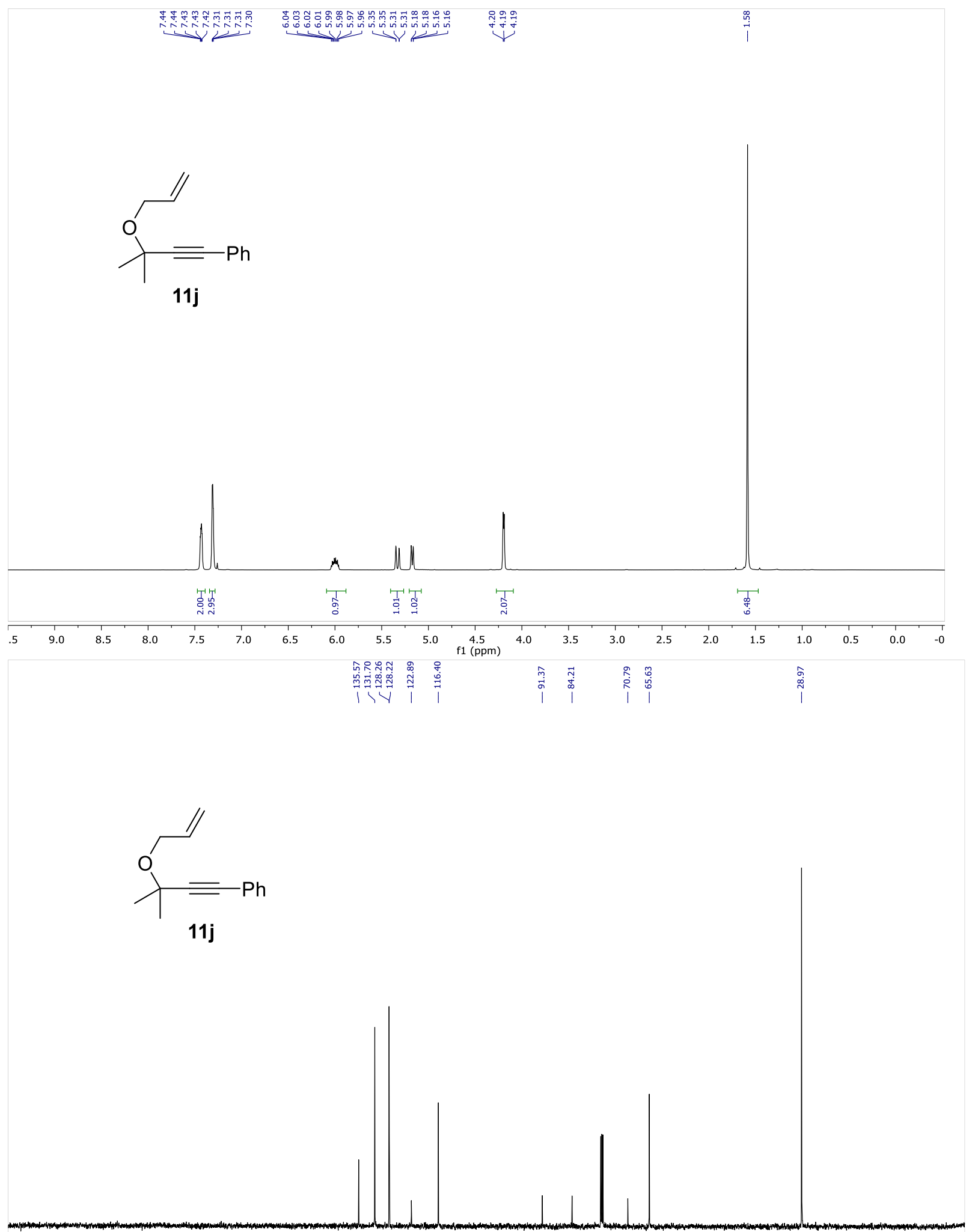

\begin{tabular}{|llllllllllllllllllllllll}
\hline 20 & 210 & 200 & 190 & 180 & 170 & 160 & 150 & 140 & 130 & 120 & 110 & 100 & 90 & 80 & 70 & 60 & 50 & 40 & 30 & 20 & 10 & 0 & -1
\end{tabular} 


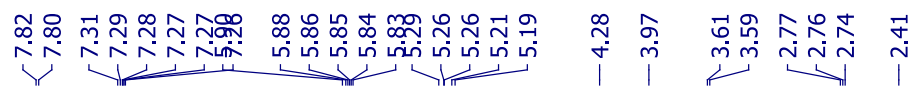
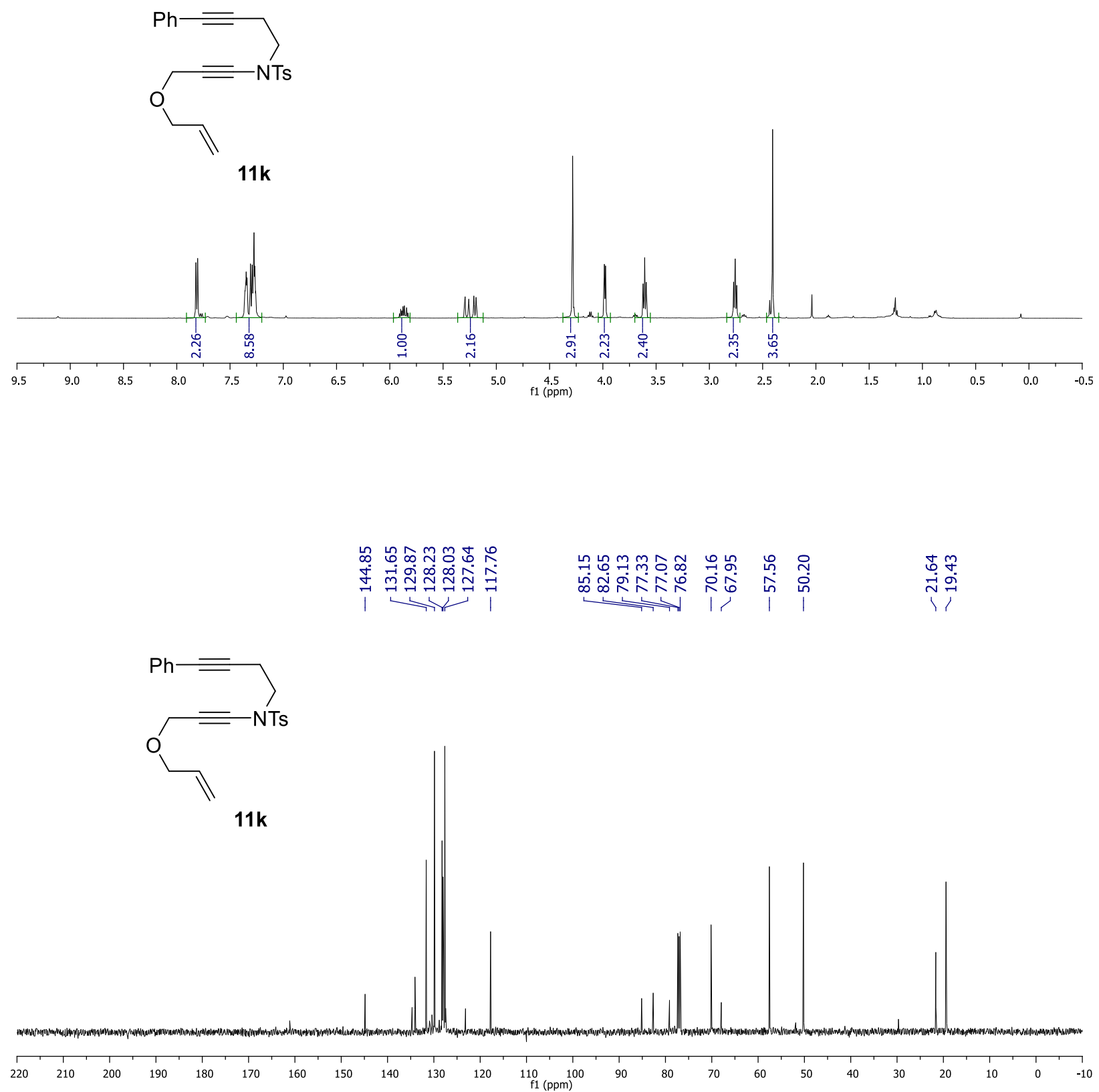


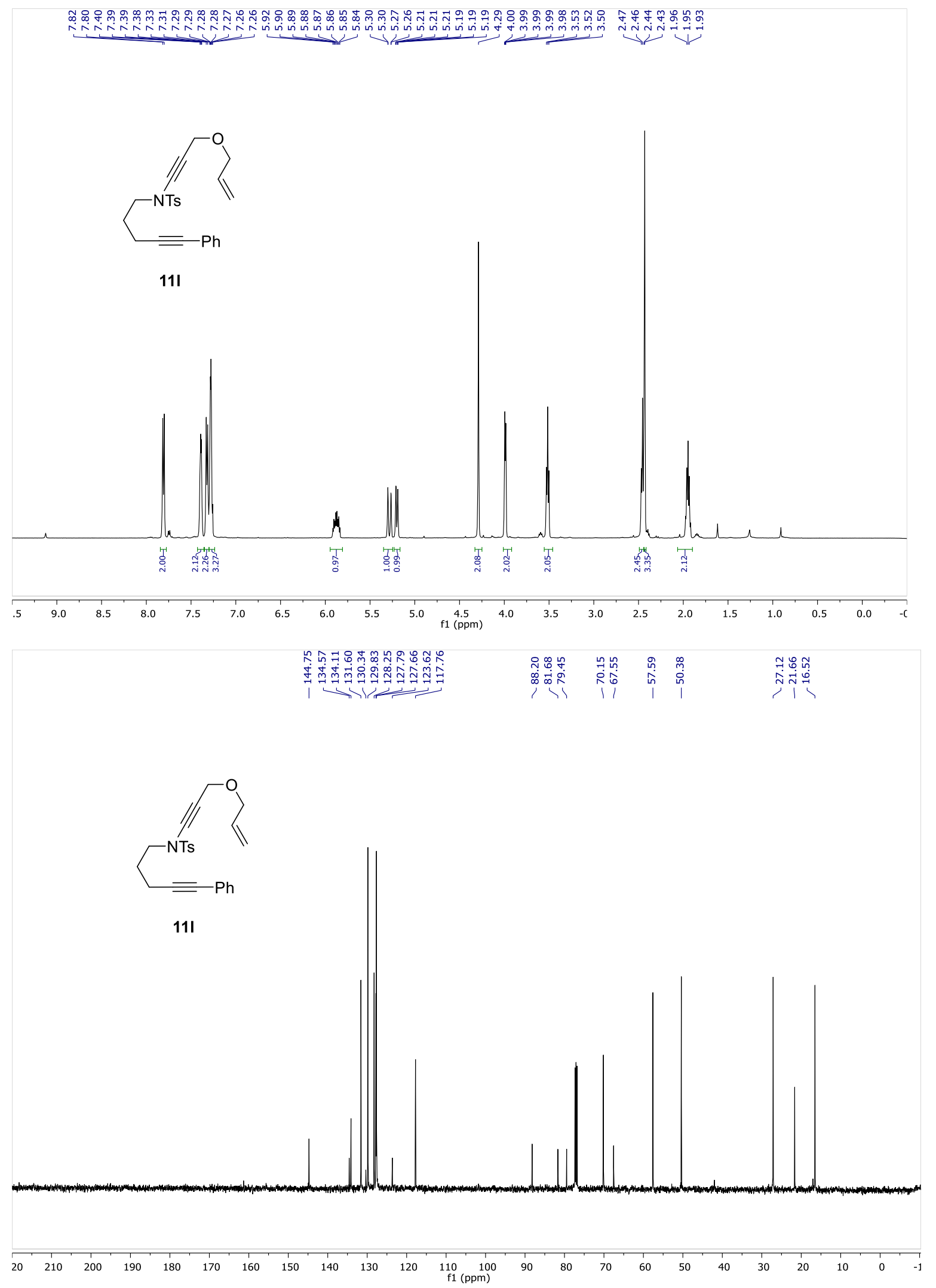




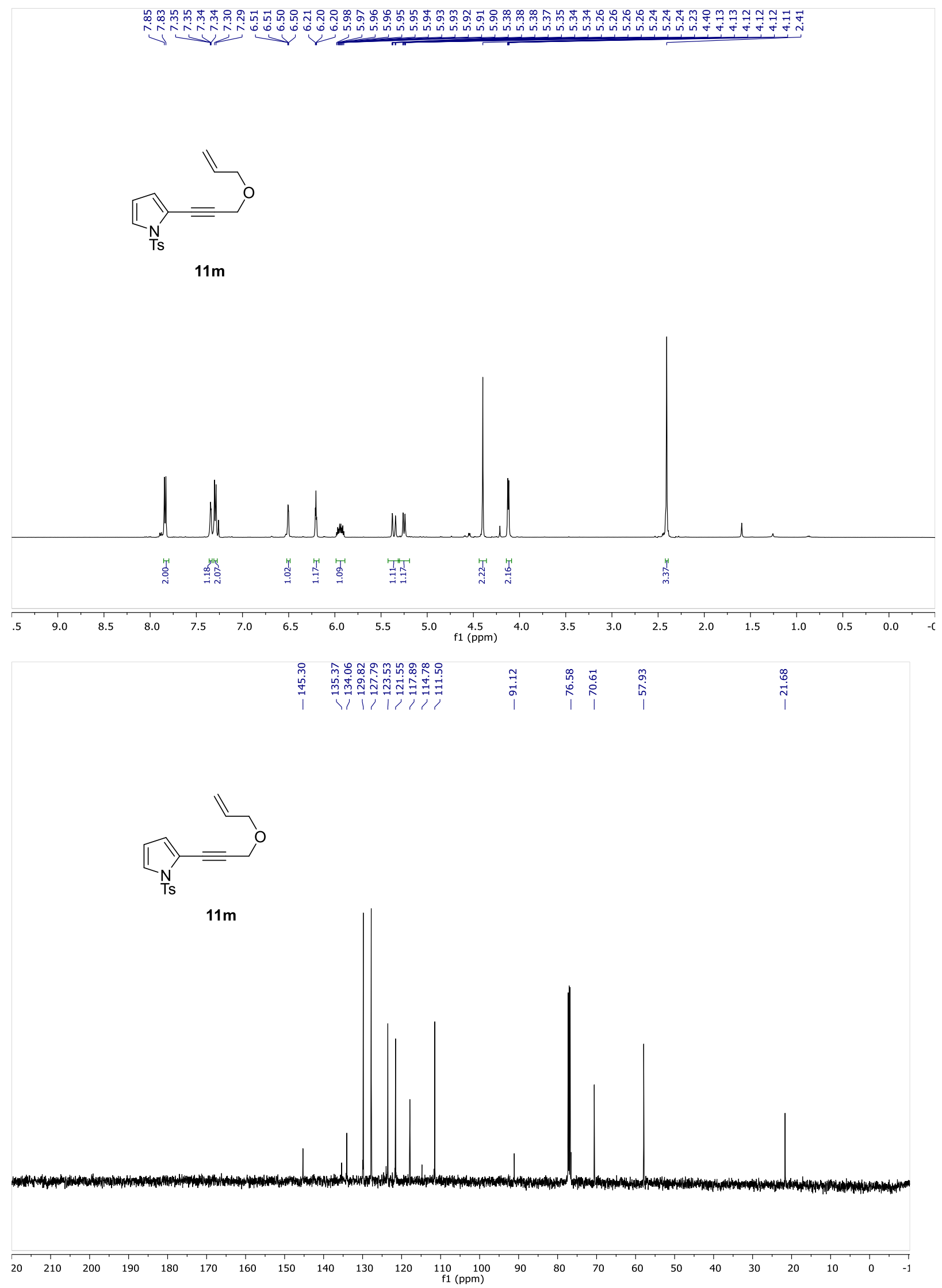




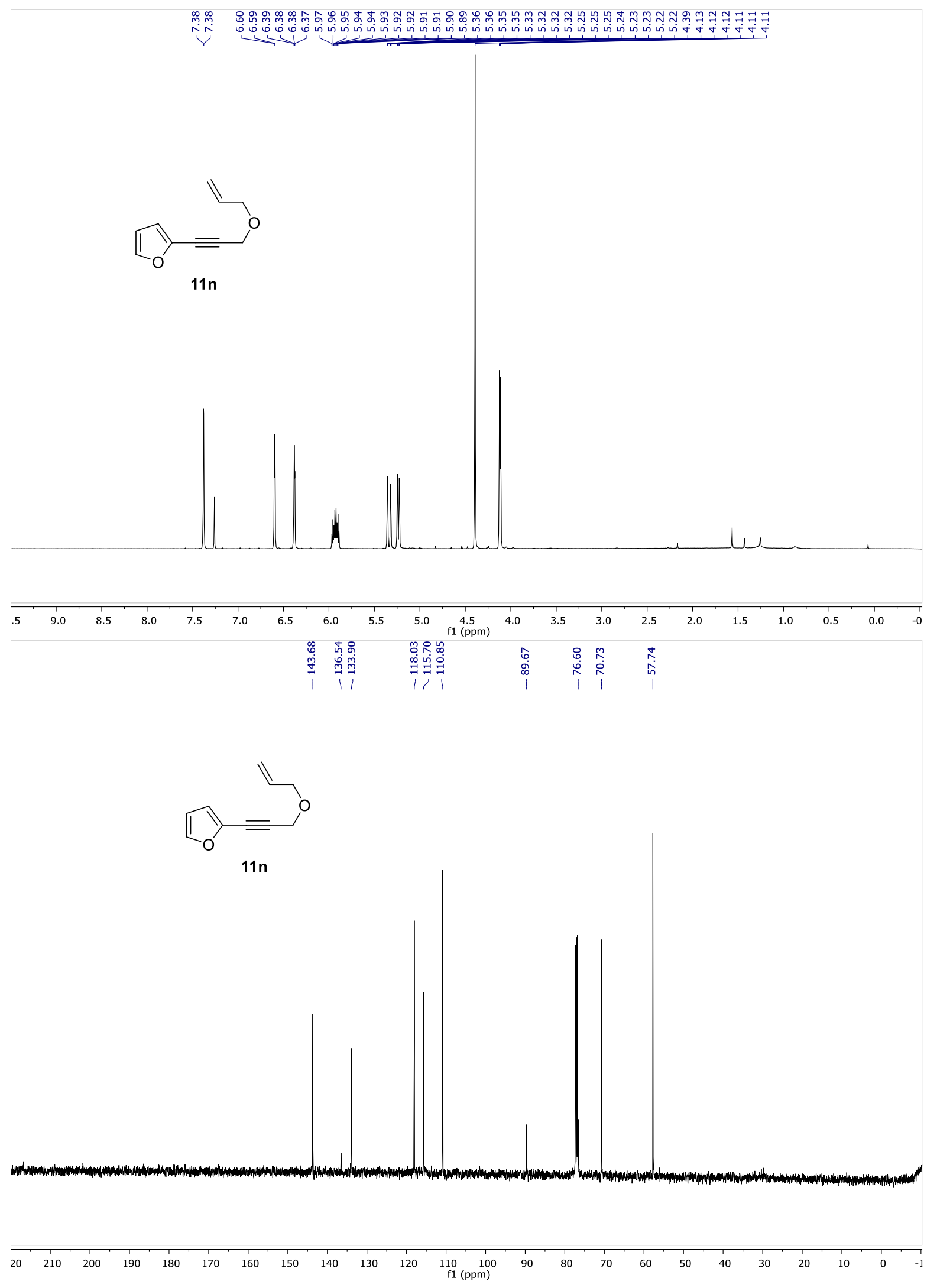




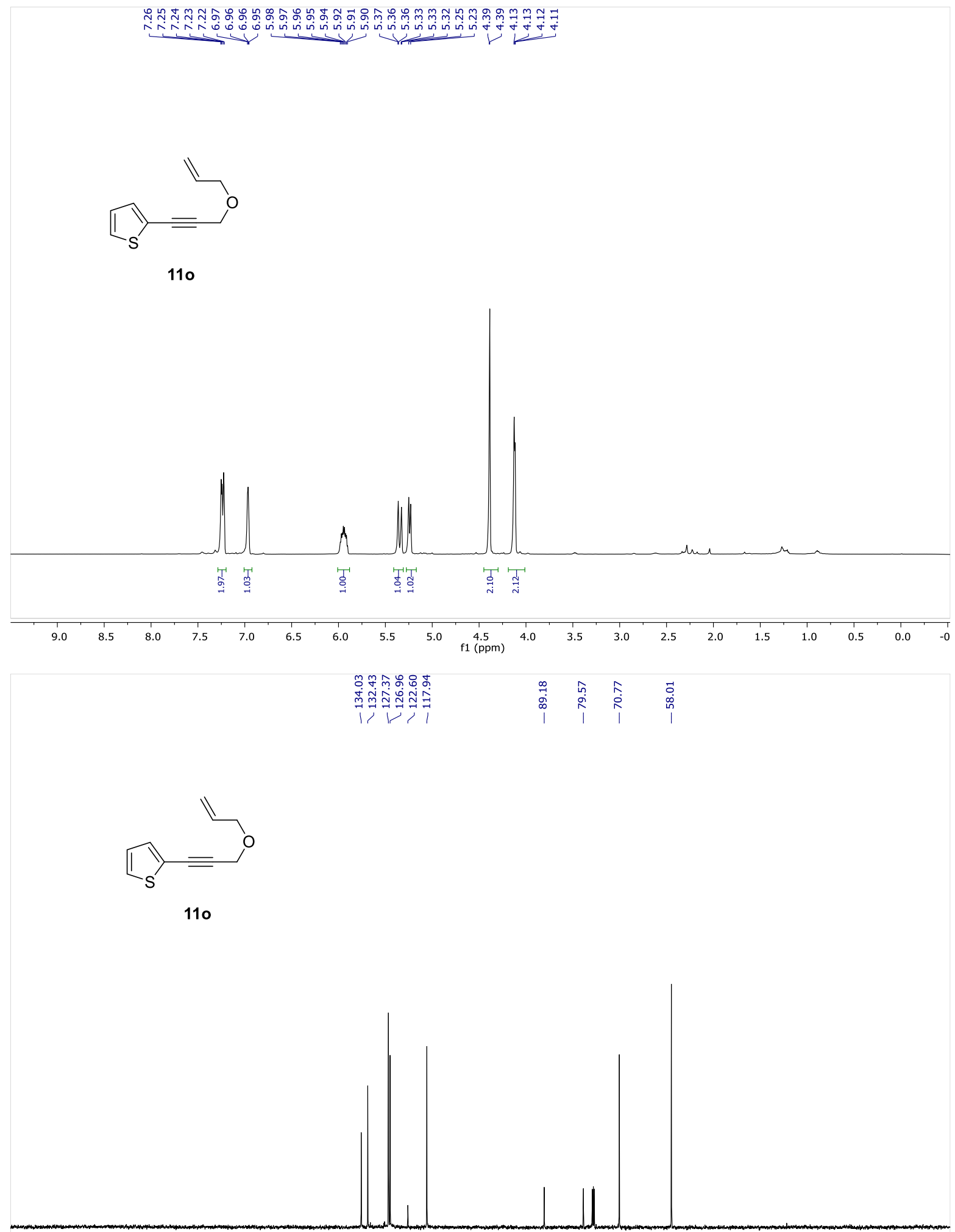

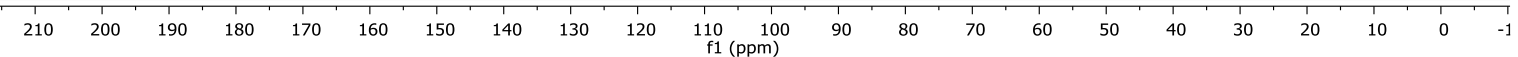



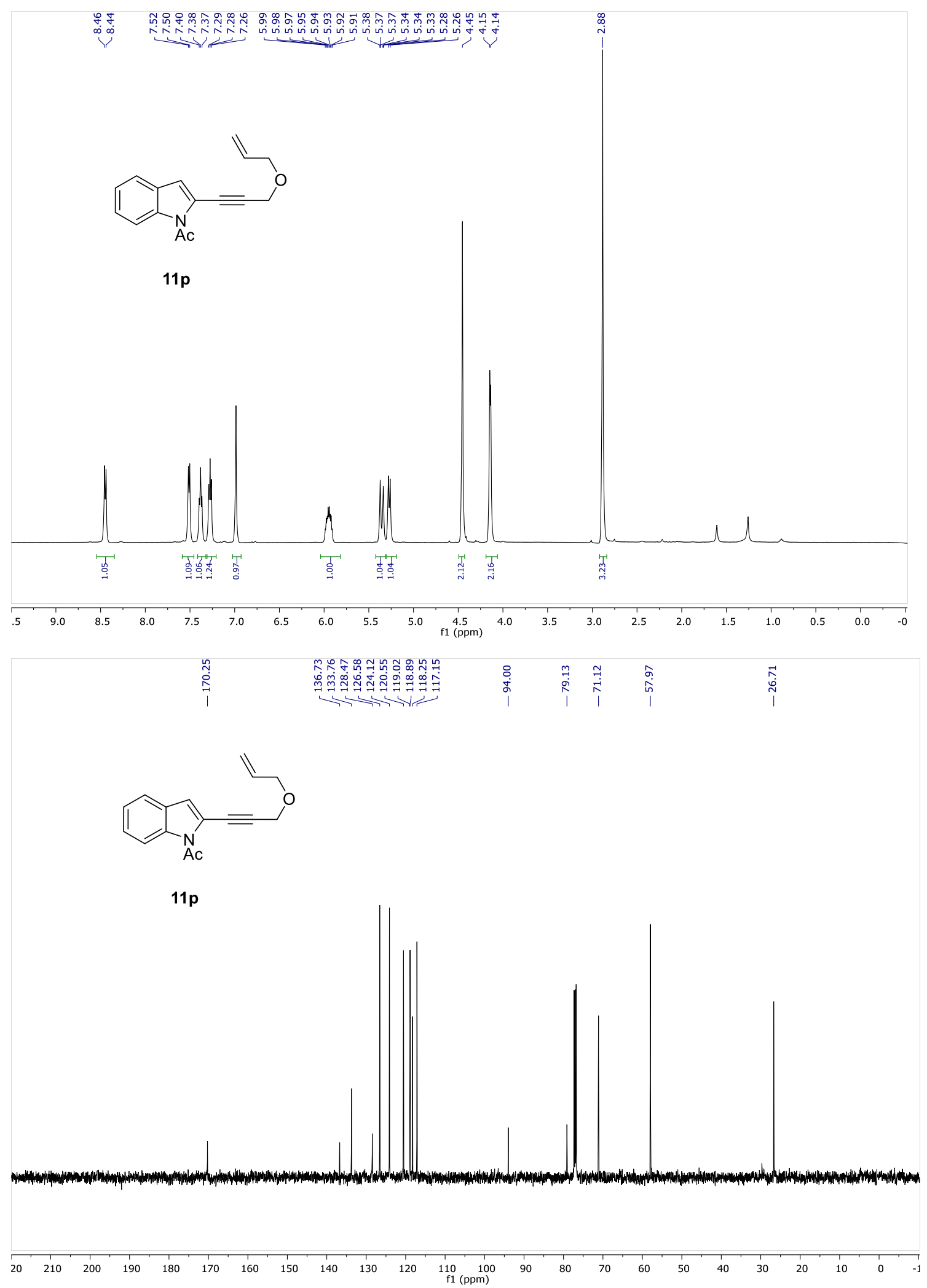


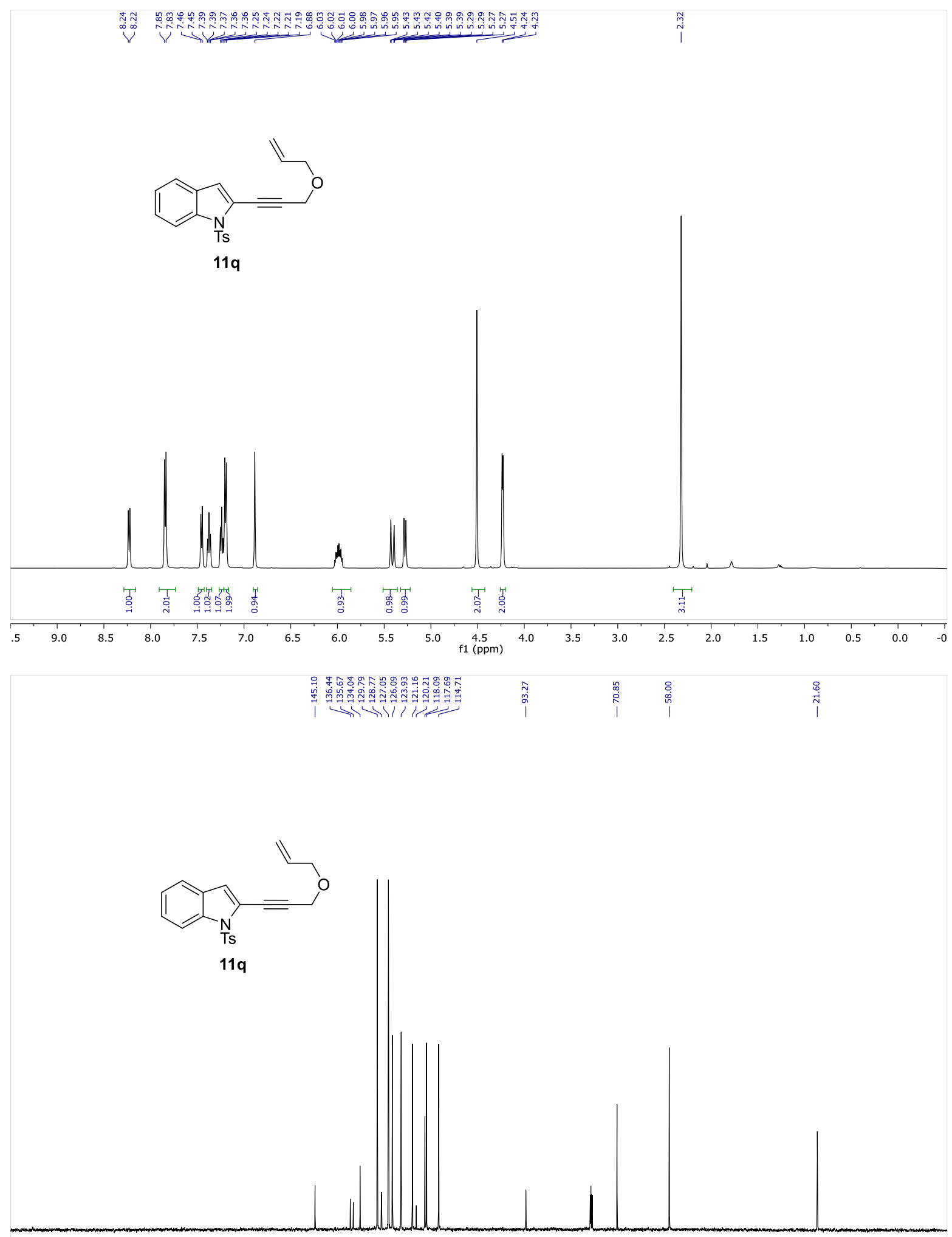

\begin{tabular}{|l|llllllllllllllllllllllll}
\hline 20 & 210 & 200 & 190 & 180 & 170 & 160 & 150 & 140 & 130 & 120 & 110 & 100 & 90 & 80 & 70 & 60 & 50 & 40 & 30 & 20 & 10 & 0 & -1
\end{tabular} 


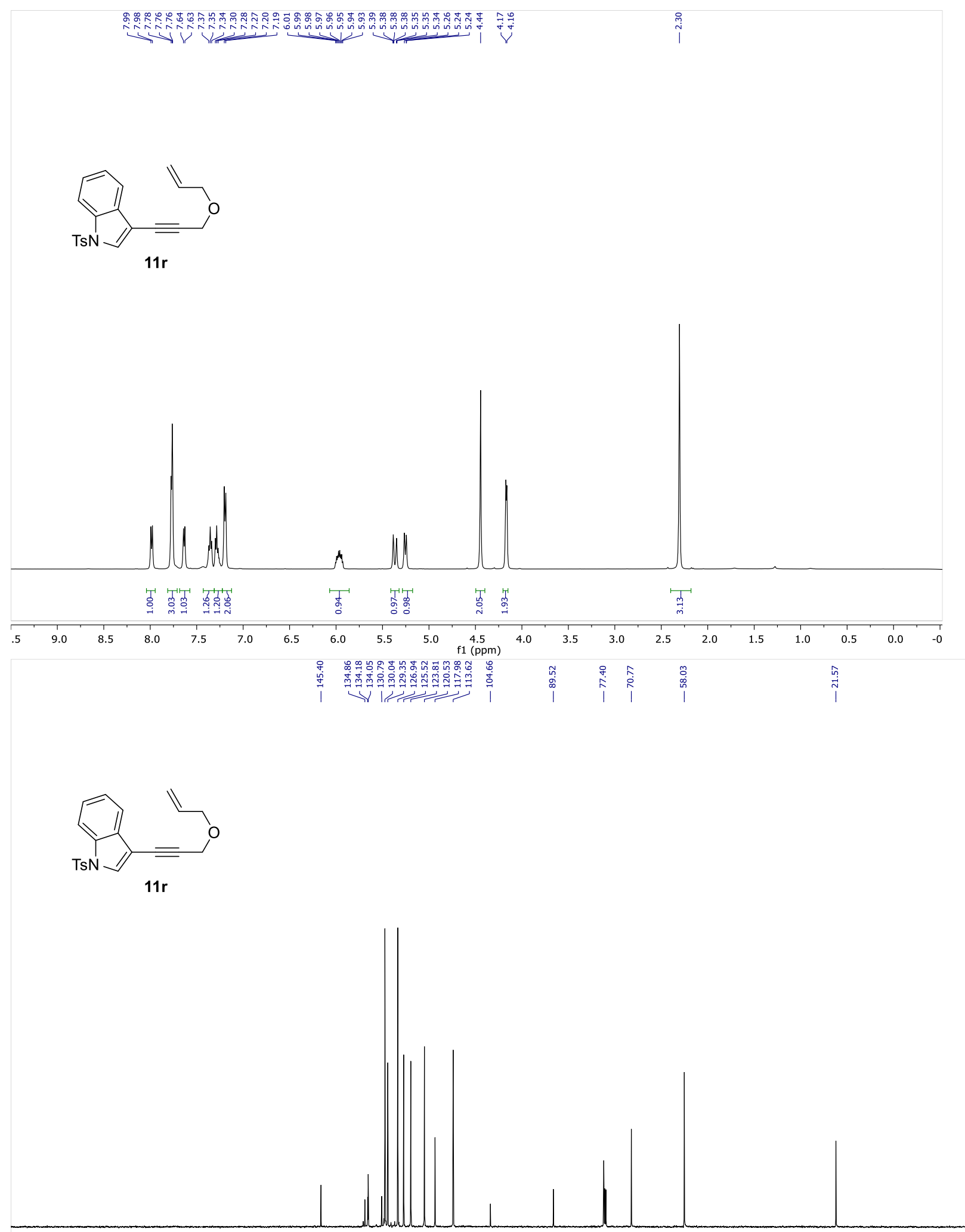

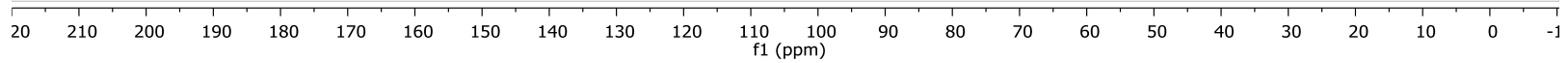




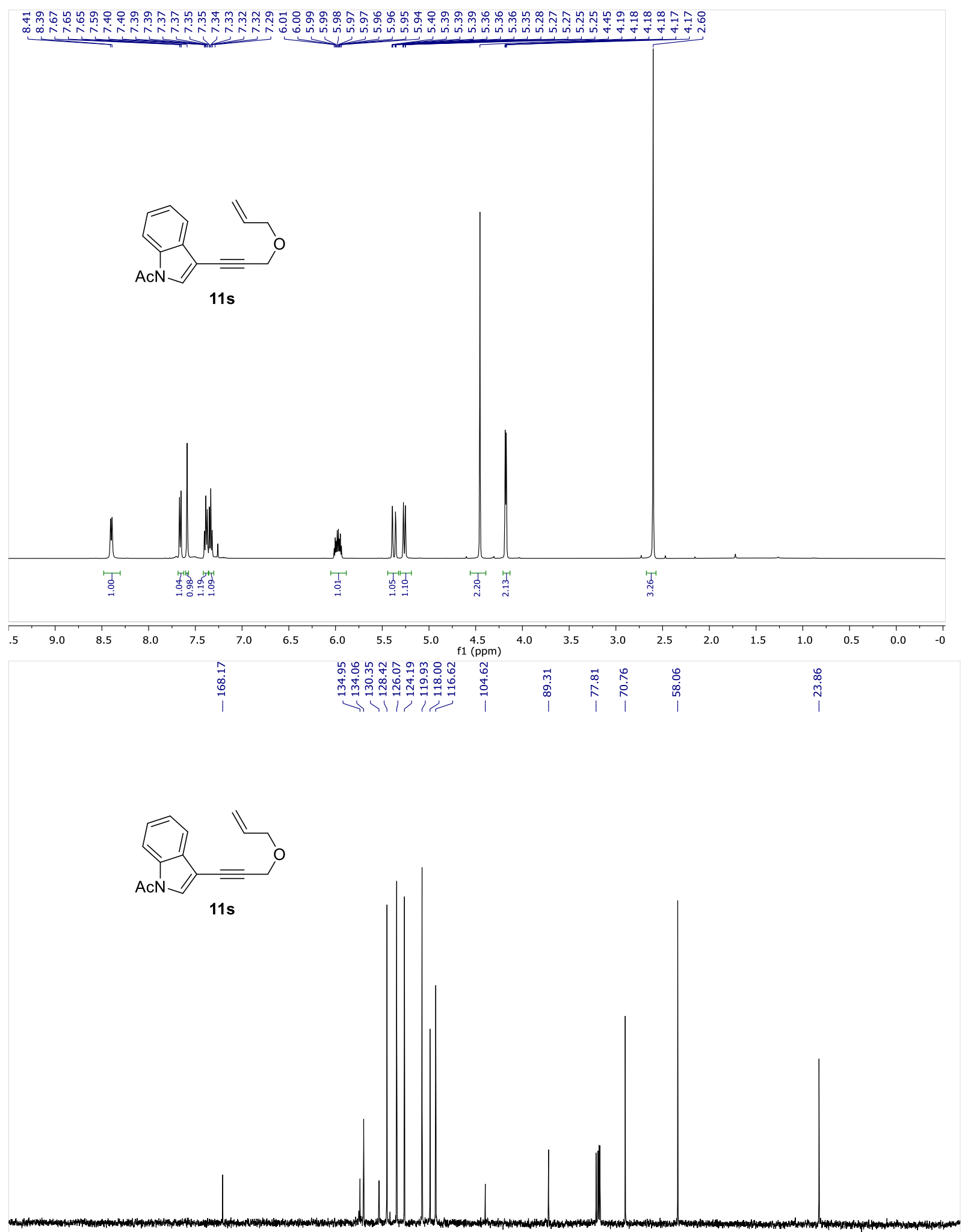

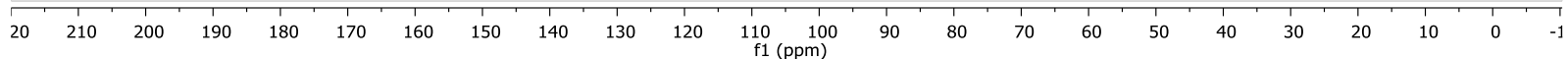



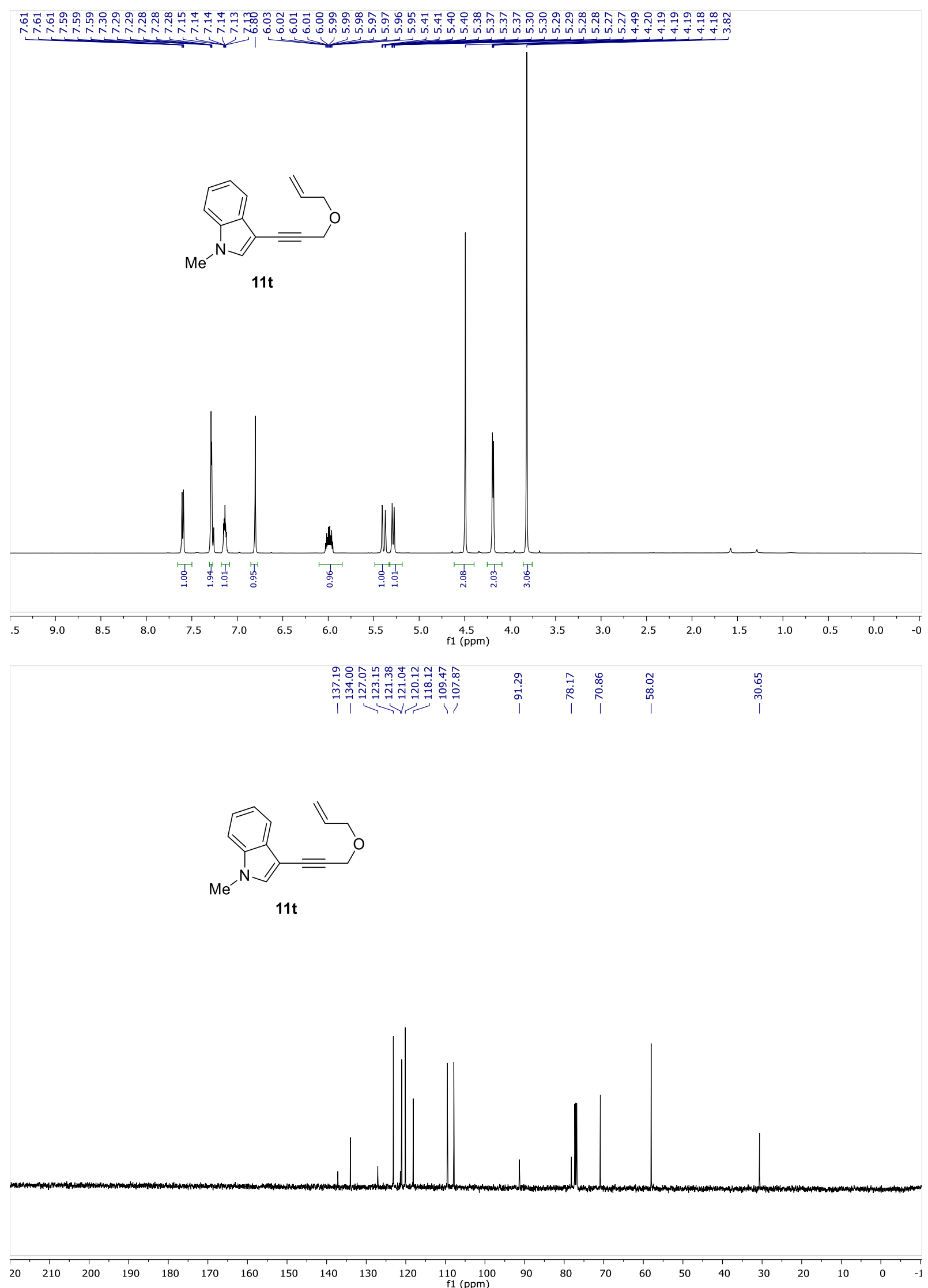

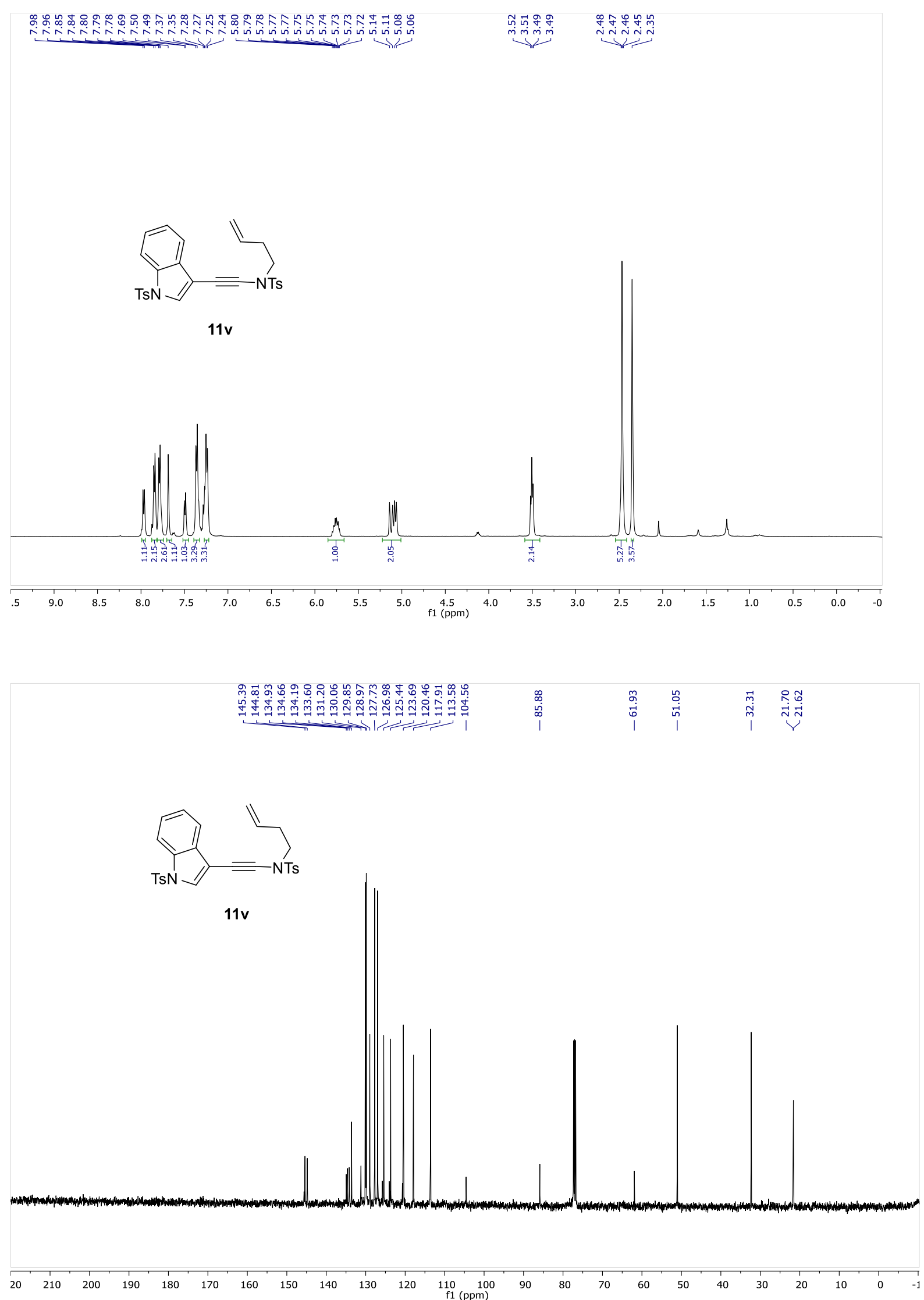


\section{${ }^{1} \mathrm{H},{ }^{13} \mathrm{C}$ NMR Spectra of Alkene-Chelates and Other Byproducts}

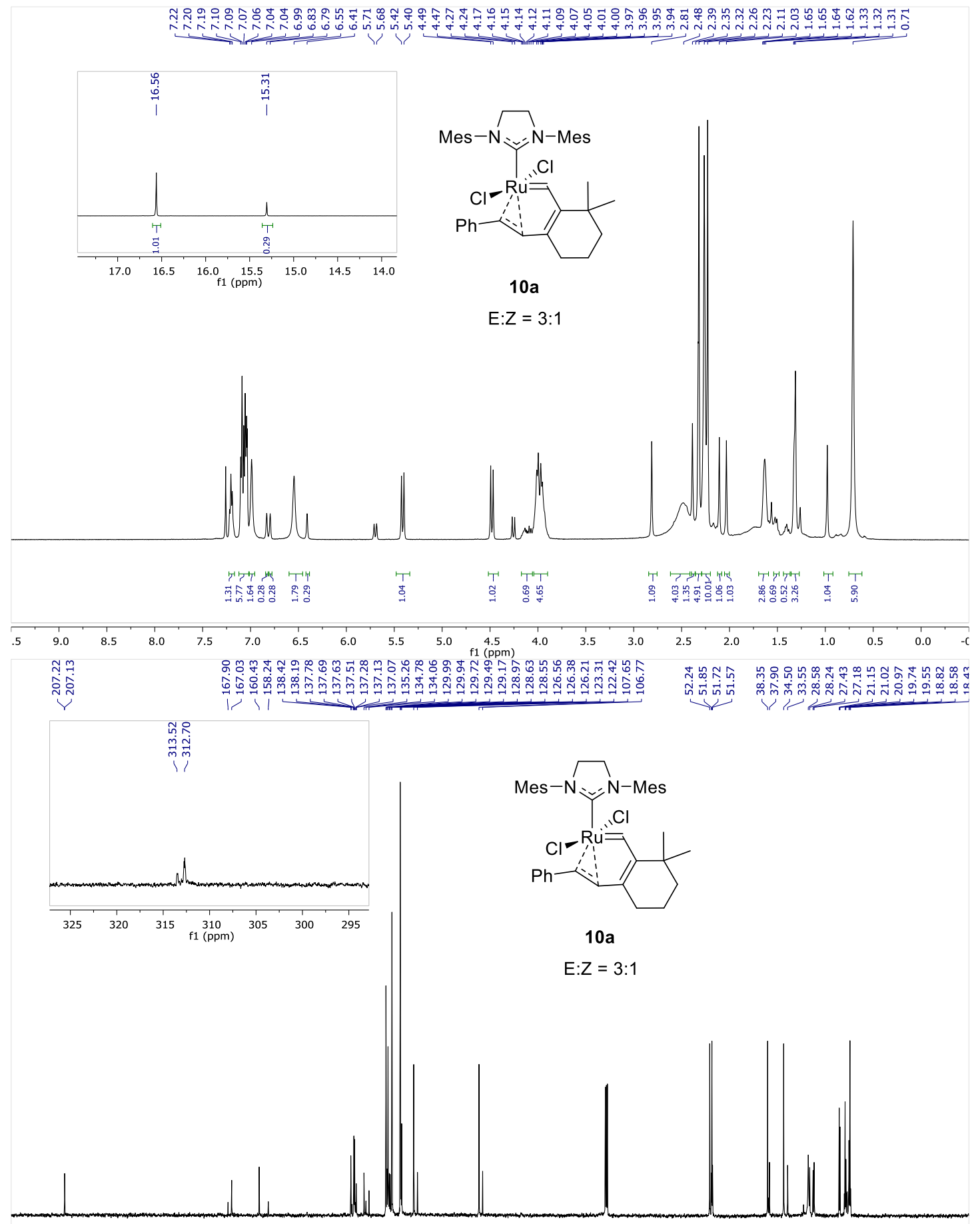



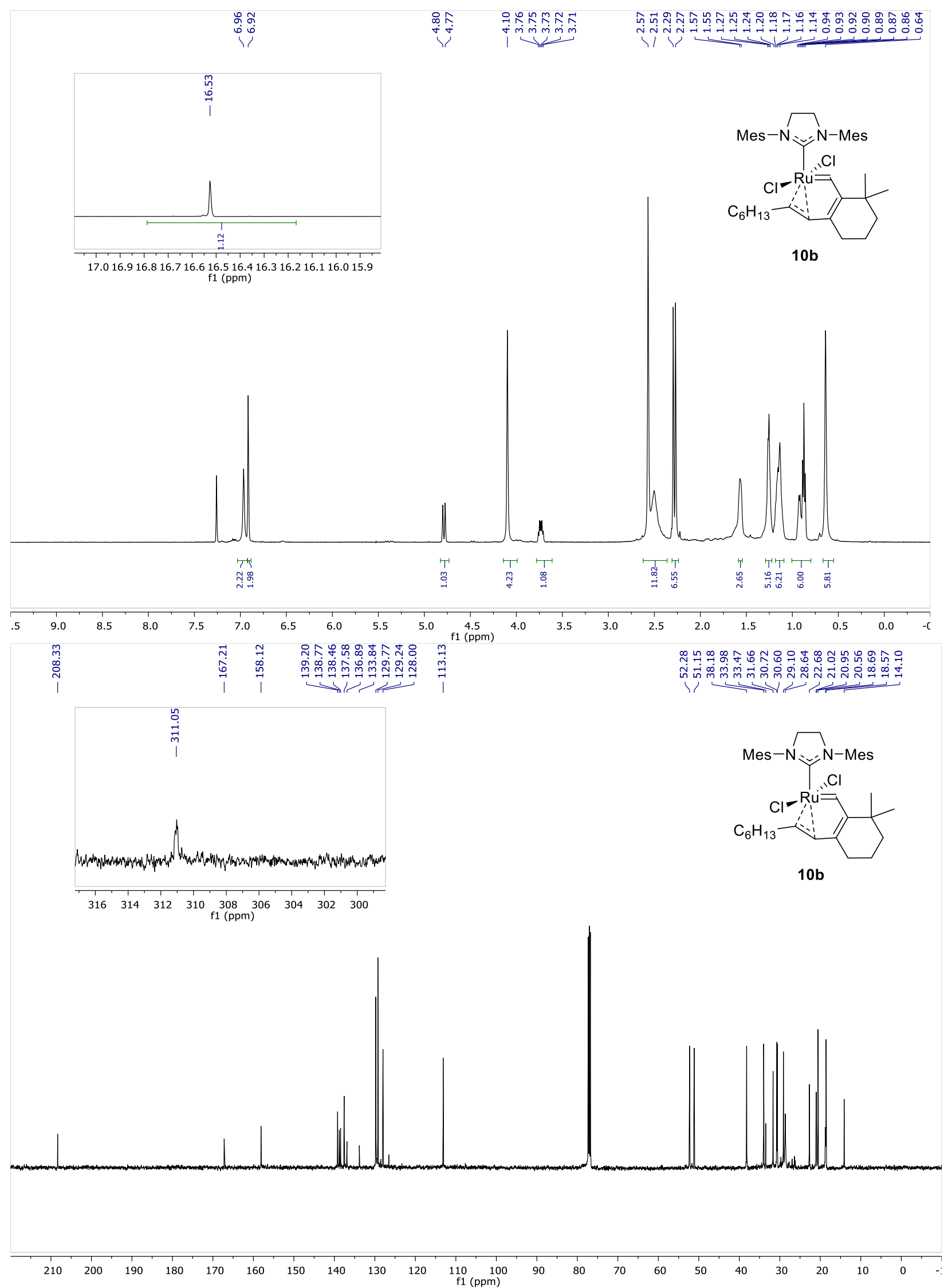

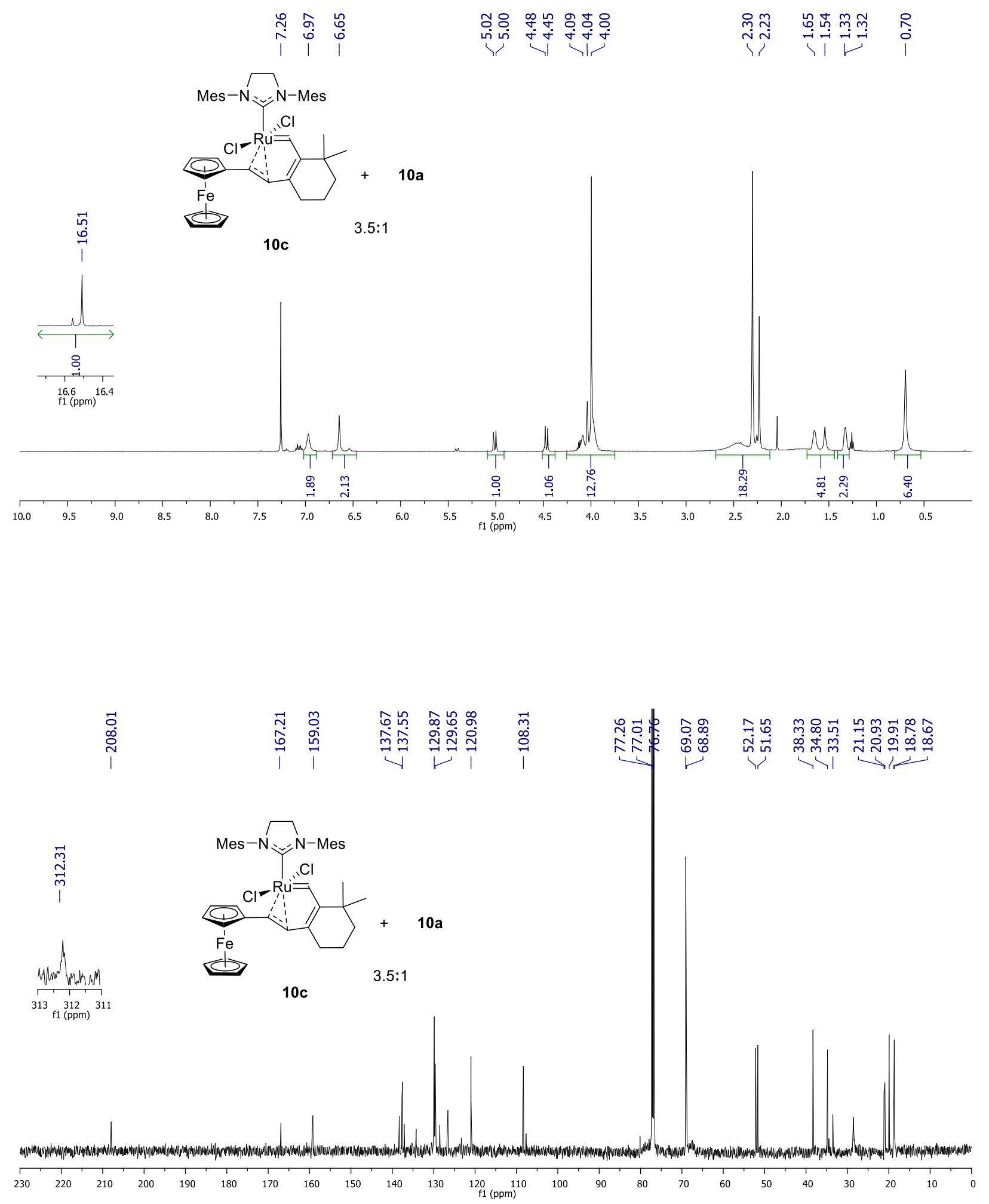

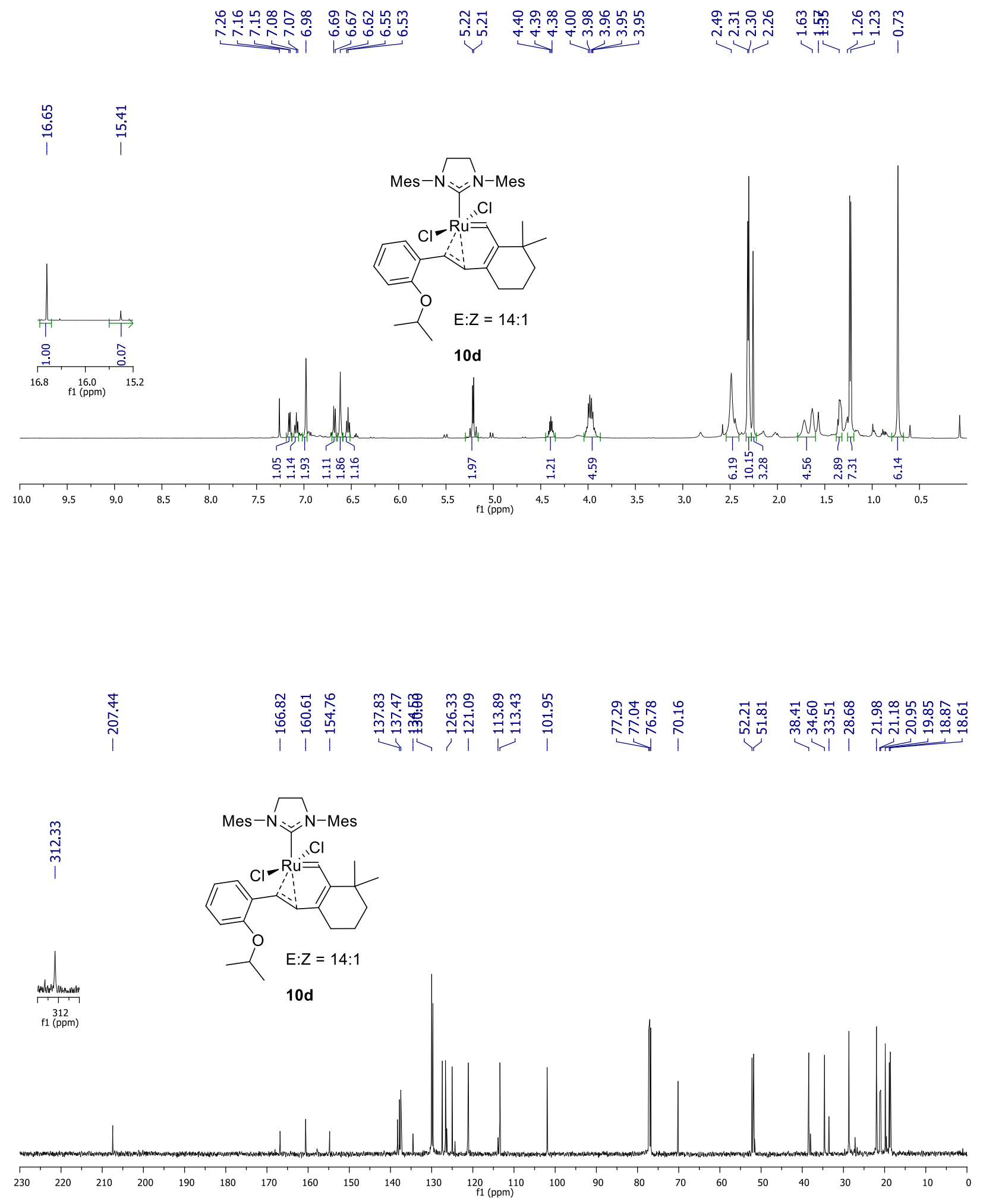

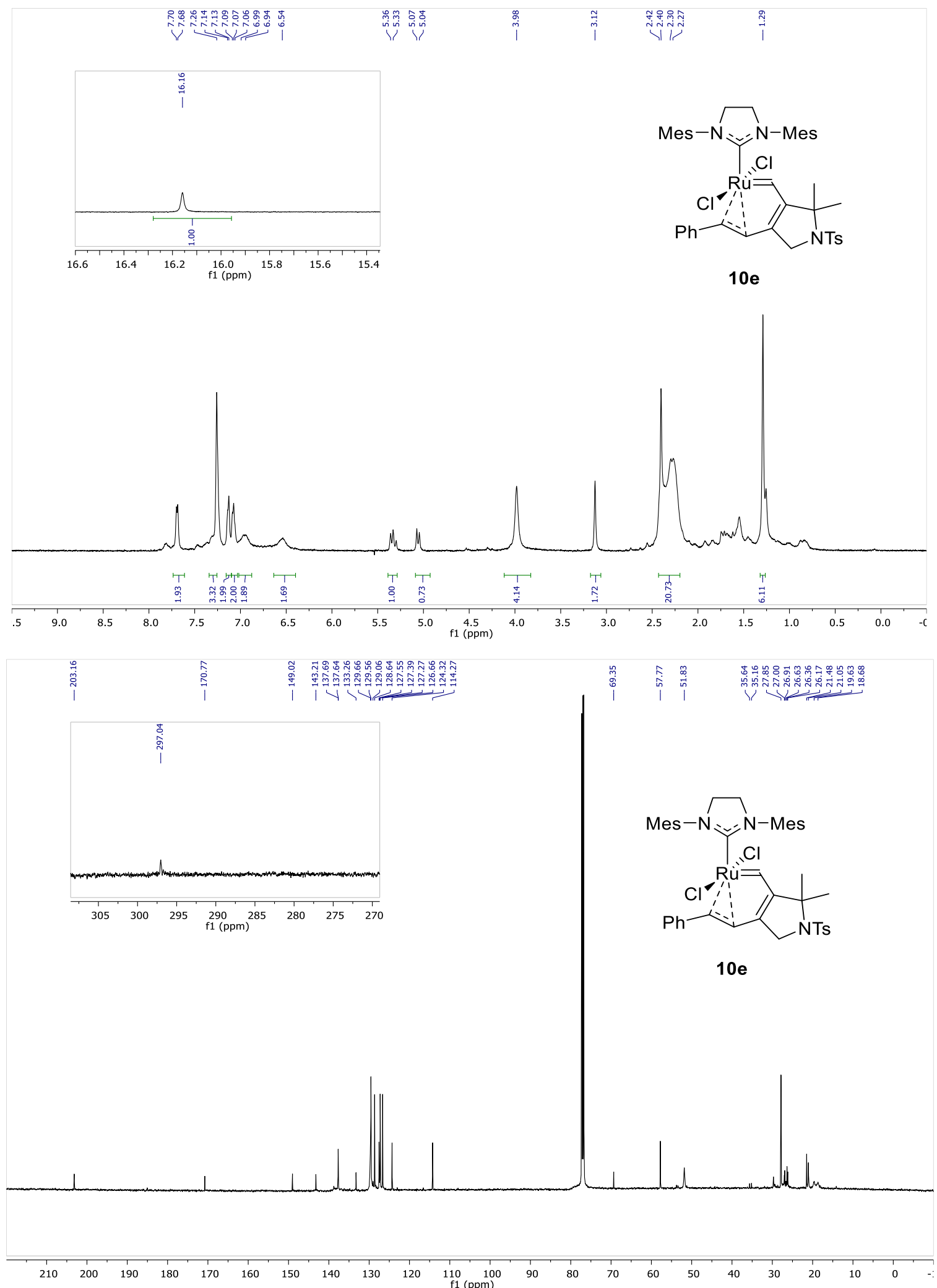

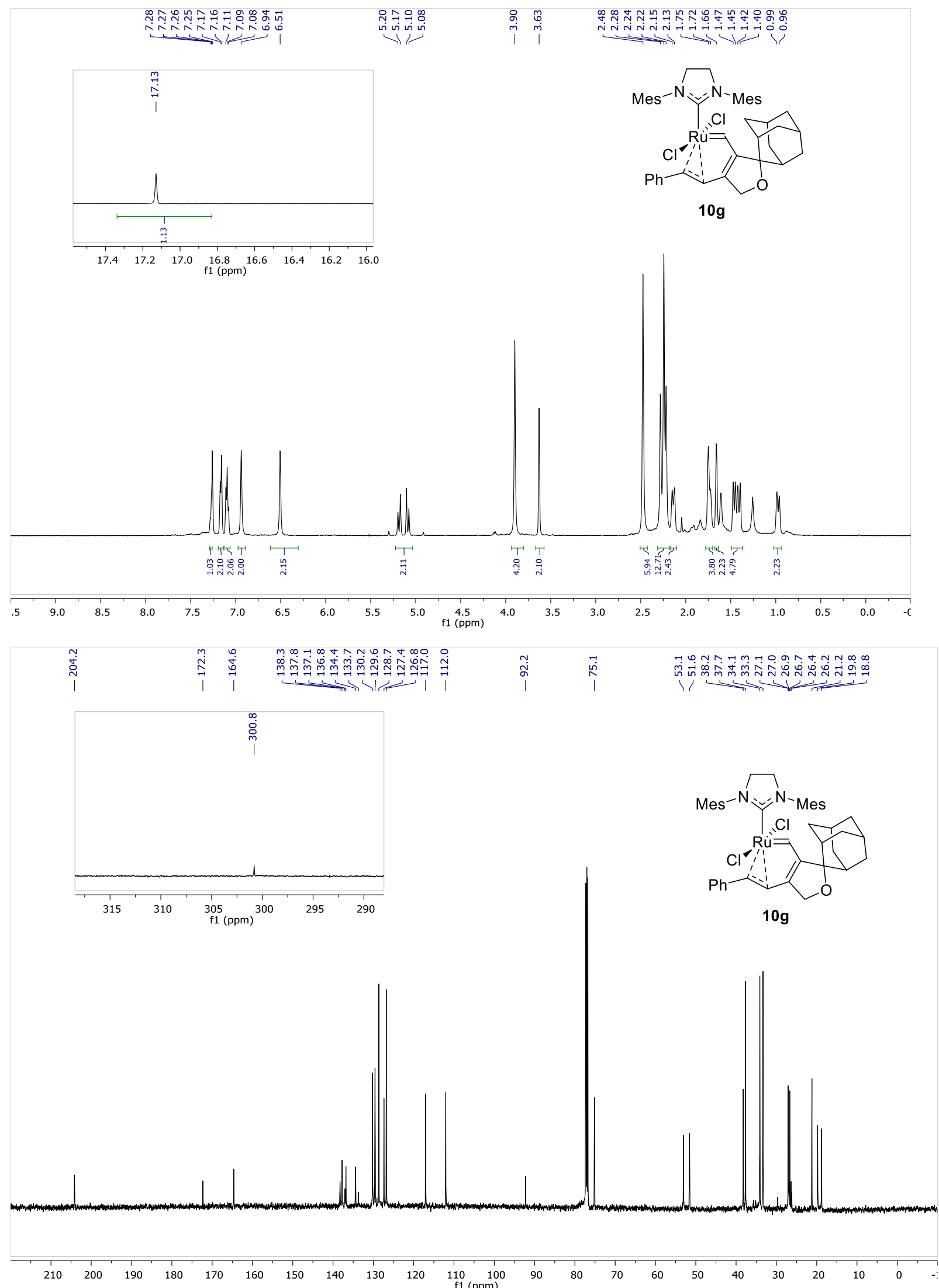


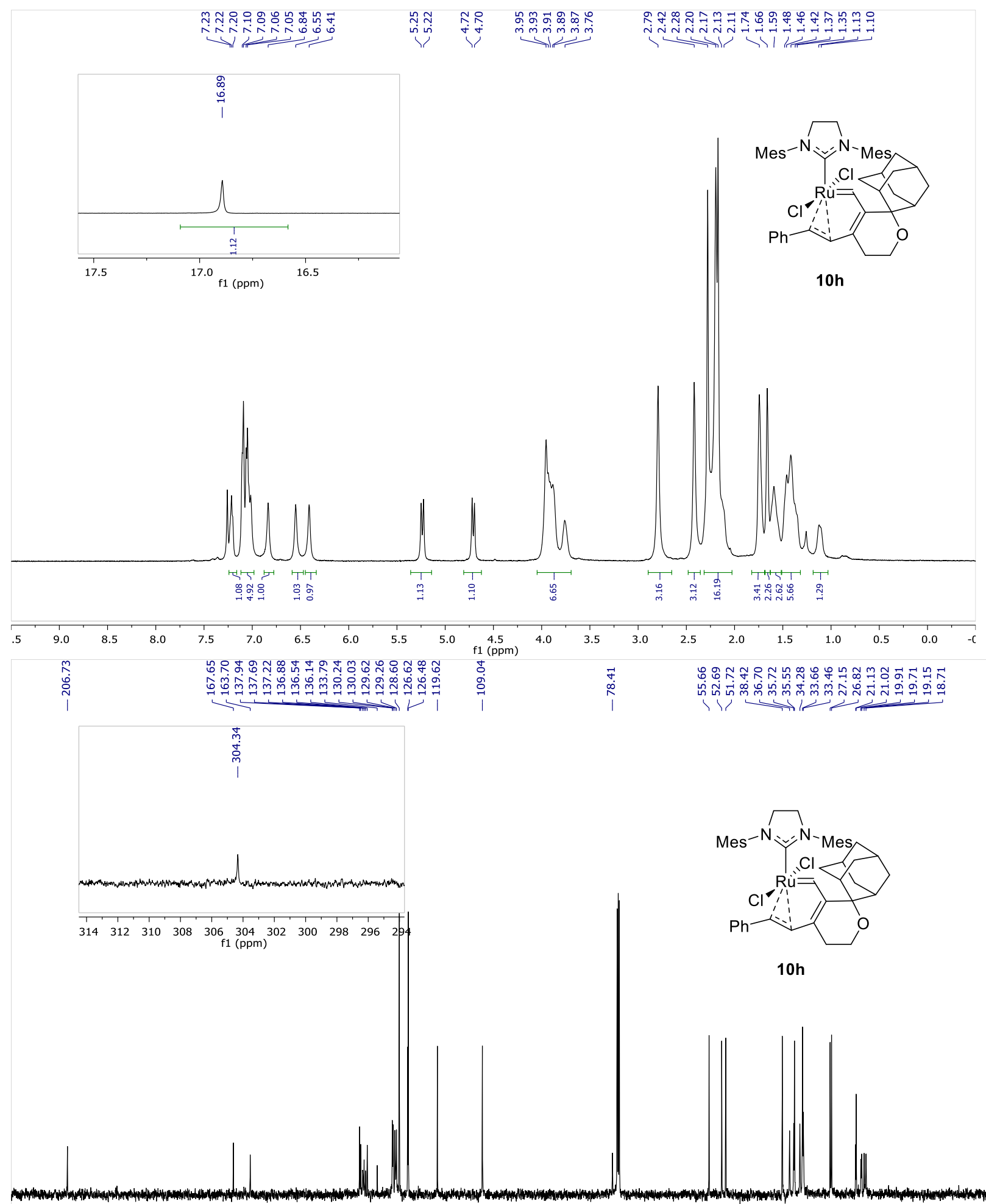

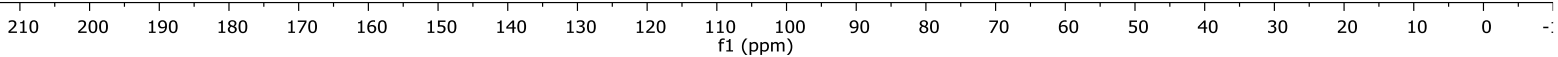



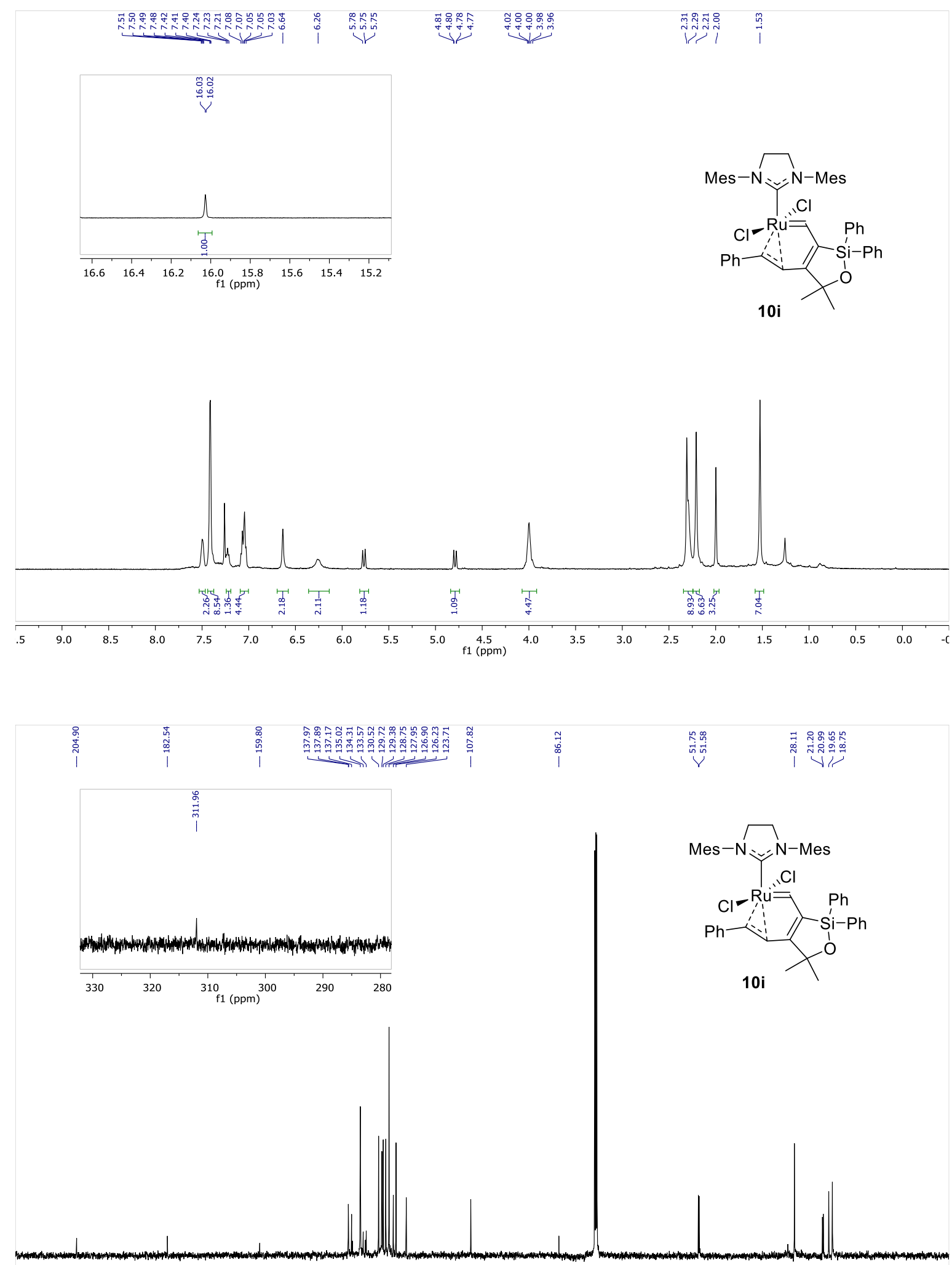

$\begin{array}{lllllllllll}210 & 200 & 190 & 180 & 170 & 160 & 150 & 140 & 130 & 120 & \begin{array}{c}110 \\ \mathrm{f} 1(\mathrm{ppm})\end{array}\end{array}$ 

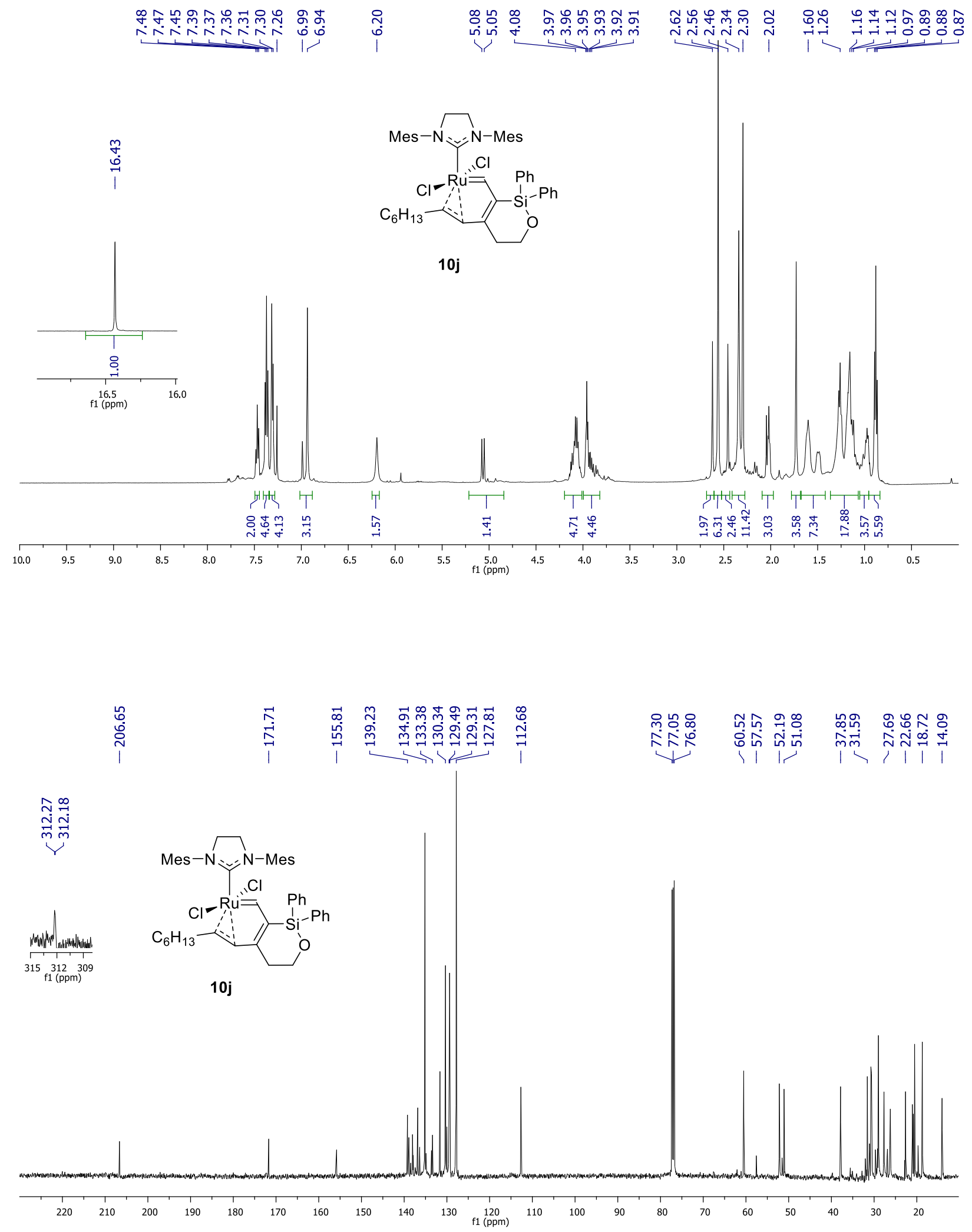


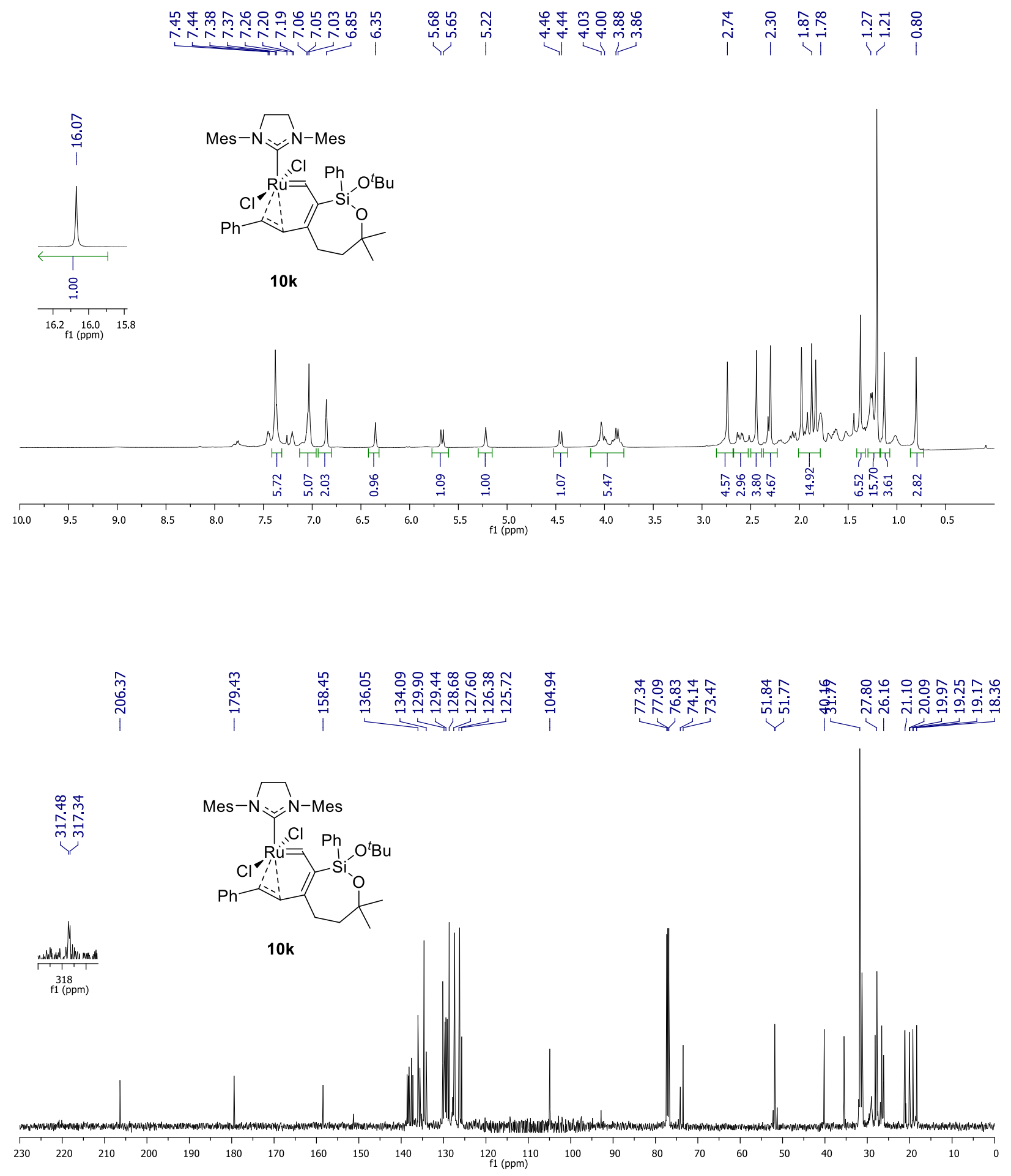



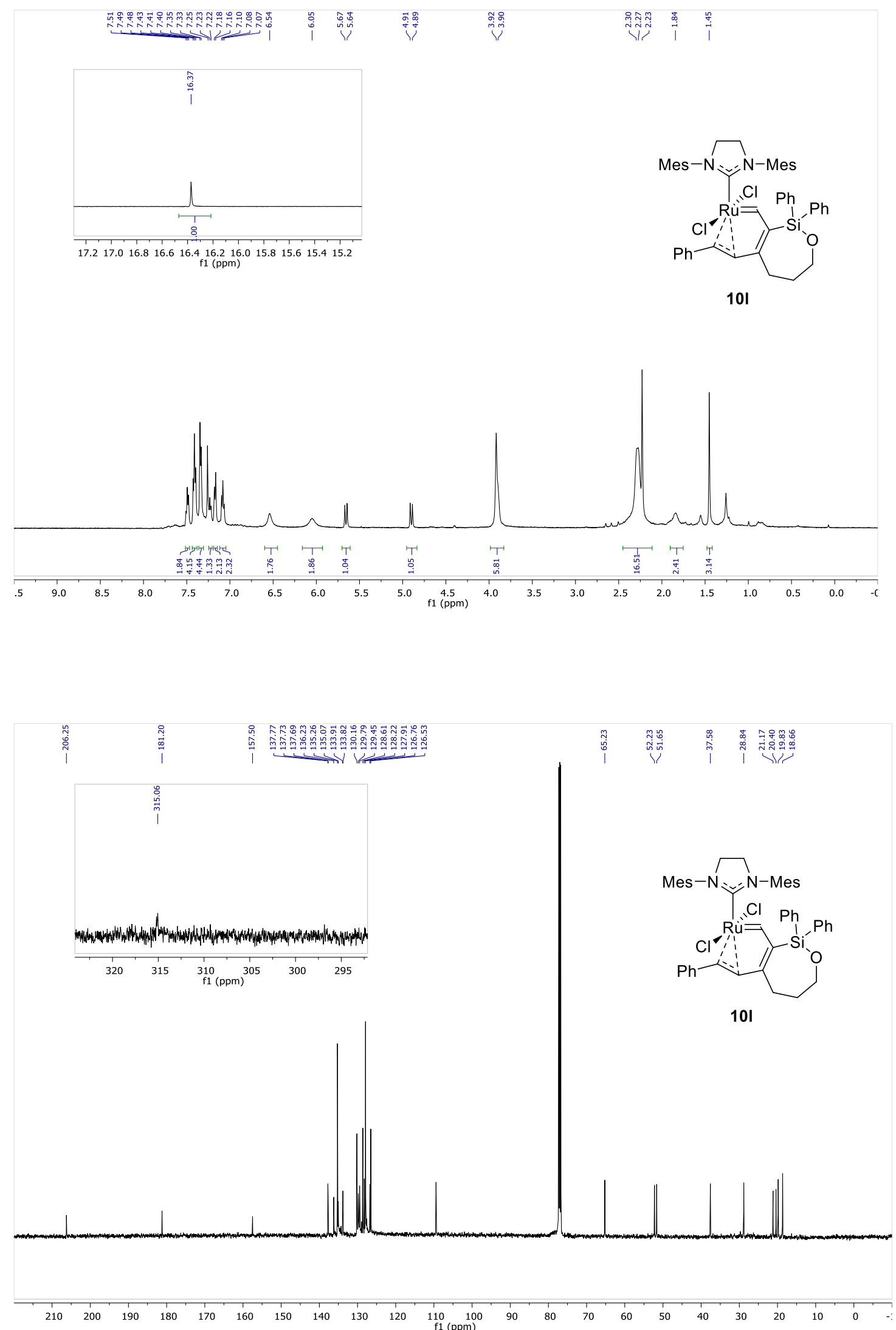

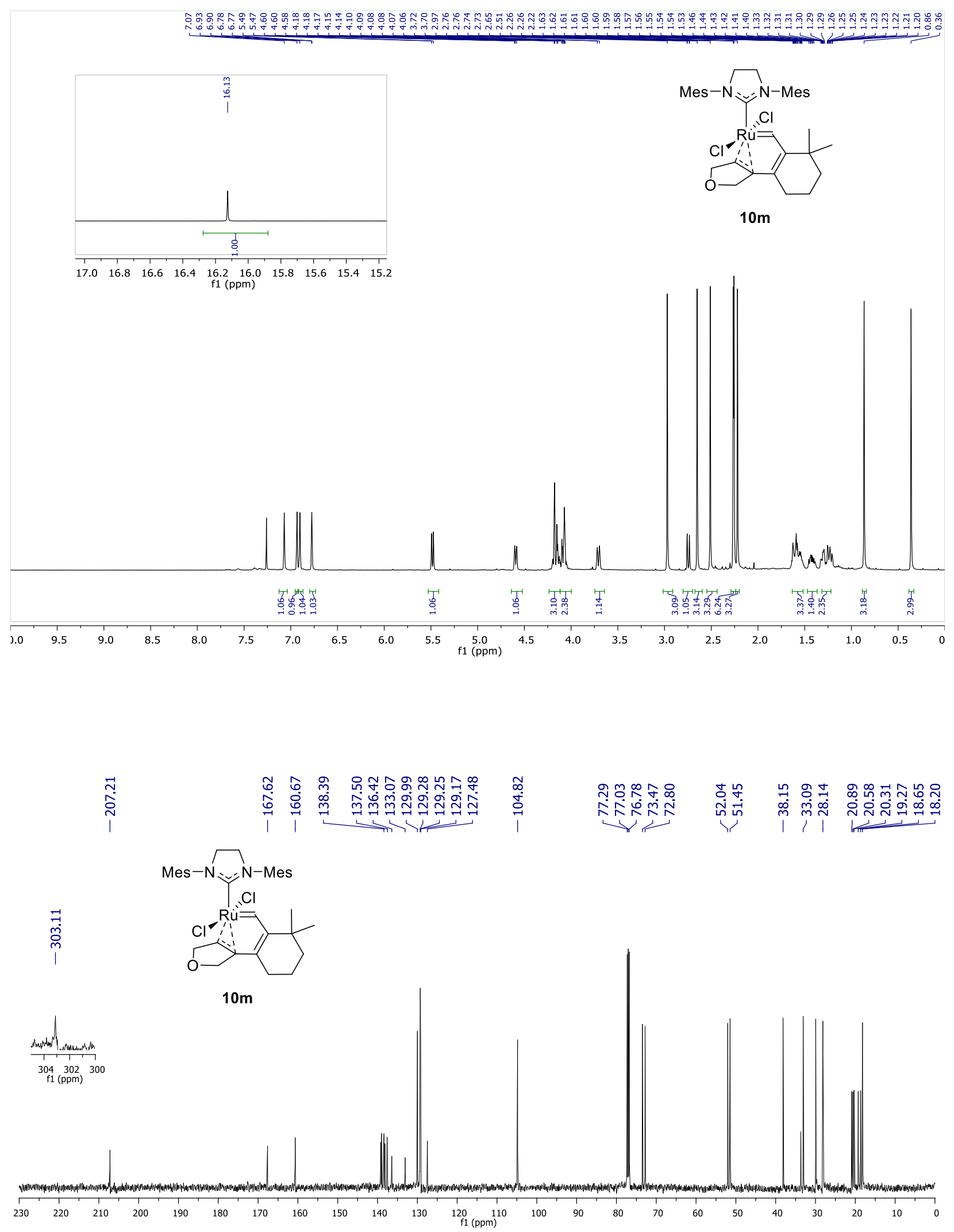


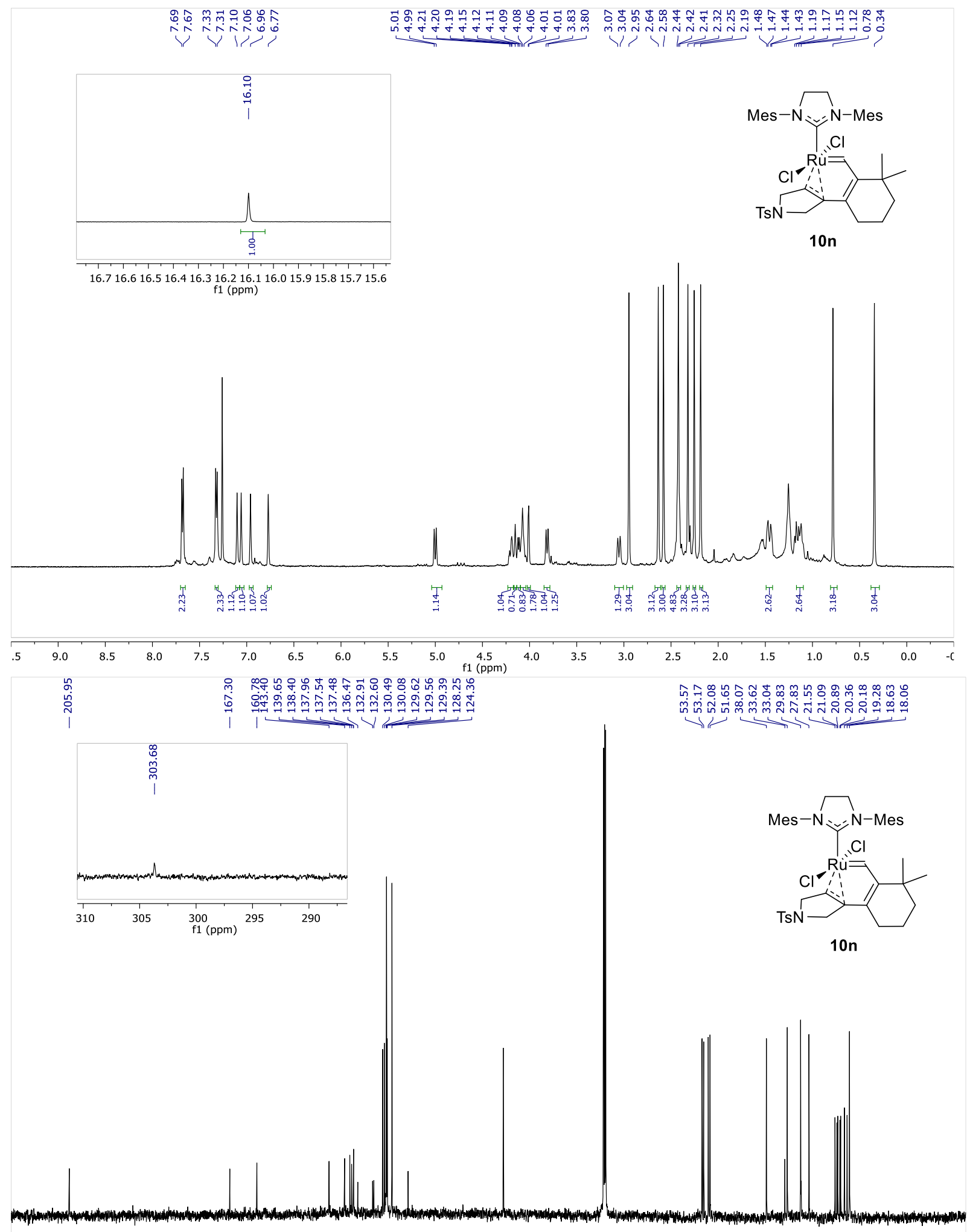

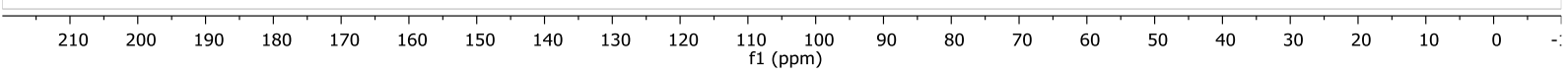




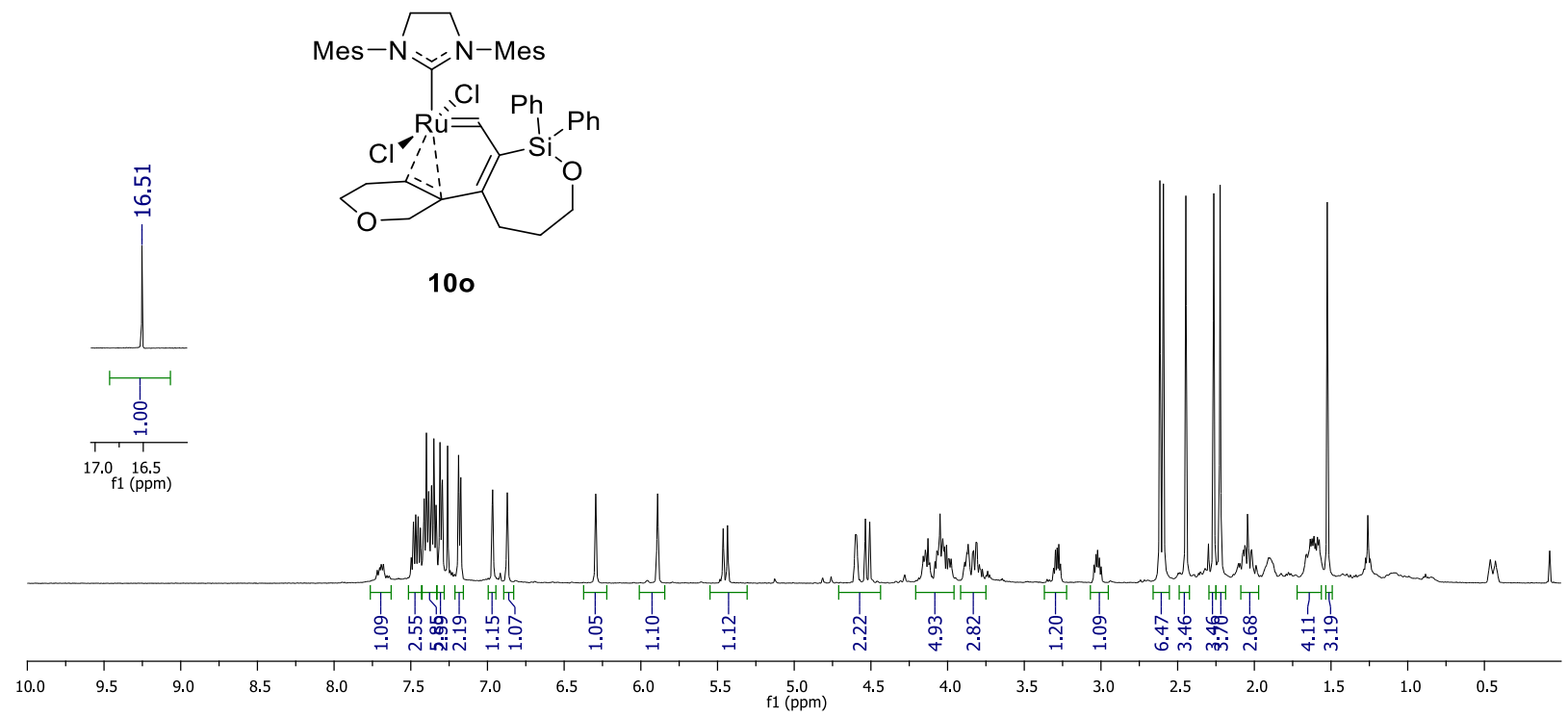

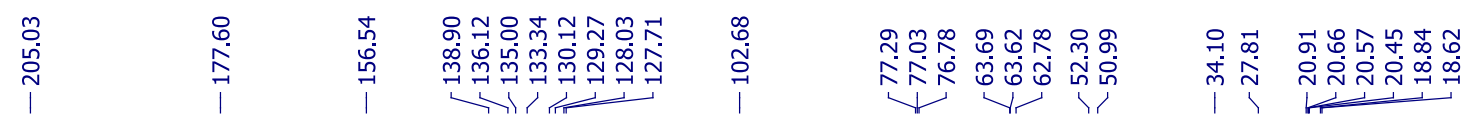

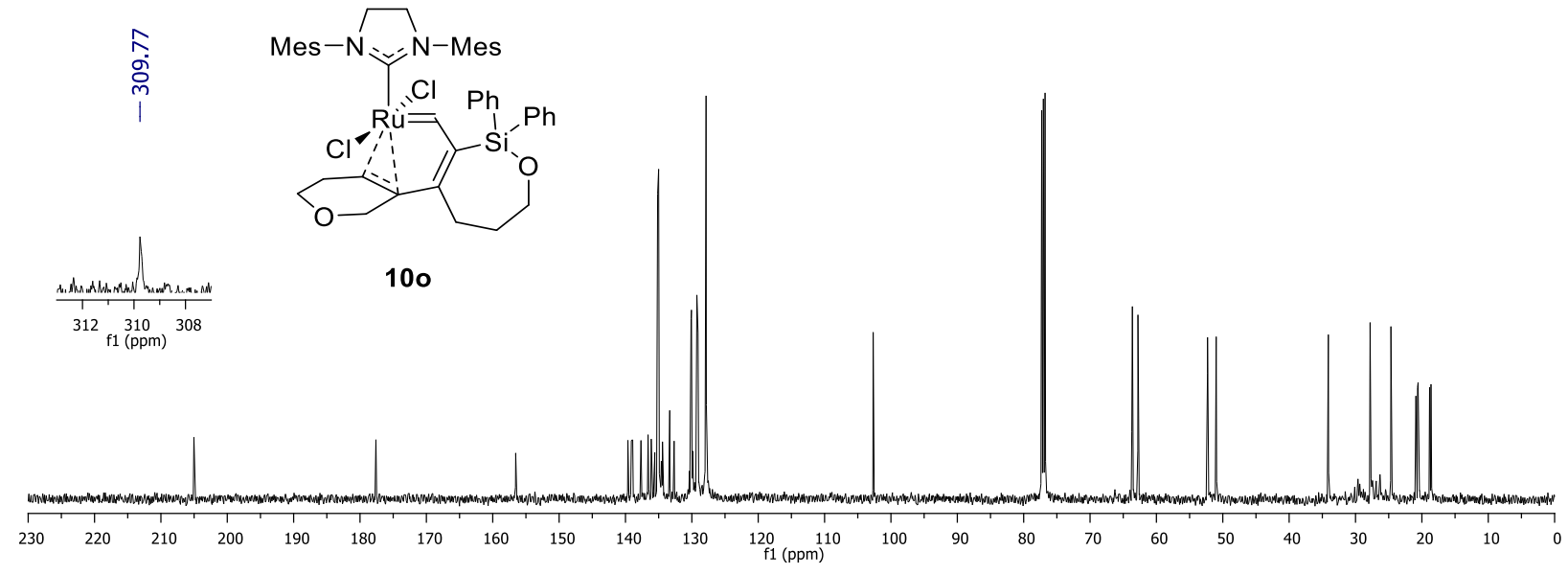



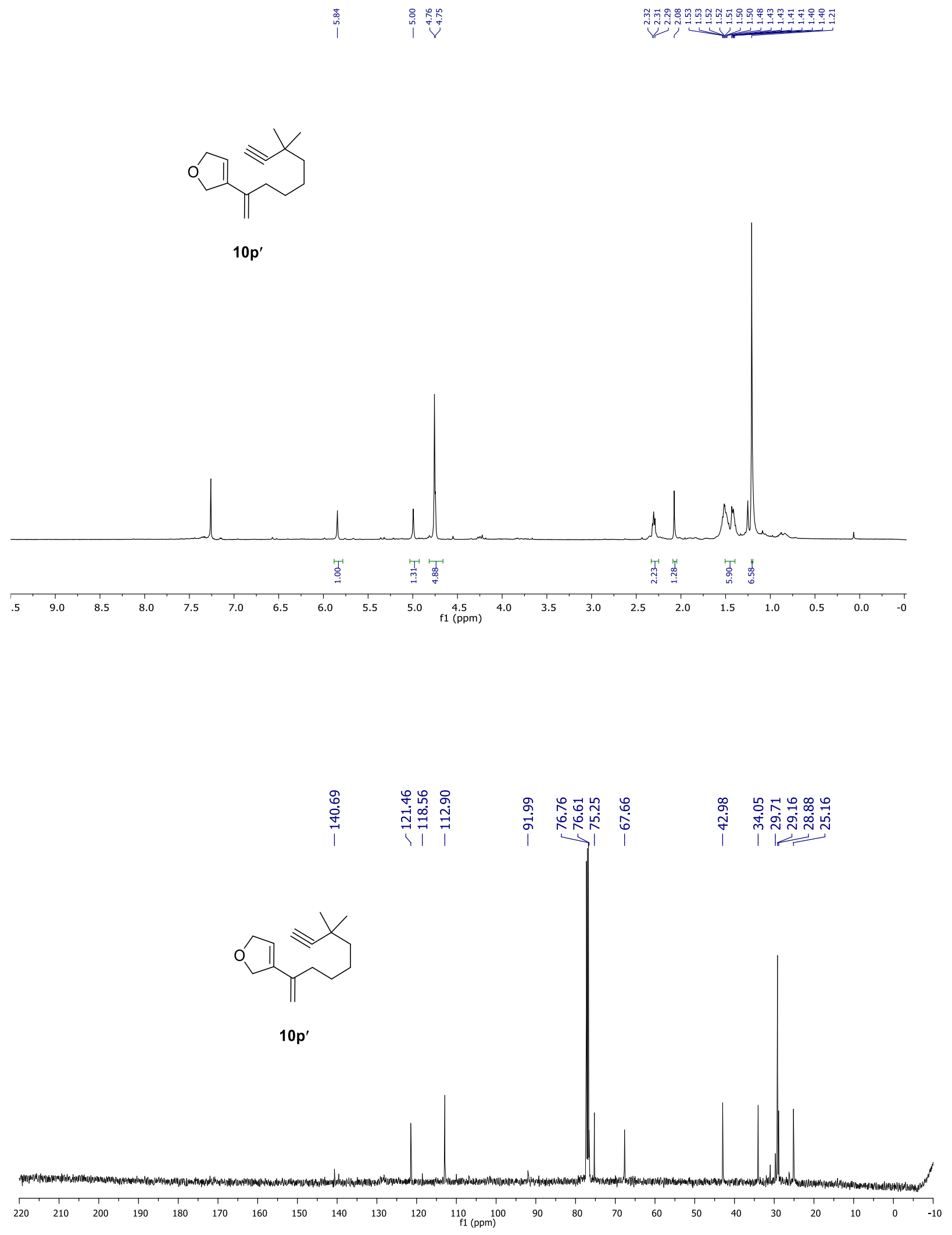

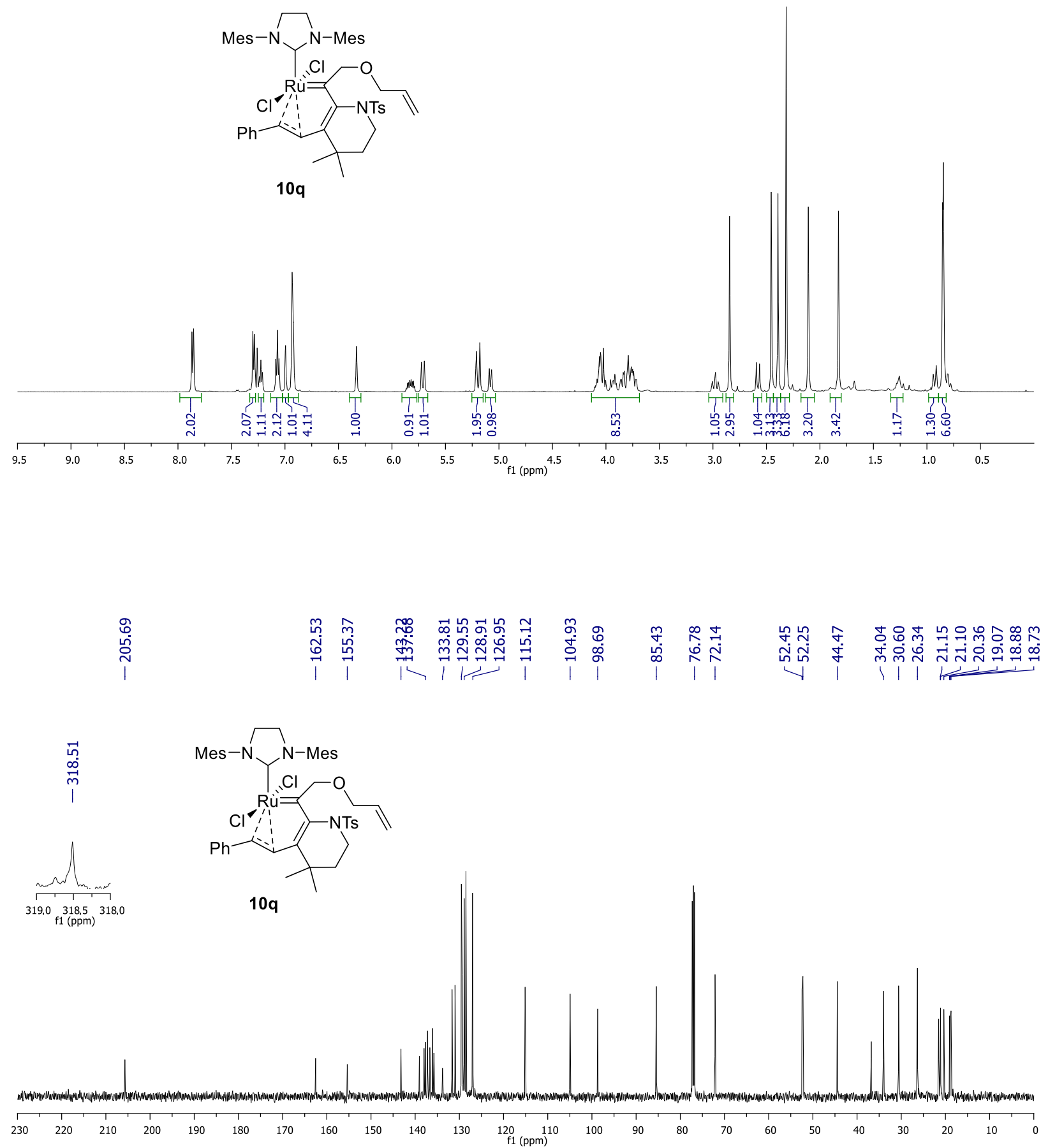

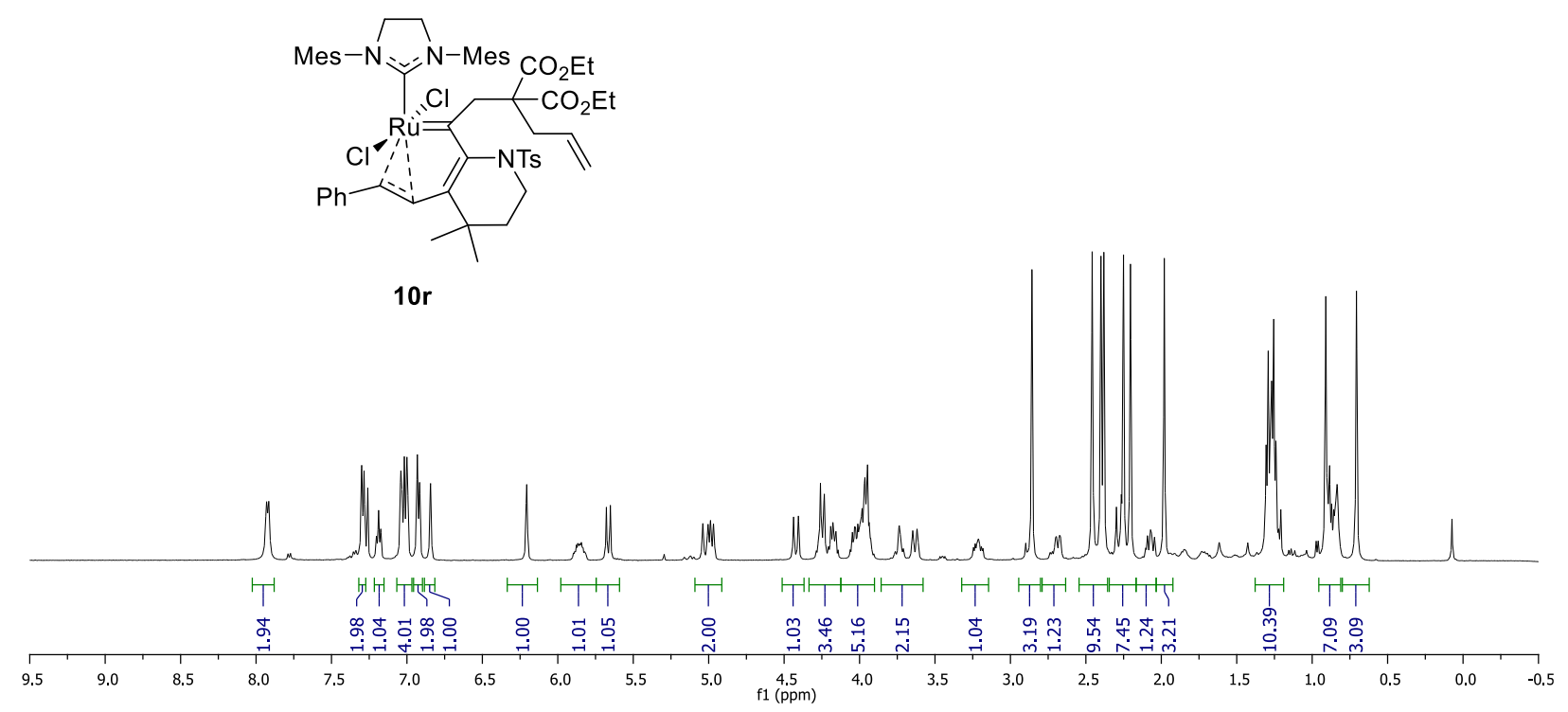

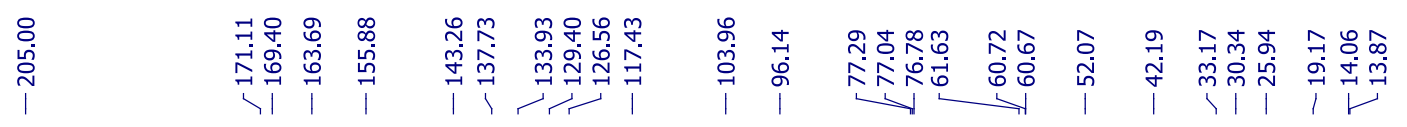

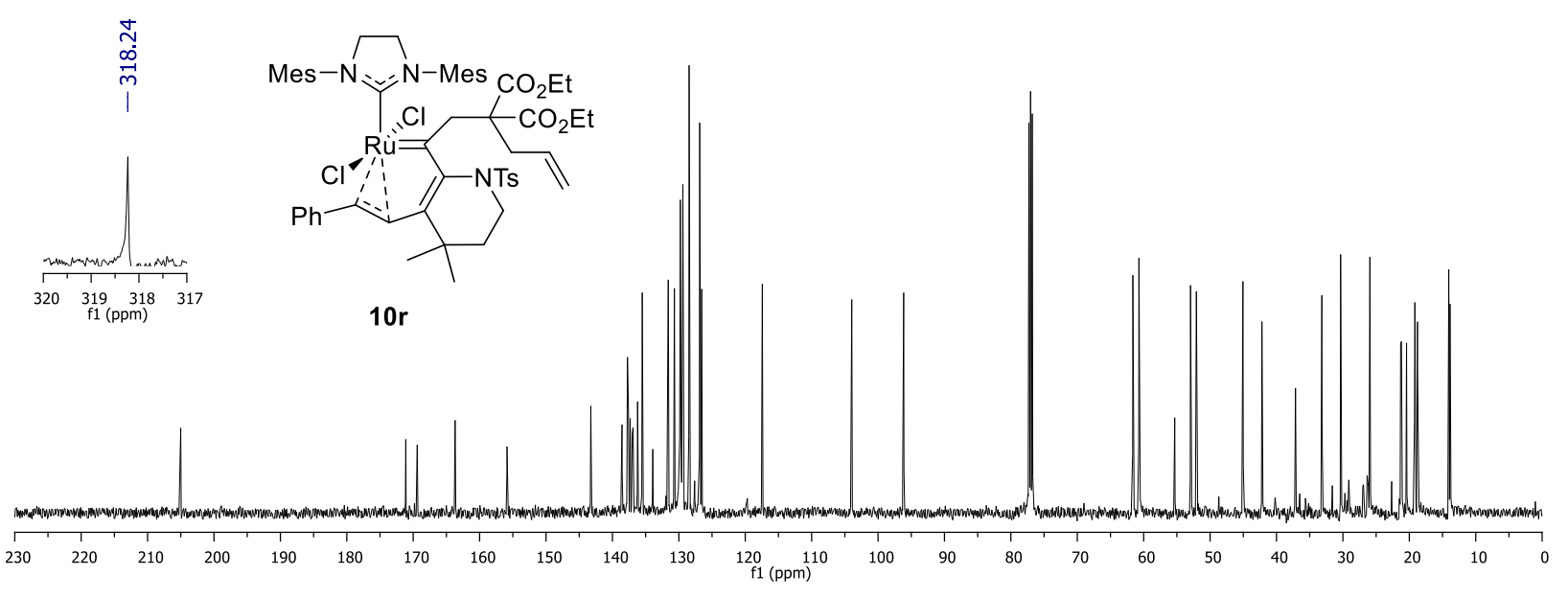



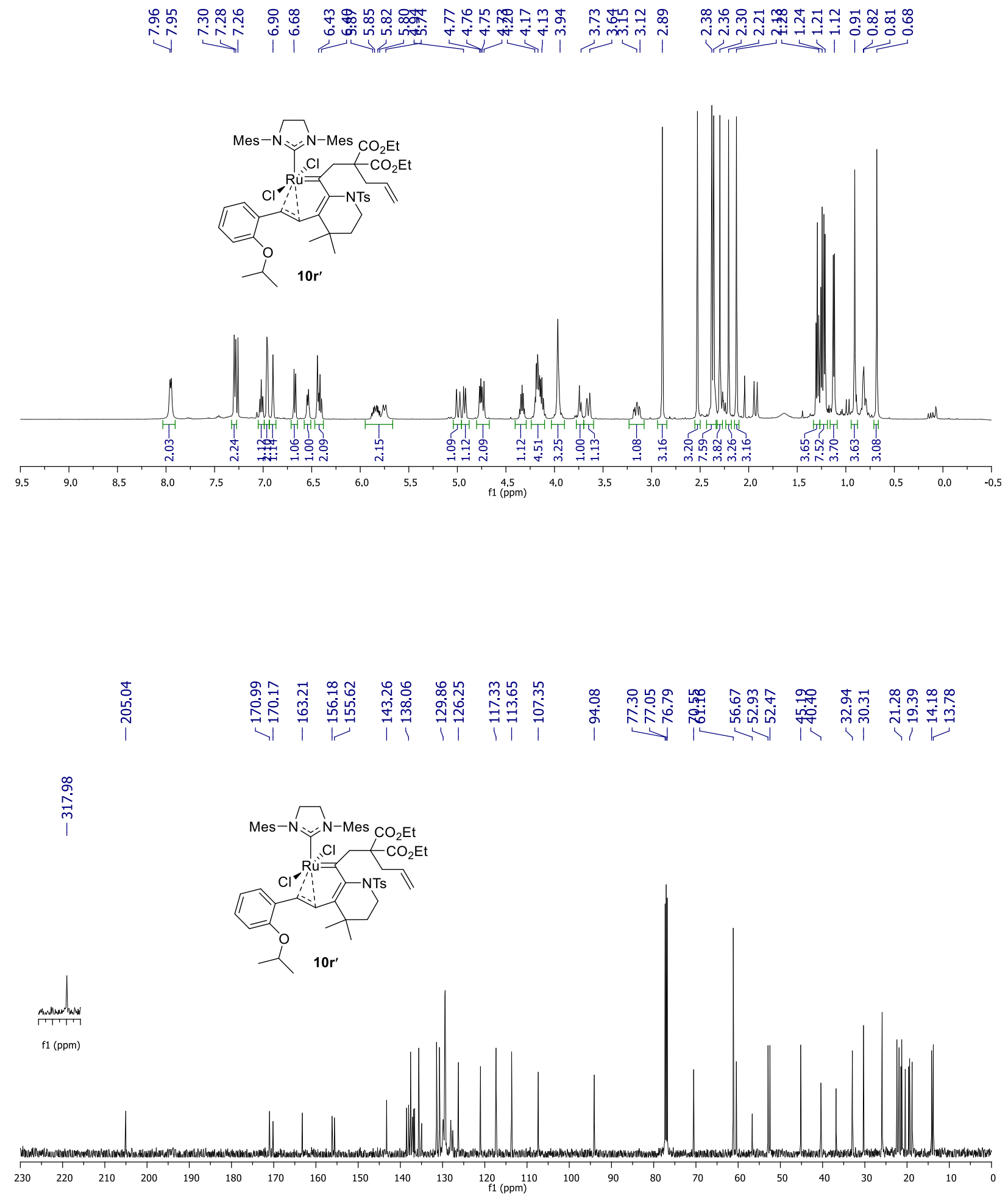


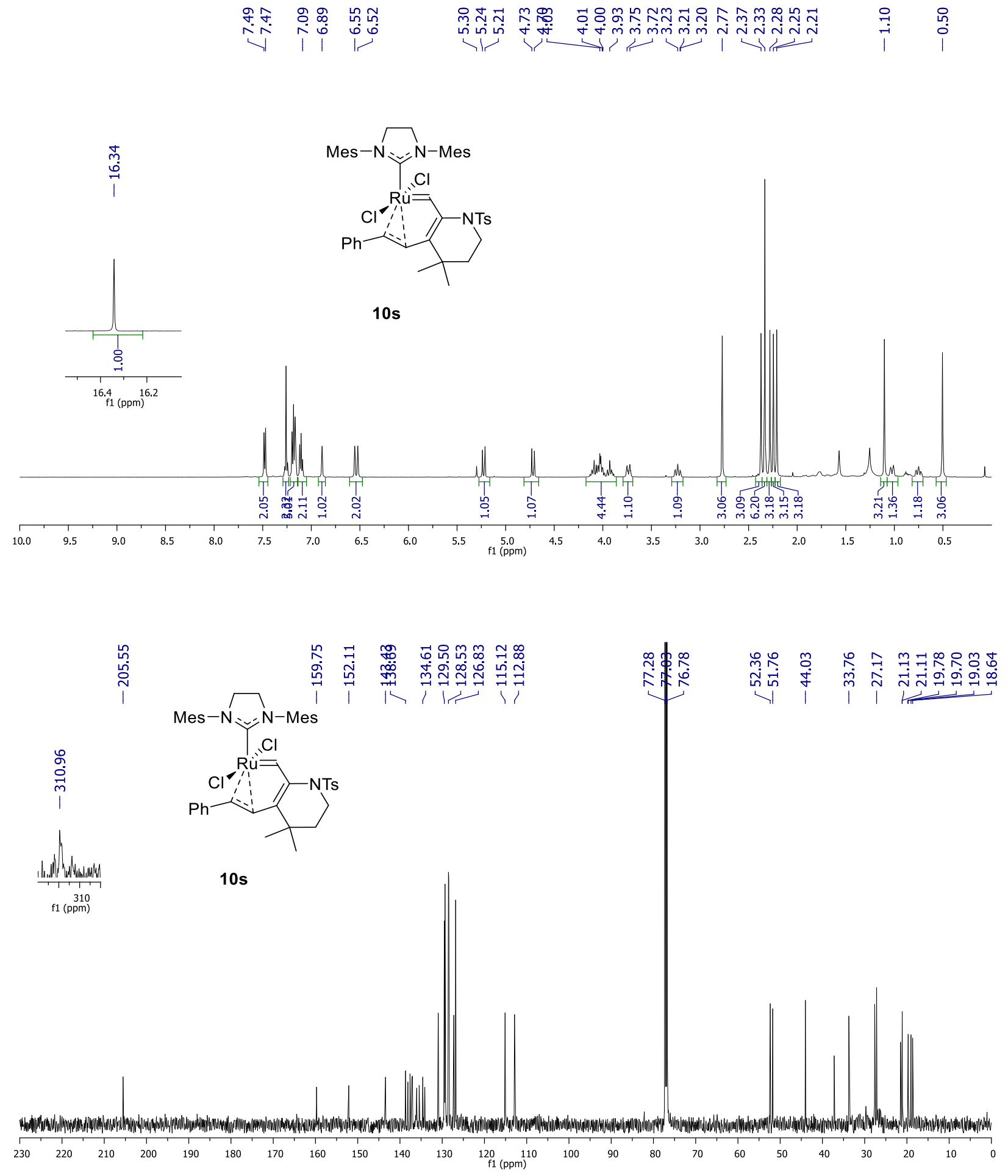



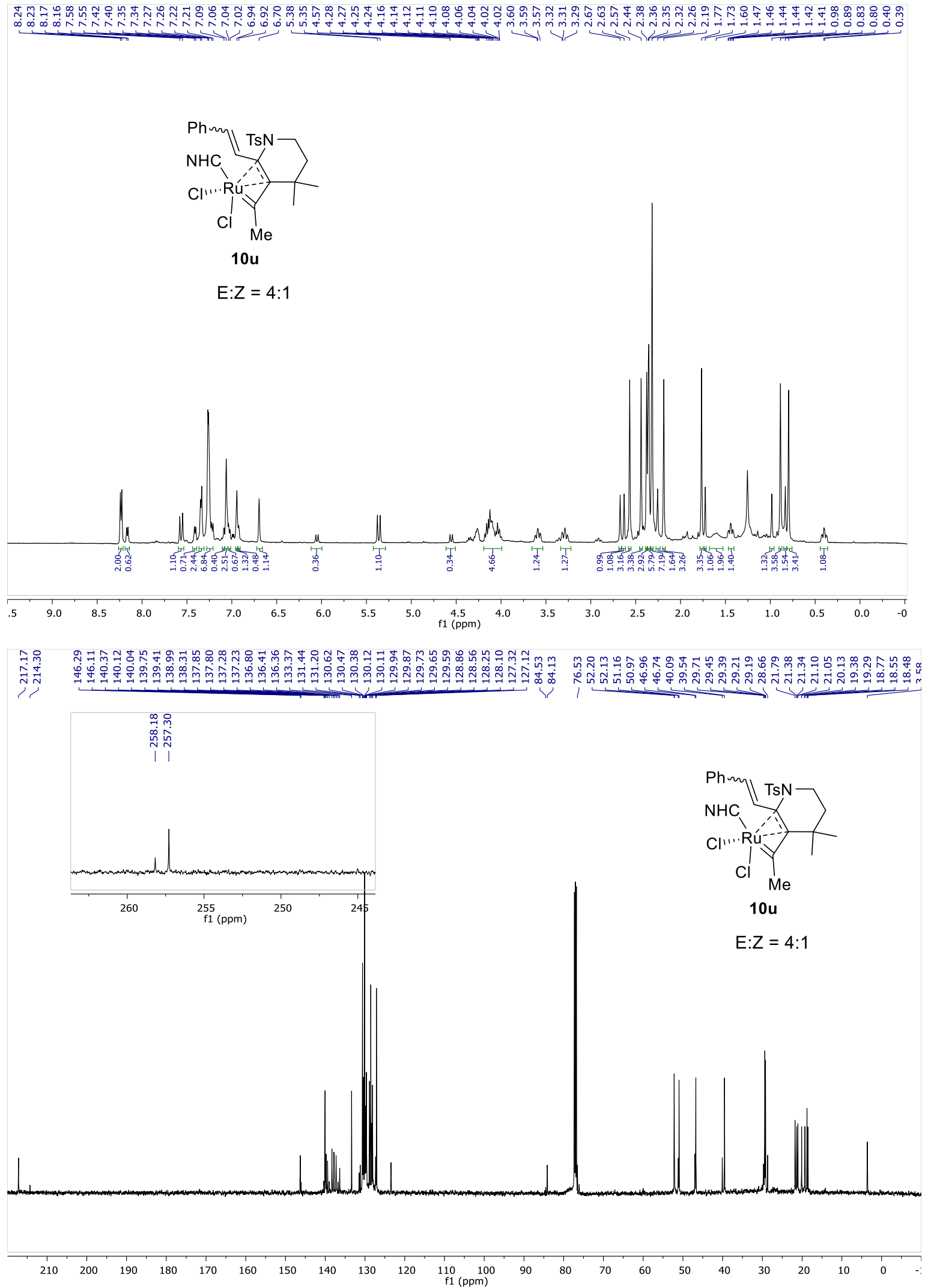

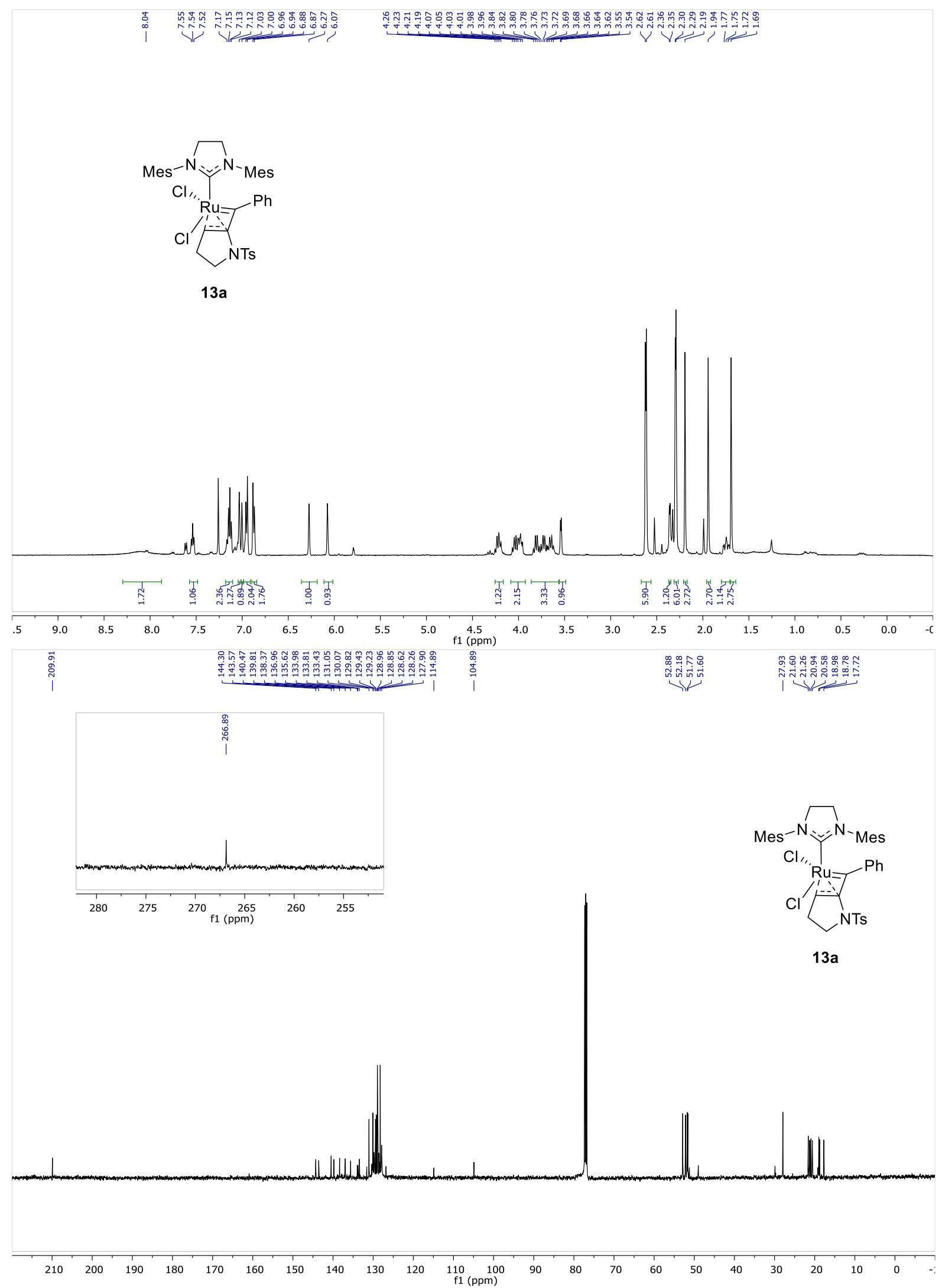

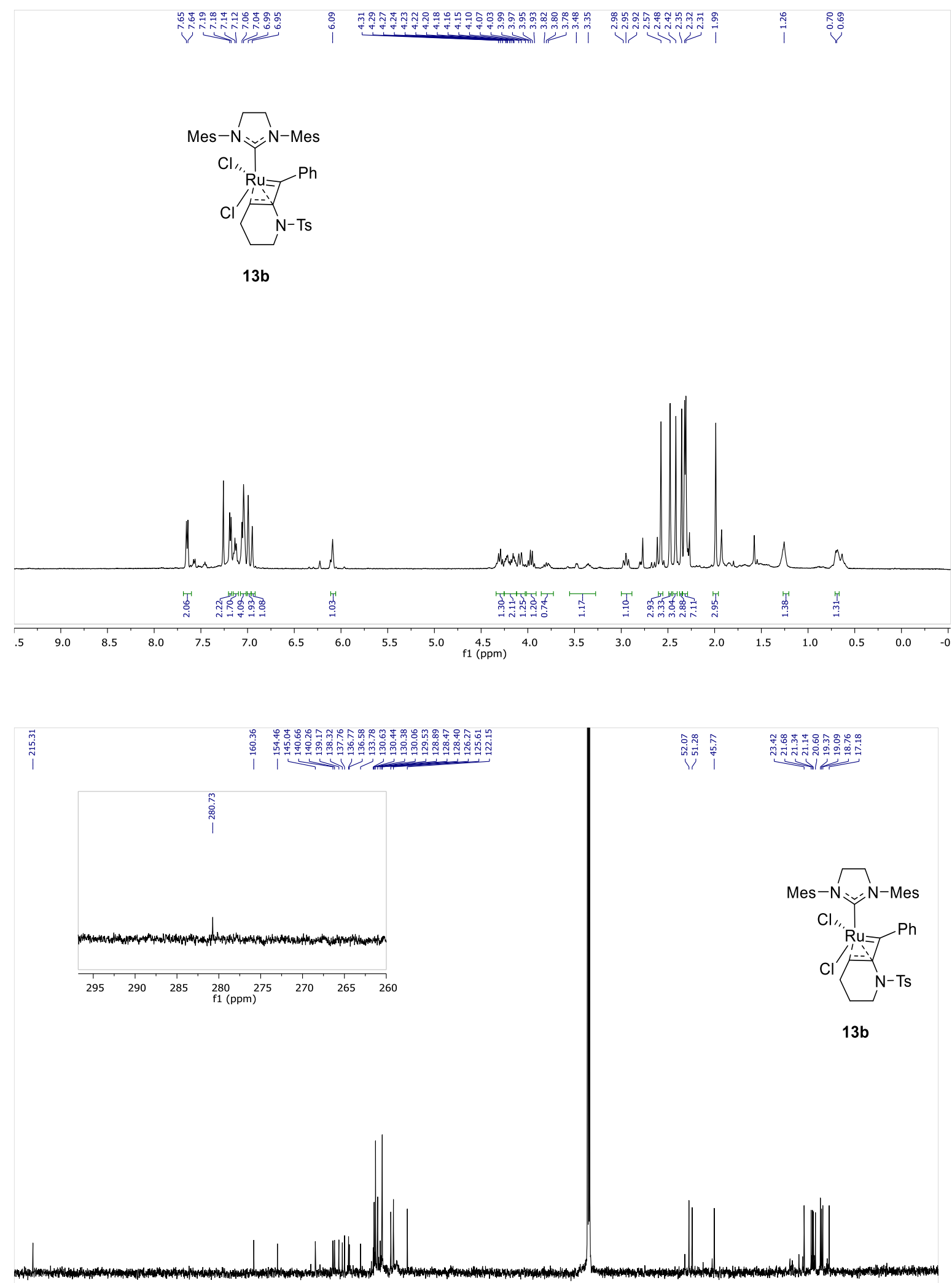

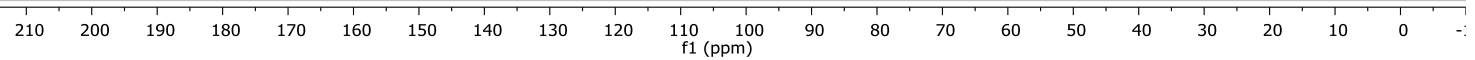



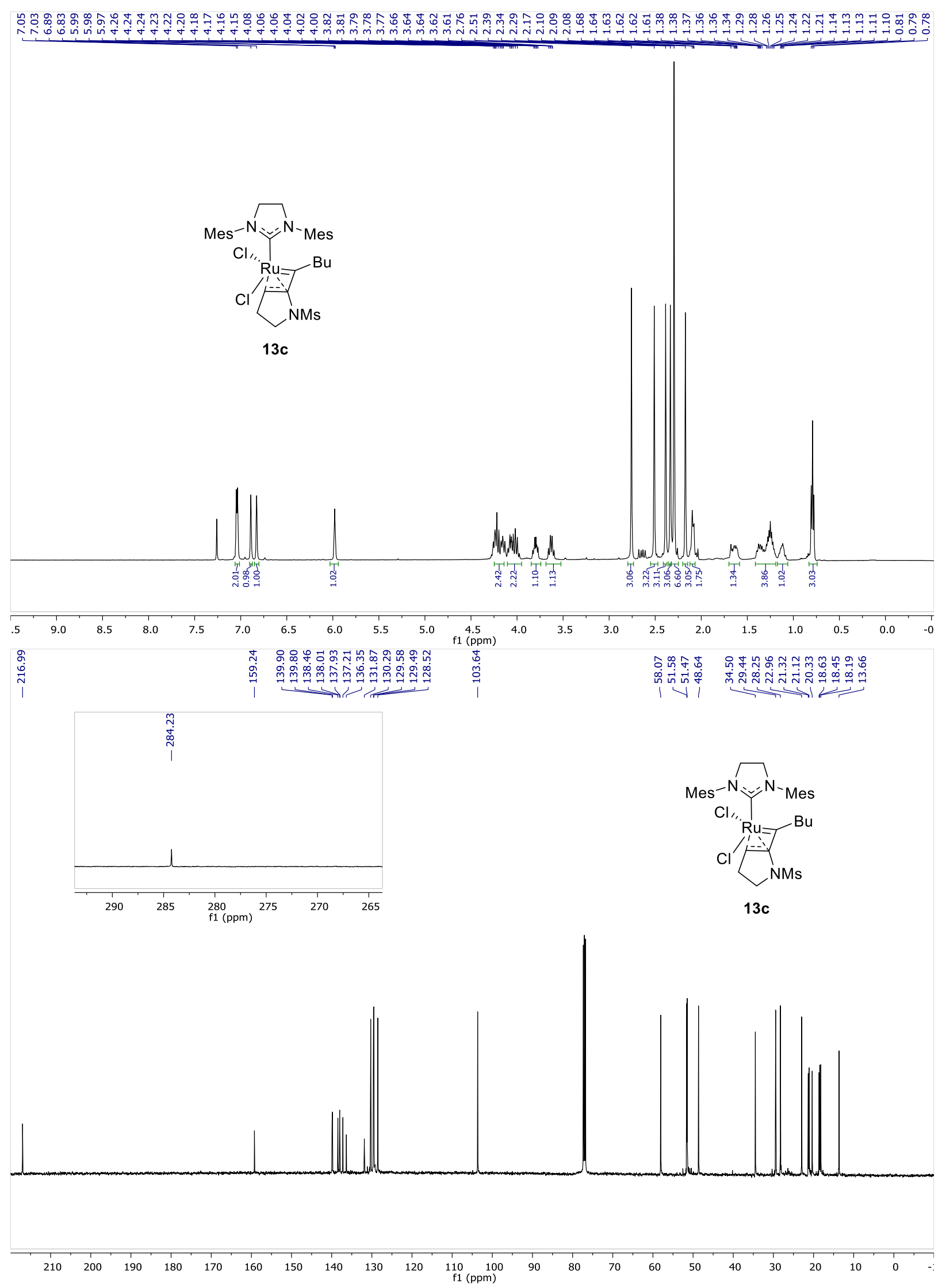


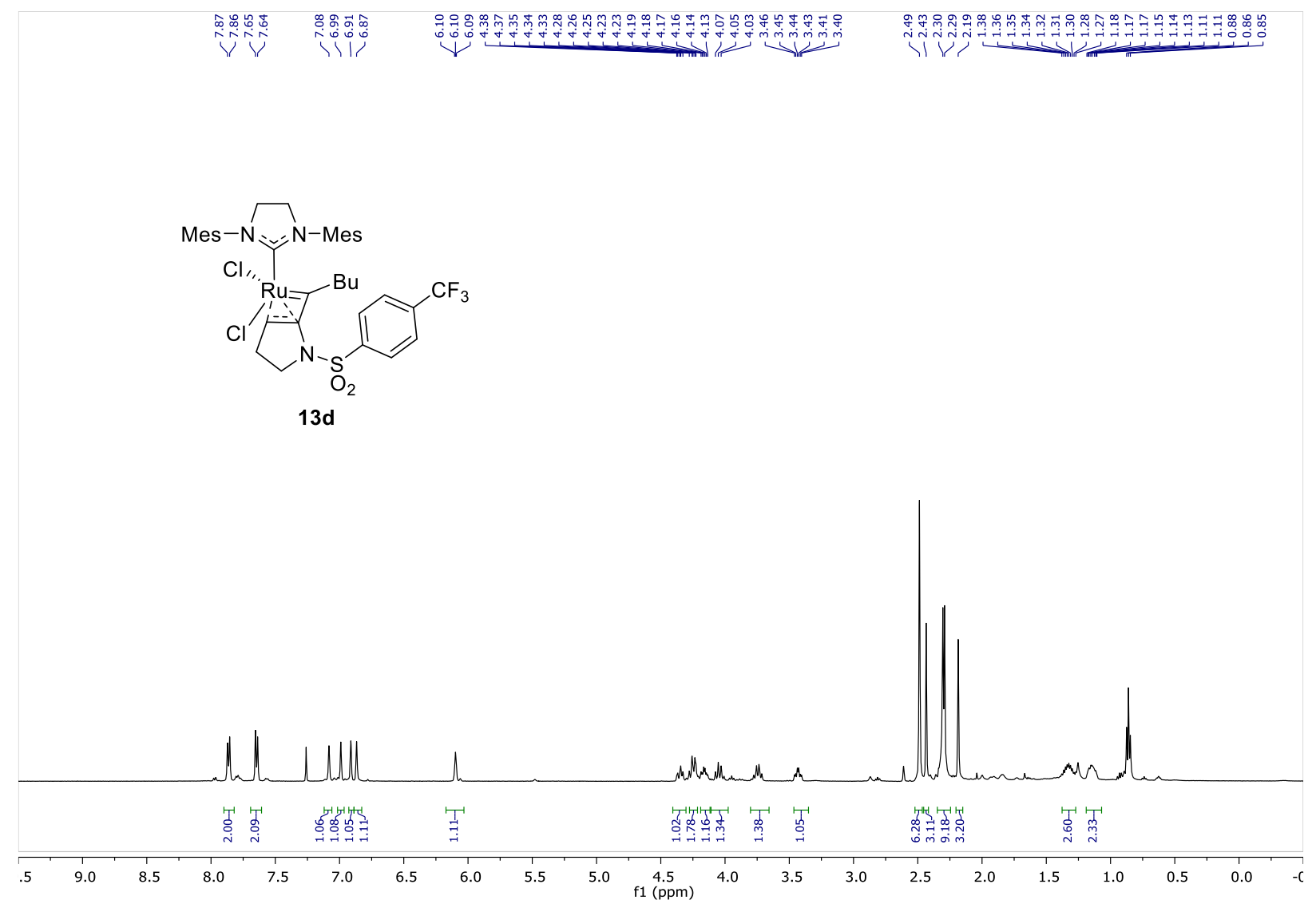

${ }^{19} \mathrm{~F}$ NMR $\left(\mathrm{CDCl}_{3}, 376 \mathrm{MHz}\right)$

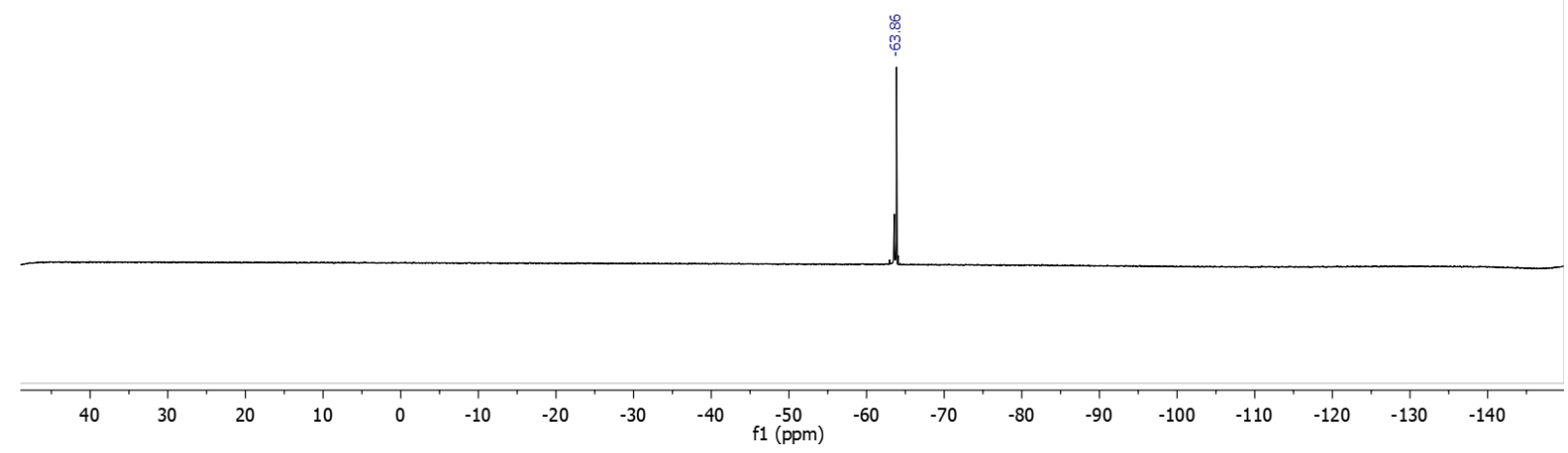




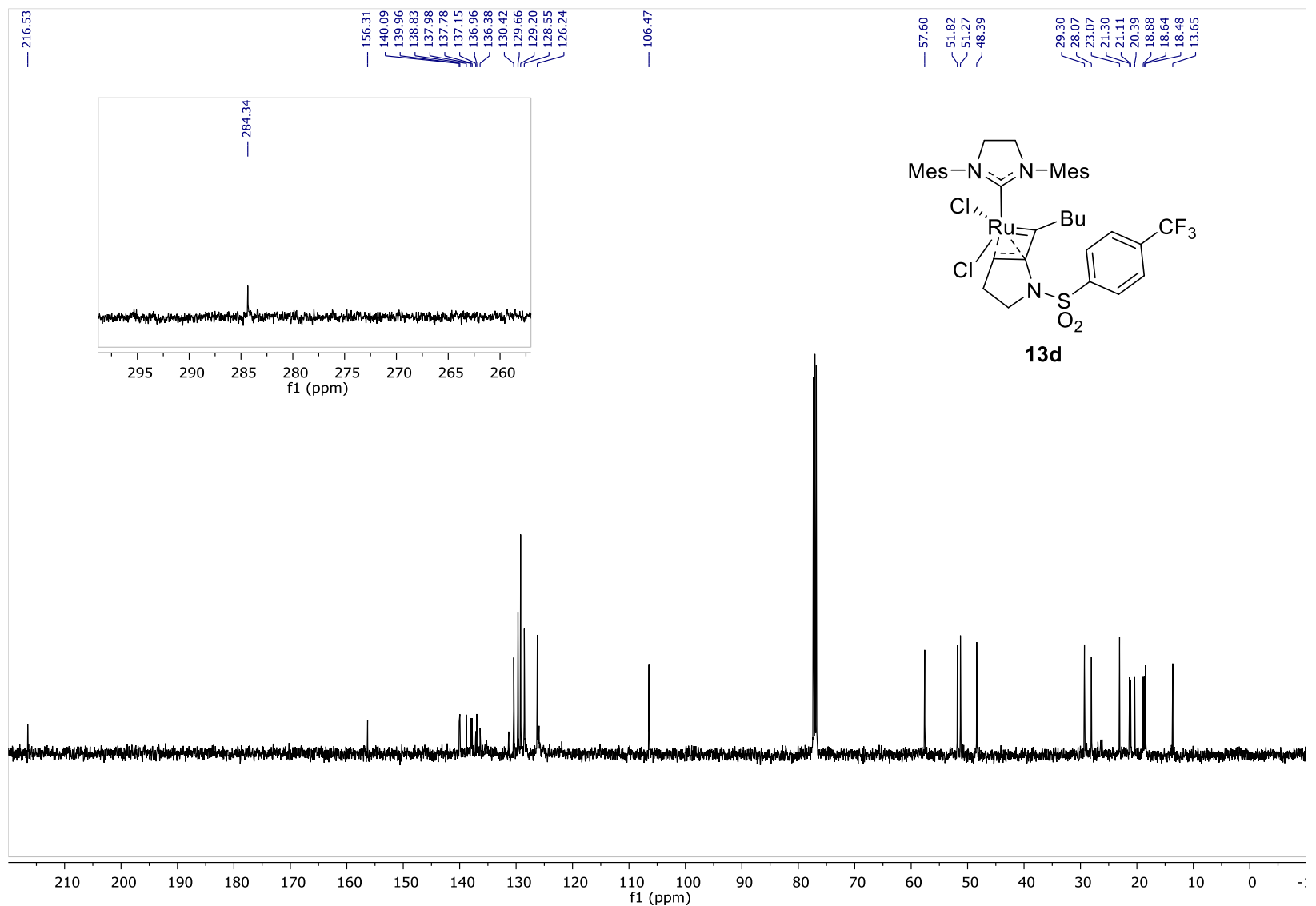



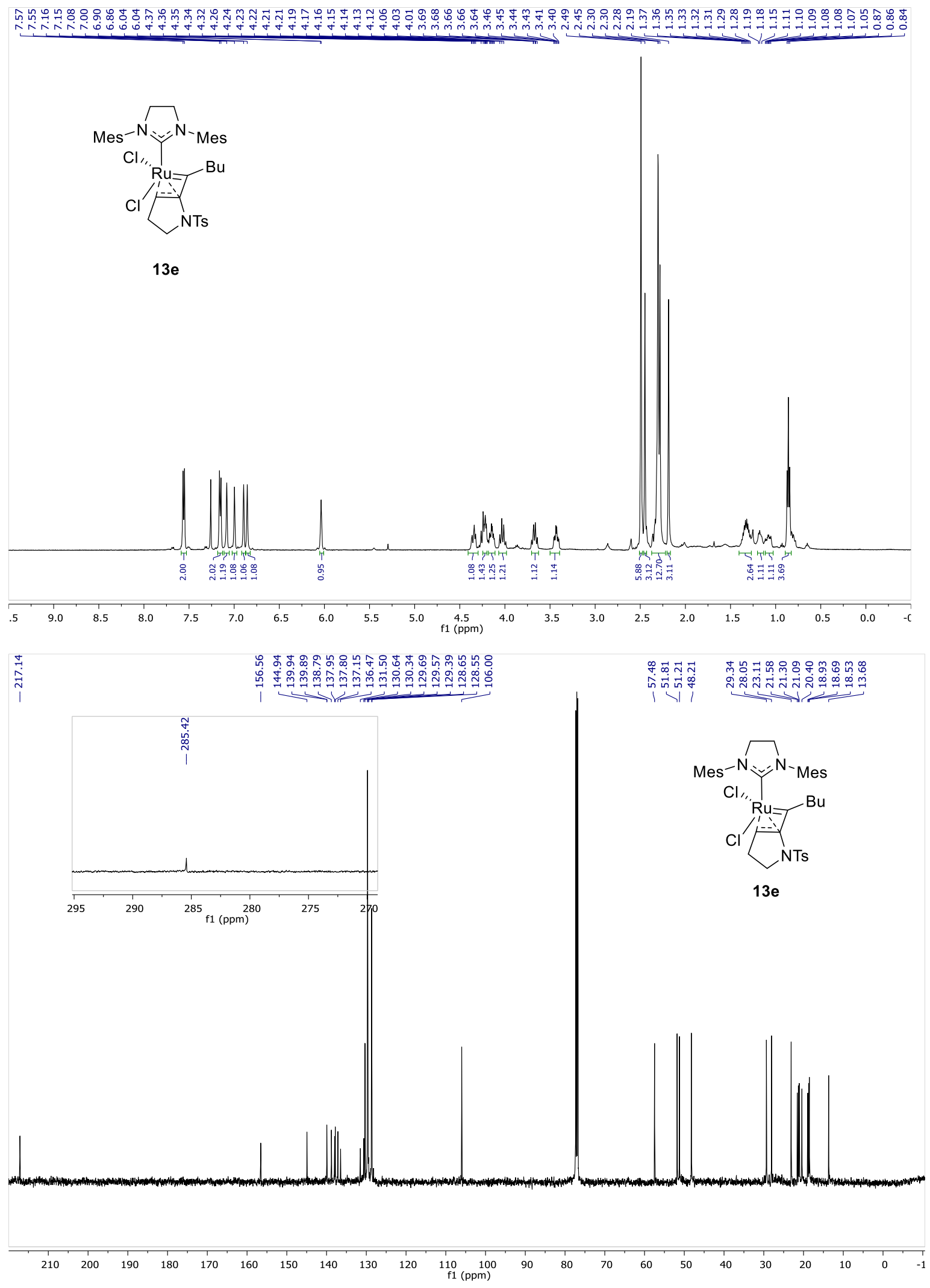

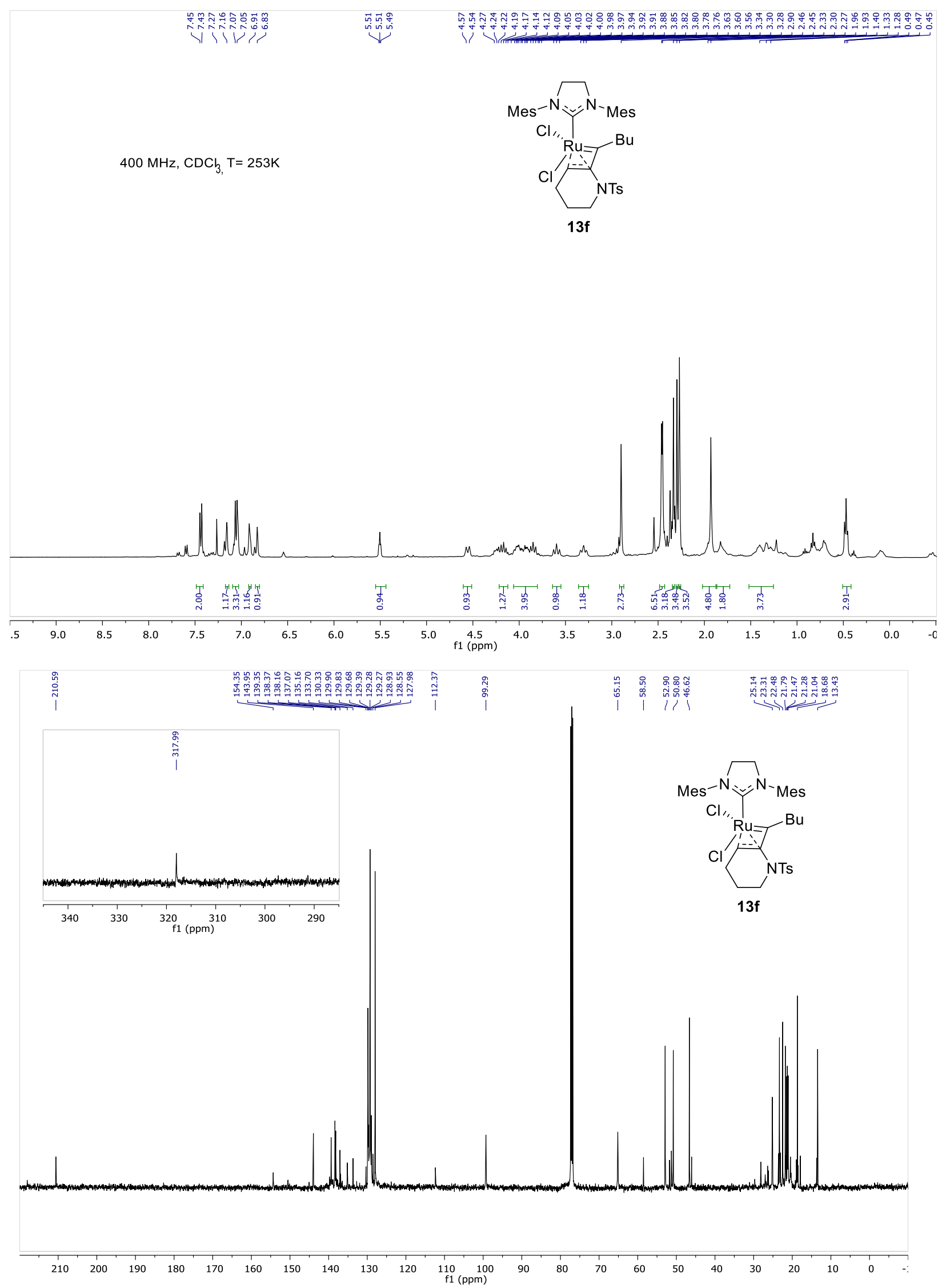


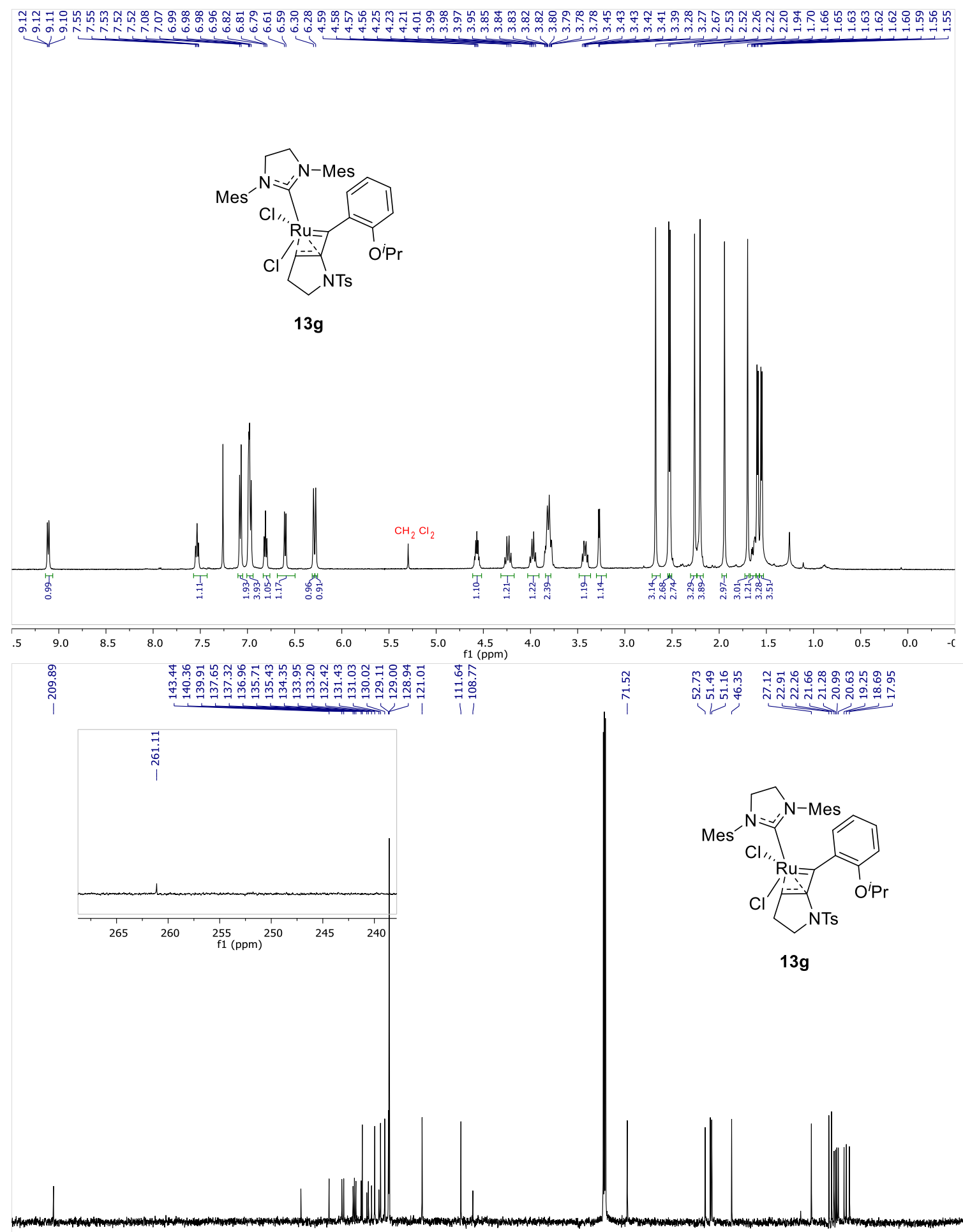

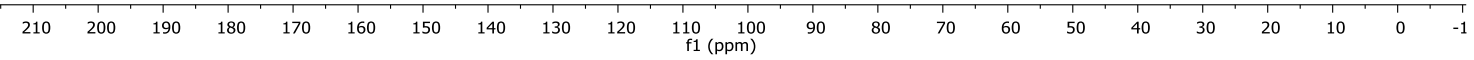




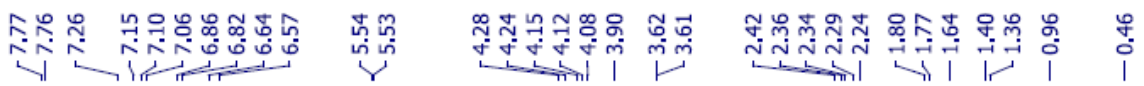
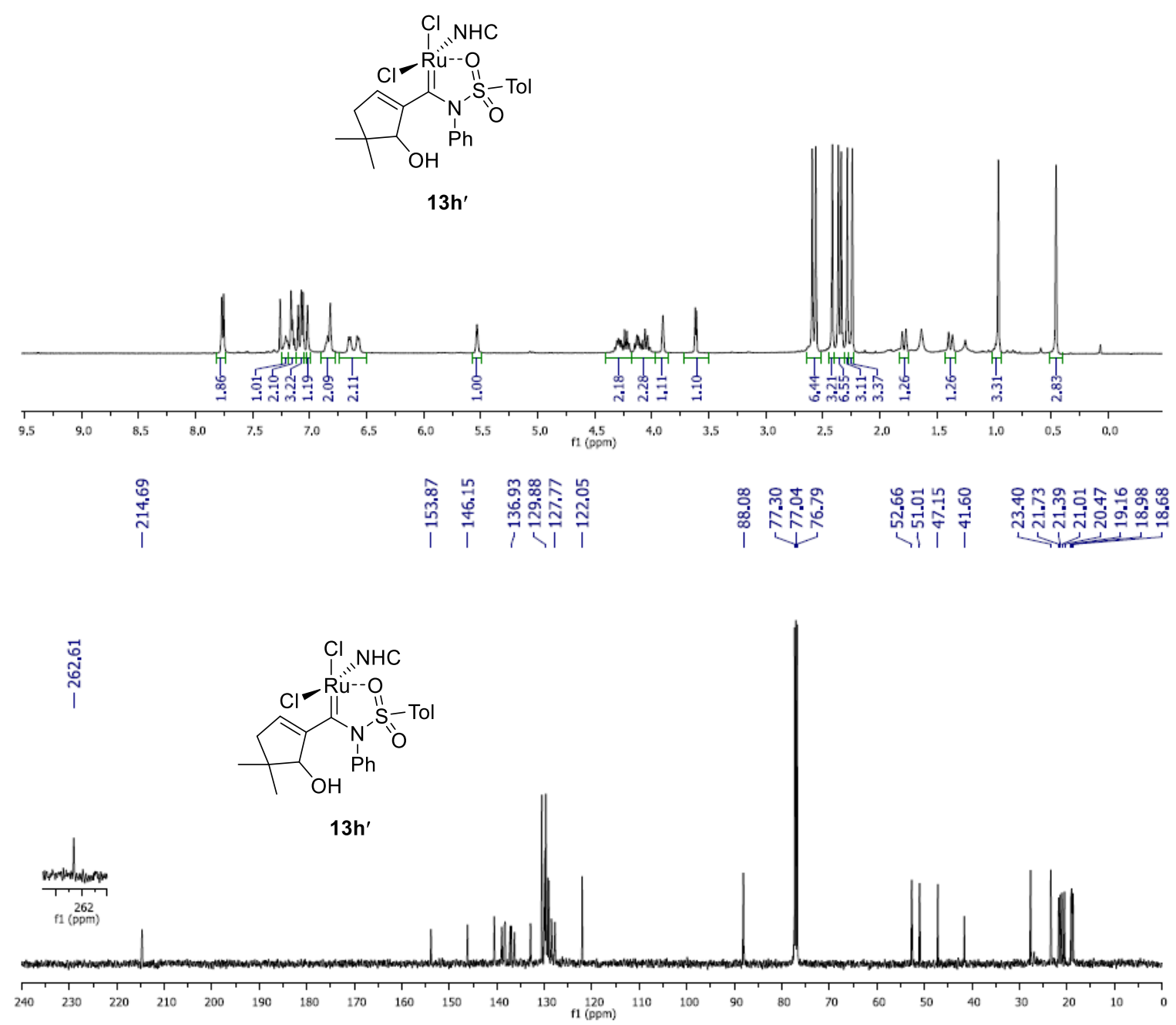


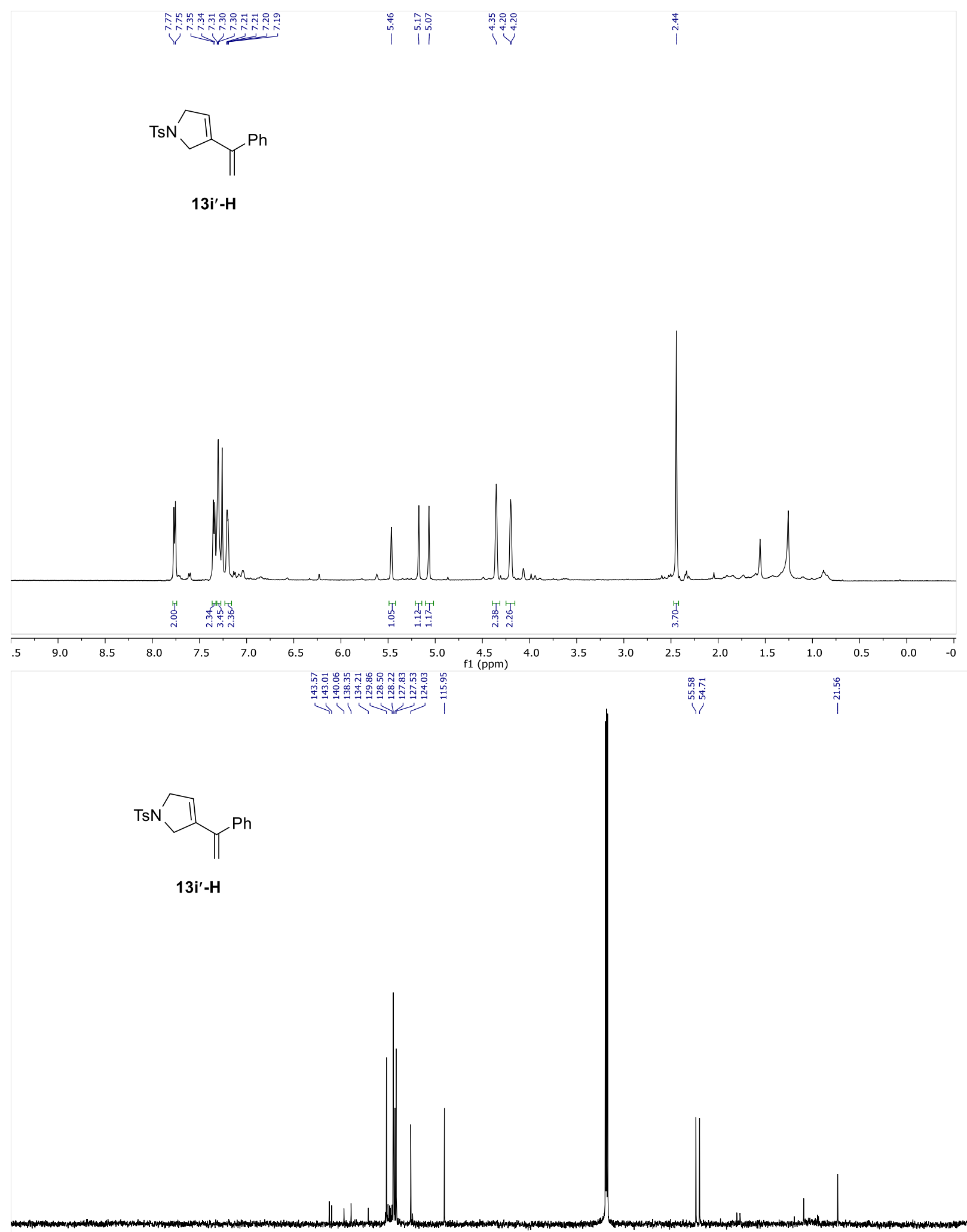

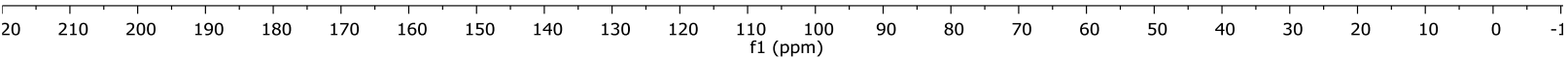



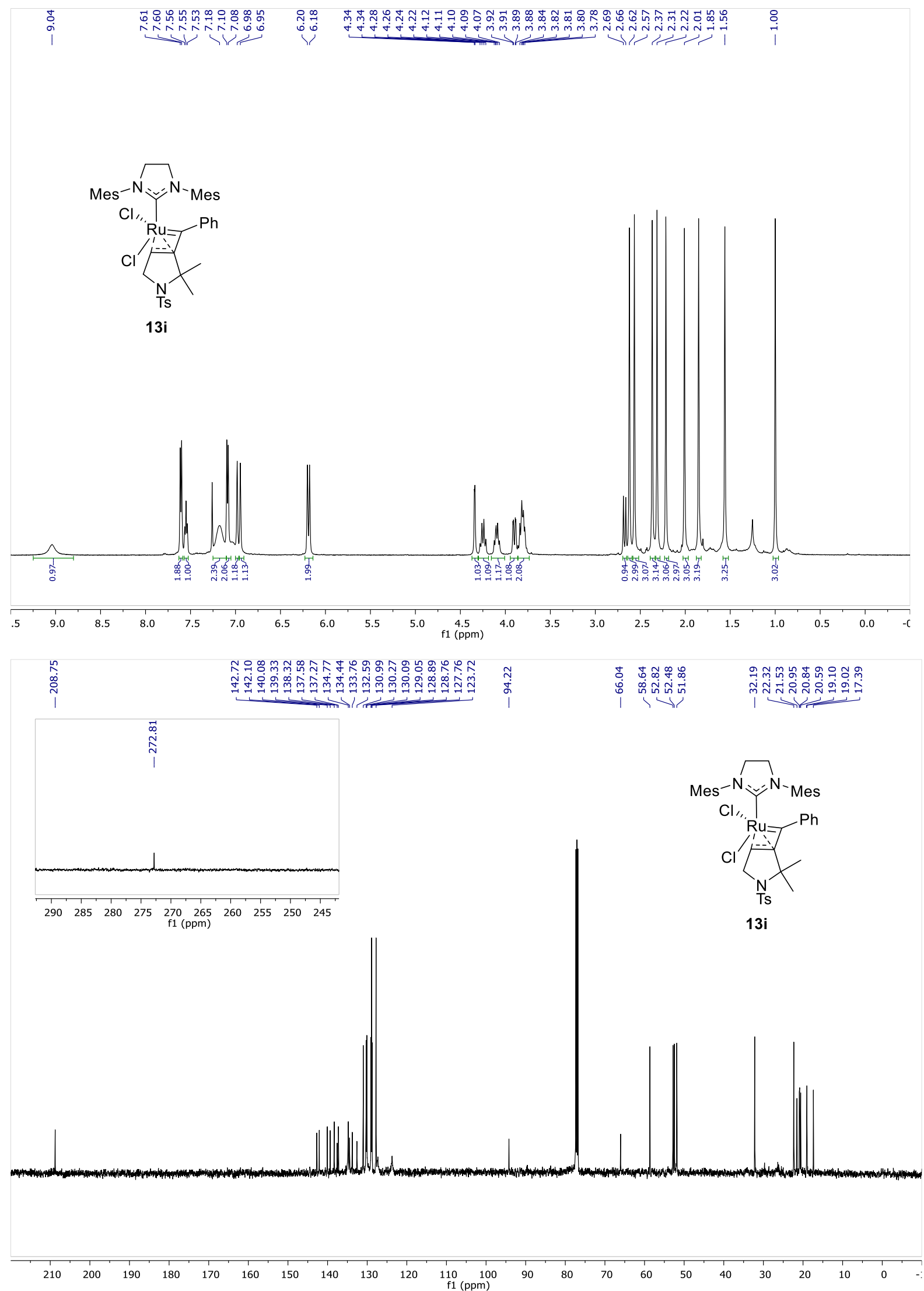


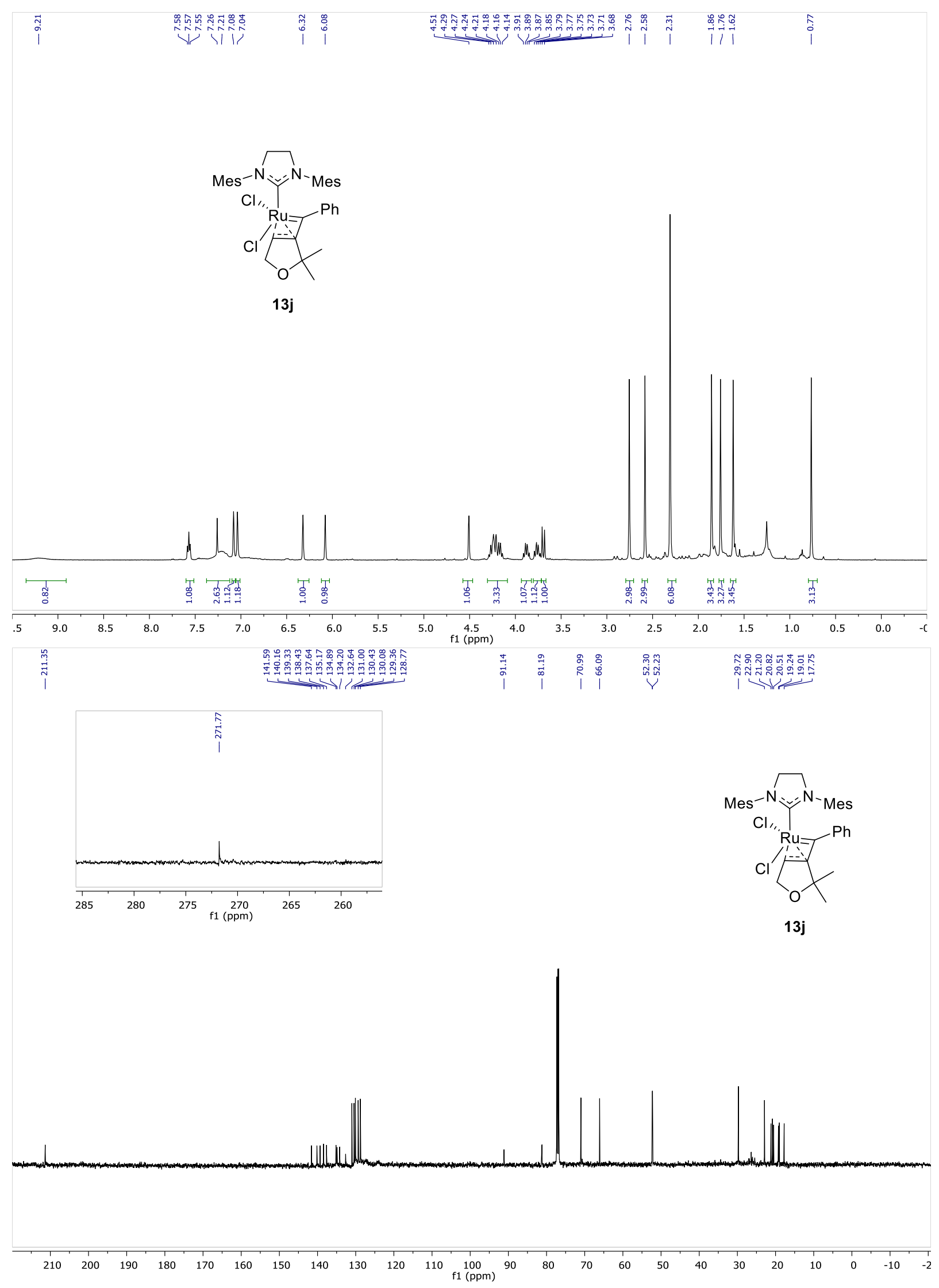



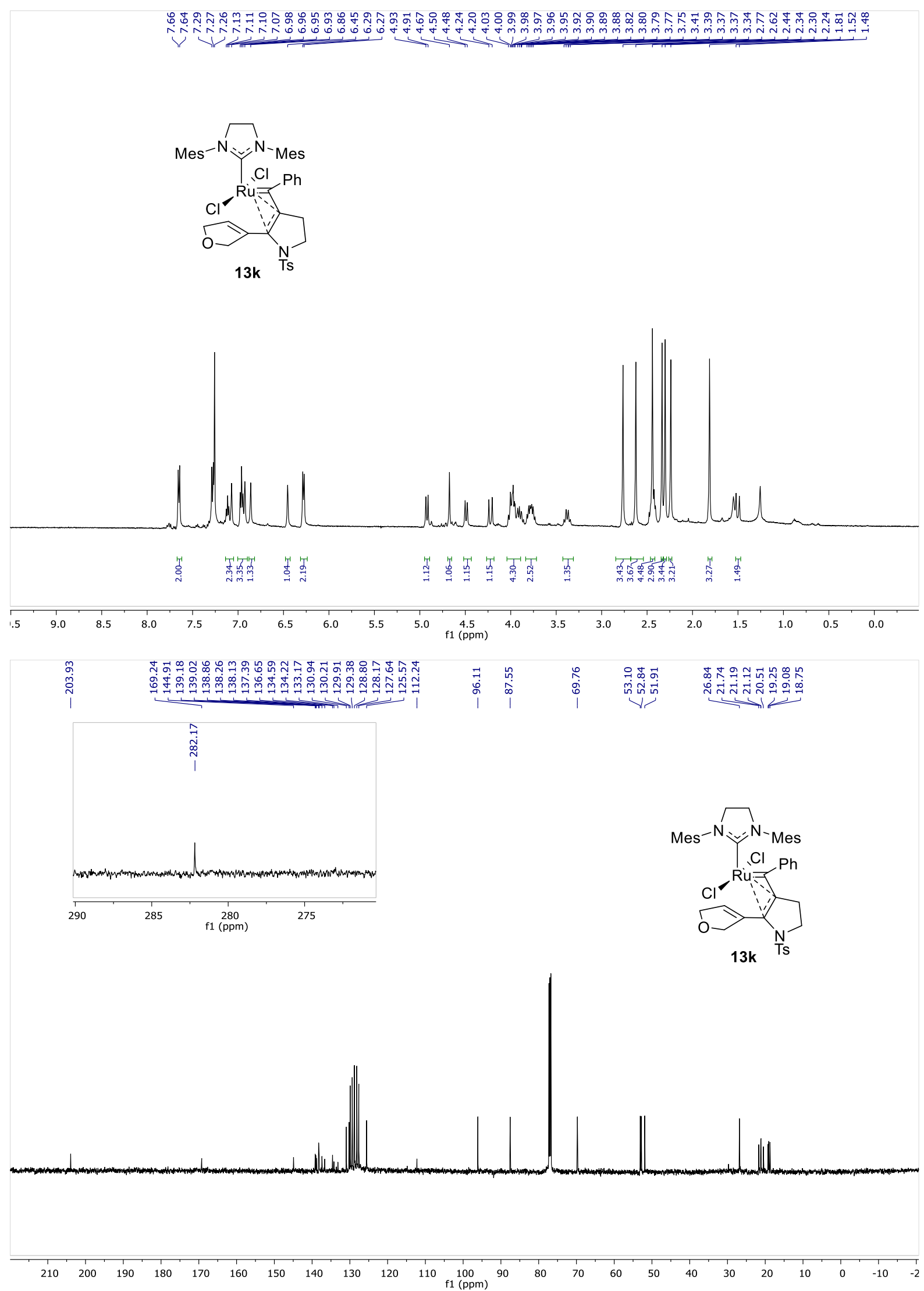


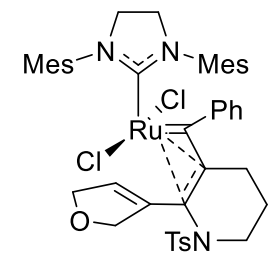

13I
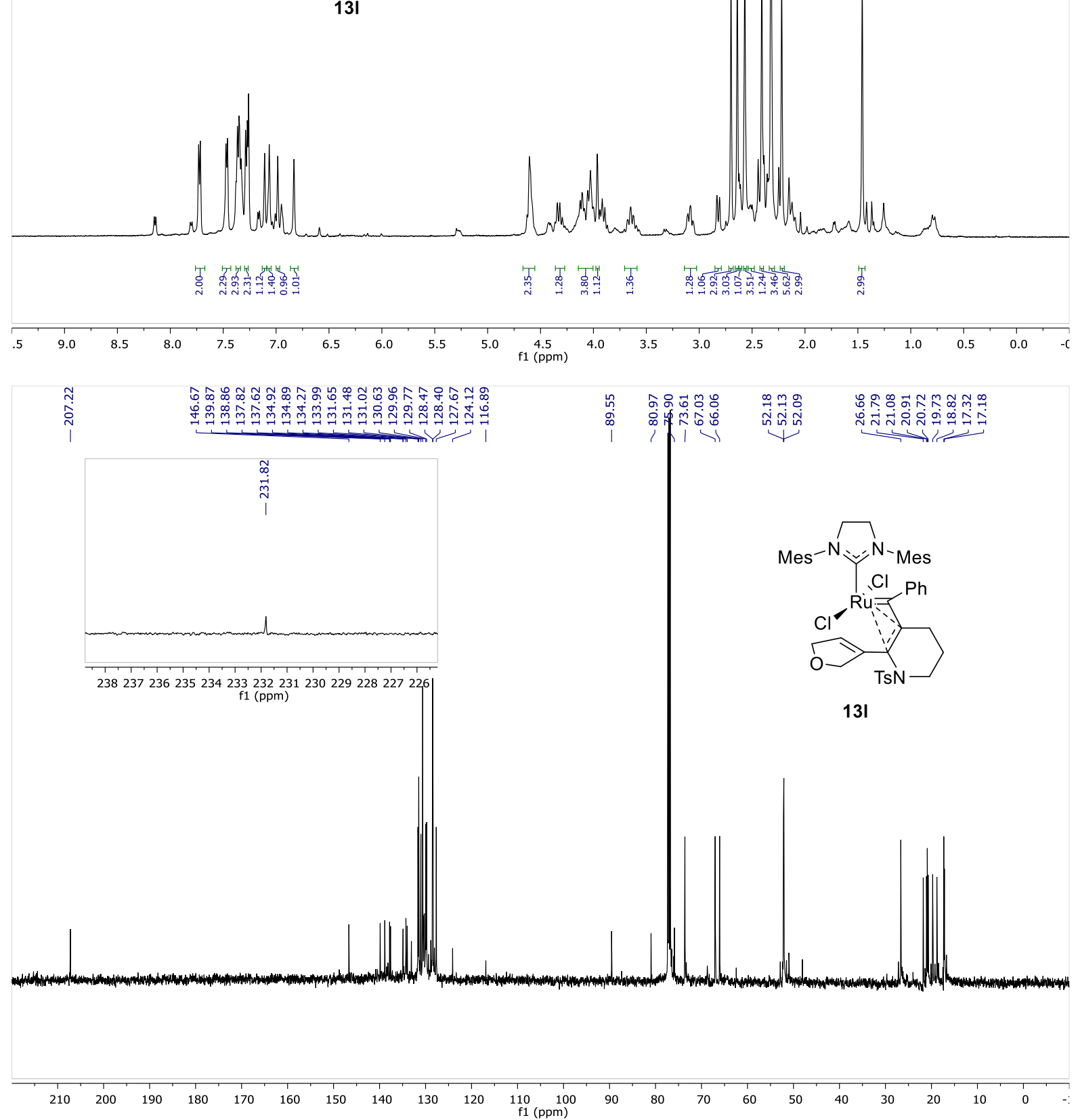


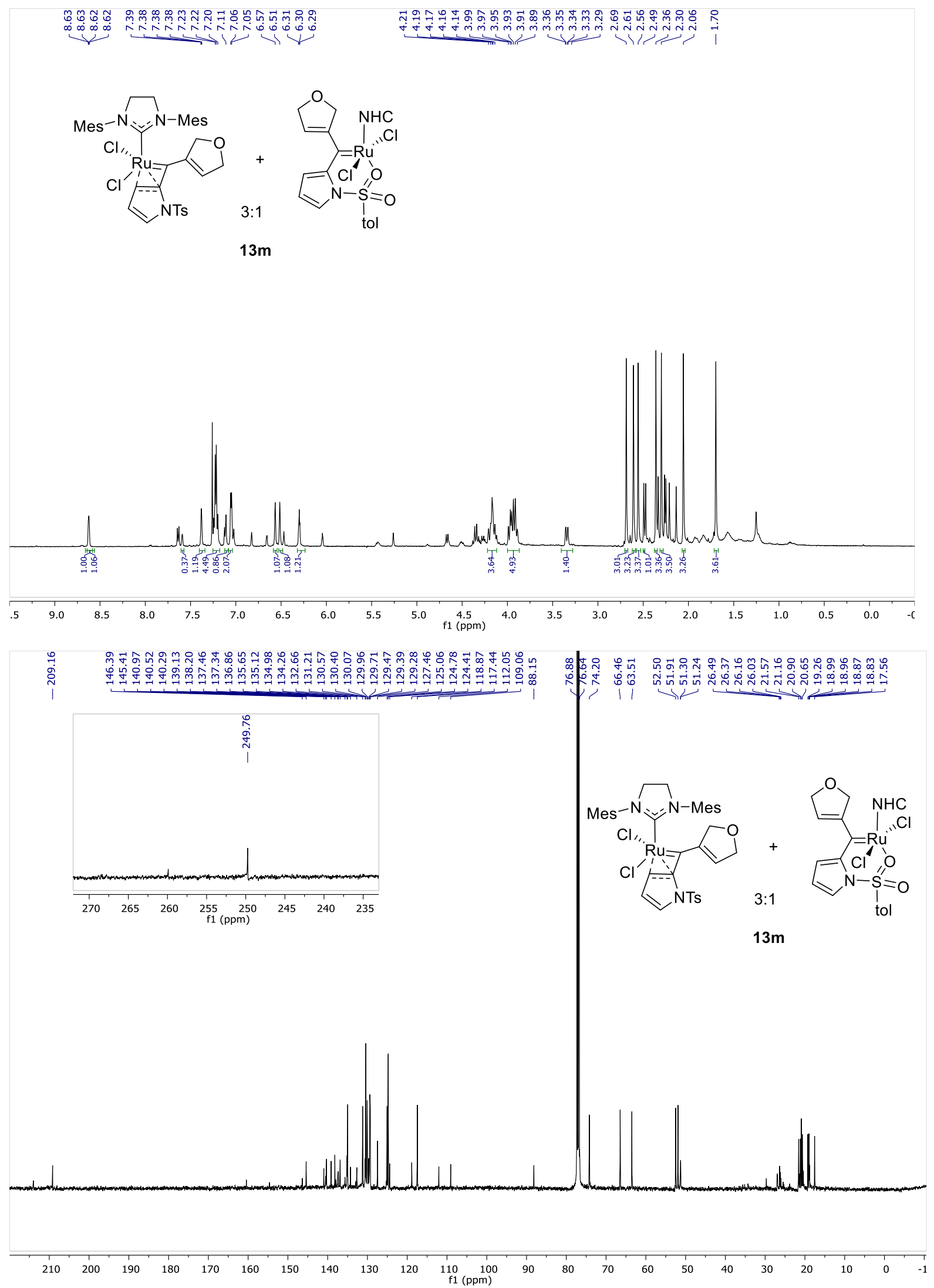




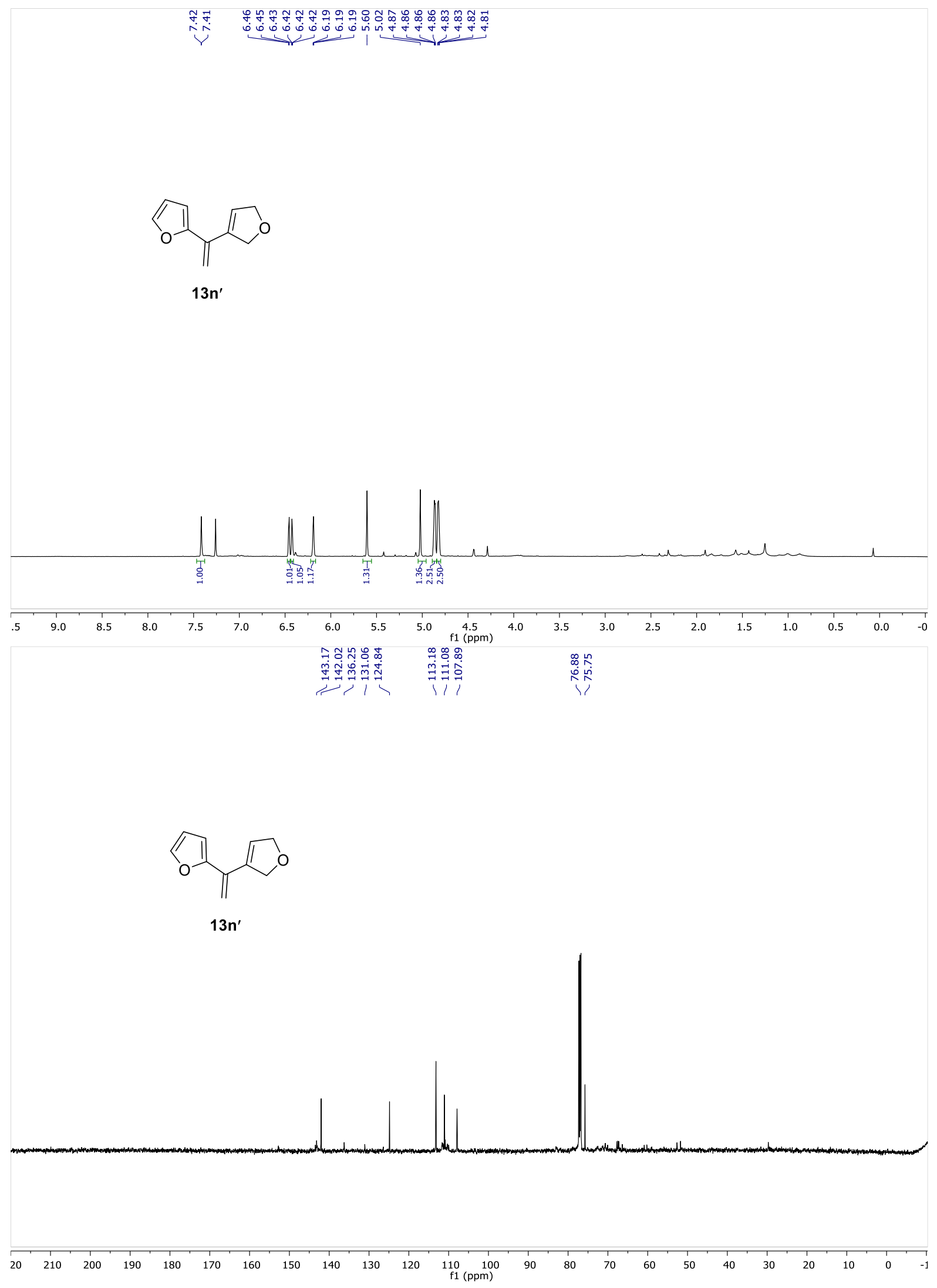




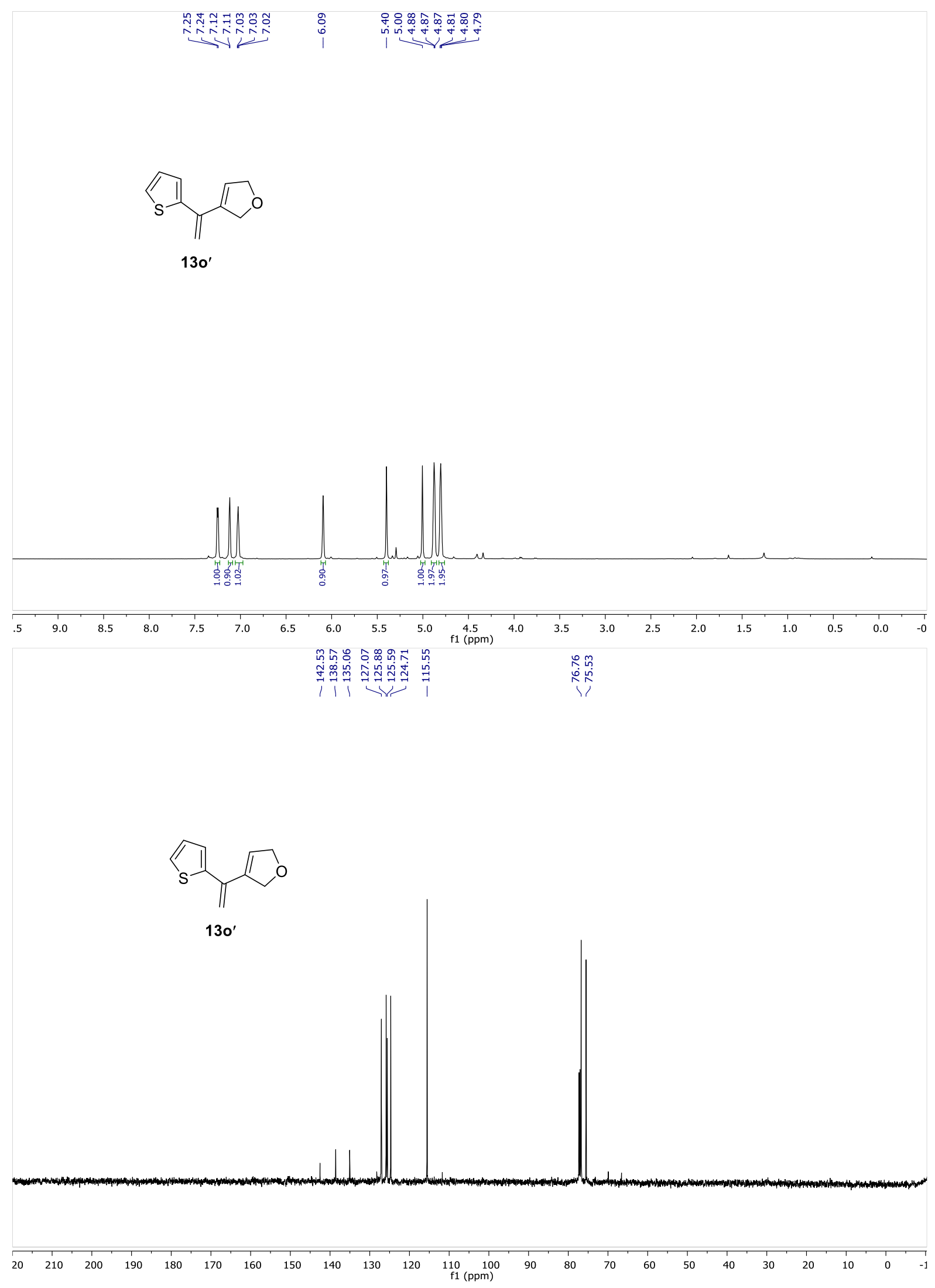



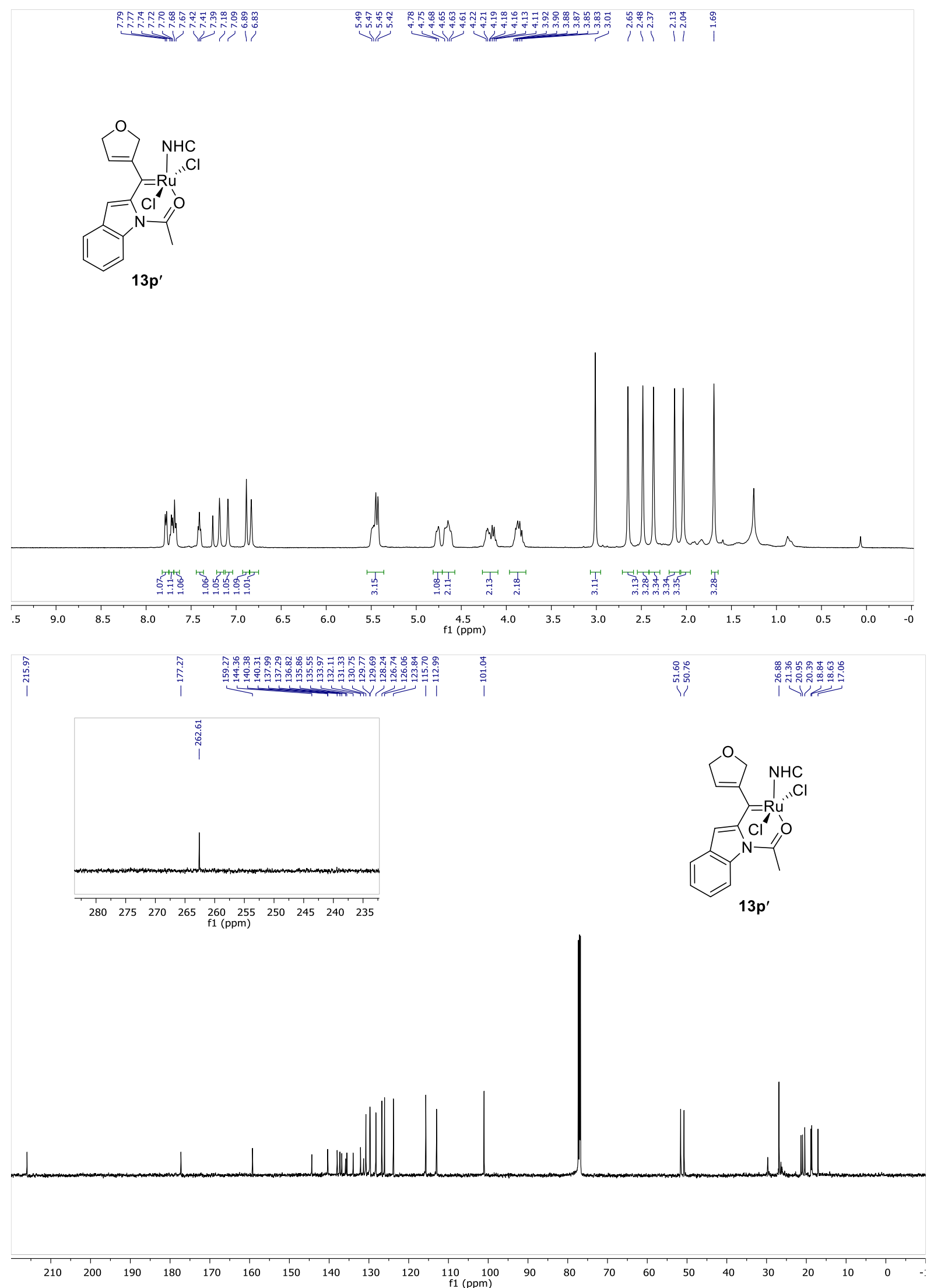


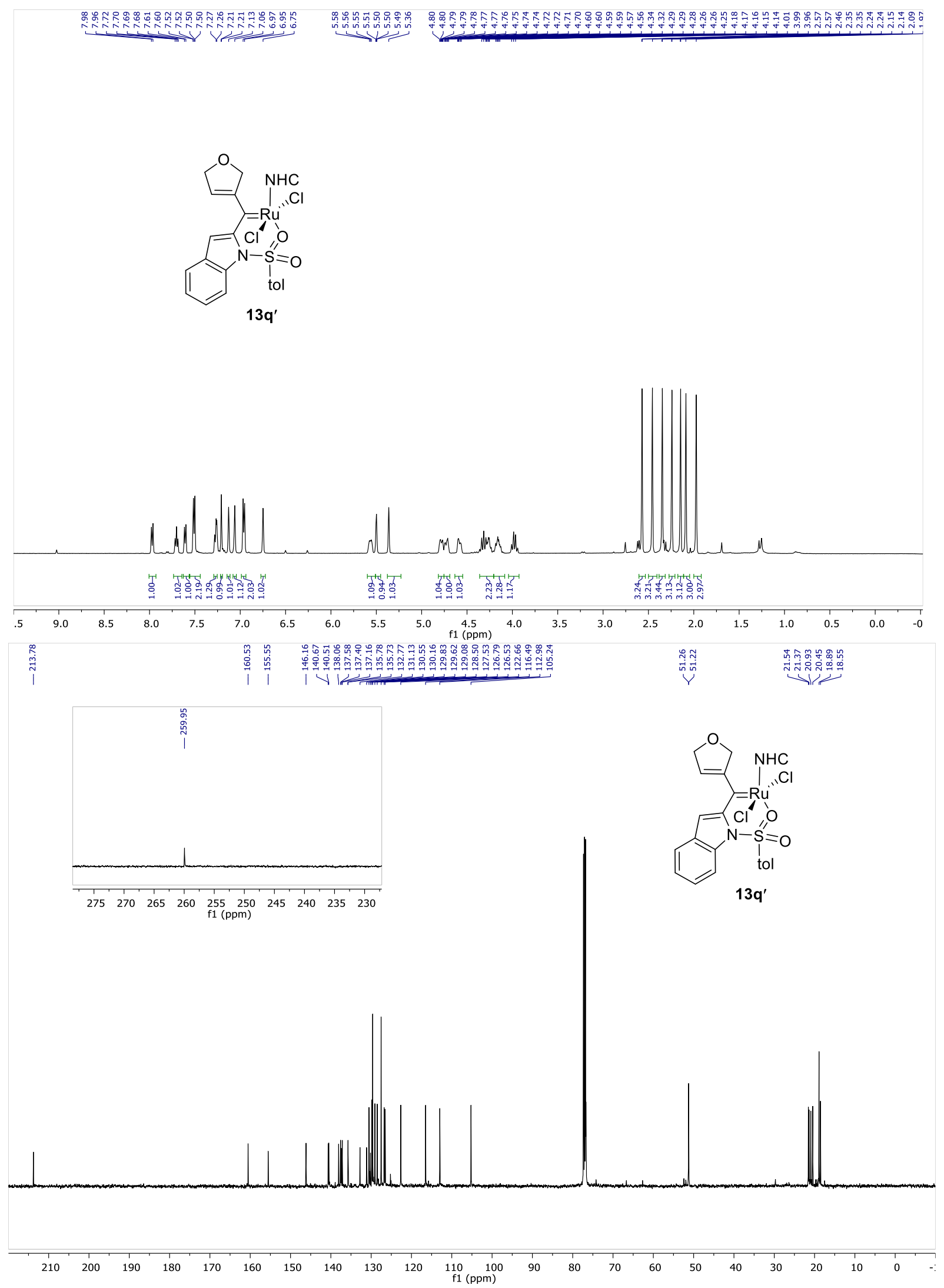



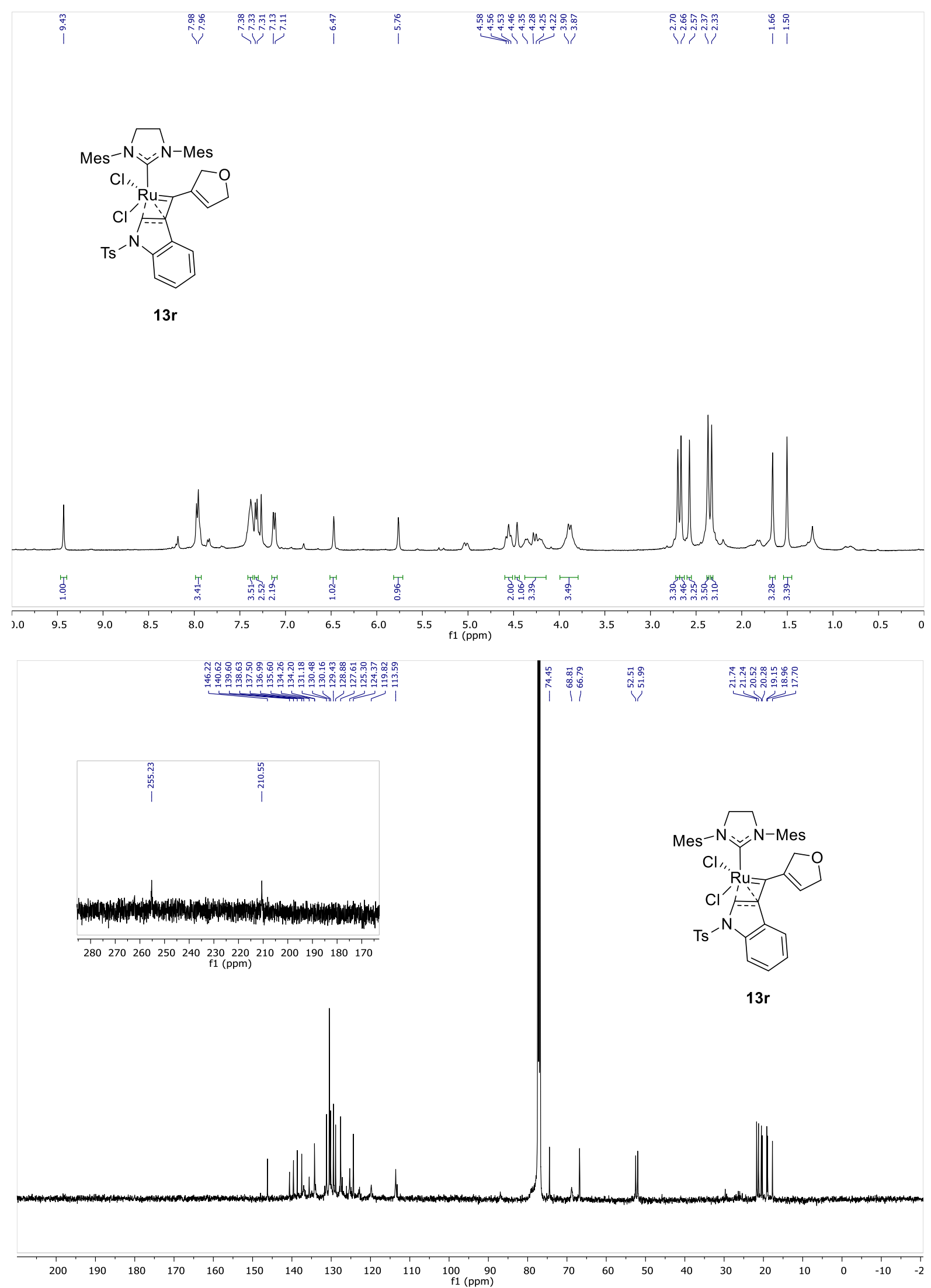

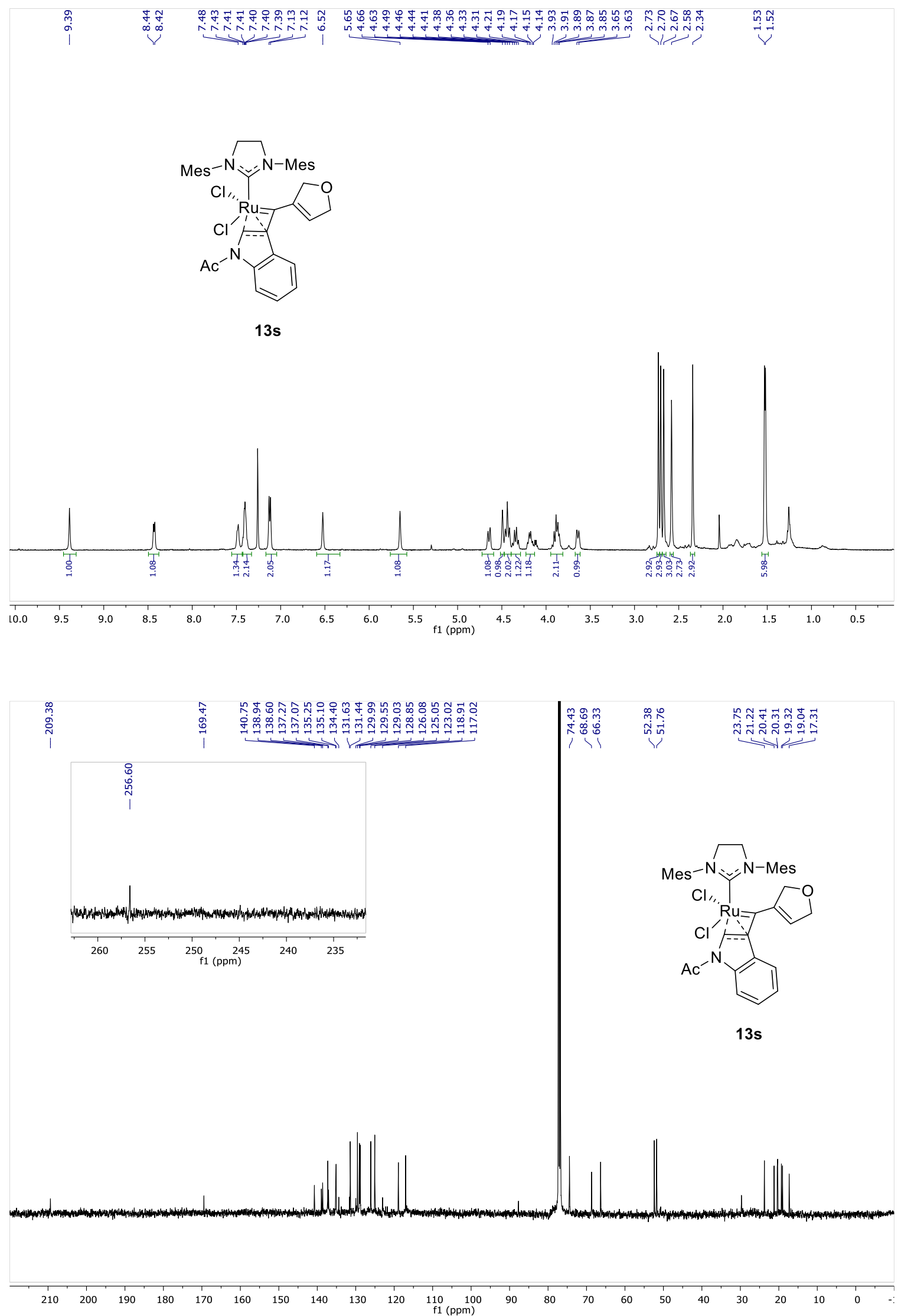

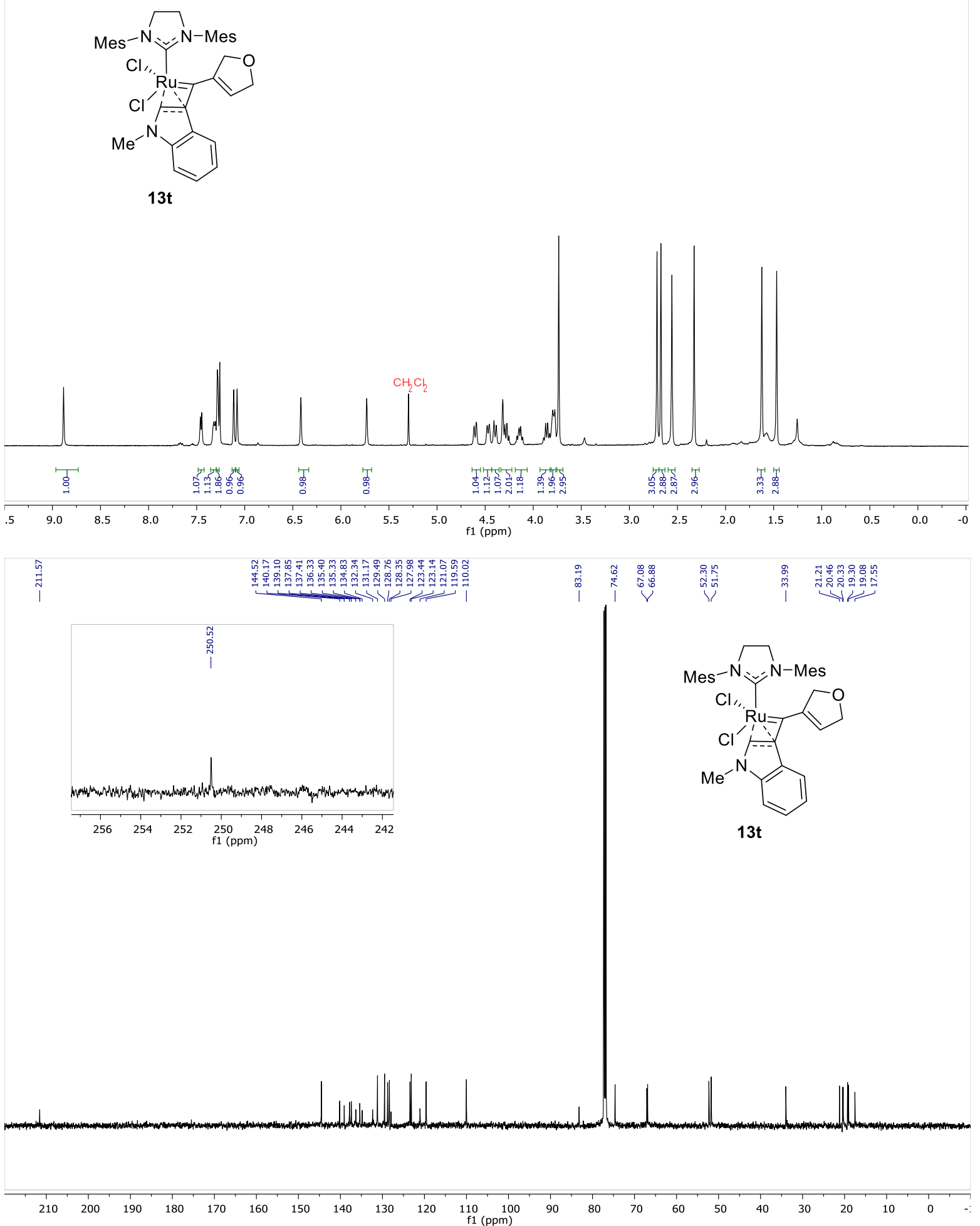

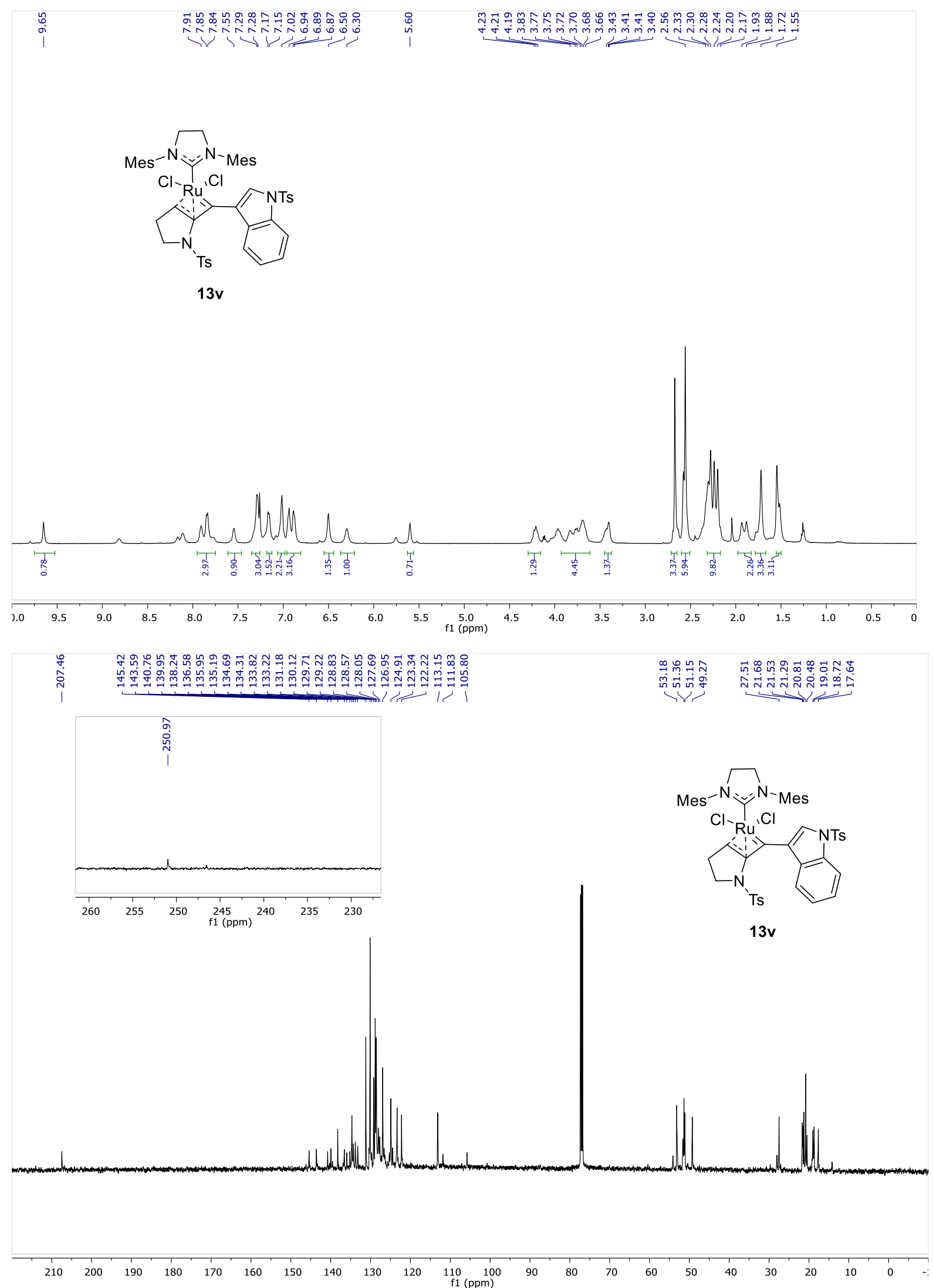


\section{X-ray Crystallographic Data}

All X-Ray data can be obtained free of charge from The Cambridge Crystallographic Data Centre via www.ccdc.cam.ac.uk/data request/cif.

\section{Summary of Xray-Crystallographic data}

\begin{tabular}{|c|c|c|c|}
\hline Crystal data & Compound 10a & Compound 10c & Compound 10d \\
\hline CCDC Deposition & 2018298 & 2021743 & 2018914 \\
\hline Chemical formula & $\mathrm{C}_{38} \mathrm{H}_{46} \mathrm{Cl}_{2} \mathrm{~N}_{2} \mathrm{Ru}$ & $\underline{\mathrm{C}}_{42} \underline{\mathrm{H}}_{44} \underline{\mathrm{Cl}}_{2} \underline{\mathrm{FeN}}_{2} \underline{\mathrm{Ru}}$ & $\underline{\mathrm{C}}_{41} \underline{\mathrm{H}}_{52} \mathrm{Cl}_{2} \underline{\mathrm{N}}_{2} \mathrm{ORu}$ \\
\hline$M_{\mathrm{r}}$ & 702.74 & 804.61 & $\underline{760.81}$ \\
\hline $\begin{array}{l}\text { Crystal system, space } \\
\text { group }\end{array}$ & Monoclinic, $P 2_{1}$ & Triclinic, $\underline{P}-1$ & Triclinic, $\underline{P}-1$ \\
\hline Temperature (K) & 200 & $\underline{200}$ & $\underline{200}$ \\
\hline$a, b, c(\AA)$ & $\begin{array}{c}11.9816(10), \\
10.5576(7), \\
13.8098(12)\end{array}$ & $\frac{12.269(4)}{(4), 13.206}$ & $\frac{10.2526(9)}{\underline{(13)}, \underline{14.9304(15)}}$ \\
\hline$\alpha, b, \gamma\left(^{\circ}\right)$ & $90,93.972(3), 90$ & $\frac{103.978(8)}{(9), 116.864(7)}$ & $\frac{75.270(3)}{(3), \frac{77.074}{87.610(3)}}$ \\
\hline$V\left(\AA^{3}\right)$ & $1742.7(2)$ & $1862.7(10)$ & $1864.5(3)$ \\
\hline Z & 2 & $\underline{2}$ & $\underline{2}$ \\
\hline Radiation type & Mo $K \alpha$ & Mo $K \alpha$ & Mo $K \alpha$ \\
\hline$\mu\left(\mathrm{mm}^{-1}\right)$ & 0.63 & $\underline{0.97}$ & $\underline{0.60}$ \\
\hline Crystal size (mm) & $0.60 \times 0.32 \times 0.16$ & $\underline{0.60} \times \underline{0.22} \times \underline{0.20}$ & $\underline{0.6} \times \underline{0.32} \times \underline{0.2}$ \\
\hline Diffractometer & $\begin{array}{c}\text { Bruker SMART } \\
\text { X2S }\end{array}$ & Bruker X2S & Bruker SMART X2S \\
\hline Absorption correction & Multi-scan & Multi-scan & Multi-Scan \\
\hline$T_{\min }, T_{\max }$ & $0.64,0.91$ & $\underline{0.594}, \underline{0.830}$ & $0.71,0.89$ \\
\hline $\begin{array}{c}\text { No. of measured, } \\
\text { independent and } \\
\text { observed }[I>2 \sigma(I)] \\
\text { reflections }\end{array}$ & $\begin{array}{c}17704,5926 \\
5252\end{array}$ & $\underline{23566}, \underline{6332}, \underline{5072}$ & $\underline{33272}, \underline{6550}, \underline{5893}$ \\
\hline$R_{\text {int }}$ & 0.060 & $\underline{0.108}$ & $\underline{0.043}$ \\
\hline$(\sin \vartheta / \lambda)_{\max }\left(\AA^{-1}\right)$ & 0.596 & 0.596 & 0.598 \\
\hline$R\left[F^{2}>2 \sigma\left(F^{2}\right)\right], w R\left(F^{2}\right), S$ & $0.036,0.115,0.60$ & $\underline{0.152}, \underline{0.391}, \underline{2.54}$ & $\underline{0.035}, \underline{0.107}, \underline{1.09}$ \\
\hline No. of reflections & 5926 & $\underline{6332}$ & $\underline{6550}$ \\
\hline No. of parameters & 396 & $\underline{460}$ & $\underline{434}$ \\
\hline No. of restraints & 1 & 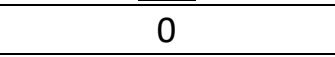 & 0 \\
\hline $\mathrm{H}$-atom treatment & $\begin{array}{c}\mathrm{H} \text {-atom } \\
\text { parameters } \\
\text { constrained }\end{array}$ & $\frac{\text { H-atom parameters }}{\text { constrained }}$ & $\frac{\mathrm{H} \text {-atom parameters }}{\text { constrained }}$ \\
\hline$\Delta \rho_{\max }, \Delta \rho_{\min }\left(\mathrm{e} \AA^{-3}\right)$ & $0.45,-0.59$ & $\underline{2.78},-3.89$ & $\underline{0.81}, \underline{-0.62}$ \\
\hline $\begin{array}{l}\text { Absolute structure } \\
\text { parameter }\end{array}$ & $-0.02(2)$ & $\mathrm{N} / \mathrm{A}$ & $\mathrm{N} / \mathrm{A}$ \\
\hline
\end{tabular}




\begin{tabular}{|c|c|c|c|}
\hline Crystal data & Compound 10l & Compound 10q & Compound 10r \\
\hline CCDC Deposition & 2018302 & 2018588 & 2018303 \\
\hline Chemical formula & $\mathrm{C}_{48} \mathrm{H}_{52} \mathrm{Cl}_{4} \mathrm{~N}_{2} \mathrm{ORuSi}$ & $\mathrm{C}_{48} \mathrm{H}_{57} \mathrm{Cl}_{2} \mathrm{~N}_{3} \mathrm{O}_{3} \mathrm{RuS}$ & $\mathrm{C}_{55} \mathrm{H}_{67} \mathrm{Cl}_{2} \mathrm{~N}_{3} \mathrm{O}_{6} \mathrm{RuS}$ \\
\hline$M_{\mathrm{r}}$ & 943.87 & 927.99 & 1070.14 \\
\hline $\begin{array}{l}\text { Crystal system, } \\
\text { space group }\end{array}$ & $\begin{array}{l}\text { Orthorhombic, } \\
{\text { Pna } 2_{1}}\end{array}$ & Monoclinic, $P 2_{1} / c$ & Triclinic, $P-1$ \\
\hline Temperature (K) & 200 & 200 & 200 \\
\hline$a, b, c(\AA)$ & $\begin{array}{c}15.2754(16) \\
28.119 \text { (3), } 10.4160 \\
(9)\end{array}$ & $\begin{array}{c}12.6853(18), \\
12.842(2) \\
32.754(5) \\
\end{array}$ & $\begin{array}{l}11.0039(7), \\
12.6857(9), \\
20.6490(15) \\
\end{array}$ \\
\hline$\alpha, b, \gamma\left(^{\circ}\right)$ & $90,90,90$ & $90,96.636(4), 90$ & $\begin{array}{l}101.995(2), \\
101.394(2), \\
106.335(2)\end{array}$ \\
\hline$V\left(\AA^{3}\right)$ & $4474.0(8)$ & $5299.8(13)$ & $2603.1(3)$ \\
\hline$Z$ & 4 & 4 & 2 \\
\hline Radiation type & Mo Ka & Mo Ka & Mo $K \alpha$ \\
\hline$\mu\left(\mathrm{mm}^{-1}\right)$ & 0.65 & 0.47 & 0.50 \\
\hline Crystal size $(\mathrm{mm})$ & $0.44 \times 0.40 \times 0.07$ & $0.46 \times 0.4 \times 0.31$ & $0.40 \times 0.31 \times 0.29$ \\
\hline Diffractometer & Bruker SMART X2S & $\begin{array}{c}\text { Bruker APEX-II } \\
\text { CCD }\end{array}$ & Bruker SMART X2S \\
\hline $\begin{array}{l}\text { Absorption } \\
\text { correction }\end{array}$ & Multi-scan & Multi-scan & Multi-scan \\
\hline$T_{\min }, T_{\max }$ & $0.71,0.96$ & $0.534,0.867$ & $0.74,0.87$ \\
\hline $\begin{array}{c}\text { No. of measured, } \\
\text { independent and } \\
\text { observed }[I>2 \sigma(I)] \\
\text { reflections }\end{array}$ & $78579,7870,7294$ & $\begin{array}{c}54576,9368, \\
8058\end{array}$ & $43746,7950,7272$ \\
\hline$R_{\text {int }}$ & 0.076 & 0.058 & 0.038 \\
\hline$(\sin \vartheta / \lambda)_{\max }\left(\AA^{-1}\right)$ & 0.596 & 0.596 & 0.568 \\
\hline $\begin{array}{c}R\left[F^{2}>2 \sigma\left(F^{2}\right)\right] \\
w R\left(F^{2}\right), S\end{array}$ & $0.041,0.128,1.01$ & $0.054,0.133,1.10$ & $0.029,0.102,1.16$ \\
\hline No. of reflections & 7870 & 9368 & 7950 \\
\hline No. of parameters & 520 & 532 & 624 \\
\hline No. of restraints & 1 & 0 & 0 \\
\hline $\mathrm{H}$-atom treatment & $\begin{array}{l}\mathrm{H} \text {-atom parameters } \\
\text { constrained }\end{array}$ & $\begin{array}{c}\text { H-atom } \\
\text { parameters } \\
\text { constrained }\end{array}$ & $\begin{array}{l}\text { H-atom parameters } \\
\text { constrained }\end{array}$ \\
\hline$\Delta \rho_{\max }, \Delta \rho_{\min }\left(\mathrm{e} \AA^{-3}\right)$ & $1.70,-0.54$ & $0.59,-1.09$ & $0.94,-0.76$ \\
\hline $\begin{array}{c}\text { Absolute structure } \\
\text { parameter }\end{array}$ & $-0.036(14)$ & N/A & $\mathrm{N} / \mathrm{A}$ \\
\hline
\end{tabular}




\begin{tabular}{|c|c|c|c|}
\hline Crystal data & Compound 10r' & Compound 10s & Compound 13a \\
\hline CCDC Deposition & 2018594 & 2018596 & 2018283 \\
\hline Chemical formula & $\mathrm{C}_{59} \mathrm{H}_{75} \mathrm{Cl}_{4} \mathrm{~N}_{3} \mathrm{O}_{7} \mathrm{RuS}$ & $\mathrm{C}_{46} \mathrm{H}_{55} \mathrm{Cl}_{6} \mathrm{~N}_{3} \mathrm{O}_{2} \mathrm{RuS}$ & $\begin{array}{c}\mathrm{C}_{40} \mathrm{H}_{45} \mathrm{Cl}_{4} \mathrm{~N}_{3} \mathrm{O}_{2} \mathrm{Ru} \\
\mathrm{S}\end{array}$ \\
\hline$M_{\mathrm{r}}$ & 1213.15 & 1027.76 & 874.72 \\
\hline $\begin{array}{c}\text { Crystal system, space } \\
\text { group }\end{array}$ & Triclinic, $P-1$ & Monoclinic, $P 2_{1} / n$ & $\begin{array}{c}\text { Monoclinic, } \\
P 2_{1} / c\end{array}$ \\
\hline Temperature (K) & 200 & 200 & 300 \\
\hline$a, b, c(\AA)$ & $\begin{array}{c}12.580(3), 13.814 \\
(4), 19.537(5)\end{array}$ & $\begin{array}{c}15.3127(15), \\
13.8064(11), \\
22.985(2) \\
\end{array}$ & $\begin{array}{l}8.471(2), \\
26.951(8), \\
18.341(6) \\
\end{array}$ \\
\hline$\alpha, b, \gamma\left({ }^{\circ}\right)$ & $\begin{array}{c}90.610(9), \\
105.904(8), \\
113.609(8)\end{array}$ & $90,91.300(3), 90$ & $\begin{array}{c}90,97.843(9), \\
90\end{array}$ \\
\hline$V\left(\AA^{3}\right)$ & $2963.8(13)$ & $4858.1(8)$ & $4148(2)$ \\
\hline$Z$ & 2 & 4 & 4 \\
\hline Radiation type & Mo $K \alpha$ & Mo $K \alpha$ & Mo $K \alpha$ \\
\hline$\mu\left(\mathrm{mm}^{-1}\right)$ & 0.53 & 0.74 & 0.72 \\
\hline Crystal size $(\mathrm{mm})$ & $0.60 \times 0.38 \times 0.16$ & $0.60 \times 0.53 \times 0.07$ & $\begin{array}{c}0.60 \times 0.20 \times \\
0.20\end{array}$ \\
\hline Diffractometer & Bruker SMART X2S & Bruker SMART X2S & $\begin{array}{c}\text { Bruker SMART } \\
\text { X2S }\end{array}$ \\
\hline Absorption correction & Multi-scan & Multi-scan & Multi-scan \\
\hline$T_{\min }, T_{\max }$ & $0.49,0.92$ & $0.63,0.95$ & $0.67,0.87$ \\
\hline $\begin{array}{c}\text { No. of measured, } \\
\text { independent and } \\
\text { observed }[I>2 \sigma(I)] \\
\text { reflections }\end{array}$ & $\begin{array}{c}60461,12790 \\
8360\end{array}$ & $81174,8620,6868$ & $\begin{array}{c}17685,7945 \\
7945\end{array}$ \\
\hline$R_{\text {int }}$ & 0.169 & 0.124 & 0.134 \\
\hline$(\sin \vartheta / \lambda)_{\max }\left(\AA^{-1}\right)$ & 0.641 & 0.598 & 0.625 \\
\hline $\begin{array}{c}R\left[F^{2}>2 \sigma\left(F^{2}\right)\right], w R\left(F^{2}\right) \\
S\end{array}$ & $0.087,0.242,1.20$ & $0.053,0.170,1.09$ & $\begin{array}{c}0.134,0.325 \\
1.25 \\
\end{array}$ \\
\hline No. of reflections & 12790 & 8620 & 7945 \\
\hline No. of parameters & 689 & 541 & 468 \\
\hline No. of restraints & 0 & 0 & 146 \\
\hline $\mathrm{H}$-atom treatment & $\begin{array}{c}\mathrm{H} \text {-atom } \\
\text { parameters } \\
\text { constrained } \\
\end{array}$ & $\begin{array}{l}\mathrm{H} \text {-atom parameters } \\
\text { constrained }\end{array}$ & $\begin{array}{c}\mathrm{H} \text {-atom } \\
\text { parameters } \\
\text { constrained }\end{array}$ \\
\hline$\Delta \rho_{\max }, \Delta \rho_{\min }\left(\mathrm{e} \AA^{-3}\right)$ & $1.45,-1.68$ & $3.03,-0.77$ & $2.28,-2.31$ \\
\hline $\begin{array}{l}\text { Absolute structure } \\
\text { parameter }\end{array}$ & $\mathrm{N} / \mathrm{A}$ & $\mathrm{N} / \mathrm{A}$ & $\mathrm{N} / \mathrm{A}$ \\
\hline
\end{tabular}




\begin{tabular}{|c|c|c|c|}
\hline Crystal data & Compound 13b & Compound 13g & Compound 13h' \\
\hline CCDC Deposition & 2018831 & 2018917 & 2021918 \\
\hline Chemical formula & $\underline{\mathrm{C}}_{41} \underline{\mathrm{H}}_{47} \underline{\mathrm{Cl}}_{4} \underline{\mathrm{N}}_{3} \underline{\mathrm{O}}_{2} \underline{\mathrm{RuS}}$ & $\underline{\mathrm{C}}_{43} \underline{\mathrm{H}}_{51} \mathrm{Cl}_{4} \underline{\mathrm{N}}_{3} \underline{\mathrm{O}}_{3}{ }_{\mathrm{RuS}}$ & $\underline{\mathrm{C}}_{43} \underline{\mathrm{H}}_{51} \mathrm{Cl}_{4} \underline{\mathrm{N}}_{3} \mathrm{O}_{3} \mathrm{RuS}$ \\
\hline$M_{\mathrm{r}}$ & 888.74 & $\underline{932.80}$ & $\underline{932.80}$ \\
\hline $\begin{array}{l}\text { Crystal system, } \\
\text { space group }\end{array}$ & $\frac{\text { Orthorhombic, }}{P b c a}$ & $\underline{\text { Monoclinic }}, \underline{P 2_{1} / n}$ & Monoclinic, $\underline{P 2} 1 / n$ \\
\hline Temperature (K) & $\underline{\underline{200}}$ & $\underline{\underline{296}}$ & $\underline{\underline{300}}$ \\
\hline$a, b, c(\AA)$ & $\frac{21.747(7)}{(5), 23.171(8)}$ & $\begin{array}{l}\frac{13.6581(8),}{10.7783(6),} \\
\underline{30.3452(16)} \\
\end{array}$ & $\begin{array}{l}\frac{13.3662(8),}{19.3514(12),} \\
18.2778(11) \\
\end{array}$ \\
\hline$\alpha, \beta, v\left(^{\circ}\right)$ & $90,90,90$ & $90,96.306(1), 90$ & $90,107.451(2), 90$ \\
\hline$V\left(\AA^{3}\right)$ & $8307(5)$ & $4440.1(4)$ & $4510.0(5)$ \\
\hline Z & 8 & $\underline{4}$ & $\underline{4}$ \\
\hline Radiation type & Mo $K \alpha$ & Mo $K \alpha$ & Mo $K \alpha$ \\
\hline$\mu\left(\mathrm{mm}^{-1}\right)$ & $\underline{0.72}$ & $\underline{0.68}$ & $\underline{0.67}$ \\
\hline Crystal size $(\mathrm{mm})$ & $\underline{0.60} \times \underline{0.26} \times \underline{0.05}$ & $\underline{0.25} \times \underline{0.18} \times \underline{0.10}$ & $\underline{0.40} \times \underline{0.40} \times \underline{0.40}$ \\
\hline Diffractometer & Bruker SMART X2S & $\overline{\mathrm{CCD}}$ & Bruker SMART X2S \\
\hline $\begin{array}{l}\text { Absorption } \\
\text { correction }\end{array}$ & Multi-scan & Multi-scan & Multi-scan \\
\hline$T_{\min }, T_{\max }$ & $\underline{0.67}, \underline{0.96}$ & $\underline{0.850,0.934}$ & $\underline{0.775}, \underline{0.775}$ \\
\hline $\begin{array}{c}\text { No. of measured, } \\
\text { independent and } \\
\text { observed }[I>2 \sigma(I)] \\
\text { reflections }\end{array}$ & $\underline{36892}, \underline{9861}, \underline{4758}$ & $\underline{37672}, \underline{7129}, \underline{5696}$ & $\underline{92644}, \underline{9210}, \underline{7407}$ \\
\hline$R_{\text {int }}$ & $\underline{0.305}$ & $\underline{0.038}$ & $\underline{0.050}$ \\
\hline$(\sin \vartheta / \lambda)_{\max }\left(\AA^{-1}\right)$ & 0.658 & 0.578 & 0.625 \\
\hline $\begin{array}{c}R\left[F^{2}>2 \sigma\left(F^{2}\right)\right] \\
w R\left(F^{2}\right), S\end{array}$ & $\underline{0.092}, \underline{0.237}, \underline{1.01}$ & $\underline{0.045}, \underline{0.134}, \underline{0.99}$ & $\underline{0.058}, \underline{0.190}, \underline{1.35}$ \\
\hline No. of reflections & $\underline{9861}$ & 7129 & $\underline{9210}$ \\
\hline No. of parameters & $\underline{476}$ & 509 & 506 \\
\hline No. of restraints & 0 & 0 & $\underline{155}$ \\
\hline $\mathrm{H}$-atom treatment & $\begin{array}{c}\begin{array}{c}\mathrm{H} \text {-atom } \\
\text { parameters } \\
\text { constrained }\end{array} \\
\end{array}$ & $\begin{array}{c}\begin{array}{c}\mathrm{H} \text {-atom } \\
\text { parameters } \\
\text { constrained }\end{array} \\
\end{array}$ & $\begin{array}{c}\begin{array}{c}\mathrm{H} \text {-atom } \\
\text { parameters } \\
\text { constrained }\end{array} \\
\end{array}$ \\
\hline$\Delta \rho_{\max }, \Delta \rho_{\min }\left(\mathrm{e} \AA^{-3}\right)$ & $\underline{1.06}, \underline{-0.84}$ & $\underline{0.50},-1.08$ & $\underline{2.14},-0.96$ \\
\hline $\begin{array}{l}\text { Absolute structure } \\
\text { parameter }\end{array}$ & N/A & N/A & $\mathrm{N} / \mathrm{A}$ \\
\hline
\end{tabular}




\begin{tabular}{|c|c|c|c|}
\hline Crystal data & Compound 13p' & Compound 13q' & Compound 13v \\
\hline CCDC Deposition & 2019504 & 2019503 & 2019480 \\
\hline Chemical formula & $\underline{\mathrm{C}}_{75} \underline{\mathrm{H}}_{78} \underline{\mathrm{Cl}_{1}} \underline{\mathrm{N}}_{6} \underline{\mathrm{O}}_{4} \underline{\mathrm{Ru}}{ }_{2}$ & $\mathrm{C}_{41} \mathrm{H}_{42} \mathrm{Cl}_{2} \mathrm{~N}_{3} \mathrm{O}_{3} \mathrm{RuS}$ & $\mathrm{C}_{48} \underline{\mathrm{H}}_{15} \mathrm{Cl}_{2} \underline{\mathrm{N}}_{4} \underline{\mathrm{O}}_{4}{ }_{2} \mathrm{RuS}_{2}$ \\
\hline$M_{\mathrm{r}}$ & 1684.07 & 828.81 & 984.02 \\
\hline $\begin{array}{c}\text { Crystal system, space } \\
\text { group }\end{array}$ & Monoclinic, $P 2_{1} / n$ & Monoclinic, $\underline{P 2} 1 / C$ & Monoclinic, $P 2{ }_{1} / n$ \\
\hline Temperature (K) & $\underline{296}$ & $\underline{296}$ & $\underline{296}$ \\
\hline$a, b, c(\AA)$ & $\begin{array}{l}\frac{19.5436(12),}{16.2686(10),} \\
\underline{25.2292(14)} \\
\end{array}$ & $\begin{array}{l}\frac{10.9001(7)}{16.3943(10)}, \\
\underline{21.6403(14)} \\
\end{array}$ & $\begin{array}{l}\frac{11.6701(9),}{29.6716(19),} \\
\underline{13.7738(10)} \\
\end{array}$ \\
\hline$\alpha, b, \gamma\left(^{\circ}\right)$ & $90,92.134(2), 90$ & $90,98.664(2), 90$ & $90,93.446(2), 90$ \\
\hline$V\left(\AA^{3}\right)$ & $8016.0(8)$ & $3823.0(4)$ & $4760.8(6)$ \\
\hline$Z$ & $\underline{4}$ & $\underline{4}$ & $\underline{4}$ \\
\hline Radiation type & Mo $K \alpha$ & Mo $K \alpha$ & Mo $K \alpha$ \\
\hline$\mu\left(\mathrm{mm}^{-1}\right)$ & $\underline{0.76}$ & $\underline{0.65}$ & $\underline{0.58}$ \\
\hline Crystal size $(\mathrm{mm})$ & $\underline{0.54} \times \underline{0.33} \times \underline{0.07}$ & $\underline{0.35} \times \underline{0.20} \times \underline{0.10}$ & $\underline{0.38} \times \underline{0.18} \times \underline{0.04}$ \\
\hline Diffractometer & $\underline{\mathrm{CCD}}$ & $\underline{\mathrm{CCD}}$ & $\frac{\mathrm{CCD}}{\text { diffractometer }}$ \\
\hline $\begin{array}{l}\text { Absorption } \\
\text { correction }\end{array}$ & Multi-scan & Multi-scan & $\frac{\text { Multi-scan }}{S A D A B S}$ \\
\hline$T_{\min }, T_{\max }$ & $\underline{0.686}, \underline{0.950}$ & $\underline{0.805}, \underline{0.938}$ & $\underline{0.810}, \underline{0.976}$ \\
\hline $\begin{array}{c}\text { No. of measured, } \\
\text { independent and } \\
\text { observed }[I>2 \sigma(I)] \\
\text { reflections }\end{array}$ & $\frac{60908}{\underline{8422}}, \frac{12846}{2}$ & $\underline{47856}, \underline{6063}, \underline{4182}$ & $\underline{17303}, \underline{7347}, \underline{4976}$ \\
\hline$R_{\text {int }}$ & $\underline{0.084}$ & $\underline{0.100}$ & $\underline{0.040}$ \\
\hline$(\sin \vartheta / \lambda)_{\max }\left(\AA^{-1}\right)$ & 0.578 & 0.575 & 0.575 \\
\hline $\begin{array}{c}R\left[F^{2}>2 \sigma\left(F^{2}\right)\right] \\
w R\left(F^{2}\right), S\end{array}$ & $\underline{0.148}, \underline{0.325}, \underline{1.88}$ & $\underline{0.050}, \underline{0.142}, \underline{0.90}$ & $\underline{0.071}, \underline{0.184}, \underline{1.13}$ \\
\hline No. of reflections & 12846 & $\underline{6063}$ & 7347 \\
\hline No. of parameters & $\underline{874}$ & $\underline{467}$ & $\underline{558}$ \\
\hline No. of restraints & 0 & 0 & 0 \\
\hline $\mathrm{H}$-atom treatment & $\frac{\frac{\mathrm{H} \text { atoms treated }}{\text { by a mixture of }}}{\frac{\text { independent and }}{\text { constrained }}}$ & $\begin{array}{l}\begin{array}{c}\mathrm{H} \text {-atom } \\
\text { parameters } \\
\text { constrained }\end{array} \\
\end{array}$ & $\begin{array}{l}\frac{\mathrm{H} \text {-atom }}{\text { parameters }} \\
\text { constrained } \\
\end{array}$ \\
\hline$\Delta \rho_{\max }, \Delta \rho_{\min }\left(\mathrm{e} \AA^{-3}\right)$ & $\underline{1.93},-1.20$ & $\underline{0.60}, \underline{-0.68}$ & $\underline{0.49}, \underline{-0.90}$ \\
\hline $\begin{array}{c}\text { Absolute structure } \\
\text { parameter }\end{array}$ & N/A & $\mathrm{N} / \mathrm{A}$ & N/A \\
\hline
\end{tabular}




\section{Crystal structure of $10 a$}

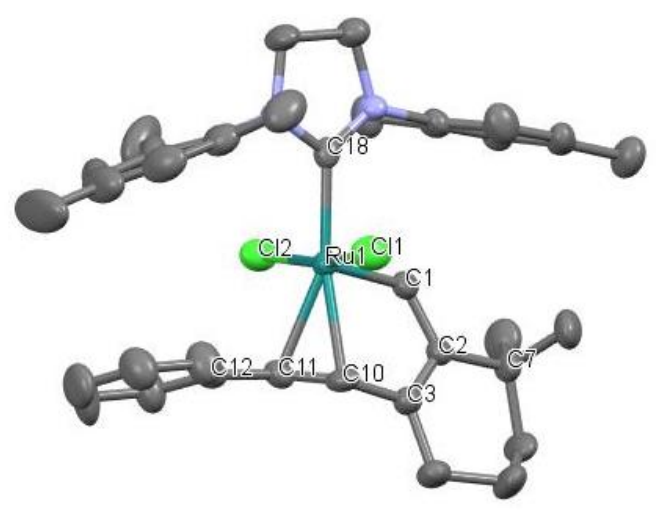

Fig. S16. Molecular Structure of 10a (CCDC 2018298) showing 50\% thermal probability ellipsoids. Hydrogen atoms are not shown for clarity. Selected bond distances [Å]: Ru1-Cl1 2.360(2), Ru1-Cl2 2.385(2), Ru1-C1 1.846(6), Ru1-C10 2.302(7), Ru1-C11 2.277(7), Ru1-C18 2.106(7), C1-C2 1.42 (1), C10-C11 1.37(1), C11-C12 1.46(1). Selected bond angles [ ${ }^{\circ}$ ]: Cl1-Ru1-Cl2 166.59(7), Cl1-Ru1-C1 101.2(2), Cl1-Ru1C18 87.2(2), C1-Ru1-C10 78.8(2), Ru1-C1-C2 123.7(5), C10-Ru1-C11 34.7(3), Ru1-C11-C10 73.7(4), Ru1C10-C11 71.6(4), Ru1-C11-C12 109.3(5), C1-C2-C7 122.1 (6).

\section{Crystal structure of $10 \mathrm{c}$}

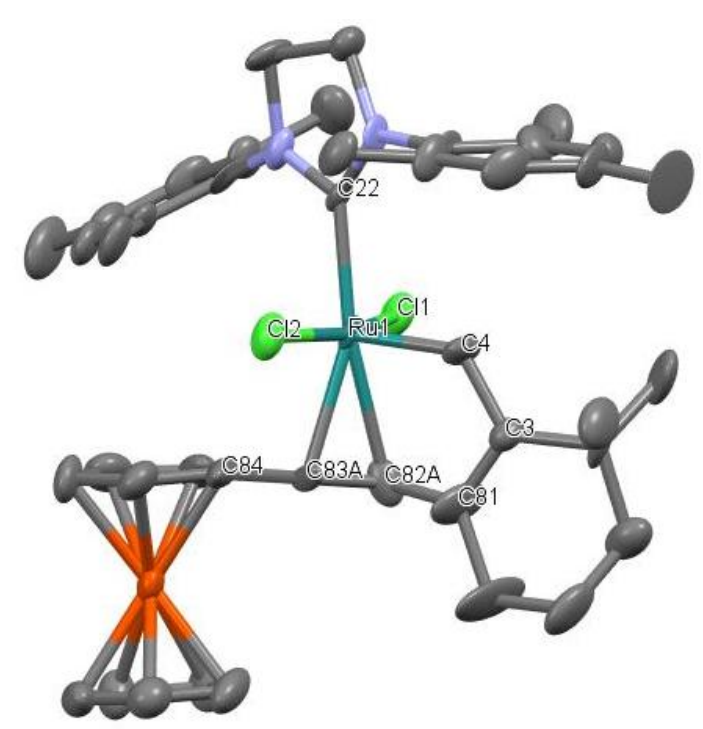

Fig. S17. Molecular Structure of 10c (CCDC 2021743) showing 50\% thermal probability ellipsoids. Hydrogen atoms are not shown for clarity. Selected bond distances [Å]: Ru1-Cl1 2.360(5), Ru1-Cl2 2.372(5), Ru1-C4 1.86(1), Ru1-C22 2.08(1), Ru1-C82A 2.35(3), Ru1-C83A 2.25(3), C82A-C83A 1.32(6), C83AC84 1.65(4), C3-C4 1.43(2). Selected bond angles [']: Cl1-Ru1-Cl2 165.7(1), Cl1-Ru1-C4 97.6(5), Cl1-Ru1C22 87.3(4), C4-Ru1-C82A 76.0(9), Ru1-C4-C3 124(1) C82A-Ru1-C83A 33(1), Ru1-C82A-C83A 69(2), Ru1C83A-C82A 77(2), Ru1-C83A-C84 109.3(5), C4-C3-C5 123(1). 


\section{Crystal structure of $10 \mathrm{~d}$}

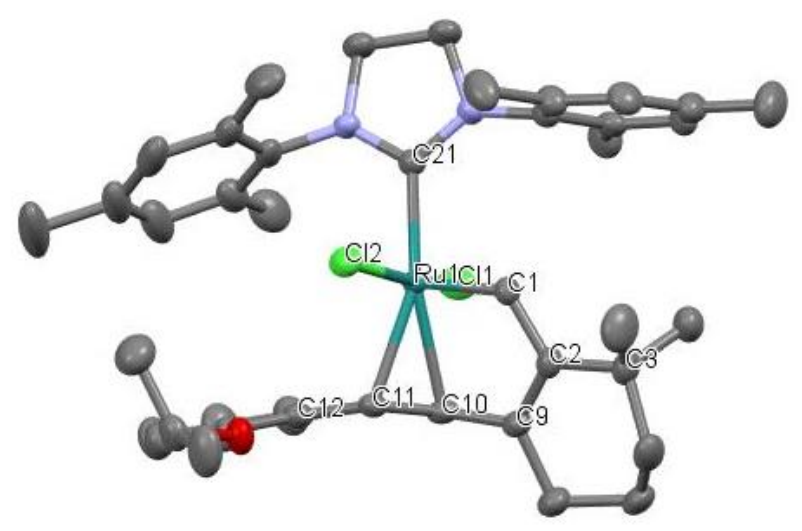

Fig. S18. Molecular Structure of 10d (CCDC 2018914) showing 50\% thermal probability ellipsoids. Hydrogen atoms are not shown for clarity. Selected bond distances [Å]: Ru1-Cl1 2.374(9), Ru1-Cl2 2.368(7), Ru1-C1 1.840(3), Ru1-C10 2.307(3), Ru1-C11 2.282(3), Ru1-C21 2.804(3), C1-C2 1.436(4), C10C11 1.379(3), C11-C12 1.474(4). Selected bond angles [ [ ${ }^{\circ}: \mathrm{Cl} 1-\mathrm{Ru} 1-\mathrm{Cl} 2$ 164.90(3), Cl1-Ru1-C1 102.02(9), Cl1-Ru1-C21 83.76(8), C1-Ru1-C10 78.4(1), Ru1-C1-C2 123.3(2) C10-Ru1-C11 35.0(1), Ru1-C11-C10 73.5(2), Ru1-C10-C11 71.5(2), Ru1-C11-C12 108.7(2), C1-C2-C3 121.6(2).

\section{Crystal structure of 10 l}

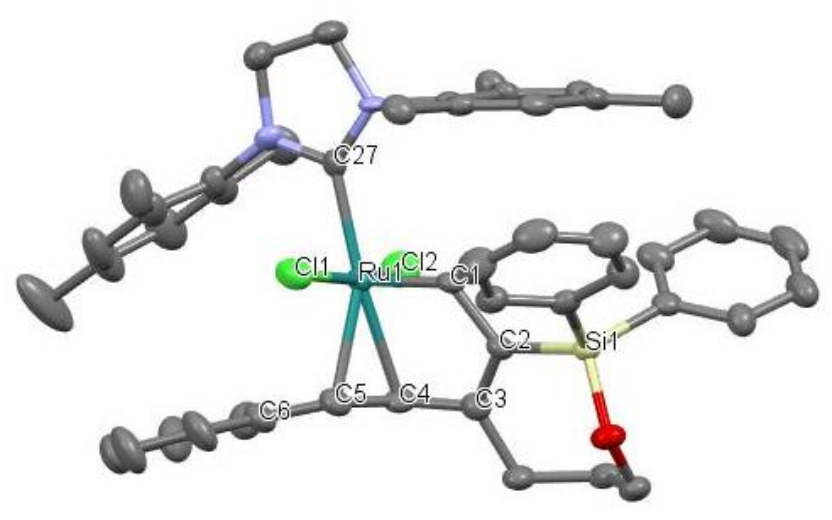

Fig. S19. Molecular Structure of 10I (CCDC 2018302) showing 50\% thermal probability ellipsoids. Hydrogen atoms are not shown for clarity. Selected bond distances [Å]: Ru1-Cl1 2.395(2), Ru1-Cl2 2.354(2), Ru1-C1 1.848(5), Ru1-C4 2.295(7), Ru1-C5 2.225(7), Ru1-C27 2.109(6), C1-C2 1.446(9), C4-C5 1.36(1), C5-C6 1.48(1). Selected bond angles [ ${ }^{\circ}$ ]: $\mathrm{Cl} 1-\mathrm{Ru1}-\mathrm{Cl} 2$ 166.93(7), Cl1-Ru1-C1 89.4(2), Cl1-Ru1-C27 82.0(2), C1-Ru1C4 78.7(4), Ru1-C1-C2 123.4(5), C4-Ru1-C5 35.0(3), Ru1-C5-C4 75.3(4), Ru1-C4-C5 69.7(4), Ru1-C5-C6 107.8(5), C1-C2-Si1 120.8(4). 


\section{Crystal structure of $10 q$}

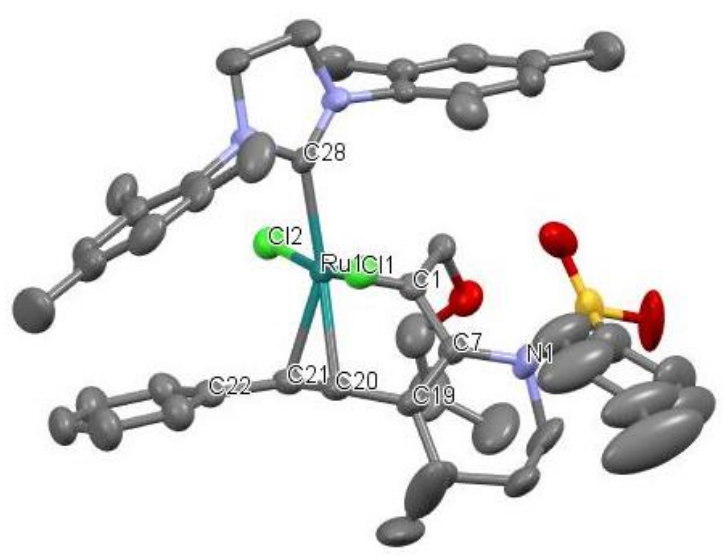

Fig. S20. Molecular Structure of $\mathbf{1 0 q}$ (CCDC 2018588) showing 50\% thermal probability ellipsoids. Hydrogen atoms are not shown for clarity. Selected bond distances [Å]: Ru1-Cl1 2.380(1), Ru1-Cl2 1.866(1), Ru1-C1 1.866(4), Ru1-C20 2.234(4), Ru1-C21 2.243(4), Ru1-C28 2.119(4), C1-C7 1.467(5), C20C21 1.379(5), C21-C22 1.484(5). Selected bond angles [ ${ }^{\circ}$ ]: Cl1-Ru1-Cl2 166.16(4), Cl1-Ru1-C1 96.1(1), Cl1Ru1-C28 85.1(1), C1-Ru1-C20 79.5(4), Ru1-C1-C7 117.2(3), C20-Ru1-C21 35.9(1), Ru1-C20-C21 72.4(2), Ru1-C21-C20 71.7(2), Ru1-C21-C22 112.8(2), C1-C7-N1 122.7(3).

\section{Crystal structure of $10 \mathrm{r}$}

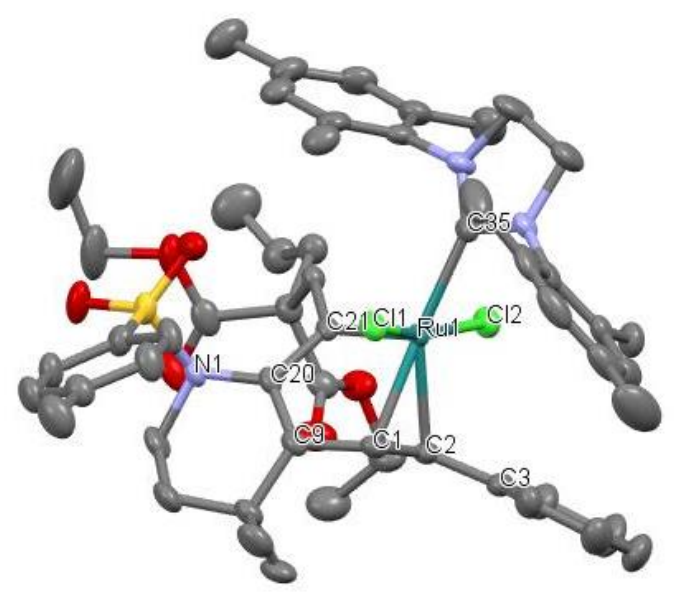

Fig. S21. Molecular Structure of 10r (CCDC 2018303) showing 50\% thermal probability ellipsoids. Hydrogen atoms are not shown for clarity. Selected bond distances [Å]: Ru1-Cl1 2.3772(7), Ru1-Cl2 2.4161(1), Ru1-C1 2.219(2), Ru1-C2 2.230(3), Ru1-C21 1.877(4), Ru1-C35 2.152(2), C1-C2 1.388(4), C2-C3 1.479(4), C20-C21 1.462(3). Selected bond angles [ ${ }^{\circ}$ ]: Cl1-Ru1-Cl2 165.59(3), Cl1-Ru1-C21 97.11(8), Cl1Ru1-C35 84.92(8), C1-Ru1-C21 79.5(1), C1-Ru1-C2 36.4(1), Ru1-C1-C2 72.2(2), Ru1-C2-C1 71.4(2), Ru1-C2C3 113.1 (2), Ru1-C21-C20 116.4(2), C21-C20-N1 120.9(2). 


\section{Crystal structure of $10 r^{\prime}$}

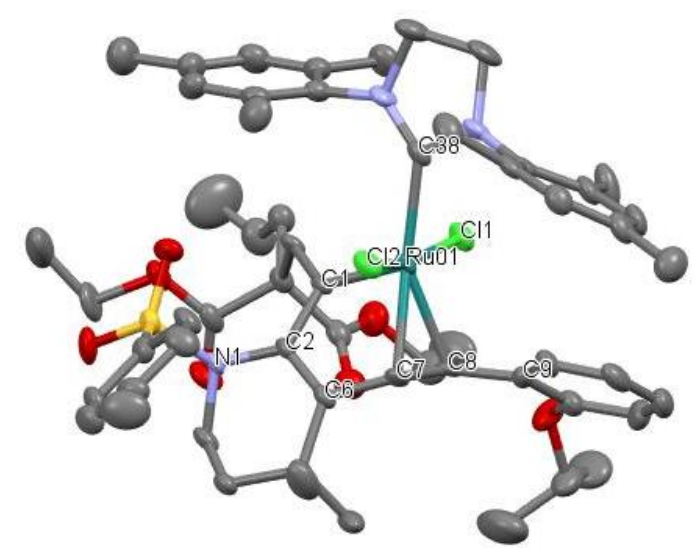

Fig. S22. Molecular Structure of 10r' (CCDC 2018594) showing 50\% thermal probability ellipsoids. Hydrogen atoms are not shown for clarity. Selected bond distances [Å]: Ru01-Cl1 2.410(1), Ru01-Cl2 2.369(1), Ru01-C1 1.876(6), Ru01-C7 2.223(7), Ru01-C8 2.229(8), Ru01-C38 2.159(7), C1-C2 1.461(9), C7C8 1.399(9), C8-C9 1.48(1). Selected bond angles [']: Cl1-Ru01-Cl2 165.07(6), Cl1-Ru01-C1 99.1(2), Cl1Ru01-C38 82.4(2), C1-Ru01-C7 79.4(3), C7-Ru01-C8 36.6(2), Ru01-C1-C2 117.0(4), Ru01-C7-C8 71.9(4), Ru01-C8-C7 71.5(4), Ru01-C8-C9 112.3(5), C1-C2-N1 121.7(5).

\section{Crystal structure of $10 \mathrm{~s}$}

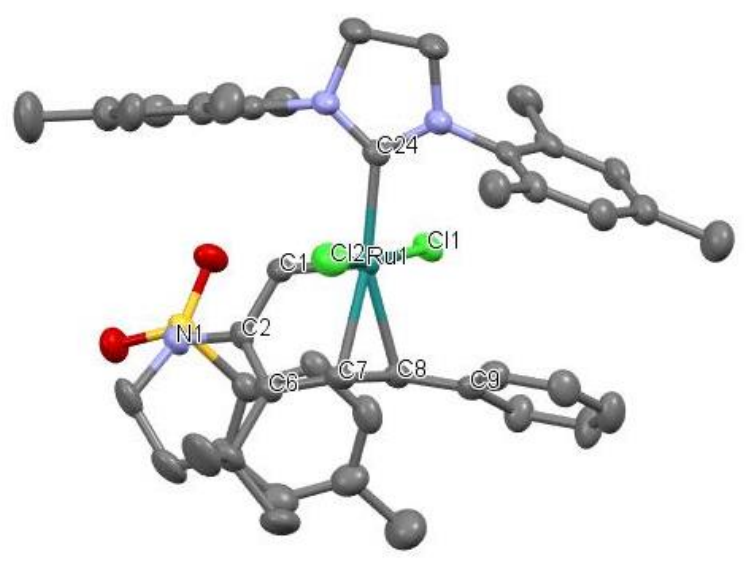

Fig. S23. Molecular Structure of 10s (CCDC 2018596) showing 50\% thermal probability ellipsoids. Hydrogen atoms are not shown for clarity. Selected bond distances [Å]: Ru1-Cl1 2.392(1), Ru1-Cl2 2.351(1), Ru1-C1 1.844(5), Ru1-C7 2.263(4), Ru1-C8 2.2250(4), Ru1-C24 2.103(4), C1-C2 1.431(6), C7-C8 1.363(6), C8-C9 1.477(6). Selected bond angles [ ${ }^{\circ}$ ]: Cl1-Ru1-Cl2 165.30(4), Cl1-Ru1-C1 92.4(1), Cl1-Ru1-C24 86.4(1), C1-Ru1-C7 79.0(2), C7-Ru1-C8 35.2(2), Ru1-C1-C2 121.6(3), Ru1-C7-C8 71.9(2), Ru1-C8-C7 73.0(2), Ru1-C8-C9 109.2(3), C1-C2-N1 120.6(4). 


\section{Crystal structure of $13 a$}

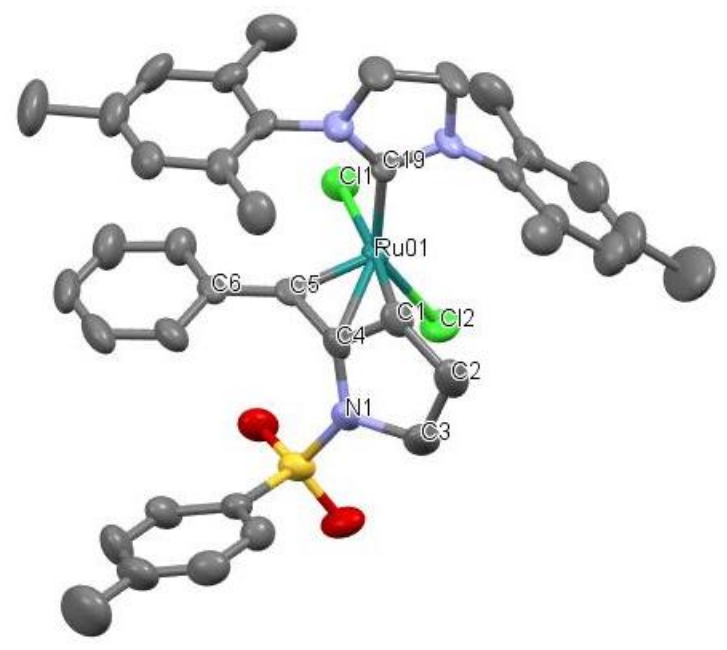

Fig. S24. Molecular Structure of 13a (CCDC 2018283) showing 50\% thermal probability ellipsoids. Hydrogen atoms are not shown for clarity. Selected bond distances [Å]: Ru1-Cl1 2.380(4), Ru1-Cl2 2.347(4), Ru1-C1 2.27(1), Ru1-C4 2.18(1), Ru1-C5 1.84(1), Ru1-C19 2.04(), C1-C4 1.39(2), C4-C5 1.44(2), C4-N1 1.41(2), C5-C6 1.47(2) Selected bond angles [ ${ }^{\circ}$ ]: Cl1-Ru1-Cl2 87.6(1), Cl1-Ru1-C5 103.1(4), Cl1-Ru1C19 92.7(4), C4-Ru1-C5 40.9(5), C4-Ru1-C1 36.3(5), Ru1-C1-C4 71.4(8), Ru1-C5-C4 82.6(8), Ru1-C5-C6 144(2), C1-C2-C3 105(1), N1-C4-C1 109(1).

\section{Crystal structure of $13 b$}

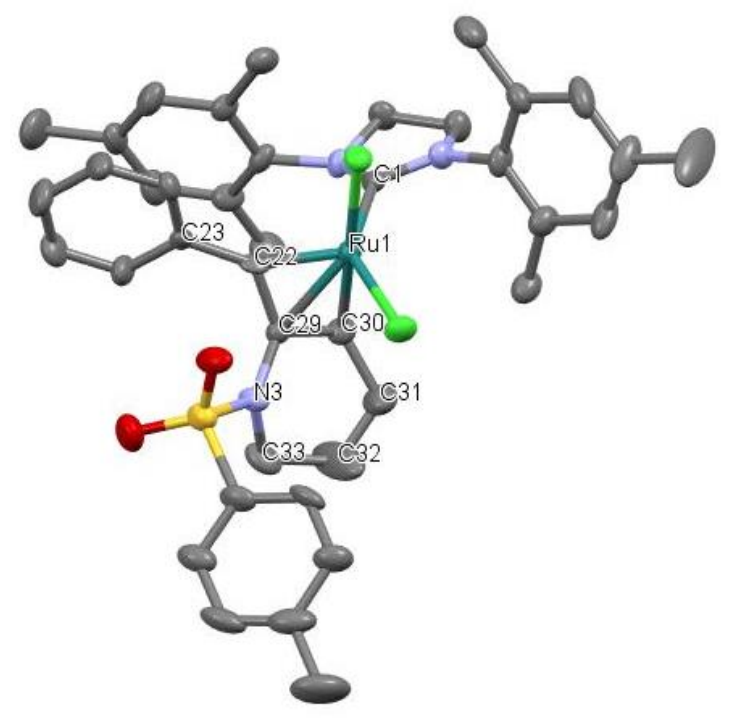

Fig. S25. Molecular Structure of 13b (CCDC 2018831) showing 50\% thermal probability ellipsoids. Hydrogen atoms are not shown for clarity. Selected bond distances [Å]: Ru1-Cl1 2.380(2), Ru1-Cl2 2.356(2), Ru1-C1 2.024(7), Ru1-C22 1.838(7), Ru1-C29 2.219(7), Ru1-C30 2.282(7), C22-C29 1.44(1), C29- 
C30 1.395(9), C22-C23 1.459(9), C29-N3 1.404(8). Selected bond angles [ ${ }^{\circ}$ ]: Cl1-Ru1-Cl2 86.97(6), Cl1-Ru1C1 97.0(3), Cl1-Ru1-C22 108.1(2), C22-Ru1-C29 40.3(3), C29-Ru1-C30 36.1(2), Ru1-C22-C29 84.2(4), Ru1C30-C29 69.5(4), Ru1-C22-C23 143.9(5), C30-C31-C32 115.8(9), N3-C29-C30 124.4(6).

\section{Crystal structure of $13 \mathrm{~g}$}

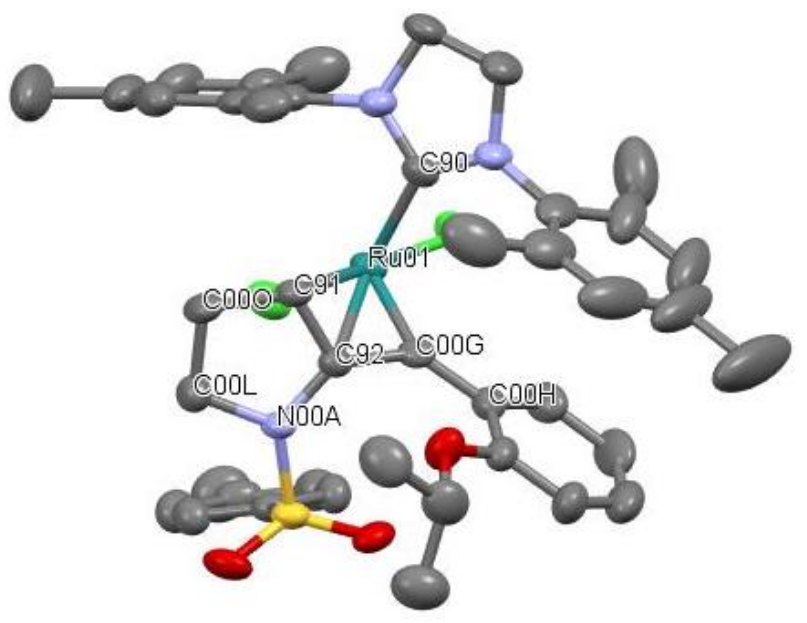

Fig. S26. Molecular structure of 13g (CCDC 2018917) showing 50\% thermal probability ellipsoids. Hydrogen atoms are not shown for clarity. Selected bond distances [Å]: Ru01-Cl03 2.349(1), Ru01-Cl04 2.396(1), Ru01-C90 2.039(7), Ru01-C91 2.237(4), Ru01-C92 2.169(3), Ru01-C00G 1.842(3), C91-C92 1.400(5), C92-COOG 1.436(5), COOG-COOH 1.451(5), C92-NOOA 1.415(4). Selected bond angles [ ${ }^{\circ}$ ]: Cl03Ru01-Cl04 88.28(4), Cl04-Ru01-C00G 105.2(1), Cl04-Ru1-C90 86.98(9), C91-Ru01-C92 37.0(1), C92-Ru01C00G 40.9(1), Ru01-C91-C92 68.9(2), Ru01-C00G-C92 81.9(2), Ru01-C00G-C00H 132.9(3), C91-C00O-C00L 104.3(3), NOOA-C92-C91 108.9(3).

\section{Crystal structure of $13 h^{\prime}$}

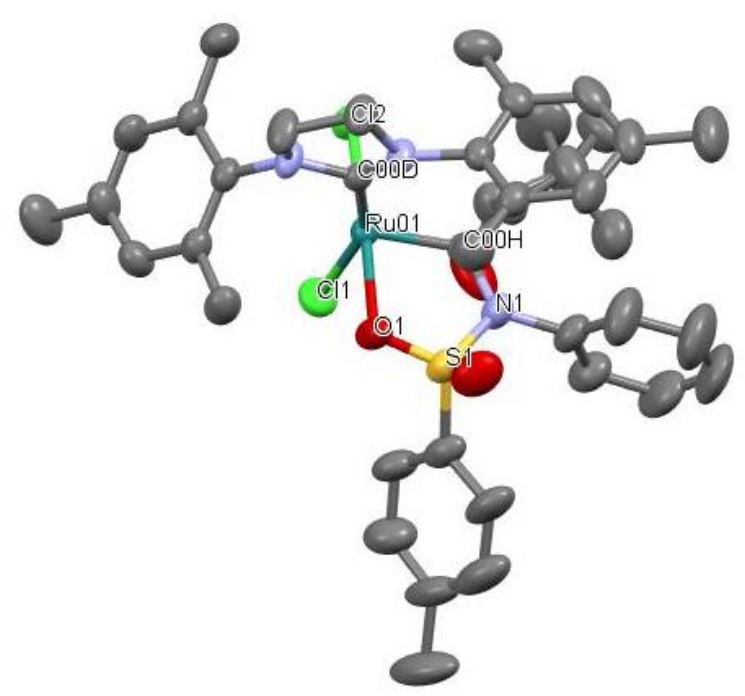


Fig. S27. Molecular Structure of $\mathbf{1 3 h}^{\prime}$ (CCDC 2021918) showing $50 \%$ thermal probability ellipsoids. Hydrogen atoms are not shown for clarity. Selected bond distances [Å]: Ru01-Cl1 2.415(1), Ru01-Cl2 2.343(1), Ru01-COOD 2.019(3), Ru01-O1 2.172(3), Ru01-COOH 1.848(4), Ru01-C00G 1.842(3), S1-O1 1.454(5). Selected bond angles [ ${ }^{\circ}$ ]: Cl1-Ru01-Cl2 89.85(4), Cl1-Ru01-COOD 154.8(1), Cl1-Ru1-COOH 99.3(1), O1-Ru01-C00H 81.4(1), Ru01-O1-S1 117.3(2), O1-S1-N1 103.0(2), Ru01-C0OH-N1 120.8(3).

\section{Crystal structure of 13p'}

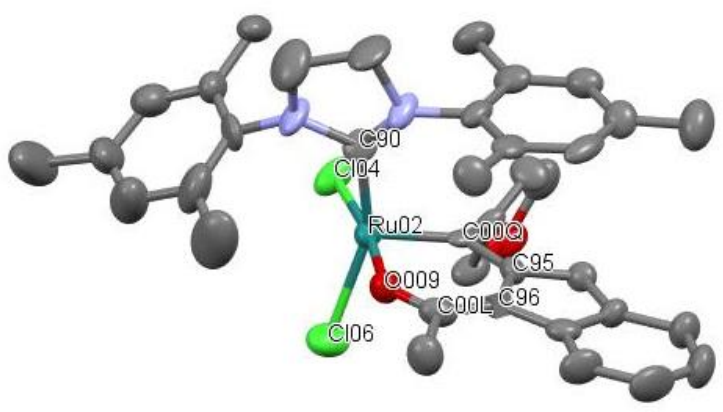

Fig. S28. Molecular Structure of 13p' (CCDC 2019504) showing 50\% thermal probability ellipsoids. Hydrogen atoms are not shown for clarity. Selected bond distances [Å]: Ru02-Cl04 2.363(3), Ru01-Cl06 2.374(4), Ru02-C90 2.02(2), Ru02-O009 2.086(8), Ru02-C00Q 1.86(1), C00Q-C95 1.44(2), O009-C00L 1.24(2) Selected bond angles [ ${ }^{\circ}$ ]: Cl04-Ru02-Cl06 90.5(1), Cl04-Ru02-C90 92.8(4), Cl04-Ru02-C00Q 98.6(3), C00Q-Ru02-O009 88.6(4), Ru02-O009-C00L 124(1), Ru02-C00Q-C95 125.0(8), O009-C00L-C96 123(1).

\section{Crystal structure of $13 q^{\prime}$}

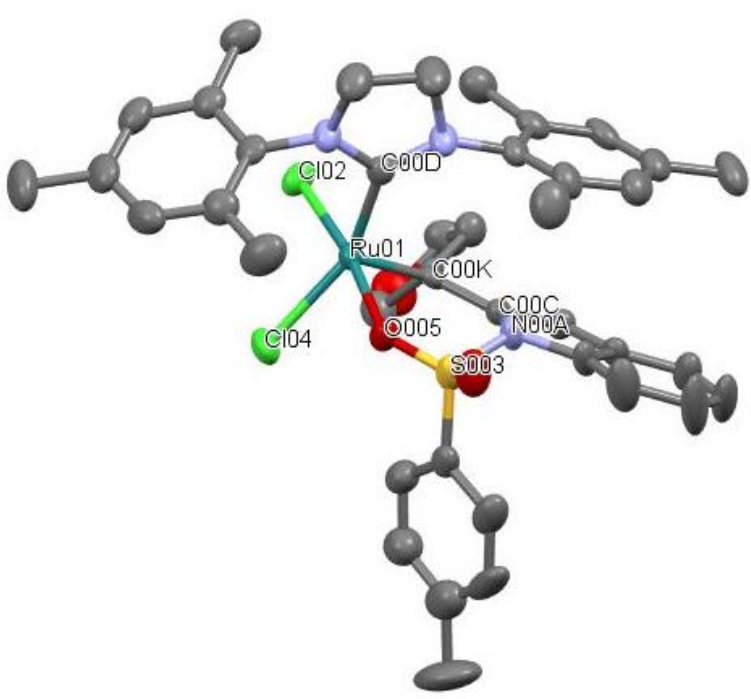


Fig. S29. Molecular Structure of 13q' (CCDC 2019503) showing 50\% thermal probability ellipsoids. Hydrogen atoms are not shown for clarity. Selected bond distances [Å]: Ru02-Cl02 2.328(1), Ru01-Cl04 2.360(2), Ru01-C00D 2.034(4), Ru01-C00K 1.856(5), Ru01-O005 2.170(3), S003-O005 1.439(4), CO0C-C00K 1.455(6) Selected bond angles [ ${ }^{\circ}$ ]: Cl02-Ru01-Cl04 90.33(5), Cl02-Ru01-COOD 90.5(1), Cl02-Ru01-COOK 96.9(1), C00K-Ru01-O005 88.8(2), Ru01-C00K-C00C 127.4(3), Ru01-O005-S003 135.4(2), O005-S003-N00A 105.4(2).

\section{Crystal structure of $13 \mathrm{v}$}

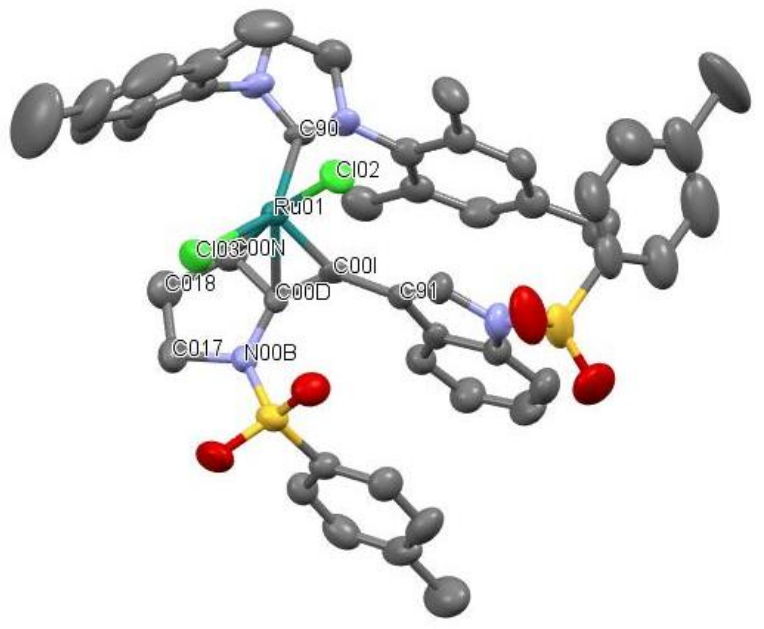

Fig. S30. Molecular Structure of 13v (CCDC 2019480) showing 50\% thermal probability ellipsoids. Hydrogen atoms are not shown for clarity. Selected bond distances [Å]: Ru01-C02 2.418(2), Ru01-Cl04 2.359(2), Ru01-C90 2.0416(6), Ru01-CO0D 2.173(7), Ru01-C00I 1.827(6), Ru01-C00N 2.250(7), COOD-C00I 1.400(5), COOD-COON 1.436(5), COOD-NOOB 1.409(9), C00I-C91 1.437(8). Selected bond angles [ ${ }^{\circ}$ ]: Cl02Ru01-Cl03 87.8(6), Cl02-Ru01-C90 95.8(2), Cl02-Ru01-C00I 99.1(2), COOI-Ru01-COOD 40.8(2), COOD-Ru01C00I 37.0(2), Ru01-COOI-COOD 82.7(4), Ru01-CO0N-CO0D 68.5(4), Ru01-C00I-C91 143.8(5), CO0N-C018C017 106.0(7), NOOB-COOD-COON 110.0(5). 


\section{References}

(1) Wang, K. P.; Yun, S. Y.; Lee, D.; Wink, D. J. Structure and reactivity of alkyne-chelated ruthenium alkylidene complexes. J. Am. Chem. Soc. 2009, 131, 15114-15115.

(2) Mitsunobu, O. The use of diethyl azodicarboxylate and triphenylphosphine in synthesis and transformation of natural products. Synthesis 1981, 1, 1-28.

(3) Zhang, Y.; Hsung, R. P.; Tracey, M. R.; Kurtz, K. C.; Vera, E. L. Copper sulfate-pentahydrate-1,10phenanthroline catalyzed amidations of alkynyl bromides. Synthesis of heteroaromatic amine substituted ynamides. Org. Lett. 2004, 6, 1151-1154.

(4) Sonogashira, K. Development of Pd-Cu catalyzed cross-coupling of terminal acetylenes with $\mathrm{sp}^{2}$ carbon halides. J. Organomet. Chem. 2002, 653, 46-49.

(5) Müller, S.; Liepold, B.; Roth, G. J.; Bestmann, H. J. An improved one-pot procedure for the synthesis of alkynes from aldehydes. Synlett 1996, 1996, 521-522.

(6) Cera, G.; Lanzi, M.; Bigi, F.; Maggi, R.; Malacria, M.; Maestri, G. Bi-directional alkyne tandem isomerization via $\mathrm{Pd}(0) /$ carboxylic acid joint catalysis: expedient access to 1, 3-dienes. Chem. Commun. 2018, 54, 14021-14024

(7) Casey, C. P.; Dzwiniel, T. L.; Kraft, S.; Guzei, I. A. Synthesis of cyclic cis-enediynes from manganese carbyne complexes and $\alpha$, $\omega$-diynes. Organometallics 2003, 22, 3915-3920.

(8) Hosseini, A.; Seidel, D.; Miska, A.; Schreiner, P. R. Fluoride-assisted activation of calcium carbide: A simple method for the ethynylation of aldehydes and ketones. Org. Lett. 2015, 17, 2808-2811.

(9) Díaz, D. D.; Martín, V. S. $\mathrm{CO}_{2}(\mathrm{CO})_{8}$-Assisted synthesis of propargylic unsymmetrical ethers by reaction of alcohols with propargylic alcohols. Tetrahedron Lett. 2000, 41, 9993-9996.

(10) Maifeld, S. V.; Miller, R. L.; Lee, D. Group-selective ring-closing enyne metathesis: Concentrationdependent selectivity profile of alkynylsilyloxy-tethered dienynes. J. Am. Chem. Soc. 2004, 126, $12228-12229$.

(11) Lin, M.; Kang, G. Y.; Guo, Y. A.; Yu, Z. X. Asymmetric Rh (I)-catalyzed intramolecular [3+2] cycloaddition of 1-yne-vinylcyclopropanes for bicyclo [3.3. 0] compounds with a chiral quaternary carbon stereocenter and density functional theory study of the origins of enantioselectivity. J. Am. Chem. Soc. 2012, 134, 398-405.

(12) Corey, E. J.; Fuchs, P. L. A synthetic method for formyl $\rightarrow$ ethynyl conversion. Tetrahedron Lett. 1972, 36, 3769-3772. 
(13) Bernar, I.; Blanco-Ania, D.; Stok, S. J.; Sotorríos, L.; Gómez-Bengoa, E.; Rutjes, F. P. Synthesis of 3Amino-1-benzothiophene-1, 1-diones by Alkyne Directed Hydroarylation and 1/N $\rightarrow 3 / \mathrm{C}$-Sulfonyl Migration. Eur. J. Org. Chem. 2018, 2018, 5435-5444.

(14) Ratovelomanana, V.; Rollin, Y.; Gébéhenne, C.; Gosmini, C.; Périchon, J. DBU/DMSO promoted dehydrobromination of 1, 1-dibromoolefins. A general synthesis of 1-bromoaromatic alkynes under mild conditions. Tetrahedron Lett. 1994, 35, 4777-4780.

(15) Ritter, T.; Hejl, A.; Wenzel, A. G.; Funk, T. W.; Grubbs, R. H. A standard system of characterization for olefin metathesis catalysts. Organometallics 2006, 25, 5740-5745.

(16) Song, K.; Kim, K.; Hong, D.; Kim, J.; Heo, C. E.; Kim, H. I.; Hong, S. H. Highly active ruthenium metathesis catalysts enabling ring-opening metathesis polymerization of cyclopentadiene at low temperatures. Nat. Commun. 2019, 10, 1-9. 